

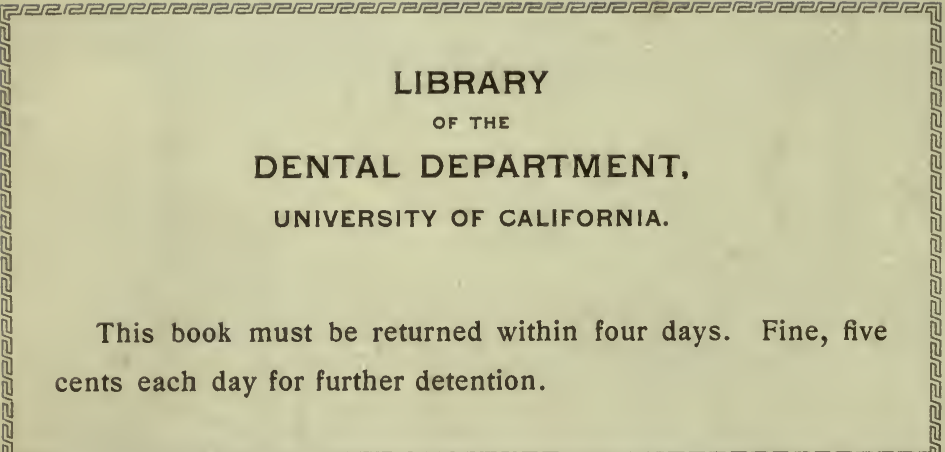

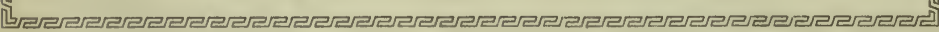



Digitized by the Internet Archive in 2007 with funding from Microsoft Corporation 



\section{A TEXT-BOOK}

OF

\section{Physiological Chenistri}

FOR

\section{STUDENTS OF MEDICINE AND PHYSICIANS.}

BY

CHARLES E. SIMON, B. A., M. D.,

Professor of Clinical Pathology at the Baltimore Medical College.

THIRD EDITION, THOROUGHLY REVISED.

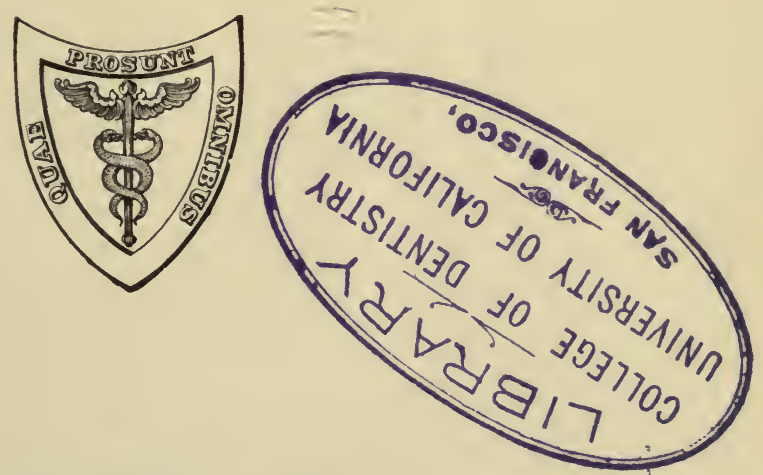

LEA BROTHERS \& CO., PHILA DELPHIA AND NEW YORK. 
Entered according to Act of Congress, in the year 1907, by LEA BROTHERS \& CO.,

In the Office of the Librarian of Congress, at Washington. All rights reserved. 


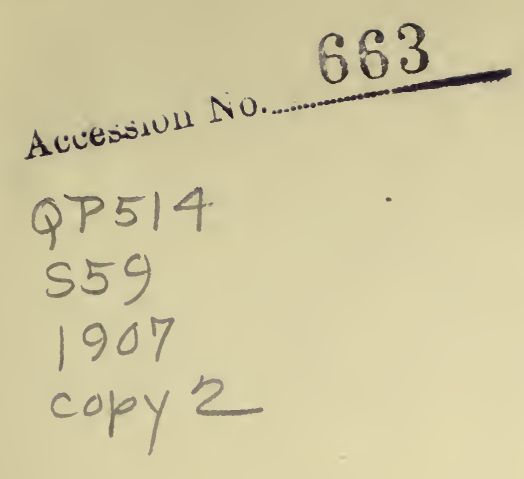

\section{PREFACE TO THE THIRD EDITION.}

Is preparing the third edition of the Physiological Chemistry the writer has attempted to bring the volume up to date without increasing its size. This has been somewhat difficult in view of the large amount of literature which had to be considered. It has been accomplished, however, by eliminating all unnecessary theoretical discussions and by presenting only such fundamental facts as seemed essential to a proper understanding of the subject matter. The aim of the writer has been, as heretofore, to present a text-book for the use of medical students and physicians who wish to keep abreast of one of the most important branches of modern medicine, and also a guide to practical work in the laboratory.

\section{Charles E. Simon.}

1302 Madison Avenue, Baltimore, Mp.,

1907.

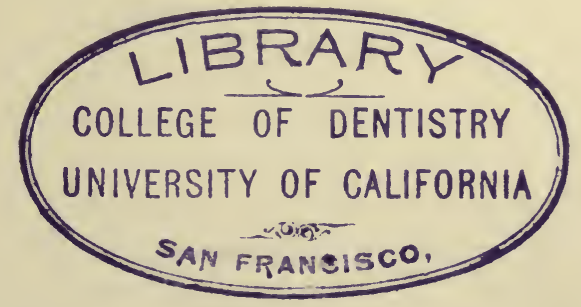





\section{PREFACE TO THE FIRST EDITION.}

In preparing the present volume on Physiological Chemistry I have endeavored to adapt the book as much as possible to the wants of the medical student, and the physician who in the past has been unable to devote the attention to the subject which it merits. The work is intended as a text-book for the lecture-room and as a guide in the physiological-chemical laboratory. Theoretical discussions have been avoided as far as possible, and it has been my aim to present ascertained facts as concisely as appeared consistent with the importance of the problems under consideration. The various chemical methods have been described with all due regard to necessary detail, but with the supposition that the student's course in physiological chemistry has been preceded by a course in general chemistry, such as is offered now in the majority of our medical colleges.

The subject-matter has been arranged in such a manner that in the first section of the work a general survey is given of the origin and the chemical nature of the three great classes of food-stuffs, and also of the most important products of their decomposition; the second section deals essentially with the processes of digestion, resorption, and excretion; while the third portion of the work is devoted to the chemical study of the elementary tissues and the various organs of the animal body, the specific products of their activity and their relation to physiological function. This arrangement has suggested itself to me as the most satisfactory for purposes of teaching.

References to literature have been omitted, as they did not appear to be necessary in a work which is intended primarily for the student. The names of the grand-masters of physiological chem- 
istry and physiology, however, have been introduced into the text as a matter of historical interest.

To my friend, Mr. Charles Glaser, of Baltimore, I wish to express my sincere thanks for many valuable suggestions and aid in the revision of the manuscript. To Messrs. Lea Bros. \& Co. I am indebted for many acts of courtesy.

1302 Madison Avenue,

BALTimore, MD., 1901. 


\section{CONTENTS.}

\section{CHAPTER I.}

INTRODUCTION.

General chemical composition of living matter . . . . . . . . . . 17

Forces at work in the living world . . . . . . . . . . . . . . . 17

Character of chemical changes .............. . . 18

Synthetic processes in plants . . . . . . . . . . . . . . 18

Oxidations and hydrations in the animal body . . . . . . . . . . 19

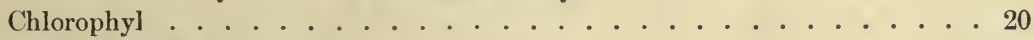

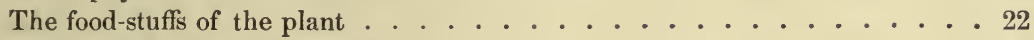

Synthesis of the carbohydrates. . . . . . . . . . . . 23

Glucosides................... . . . 24

Mannides ......................... 25

Synthesis of the fats .................. . . . 25

Synthesis of the albumins . . . . . . . . . . . . . 26

CHAPTER II.

THE ALBUMINS.

Elementary composition . . . . . . . . . . . . . . 27 27

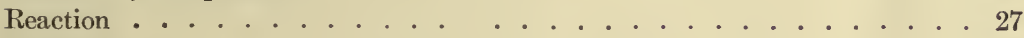

Solubility . . . . . . . . . . . . . . . . . . . . . . . 28

Crystallization . . . . . . . . . . . . . . . . . . 28

Diffusion . . . . . . . . . . . . . . . . . . . . . . . . 29

Behavior toward polarized light . . . . . . . . . . . . . 29

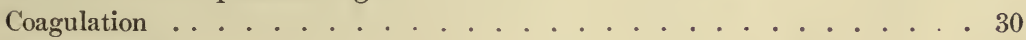

Denaturization ............................ 31

Behavior toward neutral salts .............. . . 31

Behavior toward alcohol . . . . . . . . . . . . . . . . 34

Special reactions of the albumins . . . . . . . . . . . . . . 34

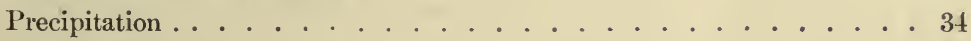

Color reactions .. . . . . . . . . . . . . . . . 35

Structural composition . . . . . . . . . . . . . . . . . . . . . . . . . . . . . . .

Molecular size . . . . . . . . . . . . . . . . . 45

Classification ....................... . . . . . 45

The Native Albunins . . . . . . . . . . . . . . . . . . . . . . . . . . . .

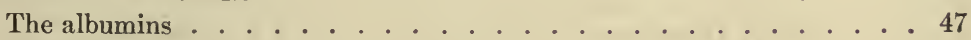

The globulins . . . . . . . . . . . . . . . . . . . . . . . . . 47

The gluco-albumins . . . . . . . . . . . . . . . . 48

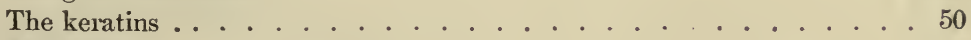

The histons ..................... . . 51 
The protanins

The nucleo-albumins . . . . . . . . . . . . . . . 53

The proteids ... . . . . . . . . . . . . . . . . . 55

The nucleoproteids . . . . . . . . . . . . . . . . . 55

The nucleins ... . . . . . . . . . . . . . . . . 56

The hæmoglobins ... . . . . . . . . . . . . . . . 57

The Albuminoids .. . . . . . . . . . . . . . . . . . 57

The Derived Albumins . . . . . . . . . . . . . . . . . . 59

Fibrin . . . . . . . . . . . . . . . . . 59

The coagulated albumins . . . . . . . . . . . . . . 59

The albuminates . . . . . . . . . . . . . . . . . . 59

The albumoses ... . . . . . . . . . . . . . . . . 59

The peptones . . . . . . . . . . . . . . . . . . 61

The protones . . . . . . . . . . . . . . . . . 62

CHAPTER III.

THE CARBOHYDRATES.

The Monosaccharides . . . . . . . . . . . . . . . . . . 64

The Hexoses . . . . . . . . . . . . . . . . . . . . . . . . . . 64

Glucose . . . . . . . . . . . . . . . . . 67

Lævulose . . . . . . . . . . . . . . . 67

Galactose . . . . . . . . . . . . 67

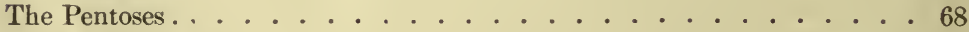

The Disaccharides . . . . . . . . . . . . . . . 68

Cane-sugar ... . . . . . . . . . . . . . . . . 69

Maltose . . . . . . . . . . . . . . . . . 70

Isomaltose . . . . . . . . . . . . . . . . . . . 70

Lactose . . . . . . . . . . . . . . . . . . . 70

The Polysaccharides . . . . . . . . . . . . . . 70

Starch . . . . . . . . . . . . . . . . . . 70

Inulin and lichenin ... . . . . . . . . . . . . . . . 72

Glycogen .. . . . . . . . . . . . . . . . 72

Dextrins . . . . . . . . . . . . . . . . . 72

Celluloses . . . . . . . . . . . . . . . . . . 72

The FAtS . . . . . . . . . . . . . . . . . 74

The Lectithins . . . . . . . . . . . . . . . . . . . 76

The Cholesterins . . . . . . . . . . . . . . . . 78

CHAPTER V.

THE CLEAVAGE PRODUCTS OF THE ALBUMINS.

The Mono-amino Acids . . . . . . . . . . . . . . 80

The Diamino Acids . . . . . . . . . . . . . 87

The Organic, Non-nitrogenous Derivatives. . . . . . . . 90 
The Nucleinic Acids . . . . . . . . . . . . . . . . . . . . . . . . . 94

The pyrimidin derivatives . . . . . . . . . . . . . . . . . . . 98

The purin derivatives................. 100

The Ureids . . . . . . . . . . . . . . . . . . 104

The Kreatins . . . . . . . . . . . . . . . . . . 107

The Ptomanss . . . . . . . . . . . . . . . . 108

\section{CHAPTER VI.}

THE FERMENTS.

General properties . . . . . . . . . . . . . . . 112

Chemical composition and general reactions . . . . . . . . 115

Mode of action . . . . . . . . . . . . . 116

Classification .................... 116

The proteolytic ferments ............. . 117

The amylolytic ferments .............. . . . 117

The inverting ferments . . . . . . . . . . . . 117

The lipolytic ferments. . . . . . . . . . . . . 117

The ureases ..................... 117

Ferments which transform amino-acids into amides . . . . . . 117

The histozyme of Schmiedeberg . . . . . . . . . 118

Ferments which cause the cleavage of glucosides . . . . . . 118

The nucleases . . . . . . . . . . . . 118

Ferments which split off carbon dioxide . . . . . . . . 118

The oxidation ferments . . . . . . . . . . . 118

The coagulating ferments . . . . . . . . . . . 119

Reducing ferments ............. . . 119

The kinases . . . . . . . . . . . . . . 119

CHAPTER VII.

THE DIGESTIVE FLUIDS.

The Saltva . . . . . . . . . . . . . . . 120

General characteristics . . . . . . . . . . 120

Amount . . . . . . . . . . . . . . . 120

Chemical composition................ 121

Ptyalin .................... 122

Mucin . . . . . . . . . . . . . . . 124

Sulphocyanides ................. 125

Nitrites . . . . . . . . . . . . . 125

Extractives . . . . . . . . . . . . . 126

Mineral constituents . . . . . . . . . . . 126

Gases .................. . . 126

The Gastric Jutce . . . . . . . . . . . . . . . . 126

General considerations . . . . . . . . . . 126

Amount . . . . . . . . . . . . . 126

Chemical composition . . . . . . . . . . . . 127

Acidity of the gastric juice. . . . . . . . . . . . 127 
Determination of the total acidity of the gastric contents -

Hydrochloric acid . . . . . . . . . . . . . . . . 129

Origin . . . . . . . . . . . . . . 129

Significance . . . . . . . . . . . . . . . . 130

Tests for hydrochloric acid . . . . . . . . . . 130

Estimation . . . . . . . . . . . . . 131

Lactic acid . . . . . . . . . . . . . . . . . 133

Tests... . . . . . . . . . . . . . 133

Estimation . . . . . . . . . . . . . . . 133

Acetic acid and butyric acid . . . . . . . . . . . . . . 134

Tests. . . . . . . . . . . . . . . . 134

Estimation . . . . . . . . . . . . . . . 134

The ferments of the gastric juice and their pro-enzymes . . . . . . 135

Pepsin . . . . . . . . . . . . . . . . . . 137

Isolation . . . . . . . . . . . . . . . . . 140

Estimation . . . . . . . . . . . . . . 140

Pepsinogen . . . . . . . . . . . . . . . . . . 141

Tests. . . . . . . . . . . . . . . . . . 141

Estimation . . . . . . . . . . . . . . . 141

Chymosin and chymosinogen . . . . . . . . . . . . 141

Tests... . . . . . . . . . . . 143

Isolation . . . . . . . . . . . . . . . . . 143

Estimation . . . . . . . . . . . . . . 143

Other constituents of the gastric juice . . . . . . . . . . . 144

Gases . . . . . . . . . . . . . . . . . 144

The Pancreatic Juice . . . . . . . . . . . . . . . . . . 144

General properties .. . . . . . . . . . . . . . 146

Amount . . . . . . . . . . . . . . . . . . 146

Specific gravity . . . . . . . . . . . . . . . . . 147

Chemical composition . . . . . . . . . . . . . . . 147

The ferments and their zymogens . . . . . . . . . . . . . . 147

Trypsin . . . . . . . . . . . . . . . . . 149

Test . . . . . . . . . . . . . . . 150

Isolation . . . . . . . . . . . . . . . . 150

The amylolytic ferment . . . . . . . . . . . . . . 151

Steapsin . . . . . . . . . . . . . . . . . . 151

Maltase... . . . . . . . . . . . . . . . . 152

Chymosin . . . . . . . . . . . . . . . . . . . 152

'Tif Secretion of the Glando of Brunner . . . . . . . . . . . . 152

The Enteric Jutce . . . . . . . . . . . . . . . . . . . 152

Enterokinase ................. . . . 154

Erepsin . . . . . . . . . . . . . . . . 155

Secretin and prosecretin . . . . . . . . . . . . 155

The BrLE... . . . . . . . . . . . . . . . . . . 155

Secretion .. . . . . . . . . . . . . . . . 156

Amount . . . . . . . . . . . . . . . . . . 157

General properties . . . . . . . . . . . . . . 157

Chemical composition ... . . . . . . . . . . . . 158 
The mucinous body of the bile.

The biliary acids . . . . . . . . . . . . . 159

Isolation . . . . . . . . . . . . . . . . 160

Tests. . . . . . . . . . . . . . 161

Pettenkofer's test . . . . . . . . . . 162

Physiological test . . . . . . . . . . . 162

Glycocholic acid . . . . . . . . . . . 162

Hyoglycocholic acid . . . . . . . . . . . 163

Taurocholic acid ... . . . . . . . . . 163

Hyotaurocholic acid . . . . . . . . . . . 164

Chenotaurocholic acid . . . . . . . . . . . 164

Cholalic acid . . . . . . . . . . . . . . 165

Hyocholalic acid and chenocholalic acid . . . . . . . 167

Choleic acid . . . . . . . . . . . . 167

Fellic acid . . . . . . . . . . . . 167

Lithofellic acid . . . . . . . . . . . . 167

Taurin ...................... 168

Isolation . . . . . . . . . . . . . 169

Glycocoll . . . . . . . . . . . . . 169

The bile-pigments . . . . . . . . . . . . 170

Bilirubin . . . . . . . . . . . . 170

Tests.................... 173

Isolation . . . . . . . . . . . . . . 174

Biliverdin . . . . . . . . . . . . 174

Isolation . . . . . . . . . . . . . 175

Biliprasin . . . . . . . . . . . . 175

Bilifuscin .................... 175

Bilicyanin . . . . . . . . . . . . 175

Bilipurpurin ................ . . 176

Choletelin ................. . . 176

Bilihumin ............... 176

Cholesterin ..................... 176

Tests.................... . . 177

Other organic constituents of the bile . . . . . . . . 178

Tbe biliary iron .................. 178

\section{CHAPTER VIII.}

THE PROCESSES OF DIGESTION AND RESORPTION.

The Digestion and Resorption of the Carbohydrates . . . . . . . 179

The Digestion of the Alibumins . . . . . . . . . . . . . 181

Digestion of the native albumins . . . . . . . . . . . . 181

Gastric digestion ................ . . 181

Tryptic digestion ... . . . . . . . . . . . 185

Digestion of the proteids . . . . . . . . . . . . 187

Digestion of the albuminoids . . . . . . . . . . . . . . 188

Resorption of the Products of Proteolytic Digestion . . . . . . 189

Erepsin . . . . . . . . . . . . . . . 189

The Dinfistion and Resorption of the Fats . . . . . . . . . . . . 192

Autolysis . . . . . . . . . . . . . . . . 193 


\section{CHAPTER IX.}

ANALYSIS OF THE PRODUCTS OF ALBUMINOUS DIGESTION.

The Products of Peptic Dighation

The Promucts of Tryptic Digestion

Reactions of the individual albumoses.

Hetero-albumose . . . . . . . . . . . . . . . . . . . . . . 197

Proto-albumose .................. . . 197

Gluco-albumose ................... . . . . 200

Deutero-fraction A . . . . . . . . . . . . . . 200

Deutero-fraction B . . . . . . . . . . . . . 200

Deutero-fraction C . . . . . . . . . . . . 201

The End-Products of Albuminous Digestion . . . . . . . . 201

Antipeptone fraction . . . . . . . . . . . 201

The mono-amido-acids .. . . . . . . . . . . . . 202

Leucin . . . . . . . . . . . . . . 202

Tyrosin ................... 203

Aspartic acid . . . . . . . . . . . . . . 205

Glutaminic acid . . . . . . . . . . . . 206

Glycocoll . . . . . . . . . . . . . . 207

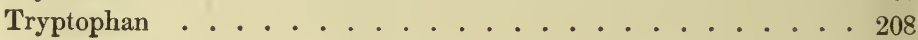

\section{CHAPTER X.}

BACTERIAL ACTION IN THE INTESTINAL TRACT.

Indol . . . . . . . . . . . . . . . . . 213

Skatol ........................ . . . . 214

Phenol . . . . . . . . . . . . . . . . . 215

Ptomains . . . . . . . . . . . . . . . . 216

Bacterial Decomposition of the Fats . . . . . . . . . . 216

Bacterial Decomposition of the Biliary Constituents. . . . . . 217

\section{CHAPTER XI.}

THE FECES.

Consistence and form . . . . . . . . . . . . . . 219

Amount . . . . . . . . . . . . . . . . . 219

Odor. . . . . . . . . . . . . . . . . . 219

Color . . . . . . . . . . . . . . . . 219

Macroscopical constituents . . . . . . . . . . . . 220

Microscopical constituents . . . . . . . . . . . . 220

Reactions . . . . . . . . . . . . . . . . 220

General chemical composition. . . . . . . . . . . . . 220

Analysis of the Products of Albuminous Putrefaction . . . . . . 221

Hydrobilirubin .............. . . . . 222

Excretin ................. . . 223

Stercorin . . . . . . . . . . . . . 223

MeconiuM ........................... 223 


\section{CHAPTER XII.}

\section{THE URINE.}

General Characteristics

General appearance ................ . . . 224

Color .. . . . . . . . . . . . . . . . . 225

Odor. . . . . . . . . . . . . . . . . . 226

Amount . . . . . . . . . . . . . . . . . 226

Specific gravity . . . . . . . . . . . . . . . . . . 227

Reaction . . . . . . . . . . . . . . . . . . 227

Determination of the acidity of the urine . . . . . . . . . 229

Chemical composition . . . . . . . . . . . . . . . . . . 230

The Inorganic Constituents of the Urine . . . . . . . . . . . . . 230

Quantitative estimation of the mineral ash . . . . . . . . . . 233

Quantitative estimation of the chlorides . . . . . . . . . . . 233

Quantitative estimation of the phosphates . . . . . . . . . . 233

Separate estimation of the earthy and alkaline phosphates . . . . . . . 234

Quantitative estimation of the sulphates . . . . . . . . . . . 234

Test for nitrates . . . . . . . . . . . . . . . . 236

The Organic Constituents of the Urine . . . . . . . . . . . . 236

The nitrogenous constituents of the urine . . . . . . . . . . 236

Urea... . . . . . . . . . . . . . . . 236

Origin . . . . . . . . . . . . . 236

Nitrogenous equilibrium . . . . . . . . . . . . 239

Properties . . . . . . . . . . . . . 241

Urea-nitrate . . . . . . . . . . . . . 241

Urea-oxalate . . . . . . . . . . . . . 241

Synthetic formation .............. . . 243

Isolation . . . . . . . . . . . . . . . 243

Quantitative estimation .............. 243

Estimation of the preformed ammonia . . . . . . . . 244

Estimation of the total urinary nitrogen . . . . . . . 245

Uric acid . . . . . . . . . . . . . . . . . . . 246

Origin . . . . . . . . . . . . . . . . . 246

Properties . . . . . . . . . . . . . . . . . 249

Tests. . . . . . . . . . . . . . . . . . 250

Isolation . . . . . . . . . . . . . . . . . . 251

Quantitative estimation .. . . . . . . . . . . . 251

The xanthin-bases . . . . . . . . . . . . . . . . 252

Origin . . . . . . . . . . . . . . . . 252

Quantitative estimation ............... . 253

Oxalic acid and oxaluric acid . . . . . . . . . . . . . . . 254

Quantitative estimation of oxalic acid . . . . . . . . . 255

Allantoin. . . . . . . . . . . . . . . . . 256

Isolation . . . . . . . . . . . . . . . . . 256

Kreatin and kreatinin . . . . . . . . . . . . . . . 257

Properties .. . . . . . . . . . . . . . . . 257

Tests. . . . . . . . . . . . . . . . . 258

Synthesis . . . . . . . . . . . . . . . . . 258

Isolation and quantitative estimation . . . . . . . . . . . . 259 
The Aromatic Constiturnts of the Urine Page

The conjugate sulphates . . . . . . . . . . . . . . 261

The phenols . . . . . . . . . . . . . . 262

Quantitative estimation .......... . . 263

Indoxyl sulphate . . . . . . . . . . . . . . 264

Tests. . . . . . . . . . . . 265

Quantitative estimation ............ 266

Skatoxyl sulphate . . . . . . . . . . . . . 267

Tests.................. . . . 267

The conjugate glucuronates . . . . . . . . . . . . . . 268

The compound glycocolls . . . . . . . . . . . . . . . . . 270

Hippuric acid . . . . . . . . . . . . . . 270

Properties . . . . . . . . . . . . 271

Synthesis . . . . . . . . . . . . . . . 271

Isolation . . . . . . . . . . . . . . 272

Quantitative estimation ........... . . 272

Phenaceturic acid . . . . . . . . . . . . . . 272

Properties .............. . . . 272

Isolation . . . . . . . . . . . . . . . 272

Ornithuric acid . . . . . . . . . . . . . . . . 273

The Aromatic Oxy-Aclds . . . . . . . . . . . . . 273

Homogentisinic acid . . . . . . . . . . . . 274

Inosit . . . . . . . . . . . . . . 276

Kynurenic acid . . . . . . . . . . . . 277

The Fatty Acids . . . . . . . . . . . . . . . . 278

The volatile fatty acids. . . . . . . . . . . . . 278

Isolation and quantitative estimation . . . . . . . 278

$\beta$-oxybutyric acid . . . . . . . . . . . . . 279

Test .................... . 280

Estimation . . . . . . . . . . . 2 281

Diacetic acid . . . . . . . . . . . . 281

Tests....................... 281

Acetone . . . . . . . . . . . . . . 282

Tests................... . . . . 282

Quantitative estimation . . . . . . . . . 284

Lactic acid . . . . . . . . . . . . . . . . . . . . . . 284

Isolation .................... 284

Mono-amino acids . . . . . . . . . . . 285

The Neutral Sulphur Bodies of the Urine. . . . . . . . . 286

Cysteïn . . . . . . . . . . . . . . . . . 287

Cystin . . . . . . . . . . . . . . . 288

Properties . . . . . . . . . . . . . . . 288

Isolation and estimation . . . . . . . . . . . 290

Preparation of . . . . . . . . . . . . 290

Estimation of neutral sulphur . . . . . . . . . . 290

The Carbohydrates. . . . . . . . . . . . . . . . 291

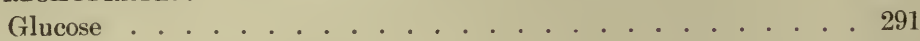

Tests................... . . 293

Quantitative estimation. . . . . . . . 296 
Lactose

Isol

Lævulose . . . . . . . . . . . . . . . . . 299

Laiose . . . . . . . . . . . . . . . . . . 300

Maltose . . . . . . . . . . . . . . . . 300

Dextrin . . . . . . . . . . . . . . . . 300

Pentoses . . . . . . . . . . . . . . . . 300

The Albumins . . . . . . . . . . . . . . . . . . . . . . 301

Tests. . . . . . . . . . . . . . . . 302

Estimation .. . . . . . . . . . . . . 306

The Pigments of the Urine . . . . . . . . . . . . . . 307

Urochrome .. . . . . . . . . . . . . 307

Isolation . . . . . . . . . . . . . . 308

Uroerythrin . . . . . . . . . . . . . . . . 308

Isolation . . . . . . . . . . . . . . . 309

Urobilin . . . . . . . . . . . . . . . . 309

Tests .................... 309

Ehrlich's reaction . . . . . . . . . . . . . 310

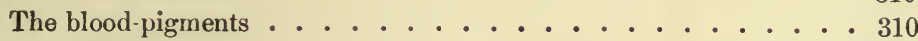

Hæmatin . . . . . . . . . . . . . . 311

Hæmatoporphyrin . . . . . . . . . . . . . 311

Urorubrohæmatin and urofuscohæmatin . . . . . . . 312

Melanins . . . . . . . . . . . . . . 312

The bile-pigments . . . . . . . . . . . . . 313

The bile-acids. . . . . . . . . . . . . . . 314

Fats, cholesterin, and lecithins . . . . . . . . . . . . . 314

Ferments . . . . . . . . . . . . . . . . . 315

Gases . . . . . . . . . . . . . . . . . 315

Ptomains . . . . . . . . . . . . . . . . . 315

CHAPTER XIII.

THE ANIMAL CELL.

Protoplasm and nucleus 818

\section{CHAPTER XIV.}

THE BLOOD.

General considerations . . . . . . . . . . . . . 322

Physical Characteristics of the Blood . . . . . . . . . 323

Color . . . . . . . . . . . . . . . 323

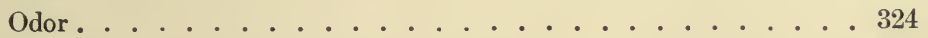

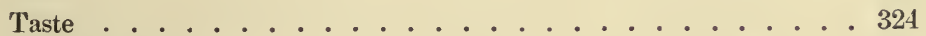

Specific gravity . . . . . . . . . . . . . . . 324

Amount . . . . . . . . . . . . . . . 325

Chemical Examination of the Blood. . . . . . . . 325

Reaction . . . . . . . . . . . 325

Chemical composition of the blood as a whole . . . . . . . . 327 
The plasma. PAGE

Fibrinogen . . . . . . . . . . . . . . 330

Isolation . . . . . . . . . . . . . . . 330

Properties . . . . . . . . . . . . 330

Serum-globulin . . . . . . . . . . . . . . 330

Isolation . . . . . . . . . . . . . . . 331

Properties . . . . . . . . . . . . . . 332

Serum-albumin . . . . . . . . . . . . . 332

Separation of the albumins from each other. . . . . . . . . 333

Quantitative estimation of the albumins ......... 333

Serum . . . . . . . . . . . . . . . . . . . . . . . . . . . . . . . . .

The coagulation of the blood . . . . . . . . . . . 335

The fibrin ferment ................ . . . . 335

Isolation . . . . . . . . . . . . . . . . . 336

Fibrin . . . . . . . . . . . . . . . . . . 337

Properties . . . . . . . . . . . . . . . . . 330

Estimation . . . . . . . . . . . . . . . . 338

Rapidity of coagulation . . . . . . . . . . . . . 339

Ferments . . . . . . . . . . . . . 339

Glycogen . . . . . . . . . . . . . 340

Fat . . . . . . . . . . . . . . . 340

Urea . . . . . . . . . . . . . . . . . . . 340

The leucocytes . . . . . . . . . . . . . . . 341

Nucleohiston . . . . . . . . . . . . . 341

Isolation . . . . . . . . . . . . . . . . . . . 341

Properties . . . . . . . . . . . . . . . . 341

Chemical composition . . . . . . . . . . . . . 342

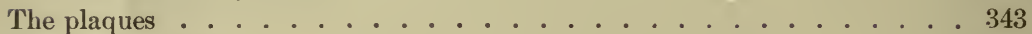

The red corpuscles . . . . . . . . . . . . . . . . 343

Isolation . . . . . . . . . . . . . . . 344

Hæmoglobin and its derivatives . . . . . . . . . . . . . 344

Hæmoglobin ................ . . . . 344

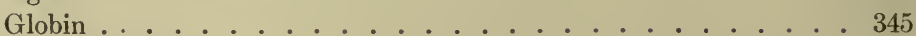

Hrmochromogen . . . . . . . . . . . . . 346

Oxyhæmoglobin ................... 347

Hrmatin ................ . . . . 347

Hrmin . . . . . . . . . . . . . . . 349

Carbon dioxide hæmoglobin . . . . . . . . . . . . . . . . 352

Carbon monoxide hrmoglobin . . . . . . . . . . . . 352

Nitric oxide hæmoglobin .............. . . 353

Cyanhæmoglobin ................ . . . 353

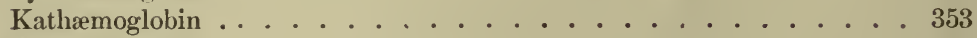

Methæmoglobin . . . . . . . . . . . . 353

Hæmatoporphyrin ............... . . . . 354

Phylloporphyrin ............... . . . 355

Hæmatoidin . . . . . . . . . . . . . 355

Hitmocyanin ................ . . . 356 


\section{CHAPTER XV.}

THE LYMPH.

The lymph .

PAGE 357

\section{CHAPTER XVI.}

THE MUSCLE TISSUE.

Analyses of fresh tissue . . . . . . . . . . . . . . 364

The muscle albumins . . . . . . . . . . . . . . . 365

Myogen ..................... 366

Myosin . . . . . . . . . . . . . 367

Ferments ............................ 370

Muscle stroma . . . . . . . . . . . . . . . 370

Muscle pigments .................. . . . 371

Glycogen . . . . . . . . . . . . . . 371

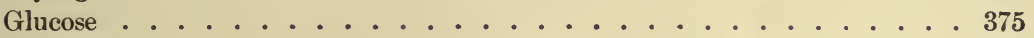

Lactic acid . . . . . . . . . . . . . . . 375

Inosit . . . . . . . . . . . . . . . . . 378

Kreatin and kreatinin . . . . . . . . . . . . 379

The xanthin bases . . . . . . . . . . . . . . . . 381

Gases . . . . . . . . . . . . . . . 385

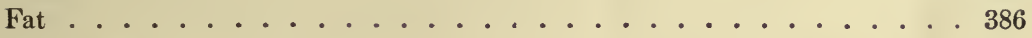

\section{CHAPTER XVII.}

THE NERVE TISSUE.

Albumins . . . . . . . . . . . . . . . . 388

Neurokeratin . . . . . . . . . . . . . . 389

Protagon .................... . . . . 390

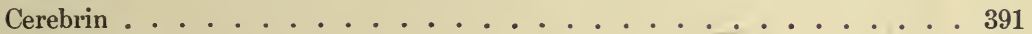

Homocerebrin . . . . . . . . . . . . . . 392

Encephalin . . . . . . . . . . . . . . . 393

Lecithins . . . . . . . . . . . . . . . 393

The cholesterins ................. . . . . 394

Extractives ............................ 394

CHAPTER XVIII.

THE EYE AND EAR.

The cornea . . . . . . . . . . . . . . . . . 396

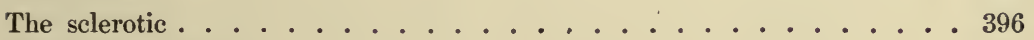

The aqueous humor................ . . . 396

The crystalline lens . . . . . . . . . . . . . . 397

The vitreous body . . . . . . . . . . . . . . . 398

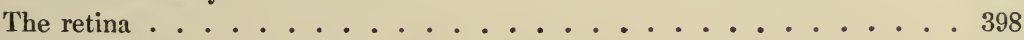

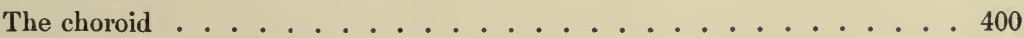

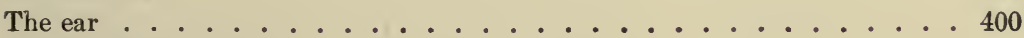




\section{CHAPTER XIX.}

\section{THE SUPPORTING TISSUES.}

White fibrous tissue ................. . . . 401

Yellow or elastic tissue . . . . . . . . . . . . . . 402

Reticulated tissue . . . . . . . . . . . . . . 402

Cartilage . . . . . . . . . . . . . . . . . . 402

Chondroitin-sulphuric acid . . . . . . . . . . . 403

Chondromucoid . . . . . . . . . . . . . . . 404

Albumoid .................... . . . . 405

Bone .................... . . . . 405

Bone marrow . . . . . . . . . . . . . . . . 407

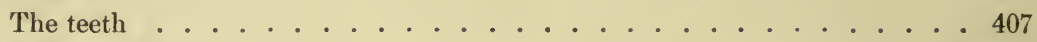

Adipose tissue .................. . . . . . . 408

Analysis of fat ................ . . . 4 410

Origin . . . . . . . . . . . . . . . 4 4 . . . . . . . . . . .

Significance ..................... 412

\section{CHAPTER XX.}

\section{THE SKIN AND ITS APPENDAGES.}

The skin . . . . . . . . . . . . . . . . . . . 414

The sweat ........................ 416

The sebum ............................. 418

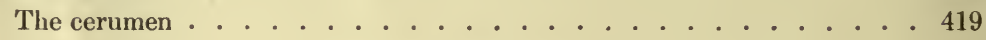

\section{CHAPTER XXI.}

THE GLANDULAR ORGANS.

The liver . . . . . . . . . . . . . . . . . . 420

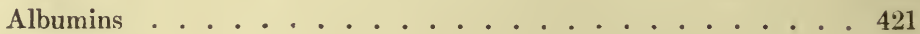

Glycogen ................... . . . . 424

Ferments ................. . . . 424

Glucose ................ . . . 425

Fat .................... 425

Extractives ................... 426

The pancreas . . . . . . . . . . . . . . . . . 426

Guanylic acid .................. . . 426

The lymph glands . . . . . . . . . . . . . . . . . 4 427

The kidneys . . . . . . . . . . . . . . . . . . . 4 428

The mammary glands . . . . . . . . . . . . . . . . 4 428

The milk . . . . . . . . . . . . . . . 429

General properties ............ . . 429

Amount ............... . . 4 430

Specific gravity . . . . . . . . . . . . . . . . 431

Reaction............... . . 4 431

Composition ................ 432

Albumins ....................... 433

Fats . . . . . . . . . . . . . . 437

Lactose . . . . . . . . . . . . . . 4 438

Extractives .................. 439 


\section{Ferments}

The colostrum .............. . . . . . 440

The reproductive glands . . . . . . . . . . . . . . 441

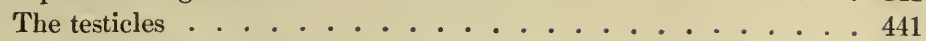

The semen . . . . . . . . . . . . . 4442

The spermatozoa ......................... 444

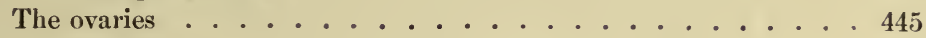

The ovum . . . . . . . . . . . . . . 445

The shell . . . . . . . . . . . . . . . 446

The albumen . . . . . . . . . . . . . 446

The yolk .............. . . 449

Incubation . . . . . . . . . . . . . 453

CHAPTER XXII.

THE DUCTLESS GLANDS.

The thyroid gland . . . . . . . . . . . . . . . . 456

The adrenal glands . . . . . . . . . . . . . . 459

\section{APPENDIX.}

LABORATORY EXERCISFS . . . . . . . . . . . . . 463 


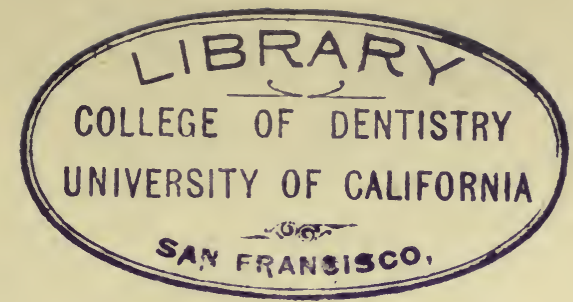

\section{PHYSIOLOGICAL CHEMISTRY.}

\section{CHA P T ER I.}

\section{INTRODUCTION.}

THE science of physiolngical chemistry has for its object the study of the various chemical processes which take place in the bodies of animals and plants, and which are more or less intimately associated with the phenomena of life. As the phenomena of life, moreover, are essentially dependent upon the transformation of living matter into non-living matter, and vice versa, physiological chemistry deals primarily with the chemical processes of nutrition in the widest sense of the term. Its study therefore comprises a consideration of the various substances which are generally designated as food-stuffs, their origin, their transformation into living tissue, and their ultimate fate.

General Composition of Living Matter.-Chemical examination shows that plants and animals consist essentially of carbon, hydrogen, nitrogen, oxygen, sulphur, phosphorus, chlorine, potassium, sodium, calcium, magnesium, and iron-that is, of elements which occur also widely distributed in the non-organized world. In the bodies of animals and plants these elements are built up to form bodies of highly complex chemical constitution, which belong to the class of albumins, carbohydrates, and fats. Upon their presence both animals and plants are dependent for their existence, and as these bodies are constantly being broken down and transformed into simpler chemical compounds, as the result of the various manifestations of life, it follows, from the law of the indestructibility of matter, that for their replacement the living body is forced to depend upon such simpler matter as is pre-existent. This matter it is capable of transforming into the complex substances of which its tissues are composed.

Forces at Work in the Living World.-The forces which are at work in effecting these various changes are apparently the same as those which are operative in the non-organized world. For the assumption of special vital forces there seems to be less necessity the more we come to understand the mechanism of vital phenomena. 
Character of Chemical Changes.-The chemical processes which are involved in the transformation of non-living matter into living tissue are qualitatively the same in plants and animals. Quantitative differences, however, exist, which are sufficiently pronounced to serve as marks of distinction between animals and plants. Thus plants are capable of evolving from relatively simple compounds those complex chemical substances which go to form their structure, while animals apparently do not possess this power. They are hence dependent for their existence upon food-stuffs which are preformed; and the potential energy which animals require for the functioning of their various organs, and which they transform into kinetic energy, is, as a matter of fact, derived in every instance, either directly or indirectly, from plant-life. Plants, in turn, obtain the potential energy which is stored in their tissues from the kinetic energy of sunlight, and in virtue of this energy can elaborate those simple chemical substances which are at their disposal as food-stuffs into the complex bodies which constitute their tissues.

We thus observe that while in plant-life synthetic chemical processes prevail, analytical processes are foremost in animal life. These analytical processes, moreover, are largely of the character of oxidations, while the syntheses which are effected in the bodies of plants are essentially of the nature of reductions. But just as synthetic processes are not absolutely characteristic of plant-life, so also do oxidation-processes occur in plants, and synthetic reductions in animals. This becomes especially noticeable as we descend in the scale of both animal and vegetable life. Primitive vegetable organisms are thus met with which, like the highly organized mammal, are almost entirely dependent for their existence upon already elaborated food-stuffs, and low forms of animal life similarly occur in which the processes of nutrition are essentially the same as those which occur in the higher plants. The differences which thus exist between animal life and plant-life are therefore, as has been stated, more of a quantitative than a qualitative kind.

Synthetic Processes in Plants.-I have said that plants are capable of elaborating from simpler compounds the complex chemical substances of which they are composed, and that the chemical processes here involved are essentially of the nature of synthetic reductions. Formerly, it was believed that the various organic substances which occur in animals and plants could be produced only through the agency of a special vital force; but we now know that this is not necessarily the case, and that as a matter of fact a large number of such bodies can be produced artificially in the chemical laboratory. Wöhler, in 1829, was the first to demonstrate this possibility by preparing urea from ammonium cyanate. This he accomplished by heating the substance to a temperature of $100^{\circ} \mathrm{C}$., when a transposition of atoms apparently takes place, and urea results. The force which is necessary to effect such a change is 
here, as in many syntheses which can artificially be brought about, a relatively high temperature. In the bodies of animals and plants a like temperature, of course, would destroy life, and there must hence be a different mechanism at the disposal of living beings to effect such a change. We know that under the influence of sunlight certain plants are capable of effecting the synthesis of carbohydrates, fats, and albumins from the carbon dioxide of the air, and the water and certain mineral salts of the soil, and that the ability to bring about these changes is in a large measure dependent upon the presence of a chemical substance which is found in the green parts of plants, and which is termed chlorophyl. We know further that chlorophyl requires exposure to sunlight to effect these changes, but of the mechanism through which these changes are brought about we know nothing.

Oxidations and Hydrations in the Animal Body.-The oxidation-processes which prevail in animals, and in consequence of which the more complex substances which go to form the various tissues and organs of the body are retransformed into those simple compounds which plants require for their existence, we are also unable to explain. We know that the oxygen of the air, as also that of the blood, exists in a neutral molecular form, and as such is incapable of effecting the oxidation of such complex substances as the albumins and fats. The older view that oxygen exists in the body as ozone, and that the various oxidation-processes take place in the animal fluids, has been abandoned, and it is now generally accepted that these changes occur in the individual cells. Here, then, a splitting up of the nentral oxygen must take place, but of the forces which effect this decomposition we know next to nothing. Whether we believe with Pflüger that the organized living albumin, in contradistinction to the non-organized circulating albumin, is characterized by a greater motility of its atoms, in consequence of which the neutral oxygen is decomposed, or whether we accept the view that reducingsubstances are formed during the decomposition of the albuminous molecule in consequence of the activity of a third factor, we are as far removed from an adequate explanation of these phenomena as in the beginning.

Within recent years numerous observations have shown that from various organs of the body certain substances can be extracted which are apparently identical with or closely related to the so-called enzymes. Certain representatives of this class, such as pepsin, trypsin, ptyalin, and others, are, as we shall see, formed in the cells of the digestive glands of the body, and serve the purpose of transforming the various food-stuffs which are furnished the animal by the plant into forms which can be absorbed and built up into its tissues. The chemical processes which are here involved are essentially of the character of hydrations. Other bodies, however, of this order which can be obtained from living tissues, and which are also 
capable of manifesting their special activity after the death of their parent-cells, apparently possess the power of oxidation, and it is hence possible that these processes in the living tissues may also be referable to such enzymatic activity. Whether this is actually the case is not definitely known. But if so, we are apparently approaching a time when what we have heretofore been forced to ascribe to the activity of a special vital force may be explained upon the basis of physical laws which are seen also at work in the non-organized world. For we know that properties which are supposedly characteristic of the enzymes are possessed also by certain elements which are found only in the inorganic world. The most notable properties of the enzymes are their ability to effect an amount of chemical change which appears to be out of all proportion to the quantity of the enzyme present, and the fact that the enzyme itself apparently does not enter into the reaction. These same properties, however, are common to certain metals and their oxides. Bredig and von Berneck showed that a gram-atomic weight (193 grams) of colloidal platinum diffused through 70,000,000 liters of water shows a perceptible action on more than $1,000,000$ times the quantity of hydrogen peroxide; and H. C. Jones demonstrated that the reaction which here takes place is a mono-molecular reaction, which indicates that the platinum itself does not enter into the reaction. Ernst has similarly shown that 0.0001 gram of colloidal platinum can catalyse 50,000 times its own weight of oxyhydrogen at ordinary temperature, without loss of efficiency on the part of itself. Curiously enough, the analogy between the action of such metallic solutions and that of the enzymes goes still further. Finely divided platinum, palladium, iridium, osmium, etc., thus have the power of inverting cane-sugar, like one of the enzymes, invertin; and certain poisons, such as hydrocyanic acid, sulphuretted hydrogen, carbon disulphide, and mercuric chloride, which inhibit or even suspend the action of the enzymes entirely, exert a similar influence upon a solution of colloidal platinum (negative catalysers, anticatalysers, or paralysers); 0.000,000,001 gram of hydrocyanic acid per c.c. will thus reduce the catalytic action of $0.000,006$ gram of colloidal platinum upon hydrogen peroxide to one-half. Without entering upon this very interesting subject further, it is clear that a path has been opened upon which it may be possible to penetrate into the mysteries of the so-called vital forces, and to show ultimately that such forces are essentially the same as those met with in the non-living world.

Chlorophyl.- In the light of more recent investigation, it seems probable that some of the synthetic processes which occur in plantlife may also be referable to the action of enzymes. As a matter of fact, such bodies are abundantly present in the vegetable world, and we know that some of these at least, and probably all, are characterized by a reversible activity. Maltase, a ferment, which, as we shall see later, causes inversion of the disaccharide maltose to 
glucose, is thus similarly able to bring about the synthetic fcrmation of maltose from two molecules of glucose. On the other hand, it appears that the primary formation of food-stuffs in plants does not occur in this manner, but is referable to the activity of a special body which, as has been stated, is present in the exposed green parts of most plants, and which is termed chlorophyl. 'This substance occurs widely distributed in the vegetable world, but is also found in those low forms of animal life in which the processes of nutrition are essentially the same as those met with in the higher plants. Of the chemical composition and structure of chlorophyl we know little that is definite. Numerous attempts have been made to isolate it from the living plant, but it is doubtful whether any of these attempts have yielded the actual substance. Only its decomposition-products, or at best very impure forms, have apparently been obtained. Gautier, it is true, claims to have isolated the substance in crystalline form by methods which are calculated to avoid its chemical alteration. Others, however, have not been successful in repeating his work.

The substance which Gautier obtained from spinach-leaves occurred in the form of small crystals of a dark-green color, which on exposure to light turned brown, then yellow, and finally became colorless. Its composition corresponded to the formula $\mathrm{C}_{40} \mathrm{H}_{64} \mathrm{~N}_{2} \mathrm{O}_{4}$. The mineral ash consisted of about 1.75 per cent. of magnesium phosphate, traces of calcium and sulphates, while iron was absent. Treated with hydrochloric acid, it was decomposed into phylloxanthin and phyllocyanic acid, $\mathrm{C}_{38} \mathrm{H}_{44} \mathrm{~N}_{4} \mathrm{O}_{6}$ or $\mathrm{C}_{36} \mathrm{H}_{40} \mathrm{~N}_{4} \mathrm{O}_{6}$. This latter is thus a homologue of bilirubin, $\mathrm{C}_{32} \mathrm{H}_{36} \mathrm{~N}_{4} \mathrm{O}_{6}$, which in turn is derived from hæmatin, and is isomeric with homatoporphyrin. A most interesting relationship between the blood coloring-matter hæmoglobin and the vegetable coloring-matter chlorophyl thus becomes apparent, and constitutes a further link connecting the animal with the vegetable world. Further investigations have shown that a substance can be obtained from chlorophyl, which is termed phylloporphyrin, and which differs from hæmatoporphyrin anhydride only in containing three atoms less of oxygen, viz., $\mathrm{C}_{32} \mathrm{H}_{34} \mathrm{~N}_{4} \mathrm{O}_{2}$. Both phylloporphyrin and hæmatoporphyrin, yield the same decomposition-product on reduction with phosphonium iodide and hydriodic acid, viz., hæmopyrrol, $\mathrm{C}_{8} \mathrm{H}_{13} \mathrm{~N}$; and if the reduction of hæmatoporphyrin is not carried on too energetically mesoporphyrin results $\left(\mathrm{C}_{32} \mathrm{H}_{36} \mathrm{~N}_{4} \mathrm{O}_{4}\right)$, which manifestly stands midway between hæmatoporphyrin and phylloporphyrin.

Moderately concentrated solutions of chlorophyl in alcohol or petroleum-ether show seven bands of absorption. The first of these, $\mathrm{I}$, is situated in the red portion of the spectrum between B and C, and is well pronounced and sharply defined on both sides. The bands II, III, and IV are rather indistinct and scattered through the orange-yellow, the yellow and the yellowish-green portion between $\mathrm{C}$ and E. From $\mathrm{F}$ off, the greater portion of the spectrum 
is absorbed by the remaining bands, V, VI, and VII, of which V is seen to the right of F, VI most marked about G, while VII occupies the extreme violet end. Very concentrated solutions allow the red rays to pass only as far as $B$, while in greater dilution the green rays likewise appear. Such solutions, therefore, appear green when viewed with transmitted light, while with reflected light they are red and fluorescent.

When a fresh leaf is similarly examined, a spectrum is obtained which is essentially the same as that just described. There is lacking, however, the band that corresponds to the red fluorescent rays of chlorophyl solutions. This is explained by the assumption that the red rays are absorbed by living chlorophyl and transformed into chemical energy. In accordance with this view, we find that when living plants are successively exposed to the various rays constituting sunlight, decomposition of earbon dioxide with liberation of oxygen-which, as we shall presently see, takes place in the green portions of every plant whenever it is exposed to sunlight-occurs with special intensity when the plant is exposed to the rays corresponding to the bands I, II, and III. In this manner, then, chlorophyl-bearing plants derive their kinetic energy from sunlight, and thus become enabled to elaborate the simple food-stuffs which are at their disposal into the complex substances which constitute their tissues.

The Food-stuffs of Plants.-The most essential elements which enter into the composition of the tissues of plants are, as has been pointed out, carbon, hydrogen, oxygen, and nitrogen. These substances are available to the plant as carbon dioxide, water, and certain nitrates. The origin of the first mentioned is, of course, obvious, while that of the last is at first sight somewhat obscure.

The nitrates are present in any soil which contains organic matter, and are known to result from this through the special activity of certain bacteria. Decomposing animal and vegetable matter is, however, not the only source of the nitrates, for it can be demonstrated that arable soil, apparently devoid of vegetable life, is capable, unless sterilized, of fixing a very considerable amount of nitrogen, which must of necessity be derived from the atmosphere. The assumption has been that this nitrogen is obtained as an anmonium compound, which the bacteria then transform into nitrates. In this connection it is interesting to note that the hydroxides of some of the heavy metals (iron, cobalt, nickel) are capable of producing nitrites in minute quantities from the nitrogen of the atmosphere, and that the first step in the formation of nitrates may hence be a purely chemical one. We do not wish to convey the impression, however, that all plants require their nitrogen in this form, for we know that Saccharomyces cerevisiæ, for example, can elaborate its nitrogen from ammonium salts directly, and is even incapable of utilizing that which is furnished in the form of nitrates. Under certain conditions, moreover, probably all plants can, for a time at least, grow in the presence of ammonium nitrogen only. 

soil.

The necessary mineral salts the plant likewise obtains from the

The question now arises: In what manner do plants effect the synthesis of those complex chemical substances which go to form their tissues from the simple bodies which serve as their food-stuffs? The kinetic energy which is necessary to effect these changes is, as has been stated, derived from the sunlight and transformed into potential energy by the chlorophyl. We should thus expect to find in those parts in which this is present the origin of those final products which we meet with in the tissues of the plant. These products may be divided into three groups, and in the following pages an attempt will be made to describe the manner in which representatives of each are formed. I shall accordingly consider the origin of the carbohydrates, the fats, albumins, and certain nonalbuminous, nitrogenous bodies, all of which are also found in the animal body, and which represent the essential food-stuffs of the animal world. In doing so, I am aware that I am trespassing to a certain extent upon what will follow in subsequent chapters; but as I shall deal with the chemistry of animal life more exclusively in the present work, it has been deemed best to consider briefly the principal syntheses which are effected by plants before proceeding to a more detailed study of the subject proper.

Synthesis of the Carbohydrates.-It has been pointed out that during exposure of chlorophyl-bearing plants to sunlight the carbon dioxide of the air is decomposed, with liberation of oxygen. The volume of gas thus set free is equivalent to the volume of carbon dioxide that is decomposed. At the same time a reduction of water takes place, as is apparent from the observation that a larger amount of hydrogen is found in the plant than is necessary to form water with all of the oxygen that is present at the same time. It thus follows that one-half of the oxygen per volume must be derived from carbon dioxide, and the other from water, according to the equation :

$$
\underset{4 \text { volumes. }}{2 \mathrm{CO}_{2}}+2 \mathrm{H}_{2} \mathrm{O}=2 r+\underset{4 \text { volumes. }}{40}
$$

in which $r$ represents one atom of carbon, one atom of oxygen, and two atoms of hydrogen, which have been retained by the plant. A combination of these atoms in one molecule, however, would represent one molecule of formic aldehyde, $\mathrm{CH}_{2} \mathrm{O}$.

Bach suggests that percarbonic acid is first formed together with water and carbon and that the percarbonic acid then yields carbon dioxide and hydrogen peroxide, while from the carbon and water formaldehyde is formed, according to the equations :

$$
\begin{aligned}
& 3 \mathrm{H}_{2} \mathrm{CO}_{3}=2 \mathrm{H}_{2} \mathrm{CO}_{4}+\mathrm{H}_{2} \mathrm{O}+\mathrm{C} \\
& 2 \mathrm{H}_{2} \mathrm{CO}_{4}=2 \mathrm{CO}_{2}+2 \mathrm{H}_{2} \mathrm{O}_{2}=2 \mathrm{CO}_{2}+2 \mathrm{H}_{2} \mathrm{O}+\mathrm{O}_{2} \\
& \mathrm{H}_{2} \mathrm{O}+\mathrm{C}=\mathrm{COH} . \mathrm{H} .
\end{aligned}
$$


Bouilhac and Trébaux, by painting a thin layer of gelatine with a benzene solution of chlorophyl, secured a mechanism which on exposure to sunlight actually decomposed carbonic acid into formic aldehyde and hydrogen peroxide. We thus have a probable explanation of the manner in which the carbohydrates may be constructed, as they are essentially polymeric compounds of formic aldehyde $\left(6 \times \mathrm{CH}_{2} \mathrm{O}=\mathrm{C}_{6} \mathrm{H}_{12} \mathrm{O}_{6}\right)$. Bouilhac and Trébaux further showed that Elodea forms starch in the dark from an 0.001 per cent. solution of formic aldehyde. The initial step in the assinilation of carbon is thus manifestly independent of living protoplasm. But since chloroform vapor prevents the polymerization of formaldehyde it would seem that the latter process cannot be caused by an enzyme.

The same writers also found that on painting the white petals of Saxafraga Wallacei, which can form starch from very dilute solutions of formic aldehyde, with their benzene-chlorophyl solution, the petals became capable of forming starch from carbon dioxide and water, directly, in the sunlight. Formic acid is formed as an intermediary product and from it Elodea has been shown to construct starch in the sunlight.

The hydrogen peroxide which is formed, together with formis aldehyde, as indicated above, is decomposed into water and oxygen by catalase, which is found in the chloroplasts of chlorophyl-bearing plants.

Glucosides.-Closely related to the carbohydrates proper, the origin of which has just been considered, is a group of substances which likewise occur widely distributed in the vegetable kingdom. These are the so-called glucosides. They are so termed from the fact that glucose is invariably formed during their hydrolytic decomposition, which, as an anhydride, thus constitutes an integral part of their molecule. This observation at once suggests their origin also from formic aldehyde.

Such substances are salicin, which on hydrolytic decomposition yields glucose and saligenin; arbutin, which yields glucose and hydroquinon; phloridzin, which gives rise to glucose and phloretin, etc.

$$
\begin{aligned}
& \underset{\substack{13 \\
\text { Salicin. }}}{\mathrm{C}_{18} \mathrm{O}_{7}}+\mathrm{H}_{2} \mathrm{O}=\underset{\text { Saligenin. }}{\mathrm{C}_{7} \mathrm{H}_{8} \mathrm{O}_{2}}+\underset{\text { Glucose. }}{\mathrm{C}_{6} \mathrm{H}_{12} \mathrm{O}_{6}} . \\
& \underset{\text { Arbutin. }}{\mathrm{C}_{12} \mathrm{H}_{16} \mathrm{O}_{7}}+\underset{\text { Hydroquinon. }}{\mathrm{H}_{2} \mathrm{O}}=\underset{\text { Glucose. }}{\mathrm{C}_{6} \mathrm{H}_{6} \mathrm{O}_{2}}+\underset{\mathrm{C}_{12} \mathrm{O}_{6}}{\mathrm{C}_{2}} \text {. }
\end{aligned}
$$

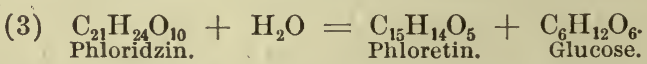

Especially interesting is a group of glucosides which are nitrogenous in character, and thus stand, as it were, midway between the carbohydrates and the albumins. A study of their decompositionproducts hence permits an insight into the manner in which the albumins are synthetically produced, and shows that here also aldehyde groups play an important part. As in the case of the albumins, the nitrogen here also occurs in combination with carbon and 
hydrogen in the group $\mathrm{CH}-\mathrm{NH}$, which in turn is structurally closely related to hydrocyanic acid. In accordance with these considerations, we thus find that amygdalin, $\mathrm{C}_{20} \mathrm{H}_{27} \mathrm{NO}_{11}$, is decomposed into glucose, hydroeyanic acid, and benzaldehyde, as shown in the equation :

$$
\mathrm{C}_{20} \mathrm{H}_{27} \mathrm{NO}_{11}+2 \mathrm{H}_{2} \mathrm{O}=2 \mathrm{C}_{6} \mathrm{H}_{12} \mathrm{O}_{6}+\mathrm{C}_{6} \mathrm{H}_{5^{\prime}} \cdot \mathrm{COH}+\mathrm{CNH} \text {. }
$$

Solanin, $\mathrm{C}_{43} \mathrm{~N}_{70} \mathrm{NO}_{16}$, similarly yields glucose and solanidin, $\mathrm{C}_{25}$ $\mathrm{H}_{39} \mathrm{NO}$.

Mannides.-Like the carbohydrates proper, the mannides or mannitides, which also occur widely distributed in the vegetable world, are likewise derived from the aldehyde radicle that is formed by chlorophyl under the influence of sunlight. They differ from the glucosides in yielding mannite, $\mathrm{C}_{6} \mathrm{H}_{14} \mathrm{O}_{6}$, instead of glucose, on hydrolytic decomposition. The origin of mannite from formic aldehyde may be represented by the equation :

$$
6 \mathrm{CH}_{2} \mathrm{O}+2 \mathrm{H}=\mathrm{C}_{6} \mathrm{H}_{14} \mathrm{O}_{6} \text {. }
$$

On the other hand, mannite may result from glucose as the result of the specific activity of certain cells, as is shown by the equation :

$$
13 \mathrm{C}_{6} \mathrm{H}_{12} \mathrm{O}_{6}+6 \mathrm{H}_{2} \mathrm{O}=12 \mathrm{C}_{6} \mathrm{H}_{14} \mathrm{O}_{6}+6 \mathrm{CO}_{2} \text {. }
$$

Synthesis of the Fats. - The fats which are found in plants are, like the carbohydrates, derived from carbon dioxide and water, and in all likelihood are formed also synthetically through the agency of the chlorophyl. The mechanism, however, by which these syntheses are effected is not so clear. It is probable that they result from the union of carbon dioxide and water, as shown by the equations :

$$
\begin{aligned}
& \text { (1) } 3 \mathrm{CO}_{2}+4 \mathrm{H}_{2} \mathrm{O} \quad=\underset{\text { Glycerin. }}{\mathrm{C}_{3} \mathrm{H}_{8} \mathrm{O}_{3}}+7 \mathrm{O} \\
& \text { (2) } 34 \mathrm{CO}_{2}+34 \mathrm{H}_{2} \mathrm{O} \quad=\underset{\text { Stearic acid. }}{\mathrm{C}_{18} \mathrm{H}_{36} \mathrm{O}_{2}} \quad+\underset{\text { Formic acid. }}{16 \mathrm{CH}_{2} \mathrm{O}_{2}}+68 \mathrm{O} \text {. }
\end{aligned}
$$

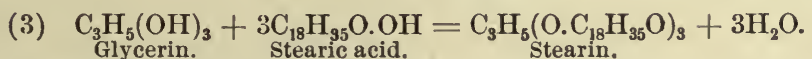

This supposition is strengthened by the observation that during certain phases in the life of some plants an actual transformation of carbohydrates into fats takes place. In the fruits and leaves of the olive tree, for example, a large amount of mannite gradually disappears during. the months of September and October, and is replaced by oil. This transformation could be explained upon the basis of the equations just given, or by the assumption that the oil results from mannite through a loss of water and carbon dioxide, as suggested by the equation :

$$
11 \mathrm{C}_{6} \mathrm{H}_{14} \mathrm{O}_{6}=\mathrm{C}_{51} \mathrm{H}_{94} \mathrm{O}_{6}+30 \mathrm{H}_{2} \mathrm{O}+15 \mathrm{CO}_{2} \text {. }
$$

In any event, the system $\mathrm{H}_{2} \mathrm{O}+\mathrm{CO}_{2}$, which gives rise to the formation of formic aldehyde and glucose, must also be regarded as the fundamental basis in the synthesis of fats. 
Synthesis of the Albumins.-Much more complicated than the synthesis of the carbohydrates and fats is that of the albumins, a class of bodies which occur widely distributed in both the animal and the vegetable world, and form the groundwork, so to speak, of all living matter. Like the carbohydrates and fats, they also consist of carbon, hydrogen, and oxygen, but in addition to these elements nitrogen and variable amounts of sulphur are constantly present. To this class belong such bodies as serum-albumin, eggalbumin, casein, fibrin, etc. They are exceedingly complex substances, and have a very high molecular weight.

The exact manner in which the albumins originate has not been determined. We are in possession of a number of observations, however, which permit some insight, at least, into the manner in which plants are capable of elaborating these complex substances from the simple material which serves as their food, and there is reason to suppose that the synthesis of the albumins also takes place, to a certain extent at least, in the chlorophyl-bearing portions of plants.

It was formerly supposed that the nitrogen necessary in these synthetic processes was furnished plants in the form of ammonium salts. Subsequent investigations have shown, however, as has been indicated, that this is usually not the ease, and we now know that through the activity of various bacteria in the soil the nitrogen required by plants is here oxidized to nitrates. These are absorbed and carried to the chlorophyl-bearing portions of the plant, where, as we have seen, formic aldehyde and glucose are constantly being formed. Here, or in the rootlets, a certain proportion of the nitrates is apparently transformed into nitric acid, which is then promptly reduced by the formic aldehyde, with the formation of a certain amount of hydrocyanic acid, as shown in the equation :

$$
2 \mathrm{HNO}_{3}+5 \mathrm{CH}_{2} \mathrm{O}=2 \mathrm{HCN}+3 \mathrm{CO}_{2}+5 \mathrm{H}_{2} \mathrm{O} .
$$

In this form, then, the nitrogen probably enters into the construction of the albuminous molecule. This supposition is strengthened by the observation that hydrocyanic acid, as such, or in the form of cyanides, occurs widely distributed in the vegetable world, and is characterized by the readiness with which it combines with a large number of organic substances to form highly complex chemical compounds. The nature of the subsequent changes will be better understood when the decomposition-products of the albumins have been studied in detail. These will be considered in a following chapter. 


\section{CHAPTER II.}

\section{THE ALBUMINS}

THe albumins, or proteins, are the most important food-stuffs which the animal requires for its existence. 'They play a predominating role in the construction of all the tissues and organs of the body, and form the groundwork of every living cell. The phenomena of life depend upon and centre in their presence.

While many different forms of albumins exist, they all present certain general chemical and physical characteristics, which serve to distinguish them as a class, and which show that a close genetic relationship exists between them.

Elementary Composition.-All albumins contain carbon, hydrogen, nitrogen, oxygen, and sulphur in certain definite proportions, which vary within fairly narrow limits in the different members of the group, but tell us very little, as such, of the structure of the albuminous molecule. The variations which do occur are shown in the following table:

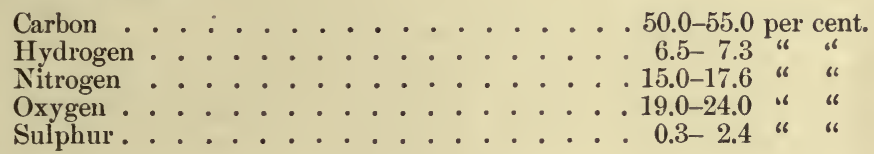

Other elements are not found in the common albumins, but occur in certain compound albumins, which result through the union of an albuminous group with other more or less complex radicles. The hæmoglobins thus contain iron, the nucleoproteids phosphorus; one highly differentiated globulin contains iodine, etc. All albumins further contain variable amounts of mineral salts, which are closely united with the albuminous molecule. The most important and constant of these are the chlorides and phosphates of the alkalies and the alkaline earths.

The structural formula of the albumins is unknown. The molecule is unquestionably very large. In the case of serum-albumin Hofmeister calculated the general formula as $\mathrm{C}_{450} \mathrm{H}_{720} \mathrm{~N}_{116} \mathrm{~S}_{6} \mathrm{O}_{140}$, which would correspond to a molecular weight of 10,166. (See molecular weight and structural composition, below.)

Reaction.-In the free state the albumins are neutral; in such solutions they are not ionized, but this results at once on contact with other ions. They then form salts, both with acids and bases which are fairly good conductors, and thus play the part of pseudoacids and pseudo-bases (Hantzsch). As acids the albumins are dibasic (Osborne, Söldner, and others). One series of salts shows a neutral reaction with the usual indicators, while the other is more 
or less markedly basic. The acid character is most pronounced in the mucins, the nucleo-albumins, and the nucleoproteids; less so in the globulins; while the albumins proper are apparently neutral or slightly alkaline. The histons and the protamins, on the other hand, are markedly basic.

Solubility.- Some albumins are soluble in water, others only in dilute saline solution, and still others in dilute acids and alkalies. In more concentrated acids and alkalies, as also in glacial acetic acid, all albumins dissolve, but are at the same time decomposed. In dilute alcohol some albumins dissolve with comparative ease, but in absolute alcohol, ether, chloroform, benzol, and all other common solvents they are insoluble.

Crystallization.-In the eggs of certain fishes and amphibia socalled yolk platelets may be observed which apparently possess a crystalline structure. Chemical examination has shown, however, that these bodies are not pure albumins, but that they contain a large percentage of lecithin and mineral salts. The same is true of the aleuronat crystals which have been found in the seeds of certain plants, and probably also of the eosinophilic crystalloids which may be seen in the blood of birds, of similar structures in the ova of the deer, and the testicular epithelium of man.

The form in which the albumins are commonly obtained in the chemical laboratory, and in which (with the exceptions mentioned) they exist in nature, is amorphous. In the dry state they form a white or but little colored non-hygroscopic powder, or they occur as yellowish, brittle, nore or less opaque lamellæ which are both odorless and tasteless.

By artificial means it is possible to obtain certain members of the group in crystalline form. To this end, a neutral salt must be present, and it is advantageous to have the reaction slightly acid. What part the salt and the acid play is not known, but there is evidence to show that either the acid or the salt enters into the composition of the crystals. In the case of edestin Osborne has shown that a salt-like body results, which contains hydrochloric acid when crystallization has taken place from a solution of the albumin in sodium chloride, or sulphuric acid if a sulphate has been employed.

Of animal albumins, crystallization has been effected in the case of serum-albumin (from horses' blood), egg-albumin, oxyhæmoglobin, hæmoglobin, methæmoglobin, and possibly also of lactalbumin and casein. Artificial crystallization of animal globulins has not led to satisfactory results; but, one substance which supposedly belongs to this class, and which was noted by Noël Paton in a pathological urine, separated out spontaneously in crystalline form.

Of vegetable albumins, the edestins (globulins) are notable examples of crystallizable albumins, but in their case also the presence of a salt is necessary. This can subsequently not be removed without impairment of the power of crystallization.

The form of the crystals seems to vary; but Wichmann has 
shown that they are all crystallographically identical, or at least isomorphous. They probably belong to the hexagonal system and are more or less markedly doubly refractive (positively). The most perfect forms are obtained on repeated crystallization, while as intermediary forms spheroids and globulits are commonly encountered. If the crystallization is repeated too often, the crystalline appearance may be lost and the body again becornes amorphous.

Diffusion.-Like the colloids of the inorganic world, so also are the albumins incapable of diffusing through animal membranes or vegetable parchment. This peculiarity Graham explained by the assumption that such bodies do not occur in a state of actual solution. This, however, is not the ease, for it has been shown that albuminous solutions are capable of conducting the electrical current and may exist both as anions and kations-i. e., that they are true solutions. Their inability to pass through animal membranes is explained most likely by the size of the albuminous molecule. This property is very important from the standpoint of chemical technique, as it renders it possible to separate the albumins from a large number of other bodies which may simultaneously be present in solution. Colloids in the soluble state are termed sols; in the solid state, gels. When dissolved in water they are known as hydrosols.

Behavior toward Polarized Light.-All true albumins are lævorotatory, the degree of rotation being different in different members of the group. This fact has been utilized in the identification of the individual albumins, but it is to be noted that unless the examinations can be made with neutral aqueous solutions the resulting data will not be constant. Bülow has definitely proved that the same albuminous solution will show a varying degree of rotation with a varying reaction. Some of the results which have been obtained are given in the following table:

Animal albumins.

Serum-albumin $(a)$ D . . . $-56^{\circ}$

Serum-globulin . . . . $-59^{\circ}-75^{\circ}$

Fribrinogen . . . . . $-43^{\circ}$

Egg-albumin . . . . . $-33^{\circ}-38^{\circ}$

Lactalbumin . . . . $-36^{\circ}-37^{\circ}$

Casein (in $\mathrm{MgSO}_{4}$ solution) . $-80^{\circ}$

Syntonin (from myosin) . $-72^{\circ}$

Alkaline albuminate . . . $-62.2^{\circ}$

Various albumoses . . . . $-70^{\circ}-80^{\circ}$
Vegetable albumins (Osborne).

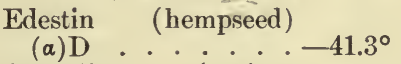

Globulins (various

forms) . . . . . $-38.78^{\circ}-45.21^{\circ}$

Excelsin (Brazilnut) $\quad-42.94^{\circ}$

Amandin (almonds) . . $-56.44^{\circ}$

Corylin (filbert) . . $-43.09^{\circ}$

Zein (maize) . . . $-28.00^{\circ}$

Gliadin (wheat) . . $-92.28^{\circ}$

Phaseolin (kidney bean) $-41.46^{\circ}$

While lævorotation is constant in the true albumins, dextrorotation probably occurs in all nucleoproteids. This has been established directly in the case of the nucleoproteids of the pancreas, the thymus and the adrenal glands (Gamgee, Jones), and Osborne has shown that this property is very likely wholly referable to the nucleinic acid component. The degree of dextrorotation in the substances which thus far have been examined varied between $+37.5^{\circ}$ (nucleohiston from thymus) and $97.9^{\circ}$ (Hammarsten's $\beta$-nucleoproteid of the pancreas). 
Coagulation.-One of the most characteristic properties of the albumins is the physical instability of their solutions and their marked tendency to revert to a solid or semisolid state. This enables them to play the important part which they take in the construction of the various tissues, and no doubt renders possible the manifold and chemically often antagonistic reactions which may simultaneously occur within the bodies of the individual cells. This change may be effected by apparently trivial factors, such as evaporation, contact with porous substances, etc. In this respect also the albumins behave very much like the inorganic colloids. When a solution of sodium silicate is thus added to large excess of dilute hydrochloric acid, the silicic acid which is formed is apparently held in solution. If then the excess of hydrochloric acid, together with the sodium chloride formed during the reaction, is removed by dialysis, a clear solution of silicic acid remains in the dialyzer. This is transformed at once into a thick, gelatinous material when a small amount of carbon dioxide is passed through the solution. Some of the albumins, such as the globulins, behave in a similar way. In undergoing such changes the albumins may retain their original properties for a while at least, but after a variable period they become insoluble, and are then said to be coagulated. 'This change can be brought about at once by the application of heat, and it is important to note that all true native albumins can be coagulated in this manner. After coagulation their solution can only be effected by influences which lead to their more or less extensive destruction. They have lost those physical properties which characterized them individually as albumins; they are permanently denaturized, as Neumeister expresses it.

The temperature of coagulation differs with the different albumins, and is fairly constant for the individual bodies, providing that the reaction of the solution is neutral, or still better very faintly acid. If the reaction is alkaline, coagulation is not complete, and in the presence of more than traces of free alkali or an alkaline carbonate it may not occur at all. A markedly acid reaction interferes in a like manner. Cohnheim explains these peculiarities as follows: On heating the albuminous solution the albumin is denaturized, no matter what the reaction may be or whether salts are present or absent. In the presence of alkalies the denaturized albumins form akaline albuminates, which are readily soluble in the case of the alkalies and with difficulty so in the case of the alkaline eartlss. With an acid reaction, on the other hand, acid albumin is formed, viz., the hydrochlorate, or acetate, of the denaturized albumins, as the case may be; this by itself is soluble in water, but insoluble in the presence of salts.

Equally important is the presence of a certain amount of salt. An albuminous solution that has been freed of salt by dialysis does not coagulate on heating; if, however, salt is subsequently added coagulation occurs. 
Spiro has demonstrated that the temperature of coagulation is materially influenced by the presence of certain nitrogenous bodies, such as cholin, piperidin, pyridin, and anilin, which are all capable of preventing the coagulation of a certain amount of albumin. Urea is even more active in this respect, and when present in sufficient concentration may prevent coagulation altogether. A similar effect is produced by the mustard oils.

Formerly the separation of the different albumins from each other and their identification by fractional coagulation was extensively employed, while at present the method has fallen somewhat into disfavor. This is largely owing to the observation that the temperature of coagulation may vary within fairly wide limits with the amount of salt present and the reaction. It is to be noted, however, that with constant conditions as regards these two factors, and especially in reference to the reaction, the result also will be quite constant. To insure complete precipitation, the reaction should be just acid; if this point has been attended to, the amount of salt is of secondary importance, providing that not too little is present. In any event, in reporting results it is well to note the concentration of the albuminous solution, the strength of the salt solution, and the reaction, together with the temperature of coagulation.

Denaturization.-It has been pointed out that heat coagulation alters the character of all true albumins in such manner that they lose their common properties and are no longer soluble in the usual neutral media. Neumeister has termed the change denaturization of the albumins. This change can also be brought about by other means than heat, such as precipitation with acids, the salts of the heavy metals, the various alkaloidal reagents, shaking with chloroform or ether, alcohol, aceton, and even by prolonged standing. Coagulation is not an essential phase of denaturization. Denaturization may indeed become manifest by the non-occurrence of coagulation. This occurs upon the addition of metallic silver or formalin to albuminous solutions, when coagulation is no longer possible. The albumins in question are then only held in solution if the reaction is acid or alkaline; in a neutral solution they are precipitated. Denaturized and coagulated albumins can only be brought into solution by means which will at the same time produce integral changes in their composition, viz., by means of proteolytic ferments, dilute mineral acids or alkalies, concentrated organic acids under the application of heat, etc.

The nature of the process which determines denaturization is possibly a primary cleavage. In favor of such a view is the observation that coagulated albumin is more readily hydrolized by ferments. Nothing certain, however, is known.

Behavior toward Neutral Salts. - All albumins and albumoses can be precipitated from their solutions by means of certain neutral 
salts, without loss of their characteristic properties or change in structure. The resulting precipitates are soluble as before. This behavior toward neutral salts is not characteristic of albumins, however, as every substance can, generally speaking, be withdrawn from its solution by a second body, but owing to the great molecular size of the albuminous molecule these bodies are thrown out of solution more readily than others.

The salts which are usually employed in the chemical laboratory for the precipitation of the albumins are sodium chloride, sodium sulphate, magnesium sulphate, and especially ammonium sulphate and zinc sulphate. The two latter are especially important, as they are universal precipitants, while the other salts and many others, which have not been mentioned, will throw down only certain individual albumins. Generally speaking, the more highly differentiated albumins can be precipitated by the least active salts, such as sodium chloride and magnesium sulphate, while for the complete precipitation of the less complex albumins (serum-albumin) and certain albumoses ammonium sulphate or zine sulphate is necessary. The more complex the albumin the more readily it is precipitated, so that with certain bodies, such as fibrinogen, casein, and other nucleo-albumins complete saturation is not necessary. In their behavior toward ammonium sulphate these bodies differ from the less complex albumins in the fact that they require a smaller amount of the salt for their precipitation. The simpler the structure, on the other hand, the more salt is necessary. The deutero-albumoses, which probably represent the least complex bodies, which still have a distinct albuminous character, can only be precipitated by complete saturation with ammonium or zinc sulphate, and with one of their number it is necessary to carry out the saturation in acid solution, while all other albumins and albumoses can be thrown down from their neutral solutions.

Magnesium sulphate occupies a position intermediate between sodium chloride and ammonium sulphate, and readily precipitates both globulins and primary albumoses.

The behavior of the different albumins to neutral salts, and notably to ammonium and zinc sulphate, is now largely utilized in their differentiation from each other and the identification of the individual bodies. It has been ascertained that under certain conditions each albumin has a definite lower and upper limit of precipitation, which for that body is quite constant-i. $e_{\text {., for a given }}$ volume of the solution a definite amount of salt is necessary, beneath which no precipitation occurs-the lower limit, and beyond which no further precipitation takes place-the upper limit. In conformity with Hofmeister's suggestion this examination is now generally conducted as follows: A series of test-tubes is prepared which are successively charged with a constant number of c.c. (2) of the albuminous solution ( 2 per cent.) and increasing amounts of 
a saturated neutral solution of ammonium sulphate, the misture being diluted in each case to 10 c.c. with distilled water. The contents of each tube are thoroughly mixed and set aside for one-half hour, when a further examination is made. In the first series it is well to start with a set of tubes containing $0.5,1.0,1.5,2.0,2.5$, etc., c.c. of the sulphate solution. At the end of a half hour the contents of each tube are passed through small filters and the filtrate treated with an additional $\frac{1}{10}$ c.c. of the salt solution. Supposing that tube 1 containing 0.5 c.c. presented no turbidity, but that this was marked in tube 2 , then it is manifest that the lower limit of precipitation lies between 0.5 and 1 . If now the filtrate of tube 1 after the addition of 0.1 c.c. of the salt solution and standing shows a turbidity, the lower limit for this particular albumin is called 0.6. In a similar manner the upper limit is determined. Supposing that the filtrate of the tube containing 3.5 c.c. of salt solution becomes turbid on adding 0.1 c.c. further, while in the filtrate of a tube containing 3.6 c.c. salt solution from the first, no turbidity developed, it is clear that 3.6 represents the upper limit.

In the following table the limits of precipitation of some of the more important albumins have been collected :

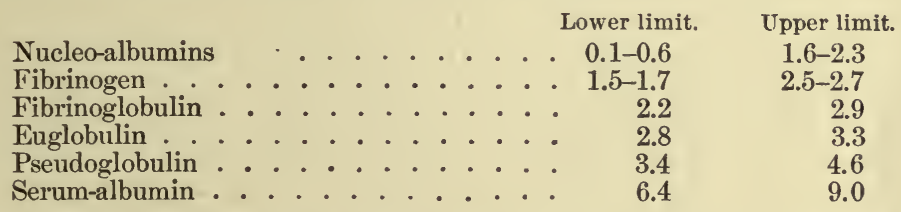

Especially characteristic and constant is the upper limit, while the lower limit occasionally fluctuates somewhat. In acid solutions there is a general lowering of the limits of precipitation; in this case the albumins are not precipitated as such, but as salts, the albumins playing the part of a base.

After precipitation the albumins tenaciously hold a certain amount of the salt, which can scarcely be removed, even on prolonged dialysis. Noteworthy also is the fact that certain albumins are capable of abstracting the acid of the particular salt which is employed, to form compounds from which the acid in question cannot be removed on washing with water. This peculiarity is especially well marked in the case of serum-albumin, and probably accounts for the observation that on repeated crystallization from ammonium sulphate solution the mixture of albumin and sulphate becomes more and more acid, while at the same time ammonia is liberated (G. Mever). Such acid compounds of proteins can apparently also occur in nature, as has been shown in the case of horn and human hair (Mörner). This property of abstracting acids from the corresponding salts and uniting therewith should not be confounded with the power of certain albumins of combining with free acids directly, which has been demonstrated by Sjöqvist, Cohn- 
heim, and others. These compounds originate only in the presence of free acids as such.

Behavior toward Alcohol.-While some of the albumins (albumoses) dissolve in dilute alcohol with comparative ease, strong alcohol acts in much the same manner as the neutral salts. But it is to be noted that after prolonged exposure, and especially in the presence of salts, the albumins are coagulated, and then remain refractory to all neutral solvents.

\section{Special Reactions of the Albumins.}

Precipitation.-It has been pointed out that with the exception of the peptones practically all albumins ean be precipitated from their neutral or feebly acid solutions by certain neutral salts. As a result of this process they apparently undergo no change in structure or in their general properties, and remain soluble in the usual neutral media. There is a large number of substances, however, which also precipitate the albumins, but which either cause their coagulation or combine with them to form compounds which are insoluble in water. Many of these reagents are extensively used in the chemical laboratory in testing for albumins ; they furnish reactions which individually are not absolutely characteristic, but the albuminous nature of a substance can usually be regarded as established when a positive result is obtained with all or at least the larger number of the reagents. The reactions are common to the true albumins, the proteids, and the majority of the albumoses.

The most important reagents are the following:

1. The mineral acids, viz., nitric, hydrochloric, sulphuric, and metaphosphoric acid. These are employed in concentrated form. The one most commonly in use is nitric acid. The test is conducted by allowing a small amount of the acid to flow beneath the fluid to be tested, when a white ring of coagulated albumin appears at the zone of contact (Heller's test). In the case of the true albumins, all of which give the reaction, the precipitate is insoluble in an excess of the acid, even on heating. But with the albumoses, which in part are also precipitated, the precipitate dissolves on boiling and reappears on cooling.

2. The Salts of the Heavy Metals.-In combining with these the albumins play the part of weak organic acids. They set free the corresponding acids of the salts and combine with the metallic oxides to form compounds which are insoluble in neutral, alkaline, and acid solutions. With the exception of myogen, hæmoglobin, and certain albumoses, the resulting precipitates are as a rule insoluble in an excess of the reagent.

The salts which are usually employed are the sulphate and acetate of copper, the chloride and acetate of iron, the neutral and basic acetate of lead, the bichloride of mercury, nitrate of silver, acetate of uranium, acetate of zinc, chloride of platinum, etc. All 
of these may be used in moderately concentrated solution, and are added directly to the albuminous fluid.

Especially important are the salts of iron, copper, and lead. If ferric chloride is added to an albuminous solution containing an excess of sodium acetate, until a distinct red color results, the albumins are completely precipitated on boiling. An excess of the iron must be avoided, as the precipitated albumins will otherwise dissolve.

Acetate of copper precipitates all true albumins and serves to separate the primary from the secondary albumoses; an excess should be avoided.

On boiling albuminous solutions with hydroxide of lead in the presence of acetate of lead complete precipitation occurs.

Bichloride of mercury precipitates not only the true albumins and albumoses, but also the peptones.

The Alkaloidal Reagents. - The most important of these are phosphotungstic and phosphomolybdenic acid, mercuropotassic iodide, bismuthopotassic iodide, and cadmium-potassic iodide, all of which precipitate albumins in the presence of a mineral acid. Further, tannic acid, picric acid, and potassium ferrocyanide as well as ferricyanide, in the presence of acetic acid, trichloracetic acid, etc. These various reagents are used in from 5 to 10 per cent. solutions, after acidifying the albuminous solution with a mineral acid, or acetic acid of moderate strength as indicated.

The histons and protamins, which are more markedly basic than the other classes of albumins, are precipitated even with a neutral or slightly alkaline reaction. But all precipitates dissolve when the reaction is markedly alkaline. In an excess of the alkaloidal reagent only the peptones and some of the albumoses dissolve.

The precipitation by the alkaloidal reagents is attributed to the diamino-complexes of the albuminous molecule, viz., the more markedly basic radicles.

Color Reactions.- The color reactions to be described are individually not peculiar of the albumins, but merely indicate the presence of certain atomic complexes which in themselves are capable of producing the reactions. Collectively they are characteristic, however, and are of special interest as they permit a partial insight into the structure of the albuminous molecule.

1. The Biuret Reaction.-The test is conducted as follows: A few c.c. of the solution to be examined are treated with an excess of a concentrated solution of sodium or potassium hydrate, and then drop by drop with a 2 per cent. solution of copper sulphate. In the presence of native albumins a bluish or reddish-violet color results, while with albumoses, peptones, histons, and certain vitellins the color is a pure red. With larger amounts of albumin the reaction is obtained without difficulty; if traces only are present, great eare. must be had not to add too much of the copper solution as otherwise the blue color of the reagent may obscure the reaction. In 
such an event it is well to use a still more dilute solution of the copper sulphate. Where larger amounts are present, it is necessary to add more of the reagent. An excess of the neutral salts which are often present when the test is employed does not interfere with the reaction. With ammonium sulphate, however, it is necessary to use a large quantity of the caustic alkali to bring out the color. Should magnesium sulphate be present, a precipitate of magnesium hydroxide results on adding the alkali, and is allowed to settle.

The resulting color, according to Schiff, is due to the formation of biuret potassium cupric oxide:<smiles>[X]NC(=O)NC(=O)C(=O)NO</smiles>

In the place of sodium or potassium hydrate other substances may also be used in the test, some of which are only feebly or indeed scarcely alkaline, such as barium and calcium hydrate, the carbonates of the alkalies, ammonia, magnesium oxide, trimethylamin, coniin, piperidin, atropin, etc. Instead of copper salts, nickel salts may also be used; in that case no red color but an orange yellow is obtained.

The biuret reaction is dependent upon the presence in the albuminous molecule of $\mathrm{CH}_{2} \cdot \mathrm{NH}_{2}-$

$\mathrm{CO}-\mathrm{NH}$ groul such radicles are present-e. $g$.,

glycinamin :

sarcosinamin :<smiles>CC(N)CN</smiles>

$\mathrm{CH}_{2}-\mathrm{NH}\left(\mathrm{CH}_{3}\right)$

$\stackrel{\mathrm{CO}}{\mathrm{C}-\mathrm{NH}_{2}}$

the diamin of asparaginic acid:

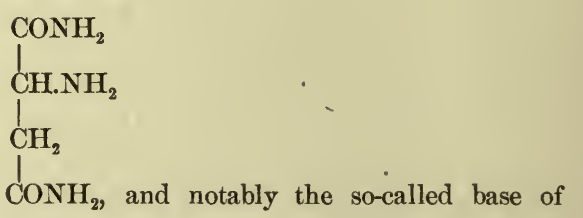

Curtius: $\mathrm{NH}_{2} \cdot\left(\mathrm{CH}_{2} \cdot \mathrm{CO} \cdot \mathrm{NH}\right)_{6} \cdot \mathrm{CH}_{2} \cdot \mathrm{CO}_{2} \cdot \mathrm{C}_{2} \mathrm{H}_{5}$.

Hofmeister has pointed out that there is excellent evidence to support the belief that in the albuminous molecule the component anhydrides of the $\alpha$-amino acids are united to each other by $-\mathrm{CO}-\mathrm{NH}-\mathrm{CH}=$ groups, and it appears that the same atomic grouping which in the albumins forms the basis of the biuret reaction is also the point of attack in their cleavage by the digestive 
ferments. This supposition is materially strengthened by the interesting observations of Schwarzschild that pure trypsin is capable of altering the base of Curtius in such manner that this no longer gives the biuret reaction, while it is incapable of splitting off ammonia from acid amides, such as acetamide, asparagin, and others.

2. The Xanthoproteic Reaction.-A few c.c. of the solution to be tested are treated with concentrated nitric acid, when in the presence of albumin a yellow color develops either at once or upon heating. With some albumins a white flaky precipitate develops at the same time, while with others the solution remains clear. Upon the subsequent addition of an excess of ammonia the color turns orange, or with caustic soda, a reddish brown.

The reaction depends upon the formation of certain nitro derivatives, and is referable to the presence in the albuminous molecule of a tyrosin group or of an indol complex. It is accordingly not characteristic of albumins alone, and can be obtained with many other substances.

3. Millon's Reaction.-The reagent is a solution of mercuric nitrate containing a little nitrous acid. It is prepared by dissolving a few grammes of mercuric nitrate in an amount of water which is just sufficient for its solution. Any kasic salt that may be present is dissolved with fuming nitric acid, when a solution of sodium acetate is added, drop by drop, until the reagent gives a red color on boiling with a few drops of a dilute solution of phenol.

The test is conducted by adding a few drops of the solution to be examined to a few c.c. of the reagent, when in the presence of albumins a white precipitate forms, which turns a brick red on boiling. If the substance to be examined is a solid, this is suspended in a few drops of water and treated in the same manner.

Millon's reaction is common to all benzol derivatives, in which one hydrogen atom has been replaced by a hydroxyl group; it is consequently obtained with all albumins which on tryptic digestion yield tyrosin. This radicle is absent in glutin and in those albumoses which contain the hemi-group (see later), and these bodies accordingly do not give the reaction.

4. The Reaction of Adamkiewicz.-A particle of the dry albuminous substance which should contain as little fat as possible is dissolved in about 6 c.c. of glyoxylic acid by the aid of heat, and underlayed with an equal volume of concentrated sulphuric acid. Immediately or on boiling red, green, and violet rings form at the zone of contact with the sulphuric acid, and on shaking the entire fluid turns violet. At the same time it becomes slightly fluorescent, and on spectroscopic examination gives a broad band extending from yellow to blue. Albuminous solutions are similarly treated.

The reaction is referable to the tryptophan complex, viz., to skatol-amino-acetic acid (Hopkins and Cole).

Neubauer and Rohde suggest the following reaction as a test for the tryptophan complex: An aqueous solution or suspension of 
albuminous material is treated with 5-10 drops of a 5 per cent. solution of $p$-dimethyl-amino-benzaldehyde (in 10 per cent. sulphuric acid); upon the further addition of concentrated sulphuric acid (drop by drop, quick shaking) a reddish violet results, which soon changes to a beautiful dark violet. On spectroscopic examination a broad band is seen in the orange, and a second one, less distinct, in the green portion.

5. Liebermann's Reaction.-The albuminous material after extraction with hot alcohol and subsequently with ether (to remove fats) is boiled for a few minutes with concentrated hydrochloric acid to which a drop of concentrated sulphuric acid has been added. The albumin passes into solution and the fluid assumes a deep-blue or violet-blue color.

The reaction is likewise a furfurol reaction, and has also been ascribed to the simultaneous presence of a carbohydrate and an oxyphenyl group. Like the Adamkiewicz reaction, it may be dependent upon the tryptophan complex.

6. Molisch's Reaction. - A small amount of the albuminous material is suspended in about 1 c.c. of water, treated with a few drops of a 15 per cent. alcoholic solution of $\alpha$-naphthol, and underlayed with concentrated sulphuric acid. A violet color results at the zone of contact, and is assumed by the entire bulk on shaking. Upon the addition of alcohol, ether, or caustic alkali it turns to yellow (on standing).

Thymol can be used in the place of the $\alpha$-naphthol; it gives a carmin color, which on dilution turns to green.

Like the two preceding reactions this one also is a furfurol reaction; but unlike the others it is referable exclusively to the presence of a carbohydrate group. It is positive with most albumins, but negative with casein.

7. The Sulphur Reaction.-A small amount of the albuminous solution or suspension is treated with an excess of a concentrated solution of sodium hydrate and a few drops of lead acetate solution. On boiling, according to the amount of sulphur present, the entire fluid turns a more or less marked brown, and on standing a precipitate of sulphide of lead results.

The reaction is referable to a sulphur group, which is present in the albuminous molecule as a cystin radicle. As all albumins contain sulphur it is obtained with all, though to a more or less marked degree.

Structural Composition.-Our knowledge of the structural composition of the albumins is largely the outcome of a study of the decomposition-products obtained by hydrolysis, with boiling mineral acids and alkalies, by boiling with water under pressure, and by digestion with proteolytic ferments. As a result it has been possible to establish the existence in the albuminous molecule of certain radicles which, with few exceptions, are common to all forms. The physical differences of the various forms are probably the 
outcome of quantitative variations and of differences of chemical union, rather than of qualitative composition. These radicles may be classified as follows :

I. Radicles of the aliphatic series.

1. Amino acids :

(a) Mono-amino acids.

(a) Radicles of mono-amino-monocarbonic acids; the leucin group. The most notable radicle of this order is leucin ( $\alpha$-aminoisobutyl-acetic acid); it predominates quantitatively and is present in all typical albumins. Next in frequency follow glycocoll (aminoacetic acid) and alanin ( $\alpha$-amino-propionic acid) ; more rarely aminovalerianic acid and possibly also isoleucin ( $\alpha$-amino-methyl-ethyl propionic acid).

( $\beta$ ) Radicles of mono-amino-dicarbonic acids; the glutaminic acid group. Of these, aspartic acid ( $\alpha$-amino-succinic acid) and glutaminic acid ( $\alpha$-amino-glutaric acid) are probably never absent in typical albumins, the latter predominating in quantity.

(r) Radicles of mono-amino-oxymonocarbonic acids; only one member of this group has been found, but is probably present in all albumins, viz., serin ( $\alpha$-amino- $\beta$-oxypropionic acid).

(b) Diamino acids.

Two radicles belonging to the diamino-monocarbonic acids have been found, viz., ornithin ( $\alpha$ - $\delta$-diamino-valerianic acid), which is obtained on hydrolysis with acids in combination with the guanidin remnant in the form of arginin; and lysin ( $\alpha$ - $\varepsilon$-diamino-capronic acid).

In addition, a representative of the diamino-oxymonocarbonic acids has been encountered (in casein), viz., diamino-trioxy-dodecanic acid.

(c) Sulphur-containing amino acids.

The sulphur complex of the albumins is usually obtained on hydrolysis as cystin, viz., as the disulphide of cystein ( $\alpha$-amino- $\beta$ thiolactic acid; hence $\alpha$-diamino- $\beta$-dithio-dilactic acid). From serum-albumin and the keratins Friedmann further obtained the non-nitrogenous $\alpha$-thiolactic acid, and suggests that this also may occur in the albuminous molecule preformed. Some albumins further contain sulphur radicles, which are obtained as mercaptans or as ethyl sulphide.

(d) Histidin ( $\alpha$-amino- $\beta$-imidazol-propionic acid).

2. Amino alcohols (hexosamins, the carbohydrate group of the albumins).

In many but not in all albumins there is a radicle which on hydrolysis appears as a hexosamin and apparently always as chitosamin (glucosamin). In its place or in addition there may be other nitrogenous or non-nitrogenous carbohydrate radicles.

II. Radicles of the aromatic series.

1. To judge from the constancy with which phenyl-alanin is encountered among the hydrolytic decomposition-products there 
can be no doubt that a phenyl- $\alpha$-amino-propionic acid radicle is present in all albumins.

2. The same is true of a tyrosin radicle (oxyphenyl-alanin$p$-oxyphenyl- $\alpha$-amino-propionic acid).

III. Heterocyclic radicles.

1. Radicles of the pyrrol group. Through the researches of E. Fischer and his pupils it has been shown that a prolin radicle ( $\alpha$-pyrrolidin carbonic acid) is represented in all albumins; oxyprolin (oxy-a-pyrrolidin carbonic acid) is usually obtained at the same time.

2. Radicles of the indol group. The indol group is probably represented in the albuminous molecule by the tryptophan complex, which Hopkins and Cole have identified as skatol-amino-acetic acid. This radicle is the mother-substance of indol, skatol, skatol-carbonic and skatol-acetic acids, all of which are formed during albuminous putrefaction and in part also on fusion with caustic alkali. The kynuric acid of dogs' urine likewise ( $\alpha$-oxy- $\beta$-quinolin carbonic acid) is a derivative of tryptophan, as also possibly skatosin, which has been encountered among the products resulting from pancreatic autolysis, as also on prolonged peptic digestion of serum-albumin.

The presence of the various radicles just considered has been largely estahlished by a study of the decomposition-products of the albumins which result on hydrolysis, by means of boiling mineral acids and alkalies, proteolytic ferments, etc. Other methods in part at least yield other end-products, which, however, so far as they have been studied, can readily be reduced to the same radicles that are furnished by hydrolysis.

As a result of putrefactive changes indol, skatol, skatol-carbonic acid, and skatol-acetic acid are obtained, all of which are derivatives of the tryptophan complex ; further, phenyl-propionic (hydrocinnamic) acid, phenyl-acetic acid, and phenyl-ethylamin, all three derivatives of phenyl-amino-propionic acid; further, para-oxyphenyl-propionic (hydroparacumaric) acid, para-oxyphenyl-acetic acid, cresol, and phenol, all derivatives of para-oxyphenyl-amino-propionic acid (tyrosin); then $\alpha$-amido-valerianic acid and putrescin, derivatives of arginin; cadaverin from lysin; hydrogen sulphide from cystin, etc.

On oxidation with potassium bichromate and sulphuric acid benzoic acid has been obtained ; with barium permanganate guanidin is formed.

On fusion with caustic alkali leucin, tyrosin, indol, skatol, hydrogen sulphide, and methyl mercaptan are quite constantly obtained, the most notable being indol and skatol, whose formation under these conditions is generally regarded as one of the most characteristic reactions of the albumins.

With any method, of course, products are also obtained which are not primary components of the albuminous molecule, but which result on further destruction of the essential radicles. Such bodies are carbon dioxide, oxalic acid, formic acid, acetic acid, butyric acid, isovaleric aldehyde, acetone, ammonia, hydrogen sulphide, etc., 
. As regards the manner in which the various radicles of the albuminous molecule are linked together, our knowledge, while still imperfect, has been materially advanced within recent years through the researches of $\mathrm{E}$. Fischer and his pupils. As a result there is evidence to show that the $\alpha$-amino acids exist in combination with each other as so-called peptids, which have the general structure $\mathrm{NH}_{2} \cdot\left(\mathrm{CH}_{2} \cdot \mathrm{CO} \cdot \mathrm{NH}\right)_{n} \cdot \mathrm{CH}_{2} \cdot \mathrm{COOH}$, the amino group of one amino acid having united with the carboxyl group of the other (with coincident loss of water). The simplest product of this order, glycyl-glycin, would be formed according to the equation :

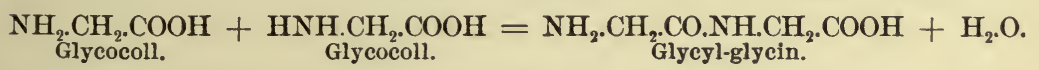

That the albuminous nitrogen is largely present in the intact molecule as an imido group is shown by the fact that only a small fraction can he readily split off as ammonia, while approximately 90 per cent. remains and on hydrolytic decomposition appears in the form of amido-acids. On treating with nitrous acid similarly only a small amount of nitrogen is split off, while a body remains in which the albuminous character is still preserved to a large extent, but which only gives an imperfect biuret reaction-the desamidoalbumin of Schiff.

Fischer's theory of the presence in the albuminous molecule of the amino-acids in the form of peptids, constructed on the plan just outlined, is strengthened by his successful synthetic preparation not only of dipeptids (glycyl-alanin, alanyl-leucin, leucyl-glycin, etc.), but also of tripeptids (leucyl-glycyl glycin, leucyl-alanyl alanin), tetrapeptids (dileucyl-glycyl-glycin), and even pentapeptids (pentaglycin, leucyl-tetraglycin, etc.). The higher peptids, it is interesting to note, give the biuret reaction (e. g., the tetrapeptid of glycin, dialanyl cystin, leucyl-pentaglycin). On careful hydrolysis of silk collagen with cold hydrochloric acid and subsequent digestion with pancreatin Fischer obtained a peptone-like body, moreover, which could be shown to be glycyl- $\alpha$-alanin.

Still more recently Fischer and Abderhalden have shown that on digestion of various albumins (casein, edestin, serum-globulin, eggalbumin, hæmoglobin, and fibrin) with pancreatin a polypeptid-like body remains, which, on hydrolysis with acids, yields a large amount of $\alpha$-pyrrolidin carbonic acid, phenylalanin, and all the glycocoll which was originally present in the albuminous molecule.

Through the union of the $\alpha$-amino-peptids with diamino-acids, the dicarbonic acids, the sulphur-containing cystin, and the glucosamin group, etc., still more complex radicles result, of which we are in comparative ignorance as yet. But we can conceive that these various complexes may then be further united to form still more complicated radicles, until at last the complete molecule is constructed. In the typical albumins we can distinguish three such complicated radicles, two of which, in conformity with the nomen- 
clature established by the Kühne school, we may designate as the hemi- and the anti-complex respectively, while the third is less constant and differs from the hemi- and anti-group in the presence of a carbohydrate radicle and a larger percentage of oxygen, while the amount of nitrogen and carbon is less than in the two other groups. These three fundamental radicles are represented by the three albumoses, which result primarily on proteolytic digestion, viz., proto-albumose, hetero-albumose, and gluco-albumose. Of these, proto-albumose represents the hemi-group, hetero-albumose the anti- group, and gluco-albumose the third complex, which contains the carbohydrate radicle. The essential points of difference between the hemi- and anti- complex are the following:

Hemi-group.

Diamino-acids in small amount.

Mono-amino acids in large amount.

Little or no leucin.

No glycocoll.

Much tyrosin.

Much skatolamino-acetic acid (tryptophan).

No carbohydrate group.

Contains same amount of sulphur as original albuminous molecule, but only in loosely combined form.
Anti-group.

Diamino-acids in large amount.

Much leucin.

Entire amount of glycocoll of original albumin.

No tyrosin.

No skatolamino-acetic acid.

No carbohydrate group.

Sulphur the same as in hemi- group.

The anti- group owes its name to the great resistance which it offers to further decomposition, by oxidizing agents, mineral acids, alkalies, and proteolytic ferments, notably trypsin, all of which readily decompose the hemi- group into its components.

The extent to which the hemi-, the anti-, and the gluco-group are represented in the different albumins differs to a considerable extent. Caseïn is generally regarded as a pure hemi- body ; it contains no glycocoll, but more tyrosin than all true albumins. Collagen contains much glycocoll, but no tyrosin, indol, or tryptophan radicles, etc.

Of the various radicles which do not belong to the amino-acids, either of the aliphatic or the aromatic series, two complexes deserve especial consideration, namely, those which represent the sulphur group and the carbohydrate group of the albuminous molecule.

The Sulphur Group.-Sulphur is present in all true albumins and in most albumoses. According to older views, it exists in two forms, one of which can be readily split off as hydrogen sulphide hy means of caustic alkali of moderate strength, while the other can only be demonstrated after the complete destruction of the albuminous molecule and then appears in the form of sulphuric acid. It has therefore been customary to speak of a loosely combined and a firmly combined group. It has further been regarded as an established fact that the loosely combined sulphur is present in the intact molecule in the form of a cystin complex, while nothing was known 
of the form in which the so-called oxidized sulphur existed. Mörner has shown that in certain albunins the cystin-yielding complex comprises not only the lonsely combined, but also a portion at least of the firmly united variety. In the case of the keratins of horn and hair it seems quite likely indeed that the sulphur is present only in this form. 'The shells of birds' eggs, on the other hand, if they represent a chemical unity at all, contain several sulphur atoms, which are present in at least two forms, and only one of which appears to be represented by the cystin-yielding group; this portion corresponds to three-quarters of the total sulphur, while one-quarter is present in some other form. But even here the cystin sulphur includes a certain portion of the firmly combined sulphur. The same is true of serum-globulin, while in fibrinogen and ovalbumin at least one other sulphur complex appears to exist in addition to the one which is obtained on hydrolysis as cystin. The latter in the case of fibrinogen represents one-half of the total sulphur, and in the case of ovalbumin only one-third, while the remaining two-thirds exist in a yet unknown form ; onethird of this in turn may under certain circumstances escape in volatile form, which, however, is not hydrogen sulphide.

In the albuminous molecule the disulphide cystin is the primary radicle, and not cysteïn ; it hence contains at least two molecules of sulphur. Of other sulphur compounds which have been obtained from the albumins, there may be mentioned thiolactic acid, thioglycolic acid, ethyl sulphide, and ethyl- and methyl-merkaptan. To what extent these bodies occur as radicles in the albuminous molecule is an open question. In the case of those albumins in which the sulphur is only present in the cystin form they could only be secondary, but in the albumins which contain more than one sulphur group some of them at least might occur preformed.

Oxidized sulphur-viz., sulphur combined with oxygen-does not occur in the albumins.

The amount of sulphur differs in the different albumins, as seen in the following table (see also table on p. 46):

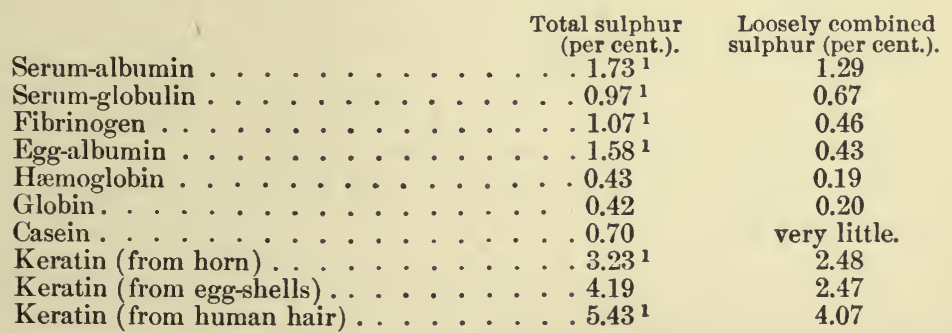

The Carbohydrate Group.-To judge from recent researches a carbohydrate group is present with few exceptions in all albumins. It

\footnotetext{
${ }^{1}$ After deducting sulphur present as $\mathrm{SO}_{3}$.
} 
characterizes one of the three large molecular complexes which together form the albuminous molecule, viz., the gluco-complex. As has been seen, this can be obtained as a primary product of proteolysis in the form of a gluco-aibumose, which contains the entire carbohydrate group of the original molecule. Notably in the mucins and mucoids, but also in some of the native albumins in the narrower sense of the term, such as ovalbumin, serum-albumin, and the mixed globulins of the blood-serum, this complex is obtained on hydrolysis as an amino-sugar, and usually as glucosamin; less commonly, as in the case of the mucin derived from the frog's socalled albuminous gland, as a galactosamin. These bodies, however, probably do not occur preformed in the albuminous molecule, but as higher carbohydrates, viz., as disaccharides or polysaccharides. According to Müller, the mother-substance of glucosamin is a polymeric acetylated glucosamin (Fränkel's albamin).

Whether or not the glucosamin complex is found in all albumins in which a carbohydrate radicle can be demonstrated, is not known. In any event it is not the only carbohydrate group that may occur. This is notably the case with the nucleoproteids, all of which apparently contain a pentose group. This group is a part of the nucleinic acid radicle of the nucleoproteids, and it is possible that an additional carbohydrate group (perhaps glucosamin) may be contained in the albuminous radicle which is in combination with the nucleinic acid complex. Kossel could demonstrate the presence of a hexose also in the nucleoproteid of the thymus, of yeast, and in the nucleinic acid of the sturgeon's testicles, by isolating lævulinic acid from the products of decomposition. The pentose obtained from the nucleoproteid of the pancreas was $l$-xylose.

The amount of reducing substance (glucosamin) which can be obtained from the different albumins differs considerably. The largest quantities have been obtained from mucins and mucoids (30-40+ per cent.). This fact has led to the establishment of a separate group of albumins, the gluco-albumins (glucoproteids), which is distinguished in this manner from the common albumins. This division is quite arbitrary, as the latter also contain a carbohydrate group, though it is present. in most of them in much smaller amount (serum-albumin 0.5 per cent.). Crystallized ovalbumin with 15 per cent. occupies an intermediate position, inclining toward the mucoids. Casein is apparently the only animal albumin which is without a carbohydrate group, while among vegetable albumins, according to Osborne, this is more frequently the case.

Regarding the quantitative distribution of the primary radicles in the different albumins our knowledge is still quite defective, but with better methods is rapidly becoming extended. The more important data have been embodied in the accompanying table. From this it will be seen that barring the protamins all albumins have essentially the same component radicles, but that the individual 
amino-acids are present in the different substances in somewhat different quantitative proportions. To these variations the varying properties of the different substances are no doubt due. Noteworthy is the absence of glycocoll in serum-albumin and egg-albumin, and of tyrosin and tryptophan in glutin. Most remarkable is the extraordinary predominance of the diamino-acids in the protamins and the low values found in the albuminoids. Note further the high value of glycocoll and alanin in silk fibroin, and of cystin in keratin. The percentage values of the resolved portion of the different albumins, when compared with our knowledge of a few years ago, show a most encouraging advance all along the line, but also disclose deep gaps which must yet be bridged.

Molecular Size.-Our knowledge of the molecular size of the protein molecule is as yet very imperfect, and the figures given should only be regarded as approximative, minimal values :

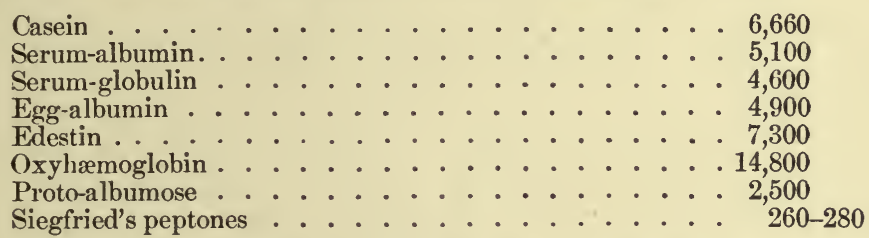

For cystallized egg-albumin Hofmeister calculated the formula $\mathrm{C}_{239} \mathrm{H}_{386} \mathrm{~N}_{58} \mathrm{~S}_{2} \mathrm{O}_{78}$, which would correspond to a weight of 5378 .

Classification of the Albumins.-A satisfactory classification of the proteins upon a chemical basis is not yet possible, and we are hence forced to adhere to their old division into groups, which is essentially based upon their gross physical properties. We accordingly divide them into five classes, viz., the native albumins, the nucleo-albumins, the proteids, the albuminoids, and the derived albumins. These can be subdivided further as follows:

I. The native albumins :

1. The albumins proper (serum-albumin, egg-albumin, lactalbumin).

2. The globulins (serum-globulin, fibrinogen, ovoglobulin, myosin, myogen, lactoglobulin, various cell-globulins, and vegetable globulins).

3. The gluco-albumins (mucins, mucoids, helicoproteid).

4. The keratins.

5. The histons.

6. The protamins.

II. The nucleo-albumins (caseïn, the vitellins, phytovitellins, the nucleo-albumins of the cell-protoplasm, mucinous nucleo-albumins).

III. The proteids :

1. The nucleoproteids (compounds of nucleinic acid with $(a)$ histon, $(b)$ protamin, $(c)$ other albumins.

2. The hæmoglobins (compounds of a histon with hæmatin). 


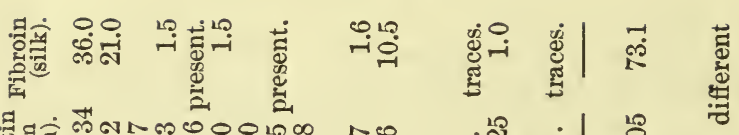

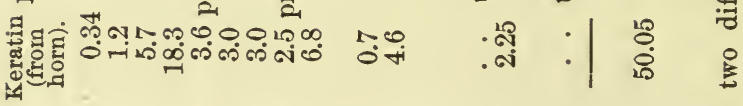

苞

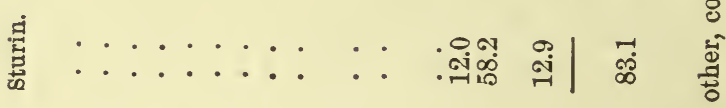

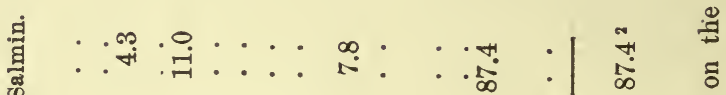
同

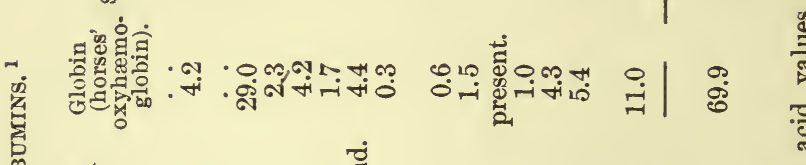

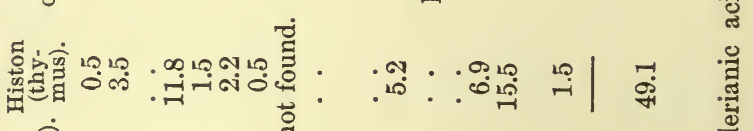

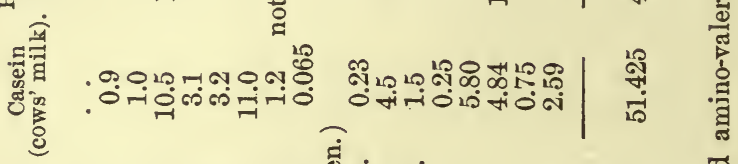

商 竞

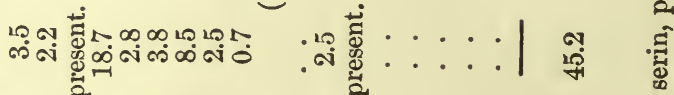

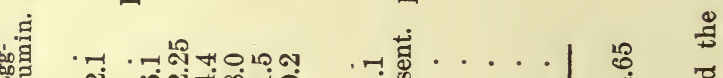

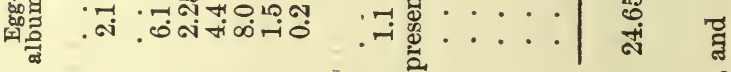

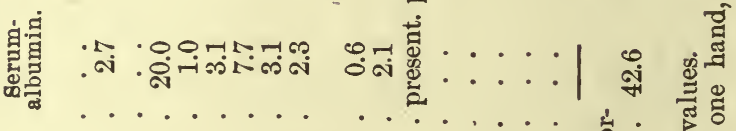

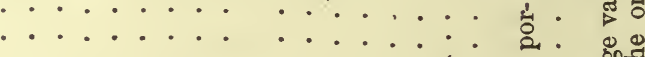

........... . . . . .

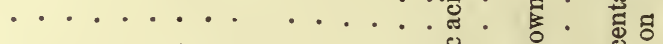

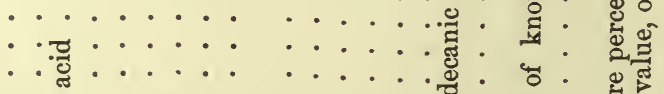

$\because \because \frac{0}{0} \cdot \ldots$

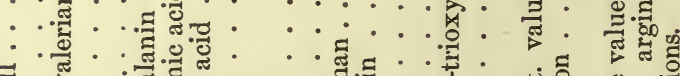
हี.

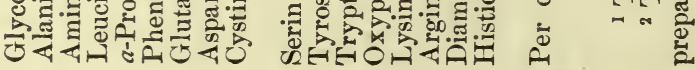


IV. The albuminoids (collagen, elastin, spongin, fibroin, amyloid, albumoid, pigments which are derived from albumins).

V. The derived albumins :

1. The coagulated albumins.

2. The albuminates.

3. The albumoses.

4. The peptones and protones.

\section{THE NATIVE ALBUMINS.}

The general physical and chemical properties of the native albumins as a class have been described in the foregoing pages. The remarks which follow apply more particularly to the various subdivisions, as follows :

The Albumins.-The albumins, in the narrower sense of the term, comprise serum-albumin, egg-albumin, and lactalbumin. They are soluble in water, dilute saline solution, and in dilute acids and alkalies. They are precipitated from their solutions with greater difficulty than the globulins and many proteids. Sodium chloride and magnesium sulphate cause their precipitation only if the reaction is acid; in neutral solutions no precipitation occurs. Ammonium sulphate precipitates the albumins upon complete saturation; the limits of precipitation are 6.4 and 9 , the reaction being neutral; if acid, they are a little lower.

All the members of the group are coagulated by heat.

One of the most interesting properties of the albumins is their ability to crystallize.

To hydrolytic decomposition by mineral acids and proteolytic ferments the albumins are comparatively resistant. Notable is the large amount of sulphur of serum-albumin and egg-albumin; with the exception of the keratins these bodies contain more sulphur (1.6 to 2.2 per cent.) than any other proteins. The end-products of hydrolysis are typical of the proteins as a class.

The Globulins.-These comprise the various globulins of the blood (the serum-globulins and fibrinogen), ovoglobulin, lactoglobulin, myosin, myogen, various cell-globulins, and vegetable globulins. They are all soluble in dilute solutions of the alkalies and neutral salts, while as a class they are insoluble in distilled water and dilute acids. There is one notable exception, however. If a dilute saline solution of the common serum-globulin of the blood-plasma, for example, is subjected to dialysis, a certain portion of the globulin is precipitated (euglobulin), while another portion remains in solution, and may be demonstrated by saturating with magnesium sulphate or by halfsaturation with ammonium sulphate (pseudoglobulin). With the exception mentioned, the globulins are precipitated from their solutions by dialysis, on copious dilution with water, by acidifying with a dilute mineral acid or with acetic acid, and even by passing a stream of carbon dioxide through the solution. By saturation with 
magnesium sulphate they are completely precipitated; with sodium chloride only in part. For ammonium sulphate the limits are 2.9 and 4.6. It will thus be noted that as a class the globulins can be readily separated from the albumins by half-saturation with ammonium sulphate. Immediately after precipitation by the various methods indicated the globulins are still soluble in dilute saline solution; but it is noteworthy that after a while they tend to lose their solubility and become coagulated (denaturized); this also occurs when they are long kept under water. A number of the globulins show an acid reaction.

Like the albumins, the globulins are coagulated by heat; and on hydrolysis, qualitatively at least, they yield the same end-products. Their sulphur content is less than that of the albumins, but not less than 1 per cent.

Certain members of the group are apparently much more highly differentiated than serum-albumin (lactoglobulin, ovoglobulin, thyreoglobulin, edestin).

The Gluco-albumins.-These comprise the mucins and mucoids, the most notable members of which are the metalbumin and paralbumin of ovarian cysts (pseudomucin), the mucin of snails, submaxillary mucin, the mucin of frogs' eggs, and of sputum, the mucoid of tendon and of the umbilical cord, the chondromucoid and ovimucoid, etc. These bodies are usually described in text-books under a subdivision of the proteids, viz., as glucoproteids. Their consideration under that heading was based on the assumption that they represented compound albumins, resulting from the union of an albuminous radicle with a special carbohydrate group. Since more recent investigation has shown that this group is probably represented in all albumins as well, and is merely present in the "glucoproteids" in especially large amount, there seems to be no adequate reason for separating them from the albumins proper. It must ke added, however, that a carbohydrate group has thus far been demonstrated satisfactorily only in the mucins and mucoids. If present in the other albumins, the amount is certainly very small and totally insufficient to serve as the direct source of the large quantities of sugar which in certain eases of diabetes are known to be derived from the albumins.

The carbohydrate radicle in question is in most members of the group obtained in the form of chitosamin (glucosamin), which in turn probably exists in the intact molecule as a polysaccharide (Fränkel's albamin, for example, see page 45). The structure of this complex mother-substance is but little known, and it appears that in different members of the group it may be different. In the case of the mucins and mucoids Müller always obtained the chitosamin in association with acetic acid, which suggests that the mother substance might possibly be an acetylated polysaccharide. Other gluco-albumins, such as chondromucin, apparently contain even more complex carbohydrate groups, which yield glucuronic acid besides 
chitosamin on hydrolytic decomposition. Landwehr's animal gum, which has figured very largely as a decomposition product of this order in the older text-books, is probably no unity; on decomposition with strong mineral acids it yields lævulinic acid, leucin, tyrosin, and other bodies of this order.

The amount of reducing substance which can be split off from some of the mucins and mucoids is quite considerable. Ovimucoid thus yields about 30 per cent., submaxillary mucin 34 per cent., the mucin derived from the respiratory tract 34 per cent., and the pseudomucin of ovarian cysts 30 per cent. From the mucoid of sepia eggs v. Fürth obtained 36-39 per cent.

As a class the gluco-albumins contain much less carbon and nitrogen, but more oxygen, than the common albumins, which is accounted for by the large amount of carbohydrate present. ${ }^{1}$

According to Levene, the mucins all contain the chondroitinsulphuric acid complex, which was formerly supposed to be peculiar to chondromucoid. Chondroitin-sulphuric acid is a conjugate sulphate, and on boiling with dilute hydrochloric acid is decomposed into sulphuric acid and chondroitin. The latter further yields acetic acid and chondrosin, which in turn supposedly gives rise to glucosamin and glucuronic acid (Schmiedeberg). This, however, has been disproved. Neuberg has shown that neither glucosamin nor glucuronic acid results on hydrolysis, but a tetra-oxyamino-capronic acid and a carbohydrate-like substance of unknown character. These decompositions can be expressed by the equations :

$$
\begin{aligned}
& \text { (1) } \mathrm{C}_{18} \mathrm{H}_{27} \cdot \mathrm{NO}_{14} \cdot \mathrm{SO}_{3}+\mathrm{H}_{2} \mathrm{O}=\mathrm{C}_{18} \mathrm{H}_{27} \mathrm{NO}_{14}+\mathrm{H}_{2} \mathrm{SO}_{4} \\
& \text { Chondroitin-sul- Chondroitin. } \\
& \text { phuric acid. } \\
& \text { (2) } \mathrm{C}_{18} \mathrm{H}_{27} \mathrm{NO}_{14}+3 \mathrm{H}_{2} \mathrm{O}=\mathrm{C}_{12} \mathrm{H}_{21} \mathrm{NO}_{11}+3 \mathrm{CH}_{3} \cdot \mathrm{COOH} \\
& \text {-Chondroitin. Chondrosin. Acetic acid. } \\
& \text { (3) } \mathrm{C}_{12} \mathrm{H}_{21} \mathrm{NO}_{11}+x \mathrm{H}_{2} \mathrm{O}=\mathrm{C}_{6} \mathrm{H}_{13} \mathrm{NO}_{6}+x \\
& \text { Chondrosin, Tetraoxy-amido-capronic }
\end{aligned}
$$

The mucins and mucoids are soluble with great difficulty in water and dilute saline solutions, and are insoluble in dilute acids. They possess markedly acid properties and dissolve in dilute alkalies with a neutral or feebly acid reaction. All mucinous solutions

\begin{tabular}{|c|c|c|c|}
\hline Mucoid of sepia eggs . . . . & $\begin{array}{c}\text { Carbon. } \\
\text {. } 49.7\end{array}$ & $\begin{array}{c}\text { Hydrogen. } \\
6.96\end{array}$ & $\begin{array}{c}\text { Nitrogen } \\
10.75\end{array}$ \\
\hline Pseudomucin (from ovarian cysts) & . 49.7 & 6.90 & 10.28 \\
\hline Mucin of frog's eggs ..... & . 52.7 & 7.10 & 9.30 \\
\hline
\end{tabular}
are more or less viscid and extremely difficult to filter.

The solutions do not coagulate on boiling, but on acidifying with acetic acid they are precipitated, the precipitate being insoluble in an excess of the acid. This precipitation by acids only occurs if sodium chloride or other neutral salts are absent or present only in

${ }^{1}$ Some of the analytical results which have been obtained are the following: 
small amounts. Neutral solutions of the mucins are precipitated by alcohol in the presence of neutral salts. Similar results are obtanned with some of the salts of the heavy metals.

On heating with dilute hydrochloric acid (2 per cent.) on a water-bath the carbohydrate group is split off and can be demonstrated with Fehling's test (see Urine).

Certain mucoids, after partial hydrolysis with alkalies, give a fine cherry-red color on treating with dimethyl-amido-benzaldehyde and heating (Ehrlich).

Whether or not some of the hyalogens belong to the order of the gluco-albumins remains to be seen. Like these, they contain a nitrogenous carbohydrate radicle which is probably chitosamin. Such bodies have been described especially by Krukenberg. They comprise the neossin of edible Chinese swallow-nests; the membranin found in Descemet's membrane, and the capsule of the crystalline lens; the spirographin of the spirographic membrane; the holothurian mucin; the chondrosin of certain mushrooms, and others. The hyalin which is found in echinococcus cysts, and the onuphin of the tubes of Onuphis tubicola, are probably not albuminous. The same is true of ehitin, which with other hyalins has been found in the extraskeletal and intraskeletal parts of various animals.

The Keratins.-Formerly the keratins were classed with the albuminoids, and were not regarded as true albumins. It has been conclusively established, however, that, like these, they contain the guanidin remnant, leucin, radicles of the glutaminic acid group, ornithin (as arginin), the cystin group, as well as an $a$-thiolactic acid nucleus; further, phenyl-alanin, a tyrosin and indol radicle, as also a carbohydrate group. Especially characteristic is the large amount of sulphur (3-5 per cent.) which is obtained in toto as cystin on hydrolytic decomposition. Tyrosin, in some members of the group, notably in human hair, is obtained only in small amount. Analysis of some keratins has given the following results :

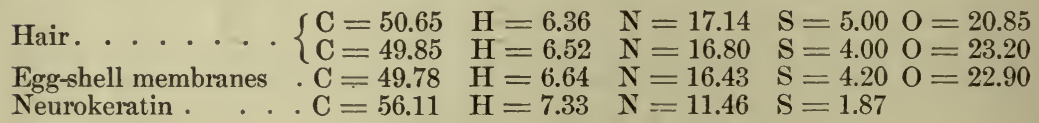

The keratins are the most important constituents of the epidermal structures of the body. They are found in the horny layer of the epidermis, in hair, in hoofs, horns, nails, and feathers ; further in the egg membranes of birds, of various reptiles and fishes, etc. A neurokeratin is found in the axillary sheath of the medullated nerves.

The keratins are all insoluble in water, dilute acids, and alkalies. Strong acids and alkalies cause their solution, but at the same time bring about their disintegration. A solution of keratin can therefore not exist. 
The Histons.-Although the histons do not occur in the animal body independently, but in combination with certain prosthetic groups, such as hæmatin (in hæmoglobin) and nucleinic acid (certain nucleoproteids), they are considered here nevertheless, as they are well-defined bodies which are capable of isolation from their pairlings as such. They comprise the common histon of the thymus, the lymph-glands, and the spleen, the histon of the red corpuscles of the goose, the salmon and scombron from the testicles of the sal$\mathrm{m} \sim \mathrm{n}$ and herring respectively, arbacin from the spermatozoa of Arbacia pustulosa, the lota histon and gadus histon, Fleroff's parahiston and globin, the albuminous radicle of hæmoglobin.

According to Kossel, the histons are compounds of protamins and albumins, and as a matter of fact compounds of this order result artificially on treating solutions of albumins with protamins. The histons are in turn capable of uniting with albumins, and may accordingly be viewed as non-saturated compound albumins.

There are many points of similarity between the histons and the protamins (see below). Both are markedly basic substances, which combine with acids to form salts. Both form precipitates with the true albumins. Both are precipitated by the alkaloidal reagents, in neutral or alkaline media. Protamins and histons are both rich in basic radicles (diamido-acids); the histons yield 40 per cent. and more on hydrolysis, and the protamins from 68 to 88 per cent. With the exception of cyclopterin the protamins do not give Millon's reaction and the histons only feebly.

Histons and protamins frequently replace each other; immature fish sperm thus contains nucleinate of histon, while in the mature material the corresponding protamin salt is found. It may, however, persist as such.

The elementary composition of the histons is different in different members of the group. Common to all is a large amount of nitrogen. In the best known representative of the group, the thymus histon, elementary analysis has given the following results: $\mathrm{C}=$ $52.35 ; \mathrm{H}=7.5 ; \mathrm{N}=18.1$; and $\mathrm{S}=0.62$. Its elementary formula, according to Bang, is $\mathrm{C}_{273} \mathrm{H}_{459} \mathrm{~N}_{81} \mathrm{SO}_{84}$.

The histons all gave a violet biuret reaction and the xanthoproteic reaction, while Millon's reaction is feeble and Molisch's reaction absent.

The histons are soluble in water, but are precipitated on the addition of a very small amount of ammonia (feebly but distinctly alkaline reaction). In the presence of an excess, even if slight, they are again dissolved, unless an ammonium salt is present, in which case they are completely or almost completely insoluble in an excess of the reagent. This reaction is very characteristic, but not altogether diagnostic; parahiston does not give it, arbacin only incompletely so, and in the case of scombron redissolution does not occur even though ammonium salts be absent.

On boiling a solution of histon in the absence of salts coagulation 
does not occur; this takes place if salts are present, but it is noteworthy that as a result of the coagulation denaturization has not occurred. For if now the precipitated histon is dissolved by the aid of an acid the solution is neutralized; the histon (unlike acir albumin) remains in solution and can again be precipitated on boiling.

Nitric acid precipitates the histons, but unlike the common albumins the precipitate dissolves on boiling and reappears on cooling. In this respect they resemble the albumoses.

The ability of the histons to precipitate other albumins (ovalbumin, caseïn, serum-globulin) from dilute saline solutions has been mentioned. This reaction, together with the behavior of the histons toward ammonia, and their precipitation by the alkaloidal reagents in neutral media, serve for their recognition and identification.

The Protamins.-The protamins which have been obtained thus far do not occur in the animal body independently, but in combination with certain nucleinic acids. They are described at this place nevertheless, as, like the histons, they represent well-defined chemical substances, and can be isolated as such.

The few known members of the group have all been obtained from the testicles of various fishes: salmin from the salmon, sturin from the sturgeon, clupeïn from the herring, scombrin from the mackerel, cyclopterin from Cyclopterus lumpus, silurin from Silurus glanus, accipenserin from Accipenser stellatus, cyprinin, $\alpha$ and $\beta$, from Cyprinus carpio, and others. In other animals protamins have not been found. Whether or not Ruppel's tuberculosamin (from tubercle bacilli) also belongs to this order is not known.

According to Kossel, the protamins are albumins of the lowest order, and he assumes that a radicle of this kind forms the nucleus of all the more complex albumins. This assumption primarily was based upon the observation that all protamins yield certain decomposition-products, which are also obtained from the albumins. These products are the diamino-acids arginin, lysin, and histidin (Kossel's hexon bases). They are present in the protamins in such large amounts (89-93.3 per cent. of the total nitrogen) that Kossel at first inclined to the view that they alone entered into the construction of the protamin molecule. Later studies, however, showed that still other radicles are present. Amino-valerianic acid has thus been found; so also tyrosin, serin, the tryptophan complex, and in salmin at least $\alpha$-pyrrolidin-carbonic acid. The protamin molecule thus proved to be more complex than Kossel at first thought, and other investigators accordingly do not share his views regarding the existence of a central nucleus of this order in the complex albumins, to which the di-amino-acids resulting on hydrolysis are referable. It has been shown, moreover, that all three hexon bases cannot be obtained from all albumins. More recently Kossel has modified his conception of the central protamin nucleus. He now regards the simplest protamin nucleus as com- 
posed of arginin in combination with amino-valerianic acid and an unknown third substance (possibly $\alpha$-pyrrolidin-carbonic acid). To these, in the higher protamins, still other radicles are united, such as lysin, histidin, and tyrosin.

However this may be, the protamins have properties which warrant their classification as albumins. They all give the biuret reaction, but neither that of Millon nor that of Adamkiewicz. They contain no sulphur. They are precipitated from their aqueous solutions by means of the alkaloidal reagents no matter whether the reaction is acid, neutral, or alkaline. Like the albumins they can be precipitated by salting (with sodium chloride and ammonium sulphate). For salmin the limits of precipitation with ammonium sulphate are 5.5 and 7.5. On heating they are not coagulated. Like the histons, the protamins are markedly basic and combine with acids to form salts ; these can be obtained in crystalline form. The sulphate is soluble in water and separates out on cooling or upon the addition of ether as an oil. With albumins and the primary albumoses they give rise to precipitates which, according to Kossel, are very similar to the histons.

The protamins are markedly toxic ; they impair the coagulability of the blood and cause a material diminution of the leucocytes.

The composition of some of the protamins is expressed by the formulæ :

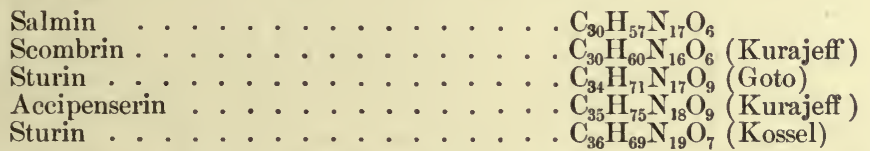

Cyclopterin differs somewhat from the ordinary protamins, and occupies a position intermediate between the histons and the protamins. It gives Millon's reaction, but does not form a precipitate when the mixture is heated; on cooling, it separates out, and then presents a rose color. It contains much less oxygen than the protamins. Its formula has not been ascertained; elementary analysis has given the following results :

$$
\text { Cyclopterin . . . . C }=42.0 \quad \mathrm{H}=6.9 \quad \mathrm{~N}=22.0
$$

On hydrolytic decomposition the protamins are first transformed into protones (which see).

\section{THE NUCLEO-ALBUMINS (PHOSPHOGLOBULINS).}

The nucleo-albumins were formerly regarded as a group of the nucleoproteids, in which the albuminous component was united with a special phosphorus-containing radicle. This latter was thought to be analogous to the nucleinic radicle of the true nucleoproteids; but as it did not yield the characteristic decompositionproducts of these, viz., xanthin bases, pyrimidin derivatives, and 
pentoses, it was termed paranuclein (Kossel) or pseudonuclein (Hammarsten). At present there is a tendency to separate the nucleo-albumins from the nucleoproteids, as further investigation has shown that barring the presence of phosphorus there is very little similarity between the two. It has been ascertained, moreover, that they probably do not enter into the construction of the nucleins; and as they resemble the globulins in many respects, Cohnheim has suggested that the term nucleo-albumins be abandoned and replaced by phosphoglobulins. They are nevertheless related to the nucleoproteids, as there are conditions (as in the developing organism) under which the body unquestionably constructs its true nucleoproteids from this source. For this reason and the phosphorus content I have given the group an independent position intermediate between the native albumins and the proteids.

The group comprises some of the most important food-stuffs, such as casein, the vitellin of egg-yolk, certain nucleo-albumins of cellprotoplasm, the phytoglobulins or phytovitellins of the leguminous plants, the ichthulin of fish eggs, etc. Some of these can be obtained in crystalline form. All of them probably contain iron. Elementary analysis of some of the bodies has given the following results :

Caseïn (Hammarsten) * $\mathrm{C}=52.90 \mathrm{H}=7.05 \mathrm{~N}=15.65 \mathrm{~S}=0.758 \mathrm{P}=0.847$

Vitellin (birds' eggs). . $\mathrm{C}=42.11 \quad \mathrm{H}=6.08 \quad \mathrm{~N}=14.73 \quad \mathrm{~S}=0.55 \quad \mathrm{P}=5.19 \quad \mathrm{Fe}=0.39$ (Bunge's hæmatogen)

Ichthulin (fish eggs) . $\mathrm{C}=53.52 \mathrm{H}=7.71 \mathrm{~N}=15.64 \mathrm{~S}=0.41 \quad \mathrm{P}=0.43 \mathrm{Fe}=0.1$

Cellular albumin $. . \mathrm{C}=52.37 \quad \mathrm{H}=6.81 \quad \mathrm{~N}=17.23 \quad \mathrm{~S}=1.06 \quad \mathrm{P}=0.42$ (snail liver)

Phytovitellin(Paranuts). $\mathrm{C}=52.43 \mathrm{H}=7.12 \mathrm{~N}=18.1 \quad \mathrm{~S}=0.55 \quad \mathrm{P}-$

Legumin (peas) . . . $\mathrm{C}=51.74 \quad \mathrm{H}=6.90 \quad \mathrm{~N}=18.0 \quad \mathrm{~S}=0.42 \quad \mathrm{P}-$

The ichthulin and the helicoproteid which Hammarsten discovered in the albuminous gland of a snail (Helix pomatia) were formerly placed in a separate category, the so-called phosphoglucoproteids, as they contain a large amount of reducing substance in their molecule; they are both, no doubt, true nucleo-albumins.

The nucleo-albumins have markedly acid properties and are almost insoluble in water. They form salts with the alkalies and ammonia, and these are readily soluble; on treating such solutions with acids the free nucleo-albumins are again precipitated. The solutions of the salts are not coagulated by heat, but with some this can be effected when the reaction is very faintly acid, so faintly that acid precipitation does not occur as yet. They are precipitated from their solutions by salting, and more readily so even than the globulins.

On peptic digestion pseudonuclein is thrown down at a certain phase of the process; this contains more phosphorus than the mother-substance. Apparently it contains a paranucleinic acid radicle. Levene has isolated a substance belonging to this order from birds' eggs (his avivitellinic acid), and there is evidence to show 
that analogous bodies also occur in plants. It appears quite probable that the paranucleinic acid of the egg-yolk represents the antecedent of the true nucleinic acids of the growing embryo. Osborne suggests that both may be ethers of a pentahydroxyl phosphoric acid- $\mathrm{H}_{5} \mathrm{PO}_{5}$-or its first anhydride, $\mathrm{H}_{8} \mathrm{P}_{2} \mathrm{O}_{7}$.

Wildenow further speaks of a phosphorus-containing substance which she obtained from caseïn, and which, like the true nucleinic acids, precipitated albumin. This has been confirmed by Salkowski, who isolated the substance and gives the following values as expressing its elementary composition : $\mathrm{C}=31.9, \mathrm{H}=4.43, \mathrm{~N}=9.72$, and $\mathrm{P}=2.55$.

\section{THE PROTEIDS.}

The proteids are conjugate albumins in which an albuminous group is united with a nuclein, a nucleinic acid, or a pigment radicle. The group comprises the nucleoproteids, the nucleins, and the hæmoglobins.

The Nucleoproteids.-The nucleoproteids are the most important constituents of nuclear structures and represent highly differentiated albumins, which are intimately concerned in the various manifestations of cell-life. They are characterized by their phosphorus content and the fact that on hydrolysis they all give rise to the formation of xanthin bases, pyrimidin derivatives, and pentoses. These in turn are derived from the nucleinic acid component of the nucleoproteids, which in turn may be directly combined with the primary albuminous radicle, or indirectly as a nuclein, viz., as an unsaturated nucleoproteid, within the larger group. These relations are shown in the following schema:

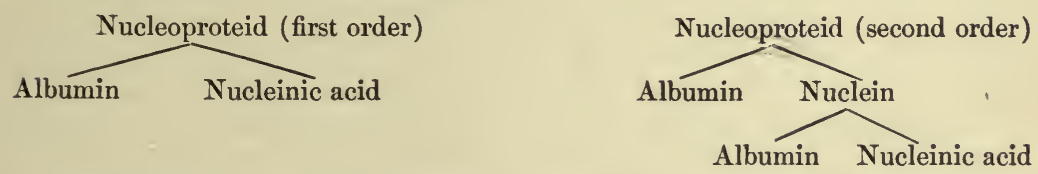

From this it will be seen that both nucleins and nucleinic acids are derivatives of the nucleoproteids. They can be isolated from their mother-substance, but do not occur in nature as such. The nucleins are split off and precipitated on digestion with pepsinhydrochloric acid, or by treating with acids and alkalies, by boiling with water, etc.

Detailed investigations into the structure of the complex nucleoproteids, in which an albuminous radicle is in combination with a nuclein, are still wanting, so that it is impossible to make any definite statements regarding the albuminous group in either the nuclein complex or in the nucleoproteid as a whole. In the simpler bodies, on the other hand, which have been obtained from the testicles of various fishes the albuminous component is apparently always a 
histon or a protamin. To this group also belongs the nucleinate of histon which has been obtained from the cellular elements of the thymus gland. Elementary analysis of some of these simpler bodies has given the following results:

Nucleinate of arbacin :

Nucleinate of salmin:

$$
\begin{aligned}
& \mathrm{C}=39.85 \quad \mathrm{H}=4.86 \quad \stackrel{\mathrm{N}=15.9}{\mathrm{~N}=18.81} \quad \mathrm{P}_{2} \mathrm{O}_{5}=17.3 \\
& 10\left(\mathrm{C}_{40} \mathrm{H}_{54} \mathrm{~N}_{14} \mathrm{O}_{27} \mathrm{P}_{4} \cdot \mathrm{C}_{16} \mathrm{H}_{28} \mathrm{~N}_{9} \mathrm{O}_{2}\right)+\mathrm{C}_{40} \mathrm{H}_{54} \mathrm{~N}_{14} \mathrm{O}_{27} \mathrm{P}_{4} \\
& \text { Nucleinate of salmin Nucleinic acid }
\end{aligned}
$$

Nucleinate of clupein : $\quad \mathrm{C}=41.2 \quad \mathrm{H}=5.75 \quad \mathrm{~N}=21.07 \quad \mathrm{P}=6.08 \quad \mathrm{O}=25.92$

$$
\left(\mathrm{C}_{30} \mathrm{H}_{57} \mathrm{~N}_{17} \mathrm{O}_{6} \cdot \mathrm{C}_{40} \mathrm{H}_{54} \mathrm{~N}_{14} \mathrm{P}_{4} \mathrm{O}_{29}\right)
$$

All or nearly all nucleoproteids contain iron, which, according to Kossel and Ascoli, is united with the phosphorus of the nucleinic acid and not with the albumin.

More complex nucleoproteids occur in the pancreas, the thyroid, the adrenal glands, the mammary glands, the spleen, etc. Some ferments also are supposedly of nucleoproteid nature.

The nucleoproteids are all markedly acid bodies. They are soluble in water and saline solutions and even more so in alkalies. They are precipitated by acids, but dissolve in an excess (being at the same time decomposed). Like the native albumins, they are coagulated by heat and the usual reagents, and undergo denaturization; during this process only the albuminous radicle, however, is affected. They can be precipitated by salting, and give the characteristic color reactions of the albumins. Noteworthy is their behavior toward polarized light. Whereas the albumins are all lævorotatory, the nucleoproteids turn the plane of polarization to the right (Gamgee, Jones). Osborne has shown that this property is very likely wholly referable to the nucleinic acid component. The degree of dextrorotation may vary between $+37.5^{\circ}$ and $+97.9^{\circ}$.

On hydrolysis the nucleoproteids yield the decomposition-products of the albuminous component, besides those of the nucleinic acid radicle, viz., xanthin bases and phosphoric acid.

The Nucleins.-The nucleins, as has been stated, do not occur in nature in the free state, but only in combination with albumins as nucleoproteids. Their acid properties, as would be expected, are even more marked than those of the nucleoproteids, and they are scarcely soluble in acids, even when present in excess. They give the reactions of the albumins, but differ from these to a great extent in their elementary composition; they contain only about 40 per cent. of carbon, but 4-7 per cent. of phosphorus. In gastric juice most of them are insoluble, while they are readily digested by trypsin. They are soluble in solutions of the hydrates of the alkalies, less readily so in dilute solutions of the alkaline carbonates. In water and alcohol they are for the most part insoluble. They are coagulated by heat, as also by alcohol, and are then insoluble in solutions of the alkaline hydrates. Like the nucleoproteids proper the nucleins also are dextrorotatory, which fact fincls its explanation in the observation 
of Osborne that this property is referable to the nucleinic acid component. As would be expected, the degree of dextrorotation is greater in the nucleins than in the corresponding nucleoproteids.

The Hæmoglobins.-The group of the hæmoglobins is essentially represented by the common blood-pigment of vertebrate animals, viz., hæmoglobin. Closely related to it is the so-called hæmocyanin, which is found in crabs and other invertebrates, and the chlorophyll of plants. The hæmoglobin has been studied with special care, and we now have a fairly clear idea of its structure. In it an albuminous radicle belonging to the histon group, viz., globin, is combined with the iron-containing pigment hæmatin. Both, as also the hæmocyanin, will be considered in detail in the section on the blood, where the chemical relation of the blood-pigment to the common respiratory pigment of the plant will likewise be pointed out.

\section{THE ALBUMINOIDS.}

While the albumins and proteids, which have been considered in the foregoing pages, are essentially constituents of the animal cell, viz., nucleus and protoplasm, the albuminoids or glutinoids, as they are also termed, represent the principal components of the intercellular structures. They are thus largely found in the supporting tissues of the body, viz., the connective tissue, cartilage, and bone. In the nutritive fluids, viz., blood and lymph, they do not occur. The group comprises the collagens or glutins, certain vegetable albuminoids, reticulin, elastin, the ichthylepidin of fish scales, various substances which have been found in the supporting structures of invertebrates, and which are collectively termed skeletins (fibroin, sericin, spongin, conchiolin, corneïn, elastoidin) and amyloid, which is a pathological product and not normally encountered in the body. The keratins have also been classed as albuminoids in the past, but there is a tendency at present to regard them as true albumins and as such they have been considered in this work.

The albuminoids are albuminous derivatives, which are produced in the body from albumins throngh cellular activity. As a class they do not contain all the typical radicles of the albumins, while in some certain complexes are more largely represented. The collagens thus differ from the true albumins in the absence of the cystin and carbohydrate group; they further lack the tyrosin and tryptophan group; elastin, fibroin, and sericin lack the glutaminic: acid radicle, etc. Collagen, on the other hand, contains much more glycocoll than the true albumins.

The nutritive value of the albuminoids is distinctly less than that of the albumins. Voit has shown that gelatin (glutin), for example, is not capable of maintaining life. Certain members of the group, moreover, cannot be regarded as food-stuffs in any sense owing to the extreme resistance which they offer to most solvents, including the digestive fluids. All albuminoids in fact are insoluble in the 
common solvents of the albumins (water and dilute saline solutions, and for the most part also in dilute acids and alkalies). Their solution involves their destruction.

The elementary composition of some of the more important members of the group follows:

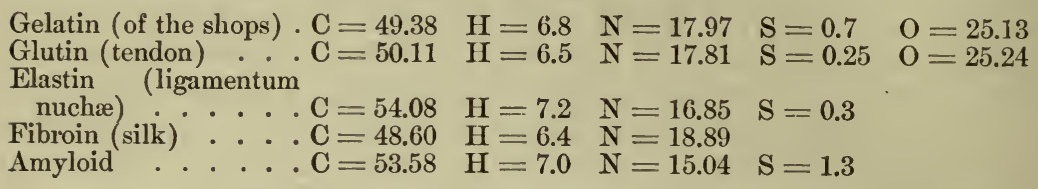

Of special interest among the albuminoids is collagen and its hydrate glutin or gelatin. Solutions of collagen gelatinize on cooling and redissolve on the application of heat. The mineral acids, potassium ferrocyanide and acetic acid, and most mineral salts do not precipitate the substance from its solutions. Pure solutions of gelatin give the biuret reaction and the xanthoproteic reaction, while those of Millon and Adamkiewicz are negative. An aromatic complex, however, is present nevertheless, for on hydrolysis with mineral acids phenyl-alanin may be obtained, though only in small amount ( 0.4 per cent.). Noteworthy is the large amount of glycocoll (16.5 per cent.). Sulphur is present, but cannot be split off as hydrogen sulphide by boiling with canstic alkali.

On tryptic digestion collagen yields traces of leucin, but no other amido-acids. Solutions of cartilaginous glutin (formerly termed chondrin) possess characteristics which are different from those of the glutin which is obtained from connective tissue or decalcified bone. These differences are not referable to the glutin as such, but to the presence of certain soluble compounds of chondroitin-sulphuric acid.

The amyloid substance, finally, occupies a unique position among. the albuminoids. It is met with only under pathologic conditions, and may then be found in the parenchyma of the liver, the spleen, the kidneys, etc. Iike the true albumins, it consists of carbon, hydrogen, nitrogen, oxygen, and sulphur, and on decomposition yields both leucin and tyrosin. It gives Millon's reaction, that of Adamkiewiez, and the xanthoproteic reaction. It is insoluble in water, alcohol, ether, dilute hydrochloric acid, and acetic acid. Concentrated hydrochloric acid and solutions of the alkaline hydrates cause its solution, but at the same time transform it into acid albumin or alkaline albuminate. The gastric juice, contrary to what has been claimed, likewise causes the substance to dissolve. Most characteristic is its behavior toward iodine and aniline green. The latter is colored red. Dilute aqueous solutions of iodine color the substance a brownish red or a bluish violet, which passes into blue on treating with sulphuric acid. Iodomethyl-anilin stains the substance red, especially after previous treatment with acetic acid. 


\section{THE DERIVED ALBUMINS.}

Fibrin.-Fibrin occupies a unique position among the albumins. So far as its general chemical composition goes, it is unquestionably closely related to the albumins proper. It contains carbon, hydrogen, nitrogen, oxygen, and sulphur in very much the same proportion as the true albumins, and, like these, yields the usual decomposition-products on hydrolysis. On the other hand, fibrin is insoluble in the common solvents of the true albumins, viz., in water and neutral saline solutions, while acids and alkalies cause its solution, but at the same time also its denaturization with the formation of acid albumin or alkaline albuminate. In this respect fibrin is closely related to the coagulated albumins. It merits its place among the deriver albumins by reason of its being itself a derivative of a true albumin, namely, fibrinogen. (Its relation to this and its rôle in the coagulation of the blood will be considered in a future chapter.)

The Coagulated Albumins.-The coagulated albumins result from the albumins proper through the influence of heat, prolonged exposure to strong alcohol, especially in the presence of a neutral salt, and in the case of fibrin at least, which, as we have just seen, is closely related to this group, through the activity of a specific ferment. They differ from the true albumins in their extreme resistance to all neutral solvents, and also to dilute acids and alkalies. Stronger acids and alkalies cause their dissolntion, with the simultaneous formation of acid albumins or alkaline albuminates.

The Albuminates.-The albuminates, as has been pointed out, result from the native albumins after their denaturization by acids and alkalies. Aside from their quantitative composition, they differ from each other only in so far as they have resulted through the action of an acid or an alkali. The alkaline albuminates thus contain less sulphur and less nitrogen than the acid albumins, as a portion of the sulphur and the so-called amino-nitrogen have been split off. Both the acid albumins and the alkaline albuminates are insoluble in neutral solvents, and are therefore precipitated from their solutions on neutralization. They are soluble, on the other hand, in solutions of the alkaline hydrates, in dilute solutions of sodium carbonate, in hydrochloric acid, and with a little more difficulty in strong acetic acid. From their acid solutions they are precipitated by salting with ammonium sulphate or sodium chloride. Through the action of an alkali acid albumin can be transformed into alkaline albuminate, but it is, of course, manifest that the reverse cannot occur. In the living body the denaturization of all albumins is effected during the process of digestion, and invariably precedes the formation of albumoses and peptones.

The Albumoses.-The albumoses result from the albumins proper, and also from the albuminoids and the albuminous radicles of the proteids, through the action of the so-called proteolytic ferments, or cluring their hydrolytic decomposition by means of acids 
or alkalies. In every case their formation is preceded by the denaturization of the original molecule.

Collectively the albumoses, which are derived from the true albumins in contradistinction to those which are obtained from the albuminoids, are termed proteoses. According to their origin, we distinguish between globulinoses, vitelloses, caseoses, myosinoses, keratinoses, elastoses, gelatoses, etc. During the process of digestion primary albumoses first result, which then give rise to secondary albumoses, and these in turn to simpler products, which collectively are termed peptones. Of primary albumoses, at least three are known, viz., proto-albumose, hetero-albumose, and gluco-albumose (synalbumose, Hofmeister). The secondary albumoses are designated as deutero-albumoses, and of these again several varieties exist (see Products of Digestion).

In their quantitative composition the albumoses do not differ materially from the original albumins ; their molecular weight, however, is materially less. Most likely they represent depolymerizationproducts of the albumins, and oceur preformed in the original molecule, as has been suggested (page 43).

The albumoses give the same color-reactions as their mother-substances. With the biuret test, however, instead of the original violet, a beautiful rose color is obtained. Their final decomposition-products are in general those of the original albumins ; there are certain differences, however, largely of a quantitative character, which are very important. These are shown in a subsequent table (sce page 202). Noteworthy is the absence of the glucosamin complex in the proto-albumose and hetero-albumose, and its presence in the gluco-albumose; further the large amount of sulphur in the thioalbumose ; then the presence of 39 per cent. of the total nitrogen, in the form of diamino-acids in hetero-albumose, as compared with 25 per cent. in proto-albumose, etc.

Unlike the albumins, the albumoses are not entirely indiffusible, and it appears that the power to pass through animal membrane increases as they become structurally further and further removed from their mother-substances.

As a class the albumoses are much more readily soluble than the albumins. Most of them are soluble in water or in dilute saline solutions, and also in dilute acids and alkalies. From their solutions they are readily precipitated by neutral salts, notably ammonium sulphate, which precipitates all albumoses when added to saturation, the reaction being slightly acid. Most of them, indeed, are thrown down when the salt is added to the extent of 75 per cent. Each albumose, in fact, appears to possess certain special limits of precipitation with ammonium sulphate which renders possible the separation of the individual substances from each other, and also from other albumins which may be present at the same time. Zinc sulphate behaves in a similar manner. Sodium chloride when added in substance to saturation causes a partial precipitation of the albumoses from their neutral solutions, while a fairly complete sep- 
aration is obtained if to the saturated fluid is added a small amount of acetic acid that has been saturated with the salt.

Neutral or acid solutions of albumoses are not coagulated by heat nor on treating with alcohol, although they are precipitated when this is present in considerable concentration. After precipitation, however, they are as soluble as before, and in this respect they differ markedly from the albumins proper.

Like the native albumins, the albumoses are precipitated by nitric acid, potassium ferrocyanide and acetic acid, metaphosphoric acid, phosphotungstic acid in the presence of hydrochloric acid, tannic acid, picric acid, trichloracetic acid, etc. ; but it must be noted that on the subsequent application of heat the precipitate redissolves, but reappears on cooling. The same result is obtained by treating a solution of albumoses with an equal volume of a saturated solution of sodium chloride and acidifying with acetic acid.

The Peptones. - In conformity with Kühne's teachings, the term peptone has been used to designate those products of proteolytic digestion which can no longer be salted out with ammonium sulphate, either in neutral, acid, or alkaline media, but which still give the biuret reaction. Products of this order are formed both during peptic and tryptic digestion. Those resulting in the former instance were designated as amphopeptone on the assumption that in it the hemi- and the anti-groups were still united, and it was supposed that during tryptic digestion the hemi- group was further decomposed, while the more resistent antipeptone remained as such. More recent researches have materially modified this conception. Kühne's amphopeptone and antipeptone have now been definitely proved to be no unities, and a hemipeptone as such is no longer recognized. It has been shown that the differences between the endproducts of peptic and tryptic digestion are quantitative, rather than qualitative, and that the term peptone comprises a number of hydrolytic decomposition-products which are essentially the same as those which result from the albumins on hydrolysis with boiling mineral acids and other means. Some of these bodies still give the biuret reaction (the peptones proper), while this is absent in others (the peptids).

Fischer's polypeptids which no longer give the reaction represent molecular complexes in which several amino-acid radicles are still united, and from which simpler peptids and free amino-acids may result on further hydrolysis. On digestion with proteolytic ferments (trypsin) products of this character are formed which energetically resist further cleavage by the ferment, but which can be decomposed by boiling mineral acids with the liberation, in addition to small amounts of alanin, leucin, aspartic acid, and glutaminic acid, of large quantities of $\alpha$-prolin, phenyl-alanin, and all the contained glycocoll.

Siegfried's kyrins are probably analogous to Fischer's polypeptids.

With the exception of $\alpha$-prolin, phenyl-alanin, and glycocoll, the remaining amino-acid radicles of the albuminous molecule are split off as such during the process of digestion with more or less readiness. In the case of tyrosin 97.6 of the total amount can already 
be isolated after forty-eight hours (in the case of edestin), while of glutaminic acid only 7.4 per cent. is obtained at that time. Cystin and tryptophan appear as rapidly as tyrosin, while alanin, leucin, amino-valerianic acid, and aspartic acid are split off more gradually, like glutaminic acid.

The interrelation between these various products is seen in the following schema :

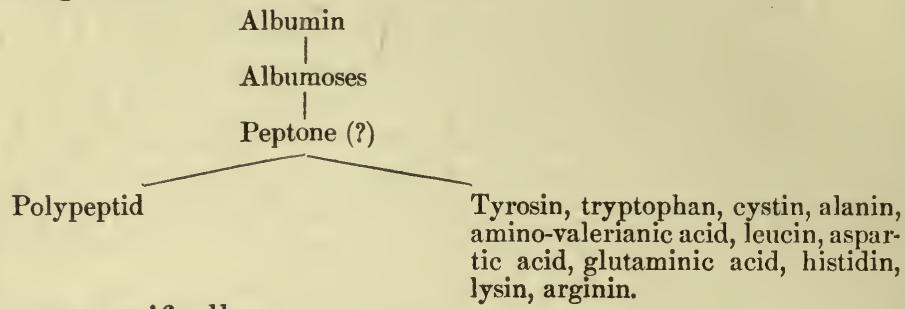

Or, more specifically : lysin, arginin.

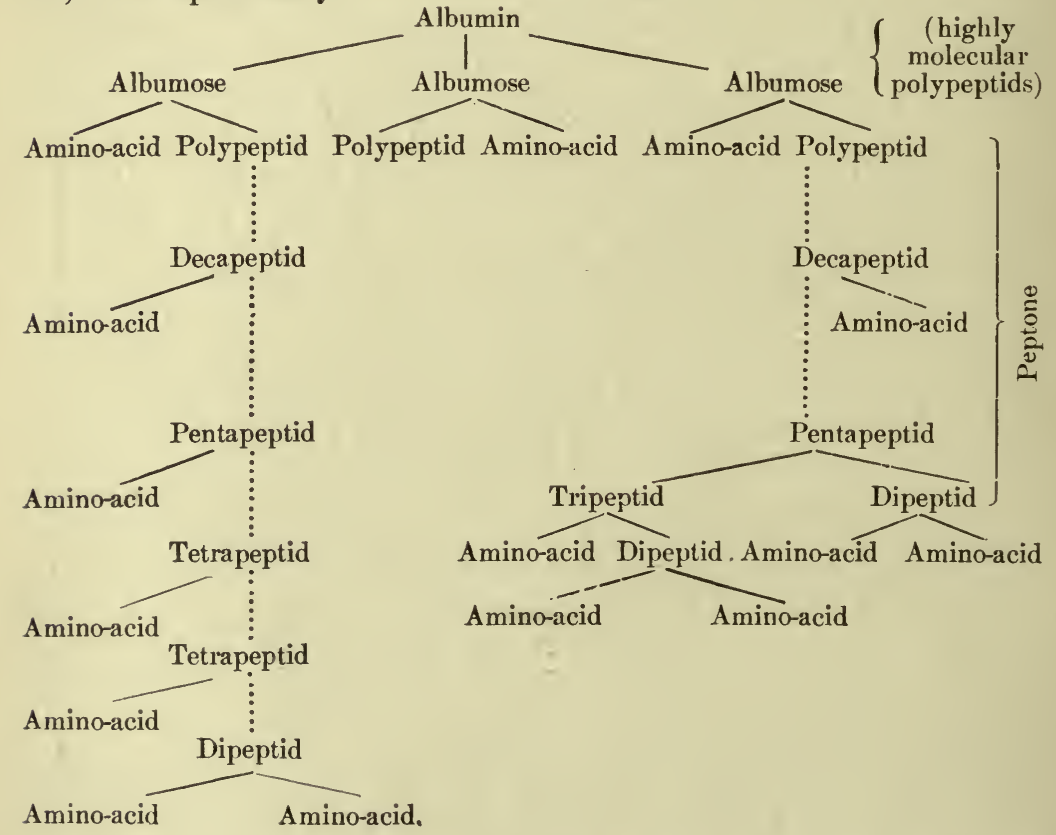

The term protones has been applied by Kossel to products which result from the protamins on digestion with trypsin and which stand intermediate between these and the end-products. Like the protamins, they turn the plane of polarization to the left, but to a less extent, and with cupric oxide they form a violet compound. From clupein Goto obtained a clupeon which corresponded to the formula $\mathrm{C}_{28} \mathrm{H}_{56} \mathrm{~N}_{14} \mathrm{O}_{8}$; in this 80 per cent. of the total nitrogen was present as arginin. A corresponding sturon gave the formula $\mathrm{C}_{30} \mathrm{H}_{61} \mathrm{~N}_{17} \mathrm{O}_{8}$. As a class the protones are but little known. 


\section{CHA PTER III.}

\section{THE CARBOHYDRATES.}

IT has been pointed out in the preceding chapter that while plants are capable of effecting from relatively simple compounds the synthesis of those complex albumins which are found in their various tissues and organs, animals do not possess this power, and are therefore dependent for their supply of nitrogen upon the albuminous food-stuffs that have been elaborated by plants. The carbohydrate supply of animals is also derived from plants, but for the maintenance of life it is not necessary that this should be furnished as such, as animals are not only capable of forming carbohydrates from the albumins as occasion demands, but, as we shall see later, they can also form carbohydrates directly from the fats which are stored in their tissues. The carbohydrates cannot therefore be regarded as essential food-stuffs, and we see, as a matter of fact, that carnivorous animals, at least, are capable of existing on albuminous food exclusively. They are important, however, as the stored energy which is thus supplied to animals represents a considerable caloric value, and they can hence protect the albumins from undue destruction. The importance of the carbohydrates as food-stuffs is thus secondary, and they are totally unable to take the place of the albumins. All living matter requires a definite amount of nitrogen so that life may be maintained, and if this is withdrawn death inevitably results. It is to be noted, however, that whereas animals can exist without carbohydrate food, and whereas the albumins largely predominate in their tissues, the reverse holds good for plants. Here the carbohydrates prevail, while the albumins are much less abundant. Consequently we may expect to find a far greater diversity of carbohydrates in the vegetable than in the animal world. This is actually the case. As it would lead too far, in a work of this scope, to consider all those carbohydrates which occur in the vegetable world, we shall confine our attention in the subsequent pages to those forms which may be regarded as common food-stuffs, or those which are more or less peculiar to the animal body.

All carbohydrates consist of carbon, hydrogen, and oxygen, and in most members of the group the elements hydrogen and oxygen are present in such proportion as to form water. In others this is not the case; and there are substances, such as lactic acid and acetic acid, which likewise contain hydrogen and oxygen in this proportion, but which are manifestly not carbohydrates. As 
there are no specific properties peculiar to these substances as a class, it is impossible to give an adequate definition of what is meant by the term carbohydrate. Chemically speaking, they are derivatives of polyatomic alcohols, and of the nature of aldehydes or ketones. They are conveniently divided into monosaccharides, disaccharides, and polysaccharides. The disaccharides and polysaccharides differ from the monosaccharides in being more complex substances and apparently built up from the monosaccharides through a condensation of monosaccharine anhydrides to form a double or a multiple group. Accordingly, on hydrolytic decomposition they yield two or more monosaccharine molecules for every original molecule, as is shown below:

(1) $\mathrm{C}_{6} \mathrm{H}_{12} \mathrm{O}_{6}-$ glucose, viz., lævulose.

(2) $\underset{\text { Cane-sugar }}{\mathrm{C}_{12} \mathrm{H}_{22} \mathrm{O}_{11}}+\mathrm{H}_{2} \mathrm{O}=\underset{\text { Glucose. }}{\mathrm{C}_{6} \mathrm{H}_{12} \mathrm{O}_{6}}+\underset{\text { Lævulose. }}{\mathrm{C}_{6} \mathrm{H}_{12} \mathrm{O}_{6}}$.

(Disaccharide.) (Monosaccharides.)

\section{THE MONOSACCHARIDES.}

According to the number of oxygen atoms which are present in the molecule, the monosaccharides can be divided into dioses, trioses, tetroses, pentoses, hexoses, heptoses, octoses, etc. Of these, the hexoses and pentoses only will be considered, as the remaining groups are not found in the animal body.

The Hexoses.-The most important representatives of the hexoses are glucose, which is also termed dextrose; lævulose or fructose; mannose and galactose. Some of these, such as glucose and lævulose, are found free in nature, or they result as hydrolytic decomposition-products from the more complex carbohydrates and related nitrogenous substances, the so-called glucosides. They are all derivatives of the stereo-isomeric hexatomic alcohols of the composition $\mathrm{CH}_{2} \cdot \mathrm{OH}-(\mathrm{CH} . \mathrm{OH})_{4}-\mathrm{CH}_{2} \cdot \mathrm{OH}$. Of these, three are known to occur in the natural state, viz., sorbite, or glucite, mannite, and dulcite. As has been pointed out above, the monosaccharides are either aldehydes or - ketones, and we accordingly find that glucose, mannose, and galactose represent the aldehydes (aldoses) of sorbite, manuite, and dulcite, respectively, while lævulose is the ketone (ketose) of mannite. They can therefore be represented by the structural formulæ :

(1) $\mathrm{CH}_{2}(\mathrm{OH}) \cdot \mathrm{CH}(\mathrm{OH}) \cdot \mathrm{CH}(\mathrm{OH}) \cdot \mathrm{CH}(\mathrm{OH}) \cdot \mathrm{CH}(\mathrm{OH}) \cdot \mathrm{CHO}$ (glucose, mannose, and galactose).

(2) $\mathrm{CH}_{2}(\mathrm{OH}) \cdot \mathrm{CH}(\mathrm{OH}) \cdot \mathrm{CH}(\mathrm{OH}) \cdot \mathrm{CH}(\mathrm{OH}) \cdot \mathrm{CO}^{-\mathrm{CH}_{2}}(\mathrm{OH})$ (lævulose).

As a matter of fact it is possible to transform these hexoses into their corresponding alcohols by careful reduction, and vice versa.

In accordance with their character as aldehydes or ketones, the aldoses on oxidation yield oxyacids, which have the same number 
of carbon atoms as the original substances, while the ketoses give rise to acids which have a smaller number of carbon atoms. The oxyacids which are derived from the aldoses, moreover, are either monobasic or dibasic, according to the extent to which the oxidation has been carried.

These changes are represented by the equations:

$$
\underset{\text { Glucose. }}{\mathrm{CH}_{2}(\mathrm{OH}) \cdot \mathrm{CH}(\mathrm{OH}) \cdot \mathrm{CH}(\mathrm{OH}) \cdot \mathrm{CH}(\mathrm{OH}) \cdot \mathrm{CH}(\mathrm{OH})} \stackrel{\mathrm{CHO}}{\mathrm{CH}_{2}(\mathrm{OH}) \cdot(\mathrm{CH} \cdot \mathrm{OH})_{4} \cdot \mathrm{COOH} .}
$$

$$
\begin{aligned}
& \mathrm{CH}_{2}(\mathrm{OH}) \cdot \mathrm{CH}(\mathrm{OH}) \cdot \mathrm{CH}(\mathrm{OH}) \cdot \mathrm{CH}(\mathrm{OH}) \cdot \mathrm{CH}(\mathrm{OH}) \cdot \mathrm{CHO}+3 \mathrm{O}= \\
& \text { Glucose. } \\
& \text { COOH. (CH.OH })_{4} \cdot \mathrm{COOH}+\mathrm{H}_{2} \mathrm{O} \text {. }
\end{aligned}
$$

The acids which can thus be obtained from the aldoses glucose, mannose, and galactose, are the monobasic acids-gluconic, mannonic, and galactonic acid ; and the dibasic acids-saccharinic, mannosaccharinic, and mucinic acid. Of these, saccharinic acid is of special interest, as it can readily be transformed into saccharolactonic acid, which in turn yields glucuronic acid. This latter, as we shall see later, is found also in the animal body. It is an aldehydic acid, and stands midway between gluconic acid and saccharinic acid. It is represented by the formula $\mathrm{COOH} \cdot \mathrm{CH}(\mathrm{OH}) \cdot \mathrm{CH}(\mathrm{OH}) \cdot \mathrm{CH}(\mathrm{OH})$. $\mathrm{CH}(\mathrm{OH}) \cdot \mathrm{COH}$.

The hexoses are colorless and odorless substances of a sweetish taste; they present a neutral reaction, and are readily soluble in water, with difficulty in absolute alcohol, and insoluble in ether. They can be obtained in crystalline form, and diffuse through animal membranes. Some of them are dextrorotatory, others lævorotatory, while still others are optically inactive. They are strong reducingsubstances, and for the most part fermentable with yeast. Especially interesting further is the behavior of the hexoses toward the hydrazins in the presence of acetic acid, with which they form hydrazons. These can be further transformed into osazons, which are very characteristic substances, and may serve to distinguish the various sugars from each other. On decomposition with fuming hydrochloric acid the osazons then give rise to the formation of osons-i.e., keto-aldehydes, which can be further reduced to ketoses. By starting with an aldose, it is thus possible to obtain an isomeric ketose. These changes may be represented by the equations:

$$
\begin{gathered}
\mathrm{CH}_{2}(\mathrm{OH}) \cdot \mathrm{CH}(\mathrm{OH}) \cdot \mathrm{CH}(\mathrm{OH}) \cdot \mathrm{CH}(\mathrm{OH}) \cdot \mathrm{CH}(\mathrm{OH}) \cdot \mathrm{CHO}+\underset{\text { Plucose }}{\mathrm{P}_{6} \mathrm{H}_{5} \cdot \mathrm{NH}_{2} \cdot \mathrm{NH}_{2}=} \\
\mathrm{CH}_{2}(\mathrm{OH}) \cdot \mathrm{CH}(\mathrm{OH}) \cdot \mathrm{CH}(\mathrm{OH}) \cdot \mathrm{CH}(\mathrm{OH}) \cdot \mathrm{CH}(\mathrm{OH}) \cdot \mathrm{CH}: \mathrm{N} \cdot \mathrm{NH}_{2} \mathrm{C}_{6} \mathrm{H}_{5}+\mathrm{H}_{2} \mathrm{O} \\
\text { Phenylglucohydrazon. }
\end{gathered}
$$

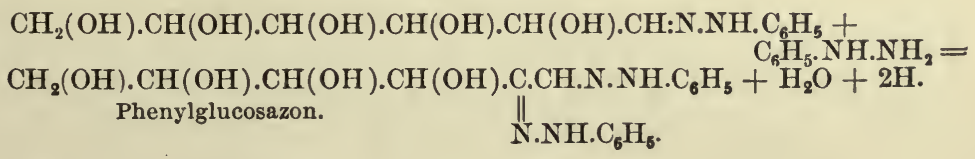


(3)

$$
\begin{gathered}
\mathrm{CH}_{2}(\mathrm{OH}) \cdot \mathrm{CH}(\mathrm{OH}) \cdot \mathrm{CH} \cdot(\mathrm{OH}) \cdot \mathrm{CH}(\mathrm{OH}) \cdot \mathrm{C} \cdot \mathrm{CH} \cdot \mathrm{N} \cdot \mathrm{NH}_{6} \mathrm{C}_{6} \mathrm{H}_{5}+2 \mathrm{H}_{2} \mathrm{O}+2 \mathrm{HCl}= \\
\text { I. } \mathrm{NH} \cdot \mathrm{C}_{6} \mathrm{H}_{5} \cdot \\
2 \mathrm{NH}_{2} \cdot \mathrm{NH} \cdot \mathrm{C}_{6} \mathrm{H}_{5} \cdot \mathrm{HCl}+\mathrm{CH}_{2}(\mathrm{OH}) \cdot \mathrm{CH}(\mathrm{OH}) \cdot \underset{\text { Oson. }}{\mathrm{CH}(\mathrm{OH})} \cdot \mathrm{CH}(\mathrm{OH}) \cdot \mathrm{CO} \cdot \mathrm{COH} .
\end{gathered}
$$

(4) $\mathrm{CH}_{2}(\mathrm{OH}) \cdot \mathrm{CH}(\mathrm{OH}) \cdot \mathrm{CH}(\mathrm{OH}) \cdot \mathrm{CH} \cdot(\mathrm{OH}) \cdot \mathrm{CO} \cdot \mathrm{CHO}+2 \mathrm{H}=$

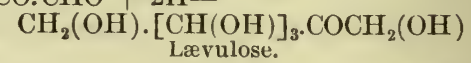

The same result may be reached when the corresponding osazon of the aldose is directly reduced, and the resulting osamin is treated with nitrous acid :

(1) $\mathrm{CH}_{2}(\mathrm{OH}) \cdot \mathrm{CH}(\mathrm{OH}) \cdot \mathrm{CH}(\mathrm{OH}) \cdot \mathrm{CH}(\mathrm{OH}) \cdot \mathrm{C} \cdot \mathrm{CH} \cdot \mathrm{N} \cdot \mathrm{NH} \cdot \mathrm{C}_{6} \mathrm{H}_{5}+\mathrm{H}_{2} \mathrm{O}+4 \mathrm{H}=$

$$
\begin{aligned}
& \begin{array}{ll}
\text { Phenylglucosazon. } & \text { N.NH. } \\
\mathrm{CH}_{2}(\mathrm{OH}) \cdot \mathrm{CH}(\mathrm{OH}) \cdot \mathrm{CH}(\mathrm{OH}) \cdot \mathrm{CH}(\mathrm{OH}) \cdot \mathrm{CH}\left(\mathrm{NH}_{2}\right) \cdot \mathrm{COH}+
\end{array} \\
& \text { Glucosamin. } \quad \mathrm{C}_{6} \mathrm{H}_{5} \cdot \mathrm{NH}_{2} \cdot \mathrm{NH}+\mathrm{C}_{6} \mathrm{H}_{5} \cdot \mathrm{NH}_{2} \\
& \text { (2) } \mathrm{CH}_{2}(\mathrm{OH}) \cdot \mathrm{CH}(\mathrm{OH}) \cdot \mathrm{CH} \cdot(\mathrm{OH} .) \mathrm{CH}(\mathrm{OH}) \cdot \mathrm{CH}\left(\mathrm{NH}_{2}\right) \cdot \mathrm{COH}+\mathrm{HNO}_{2}= \\
& \underset{\mathrm{H}_{2} \mathrm{O}+2 \mathrm{~N}}{\text { Gluensamin }}+\mathrm{CH}_{2}(\mathrm{OH}) \cdot \mathrm{CH}(\mathrm{OH}) \cdot \mathrm{CH}(\mathrm{OH}) \cdot \mathrm{CH}(\mathrm{OH}) \cdot \mathrm{CO}_{\text {Lævilose. }} \mathrm{CH}_{2}(\mathrm{OH})
\end{aligned}
$$

The glucosamin thus obtained as an intermediary product is of special interest in so far as it also results from the decomposition of the hyalins chitin and chondroitin. By oxidation with bromine glucosamin then yields chitonic acid, from which the corresponding sugar, chitose, can be obtained on reduction :

(1) $\mathrm{C}_{18} \mathrm{H}_{30} \mathrm{~N}_{2} \mathrm{O}_{12}+4 \mathrm{H}_{2} \mathrm{O}=2 \mathrm{CH}_{2}(\mathrm{OH}) \cdot \mathrm{CH}(\mathrm{OH}) \cdot \mathrm{CH}(\mathrm{OH}) \cdot \mathrm{CH}(\mathrm{OH})$.

(2) $\mathrm{CH}_{2}(\mathrm{OH}) \cdot \mathrm{CH}(\mathrm{OH}) \cdot \mathrm{CH}(\mathrm{OH} \cdot) \mathrm{CH}(\mathrm{OH}) \cdot \mathrm{CH}\left(\mathrm{NH}_{2}\right) \cdot \mathrm{COH}+4 \mathrm{O}=$

$$
\mathrm{CH}_{2}(\mathrm{OH}) \cdot \mathrm{CH}(\mathrm{OH}) \cdot \mathrm{CH}(\mathrm{OH}) \cdot \mathrm{CH} \cdot(\mathrm{OH}) \cdot \mathrm{CH}(\mathrm{OH}) \cdot \mathrm{COOH}+\mathrm{HNO}_{2}
$$

(3) $\mathrm{CH}_{2}(\mathrm{OH}) \cdot \mathrm{CH}(\mathrm{OH}) \cdot \mathrm{CH}(\mathrm{OH}) \cdot \mathrm{CH}(\mathrm{OH}) \cdot \mathrm{CH}(\mathrm{OH}) \cdot \mathrm{COOH}+2 \mathrm{H}=$

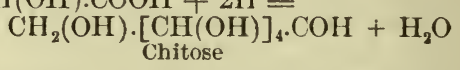

On boiling with dilute mineral acids the hexoses are decomposed into formic acid, lævulinic acid, and certain humin substances. With the alkalies, on the other hand, they yield, besides other products, also lævulinic acid and a ketonic acid of the composition $\mathrm{CH}_{3} . \mathrm{CO}$. $\mathrm{CH}_{2} \cdot \mathrm{CH}_{2} \cdot \mathrm{COOH}$. This formation of lævulinic acid, especially on boiling with concentrated hydrochloric acid, may be regarded as one of the characteristics of carbohydrates which are composed of 6 atoms of carbon. On the application of dry heat they form so-called caramel, and are finally carbonized. During the process of dry distillation, as also on distillation with hydrochloric acid, a slight formation of furfurol occurs.

On treating a small amount of a hexose solution ( 0.5 c.c.) with a drop of a 10 per cent. solution of $\alpha$-naphthol in alcohol (free from acetone) and underlaying with concentrated sulphuric acid (1 c.c.) a reddish-violet color develops at the zone of contact. The reaction, which is known as Molisch's reaction, depends upon the formation of furfurol from the sugar under the action of sulphuric acid. 
As stated above, most of the hexoses are capable of undergoing fermentation-i.e., a decomposition which is effected through the activity of certain minute organisms. According to the character of the specific organism present, we distinguish between alcoholic and acid fermentation, such as lactic acid, butyric acid, and acetic acid fermentation. The former is brought about through the influence of various varieties of yeast, while the latter is referable to the activity of certain bacteria, such as Bacterium lactis aërogenes, Bacillus acidi butyrici, Mycoderma aceti, etc. The decompositions which are thus effected may be represented by the equations :

$$
\begin{aligned}
& \text { (1) } \mathrm{C}_{6} \mathrm{H}_{12} \mathrm{O}_{6}=\underset{\text { Ethyl alcohol. }}{2 \mathrm{C}_{2} \mathrm{H}_{5}(\mathrm{OH})}+\mathrm{CO}_{2} \cdot \\
& \text { (2) } \mathrm{C}_{6} \mathrm{H}_{12} \mathrm{O}_{6}=\underset{\text { Lactic acid. }}{2 \mathrm{CH}_{3}-\mathrm{CH} . \mathrm{OH}-\mathrm{COOH} .} \\
& \text { (3) } \underset{\text { Lactic acid. }}{2 \mathrm{C}_{3} \mathrm{H}_{6} \mathrm{O}_{3}}=\underset{\text { Butyric acid. }}{\mathrm{C}_{3} \mathrm{H}_{7} \cdot \mathrm{COOH}}+2 \mathrm{CO}_{2}+4 \mathrm{H} \text {. }
\end{aligned}
$$

Upon the readiness with which the hexoses are oxidized the socalled reduction tests are based, viz., the reduction of metallic oxids on heating in alkaline solution. Of the hexoses, glucose, galactose, and lævulose are the only ones which occur in the animal kingdom, while mannose is found only in the vegetable world. Of the first three, moreover, glucose is by far the most important.

Glucose will be considered in detail in another chapter, where the methods of testing for the simple sugars in general, as also their quantitative estimation, will be described.

Lævulose (fructose) occurs in nature together with glucose, most abundantly in various fruits, the roots and seeds of many vegetables, and also in honey. It further results during the hydrolytic decomposition of cane-sugar, inulin, and other carbohydrates. In the animal body it has been found only after its introduction from without (in the urine) and in rare cases of diabetes. It is readily soluble in water, and its aqueous solutions, in contradistinction to common glucose, are lævorotatory. It may be obtained in crystalline form, but with difficulty. It is fermentable, and gives the same reduction-tests as glucose (which see). With phenylhydrazin lævulose yields the same osazon, but with methyl-phenyl hydrazin $\mathrm{C}_{6} \mathrm{H}_{5}>\mathrm{N} . \mathrm{NH}_{2}$ it forms a fructose-methyl-phenyl osazon, which cannot be obtained either with glucose, mannose, or glucosamin. Its formula is :

$$
\begin{gathered}
\mathrm{CH}_{2} \cdot \mathrm{OH} .(\mathrm{CHOH})_{3} \cdot \mathrm{C} . \mathrm{CH}: \mathrm{N} \cdot \mathrm{N}\left(\mathrm{CH}_{3}\right) \cdot \mathrm{C}_{6} \mathrm{H}_{5} \\
\text { N.N }\left(\mathrm{CH}_{3}\right) \cdot \mathrm{C}_{6} \mathrm{H}_{5}
\end{gathered}
$$

Galactose is formed during the hydrolytic decomposition of lactose and many other carbohydrates. It is also obtained from cerebrin on heating with dilute mineral acids. It is not so readily soluble in water as glucose, but like it is dextrorotatory. Galactose crystallizes in needles and platelets which melt at $168^{\circ} \mathrm{C}$. It is 
fermentable, and yields an osazon which melts at $193^{\circ} \mathrm{C}$. It reduces alkaline solutions of cupric oxide, but to a less marked degree than glucose. On oxidation it yields first galactonic acid and later mucinic acid.

The Pentoses. - The pentoses occur widely distributed in nature. In the animal body they have been demonstrated in the pancreas, the liver, thymus, thyroid, spleen, kidneys, salivary glands, brain, and muscle. The total amount which can be obtained from the adult human body probably represents about 10 grammes. The largest amount is found in the pancreas, where it is present to the extent of about 2.48 per cent. of the moist organ. In the tissues the pentoses do not exist in the free state, but form an integral part of the nucleoproteids, of which they are in a measure characteristic. Traces of pentoses occur in the urine under normal conditions; abnormally much larger amounts may be encountered (see Urine).

The pentoses which have been found in nature are arabinose, xylose, and rhamnose. Neuberg has shown that of these $l$-xylose is the common pentose of the nucleoproteids of the organs. It is an aldose and has the structural formula:<smiles>O=C(O)C(O)C(O)CO</smiles>

The optically inactive arabinose occurs in the urine, where $d$-rhamnose has also been found.

On prolonged boiling with dilute hydrochloric acid the pentoses lose water and form furfurol, viz., methyl-furfurol, according to the equation:

$$
\mathrm{C}_{5} \mathrm{H}_{10} \mathrm{O}_{5}-3 \mathrm{H}_{2} \mathrm{O}=\underset{\text { Furfurol. }}{\mathrm{C}_{5} \mathrm{H}_{4} \mathrm{O}_{2}}
$$

This fact is utilized in their quantitative estimation. On heating with hydrochloric acid and phloroglucin, or orcin, characteristic color-reactions result (see Urine). Like the hexoses they form compounds with phenylhydrazin. They are not fernentable.

Of special interest is the observation that on fermentative decomposition glucuronic acid passes over into the aldopentose $l$-xylose :

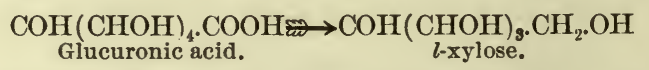

This demonstrates the possible transformation of a sugar of the $d$ - into one of the $l$-series, which is quite analogous to what occurs in the animal body.

\section{THE DISACCHARIDES.}

The disaccharides result from the monosaccharides through a condensation of the anhydrides of two monosaccharine molecules, analo- 
gous to the formation of ethers from alcohols. On hydrolytic decomposition they accordingly yield two monosaccharine molecules, which represent either one and the same substance or two isomeric bodies :

$$
\begin{aligned}
& \mathrm{C}_{12} \mathrm{H}_{22} \mathrm{O}_{11}=\left[\mathrm{C}_{6} \mathrm{H}_{12} \mathrm{O}_{6}\right]_{2}-\mathrm{H}_{2} \mathrm{O} \text { or } \\
& \mathrm{C}_{12} \mathrm{H}_{22} \mathrm{O}_{11}+\mathrm{H}_{2} \mathrm{O}=2 \mathrm{C}_{6} \mathrm{H}_{12} \mathrm{O}_{6} .
\end{aligned}
$$

Some of the disaccharides occur in nature as such, while others result from the decomposition of still more complex carbohydrates, viz., the polysaccharides proper.

The most important members of the group are cane-sugar or saccharose, lactose, and maltose. They are all hexo-bioses-i.e., they represent the union of the anhydrides of two hexoses, and can therefore be represented by the general formula $\mathrm{C}_{12} \mathrm{H}_{22} \mathrm{O}_{11}$. Of these, cane-sugar is formed through the union of one molecule of glucose and one molecule of lævulose; lactose from glucose and galactose; while maltose contains two molecules of glucose. Other members of the group which, however, do not occur in the animal body are trehalose, gentiobiose, cellose (cellobiose), and melibiose.

In their general properties the disaccharides closely resemble the monosaccharides. Like these, they have a sweet taste. They are crystallizable, capable of passing through animal membranes, and are optically active. In certain particulars, however, differences exist. Lactose, maltose, and isomaltose are thus capable of reducing metallic oxides in alkaline solution, and yield osazons with phenylhydrazin, while saccharose does not react in this manner.

The disaccharides as such are not fermentable, but become so after inversion to monosaccharides. Emil Fischer has shown that for the inversion of a special disaccharide a specific ferment is necessary. As the various fermentative agents, however, possess a varying number of inverting ferments, it is elear that a certain disaccharide may be inverted by one form of yeast, but not by another; while, on the other hand, one special form may be capable of inverting all forms of the disaccharides. This is actually the case, and we thus find that the so-called kefir granules, as also the Bacterium lactis, can invert cane-sugar as well as maltose and lactose. Common yeast, on the other hand, inverts only cane-sugar and maltose, while lactose is not attacked. According to their specific power of inversion, these ferments are spoken of as invertin, maltase, and lactase. The derivation of the two latter names is, of course, apparent. The significance of the term invertin, however, is not so clear. It has reference to the mixture of glucose and lævulose which is obtained from cane-sugar by inversion, and which was originally spoken of as invert-sugar. Invertin is thus a ferment which inverts cane-sugar.

After inversion the disaccharides undergo fermentation, like the monosaccharides, and here, as there, we may distinguish between alcoholic, lactic acid, butyric acid, and acetic acid fermentation.

Cane-sugar (saccharose, saccharobiose) is found in nature most 
abundantly in sugar-cane, in the roots of the sugar beet, and in the juices of various trees (palms, sugar maple, birch, etc.). In the animal organism it does not occur. The pure substance is crystalline, and melts at $160^{\circ} \mathrm{C}$. On further heating it turns brown and forms so-called caramel. It is easily soluble in water and turns the plane of polarization to the right. As stated, it does not yield an osazon and does not reduce metallic oxides. After inversion with invertin it undergoes the same fermentations as the resulting monosaccharides. On oxidation it yields, in addition to other substances, saccharinic and oxalic acids.

Maltose (maltobiose, ptyalose, cerealose) does not occur in nature as such, but results during the digestion of starch and glycogen in the alimentary canal through the action of diastase. It is a crystalline substance, which is easily soluble in water and turns the plane of polarization to the right. With phenylhydrazin it yields an osazon -maltosazon-which melts at $206^{\circ} \mathrm{C}$. It readily undergoes fermentation, and like glucose reduces metallic oxides in alkaline solution, but to a less degree.

An isomaltose results from starch through the action of a diastatic ferment. It is also formed (synthetically) by the maltoglucase of yeast and the taka-diastase of Aspergillus oryzæ from glucose. In the intestinal canal it is found together with maltose. It is easily soluble in water and turns the plane of polarization to the right. Its osazon melts at $150^{\circ}$ to $153^{\circ} \mathrm{C}$. It undergoes fermentation, but much more slowly than maltose.

Lactose (lactobiose), which is almost exclusively found in the animal world, will be considered in a subsequent chapter (see Milk).

An isolactose is formed (synthetically) as a result of the action of the lactoglucase of kefir upon a mixture of glucose and galactose.

\section{THE POLYSACCHARIDES.}

The higher polysaccharides result from the monosaccharides in the same way as the disaccharides. In other words, they represent the anhydrides of the monosaccharides, of which many molecules, however, are condensed to form the resulting polysaccharine molecule. Their general formula therefore is $\left(\mathrm{C}_{6} \mathrm{H}_{10} \mathrm{O}_{5}\right)_{x}$. The value of $x$ is unknown, but it is probably always large. From a determination of the size of the starch molecule, for example, we may conclude that $x$ is equivalent to at least 108. In others, such as glycogen and the dextrins, however, it is certainly much smaller. In conformity with their structure, the polysaccharides all yield monosaccharides on hydrolytic decomposition. During this process, however, a variable number of intermediary products are formed, which may themselves be polysaccharides, though of a lower order, and which in turn yield disaccharides and finally monosaccharides. Starch is thus first transformed into erythrodextrin, which in turn yields achroodextrin; this is further changed to isomaltose, and then 
to maltose, which finally yields glucose. In other cases, as with glycogen, the disaccharides isomaltose and maltose are formed directly. Cellulose likewise yields glucose as a final product, while lævulose results from inulin, and mannose from the so-called reserve celluloses, which are found in the cell-walls of many seeds. Galactose is similarly obtained from many gums, and from a variety of cellulose, which Schultze has termed galactose-cellulose, in contradistinction to the mannose-cellulose and the true dextrose-celluloses. In many instances, however, the exact mode of decomposition, as also the character and number of the intermediary products, is but imperfectly understood.

In their physical and chemical properties considerable differences exist between the polysaccharides and the other carbohydrates which have so far been described. They are thus (with the possible exception of glycogen) non-crystallizable substances and devoid of a sweet taste. In alcohol and ether they are insoluble. In water most of them are more or less soluble, but as a class they are incapable of diffusing through animal membranes, for which reason they are termed saccharocolloids. From their solutions they can be precipitated by saturation with neutral salts, and notably ammonium sulphate. Like the monosaccharides and disaccharides, they are optically active, but with the exception of the dextrins they do not reduce metallic oxides in alkaline solution, and none of them combine with phenylhydrazin to form osazons. As such, they are incapable of undergoing fermentation; but, like the disaccharides, they may be inverted to monosaccharides through various ferments or acids, and can then be further decomposed.

Especially important is their behavior toward iodine, with which most of the polysaccharides combine to form colored compounds that are quite characteristic. Starch is thus colored blue, glycogen a mahogany brown, erythrodextrin red, inulin and lichenin yellow.

The polysaccharides which are used as food-stuffs are conveniently divided into starches, dextrins, vegetable gums, and celluloses. Of these, the starches are by far the most important.

Starch occurs widely distributed in the vegetable world, and constitutes the most important reserve food of most of the higher plants. It is found in the form of distinct granules, which, on microscopic examination, exhibit a marked concentric striation, and which differ in size and form in different plants. 'The individual granules are enclosed in a capsule of so-called starch cellulose, which is insoluble in water, but which can be made to open by heating in the presence of much water. The contained starch-granulose can thus be obtained, and constitutes the so-called soluble starch, amylum or amylodextrin. During this process no doubt a still more complex molecular group of monosaccharine anhydrides is decomposed, but of the intermediary products which are formed nothing is known. In the alimentary canal this change is effected through the activity of diastatic ferments, which further give rise to the formation of dextrins, 
maltose, isomaltose, and glucose. On boiling with dilute acids also glucose is formed, with various dextrins as intermediary products. Among these, as has been shown, erythrodextrin is apparently the first to develop, achroödextrin appears later, and from this isomaltose, maltose, and glucose are finally obtained. It appears, however, that during the decomposition of achroödextrin still other dextrins of lower molecular weight are simultaneously formed, which in turn yield maltose and glucose. Finally a dextrin is obtained which undergoes no further change, and is termed maltodextrin. This would correspond to the polypeptid which results from the albuminous molecule through the action of trypsin and which similarly withstands the further action of the ferment. The other dextrins would correspond to the albumoses and those polypeptids which are ultimately split into the component amino-acids, while glucose would be comparable to these directly.

Most characteristic is the behavior of starch toward iodine, with which it gives an intense blue color that disappears on heating, but reappears on cooling. In a solution of sodium or potassium hydrate starch swells up and forms a paste.

Dextrins.-The dextrins, as has been shown, are formed from starch during its hydrolytic decomposition by means of ferments or on boiling with dilute mineral acids. To a certain extent they result also when starch is heated to a temperature of from $200^{\circ}$ to $210^{\circ} \mathrm{C}$. Through continued decomposition they give rise to maltose and isomaltose, and finally to glucose.

As a class the dextrins are easily soluble in water and turn the plane of polarization to the right. From the other polysaccharides they differ in their ability to dissolve cupric hydroxide in alkaline solution. With erythrodextrin iodine strikes a red color, while achroödextrin is unaffectd.

Glycogen apparently plays the same rôle in the animal metabolism which starch does in the vegetable world. It is accordingly also termed animal starch. In this form carbohydrate material is largely stored in the liver and the muscles. Its ultimate decompositionproduct is exclusively glucose, while dextrins and maltose are formed as intermediary products. Its properties will be considered in a future chapter.

Inulin and lichenin occur in the roots of various composites (Inula helenium, the dahlias) and in lichens (Icelandic moss) respectively. Both are soluble in hot water and are colored yellow with iodine, and neither is attacked by diastase. On hydrolysis inulin yields lævulose and lichenin glucose.

Celluloses. - As food-stuffs the celluloses are unimportant. They are considered at this place owing to their wide distribution in the vegetable world, where they form the greater portion of all cellenvelopes. In the animal world they are likewise encountered, and enter largely into the composition of the external skeleton of the tunicates. They are characterized by their extreme resistance to 
the most divers solvents, and are indeed soluble only in a solution of cupric oxide in strong ammonia-the so-called Schweitzer's reagent. From this solution the substance can be obtained in amorphous form on precipitation with acids. Moderately concentrated sulphuric acid transforms cellulose into vegetable amyloid, which is colored blue by iodine. When the resulting esters of cellulose and sulphuric acid are boiled in aqueous solution glucose results. With concentrated nitric acid, or with a mixture of nitric acid and concentrated sulphuric acid, it yields the highly explosive nitrocellulose.

Wood (lignin) and cork are derivatives of cellulose.

On hydrolytic decomposition the common cellulose yields glucose, while the so-called hemicelluloses give rise to galactose or mannose, as also to certain pentoses, such as arabinose and xylose. In the intestinal canal a certain portion of the ingested cellulose is unquestionably dissolved. The products, however, to which it gives rise are for the most part unknown. Certain micro-organisms which are present at the time bring about a fermentation of the substance, during which marsh gas, acetic acid, and butyric acid are formed, but the greater portion no doubt is eliminated in the feces as such.

Gums and Vegetable Mucins.-Various kinds of gums occur extensively distributed in the vegetable world, which on decomposition with dilute mineral acids yield galactose and arabinose. Examples are gum arabic, cherry gum, the agar-agar which is obtained from eastern Asiatic algæ, etc.

The vegetable mucins, unlike the gums, are more or less insoluble in water. 


\section{CHAPTER IV.}

\section{THE FATS.}

THE origin of fats in the animal body is threefold : one portion is derived from the fats which have been ingested as food; another portion is formed from the carbohydrates; while a third portion results from the decomposition of albumins. As food-stuffs the fats are of great importance, because their caloric value is quite highhigher in fact than that of the carbohydrates and albumins; but, like the carbohydrates, they are unable to take the place of the albumins. Animals that are fed exclusively on fats die sooner or later, although they may become quite fat during the period of their special diet. In the animal body they represent a variable amount of reserve food, which is conveniently stored in the subcutaneous areolar tissue, in the omentum and mesentery, in the bone-marrow, etc. In case of inanition it is utilized long before the tissues of the body proper are attacked, and we accordingly find that in persons who have died from wasting diseases every vestige of fat may have disappeared, while the muscular nutrition may still be fair.

That portion of the body fat which is derived from the fats ingested as such is really the smallest portion, and by far the greater amount results from the carbohydrates. The manner in which this transformation takes place is unknown, but it is probable that the carbohydrates which are utilized for this purpose are first decomposed, and then reduced; and that the fats finally result through a synthesis of such reduction-products. Such syntheses, however, cannot at once be compared to those which take place in plants, for here we have seen that the fats can be formed directly from water and carbon dioxide. In animals this does not occur.

The origin of the fats from carbohydrates can be demonstrated in various ways. Dumas and W. Milne Edwards have shown that bees which are fed exclusively on sugar produce three times as much wax as compared with that which was originally present in their bodies. It is a well-known fact, moreover, that cattle which are fed on nitrogenous food exclusively do not fatten, or only slightly so ; whereas they soon gain in weight when a certain proportion of carbohydrates is added to their food.

The proportion of fat which is normally derived from albumins is not very large, if we except the period of lactation in female animals, but its possible origin from this source is undoubted. 
Bitches which are fed solely on lean meats continue to furnish milk containing an abundance of butter. Pettenkoffer and Voit further showed in dogs that when the carbohydrates remained constant, but the albuminous food was increased, a steady gain in fat occurred, as shown in the table:

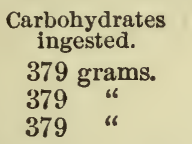

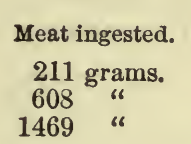

Gain in fat.

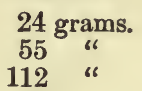

A further illustration is had in the transformation of the muscular tissue of cadavers into so-called adipocere, a substance which consists to the extent of 97 per cent. of ammonium palmitate with a small amount of stearate.

Under various pathologic conditions, finally, we can follow with the microscope the gradual transformation of albuminous material into fat.

All fats consist of carbon, hydrogen, and oxygen. They are insoluble in water, slightly soluble in cold alcohol, while in hot alcohol, ether, and benzol they dissolve with ease. Chemically speaking, they are neutral esters which are formed through the union of an acid with an alcohol according to the equation:

$$
\mathrm{C}_{2} \mathrm{H}_{5} \cdot \mathrm{OH}+\mathrm{CH}_{3} \cdot \mathrm{COOH}=\mathrm{CH}_{3} \cdot \mathrm{COO} \cdot \mathrm{C}_{2} \mathrm{H}_{5}+\mathrm{H}_{2} \mathrm{O} \text {. }
$$

The fats which are principally found in the animal world, viz., palmitin, stearin, and olein, similarly result through the union of their respective monobasic acids with the triatomic alcohol glycerin. This union is effected as shown in the equations:

$$
\begin{aligned}
& \underset{\text { Glycerin. }}{\mathrm{C}_{3} \mathrm{H}_{5}(\mathrm{OH})_{3}}+\underset{\text { Palmitic acid. }}{3 \mathrm{C}_{15} \mathrm{H}_{31} \mathrm{COOH}}=\underset{\text { Palmitin. }}{\mathrm{C}_{3} \mathrm{H}_{5}\left(\mathrm{C}_{16} \mathrm{H}_{31} \mathrm{O}_{2}\right)_{3}}+3 \mathrm{H}_{2} \mathrm{O} \\
& \mathrm{C}_{3} \mathrm{H}_{5}(\mathrm{OH})_{3}+\underset{\text { stearic acid. }}{3 \mathrm{C}_{17} \mathrm{H}_{36} \cdot \mathrm{COOH}}=\underset{3}{\mathrm{C}_{3} \mathrm{H}_{5}\left(\mathrm{C}_{18} \mathrm{H}_{35} \mathrm{O}_{2}\right)_{3}}+3 \mathrm{H}_{2} \mathrm{O} \\
& \mathrm{C}_{3} \mathrm{H}_{5}(\mathrm{OH})_{3}+\underset{\substack{17 \\
\text { Oleic acid. }}}{3 \mathrm{C}_{33} \mathrm{H}_{33} \cdot \mathrm{COOH}}=\underset{\text { Olein. }}{\mathrm{C}_{3} \mathrm{H}_{5}\left(\mathrm{C}_{18} \mathrm{H}_{33} \mathrm{O}_{2}\right)_{3}}+3 \mathrm{H}_{2} \mathrm{O}
\end{aligned}
$$

They are thus triglycerides, and are accordingly termed tripalmitin, tristearin, and triolein. Other fats have also been found in the animal world, but are of secondary importance. Such fats are the so-called cetin (the palmitic acid ester of cetyl alcohol), which is obtained from certain whales, the myricin of beeswax (the palmitic acid ester of myricyl alcohol), etc.

The animal fat as a whole usually represents a mixture of the three triglycerides, palmitin, stearin, and olein, in variable proportions, the stearin predominating in the more solid varieties, while olein prevails in the more liquid fats. In human fat olein represents about 670 to 800 pro mille of the total amount.

The triglycerides are lighter than water; they are soluble in benzol and ether, and in hot alcohol, while in water and cold 
alcohol they are insoluble. On shaking fats (containing a small amount of fatty acids) with water the fat globules are finely divided, resulting in the formation of an emulsion. They are non-volatile and burn with a luminous flame. On heating, especially in the presence of potassium bisulphate, they are decomposed with the formation of highly irritating vapors of an aldehyde, acrolein, which in turn results from glycerin, according to the equation :

$$
\mathrm{C}_{3} \mathrm{H}_{5}(\mathrm{OH})_{3}=\mathrm{C}_{2} \mathrm{H}_{3} \cdot \mathrm{CHO}+2 \mathrm{H}_{2} \mathrm{O} \text {. }
$$

On boiling with concentrated alkalies, or acids, or through the influence of superheated steam, as also through certain ferments (lipases), the fats are decomposed into glycerin and their respective acids. This decomposition is spoken of as saponification; the alkaline salts of the resulting fatty acids (in the case of hydrolysis with alkalies) are termed "soaps."

On prolonged exposure to the air, even in the absence of microorganisms, the fats become rancid-i.e., they become acid and assume a most disagreeable odor and taste. During this process a partial decomposition occurs, with the formation of glycerin and fatty acids, which latter are then oxidized to certain volatile, offensive smelling oxy-acids. The exact nature of the process which thus takes place is not well understood; as has been stated, it can occur in the absence of micro-organisms and through the influence of light and air only.

The fats which occur in the animal body generally present a more or less well-marked yellow or red color. This color is referable to the presence of certain lipochromes. These are compounds which, like the fats themselves, are devoid of nitrogen; and some of them apparently are hydrocarbons, of whose structural composition, however, nothing is known.

Closely related to the fats are the lecithins and cholesterins.

\section{THE LECITHINS.}

The lecithins are esters which result through the union of cholin with glycerin-phosphoric acid, in which the two remaining glycerin hydroxyl groups have been replaced by fatty acid radicles. This union takes place according to the equations:

(1) $\mathrm{CH}_{2} \cdot \mathrm{OH}$

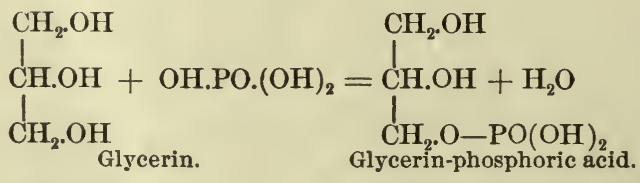

(2) $\mathrm{CH}_{2} \cdot \mathrm{OH}$

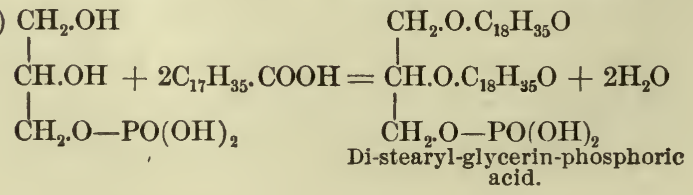


(3)

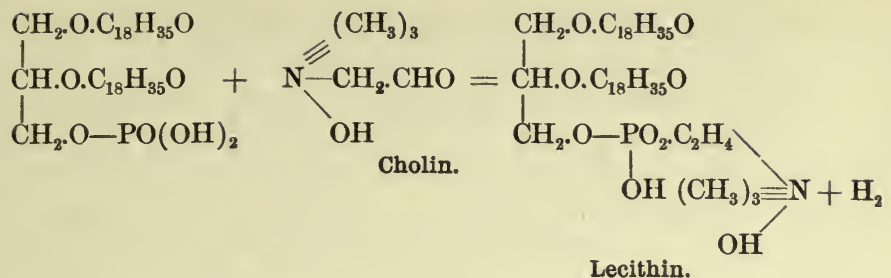

On decomposition of the lecithins with acids or alkalies we accordingly obtain glycerin-phosphoric acid, fatty acids, and cholin. At the same time, however, another basic substance, neurin, is usually found, and it is to be noted that, in contradistinction to cholin, neurin possesses extremely toxic properties. It results from cholin through the loss of two atoms of hydrogen and one atom of oxygen, and is also formed during bacterial decomposition of the lecithins in the presence of much oxygen. Chemically it is trimethyl-vinyl-ammonium hydroxide, $\underset{\mathrm{OH}}{\mathrm{N}-\mathrm{CH}=\mathrm{CH}_{2}}$, while cholin must be regarded as trimethyl-oxyethyl-ammonium hydroxide.

Another derivative of cholin which may be obtained through the action of fuming nitric acid is a basic substance that is apparently isomeric with muscarin and, like this, extremely toxic. Chemically it may possibly be represented by the formula $\mathrm{N}-\mathrm{CH}_{2} \cdot \mathrm{COH}$, and could accordingly be regarded as the aldehyde of oxyneurin (trimethyl glycocoll).

The lecithin which is most commonly found in the animal body is the cholin compound of distearyl-glycerin-phosphoric acid; other lecithins can, of course, also occur, in which the glycerin hydroxyl groups have been replaced by the radicles of oleic and palmitic acids, for example, but they are but little known.

In its dry state the common lecithin occurs as a wax-like, plastic mass, which is soluble in alcohol (at $40^{\circ}-50^{\circ} \mathrm{C}$.), ether (less readily), chloroform, benzol, carbon disulphide, and in the fatty oils, while in water it is insoluble. Placed in water it swells and becomes pasty, and on microscopical examination it will be noted that the substance occurs in the form of peculiar slimy droplets and threads, which are generally termed myelin forms. From its alcoholic solution it crystallizes in wart-like masses, which consist of small platelets.

Of special interest is the tendency of the lecithins to combine with albumins to form more or less stable compounds, which have been termed lecithalbumins. Such compounds have been found in the mucosa of the stomach, in the lungs, the liver, and the spleen. In 
the yolk of eggs it occurs in combination with vitellin, but is here apparently not closely bound. A certain similarity thus exists between the lecithins and the nucleins; both contain phosphorus in their molecules, and both combine with albumins to form more complex substances. The lecithins occur widely distributed in both the animal and vegetable world. According to Hoppe-Seyler, they are found in all cells and bodily fluids. They are abundant in nerve-tissue and also in the eggs and semen of most animals. Of special interest is the fact that the activation of certain hæmolytic substances (tetanus toxin, solanin, saponin, cobra poison) is effected by means of lecithin, and that the hæmolytic complement in normal blood-serum is probably of this nature (Kyes).

W. Koch has recently pointed out the probable import in the life of the cell of the lecithins, for which he proposes the collective term lecithanes, and summarizes his conclusions as follows: 1. In association with albumins, in colloid solutions they furnish the basis for the establishment of the necessary viscosity, by the ease with which they (the lecithanes) are influenced by ions $(\mathrm{Na}, \mathrm{Ca})$. 2. They are concerned in the metabolism of the cell, and in consequence of the presence of the unsaturated fatty acids they take part in the oxygen metabolism and by means of their methyl groups united to nitrogen in still other and unknown reactions. (Their isolation and special tests will be considered in a subsequent chapter.)

\section{THE CHOLFSTERINS.}

The cholesterins are monatomic alcohols of the formula $\mathrm{C}_{27} \mathrm{H}_{45^{\circ}}-$ $\mathrm{OH}+\mathrm{H}_{2} \mathrm{O}$. They are found in all animal cells, in the blood, lymph, etc., and are especially abundant in nerve-tissue and in the bile. In the gall-bladder they are frequently found in the form of gall-stones, and not uncommonly constitute the greater portion of their solids. Different varieties exist, such as the common cholesterin of the concretions just mentioned, the isocholesterin of woolfat (lanolin), and the phytosterins, paracholesterins, and kaulosterins of plants. While the structural composition of cholesterin has not been definitely ascertained, there is evidence to show that it may be a terpene. It is probably not formed as such in the animal body, but results in some manner from the vegetable forms. Like the fats, the cholesterins are insoluble in water, but soluble in ether, alcohol, and chloroform, from which solutions they may be obtained either in the form of very characteristic platelets or as needle-like crystals. In solutions of the alkalies, in the absence of alcohol, they are entirely insoluble, even on boiling, in which respect they differ from the fats. Like glycerin, cholesterin combines with fatty acids to form compound ethers, and in this form it is frequently found in nature. In wool-fat, for example, it is thus present in large amounts, and from it such ethers can be readily obtained. In pure form they constitute the lanolin of the shops. These ethers show a 
remarkable difference, as compared with the fats, in their behavior toward water. Of this they apparently take up one-quarter of their own weight, and on stirring give rise to a pasty, frothy mass.

From their ethereal compounds cholesterin can readily be separated by treating with diacetic-ethyl ether, which dissolves the cholesterin and leaves the ethers behind.

Of the functions of the cholesterins nothing is as yet known. It is interesting to note that they inhibit the activating properties of lecithin upon hæmolytic substances. If washed red corpuscles are thus treated with cobra poison no hæmolysis occurs, while this takes place if a trace of lecithin is added. 'Should, however, a small amount of a methyl-alcoholic solution of cholesterin be simultaneously added no hæmolysis occurs.

The isolation of the cholesterins will be described in a future chapter.

After having thus studied the three great classes of food-stuffs which plants are capable of elaborating from water, carbon dioxide, and certain mineral salts, and which are also represented in the animal body, we shall now proceed to a survey of the natural decomposition-products of these substances which are formed during their passage through the animal body, and which are of more or less interest as indicating the manner in which these decompositions are effected. Like the substances that have already been considered, these products will be taken up only in a general way at first; while their special study, as well as their methods of isolation and quantitative estimation, will be considered in succeding chapters, in connection with the chemical study of those tissues in which they are principally encountered. At this place general facts only are to be impressed upon the mind of the student, so that he will be prepared to understand the composite chemical structure of the various tissues and organs of the body, as will be described later. 


\section{CHA P TER V.}

THE CLEAVAGE-PRODUCTS OF THE ALBUMINS.

\section{THE MONO-AMINO-ACIDS.}

THE mono-amino-acid derivatives of the albumins are glyoocoll (glycin), alanin, amino-isovalerianic acid, leucin, isoleucin, serin, aspartic acid, glutaminic acid, phenyl-alanin, tyrosin, $a$-pyrrollidin carbonic acid (prolin), tryptophan, oxy-pyrrollidin carbonic acid (oxyprolin), and cystin. Of these, glycocoll, alanin, aminoisovalerianic acid, leucin, and isoleucin are amino-derivatives of monobasic acids of the formic series. They have neither marked acid nor basic properties, but in a manner unite both.

Glycocoll or glycin is amino-acetic acid: $\mathrm{CH}_{2}\left(\mathrm{NH}_{2}\right)$. $\mathrm{COOH}$.

Alanin is $a$-amino-propionic acid: $\mathrm{CH}_{3} \cdot \mathrm{CH}\left(\mathrm{NH}_{2}\right) \cdot \mathrm{COOH}$. Having an asymmetric carbon atom, it is optically active (dextrorotatory). This is true of all the decomposition-products of albumin, with the exception of glycocoll.

The amino-valerianic acid which results on decomposition of albumins is amino-isovalerianic acid :

$$
\underset{\mathrm{CH}_{3}}{\mathrm{CH}_{3}}-\mathrm{CH} \cdot \mathrm{CH}\left(\mathrm{NH}_{2}\right) \cdot \mathrm{COOH} \text {. }
$$

It is dextrorotatory.

Leucin is amino-isobutyl-acetic acid :

$$
\mathrm{CH}_{3}>\mathrm{CH} \cdot \mathrm{CH}_{2} \cdot \mathrm{CH}\left(\mathrm{NH}_{2}\right) \cdot \mathrm{COOH} \text {; }
$$

the usual product obtained on hydrolysis of albumins is lævorotatory.

Isoleucin corresponds to an $a$-amino-methyl-ethyl-propionic acid :

$$
\underset{\mathrm{C}_{2} \mathrm{H}_{5}}{\mathrm{CH}_{5}}>\mathrm{CH} \cdot \mathrm{CH}\left(\mathrm{NH}_{2}\right) \cdot \mathrm{COOH} \text {. }
$$

Serin is an $\alpha$-amino- $\beta$-oxypropionic acid. It is thus closely related to alanin and represented by the formula: $\mathrm{CH}_{2}(\mathrm{OH}) \cdot \mathrm{CH}\left(\mathrm{NH}_{2}\right) \cdot \mathrm{COOH}$. Only the racemic form has thus far been obtained.

Aspartic acid and glutaminic acid are dibasic acids of the oxalic series. Both have markedly acid properties. 
Aspartic acid is $\alpha$-amino-succinic acid :

$$
\mathrm{COOH} \cdot \mathrm{CH} .\left(\mathrm{NH}_{2}\right) \cdot \mathrm{CH}_{2} \cdot \mathrm{COOH} \text {. }
$$

Glutaminic acid has the formula :

$$
\mathrm{COOH} . \mathrm{CH}\left(\mathrm{NH}_{2}\right) \cdot \mathrm{CH}_{2} \cdot \mathrm{CH}_{2} \cdot \mathrm{COOH} \text {. }
$$

The former is lævorotatory, the latter dextrorotatory. Their amides, viz., asparagin, $\mathrm{CONH}_{2}$ and glutamin, $\mathrm{CONH}_{2}$

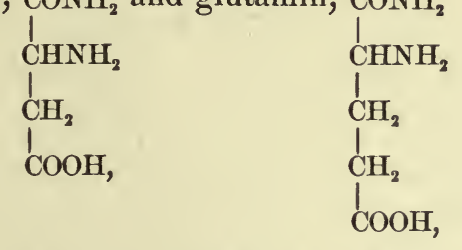

occur widely distributed in the vegetable world, but do not occur in animal tissues.

Cystin is the disulphide of $\beta$-cystein, $\alpha$-amino- $\beta$-thiolactic acidviz., $\alpha$-diamino- $\beta$-dithio-dilactic acid :<smiles>NC(CSSCC(O)C(=O)O)C(N)C(=O)OCc1ccccc1</smiles>

Phenyl-alanin and tyrosin are members of the aromatic series. Phenyl-alanin corresponds to a phenyl- $\alpha$-amino-propionic acid :

$$
\mathrm{C}_{6} \mathrm{H}_{6} \cdot \mathrm{CH}_{2} \cdot \mathrm{CH}\left(\mathrm{NH}_{2}\right) \cdot \mathrm{COOH} \text {; }
$$

it occurs in the albumins as the lævorotatory form.

Tyrosin is a corresponding oxy-acid, viz., $p$-oxyphenyl-alanin or $p$-oxyphenyl- $a$-amino-propionic acid :

$$
\mathrm{C}_{6} \mathrm{~F}_{4} \cdot \mathrm{OH} \cdot \mathrm{CH}_{2}\left(\mathrm{NH}_{2}\right) \cdot \mathrm{COOH} \text {. }
$$

Both the dextro- and the lævorotatory form occur, the latter predominating.

$a$-pyrrolidin-carbonic acid, tryptophan, and oxypyrrolidin-carbonic acid are heterocyclic compounds.

Both $a$-pyrrollidin-carbonic acid (prolin) and the corresponding oxy-compound (oxyprolin) are now recognized as primary split products of the albuminous molecule. Prolin is represented by the formula :<smiles>CCOC(=O)C1CCCN1</smiles>

while oxyprolin is $\mathrm{C}_{5} \mathrm{H}_{9} \mathrm{NO}_{3}$. 
Tryptophan is a skatol-amino-acetic acid and has the formula :

or

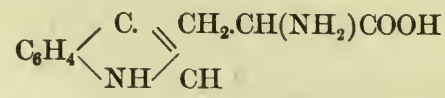

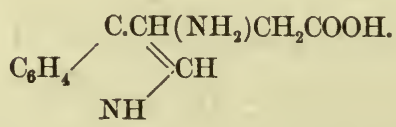

All the amino-acids mentioned are albuminous derivatives and represent integral constituents of the albuminous molecule. Their quantitative relations, as has been indicated, are not constant, however, and upon these variations no doubt the characteristics of the individual members of the group are in great part dependent.

The amino-acids of the fatty series are of special interest, as they are intimately concerned in the production of urea. Von Schröder, Nencki, and others have shown that in the liver the ammonium salt of carbonic acid, viz., amino-formic acid, is transformed into urea, and we also know that in the mammalian organism the nitrogen of leucin, glycocoll, and aspartic acid is eliminated in the form of urea. As regards the nature of the chemical changes which take place during the transformation of the amino-nitrogen into urea, our knowledge is not complete. It was formerly supposed that uric acid represented the immediate antecedent of urea and was transformed into this by oxidation. We find, as a matter of fact, that in birds and reptiles uric acid is the final decomposition-product of the nitrogenous metabolism, and is thus analogous to the urea of mammals. I have also pointed out that as a ureid, uric acid on oxidation can yield urea. But as far as is known, the uric acid of mammals is normally exclusively derived from the nucleinic bases, and is thus not formed in sufficient quantity to give rise to the large amount of urea which is daily eliminated in the urine. That a small fraction of the urea may result from uric acid by simple oxidation is possible, and indeed probable, but the greater portion must of necessity originate in a different manner.

In birds, on the other hand, some of the uric acid apparently results from glycocoll, and we thus see that in both classes of animals the amino-acids may be the antecedents of the final products of nitrogenous metabolism.

It has been suggested that in mammals cyanic acid may be produced as an intermediary product, and that urea results through a condensation of two molecules of this substance in statu nascendi, according to the equation:

$$
\mathrm{CONH}+\mathrm{CONH}=\mathrm{CO} \backslash_{\mathrm{NH}_{2}}^{\mathrm{NH}_{2}}+\mathrm{CO}_{2} \text {. }
$$

Then again we may imagine that a transformation of the amino- 
acids occurs into the ammonium salts of the fatty acids standing next in order in the downward scale, and that by further oxidation these are transformed into ammonium carbonate, and this into urea. It has been shown as a matter of fact that a fair amount of urea is produced when blood containing ammonium carbonate or ammonium formate is allowed to flow through the isolated livers of dogs.

According to Drechsel, the amino-acids are transformed into carbamic acid, from which urea may then result, as indicated by the equation :

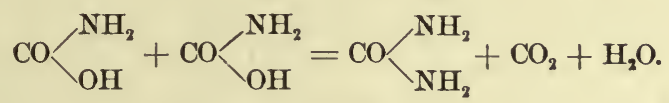

On the other hand, it is conceivable that the amino-group merely is split off and leads to the formation of urea, and that the remaining fatty acid radicles may be utilized in the synthesis of carbohydrates and fats.

In a subsequent chapter this subject will be treated in greater detail.

In addition to the important rôle which the amino-acids play in the formation of urea, they are of further interest from the part which they take in some of the syntheses that occur in the animal organism. With benzoic acid glycocoll thus combines to form hippuric acid, as shown by the equation :

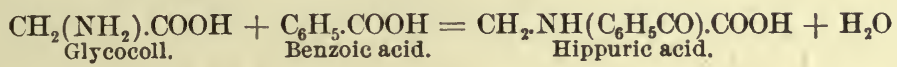

With phenyl-acetic acid glycocoll similarly combines to form phenaceturic acid :

$$
\mathrm{CH}_{2} \cdot\left(\mathrm{NH}_{2}\right) \cdot \mathrm{COOH}+\mathrm{CH}_{2} \cdot\left(\mathrm{C}_{6} \mathrm{H}_{5}\right) \cdot \mathrm{COOH}=\mathrm{C}_{6} \mathrm{H}_{5} \cdot \mathrm{CH}_{2} \cdot \mathrm{CO}-\mathrm{NH} \cdot \mathrm{CH}_{2} \cdot \mathrm{COOH}+\mathrm{H}_{2} \mathrm{O} \cdot
$$

That uric acid on hydrolytic decomposition will yield ammonia, carbon dioxide, and glycocoll has been shown. There is evidence, moreover, to show that in the organism of birds and reptiles, at least, its synthesis can similarly occur.

Ornithuric acid results through the union of benzoic acid with the diamine ornithin :

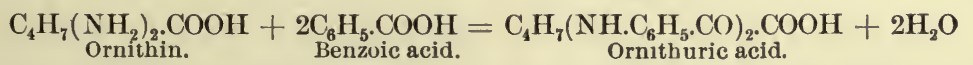

The amino-acids also are closely related to the biliary acids, as on decomposition with baryta-water, glycocholic acid is decomposed into glycocoll and cholalic acid, as shown in the equation :

$$
\underset{\substack{\text { Glycocholic } \\
\text { acid. }}}{\mathrm{C}_{26} \mathrm{H}_{43} \mathrm{NO}_{6}}+\mathrm{H}_{2} \mathrm{O}=\mathrm{CH}_{2}\left(\underset{\text { Glycocoll. }}{\left.\mathrm{NH}_{2}\right) \cdot \mathrm{COOH}}+\underset{\begin{array}{c}
\text { Cholalic } \\
\text { acid. }
\end{array}}{\mathrm{C}_{24} \mathrm{H}_{40} \mathrm{O}_{5}}\right.
$$

Taurocholic acid similarly gives rise to cholalic acid and taurin, which latter can be regarded as amino-isethionic acid-that is, as 
isethionic acid, $\mathrm{H}_{2}\left(\mathrm{C}_{2} \mathrm{H}_{4} \cdot \mathrm{OH}\right) \mathrm{SO}_{3}$, in which the hydroxyl group has been replaced by the amino-radicle:

$$
\underset{\substack{\text { Taurocholic } \\ \text { acid. }}}{\mathrm{C}_{26} \mathrm{H}_{45} \mathrm{NO}_{7} \mathrm{~S}}+\mathrm{H}_{2} \mathrm{O}=\underset{\substack{\text { Cholalic } \\ \text { acid. }}}{\mathrm{C}_{24} \mathrm{H}_{40} \mathrm{O}_{5}}+\underset{\text { Taurin. }}{\mathrm{C}_{2} \mathrm{H}_{7} \mathrm{NO}_{3} \mathrm{~S}}
$$

After the ingestion of amino-acids, moreover, the corresponding compounds of carbamide, $-\mathrm{CONH}$, appear in the urine. These are spoken of as the uramic acids, and comprise methyl-hydantoinic acid, taurocarbamic acid, uramino-benzoic acid, and tyrosin-hydantoinic acid, or hydantoin-hydroparacumaric acid. They are found after the ingestion of sarcosin or methyl-glycocoll, of taurin, aminobenzoic acid, and tyrosin, respectively. The syntheses which are thus effected may be represented by the equations :

$$
\begin{aligned}
& \underset{\text { Sarcosin. }}{\mathrm{C}_{3} \mathrm{H}_{7} \mathrm{O}_{2}}+\mathrm{CONH} \underset{\text { Methyl-hydantoinic }}{=} \underset{\text { Cacid }}{\mathrm{C}_{4} \mathrm{H}_{8} \mathrm{~N}_{2} \mathrm{O}_{3}} \\
& \underset{\text { Taurin. }}{\mathrm{C}_{2} \mathrm{H}_{7} \mathrm{NSO}_{3}}+\mathrm{CONH}=\underset{\substack{\text { Taurocarbamic } \\
\text { acid. }}}{\mathrm{C}_{3} \mathrm{H}_{8} \mathrm{~N}_{2} \mathrm{SO}_{4}} \\
& \underset{\text { Aminobenzoic }}{\mathrm{C}_{5} \mathrm{H}_{7} \mathrm{NO}_{2}}+\mathrm{CONH}=\underset{\text { Uramino-benzoic }}{\mathrm{C}_{8} \mathrm{H}_{8} \mathrm{~N}_{2} \mathrm{O}_{3}} \\
& \text { acid. acid. } \\
& \underset{\text { Tyrosin. }}{\mathrm{C}_{9} \mathrm{H}_{11} \mathrm{NO}_{2}}+\mathrm{CONH} \underset{\text { Tyrosin-hydantoinic }}{=} \underset{\mathrm{C}_{10} \mathrm{H}_{10} \mathrm{~N}_{2} \mathrm{O}_{3}}{\mathrm{H}_{2} \mathrm{O}}
\end{aligned}
$$

The cystin complex of the albuminous molecule is the mothersubstance of taurin. On oxidation it is first transformed into cysteinic, acid and by loss of $\mathrm{CO}_{2}$ this gives rise to taurin, as represented by the equations:

(1)

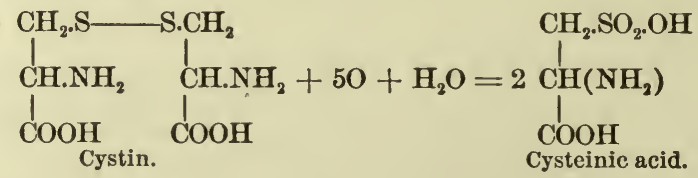

(2)

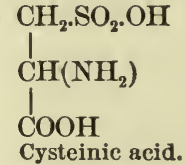

$$
-\mathrm{CO}_{3}=\quad \begin{aligned}
& \mathrm{CH}_{2} \cdot \mathrm{SO}_{2} \cdot \mathrm{OH} \\
& \mathrm{CH}_{2}\left(\mathrm{NH}_{2}\right)
\end{aligned}
$$

Analogous to the formation of taurocarbaminic acid from taurin cystin gives rise to the formation of cystin hydantoinic acid on treating with cyanic acid :

On reduction cystin yields cystein :<smiles>NC(C=CC(C(=O)O)C(N)CS)C(=O)O</smiles>

On reduction the amino-acids are transformed into the correspond- 
ing acids from which they are derived. Glycocoll is thus transformed into acetic acid, leucin into capronic acid, asparaginic acid into suocinic acid, glutaminic acid into glutaric acid, tyrosin into para-oxyphenyl-propionic acid (hydroparacumaric acid), etc., as shown by the equations:

$$
\begin{aligned}
& \mathrm{CH}_{2}\left(\mathrm{NH}_{\text {Glycocoll. }}\right) \cdot \mathrm{COOH}+2 \mathrm{H}=\underset{\text { Acetic acid. }}{\mathrm{CH}_{3} \cdot \mathrm{COOH}}+\mathrm{NH}_{3} \\
& \mathrm{CH}_{2} \cdot \mathrm{CH}\left(\mathrm{NH}_{2}\right) \cdot \underbrace{\mathrm{COOH}}_{\mathrm{COOH}}+2 \mathrm{H}=\mathrm{CH}_{2} \cdot \mathrm{CH}_{2} \cdot \underbrace{\mathrm{COOH}}_{\text {succinic acid. }}+\mathrm{NH}_{3} \\
& \mathrm{C}_{6} \mathrm{H}_{4}\left\langle\underset{\substack{\text { Tyrosin. } \\
\mathrm{CH}_{2} \cdot \mathrm{CH}\left(\mathrm{NH}_{2}\right) \cdot \mathrm{COOH}}}{\mathrm{OH}}+2 \mathrm{H}=\underset{\substack{\text { Para-oxy-phenyl-propionic } \\
\text { acid. }}}{\mathrm{C}_{6} \mathrm{H}_{4}} \underset{\substack{\mathrm{CH}_{2} \cdot \mathrm{CH}_{2} \cdot \mathrm{COOH} \\
\mathrm{OH}_{2}}}{\mathrm{OH}}+\mathrm{NH}_{3}\right.
\end{aligned}
$$

On oxidation these are further changed into the acids standing next in order in the downward scale. Acetic acid thus gives rise to the formation of formic acid, succinic acid to malonic acid, para-oxyphenyl-propionic acid to para-oxy-phenyl-acetic acid, etc., as shown by the equations :

$$
\begin{aligned}
& \underset{\text { Acetic acid. }}{\mathrm{CH}_{3} \cdot \mathrm{COOH}}+3 \mathrm{O}=\underset{\text { Formic acid. }}{\mathrm{H} . \mathrm{COOH}}+\mathrm{H}_{2} \mathrm{O}+\mathrm{CO}_{2} \\
& \mathrm{CH}_{2} \cdot \mathrm{CH}_{2} \cdot \underbrace{\mathrm{COOH}}_{\text {Succinic acid. }}+3 \mathrm{OH}=\mathrm{CH}_{2} \int_{\mathrm{COOH}}^{\mathrm{COOH}}+\mathrm{H}_{2} \mathrm{O}+\mathrm{CO}_{2}
\end{aligned}
$$

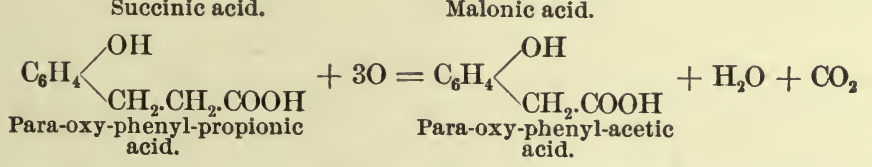

Through a splitting off of carbon dioxide para-oxy-phenyl-acetic acid then further gives rise to paracresol, from which phenol is finally obtained on oxidation :

$$
\begin{aligned}
& \mathrm{C}_{6} \mathrm{H}_{4}<\mathrm{CH}_{\mathrm{CH}_{2} \cdot \mathrm{COOH}}^{\mathrm{OH}}=\underset{\text { Paracresol. }}{\mathrm{C}_{6} \mathrm{H}_{4}} \underbrace{\mathrm{OH}}_{\mathrm{CH}_{3}}+\mathrm{CO}_{2} \\
& \mathrm{C}_{6} \mathrm{H}_{4} \succ_{\mathrm{CH}_{3}}^{\mathrm{OH}}+3 \mathrm{O}=\underset{\text { Phenol. }}{\mathrm{C}_{6} \mathrm{H}_{5} \cdot \mathrm{OH}}+\mathrm{CO}_{2}+\mathrm{H}_{2} \mathrm{O}
\end{aligned}
$$

In the animal body paracresol and phenol, which are formed from tyrosin during the process of intestinal putrefaction, then combine with sulphuric acid, and are eliminated through the urine in the form of their potassium salts:

$$
\begin{aligned}
& \mathrm{C}_{6} \mathrm{H}_{4} \succ_{\mathrm{CH}_{3}}^{\mathrm{OH}}+\mathrm{SO}_{2} \succ_{\mathrm{OH}}^{\mathrm{OH}}=\mathrm{C}_{6} \mathrm{H}_{4}<\overbrace{\mathrm{CH}_{3}}^{\mathrm{O} . \mathrm{HSO}_{3}}+\mathrm{H}_{2} \mathrm{O} \text {. } \\
& \mathrm{C}_{6} \mathrm{H}_{5} \cdot \mathrm{OH}+\mathrm{SO}_{2} \succ_{\mathrm{OH}}^{\mathrm{OH}}=\mathrm{C}_{6} \mathrm{H}_{5} \cdot \mathrm{O} \cdot \mathrm{HSO}_{3}+\mathrm{H}_{2} \mathrm{O} \text {. }
\end{aligned}
$$


Through loss of $\mathrm{CO}_{2}$ tyrosin yields para-oxyphenyl-ethylamin :

$$
\underset{\text { Tyrosin. }}{\mathrm{C}_{6} \mathrm{H}_{4}(\mathrm{OH}) \cdot \mathrm{CH}_{2} \cdot \mathrm{CH}\left(\mathrm{NH}_{2}\right) \cdot \mathrm{COOH}=\mathrm{CO}_{2}+\underset{6}{\mathrm{C}_{6} \mathrm{H}_{4}(\mathrm{OH}) \cdot \mathrm{CH}_{2} \cdot \mathrm{CH}_{2} \cdot \mathrm{NH}_{2}} \text { Oxyphenyl-ethylamin. }}
$$

In a similar manner phenyl-alanin can give rise to phenyl-ethy. lamin :

$$
\mathrm{C}_{6} \mathrm{H}_{5} \cdot \mathrm{CH}_{2} \cdot \mathrm{CH} \cdot \mathrm{NH}_{2} \cdot \mathrm{COOH}=\mathrm{CO}_{2}+\underset{\text { Phenyl-alanin. }}{\mathrm{C}_{6} \mathrm{H}_{5} \cdot \mathrm{CH}_{2} \cdot \mathrm{CH}_{2} \cdot \mathrm{NH}_{2}}
$$

Tryptophan (skatol-amino-acetic acid) is the only indol derivative which is formed from the albumins on hydrolysis by means of the common digestive ferments :

$$
\text { 等 }
$$

This can now be regarded as the mother-substance of the aromatic products indol, skatol, and skatol-carbonic acid, which are constantly formed from the albumins by putrefactive organisms. In contradistinction to tyrosin and its derivatives these bodies belong to the ortho series.

Indol, skatol, and skatol-carbonic acid are closely related to each other and to indigo. Skatol thus results from indol through a substitution of the methyl-group for a hydrogen atom of one of the $\mathrm{CH}$ groups, as shown by the formula :<smiles>C1=CCCCC1</smiles><smiles>C=CNC(C)(C)C</smiles>

Skatol-carbonic acid then results from skatol through a union with carbon dioxide :<smiles>C[C@H](C(=O)O)C(C)(C)C</smiles>

In their passage through the animal body indol and skatol are oxidized to indoxyl and skatoxyl, and are eliminated in the urine to a great extent, in combination with sulphuric acid, as potassium indoxyl sulphate (animal indican) and potassium skatoxyl sulphate, while the skatol-carbonic acid is excreted as such. These changes can be expressed by the equations :<smiles>O=CC1CCCCN1</smiles><smiles>C=CNC(C=C)C(=O)O</smiles>

(2)<smiles>O=C(O)CCCCC(=O)CCCC(=O)O</smiles> 
On decomposition with strong hydrochloric acid indican is accordingly decomposed into sulphuric acid and indoxyl, which latter can then be oxidized to indigo-blue :

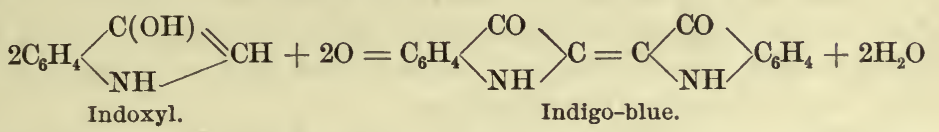

On reduction indigo-blue is transformed into indigo-white, $\mathrm{C}_{8} \mathrm{H}_{6} \mathrm{NO}$, which, when boiled with zinc and water, then further yields indol :

$$
\begin{aligned}
& \mathrm{C}_{8} \mathrm{H}_{5} \mathrm{NO}+\mathrm{H}=\mathrm{C}_{8} \mathrm{H}_{6} \mathrm{NO} . \\
& \mathrm{C}_{8} \mathrm{H}_{6} \mathrm{NO}+3 \mathrm{H}=-\mathrm{C}_{8} \mathrm{H}_{7} \mathrm{~N}+\mathrm{H}_{2} \mathrm{O} .
\end{aligned}
$$

Animal indican must not be confused with vegetable indican, which is a glucoside, and yields indigo-blue and indiglucin on hydrolytic decomposition :

$$
\underset{\text { vegetable indican. }}{\mathrm{C}_{26} \mathrm{H}_{31} \mathrm{NO}_{17}}+2 \mathrm{H}_{2} \mathrm{O}=\underset{\text { Indigo-blue. }}{\mathrm{C}_{8} \mathrm{H}_{5} \mathrm{NO}}+\underset{\text { Indiglucin. }}{3 \mathrm{C}_{6} \mathrm{H}_{10} \mathrm{O}_{6}}
$$

A small amount of skatoxyl, indoxyl, and phenol is also eliminated in the urine, in combination with glucuronic acid, as skatoxyl, indoxyl, and phenol glucuronates, respectively. This acid may be derived from glucose by the substitution of one atom of oxygen for two atoms of hydrogen, and is accordingly represented by the formula $\mathrm{COOH} .(\mathrm{CH} . \mathrm{OH})_{4} \cdot \mathrm{COH}$. On oxidation it is transformed into saccharinic acid, the relation of which to glucose has already been considered.

Glucosamin (chitosamin) occupies a position intermediary between the amino-acids and the carbohydrates. It is an amino-hexose and has the formula: $\mathrm{CH}_{2}(\mathrm{OH}) \cdot[\mathrm{CH}(\mathrm{OH})]_{3} \cdot \mathrm{CH}\left(\mathrm{NH}_{2}\right) \cdot \mathrm{CHO}$. It is the mother-substance from which chitose is built up and which is found extensively among invertebrates. The relation existing between the two is seen from the equations:

(1) $\mathrm{CH}_{2} \cdot \mathrm{OH} \cdot[\mathrm{CH} . \mathrm{OH}]_{3} \cdot \mathrm{CH} \cdot \mathrm{NH}_{2} \cdot \mathrm{COH}+4 \mathrm{O}=$ $\mathrm{CH}_{2} \cdot \mathrm{OH}[\mathrm{CH} \cdot \mathrm{OH}] \cdot \mathrm{COOH}+\mathrm{HNO}_{2}$ Chitonic acid.

(2) $\mathrm{CH}_{2} \cdot \mathrm{OH}[\mathrm{CH} \cdot \mathrm{OH}]_{4} \cdot \mathrm{COOH}+2 \mathrm{H}=$ Chitose.

\section{THE DIAMINO-ACIDS.}

The diamino-acids which have been obtained upon hydrolysis of the albumins are arginin, lysin, and diamino-trioxydodecanic acid. The two former are dianino-monocarbonic acids; the latter is a diamino-oxymonocarbonic acid. Together with arginin and lysin histidin is frequently found, and for some years it also was regarded 
as a diamino-acid. Collectively the three were termed hexon bases by Kossel. It is now known that histidin is not a diamino-acid, however, but for convenience' sake it is considered in this connection.

Arginin and lysin are common components of the albumins, while diamino-trioxydodecanic acid has only been obtained from casein. But there is evidence to show that it also or closely related bodies may occur in other albumins.

Arginin is a guanidin derivative of $\alpha, \delta$-diamino-valerianic acid (ornithin) and represented by the formula:

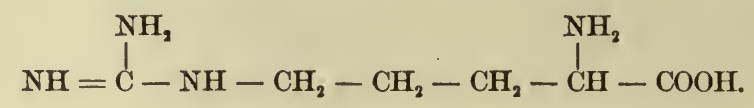

On hydrolysis it is decomposed into urea and ornithin :

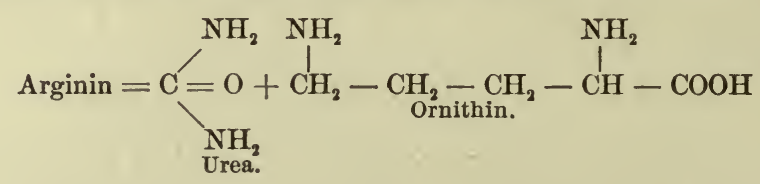

The correctness of this formula is supported by the synthesis of arginin from ornithin and cyanamide, viz. :

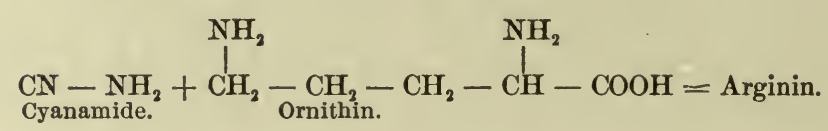

On oxidizing arginin with barium permanganate, Kutscher obtained guanidin, with guanidin-butyric acid as intermediary product.

It is very interesting to note that ornithin can give rise to putrescin, viz., to tetramethylene-diamin, a ptomain which is formed during the putrefaction of albuminous material, and which also has been found in the urine in association with cystin. Thus far this transformation has been effected only through the agency of microorganisms, but there is no reason to suppose that their presence is essential, and that in the tissues of the living body the same process cannot also occur. This transformation may be represented by the equation :

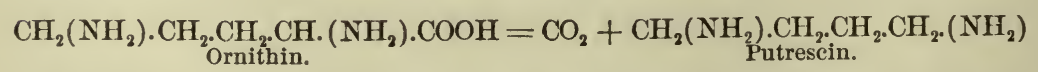

Lysin.-Lysin is apparently a homologue of ornithin, and is represented by the formula $\mathrm{CH}_{2}\left(\mathrm{NH}_{2}\right) \cdot \mathrm{CH}_{2} \cdot \mathrm{CH}_{2} \cdot \mathrm{CH}_{2} \cdot \mathrm{CH}\left(\mathrm{NH}_{2}\right)$ $\mathrm{COOH}$; it is thus $\alpha$, $\varepsilon$-diamino-capronic acid. On hydrolytic decomposition it yields ammonia, oxalic acid, propionic acid, and notably acetic acid. On oxidation of lysin with barium perman- 
ganate Zickgraf obtained hydrocyanic acid, oxalic acid, normal glutaric acid $\left(\mathrm{COOH} \cdot \mathrm{CH}_{2} \cdot \mathrm{CH}_{2} \cdot \mathrm{CH}_{2} \cdot \mathrm{COOH}\right)$, and probably also glutaminic acid. When exposed to the influence of putrefactive organisms it gives rise to the formation of cadaverin-penta-methylene-diamin-a ptomain which is frequently found together with putrescin in putrefying albuminous material, and, like this, may also appear in the urine in association with cystin. Its formation is analagous to that of putrescin from ornithin, and may be represented by the equation:

$$
\mathrm{CH}_{2}\left(\mathrm{NH}_{2}\right) \cdot \underset{\text { Lysin. }}{\mathrm{CH}_{2}} \cdot \mathrm{CH}_{2} \cdot \mathrm{CH}_{2} \cdot \mathrm{CH}\left(\mathrm{NH}_{2}\right) \cdot \underset{\mathrm{CO}_{2}}{\mathrm{COOH}}=\underset{\text { Cadaverin. }}{\mathrm{CH}_{2}}\left(\mathrm{NH}_{2}\right) \cdot \underset{\mathrm{CH}_{2}}{\mathrm{CH}_{2}} \cdot \mathrm{CH}_{2} \cdot \mathrm{CH}_{2}\left(\mathrm{NH}_{2}\right)
$$

In this manner albumins can give rise to the formation of pyridins, and, as a matter of fact, piperidin results during the dry distillation of cadaverin, as represented by the equation :

$$
\mathrm{CH}_{2}\left(\mathrm{NH}_{2}\right) \cdot \mathrm{CH}_{2} \cdot \mathrm{CH}_{2} \cdot \mathrm{CH}_{2} \cdot \mathrm{CH}_{2}\left(\mathrm{NH}_{2}\right)=\mathrm{CH}_{2} \overbrace{\mathrm{CH}_{2}-\mathrm{CH}_{2}}^{\mathrm{CH}_{2}-\mathrm{CH}_{2}} \mathrm{NH}+\mathrm{NH}_{3} \text {. }
$$

On treating lysin with benzoyl chloride Drechsel obtained a body, of the formula $\mathrm{C}_{6} \mathrm{H}_{12}\left(\mathrm{COC}_{6} \mathrm{H}_{5}\right)_{2} \mathrm{~N}_{2} \mathrm{O}_{2}$, which he termed lysuric acid, and which is thus homologous with the dibenzoyl derivative of ornithin, $\mathrm{C}_{5} \mathrm{H}_{10}\left(\mathrm{COC}_{6} \mathrm{H}_{5}\right)_{2} \mathrm{~N}_{2} \mathrm{O}_{2}$, ornithuric acid.

An inactive lysin has been obtained synthetically from $\gamma$-cyanpropyl-malonyl ether $\left(\mathrm{NC} .\left(\mathrm{CH}_{2}\right)_{3} \cdot \mathrm{CH}<\mathrm{CO}_{2} \mathrm{CO}_{2} \mathrm{H}_{5}\right)$, over $\alpha$-oximido$\delta$-cyan-valerianic ethyl ether $\left(\mathrm{NC} .\left(\mathrm{CH}_{2}\right)_{3} \cdot \mathrm{C}<{ }_{\mathrm{NOH}}^{\mathrm{CO}_{2} \mathrm{C}_{2} \mathrm{H}_{5}}\right)$. On racemisation this was shown to be identical with the active lysin.

Diamino-trioxydodecanic Acid $\left(\mathrm{C}_{12} \mathrm{H}_{26} \mathrm{~N}_{2} \mathrm{O}_{5}\right)$. - As has been pointed out above, this acid has thus far only been obtained from casein; but there is reason to think that it or similar products may also be contained in other proteins. Its constitution has not yet been established.

Histidin.-Histidin is formed in very small amounts only during the decomposition of proteins and may be absent. It was long regarded as a diamino-acid, but is now viewed as $\alpha$-amino- $\beta$-imidazol propionic acid, and is probably represented by the formula:

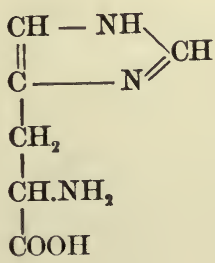


According to Gulewitch, it results from carnosin together with alanin, according to the equation :

$$
\mathrm{C}_{9} \mathrm{H}_{14} \mathrm{~N}_{4} \mathrm{O}_{3}+\mathrm{H}_{2} \mathrm{O}=\mathrm{C}_{6} \mathrm{H}_{9} \mathrm{~N}_{3} \mathrm{O}_{2}+\mathrm{C}_{3} \mathrm{H}_{7} \mathrm{NO}_{2} \text {. }
$$

It gives the biuret reaction-at first a violet color, which gradually changes to red.

\section{THE ORGANIC NON-NITROGENOUS DERIVATIVES.}

The organic non-nitrogenous acids which are formed in the animal body are largely members of the fatty acid series. Others belong to the glycolic series, still others to the acrylic series, some are representatives of the oxalic series, and still others belong to the aromatic oxy-acids. The fatty group comprises the following acids, which as a class may be represented by the formula $\mathrm{C}_{n} \mathrm{H}_{2 n} \mathrm{O}_{2}$ :

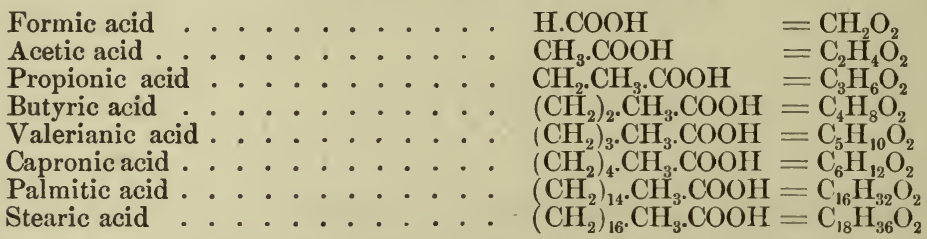

The two last, as has been seen, are integral constituents of the fats, in which they are present in combination with glycerin as triglycerides. From these the others may in part be derived, but to the greatest extent no doubt they result from the amido-acids through a process of oxidation or reduction, as illustrated by the equations :

$$
\begin{aligned}
& \mathrm{CH}_{2}\left(\underset{\text { Glycocoll. }}{\left.\mathrm{NH}_{2}\right) \cdot \mathrm{COOH}}+2 \mathrm{O}=\underset{\text { Formic }}{\mathrm{H} \cdot \mathrm{COOH}}+\mathrm{NH}_{3}+\mathrm{CO}_{2}\right. \\
& \mathrm{CH}_{2}\left(\mathrm{NH}_{2}\right) \cdot \mathrm{COOH}+2 \mathrm{H}=\underset{\text { Acetic acid. }}{\mathrm{CH}_{3} \cdot \mathrm{COOH}}+\mathrm{NH}_{3}
\end{aligned}
$$

Through further oxidation these acids can be transformed into those standing next in order in the downward scale, and so on, until finally carbon dioxide and water result, as seen in the equations :

(1) $\underset{\text { Propionic acid. }}{\mathrm{CH}_{2} \cdot \mathrm{CH}_{3} \cdot \mathrm{COOH}}+3 \mathrm{O}=\underset{\text { Acetic acid. }}{\mathrm{CH}_{3} \cdot \mathrm{COOH}}+\mathrm{CO}_{2}+\mathrm{H}_{2} \mathrm{O}$

(2) $\underset{\text { Acetic acid. }}{\mathrm{CH}_{3} . \mathrm{COOH}}+3 \mathrm{O}=\underset{\substack{\text { Formic } \\ \text { acid. }}}{\mathrm{H} \cdot \mathrm{COOH}}+\mathrm{CO}_{2}+\mathrm{H}_{2} \mathrm{O}$

(3) $\underset{\text { Formic acid. }}{\mathrm{H} . \mathrm{COOH}} \quad+\mathrm{O}=\mathrm{CO}_{2} \quad+\mathrm{H}_{2} \mathrm{O}$

To some extent the fatty acids are derived also from lactic acid and related acids. The transformation of these into fatty acids is probably analogous to the production of butyric acid during the process of butyric acid fermentation.

$$
\underset{\substack{\text { Lactic } \\ \text { acid. }}}{2 \mathrm{C}_{3} \mathrm{H}_{6} \mathrm{O}_{3}}=\underset{\substack{\text { Butyrie } \\ \text { acid. }}}{\mathrm{C}_{4} \mathrm{H}_{8} \mathrm{O}_{2}}+2 \mathrm{CO}_{2}+4 \mathrm{H}
$$


The glycolic acids which are found in the animal body may be represented by the general formula $\mathrm{C}_{n} \mathrm{H}_{2 n} \mathrm{O}_{3}$. They comprise the following bodies:

Glycolic acid . . . . . . . $\mathrm{CH}_{2} . \mathrm{OH} . \mathrm{COOH}$

Ethylidene-lactic acid (paralactic acid) . $\mathrm{CH}_{3} \cdot \mathrm{CH}(\mathrm{OH}) \cdot \mathrm{COOH}$

Ethylidene-lactic acid (optically inactive) $\mathrm{CH}_{3} . \mathrm{CH}(\mathrm{OH}) . \mathrm{COOH}$

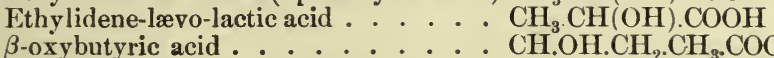

$=\mathrm{C}_{2} \mathrm{H}_{4} \mathrm{O}_{3}$

$=\mathrm{C}_{3} \mathrm{H}_{6} \mathrm{O}_{3}$

Leucinic acid . . . . . . . $\left(\mathrm{CH}_{3}\right)_{2} \cdot \mathrm{CH} \cdot \mathrm{CH}_{2} \cdot \mathrm{CH} \cdot \mathrm{OH} \cdot \mathrm{COOH}=\mathrm{C}_{6}^{4} \mathrm{H}_{12} \mathrm{O}_{3}$

Of these acids, glycolic acid does not occur in the animal body, so far as is known, but it is of interest owing to the fact that it is closely related to glycocoll, and is derived from this in the same manner in which leucinic acid is obtained from leucin, viz., by the substitution of a hydroxyl group for the amido-group, as shown by the equations :

$$
\begin{aligned}
& \mathrm{CH}_{2} \cdot \underset{\text { Glycocoll. }}{\left.\mathrm{NH}_{2}\right) \cdot \mathrm{COOH}}+\mathrm{H}_{2} \mathrm{O}=\underset{\text { Glycolic acid. }}{\mathrm{CH}_{2}(\mathrm{OH}) \cdot \mathrm{COOH}}+\mathrm{NH}_{3} \\
& \left(\mathrm{CH}_{3}\right)_{2} \cdot \mathrm{CH} \cdot \mathrm{CH}_{2} \cdot \mathrm{CH} \cdot\left(\mathrm{NH}_{2}\right) \cdot \mathrm{COOH}+\mathrm{H}_{2} \mathrm{O}= \\
& \text { Leucin. }\left(\mathrm{CH}_{3}\right)_{2} \cdot \mathrm{CH}_{\text {Leucinic acid. }}^{\mathrm{CCH}} \cdot \mathrm{CH}(\mathrm{OH}) \cdot \mathrm{COOH}+\mathrm{NH}_{3}
\end{aligned}
$$

$\beta$-oxybutyric acid is found only under pathologic conditions.

Lactic acid and its isomeric compounds, as well as leucinic acid and $\beta$-oxybutyric acid, are all albuminous decomposition-products, and in part, at least, derived from the amido-acids. The origin of lactic acid, however, is not so clear, but we shall consider this question in greater detail in a subsequent chapter.

On reduction these acids can be transformed into fatty acids. Lactic acid, as has just been shown, thus gives rise to butyric acid, leucinic acid is similarly changed to capronic acid, etc.

On oxidation $\beta$-oxybutyric acid is transformed into diacetic acid, which in turn is decomposed, with the formation of acetone and sarbon dioxide:

$$
\begin{gathered}
\underset{\text { B-oxybutyric acid. }}{\mathrm{CH}_{3} \cdot \mathrm{CH} . \mathrm{OH} . \mathrm{CH}_{2} \cdot \mathrm{COOH}}+\mathrm{O}=\underset{\text { Diacetic acid. }}{\left(\mathrm{CH}_{3} \cdot \mathrm{CO}\right) \mathrm{CH}_{2} \cdot \mathrm{COOH}}+\mathrm{H}_{2} \mathrm{O} \\
\left(\mathrm{CH}_{3} \cdot \mathrm{CO}\right) \mathrm{CH}_{2} \cdot \mathrm{COOH}=\underset{\text { Acetone. }}{\mathrm{CO}\left(\mathrm{CH}_{3}\right)_{2}+\mathrm{CO}_{2}}
\end{gathered}
$$

The bodies which are thus formed are of special interest to the pathologist, as their accumulation in the animal body is apparently capable of causing very serious disturbances. Acetone, in traces at least, is also met with under normal conditions.

On boiling with dilute mineral acids $\beta$-oxybutyric acid is transformed into an acid of the acrylic series (see below), viz., $\alpha$-crotonic acid :

$$
\mathrm{CH}_{3} \cdot \mathrm{CH}(\mathrm{OH}) \cdot \mathrm{CH}_{2} \cdot \mathrm{COOH}=2 \mathrm{H}_{2} \mathrm{O}+\underset{a \text {-Crotonic acid. }}{\mathrm{CH}_{3}}-\mathrm{CH} \cdot \mathrm{COOH}
$$

The acids of the acrylic series can be represented by the general formula $\mathrm{C}_{n} \mathrm{H}_{2 n \rightarrow 2} \mathrm{O}_{2}$. Representatives of these are the $\alpha$-capronic acid, just referred to, and oleic acid, which as a triglyceride rep- 
resents a most important constituent of many of the vegetable and animal fats. On heating with hydriotic acid and red phosphorus to a temperature of $210^{\circ} \mathrm{C}$, , oleic acid takes up two atoms of hydrogen, and is thus reduced to stearic acid:

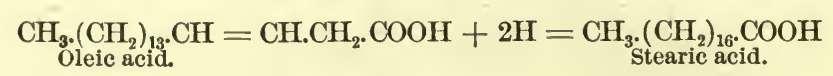

On treating with nitrous acid, in statu nascendi, it is transformed into the solid elaidinic acid, which is isomeric with oleic acid and belongs to the same series.

The dibasic acids of the oxalic series can be represented by the formula $\mathrm{C}_{n} \mathrm{H}_{2 n-2} \mathrm{O}_{4}$. They include oxalic acid, succinic acid, and glutaric acid, of which the two latter are generally met with in the form of their amides, viz., as aspartic acid and glutaminic acid, respectively. In this form they are invariably obtained, together with oxalic acid, as decomposition-products of most albumins. Oxalic acid and succinic acid, however, may also be observed as such.

The relation of oxalic acid to the ureids has already been considered. They are represented by the formulæ:

$$
\begin{aligned}
& \text { Oxalic acid . . . . . . ( }(\mathrm{COOH})_{2}=\mathrm{C}_{2} \mathrm{H}_{2} \mathrm{O}_{4} \\
& \text { Succinic acid ........ }\left(\mathrm{CH}_{2}\right)_{2^{\circ}}(\mathrm{COOH})_{2}=\mathrm{C}_{4} \mathrm{H}_{6} \mathrm{O}_{4} \\
& \text { Glutaric acid ........... }\left(\mathrm{CH}_{2}\right)_{3} \cdot(\mathrm{COOH})_{2}=\mathrm{C}_{5} \mathrm{H}_{8} \mathrm{O}_{4}
\end{aligned}
$$

On oxidation they are decomposed into water and carbon dioxide, but it is probable that during this transformation fatty acids are formed as intermediary products:

$$
\begin{aligned}
& \mathrm{C}_{2} \mathrm{H}_{2} \mathrm{O}_{4}=\mathrm{CO}_{2}+\mathrm{H} . \mathrm{COOH} . \\
& \mathrm{C}_{4} \mathrm{H}_{6} \mathrm{O}_{4}=\mathrm{CO}_{2}+\underset{\substack{\text { Propionic } \\
\text { acid. }}}{\mathrm{C}_{3} \mathrm{H}_{6} \mathrm{O}_{2}}
\end{aligned}
$$

The principal hydroxylated aromatic oxy-acids which may be found in the animal body are hydroparacumaric acid or para-oxyphenyl-propionic acid, para-oxy-phenyl-acetic acid, para-oxy-phenyl-lactic acid or oxy-hydroparacumaric acid, and para-oxy-phenyl-glycolic acid. They are derivatives of phenol, in which one hydrogen atom has been replaced by the radicle of the corresponding non-aromatic acid, as shown by the equation:

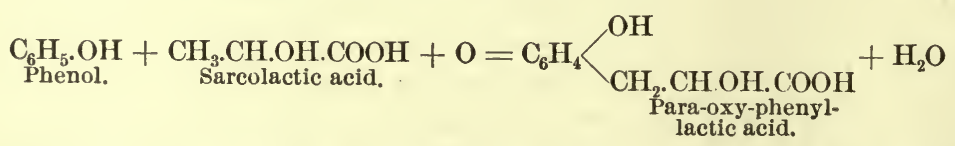

They are probably all derivatives of tyrosin, and it has already been shown how hydroparacumaric acid results from this on reduction, and is then transformed into para-oxy-phenyl-acetic acid by oxidation.

The formulæ of these acids and their relation to tyrosin are as follows : 
Tyrosin (para-oxy-phenyl-amino-propionic acid $)=\mathrm{C}_{6} \mathrm{H}_{4} \overbrace{\mathrm{CH}_{2} \cdot \mathrm{CH}\left(\mathrm{NH}_{2}\right) \cdot \mathrm{COOH}}^{\mathrm{OH}}$

$$
\text { Para-oxy-phenyl-propionic acid }=\mathrm{C}_{6} \mathrm{H}_{4} \overbrace{\mathrm{CH}_{2} \cdot \mathrm{CH}_{2} \cdot \mathrm{COOH}}^{\mathrm{OH}}
$$

On decomposition these acids yield phenol or cresol, water, and carbon dioxide, as shown by the equations :

(1)

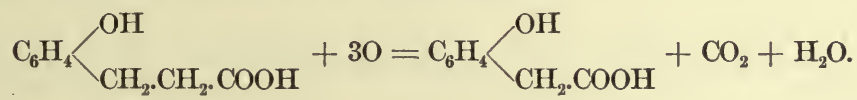

(2)

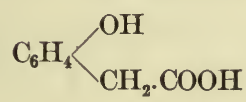

(3)<smiles>O=C(O)c1ccccc1</smiles>

$$
+3 \mathrm{O}=\mathrm{C}_{6} \mathrm{H}_{4} \int_{\substack{\text { Para-oxy- } \\ \text { benzoic acid. }}}^{\mathrm{OOH}}+\mathrm{CO}_{2}+\mathrm{H}_{2} \mathrm{O}
$$

or

$$
=\underset{\text { Phenol. }}{\mathrm{C}_{6} \mathrm{H}_{5} \mathrm{OH}}+\mathrm{CO}_{2}
$$

(1)

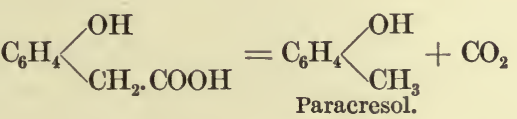

(2)

$$
\mathrm{C}_{6} \mathrm{H}_{4} \overbrace{\mathrm{CH}_{3}}^{\mathrm{OH}}+3 \mathrm{O}=\underset{6}{\mathrm{C}_{6} \mathrm{H}_{5} \cdot \mathrm{OH}}+\mathrm{CO}_{2}+\mathrm{H}_{2} \mathrm{O}
$$

From phenol the two dioxybenzols pyrocatechin and hydroquinon can then result, and appear in the urine together with phenol as conjugate sulphates or glucuronates.

$$
\mathrm{C}_{6} \mathrm{H}_{5} \cdot \mathrm{OH}+\mathrm{O}=\mathrm{C}_{6} \mathrm{H}_{4}<\begin{aligned}
& \mathrm{OH}(1) \\
& \mathrm{OH}(2) \text { or (4). }
\end{aligned}
$$

Of the non-hydroxylated aromatic acids, two are found in the animal body, and, like the hydroxylated compounds, originate during the process of albuminous putrefaction. These are phenyl-propionic acid (hydrocinnamic acid) and phenyl-acetic acid. They are rep resented by the formulæ:

$$
\begin{aligned}
& \mathrm{C}_{6} \mathrm{H}_{5} \cdot \mathrm{CH}_{2} \cdot \mathrm{CH}_{2 \cdot} \cdot \mathrm{COOH}=\text { phenyl-propionic acid } \\
& \mathrm{C}_{6} \mathrm{H}_{5} \cdot \mathrm{CH}_{2} \cdot \mathrm{COOH}=\text { phenyl-acetic acid. }
\end{aligned}
$$


By combining with glycocoll they give rise to the formation of hippuric acid and phenaceturic acid, respectively. In the first instance benzoic acid is probably formed as an intermediary body, which then unites with glycocoll, as shown in the equations below:

(1)

$$
\underset{\substack{\text { Phenyl-propionic } \\ \text { acid. }}}{\mathrm{C}_{6} \mathrm{H}_{5} \cdot \mathrm{CH}_{2} \cdot \mathrm{CH}_{2} \cdot \mathrm{COOH}}+6 \mathrm{O}
$$

(2)

$$
\underset{\text { Benzoic acid. }}{\mathrm{C}_{6} \mathrm{H}_{5} \cdot \mathrm{COOH}}+\underset{\text { Glycocoll. }}{\mathrm{CH}_{2}}\left(\underset{\mathrm{NH}_{2}}{\mathrm{Nl}_{2}} \cdot \mathrm{COOH}\right.
$$
and

$$
\underset{\substack{\text { Phenyl-acetic } \\ \text { acid. }}}{\mathrm{C}_{6} \mathrm{H}_{5}} \cdot \mathrm{CH}_{2} \cdot \mathrm{COOH}+\mathrm{CH}_{2}\left(\mathrm{NH}_{2}\right) \cdot \mathrm{COOH}=\mathrm{C}_{6} \mathrm{H}_{5} \cdot \underset{\text { Phenaceturic acid. }}{\mathrm{CH}_{2}} \cdot \mathrm{CO} \cdot \mathrm{NH} \cdot \mathrm{CH}_{3} \cdot \mathrm{COOH}+\mathrm{H}_{2} \mathrm{O}
$$$$
=\underset{\text { Benzoic acid. }}{\mathrm{C}_{6} \mathrm{H}_{5} \cdot \mathrm{COOH}}+2 \mathrm{CO}_{2}+2 \mathrm{H}_{2} \mathrm{O}
$$$$
=\mathrm{C}_{6} \mathrm{H}_{5} \cdot \underset{\text { Hippuric acid. }}{\text { COCH}} \cdot \text {. } \mathrm{NH} \cdot \mathrm{COOH}+\mathrm{H}_{2} \mathrm{O}
$$

The phenyl-acetic acid is no doubt derived from phenyl-alanin, just as para-oxyphenyl acetic acid results from tyrosin.

(1)

$$
\begin{aligned}
& \text { (1) } \mathrm{C}_{6} \mathrm{H}_{5} \cdot \underset{\text { Phenyl-alanin. }}{\mathrm{CH}_{2} \cdot \mathrm{CH} \cdot \mathrm{NH}_{2} \cdot \mathrm{COOH}}+2 \mathrm{H}=\underset{\text { Phenyl-propionic acid. }}{\mathrm{C}_{6} \mathrm{H}_{5} \cdot \mathrm{CH}_{2} \cdot \mathrm{CH}_{2} \cdot \mathrm{COOH}}+\mathrm{NH}_{3} \\
& \text { (2) } \mathrm{C}_{6} \mathrm{H}_{5} \cdot \mathrm{CH}_{2} \cdot \mathrm{CH}_{2} \cdot \mathrm{COOH} \\
& \text { Phenyl-propionic acid. }
\end{aligned}
$$

\section{THE CLEAVAGE-PRODUCTS OF THE NUCLEOPROTEIDS.}

\section{THE NUCLEINIC ACIDS.}

The nucleinic acids are specific decomposition-products of the nucleins (viz., nucleoproteids), and are characterized by the fact that on hydrolysis with certain mineral acids they yield phosphoric acid, purin bases, and frequently also pyrimidin derivatives. All nucleinic acids also contain at least one carbohydrate group.

Different nucleinic acids exist, which show a somewhat different composition according to the nucleoproteids from which they are derived. According to their origin, they are termed: spermatonucleinic acid, thymonucleinic acid, triticonucleinic acid, yeast nucleinic acid, etc. Such as they are obtained from the tissues the nucleinic acids possibly represent mixtures of different nucleinic acids of a simpler order. Neumann has pointed out that on treating the nucleoproteid of the thymus, spleen, pancreas, and the testicles of the ox, with caustic alkali, a nucleinic acid- $\alpha$ results, which is characterized by the fact that it gelatinizes in 5 per cent. or in stronger solution. This nucleinic acid- $\alpha$ apparently represents the native acid. On further treatment with caustic alkali it passes over into the nucleinic acid- $\beta$ through a process of depolymerization. This second acid does not gelatinize, and is more readily soluble than the $\alpha$ form. During this transformation two-thirds of the nucleinic bases are split off. Elementary analysis of the two forms (obtained from the thymus) has given the following results :

$$
\begin{aligned}
& \text { a-acid ................ }\left(\mathrm{C}_{41} \mathrm{H}_{74} \mathrm{~N}_{14} \mathrm{O}_{26} \mathrm{P}_{4}\right)_{n} \\
& \beta \text {-acid . . . . . . . } \mathrm{C}_{90} \mathrm{H}_{153} \mathrm{O}_{61} \mathrm{~N}_{27} \mathrm{P}_{10}
\end{aligned}
$$


Kossel has expressed the opinion that in reality only four true nucleinic acids exist, in each of which only one nucleinic base is represented. He accordingly distinguishes an adenylic acid, a guanylic acid, a sarcylic (hypoxanthylic) acid, and a xanthylic acid. In accordance with this supposition, the spermatonucleinic acid of the ox would contain three primary acids, as on decomposition it yields xanthin, hypoxanthin, and adenin. Triticonucleinic acid would similarly represent a mixture of guanylic acid and adenylic acid; thymonucleinic acid likewise contains both guanin and adenin, etc. As a matter of fact, however, only one nucleinic acid has as yet been isolated in pure form, which actually contains but one nucleinic base; the rest are hypothetical. The one acid is Bang's guanylic acid, which was obtained from the pancreas. Kossel's adenylic acid, which was first regarded as an analogous product, was shown to yield both guanin and adenin.

As a class the nucleinic acids are but little soluble in cold water, more readily so in hot water, and easily soluble in solutions of the alkalies. They have not yet been obtained in crystalline form. They are dibasic acids, and form both acid and neutral salts with the alkalies and the heavy metals. They are precipitated by the mineral acids, but not by acetic acid, with the exception of guanylic acid. They are very readily decomposed on boiling with mineral acids and even with water, while they are quite resistent to alkalies. Alcohol, especially acid alcohol, causes their precipitation; ammonium sulphate in the presence of acetic acid is equally effective. Tannic acid, picric acid, and phosphotungstic acid may also be employed.

All nucleinic acids give the reaction of Adamkiewicz, the xanthoproteic reaction, and a marked reaction with phloroglucin and hydrochloric acid (see Pentoses).

In acid solutions they form precipitates with albumins which, according to Kossel and Milroy, elosely resemble the native nucleins. In the spermatozoa of fish nucleinic acids are present as neutral or acid salts of protamins or histons. Owing to the readiness with which the nucleinic acids combine with albumins, it has been questioned whether nucleoproteids are not formed artificially during their preparation from the tissues. One of the strongest arguments in favor of their pre-existence is the fact that they can be salted out with ammonium sulphate, but it must be admitted that there is really no definite proof to show that they exist as such in the cell.

The elementary composition of the common forms is given below:

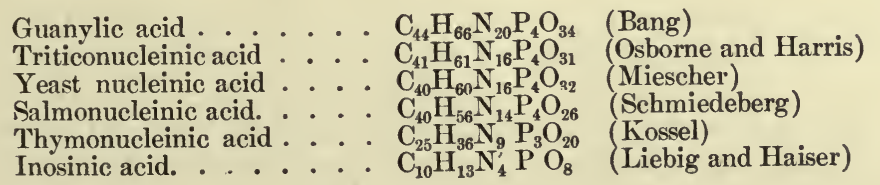


The structural composition of the nucleinic acids is still largely sub judice. For triticonucleinic acid Osborne has suggested the following formula, in which $x$ represents an as yet unresolved residue:

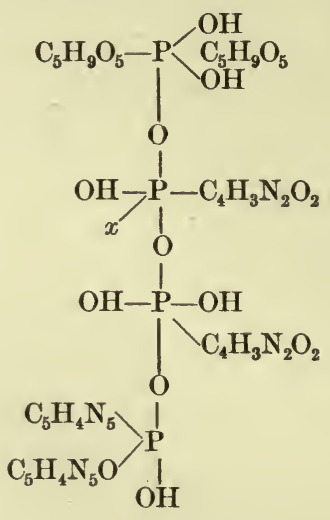

For guanylic acid Bang has suggested the structural formula :

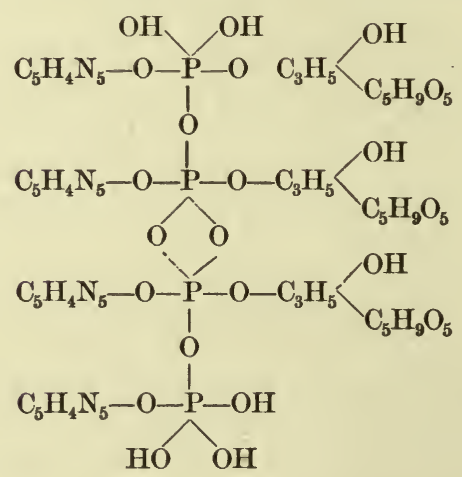

Some nucleinic acids, such as thymonucleinic acid, contain iron in masked form.

As I have already indicated, the native nucleinic acids on hydrolysis lose a portion of their xanthin bases and give rise to the formation of $\beta$-nucleinic acid. On further decomposition the remaining xanthin-bases are split off together with another basic substance which has been termed cytosin. A body then remains which contains the residual radicles of the nucleinic acid molecule. This Kossel and Neumann have termed thyminic acid. For its barium salt they obtained the formula $\mathrm{C}_{14} \mathrm{H}_{23} \mathrm{~N}_{3} \mathrm{O}_{12} \mathrm{P}_{2} \mathrm{Ba}$, and in the case of the thyminic acid resulting from triticonucleinic acid Osborne has suggested the structural formula : 


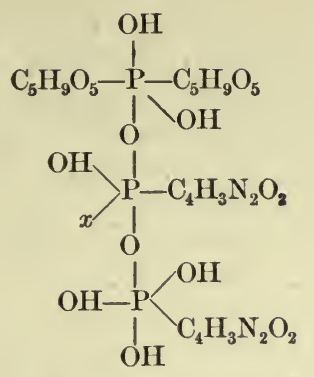

Thyminic acid differs from the nucleinic acids proper in its ready solubility in cold water, and in the fact that it is not precipitated from its solutions by the mineral acids. Like the nucleinic acids, it gives a precipitate with albumins or primary albumoses in acetic acid solution, but, in contradistinction to the nucleinic acids, this precipitate is soluble in hydrochloric acid and in solutions of many salts.

The thyminic acid radicle contains the carbohydrate group or groups of the nucleinic acid molecule; the entire amount of phosphoric acid ; a methyl-uracil complex ; and in some possibly a simple uracil, besides other bodies which are still unknown. The relation of the nucleinic acids to the nucleins and its various decompositionr roducts can accordingly be represented by the following schema:

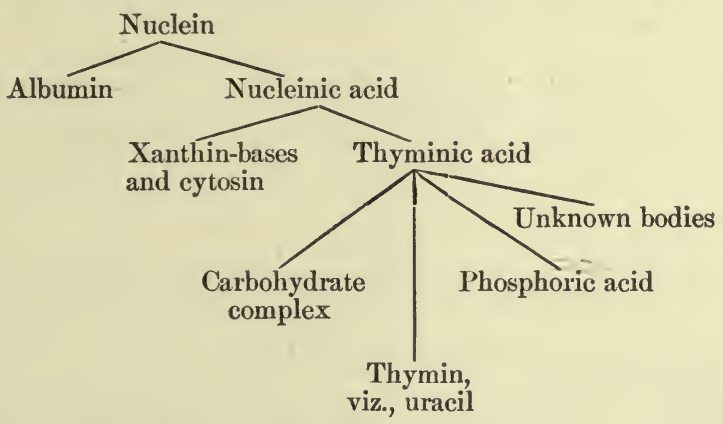

Possibly, however, the decomposition does not proceed in so simple a manner in all cases. Osborne has thus shown that on boiling tritico-nucleinic acid with dilute acids it loses about 22 per cent. of its phosphorus as orthophosphoric acid, and there remains a product which appears to be analogous to thyminic acid. It contains one pentose group less than the original molecule and no longer yields guanin and adenin, as these also have been split off. Its formula, determined by deduction of these radicles from the original molecule, would be $\mathrm{C}_{26} \mathrm{H}_{45} \mathrm{~N}_{6} \mathrm{P}_{3} \mathrm{O}_{25}$.

Schmiedeberg's nucleotin-phosphoric acid represents the corresponding thyminic acid of salmonucleinic acid.

The plasminic acid which Kossel and Ascoli obtained from yeast 
nucleinic acid apparently represents a product intermediary between thyminic acid and the original substance. Its formula is given as $\mathrm{C}_{15} \mathrm{H}_{28} \mathrm{~N}_{6} \mathrm{P}_{6} \mathrm{O}_{30}$. It still contains nucleinic bases, one or more carbohydrate groups, and possibly ammonia. According to Ascoli, the substance also contains iron in masked form.

The phosphorus of the nucleinic acids is possibly present in a form analogous to the polymetaphosphoric acids; Schmiedeberg suggests pyrophosphoric acid as a possibility.

Áccording to Kossel, at least two different carbohydrate complexes may occur in the nucleinic acids, viz., 1, a pentose, which has been shown to be $l$-xylose, first demonstrated in yeast nucleinic acid and later shown to be a common complex of the various nucleinic acids; 2 , a reducing hexose, which on decomposition yields lævulinic acid and formic acid; this was found in the thymonucleinic acid, and later in that obtained from the spermatozoa of the sturgeon. In tritico-nucleinic acid Osborne and Harris obtained evidence of three pentose groups, while a hexose was absent.

Whether or not all nucleinic acids contain a pentose group has been rendered somewhat doubtful by Bang's statement that this is absent in thymo-nucleinic acid. He adds that the thymus itself contains considerable quantities of a pentose.

The basic decomposition-products of the nucleinic acids, as has been mentioned, are the common purin bases-xanthin, hypoxanthin, guanin, and adenin; and the pyrimidin derivatives cytosin, uracil, and thymin. These will be considered below.

There remains an unresolved residue, of which nothing is known. To what extent this is concerned in the production of melanins or melanoid substances, which are constant decomposition-products of the nucleinic acids (Schmiedeberg, Osborne, and Harris), remains to be seen.

\section{The Pyrimidin Derivatives of the Nucleinic Acids.}

The pyrimidin derivatives of the nucleinic acids are cytosin, uracil, and thymin.

Cytosin.-Cytosin is apparently a constant decomposition-product of all nucleinic acids. It has been obtained from thymo-nucleinic acid, from the spermato-nucleinic acid of the sturgeon and herring, from the nucleinic acid of the spleen, the liver etc. Its formula is $\mathrm{C}_{4} \mathrm{H}_{5} \mathrm{~N}_{3} \mathrm{O}$; structurally it is 6-amino-2-oxypyrimidin :

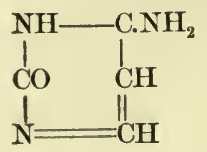

If we compare with this the structure of the purin bases, and notably of uric acid, which is a constant oxidation-product of the purin bases and formed synthetically from pyrimidin derivatives 
(see Uric Acid), the intimate relation which exists between pyrimidin and cytosin, on the one hand, and purin and the purin derivatives on the other is at once apparent. It is quite likely indeed that cytosin represents the mother-substance of the purin group. These relations are expressed by the following formulæ:

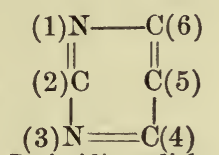

Pyrimidin radicle.

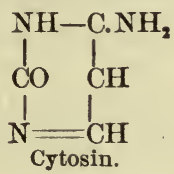

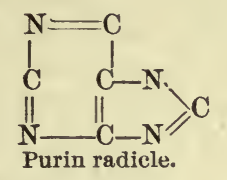

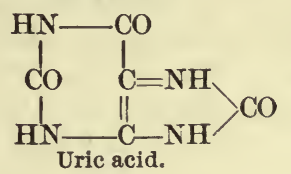

Reactions. - Nitrous acid transforms cystosin into uracil, according to the equation :

$$
\underset{\substack{4 \\ \text { Cytosin. }}}{\mathrm{C}_{5} \mathrm{H}_{5} \mathrm{~N}_{3} \mathrm{O}}+\mathrm{HNO}_{2}=\underset{\text { Uracil. }}{\mathrm{C}_{4} \mathrm{H}_{4} \mathrm{~N}_{2} \mathrm{O}_{2}}+2 \mathrm{~N}+\mathrm{H}_{2} \mathrm{O}
$$

On oxidation with barium permanganate cytosin yields biuret and oxalic acid :

$$
\underset{\text { Cytosin. }}{\mathrm{C}_{4} \mathrm{H}_{5} \mathrm{~N}_{3} \mathrm{O}}+4 \mathrm{O}+\mathrm{H}_{2} \mathrm{O}=\underset{\text { Biuret }}{\mathrm{C}_{2} \mathrm{H}_{5} \mathrm{~N}_{3} \mathrm{O}_{2}}+\underset{\text { Oxalic acid. }}{\mathrm{C}_{2} \mathrm{H}_{2} \mathrm{O}_{4}}
$$

Like many other pyrimidin derivatives, cytosin on treating with freshly prepared chlorine water and the subsequent addition of a trace of ammonia and the application of heat, gives a red color (murexid reaction).

On treating a dilute solution of cytosin with a few drops of a concentrated neutral solution of silver nitrate, needles of a double salt crystallize out on standing, which are soluble with difficulty in cold water and resemble kreatinin silver nitrate in appearance.

Uracil.-It has been pointed out in the foregoing section that cytosin yields uracil on treating with nitrous acid. It has therefore been suggested that uracil possibly does not occur preformed in the nucleinic acid complex, but is merely a secondary product. Osborne, who first encountered uracil among the decomposition-products of tritico-nucleinic acid, expressed the opinion, that possibly the substance might occupy an analogous position in the vegetable world as thymin does in animal tissues. As a matter of fact Ascoli could also demonstrate uracil among the cleavage-products of yeast, nucleinic acid. More recently, however, Kossel and Stendel also obtained the substance from thymo-nucleinic acid and the spermatonucleinic acid derived from the testicles of the herring.

Structurally it is 2.6-dioxypyrimidin; its relation to cytosin is apparent from the formulæ: 
<smiles></smiles><smiles>O=C1CNON1</smiles>

Thymin.-Thymin has been obtained from thymo-nucleinic acid, spermato-nucleinic acid, the nucleinic acids of the liver and the spleen, etc. It is generally regarded as a decomposition-product of the thyminic acid complex, but it has not yet been definitely established that a thymin radicle is present in all nucleinic acids. It is supposedly absent in guanylic acid. The amount which can be obtained is variable; some nucleinic acids apparently furnish more cytosin, others more thymin.

Like uracil, thymin is a pyrimidin derivative. It is 5 methyl,2.6-dioxypyrimidin and thus isomeric with 5,-methyl uracil.<smiles>O=C1[14CH2][14CH2][14CH2][14CH2]N1</smiles><smiles>CC1=C(C)ONN1</smiles>

\section{$\mathrm{C}_{5} \mathrm{H}_{6} \mathrm{~N}_{2} \mathrm{O}_{2}$} Thymin.

As a pyrimidin derivative thymin is closely related to the purin bases and uric acid (see the following pages). Isodialuric acid for example, which is closely related to uracil, can be readily condensed with urea to uric acid, as shown in the equation :

$$
\underset{\text { Dialuric acid. }}{\mathrm{C}_{4} \mathrm{H}_{4} \mathrm{~N}_{2} \mathrm{O}_{4}}+\underset{\text { Urea. }}{\mathrm{CO}\left(\mathrm{NH}_{2}\right)_{2}}=\underset{\text { Uric acid. }}{\mathrm{C}_{5} \mathrm{H}_{4} \mathrm{~N}_{4} \mathrm{O}_{3}}+2 \mathrm{H}_{2} \mathrm{O}
$$

\section{The Purin Derivatives of the Nucleinic Acids.}

The common purin derivatives of the nucleinic acids are xanthin, hypoxanthin (sarcin), guanin, and adenin. Derivatives of these in turn are heteroxanthin, paraxanthin, theophyllin, theobromin, caffein, epiguanin (episarcin), and carnin. Of these, paraxanthin, heteroxanthin, and epiguanin (episarcin) have thus far only been found in the urine. Theophyllin, theobromin, and caffein only occur in the vegetable world, while the remaining members of the group are common constituents of both animal and vegetable cells. Collectively they are termed purin, xanthin, or alloxuric bases. They are all closely related to each other and to uric acid, which, as we shall see later, is one of the most important end-products of nitrogenous metabolism in the animal world. They are all derivatives of E. Fischer's purin, and in this manner closely related to the pyrimidin derivatives just considered in the foregoing section. Adenin, guanin, xanthin, and hypoxanthin are direct derivatives of the nucleinic acids and possibly of the cytosin complex (see above). 
Their structural relations to each other and to purin are shown below :<smiles>C1=NC2=NC=NC2=N1</smiles>

$$
\text { Purin }=\mathrm{C}_{5} \mathrm{H}_{4} \mathrm{~N}_{4} \text {. }
$$

By substituting the group $\mathrm{NH}_{2}$ for the hydrogen atom at 6 , adenin results, and is hence spoken of as a 6-amino-purin :<smiles></smiles>

$$
\text { Adenin }=\mathrm{C}_{5} \mathrm{H}_{5} \mathrm{~N}_{5} \text {. }
$$

Hypoxanthin, according to this conception, would be 6 -oxypurin, xanthin 2,6-dioxypurin, and guanin 2-amino-6-oxypurin :<smiles>O=C1NC2NCNC1N2</smiles><smiles>O=C1NONC1NC1NONO1</smiles><smiles></smiles>

From these primary bodies the remaining ones then result through a substitution of methyl groups. Heteroxanthin is thus formed from xanthin by replacing the hydrogen atom at 7 with $\mathrm{CH}_{3}$, and is therefore 7-methyl-2,6-dioxypurin. Paraxanthin is accordingly 1,-7-dimethyl-2,6-dioxypurin, and caffein 1,3,7-trimethyl-2,6-dioxypurin. Theophyllin and theobromin are isomeric with paraxanthin, and structurally 1,3-dimethyl-2,6-dioxypurin and 3,7-dimethyl-2,6dioxypurin, respectively. Epiguanin is similarly derived from guanin by the replacement of the hydrogen atom at 7 with methyl, and is hence 7-methyl-guanin.<smiles>CN1CCNC2ONOC21</smiles>

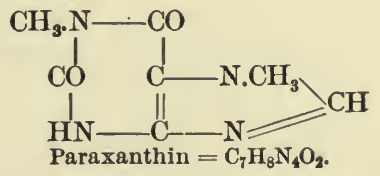

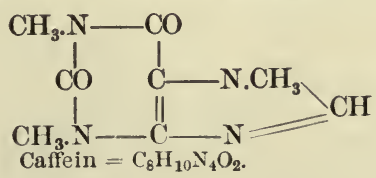


<smiles></smiles><smiles></smiles>

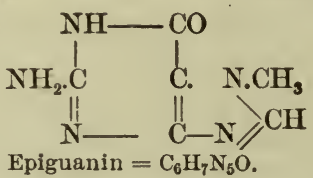

Carnin is apparently closely related to hypoxanthin, as on treatment with bromine it yields methyl bromide, carbon dioxide, and the bromhydrate of hypoxanthin, according to the equation:

$$
\mathrm{C}_{7} \mathrm{H}_{8} \mathrm{~N}_{4} \mathrm{O}_{3}+2 \mathrm{Br}=\mathrm{C}_{5} \mathrm{H}_{4} \mathrm{~N}_{4} \mathrm{O} \cdot \mathrm{HBr}+\mathrm{CH}_{3} \mathrm{Br}+\mathrm{CO}_{2} \text {. }
$$

Its structural formula is thus possibly the following:

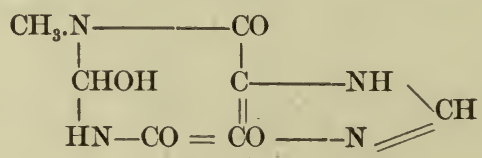

Episarcin is but little known. Its formula is given as $\mathrm{C}_{4} \mathrm{H}_{6} \mathrm{~N}_{3} \mathrm{O}$, but it is possible that this is not correct. The substance has undoubtedly many properties in common with epiguanin, and future researches indeed may possibly show that the two are identical.

The various purin bases, which Gautier once designated the xanthin leucomains, have the character of feeble alkaloids, and combine with hydrochloric acid and platinum chloride to form crystalline salts, which are dissociated only very slowly or not at all by water. When fused with alkalies they lose the greater portion of their nitrogen in the form of cyanogen, and, as a matter of fact, we find that all of them contain the group HCN. Adenin, indeed, is a polymeric compound of hydrocyanic acid, and xanthin can be obtained by direct hydration of the same body (Gautier). On heating with alkalies or with water the nucleinic bases generally do not give rise to the formation of urea, and they cannot hence be regarded as ureids, although a close relationship exists between them. By a simultaneous process of oxidation and hydration guanin thus gives rise to parabanic acid, and it is possible indeed to pass from guanin to xanthin and hypoxanthin by a series of simple reactions without the intervention of urea. These changes are represented by the equations:

$$
\begin{aligned}
& \underset{\substack{\mathrm{C}_{5} \mathrm{H}_{5} \mathrm{~N}_{5} \mathrm{O} \\
\text { Guanin. }}}{\mathrm{C}(\mathrm{NH})}\left\langle\underset{\text { Guanidin. }}{\mathrm{NH}_{2}}+\underset{\text { Parabanic acid. }}{\mathrm{CO}-\mathrm{NH}} \mathrm{H}_{2} \mathrm{O}=\mathrm{CO}+\mathrm{CO}_{2}\right. \\
& \underset{\text { Guanin. }}{\mathrm{C}_{5} \mathrm{H}_{5} \mathrm{~N}_{5} \mathrm{O}}+\mathrm{HNO}_{2}=\underset{\text { Xanthin. }}{\mathrm{C}_{5} \mathrm{H}_{4} \mathrm{~N}_{4} \mathrm{O}_{2}}+2 \mathrm{~N}+\mathrm{H}_{2} \mathrm{O} \text {. } \\
& \underset{\text { Xanthin. }}{\mathrm{C}_{5} \mathrm{H}_{4} \mathrm{~N}_{4} \mathrm{O}_{2}}+2 \mathrm{H}=\underset{\text { Hypoxanthin. }}{\mathrm{C}_{5} \mathrm{H}_{4} \mathrm{~N}_{4} \mathrm{O}}+\mathrm{H}_{2} \mathrm{O} .
\end{aligned}
$$


Xanthin, however, on oxidation and hydration yields urea and alloxan, which latter is further oxidized to parabanic acid and carbon dioxide, as shown in the equations :

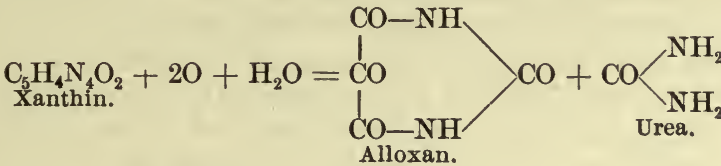

$$
\begin{aligned}
& \begin{array}{l}
\mathrm{CO} \text { Alloxan. } \\
\mid \mathrm{NH}
\end{array}>\mathrm{NO}+\mathrm{NH}=\underset{\mathrm{CO}-\mathrm{NH}}{\left.\right|_{\text {Parabanic acid. }} ^{\mathrm{CO}-\mathrm{NH}}}>\mathrm{CO}+\mathrm{CO}_{2}
\end{aligned}
$$

The close relationship which exists between uric acid and the nucleinic bases thus becomes apparent, as uric acid on oxidation and hydration gives rise to the same products as xanthin:

$$
\underset{\text { Uric acid. }}{\mathrm{C}_{5} \mathrm{H}_{4} \mathrm{~N}_{4} \mathrm{O}_{3}}+\mathrm{O}+\mathrm{H}_{2} \mathrm{O}=\underset{\text { Alloxan. }}{\mathrm{C}_{4} \mathrm{H}_{2} \mathrm{~N}_{2} \mathrm{O}_{4}}+\underset{\text { Urea. }}{\mathrm{CON}_{2} \mathrm{H}_{4}} \text {. }
$$

As a matter of fact, it is now known that uric acid is formed from the purin bases in the mammalian organism through the agency of a specific ferment. Guanin thus gives rise to xanthin, and this to uric acid, while adenin is transformed into hypoxanthin, and the latter into uric acid with the intermediary production of xanthin. In the transformation of guanin into xanthin a desamidizing ferment is active, while the subsequent transformation into uric acid is referable to an oxidizing ferment. This relationship is further shown by decomposing the primary xanthin bases and uric acid with fuming hydrochloric acid or hydriodic acid under high pressure. Qualitatively the same products are thus obtained, viz., ammonia, carbon dioxide, glycocoll, and formic acid, while the quantitative relations, of course, vary with the nature of the individual substance.

$$
\begin{aligned}
& \underset{\text { Adenin. }}{\mathrm{C}_{5} \mathrm{H}_{5} \mathrm{~N}_{5}}+8 \mathrm{H}_{2} \mathrm{O}=4 \mathrm{NH}_{3}+\mathrm{CO}_{2}+\mathrm{CH}_{2} \cdot \mathrm{NH}_{2} \cdot \mathrm{COOH}+\underset{\text { Flycocoll. }}{2 \mathrm{H} . \mathrm{COOH}} . \\
& \mathrm{C}_{5} \mathrm{H}_{4} \mathrm{~N}_{4} \mathrm{O}+7 \mathrm{H}_{2} \mathrm{O}=3 \mathrm{NH}_{3}+\mathrm{CO}_{2}+\mathrm{CH}_{2} \cdot \mathrm{NH}_{2} \cdot \mathrm{COOH}+2 \mathrm{H} \cdot \mathrm{COOH} \text {. } \\
& \text { Hypoxanthin. } \\
& \mathrm{C}_{5} \mathrm{H}_{5} \mathrm{~N}_{5} \mathrm{O}+7 \mathrm{H}_{2} \mathrm{O}=4 \mathrm{NH}_{3}+2 \mathrm{CO}_{2}+\mathrm{CH}_{2} \cdot \mathrm{NH}_{2} \cdot \mathrm{COOH}+\mathrm{H} \cdot \mathrm{COOH} \text {. } \\
& \text { Guanin. } \\
& \mathrm{C}_{5} \mathrm{H}_{4} \mathrm{~N}_{4} \mathrm{O}_{2}+6 \mathrm{H}_{2} \mathrm{O}=3 \mathrm{NH}_{3}+2 \mathrm{CO}_{2}+\mathrm{CH}_{2} \cdot \mathrm{NH}_{2} \cdot \mathrm{COOH}+\mathrm{H} \cdot \mathrm{COOH} . \\
& \mathrm{C}_{5} \mathrm{H}_{4} \mathrm{~N}_{4} \mathrm{O}_{3}+5 \mathrm{H}_{2} \mathrm{O}=3 \mathrm{NH}_{3}+3 \mathrm{CO}_{2}+\mathrm{CH}_{2} \cdot \mathrm{NH}_{2} \cdot \mathrm{COOH} \text {. }
\end{aligned}
$$

According to Emil Fischer, the structural formula of uric acid can be represented as follows :<smiles>O=C1NC2CNC(=O)C2NC1=O</smiles> 
and it is thus seen that, like the nucleinic bases, it contains the purin radicle.

All the nucleinic bases combine with acids and alkalies to form salts, many of which are readily crystallizable. On boiling with acetate of copper most of them are thrown down as insoluble compounds. From their neutral solutions, or in the presence of a little ammonia, they are precipitated by ammoniacal silver nitrate solution. This precipitate dissolves in nitric acid on the application of heat, but reappears on cooling, Most of the nucleinic bases, moreover, when evaporated to dryness in the presence of nitric acid, leave a yellowish residue, which changes to orange and often to a temporary purple on the addition of an alkali. In this respect also these substances resemble uric acid, which gives a very similar reaction (see Murexid test). When exposed to the action of putrefactive organisms adenin is transformed into hypoxanthin, and guanin into xanthin, so that these two only are found in decomposed material.

For a description of the individual members of this group which are found in the animal body, as well for the method of their isolation and quantitative estimation, the reader is referred to subsequent chapters.

\section{THE UREIDS.}

The ureids comprise a number of nitrogenous crystallizable bodies characterized by the fact that on hydrolytie decomposition alone, or on simultaneous oxidation, they yield urea. They are hence derivatives of urea, and may contain one or more molecules of this substance, in which one or more hydrogen atoms are replaced by radicles of monobasic or polybasic acids. On decomposition they yield either urea and a non-nitrogenous acid directly, or they give rise to urea and a less complex ureid, which is then further decomposed, as in the first instance. They are accordingly divided into mono-ureids and di-ureids. The former contain two atoms of nitrogen in their molecule, while the latter possess four atoms of nitrogen. All these bodies are closely related to each other and to the nucleinic bases, from which they are, in part at least, derived.

The mono-ureids comprise alloxan, alloxanic acid, dialuric acid, barbituric acid or malonyl-urea and its derivatives, all of which can be decomposed into urea and non-nitrogenous acids containing three atoms of carbon. From these another series of mono-ureids is derived, which also yield urea and non-nitrogenous acids; but the latter, in contradistinction to the first group, contain only two atoms of carbon. These include parabanic acid, oxaluric acid, allanturic acid, and hydantoin and its derivatives, viz, hydantoic acid or glycoluric acid, and methyl-hydantoin. The di-ureids are similarly divided into two groups, the first of which comprises uric acid, alloxantin, murexid or ammonium purpurate, and hydurilic acid; while the second is represented by allantoin, allantoic acid, and allituric acid. 
The best known representative of the ureids is uric acid. From it all others can be derived, and a description of its general properties and reactions may serve as an illustration of the entire class.

Uric acid is a crystallizable dibasic acid of the formula $\mathrm{C}_{5} \mathrm{H}_{4} \mathrm{~N}_{4} \mathrm{O}_{3}$. Structurally it may be regarded as a purin derivative, and may be represented by the formula :

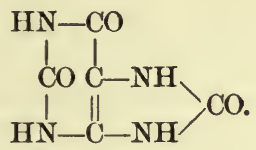

It is thus 2.6.8-trioxypurin. It was first obtained synthetically by heating a mixture of glycocoll and urea at a temperature of $220^{\circ}$ to $230^{\circ} \mathrm{C}$. The reaction may be represented by the equation:

$$
\underset{\text { Glycocoll. }}{\mathrm{CH}_{2} \cdot \mathrm{NH}_{2} \cdot \mathrm{COOH}}+\underset{\text { Urea. }}{3 \mathrm{CO}\left(\mathrm{NH}_{2}\right)_{2}}=\underset{\text { Uric acid. }}{\mathrm{C}_{5} \mathrm{H}_{4} \mathrm{~N}_{4} \mathrm{O}_{3}}+2 \mathrm{H}_{2} \mathrm{O}+3 \mathrm{NH}_{3}
$$

The same result may be reached by heating a mixture of urea and trichlorolactic amide:

$$
\underset{\text { Trichlorolactic amide. }}{\mathrm{C}_{3} \mathrm{Cl}_{3} \mathrm{O}_{2} \mathrm{H}_{2} \cdot \mathrm{NH}_{2}}+2 \mathrm{CO}\left(\mathrm{NH}_{2}\right)_{2}=\mathrm{C}_{5} \mathrm{H}_{4} \mathrm{~N}_{4} \mathrm{O}_{3}+\mathrm{H}_{2} \mathrm{O}+2 \mathrm{HCl}+\mathrm{NH}_{4} \mathrm{Cl}
$$

As indicated in the previous section, moreover, uric acid also results through the condensation of isodialuric acid and urea, according to the equation :

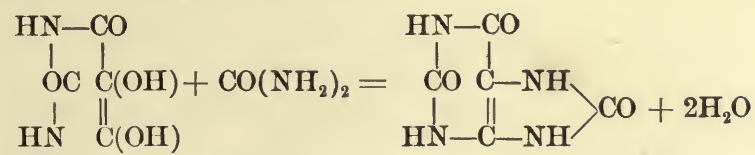

When heated in the dry state uric acid is decomposed into hydrocyanic acid, cyanuric acid, ammonium carbonate, ammonium cyanate, biuret, etc. Fused with an excess of potassium hydrate, it similarly yields ammonia, potassium carbonate, oxalate, cyanate, and cyanide.

On reduction with nascent hydrogen, in the presence of water and sodium amalgam uric acid is first transformed into xanthin, and subsequently into hypoxanthin. The elose relationship which exists between the nucleinic bases and uric acid is thus further shown (see page 104).

Analogous to the synthetic formation of uric acid from urea and glycocoll, we find that on decomposition with hydriodic acid the substance yields carbon dioxide, ammonia, and glycocoll, viz., the same products which are obtained from the nucleinic bases.

From the reactions which have thus far been described the nature of uric acid as a ureid is not apparent. If the hydrolytic decomposition of the substance is effected less energetically, this becomes manifest at once. On prolonged boiling with water it is decom- 
posed into urea and dialuric acid, which latter further yields urea and tartronic acid. In this manner the character of uric acid as a diureid of the first order is demonstrated. The reactions which take place are represented by the equations:

$$
\begin{aligned}
& \text { (1) } \underset{\text { Uric acid. }}{\mathrm{C}_{5} \mathrm{H}_{4} \mathrm{~N}_{4} \mathrm{O}_{3}}+2 \mathrm{H}_{2} \mathrm{O}=\underset{\text { Dialuric acid. }}{\mathrm{C}_{4} \mathrm{H}_{4} \mathrm{~N}_{2} \mathrm{O}_{4}}+\underset{\text { Urea. }}{\mathrm{CO}\left(\mathrm{NH}_{2}\right)_{2}} \\
& \text { (2) } \underset{\text { Dialuric acid. }}{\mathrm{C}_{4} \mathrm{H}_{4} \mathrm{~N}_{2} \mathrm{O}_{4}}+2 \mathrm{H}_{2} \mathrm{O}=\underset{\text { Tartronic acid. }}{\mathrm{C}_{3} \mathrm{H}_{4} \mathrm{O}_{5}}+\underset{\text { Urea. }}{\mathrm{CO}\left(\mathrm{NH}_{2}\right)_{2}}
\end{aligned}
$$

On treating with an oxidizing agent, in the presence of water, uric acid is similarly decomposed into urea and the mono-ureid alloxan, which can be further decomposed into urea and mesoxalic acid:

$$
\begin{aligned}
& \text { (1) } \underset{\text { Uric acid. }}{\mathrm{C}_{5} \mathrm{H}_{4} \mathrm{~N}_{4} \mathrm{O}_{3}}+\mathrm{H}_{2} \mathrm{O}+\mathrm{O}=\underset{\text { Alloxan. }}{\mathrm{C}_{4} \mathrm{H}_{2} \mathrm{~N}_{2} \mathrm{O}_{4}}+\mathrm{CO}\left(\mathrm{NH}_{2}\right)_{2}
\end{aligned}
$$

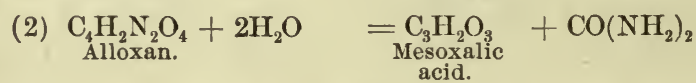

Its relation to the di-ureids of the second order is shown by oxidizing the substance with peroxide of manganese in neutral solution at a moderate temperature. In this manner allantoin is formed, from which, on further oxidation, urea and oxalic acid result:

$$
\begin{aligned}
& \text { (1) } \mathrm{C}_{5} \mathrm{H}_{4} \mathrm{~N}_{4} \mathrm{O}_{3}+\mathrm{H}_{2} \mathrm{O}+\mathrm{O}=\underset{\text { Allantoin. }}{\mathrm{C}_{4} \mathrm{H}_{6} \mathrm{~N}_{4} \mathrm{O}_{3}}+\mathrm{CO}_{2} \\
& \text { (2) } \mathrm{C}_{4} \mathrm{H}_{6} \mathrm{~N}_{4} \mathrm{O}_{3}+2 \mathrm{H}_{2} \mathrm{O}+\mathrm{O}=\underset{\substack{\text { Oxalic } \\
\text { acid. }}}{\mathrm{C}_{2} \mathrm{H}_{2} \mathrm{O}_{4}}+2 \mathrm{CO}\left(\mathrm{NH}_{2}\right)_{2}
\end{aligned}
$$

Of special interest, further, is the formation of murexid, or ammonium purpurate, which results when uric acid, even in minimal amounts, is evaporated together with nitric acid, and the reddish residue is brought in contact with ammonia. A beautiful purplishred color then develops, which is characteristic of uric acid and its salts (murexid test). The reactions which take place may be represented by the equations :

$$
\begin{aligned}
& \text { (1) } \mathrm{C}_{5} \mathrm{H}_{4} \mathrm{~N}_{4} \mathrm{O}_{3}+2 \mathrm{H}_{2} \mathrm{O}=\mathrm{C}_{4} \mathrm{H}_{4} \mathrm{~N}_{2} \mathrm{O}_{4}+\mathrm{CO}\left(\mathrm{NH}_{2}\right)_{2} \\
& \text { (2) } \mathrm{C}_{4} \mathrm{H}_{4} \mathrm{~N}_{2} \mathrm{O}_{4}+\mathrm{NH}_{4} \mathrm{OH}=\underset{\text { Ammonium }}{\mathrm{C}_{4} \mathrm{H}_{3}\left(\mathrm{NH}_{4}\right) \mathrm{N}_{2} \mathrm{O}_{4}}+\mathrm{H}_{2} \mathrm{O} \\
& \text { Ammonium } \\
& \text { (3) } \underset{\text { Ammonium dialurate. }}{2 \mathrm{C}_{4} \mathrm{H}_{3}\left(\mathrm{NH}_{4}\right) \mathrm{N}_{2} \mathrm{O}_{4}}+\mathrm{O} \quad=\underset{\text { Murexid. }}{\mathrm{C}_{8} \mathrm{H}_{4}\left(\mathrm{NH}_{4}\right) \mathrm{N}_{5} \mathrm{O}_{6}}+\underset{3 \mathrm{H}_{2} \mathrm{O}}{\text { Mmmong }}
\end{aligned}
$$

The relation of uric acid to the mono-ureids of the second order, finally, is shown by treating one part of uric acid with three parts of nitric acid (50 per cent. solution), and heating to $70^{\circ} \mathrm{C}$. On subsequent evaporation to a syrup and cooling, parabanic acid crystallizes out, and on decomposition yields urea and oxalic acid:

(1) $\mathrm{C}_{5} \mathrm{H}_{4} \mathrm{~N}_{4} \mathrm{O}_{3}+\mathrm{H}_{2} \mathrm{O}+\mathrm{O}=\underset{\text { Alloxan. }}{\mathrm{C}_{4} \mathrm{H}_{2} \mathrm{~N}_{2} \mathrm{O}_{4}}+\mathrm{CO}\left(\mathrm{NH}_{2}\right)_{2}$

(2) $\mathrm{C}_{4} \mathrm{H}_{2} \mathrm{~N}_{2} \mathrm{O}_{4} \quad+\mathrm{O}=\underset{\text { Parabanic }}{\mathrm{C}_{3} \mathrm{H}_{2} \mathrm{~N}_{2} \mathrm{O}_{3}}+\mathrm{CO}_{2}$

(3) $\mathrm{C}_{3} \mathrm{H}_{2} \mathrm{~N}_{2} \mathrm{O}_{3}+2 \mathrm{H}_{2} \mathrm{O}=\underset{\text { Oxalic acid. }}{\mathrm{C}_{2} \mathrm{H}_{2} \mathrm{O}_{4}}+\mathrm{CO}\left(\mathrm{NH}_{2}\right)_{2}$ 
Guanin, as has been shown, under the same conditions yields guanidin and parabanic acid, which further illustrates the close relationship between the two classes.

For a consideration of the methods employed in the isolation of uric acid and its quantitative estimation, the reader is referred to the chapter on the Urine.

\section{THE KREATINS.}

The kreatins, or kreatinic leucomains, as they are also termed by Gautier, are basic substances which are closely related to the nucleinic bases and to the ureids, which have just been considered. They comprise kreatin, kreatinin, crusokreatinin, xanthokreatinin, amphikreatinin, and two similar substances of doubtful composition. Kreatin, moreover, is related to arginin, and can be produced synthetically through the union of cyanamide and methyl-glycocoll, as arginin results from cyanamide and ornithin. While arginin, however, can be obtained artificially from albuminous material, kreatin has thus far not been isolated in this manner. Its formation from cyanamide and methyl-glycocoll is represented by the equation:

$$
\begin{array}{lll}
\mathrm{N} \equiv \mathrm{C}-\mathrm{NH}_{2}+\mathrm{NH}\left(\mathrm{CH}_{3}\right)-\mathrm{CH}_{2}-\mathrm{COOH}= \\
\text { Cyanamide. } & \text { Methyl-glycocoll. } & \mathrm{NH}: \mathrm{C}
\end{array}
$$

It can thus be viewed as methyl-guanidin acetic acid. It is thus a homologue of glycocyamin, which results through the union of cyanamide and glycocoll :

$$
\begin{aligned}
& \mathrm{N} \equiv \mathrm{C}-\mathrm{NH}_{2}+\mathrm{CH}_{2}<\mathrm{COOH}_{\mathrm{NH}}=\mathrm{NH}: \mathrm{C}_{\mathrm{NH}-\mathrm{CH}_{2}-\mathrm{COOH}}^{\mathrm{NH}_{2}} \\
& \text { Cyanamide. Glycocoll. Glucocyamin. }
\end{aligned}
$$

On dehydration kreatin loses one molecule of water and is transformed into kreatinin, which may thus be regarded as the anhydride of kreatin :

$$
\mathrm{NH}: \mathrm{C}_{\mathrm{N}\left(\mathrm{CH}_{3}\right)-\mathrm{CH}_{2}-\mathrm{COOH}}^{\mathrm{NH}}=\mathrm{NH}: \mathrm{C}\left\langle{ }_{\mathrm{N}\left(\mathrm{CH}_{3}\right)-\mathrm{CH}_{2}}^{\mathrm{NH}-\mathrm{H}_{2} \mathrm{O}}\right.
$$

On oxidation with mercuric oxide kreatin gives rise to the formation of the oxalate of methyl-guanidin or methyl-uramin, which in turn results from guanidin, and this from guanin.

$$
\underset{\substack{\text { Kreatin. } \\ \text { Ke }}}{2 \mathrm{C}_{4} \mathrm{H}_{9} \mathrm{~N}_{3} \mathrm{O}_{2}}+5 \mathrm{O}=\underset{\substack{\text { Methil-guanidin } \\ \text { oxalate. }}}{\left(\mathrm{C}_{2} \mathrm{H}_{2} \mathrm{~N}_{3}\right)_{2} \mathrm{C}_{2} \mathrm{H}_{2} \mathrm{O}_{4}}+2 \mathrm{CO}_{2}+\mathrm{H}_{2} \mathrm{O}
$$

On decomposition with baryta-water kreatin yields urea, methylglycocoll, and a small amount of hydantoic acid. Kreatinin is similarly transformed into methyl-hydantoin, which is then likewise decomposed into urea and methyl-glycocoll, thus demonstrating 
the close relationship which exists between the kreatins and the ureids.
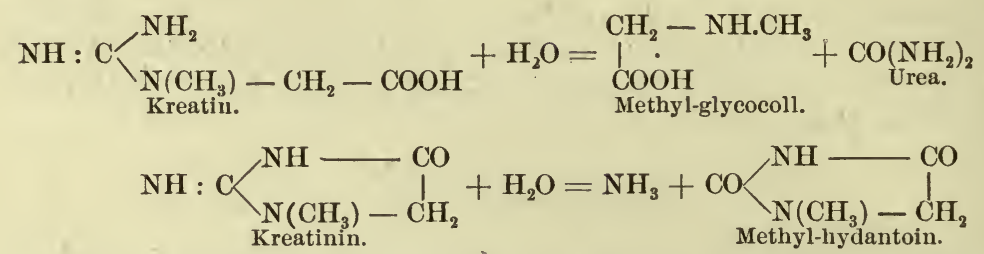

The individual representatives of the group will be considered later.

\section{THE PTOMAINS.}

The term ptomain was originally applied by Selmi to certain alkaloidal bodies which are formed during the process of albuminous putrefaction. Gautier then extended its use to include all those alkaloidal substances which result from anaërobic fermentation, as also those which are formed in the tissues of the higher animals in the absence of air, or in the presence at least of an insufficient supply of oxygen. In contradistinction to these substances, Gautier terms those alkaloidal bodies which are formed during the normal and aërobic life of the tissues leucomains. Under this latter heading, as has been seen, he comprises the nucleinic bases and the kreatins.

Gautier divides the ptomains into acyclic and cyclic ptomains, some of which are free from oxygen, while in others this is present. They are all derived from ammonia by the substitution of various radicles for one or all of the hydrogen atoms of ammonia, and are hence analogous to the amins. Some of these bodies are extremely toxic and were once regarded as specific toxins of various bacteria, to which the symptoms in the corresponding infections were essentially referable. We now know that this is true only to a limited extent.

The acyclic ptomains which are free from oxygen comprise the following substances:

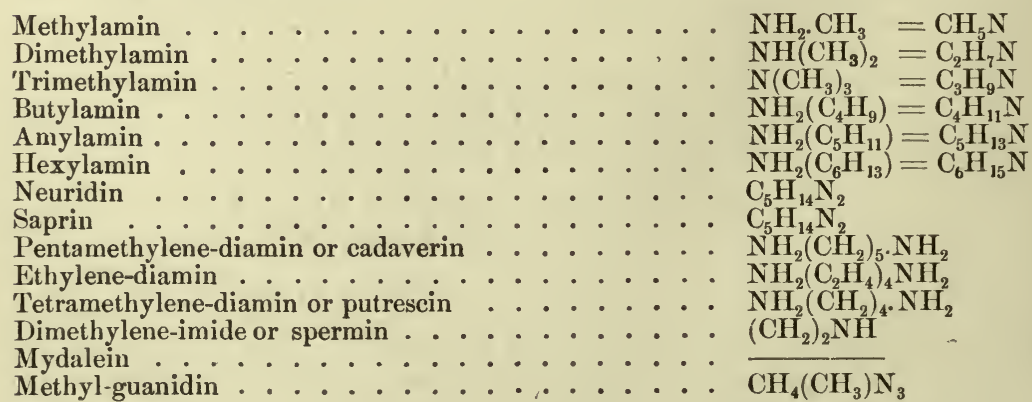


The oxygen-containing acyclic ptomains are the following:

Cholin or trimethyl-oxyethylene-am- ) monium hydrate ....... .

Neurin or trimethyl-vinyl-ammonium hvdrate ...........

Muscarin . . . . . . . .

Betain or oxycholin .......

Mydatoxin . . . . . . . .

Mydin .............

Gadinin .............

Methyl-gadinin .........

Mytilotoxin . . . . . . . . .

Propyl-glucocyamin . . . . . .

$$
\begin{aligned}
\mathrm{N}\left(\mathrm{CH}_{3}\right)_{3} \cdot\left(\mathrm{C}_{2} \mathrm{H}_{4} \cdot \mathrm{OH}\right) \cdot \mathrm{OH}= & \mathrm{C}_{5} \mathrm{H}_{15} \mathrm{NO}_{2} \\
\mathrm{~N}\left(\mathrm{CH}_{3}\right)_{3} \cdot\left(\mathrm{C}_{2} \mathrm{H}_{3}\right) \cdot \mathrm{OH}= & \mathrm{C}_{5} \mathrm{H}_{13} \mathrm{NO} \\
\mathrm{N}\left(\mathrm{CH}_{3}\right)_{3} \cdot\left(\mathrm{CH}_{2} \cdot \mathrm{COH}\right) \cdot \mathrm{OH}= & \mathrm{C}_{5} \mathrm{H}_{15} \mathrm{NO}_{3} \\
\mathrm{~N}\left(\mathrm{CH}_{3}\right)_{3} \cdot\left(\mathrm{CH}_{2} \cdot \mathrm{COOH}\right) \cdot \mathrm{OH}= & \mathrm{C}_{5} \mathrm{H}_{11} \mathrm{NO}_{2} \\
& \mathrm{C}_{6} \mathrm{H}_{13} \mathrm{NO}_{2} \\
& \mathrm{C}_{8} \mathrm{H}_{11} \mathrm{NO} \\
& \mathrm{C}_{7} \mathrm{H}_{16} \mathrm{NO}_{2} \\
& \mathrm{C}_{8} \mathrm{H}_{18} \mathrm{NO}_{2} \\
& \mathrm{C}_{6} \mathrm{H}_{15} \mathrm{NO}_{2} \\
& \mathrm{C}_{6} \mathrm{H}_{13} \mathrm{~N}_{2} \mathrm{O}_{3} \\
&
\end{aligned}
$$

The remaining ptomains are partly cyclic and in part not classified :

Collidin (iso-phenyl-ethylamin) . . .

Hydrocollidin . . . . . . . .

Parvolin .. . . . . . . . .

Corindin .. . . . . . . . .

Hydrolutidin . . . . . . . .

Hydrocornidin .. . . . . . .

Scombrin .. . . . . . . .

Morrhuin . . . . . . . . .

Asellin . . . . . . . . . .

Morrhuic acid
Typhotoxin
Tetanin

Typhotoxin
Tetanin . . . . . . . . . . . . . . . .

Tetanotoxin . . . . . . . . . .

Spasmotoxin ..........

Tyrotoxin ..........

Pyocyanin . . . . . . . . .

Pyoxanthin

Some of these substances and their origin from the albumins have already been considered, and we shall have further occasion to study them in greater detail. Others are scarcely known, and require no further description at this place. They are all intimately related to the vegetable alkaloids, with which they have many reactions in common. They have strongly basic properties, and are capable of combining with acids to form salts. Like the albumins from which they are derived, they are precipitated with the chlorides of platinum, mercury, and gold, as also with tannic acid, picric acid, phosphomolybdic acid, phosphotungstic acid, etc. With these they form well-defined crystalline salts, which serve for their differentiation from each other and as a basis for the determination of their elementary composition. The methods which are employed for the separation of ptomains will be considered in a subsequent chapter. 


\section{CHAPTER VI.}

\section{THE FERMENTS.}

In the foregoing chapters we have considered in a general way the more important characteristics of the three great classes of food-stuffs, and have studied in some detail also the various decomposition-products to which they give rise in their passage through the animal body. We have also pointed out that with few exceptions the food-stuffs, which the animal derives either directly or indirectly from the plant, cannot be utilized by the animal directly, but that they must previously undergo certain changes, which vary with the character of the individual substances. The native albumins must first be transformed into albumoses and simpler products; the disaccharides and polysaccharides must be inverted to monosaccharides, and the fats must first be decomposed into glycerin and fatty acids. We have seen also that in the chemical laboratory these changes can, for the most part, be brought about through the action of superheated steam, by boiling with acids and alkalies, etc. - that is, through agencies which manifestly are not at work in the living world. The question therefore suggests itself: What are the means at the disposal of living bodies to bring about these changes? This question has, in a measure, been answered in the introductory remarks, where it was pointed out that the animal is capable of bringing about a large number of analytical changes by means of certain ferments, or enzymes, which are furnished by the animal cells themselves. At the present time there is a tendency indeed to assume that most of the vital phenomena are referable to the action of ferments, and we know as a matter of fact that ferment action is not necessarily only destructive, but may also be constructive. The discovery of oxidation ferments has thrown further light on many processes which were formerly referred to "vital" forces inherent in the living protoplasm. The existence of such "vital" forces has become the more doubtful the more closely vital phenomena are studied. Only a few years ago the ferments furnished by the digestive glands were the only known ferments in the animal body, and our knowledge of the mechanism of the various metabolic processes was practically nil. To-day we know that ferments are present in probably every cell and are intimately concerned in all its manifestations of life. In the liver-cell, for example, not less than a dozen different ferments have been demonstrated. Still we are only on the threshold of our knowledge of intracellular chemical processes, and there are still many obscure problems which 
await their final solution. That the life of the cell is not inseparably connected with the activity of its ferments has been well shown by Buchner: If common yeast is placed in a solution of cane-sugar, this is inverted to glucose and lævulose by the contained invertin; at the same time the cell causes the further destruction of these sugars with the formation of alcohol and carbon dioxide. The fact that this latter process can be prevented by means of chloroform, which is a notable protoplasmic poison, while the inversion proceeds as before, was formerly interpreted as evidence of a special vital activity on the part of the cell. Buchner, however, has demonstrated conclusively that there is no true cause for this assumption. For on crushing the yeast-cells completely he succeeded in obtaining a fluid which could bring about the further destruction of glucose and lrvulose exactly as in the case of the living cell. He could show that this action is referable to an intracellular ferment which is termed zymase. By similar methods a large number of intracellular ferments have been discovered in the animal body (see below).

The ferments proper must be sharply distinguished from the socalled ferment-organisms, or organized ferments, which occur widely distributed in nature and comprise the important groups of bacteria, blastomycetes, and certain moulds. These are living beings themselves, and not, as the ferments proper, products of life. They contain ferments, and manifest their special activity, in a great measure, through their ferments; but they are not ferments themselves, although they are often so called. In contradistinction to these organized ferments, the ferments proper are termed nonorganized ferments, or enzymes. They are specific products of the activity of cells, and occur widely distributed in the animal and vegetable world.

For the maintenance of life, in the case of the higher plants at least, the organized ferments are of prime importance; for, as has been seen, it is through these low forms of life that the higher plants are furnished their nitrogen in a form which they can subsequently utilize. In their absence from the soil, vegetable life, such as we see it, could probably not exist. In the gastro-intestinal tract of all animals which have been examined in this direction innumerable bacteria are also found, and it was long thought that their presence here served a very definite purpose, and that animal life could not go on in their absence. This view, however, has proved erroneous, as Nuttall and Thierfelder conclusively demonstrated. They showed that when a young guinea-pig, for example, is removed from the mother animal by Cæsarean section under strict aseptic precautions, and is subsequently fed with sterile food and is furnished with sterile air, it will grow as well as a control-animal. While the presence of bacteria in the animal body is therefore not essential for the maintenance of life, and while it is very questionable, indeed, whether their presence in the alimentary canal serves any useful purpose at 
all, we know, on the contrary, that the introduction of certain forms is directly harmful, and that some of the normal inhabitants of the intestinal canal may under certain conditions develop distinct pathogenic properties.

General Properties of the Ferments.-From what has been said, it is clear that the ferments are capable of manifesting their special activity even after the death of their mother-cells, and it is noteworthy that a great many substances which are distinct protoplasmic poisons do not interfere with the action of many ferments. Such substances are chloroform, ether, thymol, toluol, salicylic acid, arsenious acid, sodium fluoride, boric acid, hydroxylamin, glycerin, etc. In the study of the ferments these bodies are of great importance, as we are thus apparently enabled to exclude the protoplasmic activity of living cells, and to determine whether certain chemical phenomena which we observe in the tissues of the body are referable to the action of ferments or not. It is conceivable, however, that the so-called protoplasmic poisons which do not interfere with most enzymic action may subsequently be shown to be protoplasmic poisons in consequence of their inhibitory action on enzymic processes which are at present unknown, and which are of vital importance to the economy. Sodium fluoride thus is a protoplasmic poison and had been thought incapable of impairing enzymic processes. Loevenhart and Peirce have shown, however, that the enzymic hydrolysis of esters is exceedingly sensitive to sodium fluoride, even in most minute quantities.

In this connection it is interesting to note that the blood-serum contains antiferments, of the nature of which still less is known than of the ferments. We can merely recognize their presence without being able to explain their action. The existence of such antiferments is of great biological importance, and to it such phenomena as the non-self-digestion of tissues may be wholly or in part due. Artificially it is possible to cause the production of antiferments by immunization with the corresponding ferments. Rennin immunization thus leads to the production of antirennin, pepsin to antipepsin, trypsin to antitrypsin, etc., in a manner which seems quite analogous to the formation of antitoxin by immunization with the corresponding toxins.

Other chemicals not only cause the death of the cell but also arrest or annihilate the action of ferments. Such substances are the bichloride of mercury, carbolic acid, ${ }^{1}$ and to a less marked degree other metallic salts, as also picric acid, tannic acid, etc. The mineral acids are variable in their action.' Some ferments are dependent upon their presence, others behave indifferently, while still others are destroyed. Some ferments are capable of destroying others. The activity of the ferments is further decreased with an increase of their specific products beyond a certain degree. Absence

1 It should be noted that bichloride and carbolic acid do not interfere with all enzymic processes. 
of water likewise inhibits the action of the ferments, but this is at once re-established when the necessary degree of moisture is supplied, and it is possible therefore to preserve the ferments in the dry state. During the process of drying, however, care must be had that the tempcrature does not exceed a certain limit. This varies with the different ferments, but it may be stated as a general rule that all animal ferments are killed by a temperature of $75^{\circ} \mathrm{C}$, while the vegetable ferments cannot survive a temperature of $80^{\circ} \mathrm{C}$. In the absence of moisture, however, they can apparently withstand much greater heat, and it is said that dry trypsin, pepsin, and diastase may be heated to a temperature of from $150^{\circ}$ to $160^{\circ} \mathrm{C}$. without losing their activity. Strong alcohol destroys the action of certain ferments, such as pepsin and diastase, while others, like the fibrinferment, are not affected unless the contact is prolonged.

A most peculiar property of the ferments, and one which is characteristic of all, is the power to bring about an amount of chemical change which is apparently out of all proportion to the quantity of the ferment present, while the ferment itself undergoes no apparent change. The common pepsin preparations of the market are of a strength that 1 part by weight of the pepsin will digest 6000 parts by weight of coagulated egg-albumin, and Petit claims that a preparation from his laboratory was capable of dissolving even 500,000 times its weight of fibrin in seven hours. Invertase has been shown capable of inverting 200,000 times its own weight of cane-sugar, and rennin at least 400,000 times its own amount of caseïn.

That the ferments themselves undergo no change while exerting their specific action can be readily shown, as it is possible to reobtain them from the various digestive mixtures and to test their efficacy as before. It is to be noted, however, that this is impaired to a certain extent, but such impairment is referable to loss of ferment, and not due to the reaction. It has been rendered quite probable that during ferment action the ferment, temporarily at least, enters into combination with the substance to which it is "tuned."

The rapidity with which the action of ferments takes place is often most remarkable, and is especially well shown during the coagulation of milk under the influence of rennin.

In order that the ferments may exhibit their activity to best advantage a definite temperature is necessary, which varies somewhat with the different ferments, but is generally about that of the body. Higher as well as lower temperatures gradually inhibit their action, and, as has been seen, destroy it entirely when $75^{\circ}-80^{\circ} \mathrm{C}$. is reached. Very low temperature has the same effect.

Oxygen has no effect upon the action of most ferments, and they thus show a distinct difference from the organized ferments, which are more or less dependent upon its presence or its absence.

The reaction of the medium in which the ferments are to display their activity is very important, and varies with the different fer- 
ments. Some of these, such as pepsin, can act to advantage only in an acid medium ; while others, such as ptyalin, require an alkaline reaction; and still others can act in acid, alkaline, and neutral media, but exhibit certain preferences.

Generally speaking, the mode of action of the ferments is specific -i.e., certain ferments will only act upon certain definite substances. Fat-splitting ferments will only act upon fats, diastases only upon carbohydrates, proteolytic ferments only upon albumins. This specificity is in some instances very pronounced and is noticeable even in individual members of a common group. The autolytic ferments, for example, will not cause the cleavage of every albumin; generally speaking those albumins which are foreign to the cell which has furnished the particular ferment are attacked with greater difficulty by the autolytic ferments of the cell. The autolytic proteolytic ferment of the liver is thus incapable of hydrolyzing the albumins of lung-tissue. For the lytic action of ferments of one organ upon material which has originated in another organ Jacoby has suggested the term heterolysis in contradistinction to autolysis.

It has long been known that the hydrolytic decomposition effected by ferments is never carried to an end, and it is usually stated that this is owing to the fact that a gradual increase in the production of decomposition-products inhibits the action of the ferments in question. In the light of more recent investigations, however, this explanation is not satisfactory. It has been demonstrated that maltase, when added to a solution of maltose, will cause inversion of the latter to glucose. An end-reaction is then not obtained; but if this solution is now added to a solution of glucose in turn, at a time when further inversion does not occur, it will be noted that a retransformation of glucose to maltose takes place, which, however, is likewise not complete. It thus appears that the ferment is not only capable of causing the hydrolytic decomposition, but also the synthesis of maltose; but that in so doing its action ceases as soon as a certain equilibrium of reaction has been established. This reversible action on the part of ferments is, of course, of the greatest interest to the physiological chemist, in showing that the complex syntheses which take place both in plant and in animal life may, to a certain extent at least, be referable to such action, and to forces which are probably at work in the non-organized world.

Croft Hill has shown that lactase is capable of synthetic action with a concentrated solution of galactose and glucose. Very curiously, however, in the use of the synthetic production of both maltose and lactose, isomaltose and isolactose are formed, which the corresponding ferments are not able to split.

Kastle and Loevenhart have demonstrated the reversible action of lipase in the case of ethyl butyrate.

In the case of the proteolytic ferments similar conditions probably 
exist. ${ }^{1}$ It has been shown that pepsin, trypsin, and papayotin, when added to concentrated solutions of albumoses, will cause a precipitation of so-called plasteïn, and Sawjalow has announced that he succeeded in coagulating such plasteïns by boiling in the presence of acetic acid, which would establish their albuminous nature.

Berumzone has further shown that an extract of renal tissue will bring about the formation of hippuric acid from benzoyl alcohol and glycocoll. By means of yeast maltase Emmerling could bring about the formation of amygdalin from glucose and amygdalic acid nitril glucoside.

Emulsin also has been shown capable of synthetic action, etc.

Further research will show whether a reversible action is common to all ferments.

Chemical Composition and General Reactions.-Of the chemical composition of the ferments but little is known that is definite. This is owing to the fact that isolation of any one of the ferments in a chemically pure form has thus far not been accomplished. They apparently contain nitrogen, and are usually regarded as albuminous substances; but it is still a matter of doubt whether this is actually the case, and it is possible that the supposition of their albuminous nature is owing to their being contaminated with albumins.

Like the albumins, they are as a class non-diffusible. They are soluble in water, and can be precipitated from their aqueous solutions by salting with ammonium sulphate or by the addition of strong alcohol, and in some cases by normal salt. But here again it is a question whether the ferments themselves are precipitated by the reagents in question or whether they are mechanically carried down by the general precipitates which are formed simultaneously.

When kept under alcohol for any length of time some of the ferments, such as pepsin and diastase, are rendered inactive and are apparently coagulated, while the activity of others, such as the fibrin ferment, remains unaffected.

Characteristic general reactions, which are common to all ferments, are unknown. Formerly it was supposed that they all possessed the power of decomposing hydrogen peroxide, but it appears that this property does not belong to the ferments proper, but to adherent particles of protoplasm. As a matter of fact, it is possible in a number of ferments to destroy this power of decomposing hydrogen peroxide without influencing their specific activity in the least. If pancreatic juice is thus heated to a temperature of $60^{\circ} \mathrm{C}$., and then allowed to cool to $40^{\circ} \mathrm{C}$., it will be observed that the fluid is still capable of digesting albumins and of inverting starch, while it has lost the power of decomposing hydrogen peroxide entirely. Similar results may be obtained on heating the dry ferments to a

\footnotetext{
${ }^{1}$ It has recently been shown that trypsin is capable of bringing about the synthesis of protamin from its split products, and from those of casein a paranuclein has been obtained by means of pepsin.
} 
somewhat higher temperature, by treating with alcohol, or by saturating their solutions with neutral salts.

Mode of Action.-The decompositions which the ferments are capable of effecting in suitable media are essentially of a hydrolytic character. This can be readily shown by comparing the decomposition-products to which the ferments give rise with the original substances, when it will be found that, practically without exception, the former contain more water. Nasse, moreover, could demonstrate a distinct increase in the electrical conductivity of watery solutions of starch, for example, when these were treated with diastase, showing that dissociated molecules of water must have been present. As to the manner, however, in which these hydrolytic phenomena are brought about we are very much in the dark. On the one hand, we may suppose that the molecular oscillations which take place in the molecules of the ferments are of such a nature as to bring about an increase in the molecular oscillations of the substances upon which the ferments exert their specific activity, and that in consequence of this increase in the oscillations the labile equilibrium of the large albuminous or polysaccharine molecules is disturbed, which in turn would lead to new combinations of atoms to form molecules that are more stable, and the oscillations of which would be more nearly like those of the ferments. According to this theory, then, the action of the ferments would be what has been termed a katalytic action, and analogous to the katalytic action of various metals, such as platinum, gold, silver, etc., which in fine suspension behave in very much the same manner as the ferments (see page $\mathrm{\Omega}$ ). On the other hand, we may suppose that the action of the ferments is an action by contact, such that one molecule of the ferment causes hydrolytic decomposition of one molecule of an albuminous substance, for example, and that this decomposition in turn causes decomposition of the adjacent albuminous molecules, and so on. The action of certain ferments, such as the fibrin ferment, and that which causes the coagulation of milk, might very well be explained upon such a basis. For here we see a rapidity of action which scarcely admits of any other explanation; and direct contact of the ferments with all parts of the surrounding material, moreover, is excluded, as each ferment-molecule must of necessity be at once surrounded by a layer of the coagulated albumin.

A second class of ferments comprises the oxidizing ferments, which either obtain their oxygen from the air or from decomposed hydrogen peroxide. The first group of these is represented by the oxydases proper, which are active within the cells and tissues as oxygen earriers.

Classification.- It has been pointed out that there are no general reactions which are characteristic of all ferments. The ferments can be separated into groups, however, which are fairly well characterized by their specific activity and the decomposition-products to which they give rise. They are accordingly divided into the following classes: 
1. The Proteolytic Ferments (Proteases).-These comprise the common digestive ferments of the stomach and pancreas, viz., pepsin and trypsin; the autolytic ferments which are responsible for the aseptic autodigestion of the various organs after death, and analogous ferments which are widely distributed in the vegetable world. They all digest the various albumins with the formation of albumoses and those end-products of hydrolysis which are collectively spoken of as peptones. While their activity is generally speaking specific (see preceding page), it is noteworthy that some proteolytic ferments will also hydrolyze the nucleinic acids (Araki) ; trypsin is a notable exception to this latter rule.

Closely related to the proteolytic ferments is the erepsin which $\mathrm{O}$. Cohnheim demonstrated in the intestinal mucosa; its specific activity is directed to the hydrolysis of albumoses, while albumins are not affected.

Rennin and thrombin are two coagulating ferments which are sometimes classified under the proteolytic ferments. The first coagulates milk, the latter the blood.

2. The Amylolytic Ferments (Amylases, Diastases).-These include the ptyalin of the saliva, the diastatic ferment of the pancreatic juice, certain endocellular diastases, the so-called vegetable diastase and related ferments, which may be obtained from bacteria. Some of these only render starch soluble, while others also cause its hydrolysis to disaccharides.

3. The Inverting Ferments (Invertases).-These are apparently related to the amylolytic ferments, and are to a certain extent identical with them. They invert the disaccharides to monosaccharides, and according to their specific effect upon cane-sugar, maltose, and lactose, they are termed invertins (invertases), maltases, and lactases, respectively. Such ferments are found in the saliva, the pancreatic juice, the enteric juice, in many of the higher plants, and also in numerous organized ferments.

4. The Lipolytic Ferments (Lipases).-Lipases are extensively distributed in the animal body. They occur in the gastric and the pancreatic juice, and are possibly represented in all tissues. In the vegetable world also they are common; lipases have been found in the seeds of ricinus, of the poppy, of cannabis sativa, in linseed, corn, etc. They cause the hydrolysis of fats to fatty acids and glycerin.

5. The ureases, viz., ferments which decompose urea with the formation of ammonia. Such ferments are present in many bacteria, such as the Micrococcus ureæ, Bacterium ureæ, Bacillus fluorescens, etc. A ferment of this order has also been demonstrated in the liver.

6. Ferments which transform amido-acids into amides occur in the liver and the kidneys, and have also been demonstrated in plants.

7. The Histozyme of Schmiedeberg. - This is found in the kidneys and is characterized by its ability to decompose hippuric acid with the formation of benzoic acid and glycocoll. 
8. Ferments which Cause the Cleavage of Glucosides.--Such ferments are quite common among invertebrates, and are especially abundant in the higher plants. Examples are the emulsin or synaptase of bitter almonds, the myrosin of mustard seeds, and other Cruciferce, etc.

9. The nucleases, viz., ferments which are capable of causing the cleavage of the nucleinic acids. This group also includes the ferments which are concerned in the transformation of guanin and adenin to xanthin or hypoxanthin respectively, viz., guanase and adenase.

All these ferments are characterized by their hydrolytic action and must be differentiated from the oxidation ferments (see below). Occupying an intermediate position between the two groups are :

10. Ferments which are capable of splitting off carbon dioxide from certain bodies.-Ferments of this order are but little known, but their presence is indicated in various ways. Emerson has shown that a ferment belonging to this class occurs in the pancreas, and is characterized by its ability to form oxyphenyl-ethylamin from tyrosin by splitting off $\mathrm{CO}_{2}$. A similar ferment apparently occurs in the liver and may be responsible for the transformation of cystin acid to taurin.

11. The Oxidation Ferments.-These are ferments which are intimately concerned in the endocellular oxidations. They can be divided into three groups.

(a) The Oxydases (oxygenases).-These take up molecular oxygen with the formation of peroxides. They occur especially widely distributed in the vegetable world.

(b) The Peroxydases.- These contain manganese and aluminium and in some instances iron and possibly copper. They are quite stable and decompose hydrogen peroxide. In themselves$i$. e., in the absence of peroxides-they are incapable of causing oxidations, but they increase the power of oxidation of the peroxides very materially.

(c) The Katalases.-These decompose hydrogen peroxide katalytically with the liberation of oxygen, but have not been shown to be oxidizing agents themselves.

Conjointly the oxygenases and peroxydases are also spoken of as oxydases. Their mode of action consists essentially in the withdrawal of two atoms of hydrogen with the formation of water, and the occasional addition of one atom of oxygen. At times, however, a more energetic oxidation is observed. The peroxydases are of supreme biological interest owing to their power of decomposing peroxides, which, according to Engler's views, form the startingpoint of all oxidations in the animal body.

Aside from the common test for oxydases, viz., blueing of tincture of guaiac, phenolphthalin may also be employed; this is oxidized to phenolphthalein, which may then be estimated colorimetrically. The 
katalases, in contradistinction to the oxydases, do not blue tincture of guaiac either directly or in the presence of hydrogen peroxide.

The oxydases occur widely distributed in the animal and especially in the vegetable world ; they include the laccase, which causes the formation of the black Japanese lacquer ; various tyrosinases, one of which causes the transformation of tyrosin to homogentisinic acid, while another is responsible for the transformation of the black pigment secreted by the octopus; an indophenol oxydase, which forms indophenol from paraphenylendiamin and $\alpha$-naphthol; aldehydases which oxidize aldehydes to the corresponding acids, etc.

To this group probably also belong the glucolytic ferments, which have been demonstrated in many organs of the animal body, notably the muscle-tissue and the liver; in both instances they are apparently activated by a kinase furnished by the pancreas.

12. The Coagulating Ferments.-These include the fibrin ferment which causes the coagulation of blood; various milk-curdling ferments which occur both in the animal and the vegetable world; a ferment which is thought to be responsible for the coagulation of myosin; pectase, which coagulates the pectins of plants and leads to the formation of pectic acid, etc.

13. Reducing Ferments (Reductases).-Such ferments apparently also exist. One has been described which reduces sulphur to hydrogen sulphide.

14. The Kinases.-These are ferment-like substances which manifest their peculiar activity by the activation of other ferments, viz., their zymogens. Enterokinase thus activates the trypsinogen of the pancreas. Another kinase furnished by the pancreas activates the glucolytic substances found in liver and muscle tissue, etc. 


\section{CHAPTER VII.}

\section{THE DIGESTIVE FLUIDS.}

As has been pointed out, the greater portion of the food-stuffs which are ingested by animals cannot be utilized as such directly, but must first be transformed into material that is capable of diffusing through animal membranes. These changes occur in the gastro-intestinal tract, and are effected by the secretions of the various digestive glands, viz., the saliva, the gastric juice, the pancreatic juice, the succus entericus, and the bile.

\section{THE SALIVA.}

General Characteristics. - The saliva is the secretory product of the salivary glands, viz., the parotid, the submaxillary, and the sublingual glands, to which the secretion of the smaller mucous glands of the oral cavity is further added.

The saliva is a colorless, inodorous, tasteless, somewhat stringy and frothy, opalescent fluid, which normally possesses a slightly alkaline reaction and a specific gravity ranging between 1.002 and 1.008 . A slightly acid reaction of the mouth secretions is, however, also frequently observed. This is not referable to a constituent of the saliva, but owing to the presence of lactic acid, which is formed through the activity of micro-organisms, from food-material that has gathered between the teeth or from desquamated epithelial cells. For this reason also we find an acid reaction of the mouth-cavity on rising in the morning.

On microscopical examination the saliva is seen to contain a variable number of pavement epithelial cells and so-called salivary corpuscles. These are identical with the mucous corpuscles, which are found in all mucous membranes, and represent young leucocytes that have not entered the blood-current. They are derived from the lymph-follicles of the mucous membrane, and in the case of the mouth-saliva, no doubt, to a great extent from the tonsils. In addition we find innumerable bacteria, and at times also schizomycetes and moulds. On standing, the liquid becomes turbid, owing to precipitation of calcium carbonate, which frequently also forms a fine, iridescent film on the surface. The phenomenon is due to the escape of carbon dioxide from the saliva, and explains the formation of tartar on the teeth, as also the origin of the somewhat uncommon salivary concretions in the larger ducts of the glands.

Amount. - The amount of saliva that is secreted in the twentyfour hours varies somewhat even in health, but probably does not 
exceed 1500 c.c. It depends upon the amount of nutriment ingested, the act of chewing, the character of the food, the mental condition, etc. Fright may arrest its flow entirely. After the administration of pilocarpin or during the inhalation of ether an abundant secretion of saliva occurs. Atropin acts in the opposite manner, and can arrest the flow entirely.

To a certain extent the amount secreted is dependent upon the blood-pressure, but it does not follow that the saliva results from the blood-plasma through a simple process of filtration. We find that in the submaxillary gland, for example, the secretion continues for some time even after decapitation of the animal. The secretory pressure, moreover, is very much greater than the blood-pressure; and after the administration of atropin, which paralyzes the secretory nerves, we further find that while electrical stimulation of the chorda calls forth an increased circulation in the gland, a secretion of saliva does not occur. These experiments show that the secretion of the saliva cannot be referable to a simple process of filtration, but must depend upon a special secretory activity on the part of the alveolar cells. We thus also find that the salivary glands are capable of eliminating certain chemical substances, such as bromides and iodides, from the body, while others, like iron compounds, for example, are not removed through this channel, if we disregard the trace which is normally present.

Chemical Composition. - The chemical composition of the saliva is qualitatively fairly constant. Quantitative variations, however, are common. This is to a certain extent owing to the fact that the different glands are not all of one kind. In the human being the parotids are thus albuminous glands, the sublinguals mucous glands, while the submaxillary glands furnish a mixed secretion. The character of the secretion, moreover, may vary with one and the same gland. The salivary glands all have a double nerve-supply, which is partly of cerebral origin and partly derived from the sympathetic system, and as the one or the other set of fibres exercises its stimulating effect, the composition of the individual secretions will vary. In the submaxillary gland of the dog, in which these relations have been especially studied, on stimulation of the sympathetic fibres a secretion is furnished which is less abundant, but contains a larger amount of solids, than the secretion obtained on stimulation of the chorda. This is well shown in the following table, which is taken from Kühne:

$$
\begin{aligned}
& \text { Sympathetic saliva. } \\
& \text { Specific gravity . . 1.007-1.018 } \\
& \text { Solids . . . . . 16-18 pro mille } \\
& \text { Chorda saliva. } \\
& \text { 1.004-1.006 } \\
& \text { 12-14 pro mille }
\end{aligned}
$$

On dividing all the nerves which supply the salivary glands, or following the administration of curare, the secretion still continues for a while, but the saliva which is thus furnished contains scarcely any solid material, and is termerl paralytic saliva. 
Qualitatively, as has just been stated, the normal mixed saliva is of fairly constant composition. The quantitative variations which may occur in health are seen from the following analyses of human saliva, which are taken from Hammarsten :

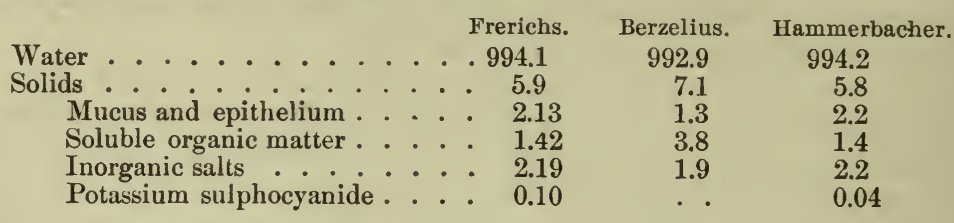

The albumins proper of the saliva are said to be similar to those of the blood-serum, but are present in only very small amount. They may, in fact, be regarded as accidental constituents, as the greater portion of the albumins which enter into the composition of the glandular cells is no doubt transformed into the specific secretory products of these glands, viz., into mucin and the amylolytic ferment ptyalin. In the cells proper, however, these substances apparently do not exist as such, but as mucinogen and ptyalingen, which are later transformed into mucin and ptyalin, respectively. As a matter of fact, it is possible to obtain the inactive ptyalinogen from the solids of the horse, and to transform it artificially into the active ferment. To this end, it is only necessary to collect the saliva from the parotid gland of the horse under aseptic precautions, and to prevent the further access of micro-organisms. A secretion is thus obtained which is perfectly inert when brought into contact with starch solution, while a corresponding specimen that has been exposed to the air at once begins to manifest the specific activity of free ptyalin. Of the manner in which this transformation is effected in the mouth we are as yet ignorant, but it appears that the bacteria which are here normally present are of importance. Similar results are reached when the finely hashed glands are extracted with chloroform-water, until the active ferment can no longer be obtained in this manner. On subsequent treatment with a very dilute solution of acetic acid other extracts can then be obtained, which are as active as the first, thus showing that a substance must have been present which could not be isolated with the chloroform-water, but which can be transformed into ptyalin by means of acetic acid.

In addition to ptyalin the saliva contains maltase, which inverts maltose to glucose.

Ptyalin.-The ptyalin or salivary diastase, as it is also termed, is an amylolytic ferment, and as such capable of causing the inversion of starch to sugar. This can be readily demonstrated as follows: A few cubic centimeters of saliva are added to a small amount of boiled starch solution and kept at a temperature of about $37^{\circ} \mathrm{C}$. If a drop of this mixture is then tested at intervals of about one minute with a dilute solution of iodine, it will be observed that the blue color, which is first obtained by bringing a drop of the two solutions together, soon gives place to a violet, and then to a red 
(erythrodextrin), and that still later no color-reaction whatever occurs (achroödextrin). As soon as this point is reached a small amount of the starch mixture is examined with Trommer's or Fehling's test, when the presence of sugar can be established. The sugar which thus results is maltose. The reaction which takes place may be represented by the schema :

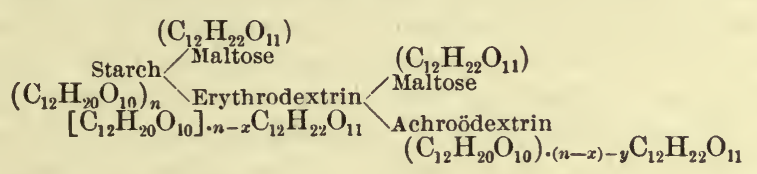

Glucose may incidentally be also demonstrable among the endproducts of salivary digestion, but is not due to the action of ptyalin, but of accompanying maltose. Its amount is rather insignificant.

We shall return to these reactions in the next chapter, when the digestion of the food is considered in detail.

To isolate the ptyalin from saliva, the following method, which has been suggested by Gautier, may be employed: To a large quantity of saliva 98 per cent. alcohol is added so long as a flocculent precipitate is seen to form. This is collected on a small filter and dissolved with a small amount of distilled water. The solution is treated with a few drops of a solution of bichloride of mercury, in order to remove any albuminous material that may be present. In the filtrate the excess of the bichloride is removed with hydrogen sulphide, when the remaining liquid is evaporated to dryness at a temperature not exceeding $40^{\circ} \mathrm{C}$., and the residue is treated with strong alcohol. The insoluble portion is then dissolved with a small amount of distilled water, filtered, dialyzed in order to remove inorganic salts, and finally precipitated with absolute alcohol, when the ptyalin will separate out in light flakes. Obtained in this manner, ptyalin is a white amorphous substance, which is soluble in water, dilute alcohol, and glycerin. In neutral or slightly alkaline solution, but not in acid solution, it rapidly transforms boiled starch into sugar at a temperature of from $36^{\circ}$ to $40^{\circ} \mathrm{C}$. Heated to a temperature of $65^{\circ}$ to $70^{\circ} \mathrm{C}$., it is destroyed, and it is thus possible to distinguish between ptyalin and vegetable diastase, for which the optimum temperature lies at $50^{\circ} \mathrm{C}$., and which is destroyed at $80^{\circ} \mathrm{C}$.

Of special interest is the fact that the transformation of starch into sugar ceases as soon as the latter is present to the extent of from 2 to 2.5 per cent. This phenomenon is common to all enzymatic processes, and is probably referable to the establishment of a certain equilibrium of reaction. A complete transformation of the starch could occur only if the resulting sugar were removed as rapidly as it is formed. So long as it is present, the reversible action of the enzyme becomes manifest. 
The amount of ptyalin which is secreted in the twenty-four hours has not been determined. Its activity, as would be expected, is subject to considerable variations. It is greatest in the morning on rising, and then steadily diminishes during the day. Immediately before meals, however, a temporary increase is observed, which is then followed by a marked decrease.

Of the chemical nature of ptyalin nothing definite is known. As it is obtained in the laboratory it gives some of the reactions of albumins (not the xanthoproteic reaction), but this may be due to contamination. From its solutions it can be precipitated with acetate and subacetate of lead, while bichloride of mercury and the salts of platinum, as also tannic acid, are without effect.

In the human being ptyalin is formed in the parotid and the submaxillary glands and, as will be seen later, also in the pancreas, while the sublingual glands apparently yield no ptyalin. In other animals its presence is variable. In the typical carnivora $(\mathrm{dog})$ it is said to be absent, while in the saliva of all herbivorous animals it is uniformly found.

Of other ferments, human saliva contains traces of maltase, and of an oxydase of unknown character; invertin has not been found.

The digestive importance of the saliva in man is now recognized to be greater than was formerly supposed; for it has been shown that food may remain in the fundus portion of the stomach for an hour or longer without coming into contact with the acid gastric juice. Salivary digestion may thus proceed uninterrupterly for a much longer time than was formerly thought to occur. The resulting maltose is probably not absorbed to any great degree until it reaches the small intestine and is then inverted by maltase.

Mucin.-The mucin of the saliva is derived from the submaxillary and sublingual glands, as also from the small mucous glands which are found imbedded in the mucous membrane of the month. Its formation in the salivary glands is apparently under the control of the sympathetic nervous system, as it is secreted in much larger amounts on stimulation of these fibres than of the corresponding cerebral fibres. According to Levene, the submaxillary mucin contains the chondroitin-sulphuric acid complex, or a closely allied group.

To the presence of the mucin the viscid, stringy character of the saliva is due. The substance can be obtained by precipitation with acetic acid, and it is to be noted that, in contradistinction to the mucin-like substances which belong to the class of the nucleo-albumins, the precipitated mucin is insoluble in an excess of the acid. In dilute solutions of the alkalies it is soluble, and it is thus possible by repeated precipitation and solution to obtain the mucin in fairly pure form. In the dry state it occurs as a white or yellowish-gray powder. In alcohol and water it is insoluble, although in the latter it swells to form a jelly-like material. Unlike the albumins, it is not coagulated by heat; but, like these, it gives the 
xanthoproteic reaction, the biuret reaction, and Millon's reaction. It contains also a small amount of sulphur. On boiling with dilute mineral acids mucin is decomposed into a substance which resembles acid albumin, and into a carbohydrate-like body which reduces Fehling's solution. This has been shown to be a glucosamin. According to Müller, it is present to the extent of 23.5 per cent.

Within the cells mucin exists as so-called mucinogen, which probably represents a compound of mucin with an additional albuminous substance.

Sulphocyanides.-Traces of sodium sulphocyanide are in man usually found in every specimen of normal saliva. It is secreted by all the salivary glands, but in largest amount by the parotids. In other animals its presence is not so constant, and in some indeed it is not found. In man also it is at times absent.

To demonstrate the presence of sulphocyanides, it usually suffices to treat a few cubic centimeters of saliva, which have been slightly acidified with hydrochloric acid, with a few drops of a very dilute solution of perchloride of iron, when a red color will be seen to develop. If no result is obtained in this manner, a larger quantity, such as 100 c.c., is evaporated to a small volume and again tested as described.

Nitrites.-Small amounts of nitrites may also at times be observed, and are no doubt derived from the nitrates ingested. To test for these, about 10 c.c. of saliva are treated with a few drops of Ilasvay's reagent, and heated to a temperature of $80^{\circ} \mathrm{C}$., when in the presence of nitrites a red color develops.

Ilasvay's reagent is prepared as follows : 0.5 gramme of sulphanilic acid in 150 c.c. of dilute acetic acid is treated with 0.1 gramme of naphtylamin dissolved in 20 c.c. of boiling water. After standing for some time the supernatant fluid is poured off, and the sediment dissolved in $\mathbf{1 5 0}$ c.c. of dilute acetic acid. The solution is kept in a sealed bottle.

Extractives.-Of extractives, normal saliva contains a small amount of urea, and traces of cholesterin, lecithin, and leucin. In gouty conditions uric acid has been found; sugar, the biliary pigments, and biliary acids are not eliminated through the saliva.

Mineral Constituents. - The mineral constituents of saliva consist to the extent of 90 to 92 per cent. of soluble salts, among which the chlorides greatly predominate, and of about 6 per cent. of salts, which are principally represented by the carbonates and phosphates of calcium and magnesium, which are held in solution by the free carbonic acid of the saliva. In addition, a trace of iron is found. Following the administration of bromides and iodides a notable elimination of these salts occurs through this channel.

Gases.-Of gases, which are present in a state of solution, we find about 20 c.c. for every 100 grammes of saliva. Of these, 19 c.c. are represented by carbon dioxide, while oxygen and nitrogen together amount to only 1 c.c. 


\section{THE GASTRIC JUICE.}

General Considerations.-The gastric juice is the secretory product of the glandular structures of the stomach, and the only digestive fluid which presents an acid reaction. In pure form it is best obtained from animals according to one of the methods devised by Pawlow and his pupils, viz., by the formation of a blind stomach pouch, with a fistulous opening to the outside; or by the establishment of a double œsophageal and a single gastric fistula, arranged in such manner that the food eaten by the animal is discharged from the œsophagus instead of entering the stomach. On feeding the animal pure gastric juice is then obtained, uncontaminated by any food material. Procured in this manner it appears as a clear or but slightly opalescent yellowish fluid, which has a very characteristic odor and a strongly acid reaction. Its density varies between 1.002 and 1.003 .

On microscopic examination epithelial cells from the lining of the glandular ducts, goblet-cells, mucous corpuscles, free nuclei, and a variable number of bacteria are found. In addition, we often observe small tapioca-like bodies, which under the microscope are seen to contain numerous formations resembling snail-shells, and which probably consist of altered mucin.

Amount.-Of the total amount of gastric juice secreted in the twenty-four hours, but little is known. Its secretion is influenced by numerous factors, such as the appetite, the act of mastication, the quality and quantity of the food ingester, the age and sex of the individual, the time of day (notably in relation to the taking of food), the psychic state of the individual, etc. ${ }^{1}$ According to Bidder and Schmidt, the amount corresponds to about one-tenth of the body-weight, so that a man weighing 70 kilogrammes would secrete about 7000 grammes in the twenty-four hours. This figure, however, I regard as too high, and am inclined to place the amount at from 2000 to 3000 c.c.

Some of the results which have been obtained by Pawlow are shown in the following table; the figures have reference to the ingestion of $200 \mathrm{gms}$. of meat, $200 \mathrm{gms}$. of bread, and $600 \mathrm{gms}$. of milk respectively :

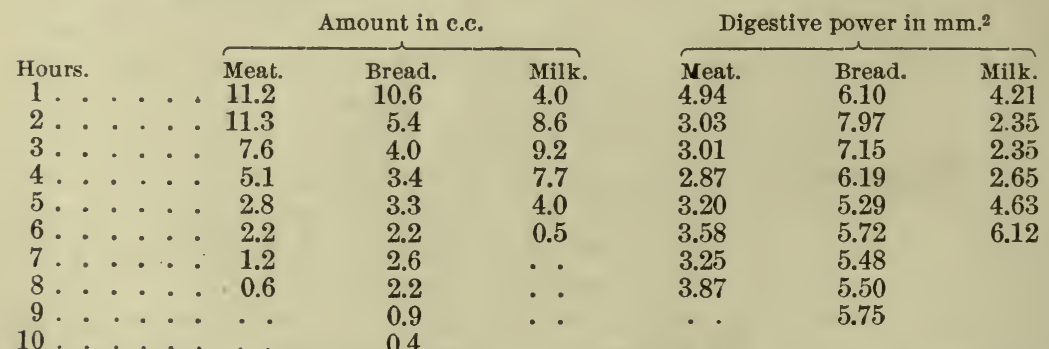

1 The secretion of the gastric juice, like that of the pancreatic juice, has been shown to be referable to a specific hormone. pepsin).

Measured by the number of mm. of digested egg-albumin in Mett tubes (see Estimation of 
From this table it is seen that with a meat diet the maximum secretion occurs in the first and second hours after eating, with bread in the first and milk in the second and third hours. With meat the strongest digestive effect is obtained during the first hour, with bread in the second and third hours, and with milk as late as the fifth and sixth hours.

The non-digesting stomach of the dog is said to contain no fluid; in man, however, a small amount of gastric juice can usually be obtained, varying between 1 and 60 c.c. Larger amounts may be found under pathologic conditions, and in the so-called Magensaftfluss of the Germans it is not rare to find as much as 1000 c.c. in the early morning, before any food has been taken.

Chemical Composition.-A general idea of the chemical composition of the gastric juice may be formed from the following analyses, which are taken from C. Schmidt; but it is to be noted that the specimen of human gastric juice was contaminated with saliva and somewhat diluted with water (the figures have reference to 1000 parts) :

\begin{tabular}{|c|c|c|c|}
\hline & & $\begin{array}{l}\text { Human gastric juice, } \\
\text { containing saliva } \\
\text { with water. }\end{array}$ & $\begin{array}{l}\text { Gastric juice of } \\
\text { dog, free } \\
\text { from saliva. }\end{array}$ \\
\hline Water & & . . 994.40 . . & . . 973.0 \\
\hline Solids . . . . & & $\begin{array}{l}.60 \\
. \quad 5.60\end{array}$ & $\therefore 27.0$ \\
\hline Organic material & & 3.10 & . 17.1 \\
\hline Mineral salts & & 2.19 & . $\quad 6.7$ \\
\hline Sodium chloride & & 1.46 . & 2.5 \\
\hline Calcium chloride & . & 0.06 . & 0.6 \\
\hline Potassium chloride. & . & 0.55 & 1.1 \\
\hline Ammonium chloride & & . . . & 0.5 \\
\hline Calcium phosphate. & . & —. & 1.7 \\
\hline Magnesium phosphate & . & -. & 0.2 \\
\hline Iron $\ldots$ & • & 0.12 . & 0.1 \\
\hline Free hydrochloric acid & & 0.20 . & 0.1 \\
\hline
\end{tabular}

Acidity of the Gastric Juice.-It has been definitely established that the acidity of normal gastric juice is referable to the presence of free hydrochloric acid, and to this only. This can be shown by estimating the amount of chlorine and all basic substances that are present, when it will be found that after the acid affinities of the latter have been satisfied, a certain amount of chlorine still remains, which can be referable only to hydrochloric acid, and corresponds in its degree of acidity to that of the gastric juice.

During the process of digestion, however, other factors enter into consideration. In the beginning of digestion lactic acid is always present when carbohydrates form part of the meal. Its amount, however, is then quite small, and after the ingestion of Ewald's test-breakfast, for example, does not exceed 0.3 pro mille. The occurrence of larger quantities of lactic acid, as from 1 to 3 pro mille, is always abnormal, and in many cases indicative of the existence of carcinoma of the stomach. During the later stages of digestion, when hydrochloric acid is found in a free state, lactic acid disappears. Its origin, under normal conditions, is usually 
referable to the action of certain bacteria, such as the Bacillus lactis aërogenes, etc., upon starches and sugars, as represented by the equations:

$$
\begin{aligned}
& \text { (1) } \underset{\text { Starch. }}{\left(\mathrm{C}_{5} \mathrm{H}_{10} \mathrm{O}_{5}\right)_{n}}+n \mathrm{H}_{2} \mathrm{O}=n\left(\underset{\text { Lactose. }}{\left.\mathrm{C}_{2} \mathrm{H}_{22} \mathrm{O}_{11}\right)}\right. \\
& \text { (2) } \mathrm{C}_{12} \mathrm{H}_{22} \mathrm{C}_{11}+\mathrm{H}_{2} \mathrm{O}=\underset{\text { Lactic acid }}{4 \mathrm{C}_{3} \mathrm{H}_{6} \mathrm{O}_{3}}
\end{aligned}
$$

Other organic acids, such as butyric acid and acetic acid, are usually not found in the gastric contents unless large amounts of milk, carbohydrates, or alcohol have been ingested. In such event, however, they may be present, and, like lactic acid, are then referable to the action of micro-organisms. They are essentially of pathological significance.

It is thus seen that even during the process of digestion the acidity of the gastric contents is, under normal conditions, scarcely influenced by acids other than hydrochloric acid. It should be noted, however, that following the ingestion of food hydrochloric acid does not appear in a free state at once, but only after the affinities of the albuminous constituents of the food have been saturated. We consequently find that while in the beginning of digestion the acidity of the stomach-contents is largely referable to such combined acid, in the later phases two factors enter into consideration, viz., free and combined hydrochloric acid. The period at which free acid appears varies with the character of the food and especially the amount of albumins ingested. After the administration of Ewald's test-breakfast (a slice of bread and a glass of water) free acid is found at the expiration of about thirty-five minutes; while after the administration of Riegel's test-dinner, which contains much larger amounts of albumin, two hours must elapse before free acid can be demonstrated.

Acid salts, finally, play only a small part in determining the total acidity of the gastric juice; and it is thus clear that unless carbohydrates, much fat, and alcohol have been ingested, hydrochloric acid, either in a free state or in combination with albumin, or both, is the sole factor which enters into consideration. Under pathological conditions, on the other hand, lactic acid, butyric acid, and acetic acid may also play a part; but then hydrochloric acid is usually not present, and the acidity of the gastric contents is hence largely referable to fermentative changes which have taken place in the stomach.

Determination of the Total Acidity of the Gastric Contents. - Five or 10 c.c. of the filtered gastric contents are titrated with a decinormal solution of sodium hydrate, using phenolphthalein as an indicator, until the rose color, which appears on the addition of each drop of the sodium hydrate solution, no longer disappears on stirring or is intensified by the addition of a further drop. The number of cubic centimeters employed to bring about this reaction, multiplied by 0.00365 , indicates the acidity of the 5 or 10 c.c. of gastric juice 
in terms of hydrochloric acid. In elinical work it is customary to express the degree of acidity in terms of the number of c.c. of $\frac{n}{10}$ alkali solution which would be necessary to neutralize 100 c.c. of stomach-contents.

Degree of Acidity.-The degree of acidity of the gastric juice is usually fairly constant, and in man varies between 0.3 and 0.5 per cent. As has been stated, it is influenced to a great extent by the character of the diet. Following the administration of a meal rich in albumins, larger amounts are obtained than after the ingestion of earbohydrates or fats. The smallest amounts are found after the ingestion of water. After Ewald's test-breakfast, which consists of from 35 to 70 grammes of wheat bread and 300 to 400 c.c. of water, or weak tea without sugar, the maximum acidity is reached in about one hour, and corresponds to 1.5 to 1.75 pro mille. Following the ingestion of Riegel's test-meal, on the other hand, which consists of a plate of soup (400 c.c.), 200 grammes of beefsteak, 50 grammes of wheat bread, and 200 c.c. of water, the amount of hydrochloric acid increases to 2.7 pro mille, after from one hundred and eighty to two hundred and ten minutes. In disease still higher figures (5 p. m.) may be observed; or its secretion may diminish below the normal, and may even cease altogether.

Hydrochloric Acid.-Origin.-The hydrochloric acid of the gastric juice is furnished by the parietal, adelomorphous, or oxyntic cells, which are principally found in the glands of the middle region of the stomach. In the pyloric region, where these are absent, the reaction is alkaline, and in the fundus also, where they are either absent or present in greatly diminished numbers, the secretion is not acid.

While it is thus quite probable that the hydrochloric acid is furnished by the parietal cells, we are ignorant of the mechanism by which this is accomplished. A free acid is manifestly not present in these cells, as can be shown by testing with litmus-paper, or still better by injecting potassium ferrocyanide and lactate of iron into the circulation of an animal, when it will be observed that Berlin-blue is formed in the stomach-cavity, while the cells themselves remain unstained. It thus follows that a substance must either be present in the cells which is capable of yielding hydrochloric acid when secreted to the outside, or a mechanism must exist by which the hydrochloric acid, though formed within the cells, is at once eliminated. The latter view is now generally held. That the hydrochloric acid is derived from the chlorides of the blood can be regarded as an established fact. It may thus be secreted even though no food-stuffs have been ingested; and Kahn has shown that animals in which the chlorides of the body have been artificially reduced to that minimum which is tenaciously retained are no longer capable of secreting hydrochloric acid. Ludwig formerly thought that hydrochloric acid resulted through electrolytic influences within the cells, and that the acid then dif- 
fused out into the lumen of the glandular duct before the alkaline elements of the cell could bring about its neutralization. This, however, is improbable. Among the various other views which have been expressed, that of Maly has attracted much attention. According to this, the hydrochloric acid results from a massaction on the part of the carbonic acid of the blood upon the chlorides in the cells, and is immediately eliminated to the outside, while the resulting bicarbonate is returned to the blood. We know that within the cells carbon dioxide is present under great pressure. Schlierbeck thus found that water which is introduced into the stomach of living dogs after a variable length of time contains a certain amount of carbon dioxide, and that its tension rises from 30 to $40 \mathrm{Hgmm}$. while fasting, to 130 to $140 \mathrm{Hgmm}$. during the process of active digestion. There are objections to this view also, and, as a matter of fact, we can offer no satisfactory explanation which rests on anything but a hypothetical basis.

The secretion of hydrochloric acid seems to be inaugurated through the activity of a specific hormone which is furnished by the mucous membrane of the pyloric portion of the stomach. Of its nature nothing is known (see also Secretin).

Significance of the Hydrochloric Acid.-One of the functions of the hydrochloric acid no doubt is the activation of pepsinogen to pepsin, and thus a digestive function. We know, however, that life can go on in the entire absence of the stomach, as has been proved not only by experiments on animals but also by operations performed on the human being. A dog whose stomach was almost entirely removed by Czerny in 1876, lived for more than six years after the operation, when it was killed in Ludwig's laboratory; and it is reported that the animal was normal in every respect, and had increased in weight from 5850 to 7000 grammes. It is thus manifest that while the hydrochloric aeid of the gastric juice no doubt aids in the process of albuminous digestion, its presence to this end is not imperative. A further function may be the prevention of putrefactive changes in the contents of the stomach, and we find, as a matter of fact, that albuminous material that has been removed from the stomach at the height of digestion can be preserved for a long time without undergoing decomposition. It has been noted, moreover, that the gastric juice is also capable of arresting putrefactive processes that have begun before the ingestion of such material. A third function, as will be shown later on, is the activation of the duodenal secretion, which in turn calls forth the secretion of the pancreatic juice.

Tests for Free Hydrochloric Acid.-A large number of tests have been devised for the purpose of demonstrating the presence of free hydrochloric acid in the stomach-contents. The most important of these are the following:

TOPFER's TEST.-A small amount of the filtered gastric contents is treated with a few drops of an 0.5 per cent. alcoholic solution of 
dimethyl-amino-azobenzol, when in the presence of free hydrochloric acid a beautiful cherry-red color develops at once, which varies in intensity with the amount of the free acid present. Combined hydrochloric acid, as well as acid salts and organic acids, in the concentration in which they may be met with in the stomachcontents, do not produce this color.

The delicacy of the reagent is such that the normal yellow color of the indicator is changed to a reddish tinge upon the addition of but one drop of a $\frac{1}{10}$ normal solution of hydrochloric acid in 5 c.c. of distilled water, viz., 0.7 per cent.

GÜNZBURG's TEST.-The reagent consists of 2 grammes of phloroglucin and 1 gramme of vanillin, dissolved in 100 grammes of 80 per cent. alcohol. It should be kept in a dark-colored, glassstoppered bottle.

A few drops of the filtered gastric contents are carefully evaporated with an equal amount of the reagent on a plate of thin porcelain or glass, when in the presence of free hydrochloric acid a rose-colored mirror is obtained, which varies in intensity with the amount of the acid.

Organic acids do not produce the reaction.

The delicacy of the test is such that the presence of 0.05 gramme of hydrochloric acid in 100 parts of water can be demonstrated.

BOAS' TEsT.-The reagent consists of 5 grammes of resublimed resorcin and 3 grammes of cane-sugar, dissolved in 100 grammes of 94 per cent. alcohol. The test is conducted like that of Günzburg, but it is necessary to heat a little more strongly, especially after the fluid has been evaporated. A similarly colored mirror is obtained, which gradually fades on cooling.

The delicacy of the test is the same as that of Günzburg.

Examination for the Presence of Combined Hydrochloric Acid.The presence of combined hydrochloric acid cannot be demonstrated by means of simple tests like those. just desçribed, but is inferred indirectly, as shown in the following method:

Separate Estimation of the Free and Combined Hydrochloric Acid of the Gastric Contents.- TöPFER's METHOD.-The total acidity of a given amount of the gastric contents is first determined, as already described, and termed A. This indicates the amount of the physiologically active hydrochloric acid, viz., the free and the combined hydrochloric acid, as well as that of any acid salts and organic acids that may be present.

In a second specimen the total amount of free acids and acid salts is determined by titrating, as before, with a $\frac{1}{10}$ normal solution of sodium hydrate, but using a few drops of a saturated aqueous solution of alizarin (alizarin-monosulphonate of sodium) as an indicator. The titration is carried to a point where a pure violet color is obtained. The result is termed B. The difference between $A$ and $B$ is thus referable to the presence of the combined hydrochloric acid, and termed C. 
In a third specimen the amount of free hydrochloric acid is determined by titrating with the decinormal solution of sodium hydrate, using a few drops of a 0.5 per cent. alcoholic solution of dimethyl-amido-azobenzol as indicator, until the red color which first appears has changed to yellow. The result is termed F. $\mathrm{F}$ plus $\mathrm{C}$ will then represent the amount of physiologically active hydrochloric acid P, viz., the combined and free acid, while the difference between $\mathrm{A}$ and $\mathrm{P}$ corresponds to the acid salts and organic acids that may also be present.

MeThod of Mörner And SJoqvist.-By this method the amount of physiologically active hydrochloric acid can also be estimated. It is somewhat more complicated and time-consuming than the one just described, but more accurate. It is based upon the fact that on evaporating the gastric contents to dryness in the presence of barium carbonate, and subsequently incinerating the residue, the organic acids are destroyed, while the hydrochloric acid combines with the barium, and can thus be estimated as barium chloride.

To this end, 10 c.c. of the filtered stomach-contents are treated with a pinch of chemically pure barium carbonate and evaporated to dryness. The residue is ignited at a moderate temperature until white, and the remaining ash extracted with hot water. After filtering the solution (about 50 c.c.) it is treated with an equal volume of alcohol (94 per cent.) and 0.75 c.c. of a 10 per cent. solution of sodium acetate in dilute acetic acid (10 per cent. solution). The barium is then estimated by titrating with a standardized solution of potassium bichromate, containing 8.5 grammes of the chemically pure substance in the liter, and using tetramethyl-paraphenyldiamin paper as an indicator, until a drop of the titrated fluid causes a distinct blue coloration of the paper within one minute. From the number of cubic centimeters employed to bring about the end-reaction the corresponding amount of hydrochloric acid can then be calculated by multiplying with 0.00405 , if the bichromate solution was standardized with a $\frac{1}{10}$ normal solution of barium chloride.

METHOD of LEO. - This method is based upon the observation that calcium carbonate combines with free and loosely bound hydrochloric acid at ordinary temperatures to form neutral calcium chloride, while the acid phosphates are not affected. If then the total acidity of the stomach-contents is first determined, and the acidity referable to acid salts deducted from this figure, the amount of physiologically active hydrochloric acid is ascertained. Organic acids, of course, must first be removed by extracting with ether (50-100 c.c. for 10 c.c. of gastric juice). As the monophosphates of potassium and sodium, however, are changed to monocalcium phosphate in the presence of calcium chloride, which requires double the quantity of sodium hydrate solution for its neutralization than the corresponding amount of the alkaline phosphates, it is either necessary to divide the number of cubic centimeters of the sodium hydrate solution which is used in the second titration by 2 , or to 
make the first titration under the same conditions as the first, viz., after adding an excess of calcium chloride solution.

To this end, then, we proceed as follows: 15 c.c. of the filtered gastric contents are treated with a pinch of dry and chemically pure calcium carbonate. The mixture is well stirred and passed at once through a dry filter. Ten c.c., from which the carbon dioxide is expelled by a current of air, are then treated with 5 c.c. of a concentrated solution of calcium chloride and titrated as usual (using phenolphthalein as indicator). The resulting value is termed $\vec{P}$, and represents the acid phosphates. The total acidity is then determined in another specimen, after adding the same amount of the calcium chloride solution, and the result termed T. T minus A will represent the amount of the physiologically active hydrochloric acid.

The combined hydrochloric acid may, of course, be readily determined with either of the two methods which have just been described, by separately estimating the amount of free hydrochloric acid by 'Töpfer's method, and deducting the result from the total amount of the physiologically active acid. More accurate results are probably reached in this manner than with 'Töpfer's method, unless some experience has been gained in the titration with alizarin.

Should organic acids also be present, their amount may be estimated by deducting from the total acidity the result reached with the above method.

Lactic Acid.-Tests for Lactic Acid.-In order to assure ourselves that any lactic acid that may be found in the gastric contents has not been introduced into the stomach from without, it is necessary to make such examinations after the administration of a testmeal, in which the acid in question does not occur preformed. The meal which is almost' exclusively used for this purpose in clinical work is the so-called test-meal of Boas. It consists of a plateful of oatmeal soup, which is prepared by adding a tablespoonful of rolled oats and a little salt to a liter of water, and boiling down to about 500 c.c. The contents of the stomach are then drawn off after one hour, filtered, and treated as described below.

UFfelmanN's TesT.-About 10 c.c. of the filtered gastric contents are extracted with ether (50-100 c.c.) by shaking in a separating funnel for from twenty to thirty minutes. The ethereal extract is then evaporated to dryness by distilling on a water-bath; the residue is taken up with a few cubic centimeters of distilled water, and treated as follows : 3 drops of a saturated aqueous solution of ferric chloride are mixed with an equal number of drops of a concentrated solution of pure carbolic acid, and diluted with water until a light-amethyst color is obtained. To this solution a portion of the ethereal extract is added, when in the presence of lactic acid a lemon or canary color develops.

The delicacy of the test is such that the presence of 0.1 per cent. of lactic acid can be demonstrated. 
Kelling's 'Test (as modified by the writer).-A solution of sesquichloride of irou is prepared, so dilute that a trace of yellow can barely be discerned. Two test-tubes are partly filled with this reagent and a small amount of the filtered gastric contents added to the one. In the presence of lactic acid a distinct yellow develops at once, which becomes especially marked when the tube is compared with the control.

BoAs' 'Test.--This test is more accurate than the two just described, but more time-consuming and complicated. It is based upon the decomposition of lactic acid into formic acid and acetic aldehyde, and the demonstration of the presence of the latter. To this end, from 10 to 20 c.c. of the filtered stomach-contents are treated with a slight excess of barium carbonate, and evaporated on a water-bath. The resulting syrup is acidified with a few drops of phosphoric acid, and freed from carbon dioxide by momentary ebullition. On cooling, it is extracted with 100 c.c. of ether by shaking for about thirty minutes. The ethereal extract is decanted, the ether distilled off, and the residue taken up with 45 c.c. of water. After filtering, the solution is treated with 5 c.c. of concentrated sulphuric acid and a pinch of manganese dioxide, and earefully heated to boiling. Should lactic acid be present, this is now decomposed, and acetic aldehyde liberated, which can be demonstrated by passing the vapor into a test-tube containing Nessler's reagent or an alkaline solution of iodopotassic iodide. In the first instance, yellowish-red aldehyde of mercury is formed, while iodoform results in the latter, and can be readily recognized from its odor, which becomes marked when the solution is heated.

Tests for Acetic Acid and Butyric Acid.-These acids can usually be recognized by their odor. Chemically they can be demonstrated as follows:

Test for Acetic Acid.-Ten c.c. of the filtered stomach-contents are extracted with ether as above. The ether is distilled off', the residue taken up with a few drops of water and accurately neutralized with sodium hydrate. To this solution a drop or two of a very dilute solution of ferric chloride is added, when in the presence of acetic acid a dark-red color develops. With nitrate of silver, on the other hand, a precipitate is obtained which is soluble in hot water.

Test for Butyric Acid.- The ethereal extract of 10 c.c. of the stomach-contents is freed from ether by distillation, the residue is dissolved in a few cubic centimeters of water, and treated with a trace of calcium chloride in substance. In the presence of butyric acid small oil droplets separate out, the nature of which is readily recognized from the pungent odor. If, in the place of calcium chloride, a slight excess of baryta-water is used, highly refractive rhombic platelets or granular, wart-like masses are obtained on evaporation, which consist of barium butyrate.

Butyric acid can also be recognized by the peculiar odor of pineapple which develops when the dry residue of the ethereal solution 
is treated with a little sulphuric acid and alcohol. The reaction is due to the formation of ethyl butyrate, $\mathrm{C}_{4} \mathrm{H}_{7} \mathrm{O}_{2} \cdot \mathrm{C}_{2} \mathrm{H}_{5}$.

Quantitative Estimation of Lactic Acid.-This is best accomplished by means of Boas' method: The decomposition of the lactic acid is effected as described above. After the addition of the sulphuric acid and manganese dioxide the flask is closed with a doubly perforated stopper. Through one aperture a bent tube passes to the enndenser, while a straight tube passes through the other opening, and is provided at its free end with a small piece of rubber tubing that is clamped; this tube should dip well into the liquid, and serves for passing a eurrent of air through the solution when the distillation is completed. The mixture is then distilled until about fourfifths of the contents have passed over, excessive heat being carefully avoided, so as to prevent decomposition of the aldehyde. The distillate, which is received in a high Erlenmeyer flask, is heated with 20 c.c. of a $\frac{1}{10}$ normal solution of iodine and the same amount of a 5.6 per cent. solution of potassium hydrate. The mixture is thoroughly shaken and set aside for a few minutes. The excess of iodine is then estimated after adding 20 c.c. of hydrochloric acid (specific gravity 1.018), by titrating with a $\frac{1}{10}$ normal solution of sodium thiosulphate. The titration is carried almost to the point of decolorization, when a little starch solution is added, and the titration continued until the last trace of blue has disappeared. The difference between the number of cubic centimeters of the thiosulphate solution employed to bring about this end and the amount of the iodine solution added, viz., 20, will indicate the number of cubic centimeters of the latter which were utilized in the formation of iodoform. By multiplying this number by 0.003388 the corresponding amount of lactic acid is ascertained.

\section{The Ferments of the Gastric Juice and their Proenzymes.}

In the gastric juice of almost all vertebrate animals two ferments are constantly found. These are termed pepsin and chymosin, or rennin, and are supposedly furnished by the so-called adelomorphous or central cells of the gastric glands. This has been established by resecting the pyloric end of the stomach and converting it into a blind pouch, with a fistulous opening on the anterior abdominal walls, while the remaining portion of the stomach was united to the duodenum. It was then noted that this resected portion of the stomach, in which no delomorphous cells are found, furnished an alkaline and markedly viscid secretion, which contained pepsin and large amounts of mucin, but no hydrochloric acid. A reversal of the experiment, in which the middle portion was thus isolated, showed that here both pepsin and hydrochloric acid are secreted. As this portion of the stomach contains both delomorphous and adelomorphous cells, the conclusion naturally suggested itself that the hydrochloric acid is furnished by the delomorphous 
cells, while the pepsin-and the same apparently holds good for chymosin-is secreted by the adelomorphous cells. The latter are hence also spoken of as pepsin cells, while the delomorphous cells are similarly termed the oxyntic cells of the stomach.

The existence of a special proteolytic ferment in the pyloric portion of the stomach, which has been termed pseudopepsin, and which supposedly resembled trypsin in its action, is doubtful. Abderhalden has shown that tryptic ferments in contradistinction to pepsin are capable of splitting peptids containing an amino-acid which is soluble with difficulty (tyrosin, eystin), such as glycyl-ltyrosin, and that the ferment of the pyloric region, after activation with hydrochloric acid, is incapable of accomplishing this.

In addition to the two ferments mentioned, the fat-splitting ferment lipase has also been found in the gastric juice of man and several mammals. (Volhard, Kastle, and Loevenhart.) It is apparently furnished by both the pyloric portion and the fundus, but more abundantly so in the latter situation.

As in the case of the ptyalin of the saliva, it appears that the ferments in question do not exist in the cells as such, but in the form of pro-enzymes or zymogens. These zymogens in the case of pepsin and chymosin are termed propepsin or pepsinogen and prorennin or chymosinogen, respectively. This is apparent from the following facts :

It has been shown that an extract of the gastric mucosa of a fasting animal when treated with 1 per cent. of soda, and which then has no digestive power whatever, when maintained aseptic with toluol may be kept for months and subsequently be rendered physiologically active by acidifying with hydrochloric acid to the extent of $0.2-0.3$ per cent. If then, however, such artificial gastric juice is alkalinized with soda to the extent of 0.5 per cent., the solution is rendered entirely inactive after a few seconds, when warmed to the temperature of the body, and it is to be noted that the subsequent addition of hydrochloric acid is now no longer capable of restoring the activity of the enzyme. This demonstrates, of course, that while the proenzyme is more or less resistent to soda, the ferment is thereby rapidly destroyed. On the other hand, it appears that pepsin is more resistant to the influence of carbonic acid than propepsin. Between chymosin and its zymogen similar relations exist.

Of the chemical nature of the proenzymes and the manner in which they are produced by the cells, practically nothing is known. Nerve-influences, no doubt, are here at work, as in the case of the salivary glands. At the same time the blood-supply is of moment, and we find that during the process of digestion the blood-vessels are dilated, and that the venous circulation is more rapid and the blood of a light-red color. But as in the salivary glands, it is certain that the height of the blood-pressure has only indirectly to do with the activity of the glands. The proenzymes here, as there, are formed 
through a specific activity on the part of the cells, from foodmaterial which is supplied by the lymph.

A solution of the pro-enzymes of the gastric mucosa that has been freed from albumins does not give the common reactions of the true albumins. From such solutions the pro-enzymes are not precipitated by dialysis, but it is interesting to note that on longcontinued dialysis they are rendered inactive; they are then apparently destroyed. To a slight extent they will pass through Chamberland filters, as well as those of Kitasato; the propepsin passes through somewhat more readily than the prochymosin.

Of interest also is the tendency of the proferments to adhere to solid substances, and it has been shown that they possess selective properties in this respect, which are not the same in both always. Lycopodium, for example, will carry down the pepsinogen, but not the chymosinogen. Charcoal, powdered marble, and calcium sulphate will carry down both.

Whether or not the transformation of the proenzymes into the corresponding ferments occurs in the bodies of the cells has not been definitely decided. It appears, however, that in the majority of animals which have been examined in this direction the glands secrete only the proenzymes, and that these are then rendered physiologically active by the hydrochloric acid of the gastric juice. A solution of propepsin, which may be obtained by macerating in glycerin the mucous membrane of a fasting animal, is thus in itself inert, but is rendered active at once when hydrochloric acid is added to the extent of from 0.1 to 0.2 per cent. It is indeed supposed that pepsin, which in itself is inactive like its zymogen, combines with hydrochloric acid, which alone is similarly inert, as regards its digestive ability, to form a compound acid, the so-called pepsinhydrochloric acid. On coming in contact with albuminous material this is supposedly decomposed, with the formation of nascent hydrochloric acid, which then acts as the active digestive principle, while the liberated pepsin combines with a new portion of hydrochloric acid, and thus serves as an acid-carrier. On this question, however, a uniformity of opinion does not exist; still, the hypothesis is an attractive one, and has a good deal in its favor. If we thus regard the action of a ferment as essentially influencing the rapidity of reaction, the action of the weak hydrochloric acid of the gastric juice could be compared to the effect of stronger solutions upon albumins under the application of heat.

Pepsin.-Pure pepsin occurs in the form of minute globules, which resemble the globulites of egg-albumin, but are somewhat smaller. Their diameter does not exceed 15-20 $\mu$. They are not doubly refracting. The substance is white when perfectly pure and is not hygroscopic. It is soluble in water, dilute acids, and glycerin. From its aqueous solutions it can be precipitated by half-saturation with ammonium sulphate, and it is also thrown down on dialysis.

When acidified with hydrochloric acid to the extent of from 0.1 
to 0.3 per cent., it is eapable of dissolving albumins, with the formation of albumoses and so-called peptones (see below). This can readily be demonstrated as follows: an artificial gastric juice is prepared by dissolving a pinch of one of the commercial preparations of pepsin in dilute hydrochloric acid (0.1-0.2 per cent.) to which a flake of boiled beef-fibrin is then added, The mixture is kept at a temperature of about $40^{\circ} \mathrm{C}$., when it will be noted that after a short time the fibrin begins to swell and is subsquently dissolved. In the solution which thus results albumoses and peptones can be demonstrated. Other acids, such as sulphuric acid, nitric acid, phosphoric acid, lactic acid, and even acetic acid, are also capable of rendering pepsin physiologically active, but much larger amounts are necessary to bring about the same result. In the case of phosphoric acid, for example, an acidity of 10-12 pro mille is necessary. Carbonic acid and hydrocyanic acid, on the other hand, are without effect.

Unlike chymosin, ordinary pepsin does not bring about the coagulation of casein, but Pekelharing has shown that in acid solution the pure substance also coagulates milk. It further causes the precipitation of so-called plastein (see below) in concentrated solutions of albumoses.

In neutral and alkaline solutions pepsin is inactive; it is rapidly destroyed by sodium carbonate, even in very small amount. Its resistance to higher temperatures is to a great extent dependent upon the reaction of its solutions. In neutral solution it is destroyed at $55^{\circ} \mathrm{C}$. ; in the presence of 0.2 per cent. of hydrochloric acid this result is reached only at $65^{\circ} \mathrm{C}$., and in the presence of peptones and certain salts a temperature of $70^{\circ} \mathrm{C}$. is necessary to bring about the same end. In the dry state, on the other hand, the ferment may be heated to $100^{\circ} \mathrm{C}$., and even higher, without being destroyed. At temperatures lower than $40^{\circ} \mathrm{C}$. pepsin is still active, but less energetically so, and at $0^{\circ} \mathrm{C}$. its action ceases altogether.

Alcohol precipitates pepsin from its solutions without effecting its subsequent activity, unless the exposure has been prolonged. Some of the salts of the heavy metals, such as the acetates of lead and platinum chloride, as also tannic acid, magnesium carbonate, and ammonium sulphate, likewise cause the precipitation of impure forms, at least, but are without effect upon the ferment itself. Uranyl acetate is an excellent reagent for the precipitation of ferments (and albumins), even with a neutral or feebly acid reaction. Like the albumins, pepsin does not diffuse through animal membranes, but is precipitated.

To a certain extent the rapidity of digestion is dependent upon the amount of pepsin that is available; but, as in the case of all ferments, very small quantities are sufficient to effect an amount of chemical change that is apparently out of all proportion to the amount present. Thus, Petit claims that a pepsin preparation, which he prepared himself, was capable of dissolving 500,000 times 
its weight of fibrin in seven hours. The much more impure commericial forms are, of course, far less active, but many of them possess remarkable digestive power.

The ability on the part of pepsin to digest albumins is, however, limited; and with an increase in the amount of digestive products formed, its activity gradually diminishes and finally ceases. This can be obviated in a measure by removing these products as they are formed, and may be artificially accomplished by allowing the digestion to take place in a parchment tube which has been suspended in dilute hydrochloric acid. The peptones which are formed then pass from the tube by dialysis, and in this manner digestion can be carried much further than under other conditions. Complete digestion, however, may even then not be achieved.

Regarding the chemical nature of pepsin, our knowledge has been greatly extended through the researches of Pekelharing, and Nencki and Sieber. From the fact that it is possible to prepare pepsin solutions which actively digest albumin, but do not show the common albumin reactions, it has been concluded that pure pepsin is probably not an albumin. The researches of the investigators just mentioned, however, seem to show conclusively that it is an albumin nevertheless, although it cannot be classed with any of the known forms. It contains no phosphorus, but nevertheless yields xanthin-bases on hydrolysis with alkalies. Both Friedenthal and Pekelharing have shown that a pentose can be obtained from it, and quite recently the latter has isolated a peculiar acid on hydrolysis with alkali which gives the biuret and xanthoproteic reaction, as also that of Adamkiewicz and Millon. This acid is termed pepsinic acid, and is derived from a coagulation-product of pepsin, which in turn is formed when acid solutions of the pepsin are rapidly heated over the free flame. Both the coagulationproduct as also the pepsinic acid, like all albumins, are lævorotatory. Elementary analysis of the pure pepsin, obtained from dogs that had been operated on by Pawlow's method (œsophageal and gastric fistulæ), as also of the coagulation-product and the acid, gave the following average results:

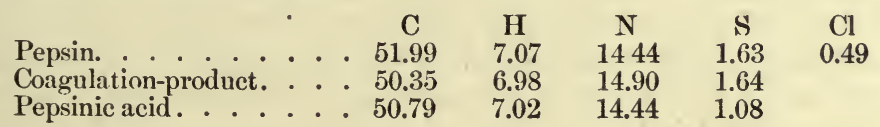

From the mode of origin of pepsinic acid it is, of course, clear that the substance can contain no loosely combined sulphur. Noteworthy is the fact that the pepsin contains chlorine, and the evidence appears to be conclusive that the chlorine is an actual constituent of the pepsin molecule.

Formalin when added to a solution of pure pepsin to the extent of 2 to 3 per cent. produces no appreciable effect upon the digestive power even after days.

While pepsin is probably constantly found in the gastric juice of 
adult vertebrate animals, it is noteworthy that it is absent from the gastric juice of sucking pups, as shown by Gmelin ; that its presence, moreover, is not essential to the maintenance of life is shown by the history of Czerny's dog and Hoffmann's observations in the case of a woman whose stomach had been removed in toto.

Specific tests for the demonstration of the pepsin of the gastric juice, as compared with other proteolytic ferments which similarly act in acid solutions, are unknown. As a result, all such ferments have been designated as pepsin, although it is very likely that they are not identical. Such ferments have been observed in the secretion of the glands of Brunner, in the muscles, the kidneys, the brain, the saliva, and the urine.

Isolation of Pepsin.-If it is merely desired to obtain an effective solution of pepsin without regard to the purity of the substance, the following procedure-may be employed:

v. WitTich's MeтноD.-The mucous membrane of a pig's stomach is carefully dissected off, freed from mucus by washing with water, hashed, rubbed together with pure quartz sand, and finally treated with glycerin, containing 0.1 per cent. of hydrochloric acid, in the proportion of 10-20 grammes for 1 part of mucous membrane. The mixture is kept at a temperature of $40^{\circ} \mathrm{C}$. for from one to two weeks, when it is filtered. The extract which is thus obtained may then be used for experimental purposes by diluting with $0.1-0.4$ per cent. of hydrochloric acid, in the proportion of $2-3: 100$. For the preparation of a pure pepsin the following method should be employed :

Pekelharing's Method.-Gastric juice uncontaminated by food-material or saliva is obtained from dogs that have been operated according to Pawlow's method (stomach and œesophageal fistulæ). The gastric juice obtained in portions after pseudofeeding, is filtered and dialyzed for about twenty hours against distilled water, at a temperature not much above $0^{\circ} \mathrm{C}$. A portion of the pepsin is thus precipitated and isolated by centrifugation. It is collected on a filter, washed with a little distilled water, and dried in the desiccator. The liquid portion of the original material, after centrifugation is half-saturated with ammonium sulphate (35 grammes for 100 c.c.). The resulting precipitate is freed from salt by dialysis, dissolved in 0.2 per cent. hydrochloric acid, reprecipitated by dialysis, collected on a filter, and dried.

Quantitative Estimation of Pepsin.-Accurate methods for the quantitative estimation of pepsin are not available. Relative values, however, can be obtained by the following method, as suggested by Mett :

Capillary glass tubes are prepared measuring from 1 to $2 \mathrm{~mm}$. in diameter. They are filled with white of egg, which is coagulated in the tubes at a temperature of $95^{\circ} \mathrm{C}$. The tubes are then cut into pieces from 1 to $2 \mathrm{~cm}$. long and placed in the digestive mixture to be examined. The length of the column digested in a given length 
of time serves as a measure of the digestive power of the specimen examined. The calculation of the corresponding amount of ferment is based upon the law of Schïtz and Borissow, viz., that corresponding amounts of ferment in two solutions bear the same ratio to each other as the square of the number of millimetres of the column of egg-albumin which has been dissolved in the same length of time.

Example.-The gastric juice of a normal individual is procured at the height of digestion after giving Ewald's test breakfast. The tube is digested for thirty minutes; at the end of this time the height of the column of albumin digested measures $3 \mathrm{~mm}$. Then the stomach contents of a second individual are obtained (the patient's) and similarly treated; in this case the column of digested albumin measures $2 \mathrm{~mm}$. The corresponding amounts of ferment are then as 9 is to 4 .

Pepsinogen.-The presence of pepsinogen in the gastric juice can be ascertained only when hydrochloric acid is absent, as it is otherwise transformed into the active enzyme. Its occurrence, as such, is hence essentially a pathologic phenomenon, and indicates the absence of free hydrochloric acid. But while the latter may be alosent in many diseases which are not associated with structural abnormalities of the gastric mucous membrane, pepsinogen, and consequently also pepsin, are found lacking only in disease of the stomach itself, and when complete atrophy of the glandular struct-. ures has occurred.

Test for Pepsinogen.-Specimens of gastric juice in which pepsinogen only is present are incapable of digesting albumins. In such cases, as I have just said, hydrochloric acid is absent. If then the solution is acidified to the extent of from 0.1 to 0.3 per cent., and the dissolution of a flake of boiled beef-fibrin now occurs, the presence of the zymogen may be inferred.

Quantitative Estimation of Pepsinogen.-The determination of the absolute quantity of pepsinogen in the gastric juice, as that of pepsin, is not possible. Relative values, however, may be obtained by the following method, as suggested by Boas : To this end, specimens of the gastric juice are variously diluted with distilled water in the proportion of $1: 5,1: 10,1: 20$, etc. A known quantity of coagulated egg-albumin or serum-albumin is then added to each tube, as also 1 or 2 drops of a 0.3 per cent. solution of hydrochloric acid, for every 10 c.c. The tubes are kept at a temperature of about $40^{\circ} \mathrm{C}$., when the degree of dilution is noted at which the albumin is still dissolved. The greater the degree of dilution at which this occurs, of course the greater is the amount of pepsinogen-that is, of pepsin-present.

Chymosin.-Chymosin, or rennin, according to the researches of Glässner, is formed only in the glands of the fundus, and, as in the case of pepsin, is secreted in the form of a pro-enzyme, which, like the pepsinogen, is then transformed into the corresponding ferment by the hydrochloric acid of the gastric juice. It is to be 
noted, however, that caleium chloride or any other soluble ealcium salt is likewise capable of bringing about this result. The specific action of chymosin is exerted upon milk or lime-containing solutions of caseïn, which are coagulated in neutral and even feebly alkaline solutions. Unlike pepsin, chymosin is not a proteolytic ferment, and its action ceases with the formation of paracaseïn. It is therefore surprising to note that ehymosin is found not only in the stomachs of mammals, but also in other vertebrate animals, and even in certain plants, where caseïn as a food-stuff certainly does not enter into consideration. Our knowledge of ferments in general, however, is as yet very defective, and, as a matter of fact, we are acquainted only with the more manifest reactions of these bodies, while it is quite possible that they possess other important properties of which we are now in ignorance. It is conceivable, moreover, that different varieties of chymosin exist, which, as a class, are all capable of coagulating casein, but which differ from each other in other respects and serve other purposes.

The view of Pawlow and Parastschuk that pepsin and chymosin are in reality one ferment and that coagulation and proteolysis are merely different manifestations of the action of one ferment, has not been generally accepted.

The question has further been raised whether pepsin and chymosin are the same in different animals or whether different varieties of the ferments occur in different groups. While such differences may exist, our knowledge of ferments generally is too defective to warrant any definite statement either way. Bang has isolated a parachymosin from calves' stomachs which is said to differ materially from that of man and the pig.

While chymosin is also active in feebly acid solution, it is gradually destroyed at a temperature of from $37^{\circ}$ to $40^{\circ} \mathrm{C}$. when exposed to the action of gastric juice containing 0.3 per cent. of hydrochloric acid. The ferment is here apparently digested by the pepsin, and it is thus easily possible to obtain solutions of pepsin which are altogether free from chymosin. In neutral solution it is more resistant, and can be heated to a temperature of $50^{\circ} \mathrm{C}$; ; at $70^{\circ} \mathrm{C}$., however, it becomes permanently inactive. In its dry state, on the other hand, it can be heated to $110^{\circ} \mathrm{C}$. without losing its activity. Alkalies when present beyond traces destroy the substance, as they do pepsin. Like all other ferments, it is eapable of effecting an extensive reaction, even when present in small amount. The quantity of ferment contained in 1 gramme of the dried and pulverized mucous membrane of the fourth stomach of the calf, when dissolved in water, is thus capable of coagulating 200 liters of milk in one minute at a temperature of $50^{\circ} \mathrm{C}$.

Of the chemical nature of chymosin, nothing is known; but, as in the ease of pepsin, the purer preparations do not give the usual reactions of albumins. It is precipitated from its neutral solutions by subacetate of lead, as also by uranyl acetate, while the acttate of 
lead and tannic acid are without effect. Alcohol likewise precipitates the ferment and gradually renders it inactive. Like pepsin, it is not dialyzable.

Under normal conditions chymosin is always present in the gastric juice of man. In certain diseases of the stomach, however, which are associated with the death of its glandular elements, the ferment, as also its zymogen, is lacking.

Tests for Chymosin and Chymosinogen.-To test for the presence of chymosin, 5 or 10 c.c. of milk are treated with a few drops of the filtered gastric juice and kept at a temperature of from $37^{\circ}$ to $40^{\circ} \mathrm{C}$. If coagulation occurs within ten or fifteen minutes, the presence of chymosin may be assumed. Should the gastric juice, however, be narkedly acid, it is necessary first to neutralize it with barium carbonate.

To test for chymosinogen, the milk is treated with 2-3 c.c. of a 1 per cent. solution of calcium chloride, and 10 c.c. of filtered gastric juice which has been rendered feebly alkaline with sodium hydrate. The mixture is kept at a temperature of from $37^{\circ}$ to $40^{\circ}$ C., when in the presence of the zymogen a thick cake of caseïn is formed within a few minutes.

Isolation of Chymosin. - To isolate chymosin in comparatively pure form, the following method, as suggested by Hammarsten, may be employed: The mucous membrane of the fourth stomach of the calf is carefully dissected off, washed with water, and extracted with an 0.1 per cent. solution of hydrochloric acid, as already described. The infusion is then neutralized and repeatedly shaken with powdered magnesium carbonate until the pepsin has been removed. The filtrate is treated with subacetate of lead, the precipitate decomposed with very dilute sulphuric acid, and the acid filtrate further precipitated with an aqueous solution of stearin soap. The ferment is thus thrown down together with the fatty acids, from which it is then separated by suspending the precipitate in water and extracting the fatty acids with ether. The chymosin remains in aqueous solution, and may now be precipitsted with strong alcohol. It is then rapidly collected on a filter and dried.

Quantitative Estimation of Chymosin and Chymosinogen.-As in the case of pepsin and pepsinogen, relative values only can be obtained. The gastric juice is nentralized with a very dilute solntion of sodium hydrate. Tubes are then prepared, containing 5 or 10 c.c. of the gastric juice, variously diluted in the proportion of $1: 10,1: 20,1: 30$, etc., to which an equal volume of neutral or amphoteric milk is further added. These tubes are kept at a temperature of from $37^{\circ}$ to $40^{\circ} \mathrm{C}$, when the degree of dilution is noted at which coagulation still occurs. Under normal conditions a positive reaction can thus be obtained in man with a degree of dilution varying between $1: 30$ and $1: 40$.

In the case of the zymogen, the gastric juice is rendered feebly alkaline, when tubes are prepared as just described. Normally a 
positive reaction can thus still be obtained with a dilution varying between $1: 100$ and $1: 150$.

Other Constituents of the Gastric Juice.-Of other constituents, the gastric juice normally contains traces of sulphocyanides, which are secreted by the stomach itself; a variable amount of mucin ; a small amount of coagulable albumin, or, if the fluid has stood for some time, a eorresponding quantity of albumoses or peptones, and, as already shown, certain mineral salts.

The gases which are found in the stomach have in part been swallowed with the food. A small portion is further referable to eructations from the duodenum, while a third portion is probably secreted by the stomach itself. This is true more especially of the carbon dioxide, and Schierbeck has shown that the tension of this gas gradually increases from 30 to $40 \mathrm{Hgmm}$. while fasting, to 130 to $140 \mathrm{Hgmm}$. during the process of digestion, and is apparently directly proportionate to the acidity of the gastric juice.

An idea of the relative amounts of the gases which are normally found in the stomach may be formed from the accompanying table, which is taken from Planer:

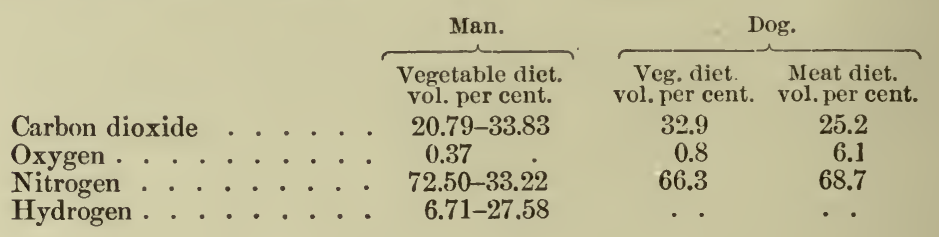

Other gases, such as marsh gas, olefiant gas, ammonia, and hydrogen sulphide, are found only under pathologic conditions, and are referable to certain fermentative and putrefactive changes which take place in the ingested food.

\section{THE PANCREATIC JUICE.}

As has been pointed out, the digestive glands which have so far been considered are not essential to the maintenance of life. The salivary glands and the stomach can in certain animals be eliminated without seriously interfering with the process of digestion, and the ferments which in man are secreted by these structures are in many animals absent. The pancreas, on the other hand, either as such or as a so-called hepatopanereas, is found in all vertebrate and invertebrate animals in which the process of digestion is carried on in a well-defined digestive tube. In many, indeed, it represents the only digestive gland of the body. Its removal, even in the higher animals, invariably leads to death. In dogs, in which this operation has been repeatedly performed, and in which life may go on for a few weeks thereafter, it has been 
observed that as a result of such interference the resorption of fats is seriously impeded, so that practically all that has been ingested reappears in the feces. In the case of the albumins, it is similarly found that but 44 per cent. is absorbed, and of the ingested starches from 20 to 40 per cent. is eliminated as such. Analogous results are obtained in the human being where atrophy of the pancreas is at times observed. As a consequence, rapid emaciation occurs, and, as has been stated, death ultimately results. It appears, however, that the fatal issue in these cases is not exclusively referable to impaired nutrition as a result of defective absorption. It is, indeed, possible to counteract this effect by administering a sufficient amount of raw pancreas together with the food, whereby the resorption of both fats and albumins is greatly improved. Death, however, takes place nevertheless. It is thus apparent that besides its digestive function the pancreas must play an additional and important rôle in the metabolism of the animal body. We find, as a matter of fact, that following the extirpation of the pancreas in dogs a severe form of diabetes rapidly develops, and is accompanied by the appearance of acetone, diacetic acid, and at times of $\beta$-oxybutyric acid in the urine. That this is not due to suspension of the pancreatic digestion can be proved in various ways. If the animal thus receives an adequate amount of raw pancreas together with its food, the absorption of albumins and fats is, as just stated, greatly increased, while the diabetes persists. It has been further noted that ligation of the secretory duct does not lead to the appearance of sugar in the urine, and that the diabetes continues after extirpation even when no food is consumed for several days. The conclusion hence suggests itself that the pancreas, like the thyroid, the adrenal body, and other glands, probably furnishes an internal secretion also, which in some manner controls the metabolism of glucose within the animal body. Arthaud and Butte, it is true, claim that diabetes does not follow ligation of the pancreatic veins; but it can readily be imagined that in such cases, and perhaps even under normal conditions, the internal secretion of the gland is removed through the lymph-channels. It has been shown, moreover, that diabetes does not occur after extirpation of the pancreas if a piece of the gland has been previously transplanted under the skin.

Of the nature of the substance which is thus secreted by the pancreas, and in the presence of which the carbohydrate metabolism continues in a normal manner, our knowledge has been greatly extended through the researches of Cohnheim and Hirsch, who could demonstrate that the pancreas furnishes a substance, possibly of the nature of a kinase, which renders possible the glucolysis in both muscle-tissue and the liver. In its absence this does not occur and diabetes is the necessary consequence. This substance, however, is probably not furnished by the pancreatic cells proper, but by the cells of the islands of Langerhans. At this place we shall deal only with the pancreatic secretion proper. 
The secretion of the pancreatic digestive fluid, like that of the saliva, is partly under the control of cerebrospinal nerve-fibres, which are derived from the vagus, and partly of sympathetic fibres. It is noteworthy, however, that even after section of the vagus and the splanchnic nerves pancreatic secretion begins when the acid contents of the stomach reach the duodenum. Bayliss and Starling have shown that this result is due to the activation, by the acid gastric juice, of a hormone present in the mucosa of the duodenum and jejunum which they term prosecretin, with the consequent production of free secretin. This latter is absorbed into the blood, is carried to the pancreas, and then stimulates the secretion of pancreatic juice. How the secretion is subsequently maintained, viz., by continued action of secretin or by nerve influences, is not known.

The material from which the secretion is elaborated through the specific activity of the glandular cells is obtained from the lymph, and ultimately, of course, from the blood. In carnivorous animals, in which the secretion of the pancreas is intermittent and dependent upon the ingestion of food, we accordingly find that in its stage of activity the gland assumes a bright rose color, and is much increased in size, while in the resting stage it is pale and shrunken.

General Properties.-Fresh pancreatic juice is best obtained by tying a cannula directly into the duct or by preparing a permanent fistula according to Pawlow's method, viz., by resecting a small piece of the duodenum (in a dog), where the pancreatic duct opens, suturing the ends into the abdominal wound and making an anastomosis between the free ends of the gut. The pancreatic juice which is then obtained represents a clear, colorless, odorless fluid of a strongly alkaline reaction; it is thick and glairy in some animals and thin and limpid in others. It shows no proteolytic activity whatever, but can be rendered physiologically active by the addition of intestinal juice or an extract of the intestinal mucosa. The activating factor is a peculiar body which has been discovered in the intestinal juice by Pawlow and his pupils, and which is termed enterokinase (which see).

When kept for a few hours at ordinary temperatures it loses its viscosity and transparency, and rapidly undergoes putrefaction. Crystals are then deposited which consist of leucin and tyrosin; they result from the digestion and subsequent decomposition of contained albumins. To prevent these changes the secretion must be placed on ice at once or treated with chloroform-water, toluol, or a similar antiseptic. Owing to the presence of the large amounts of albumin which the pancreatic juice contains, the liquid coagulates to a dense mass when heated to a temperature of $74^{\circ} \mathrm{C}$. On cooling to $0^{\circ} \mathrm{C}$., or when dropped into water, a clot is formed, which redissolves on warming the solution or on adding an excess of sodium chloride.

Amount.-The amount of pancreatic juice which is secreted in the twenty-four hours is variable, and dependent upon the quantity and quality of the food ingested. In man it varies between 700 and 
900 c.c. (Glaessner-Pfaff), while in the cow 1500 to 2000 c.c. are secreted in the twenty-four hours.

Specific Gravity.-The specific gravity of the pancreatic juice varies between 1.008 and 1.010, corresponding in man to the presence of from 2.5 to 7 per cent. of solids.

Chemical Composition.-A general idea of the chemical composition of the pancreatic juice of the dog may be formed from the accompanying analysis, which is taken from C. Schmidt. I have further added two analyses of human pancreatic juice which were made by Herter and Zawadsky. In Herter's case an accumulation of the secretion had taken place in the duct, owing to a pressurestenosis, which was produced by a carcinomatous growth; while Zawadsky's material was obtained from a pancreatic fistula which had remained after removal of a pancreatic tumor.

Of the two secretions, the first is manifestly abnormal (see below), while the analytical results in the second case conform closely to the figures which were obtained by Schmidt in what may be regarded as the normal pancreatic juice of the dog.

I have also appended an analysis of the contents of a pancreatic cyst, which in its general composition resembles the secretion obtained from permanent fistulæ in animals. Trypsin, however, was absent. Noteworthy is the low content of solids.

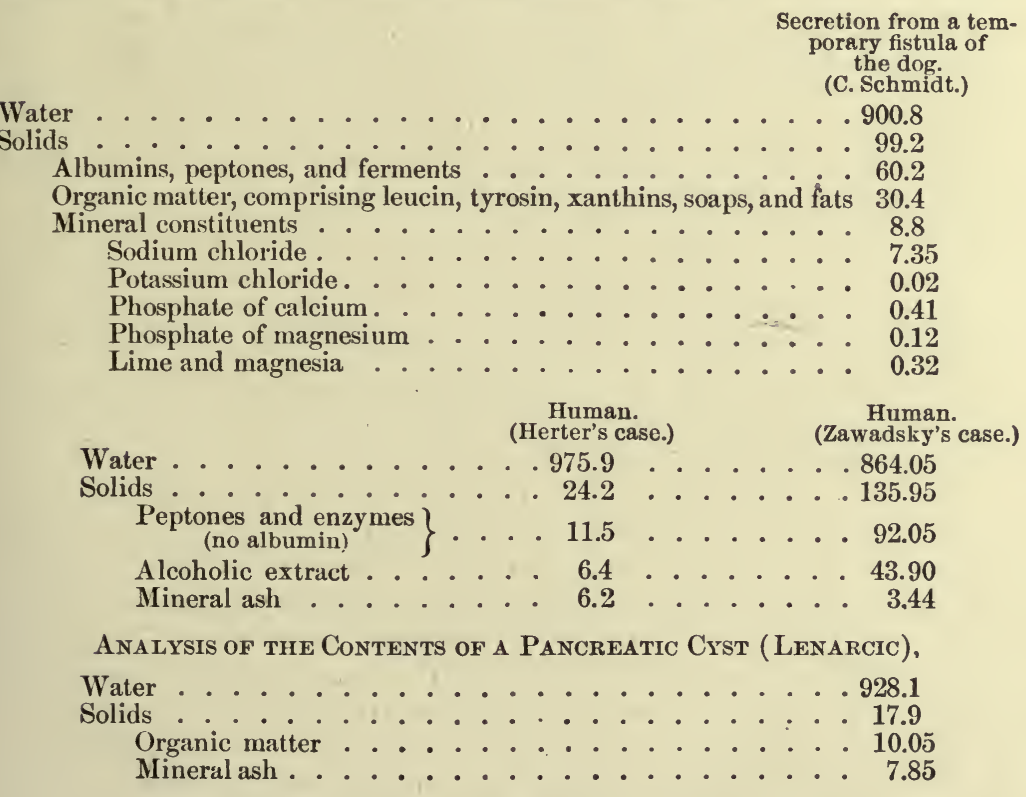

\section{The Ferments and their Zymogens.}

The pancreatic juice of all mammals contains three ferments, viz., the proteolytic trypsin, the amylolytic amylopsin, or pancreatic 
ptyalin, and the lipolytic steapsin or pancreatic lipase. Chymosin is further present in some animals, but absent in others.

Like the ferments which are furnished by the salivary glands and the central cells of the gastric glands, the enzymes of the pancreas occur in the pancreatic cells also in the form of zymogens, which are subsequently transformed into the active ferments. If a fresh pancreas is thus extracted with glycerin, it will be noted that the resulting extract has no proteolytic properties whatever, while an extract obtained after the gland has been hashed and exposed to the air for some time, or an aqueous extract of the fresh gland, digests albumins with ease. If the fresh gland, further, is hashed and briefly treated with a 1 per cent. solution of acetic acid, and then extracted with glycerin, an active preparation is obtained at once. This transformation in the case of trypsin is effected by the enterokinase of the duodenal mucosa, while steapsinogen is activated by the bile. The ptyalin also may exist in the gland cells as a zymogen, but in the pancreatic juice it apparently only appears as the free enzyme. When and how its activation takes place is not known.

The only case in which normal pancreatic juice has been obtained from the human being is reported by Glässner. The total amount of twenty-four hours varied between 700 and 900 c.c. ; the largest secretion took place during digestion, and increased steadily until the fourth or fifth hour, when a gradual decrease occurred. The amount secreted while fasting represented from one-third to a half of the total amount. The quantity was very materially influenced by the ingestion of hydrochloric acid, increasing to twice the amount. The secretion was absolutely devoid of any proteolytic properties, but manifestly contained the proferment of trypsin, as it could be readily activated by intestinal juice. Glüssner ascertained that in the fasting condition the trypsinogen secretion is practically zero, but that it slowly increases from the time when food is ingested, to reach its maximum in the fourth or fifth hour, after which it again decreases to the eighth hour. Parallel with the amount of trypsinogen runs the alkalinity curve.

The fresh pancreatic juice has marked lipolytic properties, which are increased by both the bile and the enteric juice, such that with a mixture of the three secretions the activity is from four to five times as great as in the case of the pancreatic juice alone. Like the proteolytic power, so also does the lipolytic power of the pancreatic juice reach its maximum in the fourth hour. In addition the pancreatic juice has marked diastatic properties, but it is incapable of further decomposing the resulting maltose, which is effected by the enteric juice. Neither a lactase nor an invertin is found in human pancreatic juice.

In chronic fistulæ conditions are different, and the question whether or not the digestive ferments will appear in the secretion depends here upon the character of the stimulus given, viz., upon the character of the food. With an exclusive meat diet trypsin 
appears as such, and only as such. With one of milk and bread the zymogen only appears, and must first be activated by the enteric juice. The amylolytic ferment, on the other hand, is not influenced in this manner, and is always secreted as ferment. With lipase conditions resemble those in the case of trypsin. With a diet of carbohydrates and much fat the zymogen appears, while with a meat diet the ferment is furnished directly (Lintwarew).

Of the chemical composition of the zymogens we know little, but it appears that they are of an albuminous nature and of a more complex composition than the ferments themselves. On decomposition trypsinogen is said to yield the corresponding ferment and an unknown albuminous substance. Of the origin of the zymogens, and of the manner in which they are produced in the cells, we know nothing.

Trypsin.-Trypsin is the most important proteolytic ferment which is found in the animal world, and in its action on albumins is fully capable of replacing pepsin when this is absent. Its digestive power, moreover, is much more extensive than that of pepsin, as it is capable of decomposing albumins to amino-acids. Its hydrolytic effect may thus be compared to the action of strong mineral acids under the application of heat. It is to be noted, however, that albuminous material which has been first subjected to the action of pepsin-hydrochloric acid and then to trypsin is more rapidly and more completely hydrolyzed than when acted upon by trypsin alone. The extensive digestive activity of trypsin is well shown when the gland is finely hashed and treated with a large amount of chloroform-water, so as to guard against putrefactive changes. When kept at a temperature of $40^{\circ} \mathrm{C}$. autodigestion rapidly takes place, and after several days while trypsin is still present in its full activity, the other ferments have disappeared. Together with the various albumins of the gland they have apparently been digested by the more powerful ferment.

While trypsin acts most energetically in feebly alkaline or neutral solutions (0.25-1.0 per cent. of sodium carbonate), it is also capable of digesting albumins in slightly acid media, providing that the acidity is not due to the presence of a free mineral acid; the digestive process is under such conditions, however, much less active. Free mineral acids rapidly destroy the ferment. Its optimum temperature lies between $37^{\circ}$ and $40^{\circ} \mathrm{C}$. In neutral solution it is destroyed at $45^{\circ} \mathrm{C}$, , while in feebly alkaline media, and especially in the presence of albumoses and certain ammoniacal salts, it can be heated somewhat higher without impairment of its digestive power.

One of the most important characteristics of trypsin is its inability to transform firmly combined nitrogen into the loosely combined form. On long-continued tryptic digestion of serum-albumin it will be noted that the amount of nitrogen which can be obtained on distillation with magnesia is essentially the same as the amount that can be obtained on hydrolysis with acids.

As in the case of pepsin, the digestive effect of trypsin is to a certain extent dependent upon the amount of the ferment present, 
and here as there the action of the enzyme ultimately ceases when the digestive products accumulate beyond a certain amount. Impure extracts can be prepared, as has been pointed out, by extracting the gland with glycerin after it has been left exposed to the air. The purer forms, on the other hand, which can be obtained according to Kühne's method (see below), are insoluble in glycerin and alcohol, but soluble in water.

Of the chemical nature of trypsin little is known. In acid solution it is coagulated by heat, and, according to Kühne, decomposed into an albumin and peptone. An analysis of a fairly pure preparation has given the following results : carbon, 52.75 per cent.; hydrogen, 7.51 ; nitrogen, 16.55 ; oxygen plus sulphur, 23.19 (Loew). Its composition is thus very similar to that of the peptones, and it is hence possible that, unlike the other digestive ferments which we have thus far considered, trypsin may be an albuminous substance.

Test for Trypsin.-The test for trypsin resolves itself into the demonstration of the presence of a proteolytic ferment which is capable of digesting albumins in alkaline solntion, with the ultimate formation of amino-acids. To this end (if necessary) the solution in question is rendered alkaline with sodium carbonate to the extent of from 0.25 to 1.0 per cent. A small flake of fibrin is then added and an amount of thymol sufficient to saturate the solution, so as to guard against putrefactive processes. The mixture is kept at a temperature of $40^{\circ} \mathrm{C}$. If the fibrin is then dissolved and leucin or tyrosin can be demonstrated in the resulting solution, the presence of trypsin may be inferred. To this end, it is only necessary to evaporate the solution to a thick syrup and to examine this microscopically (see tyrosin). Should the solution to be tested contain a free mineral acid, or larger amounts of organic acids, no result will be obtained, as the trypsin has in that case been destroyed.

As Abderhalden has shown, trypsin in contradistinction to pepsin is capable of splitting peptids containing an amino-acid which is soluble with difficulty (tyrosin, cystin), such as glycyl-l-tyrosin.

Isolation of Trypsin.- Unless it is desired to obtain trypsin in as pure a form as possible, alkaline-solutions of the common pancreatin preparations which are sold in the shops can be used for experimental purposes. Otherwise the method of Kühne, as modified by Gautier, should be employed: The fresh pancreas is finely hashed and washed with ice-cold water, to which 1 pro mille of salicylic acid has been added. After four hours the mass is treated with a large amount of a 5 pro mille solution of sodium carbonate, containing an excess of thymol, and is kept for twelve hours at a temperature of from $37^{\circ}$ to $40^{\circ} \mathrm{C}$. The acid and alkaline extracts are then mixed, treated with 0.5 per cent. of sodium carbonate, filtered, feebly acidulated with acetic acid, and saturated with ammonium sulphate in substance. The precipitate which then separates out contains all the trypsin. It is dissolved in water, filtered, and dialyzed, so as to remove the salt which is still present, as also traces of peptones and leucin. The resulting solution is 
concentrated at a very low temperature, and the trypsin finally precipitated with strong alcohol. It is then rapidly filtered off and dried. The amount of material which can thus be obtained from one gland is always very small, but sufficient to digest a large quantity of albumin when dissolved in about 100 c.c. of water.

The so-called dry pancreas of Kühne, from which trypsin can likewise be obtained, and which is also used in digestive experiments as such, is prepared by extracting the fresh gland with alcohol and subsequently with ether until it is free from fats. The remaining material, which contains the active ferment, is then dried and pulverized, and can be kept in this form indefinitely.

The Amylolytic Ferment of the Pancreatic Juice (Amylopsin or Pancreatic Ptyalin).-The amylolytic ferment of the pancreatic juice is by many thought to be identical with the ptyalin of the saliva. It can be isolated, according to Gautier's method, from an aqueous infusion of the fresh gland that has remained exposed to the air for about twenty-four hours, as already described. To demonstrate its action, a few cubic centimeters of such an infusion, or a glycerin extract of the gland, are added to a small amount of starch paste and kept at a temperature of $40^{\circ} \mathrm{C}$. The mixture is then tested for the presence of dextrins and maltose, as already described.

Steapsin (Pancreatic Lipase).-It has long been known that the pancreatic juice possesses the power of emulsifying fats, and of decomposing these into glycerin and the corresponding fatty acids. This phenomenon was once referred to the action of bacteria, but is now known to be referable to a specific lipolytic ferment, steapsin. Of the nature of the ferment nothing is known, but it is manifestly very unstable, as extracts prepared from glands that have been left exposed to the air for twenty-four hours are perfectly inert when brought in contact with neutral fats. Apparently it is digested during this time by the trypsin. To demonstrate the action of steapsin on fats, a small amount of perfectly fresh pancreas is finely hashed and delydrated with 90 per cent. alcohol. It is then dried between filter-paper and placed in an ethereal solution of ethyl butyrate. On digestion at the temperature of the body the odor of butyric acid will soon become manifest. Or, a small amount of neutral fat is treated with a few cubic centimeters of a feebly alkaline glycerin extract of the fresh gland (9 parts of glycerin and 1 part of a 1 per cent. solution for each gramme of the gland), to which a few drops of tincture of litmus are added. If this mixture is then kept at a temperature of $37^{\circ} \mathrm{C}$, it will be noted that the alkalinity gradually decreases, and the reaction finally becomes acid, owing to the liberation of free fatty acids (Hammarsten).

The emulsifying action of the pancreatic juice is owing to the decomposition of the neutral fats, the subsequent saponification of the resulting acids by the alkaline salts which are at the same time present, and the effect of the soaps thus formed upon the neutral fats. 
Maltase.-The presence of maltase in the pancreatic juice has been inferred from the fact that small amounts of glucose are invariably formed during the action of the aqueous or glycerin extract of the gland upon starch. Glässner, however, notes that in his case of pancreatic fistula an inversion of maltose to glucose could not be observed (see above).

Chymosin.-Chymosin can be demonstrated by adding a small amount of the pancreatic juice of the ox, pig, or sheep to milk, when coagulation results, as in the case of the chymosin of the gastric juice. The ferment, however, is not present in all mammals ; in dogs and in the human being (Glässner) it is absent.

\section{THE SECRETION OF THE GLANDS OF BRUNNER.}

In some animals, such as the rabbit, a secretion analogous to that of the pancreas is furnished also by the glands of Brunner, which are found in the upper portion of the duodenum. But in other animals, such as the dog and the pig, the function of these glands is comparable to that of the pyloric glands of the stomach. The reaction of the secretion in the dog is alkaline; the specific gravity 1.005-1.020. The amount secreted does not materially exceed 1 c.c. per hour, and is not influenced by the state of digestion or the character of the food. Ponamorew found that in the dog the fluid only digested albumin, after being acidified. This observation agrees with the results obtained by Grützner and others. Glässner, however, states that both in the $\operatorname{dog}$ and the pig the proteolytic ferment will digest albumins not only in the presence of acids $(0.2-$ 0.3 per cent. hydrochloric acid) but also with a neutral and feebly alkaline reaction ( 0.5 per cent. sodium carbonate). Pawlow regards the ferment as identical with that of the pyloric glands, viz., as pepsin.

A diastatic ferment does not occur in the secretion of Brunner's glands, but a milk-coagulating ferment is apparently present.

\section{THE ENTERIC JUICE.}

The enteric juice is essentially the secretory product of the glands of Lieberkühn, which are found imbedded in the mucous membrane of the small intestine, and to some extent also in the large intestine. It may be procured by resecting a loop of the intestine, measuring about $0.15-0.20$ meter in length, and closing the proximal end, while the distal end is sutured to the abdominal wall. The mesentery, however, is allowed to remain, so that the nerve- and bloodsupply of the portion which has thus been isolated is interfered with as little as possible. Instead of forming a blind pouch, as was first done by Thiry, both ends of the resected loop may also be sutured to the anterior abdominal wall. Such fistulæ were first established by Vella, and they accordingly bear his name. The free ends of 
the divided gut are in either case united with each other and the abdominal wound closed.

In animals which have thus been operated it is noted that after five hours following the ingestion of food a copious secretion of fluid takes place in the small intestine, which continues for about six hours. During this period of activity the mucous membrane presents a rose-red color, while it is pale when at rest. As in the case of the pancreas, the secretion is intermittent. It can be manifestly excited in a reflex manner, as a moderate secretion may be observed within an hour after the ingestion of food-that is, at a time when but little chyme has passed into the small intestine. Later, when the food has passed the stomach, its presence alone or that of the digestive products which have been formed already, apparently excites the increased secretion which is then observed. This may be further increased artificially by mechanical and especially by electrical stimulation, and it is indeed possible to cause the secretion of enteric juice in this manner even at a time when digestion is not going on.

In the upper portion of the duodenum of the dog the secretion is said to be small in amount, mucoid, and jelly-like, while further on it becomes more fluid.

The enteric juice always contains a not inconsiderable amount of mucus, which is derived from the goblet-cells that are found along the entire length of the intestinal canal. The small amorphous flakes which are always found in the secretion consist entirely of mucus. The juice itself, which can be separated from the greater portion of the nucus by filtration, is in the lower portion of the small intestine a thin light yellowish fluid, of a strongly alkaline reaction. This is largely due to the presence of considerable amounts of sodium carbonate, and we accordingly find that on the addition of an acid effervescence occurs. Sodium chloride also is present in notable quantities. The specific gravity in the dog is fairly constant, and corresponds to about 1.010-1.011.

The amount of solids is largely dependent upon the character and the quantity of the food ingested, and in the dog may vary between 12.2 and 24.1 pro mille. These variations are mainly referable to the presence of albumins, which are always found in the enteric juice, while the inorganic constituents are fairly constant. A general idea of the chemical composition of the secretion may be formed from the accompanying analyses:

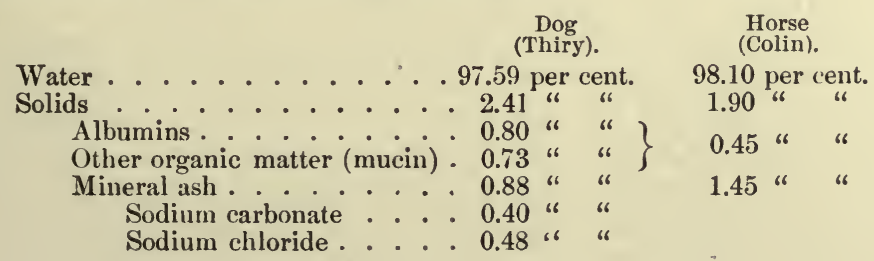


Enteric juice contains small amounts of an albumin which is coagulated only with difficulty and which has commonly been regarded as a mucin. It appears, however, that the substance is in reality a nucleo-albumin (Kutscher), and that pure enteric juice contains no mucin.

Of the amount of enteric juice which is secreted under normal conditions in twenty-four hours we know but little. In the intestinal juice of a patient with an intestinal fistula Hamburger and Hekma found a daily secretion of $50-125$ c.c., with an average of 88 c.c. The amount was the largest from the fourth to the seventh hour following the principal meal of the day. Mechanical stimulation more than donbled the flow. In disease, and notably in Asiatic cholera, exceedingly large quantities may be observed; but in such cases we are no doubt dealing with a direct transudation from the blood, and not with an actual secretory product of the cells. On section of the corresponding nerves hypersecretion can be artificially brought about. This may be compared to the paralytic saliva which is obtained from the sublingual gland on section of the chorda and of the sympathetic fibres that supply the gland.

Of ferments, the enteric juice contains invertin, ptyalin, maltase, lipase, and in sucklings lactase. While practically all the digestive ferments are thus represented, their functional value is here probably insignificant when compared with the presence of three other substances which are of fundamental importance. The one is the enterokinase to which I have already referred on several occasions, which activates the trypsin of the pancreatric juice. The other is the so-called erepsin of Cohnheim, which is characterized by the fact that it is incapable of splitting albumins to albumoses, but canses the further cleavage of the latter to amino-acids. In addition, the mucosa of the duodenum and jejunum contains a substance which, when treated with hydrochloric acid and injected into the circulation, causes a secretion of pancreatic juice. The inactive principle is termed prosecretin and its active form secretin.

Enterokinase.-The enterokinase itself has no digestive power; it merely activates the trypsin of the pancreatic juice, viz., it transforms the inactive zymogen into the active enzyme. Very interesting is the fact that the secretion of the kinase can only be effected by the introduction into the intestinal lumen of normal pancreatic secretion. Boiled pancreatic juice, purely mechanical stimulation, as also the injection of pilocarpin, only give rise to the secretion of an enteric juice, which is free from kinase. The kinase is destroyed by a temperature of $67^{\circ} \mathrm{C}$. Hamburger and Hekma were able to demonstrate the presence of enterokinase in the enteric juice of man, and ascertained that the substance is not capable of activating indefinite quantities of pancreatic juice, but that a certain quantity of enteric juice can only activate a definite amount of pancreatic juice ; they conclude that enterokinase is not a ferment, and propose to term the substance zymolysin. 
The enterokinase has not yet been obtained as such ; it is precipitated from extracts of the intestinal mucosa in impure form by means of acirls. It closely adheres to any precipitated nucleoproteids.

Delezenne states that he has demonstrated the presence of enterokinase also in the leucocytes of the blood, and he explains the digestive action of supposedly inactive pancreatic juice upon fibrin without an extra addition of enterokinase on the basis of the presence of leucocytes carrying enterokinase, contained in the meshes of the fibrin or in the pancreatic juice. The same writer claims to have found enterokinase in bacteria, in various fungi (Amanita), and in snake-venom.

Erepsin.-(This is considered in the section on Resorption).

Secretin and Prosecretin.-According to Bayliss and Starling, it is possible to extract a substance from the mucosa of the duodenum and jejunum with dilute acids (4 per cent. hydrochloric acid), which when introduced into the circulation causes a secretion of pancreatic juice. Substances of this order Bayliss and Starling term hormones. The hormone in question is known as secretin, and is supposedly formed by the acid from an inactive product, the prosecretin, which exists as such in the cells. When once formed (activated) it retains its activity even upon neutralization and alkalinization. It is not destroyed by boiling in either acid, neutral or alkaline solution, and can hence not be a ferment.

Camus has ascertained that while secretin can be obtained with various organic and inorganic acids (not with boric acid or carbonic acid), a more active preparation is obtained with hydrochloric acid, nitric acid, and sulphuric acid than with other acids.

Aside from its action upon the pancreatic cells secretin is also said to increase the secretion of bile and saliva. Atropin and anxsthethics, notably chloroform, diminish its activity.

Popielski has recently found that secretin can also be obtained from the mucosa of the rectum, the ileum, the stomach, and that it can even be isolated from the arterial blood. It appears, moreover, from his researches that the body has not only a specific action so far as the pancreatic cells are concerned, but that on intravenous or hypodermic injection it stimulates nearly all digestive glands to activity. These results have not yet been confirmed.

\section{THE BILE.}

Formerly it was supposed that the bile played an important part in the process of digestion, and was further capable of controlling the intensity of the putrefactive and fermentative processes which even normally take place in the lower intestinal tract. It has now been established, however, that, aside from its emulsifying action upon fats, the secretion possesses no digestive properties whatever, and is likewise without effect upon the bacteria 
which are normally found in the intestinal canal. We accordingly find that in animals and in man the processes of nutrition are in no way interfered with if the bile is prevented from entering the digestive tube, but is carried to the outside through the establishment of a fistulous opening in the common duct, provided that food is administered which contains but little fat. With a diet consisting of albumins and carbohydrates digestion thus continues unimpaired, and the animal is capable of maintaining its nitrogenous equilibrium practically as before. If fats, however, are given at the same time in large amounts, more or less serious digestive disturbances soon develop and the animal loses weight. In such cases it has been ascertained that whereas normally from 2 to 10 per cent. of the ingested fat is eliminated in the feces, from 31 to 47 per cent. now escapes resorption. The offensive gases which are then passed by the animal are referable to an increase of the putrefactive processes in the intestines. This is, however, not owing to the absence of bile per se, but to the fact that the unabsorbed fats envelop the albuminous material, and thus prevent its further digestion, so that in the lower portion of the digestive canal, where the putrefactive processes are most intense, the bacteria find an increased amount of pabulum at their disposal, and an increase of the putrefactive products accordingly results. In the presence of bile, on the other hand, this does not occur, as its emulsifying effect upon the fats hastens their digestion, and thus leaves the albumins exposed to the action of the digestive juices, and to their resorption in turn. Indirectly, it can thus control putrefaction, but such action is not due to any germicidal or antiseptic properties of its own. On withdrawing fats from the food and giving an adequate supply of carbohydrates, normal relations are soon re-established, though the bile is absent as before.

The bile in reality represents a most important excretory product of the animal body, and may in this sense be compared to the urine.

Secretion.-As found in the gall-bladder, the bile represents the secretory product of the liver-cells which is eliminated into the radicles of the biliary passages, together with so-called mucus which is derived from the epithelial lining of the greater trunks and the gall-bladder itself. Its secretion is continuous, but liable to exacerbations which are essentially dependent upon the ingestion of food. According to Heidenhain, the curve of secretion, in reference to amount, shows two periods of greatest activity, which in the dog correspond to the third to the fifth and the thirteenth to the fifteenth hour, respectively, after the administration of food. This curve, however, is further influenced by the character of the food ingested. With an albuminous diet three stages of maximal secretion are thus noted: The first after two to three hours, a second stage after five to eight hours, and a third stage after twelve to fourteen hours. With a diet of albumins and fats, on the other hand, the period of greatest secretion occurs after eleven to twelve hours, 
and with one of albumin and carbohydrates after from nine to fourteen hours. These figures have reference to observations which were made after the administration of only one meal in the twentyfour hours. With two meals analogous results are obtained. The individual periods, however, are shorter; and as the number of feedings is increased the secretion becomes more uniform, so that with a meal every two hours no variations of moment can be discerned.

Amount.-The amount of bile which is eliminated in the twentyfour hours is variable even under normal conditions. In dogs from 2.9 to 36.4 grammes can be obtained pro kilogramme of weight of the animal. In man the secretion apparently varies between 400 and 800 grammes; but it is possible that these figures do not represent normal values. Ranke has estimated that a man weighing 75 kilogrammes secretes about 1050 grammes even in health. To a certain extent the amount is influenced by the character of the food, and it appears to be quite generally accepted that a diet rich in albumins will call forth a greater secretion of bile, but it is noteworthy that this increased secretion does not occur at once, but only on the second or even the third day. The carbohydrates are thought to diminish its amount, or are at least incapable of increasing this, like the albumins. The fats are probably without effect in either direction.

It was formerly thought that a number of drugs could increase the flow of the bile, and physicians were wont to administer cholagogues when they supposed that the secretion of bile was deficient. This view has now been abandoned, as it has been definitely established that drugs are without effect in this direction. The only cholagogue, indeed, if it may so be termed, is the bile itself. This is readily understood, if we bear in mind that the bile is essentially an excretory product.

General Properties.-The color of the bile differs in different animals, and may vary from a bright yellow to an intense grassgreen, with various shades of brown and blue. In man it is usually of a golden-yellow color, but it may at times appear bright green even when perfectly fresh.

While pure bile, uncontaminated with mucus, is a thin transparent fluid, that which is eliminated into the intestinal tract and is found in the gall-bladder is markedly viscid and more or less turbid. Its taste is intensely bitter, with a sweetish and nauseating after-taste. The odor is in many animals peculiar, and at times suggestive of musk. The reaction is normally alkaline. After exposure to the air, however, it soon becomes acid, but becomes alkaline again owing to the development of trimethylamin and ammonia. The specific gravity varies between 1.010 and 1.033 . In the gall-bladder, where a constant resorption of water is going on, and where considerable amounts of mucus are added to the bile, it is higher $(1.026-1.033)$ than in the bile proper, which is secreted by the 
liver (1.010-1.012). We accordingly find that the amount of solids is greater in the bladder-bile than in that which may be termed the hepatic bile.

Chemical Composition.-The following analyses show the general chemical composition of the bile in different animals, and also illustrate the differences which exist between bladder-bile and hepatic bile :

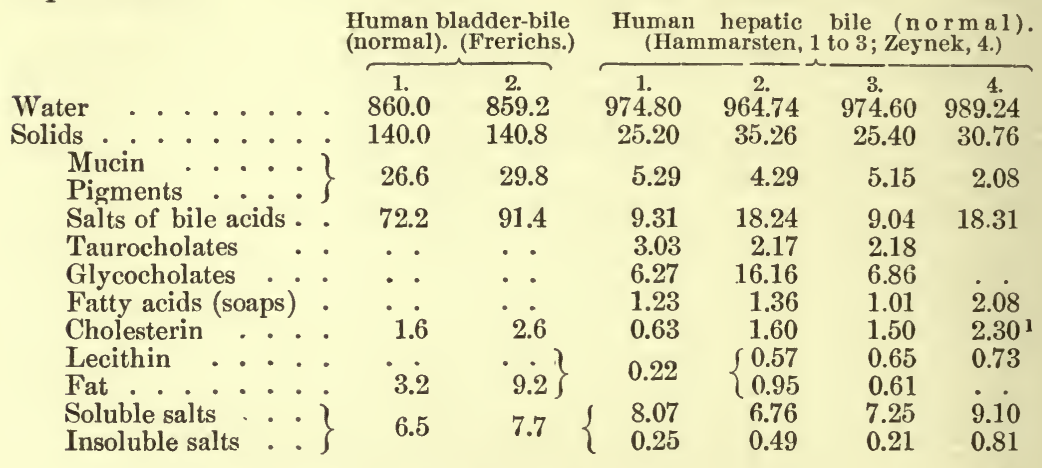

Analyses of the Bladder-bile of Animals.

\begin{tabular}{|c|c|c|c|c|c|}
\hline & $\begin{array}{l}\text { Dog. } \\
\text { (Hoppe-Seyler.) }\end{array}$ & $\begin{array}{l}\text { Pig. } \\
\text { (Grundelach } \\
\text { Strecker.) }\end{array}$ & (Berzelius.) & $\begin{array}{c}\text { Birds. } \\
\text { (MIarsson.) }\end{array}$ & $\begin{array}{l}\text { Shad. } \\
\text { (Schloss- } \\
\text { berger.) }\end{array}$ \\
\hline Water & 813.56 & 888.0 & 904.4 & 800.2 & 944.8 \\
\hline Solids . . . . . . & 186.44 & 112.0 & 95.6 & 199.8 & 55.2 \\
\hline $\begin{array}{l}\text { Sodium glycocholate } \\
\text { Sodium taurocholate }\end{array}$ & $12 \dot{2} .8\}$ & 83.8 & \} & 170.6 & 36.3 \\
\hline $\begin{array}{l}\text { Cholesterin . : } \\
\text { Fats . }\end{array}$ & $\left.\begin{array}{r}2.91 \\
15.11\end{array}\right]$ & & 80.0 & & \\
\hline Soaps . . . & 16.03 & 22.3 & & 3.6 & $2.3^{3}-x-3-1$ \\
\hline $\begin{array}{l}\text { Lecithin . . } \\
\text { Mucin }\end{array}$ & $\begin{array}{r}18.11 \\
3.49\end{array}$ & $5.9)$ & & 25.6 & 14.8 \\
\hline $\begin{array}{l}\text { Other organic solids; } \\
\text { insoluble in alcohol }\end{array}$ & 6.0 & & 3.0 & $\cdot \cdot$ & \\
\hline Inorganic solids . . & . & . & 12.6 & 21.0 & - \\
\hline
\end{tabular}

Analyses of the inorganic salts have given the following results, which are taken from Jacobsen and Hoppe-Seyler, respectively. The figures have reference to 100 parts by weight of mineral ash:

Sodium chloride

Potassium chloride . . . . . .

Sodium carbonate . . . . . . . .

Trisodium phosphate .......

Tricalcium phosphate ......

Calcium carbonate . . . . . . .

Potassium sulphate . . . . . .

Sodium sulphate . . . . . .

Iron, Silica ....................

Magnesium, copper $\therefore \therefore: \ldots$

\begin{tabular}{|c|c|}
\hline $\begin{array}{c}\operatorname{Man}_{\text {(hepatic bile). }} \\
65.16\end{array}$ & $\begin{array}{c}\text { Ox } \\
\text { (bladder-bile). } \\
7.50^{3}\end{array}$ \\
\hline 3.39 & \\
\hline 11.16 & 2.50 \\
\hline 15.90 & \\
\hline 4.44 & 40.0 \\
\hline & 9.50 \\
\hline variable & 2.0 \\
\hline . & 25.0 \\
\hline traces & traces \\
\hline
\end{tabular}

1 Jncluding fat.

2 This figure is too low, owing to the fact that Hoppe-Seyler's analysis has reference to the inorganic salts, which were not dissolved by alcohol, 


\section{The Mucinous Body of the Bile.}

The mucinous body which is found in the bladder-bile of all animals is apparently of a different nature in different animals. The mucus of the human bile largely consists of true mucin, while in ox-bile a mucinous nucleo-albumin is principally found. To isolate this latter, the bile is precipitated with 5 times its volume of absolute alcohol and immediately centrifugalized. The supernatant liquid is poured off, and the sediment rapidly dried with filter-paper and dissolved in water. By repeating this process the substance can be obtained in a fairly pure form. Its character as a nucleo-albumin becomes apparent on treating its neutral solutions with a small amount of hydrochloric acid. A flocculent precipitate then develops, which readily dissolves upon the further addition of hydrochloric acid to the extent of 0.3 per cent. This solution remains clear for a long time even when kept at the temperature of the body. On digestion with pepsin, however, a separation of pseudonuclein occurs. On fusing the dried substance with potassium hydrate and sodium nitrate, an amount of phosphoric acid is obtained which is greatly in excess of the amount required to saturate all of the mineral ash that is present when calculated as tricalcium phosphate. On boiling with a dilute mineral acid no reducing-substance is formed, as in the case of true mucin. Acetic acid precipitates the substance from its solutions, in the absence of biliary acids ; but this precipitate, unlike that of mucin, is soluble in an excess of the acid.

The mucin proper which is found in human bile may be isolated, as already described (see Saliva).

\section{The Biliary Acids.}

The biliary acids which are normally found only in the bile are essentially compound amino-acids, which are formed through the union of glycocoll on the one hand, and taurin on the other, with a cholalic acid. In the bile of sharks Hammarsten discovered the existence of a third group of biliary acids, which are rich in sulphur, and, like the conjugate sulphates of the urine, yield sulphuric acid (scymnol sulphates) on boiling with hydrochloric acid; at the same time a substance is obtained which manifestly belongs to the group of cholalic acids. Traces of these acids are said to occur also in human bile. Of their chemical nature, however, nothing is known.

Under pathologic conditions, when the free outflow of bile is impeded, owing to a swelling of the mucous membrane of the common duct or to the presence of a calculus, resorption of the bile takes place through the lymph-channels, and the secretion thus finds its way into the blood. Jaundice then results, and in such cases not only the bile-pigments but also the bile-acids can be demonstrated in the general circulation. To the presence of the latter is due the slow pulse which is so constantly observed in 
icterus. At the same time the bile-acids bring about a dissolution of the red corpuscles, and thus manifest their true nature as excretory products.

Regarding the origin of the biliary acids, we know only that they are formed exclusively in the liver, but of the manner in which their formation takes place we are ignorant.

The amido-radicles of the bile-acids are unquestionably formed in the general nitrogenous metabolism of the body, and, as such, are found in other organs besides the liver. Glycocoll is then to a certain extent further oxidized, and contributes to the formation of urea. Of the ultimate fate of taurin, we know that its sulphur can be oxidized to sulphuric acid and be eliminated in the urine in this form. In the human being traces are further excreted as taurocarbaminic acid; and in rabbits, at least, its hypodermic injection leads to the elimination of the body as such. It is thus difficult to understand why two substances like these, for the removal of which the animal body has manifestly other means at its disposal than their elimination through the bile, should here appear. We may imagine, however, that the formation of the bile-acids in the liver represents a conservation of energy on the part of the body, and further constitutes a reserve mechanism by which waste-products can be removed in a state of incomplete oxidation.

Of the origin of the non-nitrogenous acids, which combine with glycocoll and taurin to form the biliary acids, we know nothing. The synthesis, however, manifestly occurs in the liver, and here only, as after extirpation of the organ in birds and frogs bile-acids are not found in the blood. In mammals, also, neither bilepigments nor bile-acids can here be demonstrated after ligating the biliary duct and the thoracic duct.

In the bile of most animals the biliary acids occur in combination with sodium, while in sea-fish they are, curiously enough, present as potassium salts.

As regards the relative quantity of the two principal biliary acids which are found in the bile, it is to be noted that in man glycocholic acid is usually more abundant than taurocholic acid. In strictly carnivorous animals, on the other hand, the latter only is found; but the same also holds good for certain herbivora, such as the sheep and the goat. In other animals the relation is variable, and in some, it is stated, glycocholic acid only is found.

Isolation.-Collectively, the biliary acids, in the form of their salts, can be obtained in the following manner: the bile is mixed with animal charcoal, evaporated to dryness, and the residue extracted with absolute alcohol. This extract, which contains the biliary acid salts, cholesterin, fats, soaps, and lecithin, is then filtered, concentrated, and treated with a large excess of ether. In this manner the salts of the bile-acids are precipitated, while the other substances remain in solution. On standing, the precipitate gradually becomes crystalline, and is then spoken of as Platner's 
crystallized bile. In this form the biliary acids are conveniently estimated as a whole. If then it is desired to determine the relative amount of the two principal acids present, it is only necessary to estimate the sulphur in Platner's bile, from which the corresponding amount of taurocholic acid can be calculated.

To separate the two acids from each other, Platner's bile is dissolved in water and completely precipitated with a solution of neutral acetate of lead. The corresponding lead salts are thus formed, and can now be readily separated from each other, as the glycocholate is thrown down, while the taurocholate remains in solution. In the filtrate the latter is then precipitated with ammonia. To obtain the free acid from the glycocholate, the precipitated lead salt is suspended in water and evaporated to dryness in the presence of sodium carbonate. The sodium salt is thus obtained and extracted with alcohol. The alcohol is evaporated off, the residue dissolved in water and treated with hydrochloric acid, when the glycocholic acid will separate out. The taurocholic acid can be similarly obtained from its lead salt by decomposing the solution with hydrogen sulphide. The resulting plumbic sulphide is filtered off, the filtrate evaporated to dryness, the residue dissolved with a small amount of alcohol, and then precipitated with an excess of ether, when the free acid is thrown down.

Tests for the Bile-acids.-Pettenkofer's Test.-On treating the biliary acids in aqueous or alcoholic solution with a few drops of an 0.1 per cent. solution of furfurol and 1 or 2 c.c. of concentrated, chemically pure sulphuric acid, a beautiful purple color develops. As furfurol results when concentrated sulphuric acid is added to a carbohydrate, the test may also be conducted by treating the solution of the biliary acids ( 1 or 2 c.c.) with a few drops of a 10 per cent. solution of cane-sugar and then with sulphuric acid. In either case, however, care should be had that the temperature during the reaction does not exceed $70^{\circ} \mathrm{C}$., as otherwise the resulting pigment is destroyed. This may be obviated by substituting strong phosphoric acid for the sulphuric acid, and placing the solution in boiling water. The resulting pigment shows two bands of absorption on spectroscopic examination. One of these is situated at $\mathrm{F}$; the other between $\mathrm{D}$ and $\mathrm{E}$, near $\mathrm{E}$. On diluting with alcohol a green fluorescence is observed. In the presence of an excess the red pigment disappears, but reappears upon the further addition of sulphuric acid. On standing, the color gradually turns to a bluish violet.

As Pettenkofer's reaction can also be obtained with other substances, such as the phenols, the higher alcohols, certain bases of the aromatic series, the higher hydrocarbons, and acids of the fatty series and the benzol series, it is always necessary to isolate previously the biliary acids, as Platner's bile, before drawing conclusions from the reaction. Albumins, if present, must in every case first be removed. For, as has been stated, many of these contain carbohydrate groups, which on contact with concentrated sulphuric acid 
give rise to the formation of furfurol. This in turn combines with the aromatic oxy-acids and phenols that result from decomposition of the amino-acids to form colored compounds, which are either identical with or very similar to those obtained in the case of the biliary acids (see the reaction of Adamkiewicz and the hydrochloric acid test for albumins).

Pettenkofer's reaction is, in the case of the biliary acids, referable to the non-nitrogenous acid constituents of these acids, viz., to cholalic acid or one of its congeners.

Physiological Test.-Aside from their reaction with furfurol, the bile-acids, or their salts, may also be identified from their effect upon the action of the heart. To this end, the heart of a curarized frog is exposed and moistened with a small drop of a 1 per cent. solution of atropin, so as to eliminate the action of the vagus. A few drops of an aqueous solution of the bile-acids are then placed upon the heart, when their retarding action can readily be demonstrated.

Glycocholic Acid.-As has been stated, glycocholic acid is the principal biliary acid that is found in the bile of man. It is formed through the union of glycocoll and cholalic acid, as represented by the equation :

$$
\underset{\text { Glycocoll. }}{\mathrm{C}_{2} \mathrm{H}_{5} \mathrm{NO}_{22}}+\underset{\substack{\text { Cholalic } \\ \text { acid. }}}{\mathrm{C}_{24} \mathrm{H}_{40} \mathrm{O}_{5}}=\underset{\substack{\text { Glycocholic } \\ \text { acid. }}}{\mathrm{C}_{26} \mathrm{H}_{43} \mathrm{NO}_{6}}+\mathrm{H}_{2} \mathrm{O}
$$

It is accordingly decomposed into these constitutents when treated with dilute acids or alkalies under the application of heat.

In the pure state glycocholic acid occurs in the form of fine, silklike needles, which are readily soluble in alcohol, less readily so in water, and insoluble in ether. From its aqueous solutions it is readily precipitated on adding a small arnount of a mineral acid. The crystals melt at $133^{\circ} \mathrm{C}$. It is a monobasic acid, and forms salts which, with the exception of the lead and silver compounds, are all soluble in water and alcohol. The free acid and its salts give Pettenkofer's reaction, and turn the plane of polarization to the right.

On heating glycocholic acid with concentrated sulphuric acid the anhydride of glycocholic acid is precipitated in the form of oily droplets, which subsequently tend to coalesce. This anhydride is termed cholonic acid, and has the composition $\mathrm{C}_{26} \mathrm{H}_{41} \mathrm{NO}_{5}$.

According to Michailoff, glycocholic acid when treated with concentrated sulphuric acid in the presence of acetic acid is said to give an orange color with a green fluorescence. On salting with ammonium sulphate a precipitate is formed which in its reactions is identical with biliverdin. Urobilin is said to remain in solution. This observation is of special interest, as showing the possible relationship which may exist between the biliary acids and the bilepigments. We find, as a matter of fact, that an increase in the production of bile-pigments on the part of the liver is associated with a diminished formation of biliary acids. 
Closely related to the common glycocholic acid is the so-called hyoglycocholic acid, which has been found in small amounts in the bile of the pig. On decomposition it yields glycocoll and hyocholalic acid, as shown in the equation :

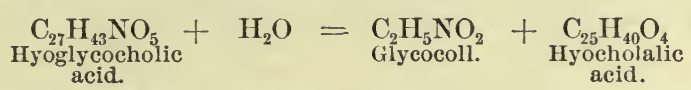

The substance itself is almost insoluble in water, but soluble in alcohol. It is crystallizable, but usually obtained as a resinous mass. Its salts are precipitated from their solutions by calcium chloride, barium chloride, and magnesium chloride. By salting with sodium sulphate they separate out like soaps. Like the common glycocholates, they give Pettenkofer's reaction.

In addition to these two forms still other glycocholates apparently exist. In the bile of rodents a glycocholate is thus found, which cannot be salted out with sodium sulphate, but which is also precipitated by the salts of the alkaline earths. Of its nature, however, as also of the so-called guano-biliary acid, which apparently belongs to this order, nothing is known.

Isolation.-The common glycocholic acid is most conveniently obtained by starting with Platner's bile, that has been prepared from human bice or from that of the ox, as already described.

Or, the following method may be employed (Bleibtreu): Starting with ox-bile the pigments are precipitated with uranium acetate; the bile acids remain in solution. In the filtrate glycocholic acid is precipitated with chloride of iron; the resulting iron compound is decomposed by heating with ammonia, the hydroxide of iron is filtered off, the filtrate acidified with hydrochloric acid and shaken with ether; the glycocholic acid separates out in crystalline form. Or, to purify the substance further, the ammoniacal solution is neutralized with acetic acid, the glycocholic acid precipitated with uranium nitrate, and the precipitate decomposed by heating with sodium phosphate solution. The resulting solution of the sodium salt is acidified with hydrochloric acid and shaken with ether, when the glycocholic acid separates out.

Hyoglycocholic acid can be isolated from the bile of the pig by first decolorizing with animal charcoal and then salting with sodium sulphate in substance. The acid is thus precipitated, and can then be filtered off. It is washed with a solution of the salt, dissolved in water, and precipitated in the form of the free acid by means of hydrochloric acid.

Taurocholic Acid.-Taurocholic acid, as has been stated, is the only biliary acid that is found in the bile of the purely carnivorous animals, but it also occurs in the bile of man and most herbivorous animals. Among these, the sheep and goat are especially noteworthy, as in these, like the pure carnivora, taurocholic acid only is found. It is formed synthetically in the liver from taurin and cholalic acid, and is accordingly resolved into its components 
by treating with dilute acids or alkalies under the application of heat. The same result indeed is reached by evaporating the bile together with water or allowing it to undergo putrefaction. The chemical change is represented by the equation:

$$
\underset{\substack{\text { Taurocholic } \\ \text { acid. }}}{\mathrm{C}_{26} \mathrm{H}_{45} \mathrm{NSO}_{7}}+\mathrm{H}_{2} \mathrm{O}=\underset{\text { Taurin. }}{\mathrm{C}_{2} \mathrm{H}_{7} \mathrm{NSO}_{3}}+\underset{\substack{\text { Cholalic } \\ \text { acid. }}}{\mathrm{C}_{24} \mathrm{H}_{40} \mathrm{O}_{5}}
$$

Tauber succeeded in effecting the synthesis of taurin and cholalic acid by using sodium cholate, while with the free acid directly no result was obtained. Pellizari and Matteucci arrived at similar results by starting with sodium-taurin instead of free taurin.

In the pure state taurocholic acid occurs in the form of fine deliquescent needles, which are soluble in water and alcohol, but insoluble in ether. It is capable of maintaining glycocholic acid in solution, and it is for this reason that the latter acid cannot be precipitated from the bile by adding a mineral acid when taurocholic acid is at the same time present in sufficient amount. Like glycocholic acid, it is a monobasic acid, and forms salts which for the most part are soluble in water and in alcohol. Its salts with the alkalies are precipitated from their solutions by subacetate of lead and ammoniacal subacetate of lead, but not by the neutral acetate, by copper sulphate, or silver nitrate. Curiously enough, the sodium salt obtained from the bile of the ox is dextrorotatory, while the same salt which is found in that of the dog turns the plane of polarization to the left. An isomerism thus apparently exists which is analogous to that observed in the case of the tartaric acids.

Its aqueous solution precipitates albumins in large white flakes. With albumose solution a milky precipitate is obtained which is largely soluble in water (Maly and Emich).

Taurocholic acid forms emulsions with the peptones, but does not precipitate them, as is generally stated ; but it does precipitate albumins, syntonins, and albumoses.

Hyotaurocholic acid is the biliary acid which, as a sodium salt, is found in the bile of pigs, and is analogous to the hyoglycocholic acid already described. Its amount; however, is small. On decomposition it yields taurin and hyocholalic acid, as shown in the equation :

$$
\underset{\substack{\text { Hyotaurocholic } \\ \text { acid. }}}{\mathrm{C}_{26} \mathrm{H}_{45} \mathrm{NSO}_{6}}+\mathrm{H}_{2} \mathrm{O}=\underset{\substack{\text { Hyocholalic } \\ \text { acid. }}}{\mathrm{C}_{25} \mathrm{H}_{40} \mathrm{O}_{4}}+\underset{\text { Taurin. }}{\mathrm{C}_{2} \mathrm{H}_{7} \mathrm{NSO}_{3}}
$$

Chenotaurocholic acid is the most important biliary acid, which is found in the bile of geese. It is indistinctly crystalline, and is soluble in water and alcohol. Like the acids already described, it gives Pettenkofer's reaction. On prolonged boiling with alkalies it is decomposed into taurin and chenocholalic acid, as shown in the equation :

$$
\underset{\substack{\text { Chenotaurocholic } \\
\text { acid. }}}{\mathrm{C}_{29} \mathrm{H}_{49} \mathrm{NSO}_{6}}+\mathrm{H}_{2} \mathrm{O}=\underset{\begin{array}{c}
\text { Chenochola- } \\
\text { lic acid. }
\end{array}}{\mathrm{C}_{27} \mathrm{H}_{44} \mathrm{O}_{4}}+\underset{\text { Taurin. }}{\mathrm{C}_{2} \mathrm{H}_{7} \mathrm{NSO}_{3}}
$$


Isolation.-Taurocholic acid is most conveniently obtained from Platner's bile of man or the ox, as already described. To isolate it from the bile of dogs, the fluid is shaken with animal charcoal and alcohol, then decanted and filtered. The filtrate is evaporated to dryness, the residue taken up with a small amount of warm absolute alcohol, and filtered. 'The clear solution is then treated with ether until it becomes cloudy. On standing, the taurocholate of sodium separates out in the form of fine crystals. These are dissolved in water and treated with ammoniacal subacetate of lead. The resulting precipitate is suspended in alcohol and decomposed with hydrogen sulphide. The free acid is thus liberated, and can be obtained as such by evaporating the filtrate to dryness or by diluting with ether.

To isolate chenotaurocholic acid, the bile of geese is first treated with strong alcohol, to remove the mucus. The filtrate is mixed with ether and set aside, when the biliary salts separate out as a glutinous mass, which is then washed, dried, redissolved in 99 per cent. alcohol, and again precipitated with ether. The crystalline deposit which is formed is dissolved in strong alcohol and decomposed with hydrogen sulphide. On evaporation the free acid is obtained.

Cholalic Acid.-Cholalic acid is the principal biliary acid which is formed in the liver, and to its presence in the molecule of glycocholic acid and taurocholic acid Pettenkofer's reaction is due. It is a product of the specific activity of the liver-cells, and is normally not found in any other tissues or organs of the animal body. In the intestinal contents, however, it may be encountered, as such, even in health, and in cases of jaundice it has been observed also in the urine. Its artificial introduction in dogs leads to an increased elimination of taurocholic acid. 'This is temporary, and ceases when the taurin, which has been stored in the liver, is exhausted.

As I have already shown, cholalic acid is liberated when glycocholic acid or taurocholic acid is decomposed with alkalies or acids, under the application of heat. Its crystalline form differs according to the medium in which crystallization has taken place. From its alcoholic solution it separates out in the form of rhombic tetrahedra or octahedra, which contain one molecule of alcohol as liquid of erystallization, $\mathrm{C}_{24} \mathrm{H}_{40} \mathrm{O}_{5}+\mathrm{C}_{2} \mathrm{H}_{6} \mathrm{O}$. On prolonged boiling with water the crystal-alcohol can be removed. When dissolved in dilute boiling acetic acid cholalic acid takes up one molecule of water, and can be obtained from such solutions in the form of rhombic plates or prisms, $\mathrm{C}_{24} \mathrm{H}_{40} \mathrm{O}_{5} \cdot \mathrm{H}_{2} \mathrm{O}$. On exposure to the air the crystals in either case soon become opaque. They melt at a temperature of $195^{\circ} \mathrm{C}$. The free acid is readily soluble in alcohol, with difficulty so in water, and is almost insoluble in ether.

According to Mylius, it is a monobasic alcoholic acid, and contains one secondary and two primary alcoholic groups, as represented by 
the formula: $\left.\mathrm{C}_{20} \mathrm{H}_{31}-\frac{\mathrm{CH} . \mathrm{OH}}{(\mathrm{COOH}} . \mathrm{OH}\right)_{2} . \quad$ Of its structure, however, and

of its origin nothing is known. Some investigators have suggested a chemical relation between it and cholesterin, but there are no definite data to support this view. It combines with alkalies and alkaline earths, as also with the heavy metals, to form salts. Its compounds with the alkalies are readily soluble in water, but with some difficulty in alcohol. The barium salt is somewhat soluble in water, and, like the lead salt, soluble in hot alcohol. The calcium salt is slightly soluble in boiling alcohol. Concentrated solutions of the alkaline hydrates and carbonates precipitate the alkaline salts from their solutions in the form of an oily material which becomes crystalline on cooling. The salts, like the free acids, are dextrorotatory.

On prolonged boiling with acids or at a temperature of $200^{\circ} \mathrm{C}$. cholalic acid loses two molecules of water and is transformed into dyslysin, as shown in the equation :

$$
\underset{\substack{\text { Cholalic } \\ \text { acid. }}}{\mathrm{C}_{24} \mathrm{H}_{40} \mathrm{O}_{5}}=\underset{\text { Dyslysin. }}{\mathrm{C}_{24} \mathrm{H}_{36} \mathrm{O}_{3}}+2 \mathrm{H}_{2} \mathrm{O}
$$

The same result is brought about through the influence of various bacteria, and there can be no doubt that the dyslysin which is constantly encountered in the stools is referable to the normal processes of putrefaction, which take place in the intestinal canal.

The common dyslysin which is met with in the feces is amorphous, and is insoluble in water and in dilute solutions of the alkalies.

Choloidinic acid, $\mathrm{C}_{24} \mathrm{H}_{38} \mathrm{O}_{4}$, probably represents an intermediary product which is formed during this process, and may be regarded as a primary anhydride of cholalic acid.

On oxidation with permanganate cholalic acid first yields dehydrocholalic acid, and then bilianic acid, together with isobilianic acid. These changes may be represented by the equations :

$$
\begin{aligned}
& \text { (1) } \begin{array}{c}
\mathrm{C}_{24} \mathrm{H}_{40} \mathrm{O}_{5} \\
\text { Cholalic } \\
\text { acid. }
\end{array} \quad 3 \mathrm{O}=\underset{\begin{array}{c}
\text { Dehydrocholalic } \\
\text { acid. }
\end{array}}{\mathrm{C}_{24} \mathrm{H}_{34} \mathrm{O}_{5}}+3 \mathrm{H}_{2} \mathrm{O} \\
& \text { (2) } \begin{array}{c}
\mathrm{C}_{24} \mathrm{H}_{34} \mathrm{O}_{5} \\
\text { Dehydrochol- } \\
\text { alic acid. }
\end{array}
\end{aligned}
$$

On further oxidation another acid has been obtained, which is termed cilianic acid, $\mathrm{C}_{20} \mathrm{H}_{28} \mathrm{O}_{8}$.

Dehydrocholalic aeid also results on oxidizing cholalic aeid with nitric acid; but it is to be noted that on further oxidation a new aeid results, which is known as choleocamphoric acid, and is thought to be isomeric with eamphoric acid. Its formula is given as $\mathrm{C}_{10} \mathrm{H}_{16} \mathrm{O}_{4}$. On oxidation with potassium bichromate and sulphuric acid, on the other hand, Tappeiner claims to have obtained cholesteric acid (not to be confounded with cholesterinic acid, sce below), $\mathrm{C}_{12} \mathrm{H}_{16} \mathrm{O}_{7}$; pyrocholesteric acid, $\mathrm{C}_{11} \mathrm{H}_{16} \mathrm{O}_{7}$; cholanic acid, $\mathrm{C}_{20} \mathrm{H}_{28} \mathrm{O}_{6}$; as also palmitic, stearic, and acetic acids. 
On reduction, as during the process of putrefaction, cholalic acid may give rise to the formation of desoxycholalic acid, $\mathrm{C}_{24} \mathrm{H}_{40} \mathrm{O}_{4}$. On boiling with concentrated solutions of the alkaline hydrates a mixture of the corresponding salts of formic acid, acetic acid, propionic acid, and palmitic acid is obtained, and it is interesting to note that the latter, like cholalic acid, gives Pettenkofer's reaction.

Hyocholalic acid and chenocholalic acid, which result from the decomposition of hyotaurocholic acid, hyoglycocholic acid, and chenotaurocholic acid, respectively, in their general properties and reactions are closely analogous to the common form of cholalic acid that has just been considered. Like the latter, they are transformed in the intestinal tract into the corresponding dyslysins, and these in turn yield the original acids on heating with sodium hydrate solution. Both are soluble in alcohol and ether, but insoluble in water.

Isolation of Cholalic Acid from the Bile.-Platner's bile, obtained from the ox, is boiled for twenty-four hours with barium hydrate. The cholalic acid which is thus set free is precipitated by adding an excess of hydrochloric acid. It is then washed with water, dissolved in a dilute solution of sodium carbonate, and reprecipitated with hydrochloric acid. The precipitate is covered with ether and allowed to stand, when crystallization will gradually occur. The crystals are freed from liquid as far as possible by filtration with the suction-pump, and are redissolved in hot alcohol. The solution is diluted with water until a persisting turbidity develops, and is then set aside in a cold place until crystallization is complete.

Choleic Acid.-When the biliary acids of ox-bile are decomposed, as just described, a small amount of choleic acid, $\mathrm{C}_{24} \mathrm{H}_{40} \mathrm{O}_{4}$, is always found associated with common cholalic acid. On oxidation it first yields dehydrocholeic acid, $\mathrm{C}_{24} \mathrm{H}_{34} \mathrm{O}_{4}$, and then cholanic acid, $\mathrm{C}_{24} \mathrm{H}_{34} \mathrm{O}_{8}$. It is possibly identical with desoxycholalic acid (see above). The substance has also been found in human bile.

Fellic Acid.-This acid has been obtained together with common cholalic acid and choleic acid from human bile, where it possibly exists in combination with glycocoll and taurin. Its amount is always small. With Pettenkofer's test it gives a reddish-blue color. On heating, it is decomposed, with the formation of vapors which are strongly suggestive of turpentine. Its formula is given as $\mathrm{C}_{23} \mathrm{H}_{40} \mathrm{O}_{4}$.

Lithofellic Acid.-Lithofellic acid is a substance which is closely related to the cholalic acids just described, and is found in certain gastro-intestinal concretions of various ruminants. It forms the greater portion of the oriental Bezaar stones, which are obtained from the stomach of the wild goat and antelope. It gives Pettenkofer's reaction, and is said to have the formula $\mathrm{C}_{20} \mathrm{H}_{36} \mathrm{O}_{4}$.

Ursocholeic acid, $\mathrm{C}_{19} \mathrm{H}_{30} \mathrm{O}_{4}$ or $\mathrm{C}_{18} \mathrm{H}_{28} \mathrm{O}_{4}$, has been found in the bile of ice-bears (Hammarsten), and is thus a homologue of the common choleic acid. 
Taurin.-As has been pointed out, taurin is not found exclusively in the bile, but occurs also in the lungs, kidneys, and in the muscletissue of many vertebrate animals. It is a derivative of cystin, and thus of albuminous origin. It is noteworthy, however, that an increased ingestion of albumins to eight times the usual amount increases the biliary sulphur (taurocholic acid) only twice. This finds its explanation in the observation of $\mathrm{v}$. Bergmann that cystin per se will not lead to an increased elimination of the bile sulphur, when fed directly, while this occurs when cholalic acid is simultaneously administered. We may accordingly conclude that under usual conditions a sufficiently large amount of cholalic acid is not available for the elimination of a larger amount of taurin than ordinary as taurocholic acid. There is evidence that in dogs there is a store of taurin (or of cystin) normally, which rapidly disappears when cholalic acid is administered, as evidenced by an increased output of the bile sulphur. Personal investigations in association with Dr. D. G. Campbell have led me to similar results in man.

Of the manner in which cystin gives rise to taurin our knowledge is not yet complete. Different possibilities exist. On the one hand, the cystin may be oxidized to cysteïnic acid, which apparently represents the sulpho-acid of cystein, and from which taurin could then result through loss of $\mathrm{CO}_{2}$ as expressed by the equations :
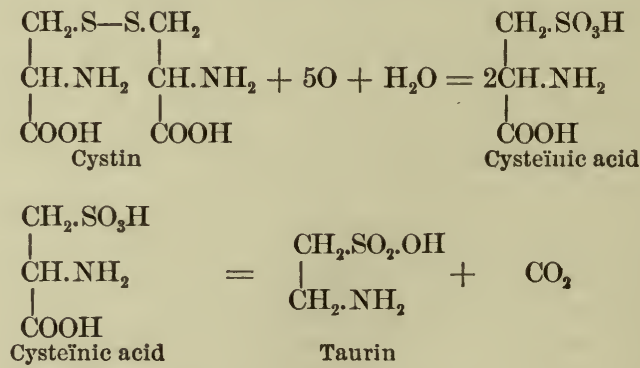

It is possible, on the other hand, that the organism forms taurin directly by oxidation of the thio- to the sulpho-group with the coincident loss of carbon dioxide.

Then again there is a possibility that cystin may unite with cholalic acid primarily, and that the resulting product is subsequently oxidized to taurocholic acid without the intermediate formation of taurin.

Unlike the loosely combined sulphur of cystin, the sulphur of taurin cannot be split off on boiling with dilute alkalies. It is present in oxidized form. Its separation necessitates the complete destruction of the taurin.

Wahlgreen has recently isolated the corresponding glycocholic acid from ox-bile, and it is possible that this is identical with Mulder's cholonic acid : 
Taurin can be formed synthetically by heating ammonium-oxyethyl-sulphonate to a temperature of $230^{\circ} \mathrm{C}$, , or from ammonia and chlor-ethyl-sulphonic acid, as represented by the equations :

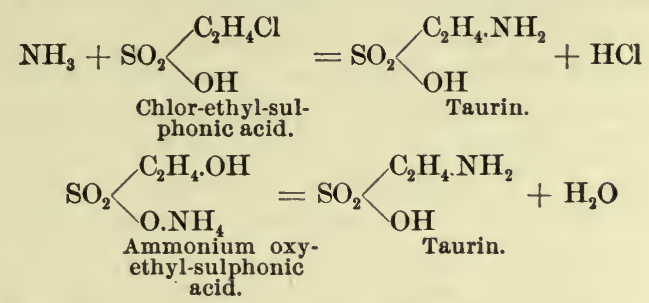

It can hence be regarded as amino-ethyl-sulphonic acid (viz., amino-isethionic acid). It crystallizes in the form of four- or sixsided prisms, and is fairly soluble in hot water, slightly soluble in common alcohol, and insoluble in absolute alcohol and ether. It has a markedly acid character, and accordingly does not combine with acids, but with alkalies, to form salts. Its compound with mercuric oxide is quite insoluble.

Isolation of Taurin.-Taurin is most conveniently prepared from the bile of animals in which taurocholic acid is present. To this end, the fluid is boiled for several hours with hydrochloric acid. The dyslysin and choloidinic acid are filtered off:- The filtrate is concentrated on a water-bath to a small volume, and freed from the sodium chloride and other substances that have separated out, by filtering while still warm. The liquid is then evaporated to dryness and the residue extracted with strong alcohol, which dissolves any glycocoll hydrochlorate that may be present, while the taurin remains behind. This is then dissolved with a little warm water, filtered while still warm, and treated with an excess of warm alcohol. The resulting precipitate is immediately filtered off. In the filtrate the taurin crystallizes out on cooling, and can be identified by the form of its crystals, their solubility in water and insolubility in alcohol, and the formation of potassium sulphate when fused with potassium hydrate and potassium nitrate.

Should glycocholic acid be present in the bile at the same time, this is likewise decomposed on boiling with hydrochloric acid. The hydrochlorate of glycocoll which thus results is found in the first alcoholic extract. To isolate the glycocoll as such, the solution is evaporated to dryness, the residue dissolved in water and treated with plumbic hydrate. On filtering, the solution, which contains the lead salt of glycocoll, is decomposed with hydrogen sulphide. The resulting lead sulphide is filtered off, and the filtrate concentrated until crystallization occurs. The crystals are then dissolved in water and decolorized with animal charcoal, and the solution is evaporated until the crystals again separate out.

Glycocoll._-As has been shown before, glycocoll is a decomposition-product of most albumins, but is obtained in especially large 
amounts from collagen, elastin, and especially silk fibroin. From its occurrence in collagen and its sweet taste it has been termed collagen-sugar (Leimzucker) or glycin (glucin). It is one of the most important decomposition-products which are formed in the nitrogenous metabolism of the animal body, and in part probably gives rise to the formation of urea in mammals and to uric acid in birds and reptiles. Another portion, as we have seen, is eliminated in the bile in combination with cholalic acid; while a third portion appears in the urine in combination with benzoic acid and phenylacetic acid, as hippuric acid and phenaceturic acid, respectively (which see).

As we have seen, glycocoll is amino-acetic acid. The pure substance crystallizes in the form of colorless rhombohedra or of foursided prisms, which are readily soluble in water, with difficulty so in warm alcohol, and insoluble in absolute alcohol and ether. It combines with acids and alkalies to form salts. The most important of these are the hydrochlorate, which is soluble in water and alcohol, and the copper salt, which results when a boiling solution of glycocoll is added to freshly precipitated cupric hydrate; the hydrate is thus dissolved, and after concentrating the solution blue needles of the copper salt separate out on cooling.

Isolation.-For purposes of study glycocoll is best obtained from collagen, on hydrolysis with dilute mineral acids, as will be described in a subsequent chapter.

\section{The Bile-pigments.}

The bile-pigments which have been obtained from the bile itself or from biliary concretions are bilirubin, biliverdin, biliprasin, bilifuscin, and others which are less well known.

Perfectly fresh hepatic bile, in contradistinction to that which is found in the gall-bladder, contains only one pigment, bilirubin, from which all other forms are derived. Such bile is of a golden-yellow color, while bladder-bile usually presents an olive-brown color, owing to the simultaneous presence of its nearest oxidation-product, biliverdin. A grass-green color is observed when the latter predominates or is exclusively present.

Bilirubin.-Bilirubin results from the decomposition of hæmatin, and normally constitutes a specific product of the activity of the hepatic cells. It appears, however, that the power of transforming the blood-pigment into bilirubin is common to other tissues as well, if we regard the hæmatoidin of Virchow, which is so often found in old extravasations of blood, as identical with bilirubin. Under normal conditions, however, the liver is apparently the only organ of the body in which the formation of bilirubin takes place. Whether or not the final dissolution of disintegrating red corpuscles also occurs at this place has not been decided, but the liver is manifestly capable of retaining the hæmoglobin which is thus set 
free. If a moderate amount of a solution of hæmoglobin is injected into the bloodvessels of an animal, an increased elimination of bilirubin results, while the blood-pigment does not appear in the urine. If larger amounts are injected, the resulting bilirubin apparently cannot be removed with sufficient rapidity through the biliary ducts. Resorption of the pigment then takes place through the lymph-vessels, jaundice results, and the bile-pigment appears in the urine. If excessive amounts of hæmoglobin finally are injected, the liver is manifestly incapable of retaining all the pigment which reaches the organ, jaundice results, and both the bile-pigment and the blood-pigment appear in the urine. Even in such extreme cases the remaining tissues of the body do not participate in the formation of bilirubin. This has been conclusively demonstrated by Minkowski and Naunyn. These observers have shown that while in normal geese poisoning with hydrogen sulphide, which causes an extensive dissolution of the red corpuscles, invariably leads to jaundice and the appearance of bile-pigment in the urine, the previous removal of the liver prevents such an occurrence, and results in simple hæmoglobinuria. In mammals such crucial tests unfortunately cannot be applied, but there is no reason to suppose that different conditions exist. The possible occurrence of a hæmatogenic icterus, in contradistinction to a hepatogenic icterus, is thus rendered extremely improbable, and it is scarcely warrantable to point to the development of bilirubin (hæmatoidin) from bloodpigment in the tissues as indicating the possibility of such a transformation in the circulating blood.

Of the manner in which this transformation is effected in the liver we know nothing. We may imagine, however, that the oxyhæmoglobin is here first decomposed into its albuminous componentglobin - and into hæmatin, which latter, after loss of the iron, then passes over into bilirubin, as shown in the equation:

$$
\underset{\text { Hæmatin. }}{\mathrm{C}_{32} \mathrm{H}_{32} \mathrm{~N}_{4} \mathrm{O}_{4} \mathrm{Fe}}+2 \mathrm{H}_{2} \mathrm{O}-\mathrm{Fe}=\underset{\text { Bilirubin. }}{\mathrm{C}_{32} \mathrm{H}_{36} \mathrm{~N}_{4} \mathrm{O}_{6}}
$$

This reaction, it will be noted, is also supposod to express the formation of hæmatoporphyrin from hæmatin (see Blood). The two substances, indeed, are now quite generally regarded as isomeric, especially since Küster has shown that the hærnatinic acids which he obtained from bilirubin are identical with those resulting from hæmatin, viz., hæmatoporphyrin (see Blood). As regards the size of the molecule, however, opinions differ, and it is possible, as Nencki and Sieber suppose, that the molecule of bilirubin, as well as of hæmatoporphyrin, is only half as large as expressed above. In that case, of course, the equation would have to be written :

$$
\mathrm{C}_{32} \mathrm{H}_{32} \mathrm{~N}_{4} \mathrm{O}_{4} \mathrm{Fe}+2 \mathrm{H}_{2} \mathrm{O}-\mathrm{Fe}=2 \mathrm{C}_{16} \mathrm{H}_{18} \mathrm{~N}_{2} \mathrm{O}_{3} \text {. }
$$


On reduction with nascent hydrogen bilirubin is transformed into urobilin, as is shown by the equation :

$$
\left.\mathrm{C}_{32} \mathrm{H}_{36} \mathrm{~N}_{4} \mathrm{O}_{5} \text { (viz., } 2 \mathrm{C}_{16} \mathrm{H}_{18} \mathrm{~N}_{2} \mathrm{O}_{3}\right)+\mathrm{H}_{2} \mathrm{O}+2 \mathrm{H}=\underset{\text { Urobilin. }}{\mathrm{C}_{32} \mathrm{H}_{40} \mathrm{~N}_{4} \mathrm{O}_{7}}
$$

This actually takes place in the intestinal canal, where nascent hydrogen is constantly being formed through the action of various bacteria, as during the process of lactic acid fermentation. During intra-uterine life, however, where no bacteria are found in the intestinal tract, bilirubin appears in the feces as such (meconium).

In the crystalline state bilirubin occurs in the form of reddishyellow rhombic platelets with rounded angles, which are soluble in benzol, carbon disulphide, amyl alcohol, glycerin, the fatty oils, and especially in warm chloroform. In alcoliol and ether they are but little soluble, and in water, as also in Platner's bile, they are insoluble. On spectroscopic examination its solutions do not give rise to any specific bands of absorption, but to a continuous absorption which extends from the red to the violet end.

Bilirubin as such is a weak acid, bilirubinic acid, and combines with bases to form salts, which for the most part are either insoluble or only slightly soluble in water and insoluble in chloroform. Its salts with the alkalies, however, are soluble in solutions of the alkaline hydrates and carbonates. In the bile bilirubin is largely present as neutral bilirubinate of sodium, and is held in solution owing to the presence of alkaline carbonates.

When perfectly fresh bile of a golden-yellow or olive-brown color is exposed to the air for a while, it will be noted that the fluid gradually assumes a bright-green color, owing to a transformation of the bilirubinate into biliverdinate of sodium. Free bilirubin, according to Dastre and Floresco, does not absorb oxygen and is thereby transformed into biliverdin, as has been supposed. The same observers state that on careful oxidation bilirubin can be transformed into biliprasin, which presents a green color as such, while its sodium salt, sodium biliprasinate, is yellowish brown. On further oxidation the biliprasin then yields biliverdin, viz., its corresponding salt.

According to former views, the relation existing between bilirubin and biliprasin were represented by the equations given below; but it is, of course, manifest that these are no longer tenable if the work of Dastre and Floresco should be confirmed.

$$
\begin{array}{cc}
\underset{\text { Bilirubin. }}{\mathrm{C}_{16} \mathrm{H}_{18} \mathrm{~N}_{2} \mathrm{O}_{3}}+\mathrm{O} & =\underset{\text { Biliverdin. }}{\mathrm{C}_{16} \mathrm{H}_{18} \mathrm{~N}_{2} \mathrm{O}_{4}} \\
\underset{\text { Bilirubin. }}{\mathrm{C}_{16} \mathrm{H}_{18} \mathrm{~N}_{2} \mathrm{O}_{3}}+\mathrm{H}_{2} \mathrm{O} & =\underset{\text { Bilifuscin. }}{\mathrm{C}_{16} \mathrm{H}_{20} \mathrm{~N}_{2} \mathrm{O}_{4}} \\
\text { Bilifuscin. } & \mathrm{C}_{16} \mathrm{H}_{20} \mathrm{~N}_{2} \mathrm{O}_{4} \\
\text { Bili } & \mathrm{H}_{2} \mathrm{O}+\mathrm{O}=\underset{\text { Biliprasin. }}{\mathrm{C}_{16} \mathrm{H}_{22} \mathrm{~N}_{2} \mathrm{O}_{6}}
\end{array}
$$

The formula of biliprasin, as here indicated, would, moreover, be an impossibility. As a matter of fact, these formulæ cannot be 
regarded as definitely established; and according to some observers, the molecule of biliverdin is only one-half as large as represented above. Much work still remains to be done in this connection, but we know at least that biliverdin constitutes a normal oxidationproduct of bilirubin. From biliverdin Küster obtained a new oxidation-product, which he termed biliverdinic acid, but which must not be confounded with the substance of the same name referred to above. This body has the formula $\mathrm{C}_{8} \mathrm{H}_{9} \mathrm{NO}_{4}$, and is identical with the dibasic hæmatinic acid, which results from hæmatin directly, and, like this, yields a substance of the composition $\mathrm{C}_{8} \mathrm{H}_{8} \mathrm{O}_{5}$, viz., the anhydride of the dibasic hæmatinic acid, $\mathrm{C}_{8} \mathrm{H}_{10} \mathrm{O}_{6}$.

Tests for Bilirubin.-GMELIN's TesT.-The fluid to be examined is treated with an amount of concentrated nitric acid, containing a trace of nitrous acid, sufficient to form a layer beneath the liquid to be tested, when in the presence of bilirubin a color-play from yellow through green, blue, violet, and red to orange will be observed at the zone of contact; the green will be noticed nearest the bile-containing solution, and the orange in the upper portion of the nitric acid.

The test is exceedingly sensitive, and is said to indicate the presence of bilirubin in a dilution of $1: 80,000$. The green color which develops is the most characteristic, but a reddish violet must also occur.

Rosenbach's Modification of Gmeinin's Test.-If bile containing bilirubin is filtered through Swedish filter-paper, and a drop of concentrated nitric acid containing a trace of nitrous acid is placed upon the paper, which is colored a bright yellow, the play of colors just described will occur. The green color is referable to the biliverdin. The blue color is ascribed to bilicyanin or cholecyanin, and the final orange to choletelin.

HuPPERT's TEST. - A few cubic centimeters of the solution to be examined are precipitated with barium chloride and ammonia. The precipitate is washed with water and suspended in a small amount of alcohol that has been acidulated with sulphuric acid. This mixture is then boiled for a few minutes, when in the presence of bilirubin a bright emerald-green color develops.

Surtu's Test.-A small amount of the fluid is placed in a testtube and treated with a few cubic centimeters of tincture of iodine which has been diluted with alcohol in the proportion of $1: 10$, so as to form a layer above the fluid to be examined. In the presence of bilirubin a distinct emerald-green ring will develop at the zone of contact.

According to some observers, the green color which thus results when bilirubin is treated with iodine is not referable to the formation of biliverdin, but to a substitution- or addition-product of biliverdin with iodine. This, however, is denied by others, and Jolles has shown that the iodine merely acts as an oxidizing agent, and that true biliverdin is formed, as indicated by the equation:

$$
\mathrm{C}_{16} \mathrm{H}_{18} \mathrm{~N}_{2} \mathrm{O}_{3}+2 \mathrm{I}+\mathrm{H}_{2} \mathrm{O}=\mathrm{C}_{18} \mathrm{H}_{18} \mathrm{~N}_{2} \mathrm{O}_{4}+2 \mathrm{HI} \text {. }
$$


Spectroscopic Test.-If a dilute solution of sodium bilirubinate in water is treated with an excess of ammonia and a small amount of a solution of chloride of zinc, the liquid at first turns a deep orange, but subsequently becomes olive brown, and finally green. If this solution is examined spectroscopically, it will be noted that the violet and blue portions of the spectrum are at first quite dark, but subsequently the bands presented by an alkaline solution of bilicyanin become apparent, and notably the one between $\mathrm{C}$ and $\mathrm{D}$, near C (see below).

Isolation of Bilirubin.-Bilirubin is most conveniently obtained from the biliary concretions which are so often found in the gallbladder of cattle, and which consist almost entirely of the calcium salt of the pigment. They are finely powdered and extracted with ether and then with hot water, so as to remove the cholesterin and the biliary acids which are present. The remaining material is treated with hydrochloric acid, so as to liberate the pigment. It is then washed free from acid with water, and subsequently with absolute alcohol to remove the water and any biliverdin that may be present. The pigment remains, and is now dissolved with boiling chloroform. From this solution the chloroform is distilled off, the residue extracted with absolute alcohol, so as to remove any bilifuscin, and the remaining bilirubin dissolved in a small amount of chloroform and precipitated with alcohol. This procedure is repeated until the substance has been obtained in pure form; it is then allowed to crystallize out from its chloroform solution on cooling.

Biliverdin.-Biliverdin is found in the bladder-bile of many animals together with bilirubin, and is especially abundant in certain herbivora, where the bile frequently presents a bright grass-green color. It is said to occur also in the placenta of the bitch, in the shells of certain mollusks, and in birds' eggs. Its relation to bilirubin has already been considered. In the bile it is present principally in the form of its sodium salt, and, like bilirubin, the free pigment possesses acid properties; this is termed biliverdinic acid, but should not be confounded with the acid of the same name which Küster obtained from biliverdin on oxidation with sodium chromate (see above). In acid bile biliverdin is found as such. Unlike bilirubin, the free pigment is readily soluble in normal as well as in neutral and acid bile. It is insoluble in water, ether, and chloroform, but dissolves in alcohol, glacial acetic acid, and solutions of the alkalies. From the latter it is precipitated by the salts of the alkaline earths and the heavy metals, as also by acids. On treating an alcoholic solution of biliverdin with ammoniacal chloride of zine solution the fluid exhibits a green fluorescence. The green color of the pigment is changed to yellow if its solution in acid alcohol is treated with zinc. If chlorine-water is added instead, a blue color develops at the bottom of the liquid, and above it layers presenting a violet, a red and a yellow color will be observed. On adding an excess of chlorine-water the solution is decolorized. 
Pure biliverdin, like bilirubin, gives no characteristic band of absorption in alkaline solution. In acid solution, however, or in pure alcoholic solution, an indistinct band is observed at $\mathrm{D}$, and one that is more pronounced near $\mathrm{F}$.

The substance is amorphous, or at least cannot be obtained in a pronounced crystalline form.

On reduction, biliverdin is supposedly transformed into bilirubin, though this is denied by some observers.

On oxidation with nitric acid biliverdin gives rise to the various colors which have already been described, beginning with blue. It gives Huppert's reaction directly.

Isolation.-To prepare biliverdin, it is most convenient to start with a solution of bilirubin in the form of its sodium salt which has been exposed to the air until the original golden-yellow color has changed to a brownish green. The biliverdin is then precipitated by adding an excess of hydrochloric acid. It is filtered off', washed free from all acid, dissolved in absolute alcohol, and precipitated by .copiously diluting with water. Any bilirubin that may be present is removed by extracting with chloroform.

Biliprasin.-Biliprasin as such, and biliprasinate of sodium, according to Dastre and Floresco, are also found in normal bladderbile, and, as has been indicated, represent intermediary products of oxidation between bilirubin and biliverdin.

The sodium salt, according to these observers, is a yellowish-brown pigment, and can be transformed into biliprasin through the agency of mineral acids, of acetic acid, and even of carbonic acid. On exposure to the air it passes over into the corresponding biliverdinate. To its presence the yellow color of the bile of calves and of other animals is supposedly due.

Biliprasin itself is green, but can be transformed into the yellow salt by adding a few drops of an alkali, and this in turn yields the green pigment on treating with an acid. This reaction, according to Dastre and Floreseo, explains the fact that yellow bile can become green without oxidation, viz., without the formation of biliverdin. According to older ideas, however, biliprasin is an oxidation-product of biliverdin, and is supposed to result from this with the intermediary formation of bilifuscin, as has already been outlined.

Bilifuscin is said to occur in gall-stones together with bilirubin. The formula which has been ascribed to it is that of bilirubin plus one or two molecules of water, viz., $\mathrm{C}_{32} \mathrm{H}_{40} \mathrm{~N}_{4} \mathrm{O}_{8}$ or $\mathrm{C}_{16} \mathrm{H}_{20} \mathrm{~N}_{2} \mathrm{O}_{4^{\circ}}$. It is of a dark-brown color, and is soluble in alcohol and the solutions of the alkaline hydrates, but insoluble in water and chloroform. In pure form it does not give Gmelin's reaction.

Bilicyanin, or cholecyanin, is the blue pigment which is formed during the oxidation of bilirubin and biliverdin with nitric acid. It has been found together with the common bile-pigments in gallstones taken from man. Its neutral and alkaline solutions give rise to three bands of absorption. One of these is located between $\mathrm{C}$ 
and $\mathrm{D}$, near C; another about $\mathrm{D}$; and a third very faint band midway between $\mathrm{D}$ and $\mathrm{E}$. In acid solutions two bands are seen between $\mathrm{C}$ and $\mathrm{E}$. On treating the alcoholic solution of the pigment with an ammoniacal solution of zinc chloride a distinct fluorescence is obtained. Its formula has not as yet been determined, and, according to some observers, indeed, bilicyanin does not represent a separate substance.

Bilipurpurin.-This term has been applied to a red pigment which is formed from bilirubin and biliverdin on treating with nitric acid. A pigment of the same name has been isolated from ox-gall by Löbisch and Fischler. Its formula is given as $\mathrm{C}_{32} \mathrm{H}_{34^{-}}$ $\mathrm{N}_{4} \mathrm{O}_{5}$, which would suggest that the substance is an anhydride of bilirubin.

Choletelin, or bilixanthin, is generally regarded as the final oxidation-product of the common bile-pigments. It is an amorphous brown substance, which is soluble in alcohol, ether, chloroform, and in solutions of the alkaline hydrates, from which latter it can be precipitated by the addition of acids. Its formula is given as $\mathrm{C}_{16} \mathrm{H}_{18} \mathrm{~N}_{2} \mathrm{O}_{6}$.

Bilihumin is a pigment of unknown composition that has been found in gall-stones. It is insoluble in all organic solvents.

\section{Cholesterin.}

Cholesterin is not exclusively a product of the activity of the hepatic cells, but is found in other tissues as well. It is thus a constituent of the red corpuscles of the blood, of the plasma, of the yolk of eggs, of the semen, of the secretion of the sebaceous glands, and is especially abundant in nerve-tissue. In the vegetable world also cholesterin is widely distributed. The liver is probably the organ through which the substance, wherever formed, is eliminated. Ultimately it appears in the feces. In the urine it is found only under exceptional conditions, and then only in very small amounts. Of its mode of formation nothing is known, but it is interesting to note that wherever cholesterin is found lecithin is likewise observed. In the brain a considerable amount of the substance occurs in combination with a fatty acid, cholic acid, from which it can only be separated by saponification.

The amount of cholesterin which is found in the bile represents about 2 per cent. of the total solids. Normally it is held in solution owing to the presence of the biliary acids, but under pathologic conditions it may separate out in crystalline form, either in the gallbladder itself or in the larger hepatic ducts, and then gives rise to the formation of stones. Of the origin of these concretions we know little. Very often they contain a nucleus of epithelial cells or of bacteria, around which the cholesterin, together with a variable amount of bile-pigment and mineral salts, becomes deposited. The stones which are usually found in man are for the most part very 
rich in cholesterin, while the pigment-stones which are so common in cattle are less frequently seen in the human being.

The common cholesterin which is found in the animal body has the composition $\mathrm{C}_{27} \mathrm{H}_{44} \mathrm{O}$ or $\mathrm{C}_{27} \mathrm{H}_{46} \mathrm{O}$, and apparently contains an alcoholic hydroxyl group. There is evidence to show that it belongs to the class of terpenes, which occur widely distributed in the vegetable world, and which had previously not been recognized as existing in the animal world. This shows at the same time that the animal organism contains hydro-aromatic compound. It combines with fatty acids to form compound esters which are analogous to the fats, and in this form also cholesterin occurs widely distributed in the animal world. The common lanolin of wool-fat thus contains large amounts of esters, both of cholesterin and its isomeric compound isocholesterin. On treating cholesterin with concentrated sulphuric acid the substance gives rise to the formation of hydrocarbons, which are termed cholesterilins, which stand in a close relation to the terpene group. With iodine these bodies give a blue color.

Cholesterin usually occurs in the form of colorless, transparent plates, with ragged margins and angles, which are very characteristic. It is practically insoluble in water, dilute acids and alkalies, but dissolves with ease in ether, chloroform, benzol, and in boiling alcohol. From its ethereal solutions it crystallizes out in the form of fine needles. It is further soluble in the essential and fatty oils, as also in the presence of biliary acids. Its crystals melt at $145^{\circ} \mathrm{C}$.

Tests.-SALkowski's TEST.-A few crystals of cholesterin are dissolved in a small amount of chloroform and treated with an equal volume of concentrated sulphuric acid. The solution of cholesterin then first assumes a blood-red color, and then gradually turns to a violet red, while the sulphuric acid appears dark red and shows a green fluorescence. On pouring the chloroform into a shallow porcelain dish it turns violet, then green, and finally becomes yellow.

The Test of Liebermann-Burckhard.-If a small amount of cholesterin is dissolved in about 2 c.c. of chloroform and is treated with 10 drops of acetic acid anhydride, and subsequently. with concentrated sulphuric acid, the solution at first assumes a red, then a blue, and finally a green color. The latter develops at once if cholesterin is present only in traces.

Isolation.-To prepare cholesterin for purposes of study, it is most convenient to isolate the substance from cholesterin stones. 'To this end, the concretions are finely pulverized and extracted with boiling water and then with boiling alcohol. From the alcoholic extract the cholesterin crystallizes out on cooling, and is then boiled with an alcoholic solution of sodium hydroxid, so as to saponify the fats which are at the same time present. The alcohol is then distilled off and the residue extracted with ether, which removes the cholesterin and leaves the soaps behind. On evaporating this extract, after filtration, the substance is obtained in crystal- 
line form, and can be further purified by recrystallization from a mixture of alcohol and ether.

Other Organic Constituents of the Bile.-In addition to the bodies already described, the bile contains small amounts of lecithin, of palmitin, stearin, olein, and the soaps of the corresponding fatty acids. In ox-bile Lassar-Cohn found also traces of myristinic acid, $\mathrm{C}_{14} \mathrm{H}_{28} \mathrm{O}_{21}$, which has heretofore only been observed in the spermaceti of whales. We further find traces of urea, and occasionally a diastatic ferment, which is by some observers regarded as identical with ptyalin. Its presence, however, is by no means constant, and it can scarcely be regarded as playing a rôle in the process of intestinal digestion. Larger amounts of urea, according to Hammarsten, are found in the bile of the shark and the sturgeon.

In decomposing bile cholin, glycerin-phosphoric acid and trimethylamin may be observed, and are referable to the decomposition of lecithin.

\section{The Biliary Iron.}

If we recall the origin of bilirubin from hæmatin, we should expect to find in the bile the iron which is liberated during the decomposition of the latter. Traces of iron, indeed, are constantly present, principally in combination with phosphoric acid. The amount, however, which is thus eliminated is far too small to represent that which must of necessity be set free. Kunkel thus found that while 100 parts of hæmatin correspond to 9 parts of iron, only 1.4-1.5 parts of iron appear in the urine for every 100 parts of bilirubin. But even if we add to this the amount which is eliminated in the urine and that which is excreted through the intestinal mucosa, we still find a very large deficit, and we are accordingly forced to the conclusion that the greater portion of the iron must be retained in the liver. But while such a retention must of necessity occur, we are ignorant of the manner in which it is accomplished. Of the form, also, in which the iron exists in the liver we know but little. That it is subsequently utilized in the construction of hæmoglobin is quite likely, but not proved. Naunyn and Minkowski have observed that following poisoning with arseniuretted hydrogen, ironcontaining pigments can be demonstrated in the liver. Latschenberger speaks of the formation of choleglobins as antecedents of bilirubin, and of the simultaneous appearance of iron-containing melanins in the liver; and Neumann has demonstrated the presence of an organic iron pigment, hæmosiderin, in old extravasations of bloorl and in thrombi together with hæmatoidin; but of the true nature of these substances we practically know nothing. It is possible that the globin, which must of necessity be liberated during the decomposition of hæmatin, takes up the iron which is set free from the latter; but this also is a supposition, and further researches in this direction are needed. 


\section{CHAPTER VIII.}

\section{THE PROCESSES OF DIGESTION AND RESORPTION.}

In the preceding chapter we have considered the various digestive fluids which are concerned in the transformation of those foodstuffs that are incapable of resorption as such into material which the body can utilize for purposes of nutrition, and we have seen that the most important agents which are here concerned belong to the class of the non-organized ferments. In the present chapter we shall study the action of these various substances upon the different classes of food-stuffs collectively and in somewhat greater detail, and shall incidentally also consider the resorption of the final products of digestion from the gastro-intestinal canal.

\section{THE DIGESTION OF THE CARBOHYDRATES.}

The digestion of the carbohydrates is essentially effected in the small intestine through the agency of the amylolytic ferment of the pancreas, ptyalin, and the inverting ferments maltase, lactase, and invertin, which are in part also furnished by the pancreas, but are principally found in the enteric juice. In those animals in which ptyalin occurs in the saliva, amylolysis to a certain degree also takes place in the mouth and continues in the stomach for a variable length of time, until the ferment is destroyed by the hydrochloric acid.

In the majority of the purely carnivorous animals, as has been pointed out, the saliva contains no digestive ferments, and, in such, carbohydrate digestion takes place exclusively in the small intestine.

Through the action of the ptyalin of the pancreatic juice or of the saliva, as the case may be, the insoluble starch is first transformed into soluble starch or amidulin (amylodextrin), and is then successively decomposed by hydrolysis into erythrodextrin, achroödextrin, and maltose, as previously shown (see Saliva).

Glycogen is similarly decomposed and, like starch, gives rise to the formation of maltose. The celluloses, on the other hand, are not affected by ptyalin nor, indeed, by any of the digestive fluids. As we shall see, however, they undergo a certain kind of fermentation under the influence of various bacteria, and as a result we find that in herbivorous animals, at least, only a fraction of the ingested cellulose reappears in the feces. Thus far a transformation into maltose or glucose has not been observed in the intestinal tract.

The inversion of disaccharides is brought about by invertase, 
maltase, and lactase, which are partly furnished by the pancreas, but principally by the enteric juice. This inversion is likewise of the nature of a hydrolytic process, and may be represented by the general equation :

$$
\underset{\text { Disaccharide. }}{\mathrm{C}_{12} \mathrm{H}_{22} \mathrm{O}_{11}}+\mathrm{H}_{2} \mathrm{O}=\underset{\text { Monosacharide. }}{2 \mathrm{C}_{6} \mathrm{H}_{12} \mathrm{O}_{6}}
$$

To judge from certain experiments which have been performed on animals, it appears that amylolysis can also take place in the absence of the principal gland by which the ptyalin is formed, viz., the pancreas. For we find that following the extirpation of this organ or ligation of the pancreatic duct dogs are still capable of utilizing as much as from 47 to 71 per cent. of the starch ingested. As the dog's saliva contains no ptyalin, the amylolysis cannot be referable to a converting activity of the salivary glands. Whether in such cases the small amount of ptyalin which is furnished by the enteric juice is sufficient to transform the ingested starch into maltose is questionable, and there is some reason for supposing that the epithelial cells of the small intestine are capable not only of causing the transformation of disaccharides to monosaccharides but also of inverting dextrin to maltose. It has been shown that in animals with Thiry-Vella fistulæ injected solutions of starch and cane-sugar rapidly disappear, although maltose cannot always be demonstrated in the fluid. In what manner this change is effected by the epithelial cells is not known. In any event it is necessary that the polysaccharides should be inverted to monosaccharides before passing beyond the mucous membrane of the gastro-intestinal tract. Resorption takes place primarily through the specific activity of the epithelial lining of the intestinal mucosa. LThe monosaccharides then enter the blood-current and are carried to the muscles and the liver, where they are transformed into glycogen and stored in a manner analogous to the reserve starch of the plant. This transformation, however, as well as the subsequent fate of the sugar, we shall have occasion to study in greater detail in a subsequent chapter.

Neither the polysaccharides nor the disaccharides when introduced into the blood-current directly can be utilized by the body as such, and they are accordingly eliminated in the urine as foreign matter.

The extent to which amylolysis can occur in the intestinal canal is remarkable, and far exceeds the ability of the liver and the muscle-tissue to transform the corresponding amount of glucose into glycogen. As a consequence, the percentage of circulating sugar rises beyond the normal and glucosuria results (alimentary glucosuria). That disaccharides may pass the intestinal mucosa without being inverted is possible, but certainly of exceptional occurrence. In such cases we must imagine that the intestinal epithelium has lost its specific power as a barrier to the passage of the sugars, as well as its ability to cause their inversion. As a result they pass 
this barrier by diffusion, and probably enter both the blood- and the lymph-current, and are then eliminated in the urine. A formation of glycogen from disaccharides directly is apparently not possible.

The rapidity with which resorption takes place in the small intestine seems to vary with the character of the sugar. In dogs Albertini thus found that of 100 grammes of glucose, 60 grammes are absorbed in the course of the first hour, while of maltose and canesugar from 70 to 80 grammes and of lactose only 20 to 40 grammes disappear within the same period of time.

The ingestion of very large amounts of disaccharides and monosaccharides leads to a general disturbance of intestinal digestion and results in diarrhœe. A corresponding amount of starch, on the other hand, is without effect in this respect. This is no doubt owing to the fact that in the latter case inversion and resorption proceed pari passu, so that the bacteria have but little chance of setting up fermentative changes, which lead to the formation of substances that directly increase the peristalsis owing to their irritating properties. In the presence of abnormally large amounts of sugars as such, on the other hand, resorption is not sufficiently rapid, and in the presence of the increased amount of pabulum an increase of bacterial fermentation beyond the normal takes place. As a result various acid decomposition-products of the carbohydrates, such as lactic acid, butyric acid, acetic acid, formic acid, succinic acid, together with carbon dioxide, methane, and hydrogen, are formed in increased amounts.

\section{DIGESTION OF THE ALBUMINS.}

The digestion of the albumins takes place in the stomach and in the small intestines under the influence of the pepsin and the hydrochloric acid of the gastric juice and the trypsin of the alkaline pancreatic juice respectively. We know that the presence of the former is not altogether necessary, however, and that the pancreatic juice is in itself sufficient to accomplish the digestion of the albumins, but under normal conditions the gastric juice also plays a part. We know, as a matter of fact, that albuminous material which has been first subjected to the action of pepsin-hydrochloric acid and then to trypsin is more rapidly and more completely hydrolyzed than when acted upon by the trypsin alone. Of the relative extent to which the one and the other enters into the process our knowledge is not complete. We may imagine that in the stomach a primary dissolution of the solid constituents of the food takes place, and that the soluble products which are thus formed are further digested by the pancreatic juice. This actually occurs to a certain extent, but we further know that in the stomach certain albuminous food-stuffs are broken down (nucleoproteids) with the liberation of constituents which are insoluble in the gastric juice, and which pass the pylorus as such and are then modified by the pancreatic juice. Tryptic di- 
gestion, moreover, is far more extensive than peptic digestion, so that we may well conclude that the latter essentially represents a preliminary phase of digestion ; and that the digestion proper, viz., the transformation of the albumins into those final products which can be directly utilized by the body in its tissue metabolism, occurs under the influence of the trypsin of the pancreatic juice.

For convenience' sake, we shall study the action of the gastric juice and of the pancreatic juice separately upon the various classes of albumins, as the digestive products which are formed are somewhat different in the different classes. In every case we shall follow the fate of these various substances to the final products, as we obtain them artificially in digestive experiments in vitro; but we must bear in mind that such experiments cannot reproduce what actually takes place in the living body, where resorption is constantly going on, and where the varions digestive processes in a manner supplement each other and conditions overlap.

\section{Digestion of the Native Albumins.}

Gastric Digestion.-In the stomach the native albumins, if introduced in the coagulated state, are first transformed into a soluble form, and at the same time or immediately following their dissolution they undergo the process of denaturization-i.e., they are transformed into syntonins or acid albumins, and as a consequence all individual characteristics which previously existed are lost. This transformation is essentially referable to the hydrochloric acid of the gastric juice, and can be brought about artificially in the absence of pepsin. In such an event, however, a higher grade of acidity and a higher temperature are required. The presence of the pepsin obviates such a necessity. A possible explanation of this phenomenon is afforded by the modern doctrine which teaches that the action of enzymes merely consists in hastening the rapidity of reaction.

On continued exposure to the acid gastric juice albumoses appear and finally peptids; amino-acids, however, cannot be demonstrated under normal conditions.

This decomposition may be compared to the inversion of the polysaccharides to monosaccharides. Kühne and his school, who have largely contributed to our knowledge of the products of digestion, have suggested their division into two classes, viz., the primary and secondary albumoses, according to their nearer or more distant relationship to the original albumins. He recognized two primary albumoses, viz., proto-albumose and hetero-albumose, each of which on further digestion was supposed to give rise to a deutero-albumose, from which in turn peptone was derived. This peptone, which resulted from peptic digestion, was regarded as a unity and termed amphopeptone. In it the hemi- and anti-complex of the albuminous molecule were still supposedly united. In a general way this concept of the course of peptic digestion has proved correct, but it has 
been modified in several important particulars. Much of our present knowledge is due to the Strassburg school and to the researches of Emil Fischer and his pupils. It has thus been shown that quite early in the course of digestion a certain proportion of nitrogen is split off from the albuminous material in the form of ammonia or of a compound which yields ammonia on distillation with magnesia. At first this is small in amount; subsequently it increases and for a time it remains constant. This nitrogen represents products which no longer give the biuret reaction and which undoubtedly are closely related to the end-products of digestion. Coincidently the primary albumoses appear, of which we now recognize three. These are proto-albumose, hetero-albumose, and a third, which Pick has designated as gluco-albumose, ${ }^{1}$ from the fact that it contains the carbohydrate group, which is absent in the first two mentioned (in the case of fibrin, at any rate).

On further digestion the primary albumoses give rise to secondary or dentero-albumoses, of which several forms exist. These are designated as deutero-albumose $A$ and $A^{\prime}$, deutero-albumose $B$ and $B^{\prime}$, and deutero-albumose C. These in turn give rise to bodies which in part give the biuret reaction and in part not. Collectively these are termed polypeptids and, in part, no doubt, they correspond to Kühne's peptones. Many of the simpler forms have been made synthetically (see p. 000), but the more complex bodies, which are more closely related to the albumoses, are comparatively unknown.

Pick speaks of an A and a B peptone which result from the secondary albumoses (with the exception of deutero-albumose $\mathrm{C}$ ), and which differ from each other in their behavior toward alcohol and iodopotassic iodide in saturated ammonium sulphate solution, peptone $\mathbf{A}$ being precipitated by both reagents, while peptone $B$ remains in solution. A further examination of the bodies in question has not been made.

Siegfried and his pupils describe two pepsin peptones which were obtained from fibrin. They are designated as pepsin-fibrin peptone $\alpha$ and $\beta$. By losing water the $\beta$-peptone passes over into the $\alpha$ peptone :

$$
\left(\mathrm{C}_{21} \mathrm{H}_{36)} \mathrm{N}_{6} \mathrm{O}_{10}\right)_{a}
$$$$
\mathrm{C}_{21} \mathrm{H}_{34} \mathrm{~N}_{6} \mathrm{O}_{9}
$$

On further decomposition with trypsin the $\alpha$-peptone then yields tyrosin, arginin, and, according to Siegfried, also two other peptones, which he terms trypsin-fibrin peptone $\alpha$ and $\beta$. He accordingly points out that the $\alpha$-peptone is a true ampho-peptone in the sense of Kühne.

Of the nature of these products, however, very little is known. They manifestly are polypeptids in the sense of Fischer.

\footnotetext{
1 The term first used for this was dentero-albumose $\dot{B}$ or albumose $B a$. Hofmeister suggests synalbumose as a better term, since there is a possibility that albumins may exist which may yleld a primary albumose of this order containing no carbohydrate group (caseïn), and that on the other hand albumins which are very rich in carbohydrate groups may possibly form proto- and hetero-albumoses in which these are represented.
} 
The end-products of peptic digestion are qualitively the same as those which result on tryptic digestion. The velocity of reaction in the case of pepsin is, however, materially less than with trypsin. On long-continued peptic digestion the following well-defined products have been obtained: leucin, amidovalerianic acid, tyrosin, phenyl-alanin, glutaminic acid, asparaginic acid, cystin, lysin, skatosin, putrescin, cadaverin, oxy-phenyl-ethylamin, but it is noteworthy that during gastric digestion anino-acids are not formed.

Among the products of digestion there must be mentioned the socalled anti-albumid of Kühne. This still bears an albuminous character and supposedly contains the anti-group of the albuminous molecule, as it is extremely resistant to the further action of pepsin hydrochloric acid. On tryptic digestion it is thrown down as a delicate jelly-like material-the so-called anti-albumid coagulum. The substance does not give Millon's reaction.

As regards the extent to which peptic digestion is carried in the stomach our knowledge is not complete. Zunz has recently studied this subject in the case of dogs fed on meat. From these observations it appears that the coagulated albumins are first brought into solution; during this process very little acid albumin is formed, but large amounts of albumoses appear, besides small amounts of peptones and peptids. The larger portion of the material which is now in solution then passes over into the small intestine, where it is rapidly further decomposed and absorbed. A smaller fraction is absorbed in the stomach directly, and here the more distant products of digestion are primarily concerned, while an absorption of albumoses, though its occurrence cannot be denied, is nevertheless much more difficult. Accordingly we find in the liquid contents of the stomach mostly albumoses and only small amounts of the more remote digestive products. Of albumoses, we find both primary and secondary forms; a definite relation between the two does not appear to exist. Peptones (according to the biuret reaction) are either present in traces or they are absent, while peptids are constantly encountered.

As regards the appearance of the individual digestive products in point of time and in relation to each other the following facts have been ascertained (the observations have reference to crystallized serumalbumin, crystallized egg-albumin, serum-globulin, euglobulin, pseudoglobulin, and casein (Zunz). The solution of the coagulated albumins begins at once after exposure to pepsin hydrochloric acid at the temperature of the body. In the case of serum-albumin and englobulin the primary albumoses appear after nine minutes, together with acid albumin. In the case of caseïn albumoses are likewise found, but no acid albumin, at the same time. Pseudoglobulin and egg-albumin appear to be more resistant, as no digestive products can be demonstrated so early. Twenty-six minutes from the beginning of digestion acid albumin and proto- and hetero-albumose can be demonstrated in the case of the egg-albumin, together with the deutero-albumose $\mathbf{B}$. 
Acid albumin is never found in the entire absence of albumoses; and it is interesting to note that primary albumoses may be present at a time when no acid albumin is as yet demonstrable. This observation may be explained by the assumption that the acid albumin, as soon as formed, is transformed into albumoses. But opposed to this interpretation is the fact that acid albumin is only transformed into albumoses after a comparatively long time, and does not give rise to all products of digestion which can be obtained from the primary albumins from which the acid albumin in turn is derived. Zunz was thus unable to isolate the deutero-albumoses $\mathrm{A}$ in all experiments in this direction. It has accordingly been suggested that acid albumin does not represent a purely denaturized albumin, but should be placed on the same level with the primary albumoses.

Goldschmidt maintains the view that the formation of acid albumin occurs with a coincident splitting off of albumose complexes. On the other hand, it has been noted that under certain conditions the formation of acid albumin can be prevented even though digestion otherwise proceeds in the normal manner, and that then the deutero-albumose $\mathrm{A}$ is likewise absent, as also that portion of the end-products of peptic digestion which give no biuret reaction. It follows that the formation of acid albumin as an intermediate product in the formation of albumoses is not essential, even though it may be of value during the process of digestion in the living organism.

Tryptic Digestion.-On entering the small intestine the acid gastric contents are rendered alkaline, the pepsin is destroyed, and tryptic digestion begins.

The material which is exposed to the action of the pancreatic juice consists in part of the primary albumoses which were formed in the stomach, in part of Kühne's anti-albumid, and in part of svntonin and of native albumins, in soluble or insoluble form, which have escaped the action of the gastric juice. The latter are first dissolved, and together with the syntonins transformed into alkaline albuminate. This result, analogous to the formation of the syntonin in acid solution, as well as the further decomposition of the alkaline albuminate, is no doubt primarily referable to the action of the alkalies of the pancreatic juice, and merely hastened by the ferment which is at the time present. But unlike the action of the gastric juice, tryptic digestion immediately leads to the formation of deutero-albumoses without the intermediary production of primary albumoses in the sense of Kühne. According to older views, amphopeptone is then formed, from which hemi- and anti-peptone finally result. This concept, as in the case of the gastric digestion, has been materially modified by more recent investigations. Following the stage of deutero-albumoses, here as there, products are formed which in part give the biuret reaction and in part not. A hemipeptone and an antipeptone in the sense of Kühne do not exist. The hemi-groups at once break down into 
amino-acids. Kühne's antipeptone, which represents that portion of the tryptic digestive products which cannot be salted out by ammonium sulphate either in neutral, acid, or alkaline media, but which can be precipitated by absolute alcohol, is no chemical unity. Kutscher has demonstrated in the case of fibrin that antipeptone consists to the extent of 30 per cent. of arginin, lysin, and histidin. In addition he found small amounts of leucin, tyrosin, aspartic acid, and still other bodies which were not identified.

Peptones, analogous to those which Siegfried has described in the case of peptic digestion, are here also formed. These peptones, according to Siegfried, do not contain the tyrosin group and energetically resist the further action of trypsin. In this sense they correspond to Kühne's concept of antipeptone. From fibrin he obtained two bodies of this order, which he terms $\alpha$ - and $\beta$-trypsinfibrin peptone, $\mathrm{C}_{10} \mathrm{H}_{17} \mathrm{~N}_{3} \mathrm{O}_{5}$ and $\mathrm{C}_{11} \mathrm{H}_{19} \mathrm{~N}_{3} \mathrm{O}_{5}$. They are both active acids; they color blue litmus paper red and decompose carbonates with the formation of $\mathrm{CO}_{2}$. On decomposition with acids they yield among other products arginin, lysin, glutaminic acid, and possibly also serin.

From glutin Siegfried obtained a glutin-trypsin-peptone, $\mathrm{C}_{19} \mathrm{H}_{30} \mathrm{~N}_{6} \mathrm{O}_{9}$. On hydrolysis this in turn yielded a basic peptone

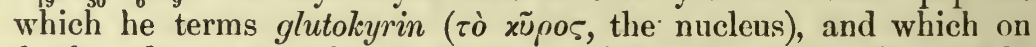
further cleavage yields arginin, lysin, glutaminic acid, and probably glycocoll. He supposes that the kyrin molecule consists of one molecule of arginin, one molecule of lysin, one of glutaminic acid, and two of glycocoll.

In this connection it is interesting to note that on tryptic digestion of various albumins $\mathrm{E}$. Fischer and Abderhalden obtained a polypeptid which was resistant to further decomposition by means of trypsin, but which yielded a large amount of $\alpha$-pyrrolidin-carbonic acid and phenyl-alanin on hydrolysis with boiling hydrochloric acid; this polypeptid no longer gave the biuret reaction. This reaction, indeed, disappears altogether on long-continued tryptic digestion, from which we can conclude that Siegfried's peptones also must have undergone further cleavage. No doubt they represent relatively complex polypeptids in the sense of Fischer.

The end-products of tryptic digestion are, as already pointed out, qualitatively the same as those which result on peptic digestion, with the exception of tryptophan-skatol-amino-acetic acid-which may in a measure be regarded as characteristic of tryptic action. The essential difference is a matter of velocity of reaction ; this is materially greater with trypsin than with pepsin.

The chyme from the stomach reaches the duodenum at the beginning of the fourth hour after a full meal ; the height of pancreatic digestion lies between the third and the fifth hour.

In the account of the process of albuminous digestion, as outlined in the foregoing pages, it has in a manner been assumed that 
the intermediary products of digestion of different albumins are the same, irrespective of their origin. Strictly speaking, this is probably not correct, although in the absence of quantitative studies of their decomposition-products it is scarcely warrantable to make positive statements. Their identity, however, is rendered unlikely by the varying degree to which the various primary radicles are represented in the different native bodies.

\section{Digestion of the Proteids.}

The digestion of the nucleoproteids and nucleo-albumins, like that of the native albumins, begins in the stomach. Here the separation of the non-albuminous pairing is first effected, and is then followed by the digestion of the liberated albumins. This digestion is in all respects analogous to that of the native albumins proper. Syntonins are first formed, then primary albumoses, subsequently secondary albumoses, and finally "peptones"-i. e., bodies which still give the biuret reaction, but which in contradistinction to the albumoses are not precipitated by salting with ammonium sulphate. The individual products which thus result from the proteids have not been studied with the same care as those which are derived from the native albumins, but there can be no doubt that here also Kühne's schema of digestion does not apply in its original form. Individual differences also probably exist between the various digestive products according to their origin, but of these also we know but little.

Of special interest are the earlier phases of digestion of the easein of milk. This normally exists in the milk in solution as a neutral calcium salt. In the stomach a transformation into the corresponding acid salt is then first effected by the hydrochloric acid of the gastric juice, and followed by the action of the chymosin. According to Hammarsten, this effects a partial decomposition of the soluble acid salt with the formation of calcium-paracasein, and a small amount of an albumose-like posset albumin. The paracasein is then precipitated and decomposed, with the formation of the corresponding paranuclein and the albuminous pairling.

Of the fate of the non-albuminous components of the proteids but little is known. The paranuclein of casein, it is stated, undergoes solution on continued digestion in vitro, but is at the same time decomposed with the formation of a small amount of orthophosphoric acid and an organic acid, which likewise contains phosphorus. Of this, however, nothing further is known.

The nucleins proper are not digested in the stomach and remain undissolved.

Under the influence of the pancreatic juice casein is digested in very much the same manner as with the gastric juice, but in this case the transformation into paracasein is brought about through the influence of the chymosin of the pancreas in an alkaline medium. 
Caseoses then result as with the common native albumins, and finally peptones are formed.

The glucoproteids and the hæmoglobins are decomposed as in the case of the gastric juice, and the albuminous components further digested like the native albumins. The individual products, however, which are thus formed have not as yet been studied in detail.

The true nucleins, which, as we have seen, escape gastric digestion and which do not undergo solution in the stomach, are dissolved by the pancreatic juice and are decomposed with the liberation of the contained nucleinic acids and the albuminous radicles. The latter are further digested in the usual manner. 'The paranucleins similarly undergo dissolution, and are probably decomposed as already indicated. Of the subsequent fate of the non-albuminous pairlings of the proteids in general, however, but little is known.

\section{Digestion of the Albuminoids.}

The only albuminoids which are digested in the stomach in the case of the higher vertebrate animals are collagen and elastin. Both give rise to protogelatose and proto-elastose respectively, while hetero-albumoses are not formed. The corresponding deuteroalbumoses then result. But while the deutero-gelatose subsequently gives rise to the formation of peptone-the so-called glutin-peptone - a similar transformation of the deutero-elastose apparently does not occur.

In the small intestine, under the influence of the pancreatic juice, collagen and elastin can also be digested, and it is noteworthy that the transformation of the gelatins into glutin-peptone is apparently more readily effected than that of any other albuminous substance. Unlike the gastric juice, however, the pancreatic secretion is in itself not capable of transforming the native collagen into gelatin. This change must hence be first effected artificially or in the stomach before its further digestion can occur. The peptonization of elastin in the pancreatic juice likewise ceases with the formation of deutero-elastose, while gelatin is transformed in vitro, at least, into glutin-peptone. Accordıng to Kühne and Chittenden, this is not further decomposed by trypsin. This is rather remarkable, as on hydrolytic decomposition with mineral acids gelatin yields leucin, aspartic acid, glutaminic acid, and considerable amounts of glycocoll. Reich-Herzberger, however, has recently announced that a slight formation of leucin takes place nevertheless during the action of trypsin on gelatin. The existence of aromatic groups in the original molecule, on the other hand, is very doubtful, and as a matter of fact it is impossible to obtain either tyrosin, indol, or skatol from the substance, even on bacterial decomposition. Diamino-groups, however, are largely present.

The fact that neither elastin nor collagen (gelatin) give rise to the formation of hetero-albumoses is of special interest in view of 
the fact that both substances cannot be regarded as true food-stuffs, viz., they are unable to maintain the nitrogenous equilibrium of the higher animals when exclusively used. The possibility that this may be due to the absence of aromatic groups in the gelatin has been rendered highly probable through the studies of Kauffmann, who found that its nutritive value could be materially increased if tyrosin and tryptophan were at the same time administered. The diamino-acids are manifestly of no moment in this connection, as gelatin, at least, yields rather more arginin than any of the common albuminous foodstuffs, and, as I have pointed out, Ellinger has shown that the diamino-acids alone are likewise not capable of maintaining nitrogenous equilibrium.

A digestion of other albuminoids, notably of the keratins, does not take place in the small intestine of the higher animals, while some of the invertebrates, such as the common house moth, are manifestly capable of utilizing these also for purposes of nutrition.

\section{RESORPTION OF THE PRODUCTS OF PROTEOLYTIC DIGESTION.}

The resorption of the products of proteolytic digestion is essentially the function of the intestinal mucosa. The gastric mucous membrane probably plays a secondary rôle-only, and, as I have already pointed out, normal nutrition can be maintained in the absence of the stomach. Of the manner in which resorption takes place we know very little. That the process is not one of simple diffusion seems to be definitely established, and there is good evidence to show that the epithelial cells lining the mucous membrane are actively concerned in the event.

In the past much uncertainty existed in reference to the form in which the albumins were absorbed, and it was generally taught that digestion proceeded as far as the formation of albumoses and "peptones," in the older sense of the term, and that the reconstruction of the albuminous molecule then occurred in the gastro-intestinal mucous membrane. This conclusion appeared to be well supported by the observation of Hofmeister, Neumeister, and Salvioli, that "peptones" will disappear from a solution in the presence of particles of intestinal mucous membrane. More recent experiments by Cohnheim have thrown a new light on this observation and have materially contributed to our understanding of resorptive processes. His researches centre in the discovery of a new ferment, erepsin, in the intestinal mucosa, which is capable of hydrolyzing acid albumin, albumoses, and peptones, but which is without affect upon the native albumins.

Erepsin.-To isolate the ferment, the intestinal mucosa of recently killed animals is scraped off with a piece of glass, triturated with sand, extracted repeatedly from one-half to twelve hours with alkaline normal salt solution, and finally pressed out in a tincture 
press. Pressings and extract are united and treated with a saturated solution of ammonium sulphate, in the proportion of three volumes of the salt solution to three of the extract. A heavy precipitate results on standing, which is filtered off, suspended in water, and dialyzed. The greater portion of the albumins remains undissolved, while a smaller amount together with the ferment passes into solution. The residue is again extracted, the extracts are united, and dialyzed free from the sulphate (three to four days). This solution is quite active and rapidly changes albumoses and peptones into material which no longer gives the biuret reaction and is in great part precipitated by phosphotungstic acid in crystalline form.

The ferment is destroyed by boiling, and is much impaired in its activity by exposure to a temperature of $63^{\circ} \mathrm{C}$. for two hours. It decomposes peptone (albumoses) in feebly alkaline or neutral media, while it is inactive in an acid medium. Acetic acid does not destroy the ferment within one hour. Prolonged contact with hydrochloric acid (of course, always in dilute solution) seems to destroy it. Alcohol impairs its activity very materially.

Among the products of decomposition resulting from the action of erepsin on syntonin Cohnheim obtained arginin, lysin, histidin, leucin, tyrosin, and ammonia. We now know that all the other cleavage products to which trypsin gives rise also appear with erepsin. Especially important is the fact that the amount of ammonia which is obtained on distillation with magnesia from the mixture of decomposition-products is the same as is obtained on decomposition with acids, as also with trypsin, showing that the decomposition of the nitrogenous molecule does not extend beyond the stage of amino-acids.

As regards the action of erepsin on the various albumoses and peptones, Cohnheim ascertained that they are all decomposed by the erepsin to the point where the biuret reaction is no longer obtained; though with varying rapidity. The native albumins are in no ways affected, but, very curiously, caseïn is decomposed. This is noteworthy in view of Gmelin's observation that suckling dogs secrete no pepsin.

The protamins are decomposed entirely like the albumoses and peptones, while the histons are only affected in part, which coincides with the position which the histons occupy midway between the protamins and the true albumins.

While Cohnheim assumes that the activity of erepsin is mainly displayed within the cells of the intestinal mucosa, there is some evidence to show that a portion of the ferment is secreted into the lumen of the gut and may thus become active outside of the cells also (Salaskin).

To demonstrate the action of erepsin, it is only necessary to add from 15 to 20 c.c. of an extract of the intestinal mucosa, prepared as described above, to a small amount of a solution of peptone (Witte), and to test every ten to fifteen minutes for the biuret reaction. 
As this disappears, it may be shown that the amount of material which yields a crystalline precipitate with phosphotungstic acid gradually increases, while the gelatinous precipitate, referable to the albumoses, diminishes in amount and ultimately disappears. Any coagulable albumins that may be present should be removed before testing.

Cohnhein's experiments thus place the observation that peptone disappears from its solutions in the presence of pieces of intestinal mucous membrane, in a new light. For it disappears, not because the epithelial cells reconstruct albumins from this source, but because the erepsin causes its further cleavage to products which no longer give the biuret reaction. Where and how the reconstruction of the albuminous molecule then takes place is not known; but there can be no reasonable doubt that this occurs from the endproducts of proteolytic digestion, and that albuminous cleavage and reconstruction thus go on in a manner perfectly analogous to what we have already seen in the ease of the carbohydrates. Some observers believe that the reconstruction of the albuminous molecule occurs in the intestinal mucosa and that the blood albumins, notably serum-albumin, are here formed, and become the carriers of the different radicles, from which the more highly differentiated albumins in turn are built up in situ. Others maintain that the ultimate cleavage-products are absorbed as such when they enter the portal circulation and are reunited to form the albuminous molecule on the distal side of the intestinal epithelial barrier. As a matter of fact, Howell has shown that such simple products actually occur in the circulating blood.

Of great interest in this connection are certain experiments of Löwy, in which he succeeded in maintaining the nitrogenous equilibrium of a $\operatorname{dog}$ by feeding with a mixture of the crystalline decomposition-products, the result of pancreatic autolysis, in which the biuret reaction could no longer be demonstrated. Abderhalden and Rona obtained analogous results with hydrolyzed casein. Henderson and Dean also performed similar experiments with similar results, although they merely conclude that they established a marked protein-sparing action on the part of the cleavageproducts. The hexon bases alone, as Ellinger has shown, are not (apable of maintaining nitrogenous equilibrium.

The question whether or not native albumins can be absorbed as such by the gastro-intestinal mucosa can be answered in the affirmative. Ascoli and Vigano have thus shown by the aid of the precipitin reaction that native as well as denaturized albumins can pass the gastro-intestinal mucosa and enter the lymph and blood, while retaining at least a portion of their biological characteristics. To what extent this passage of native albumins occurs under normal conditions remains to be seen.

Whether or not albumoses can pass the intestinal mucosa without undergoing further cleavage has not been satisfactorily ascer- 
tained. Embden and Knoop maintain that this is possible, and Langstein has shown that one or more albumoses can actually be demonstrated in the blood. Further investigations in this direction, however, are necessary before any definite conclusions can be reached.

The observation that albumins when introduced into the bloodcurrent directly can be utilized to a very great extent cannot be surprising in the case of those albumins which are normally found here. But Oppenheimer has shown that this also occurs to a certain extent in the case of albumins which are foreign to the animal body, such as egg-albumin. When introduced beyond a certain amount, however, such albumins are eliminated in the urine; but very curiously the power of retaining egg-albumin, for example, can be greatly increased by frequently repeated hypodermic or intravenous injections.

In connection with the question regarding the reconstruction of the albuminous molecule, certain observations made by Russian writers especially deserve consideration. Danilewsky has thus shown that it is possible by means of chymosin to produce precipitates in concentrated solutions of albumoses, and Okunew could demonstrate that this action is referable to the chymosin itself and not to any other contaminating ferment. It was further shown that both proto- and hetero-albumose are affected by the chymosin and not the deutero-albumoses. The resulting products Sawjalow has termed plasteïns, and he regards these as albumins sui generis. Later Kurajeff showed that papayotin has a similar action, but that the secondary albumoses represent the most appropriate material from which papayotin-plasteïn can be prepared. On peptic or papayotin digestion this product again yields secondary albumoses.

Preceding Cohnheim's investigations, these experiments were interpreted as indicating the manner in which the reconstruction of the albuminous molecule might possibly occur in the intestinal mucosa. In the light of Cohnheim's discovery of erepsin, however, this construction falls away.

\section{THE DIGESTION OF FATS.}

The important rôle of the pancreas in the digestion of fats is apparent from the experiments of Minkowski and Abelmann, who could show that following extirpation of the pancreas in dogs the absorption of fats ceases altogether, with the exception of butterfat, of which from 28 to 53 per cent. can still be utilized. Other observers have obtained results which differ somewhat from those of Minkowski ; but in all investigations it could at least be demonstrated that in the absence of the pancreatic juice the absorption of fats is impeded. This is due to the absence of lipase (steapsin). Under normal conditions the lipase of the pancreatic juice causes 
the cleavage of fats into glycerin and fatty acids, which are then absorbed and reconstructed into neutral fats by the intracellular lipase, in consequence of the reversible action of which this is capable (Kastle and Loevenhart).

The digestion of fats is facilitated by the presence of bile. This is apparent from experiments in which the bile was prevented from entering the intestinal tract, when it could be demonstrated that only the seventh part to one-half of the fat was resorbed, while the remainder, principally in the form of fatty acids, appeared in the feces. The former view, according to which the bile facilitates the emulsification of the fats and renders the intestinal wall more permeable, does not express the actual function of the bile in this respect. Moore, Rockwood, and notably Pflüger have shown that its principal import in this direction is referable to the readiness with which it dissolves soaps, and through their aid also free fatty acids, especially oleic acid, which in turn dissolves stearic acid and palmitic acid.

The lecithins, like the fats, are decomposed by steapsin into their components, viz., into glycerin-phosphoric acid, the corresponding fatty acids, and cholin. The former is then absorbed, and appears in part at least in the urine as such. The fatty acids after saponification are similarly absorbed and reconstructed into neutral fats, while cholin is decomposed by the bacteria which are present in the intestines, with the formation of carbon dioxide, methane, and ammonia.

\section{AUTOLYSIS.}

By autolysis is meant the self-digestion of tissues in the absence of micro-organisms. The phenomenon is referable to the action of intracellular ferments, and is of universal occurrence both in the animal and the vegetable world. The changes which occur are essentially postmortem changes; but it is possible that something similar occurs during life.

As I have already pointed out, the ferments in question are more or less specific in their action, and albumins which are foreign to a certain cell are, generally speaking, attacked with greater difficulty by the autolytic ferments of that cell than the albumins of homologous cells. The proteolytic ferments of hepatic tissue thus attack the albumins of lung-tissue only very slowly.

The proteolysis proceeds as in the case of the digestive ferments and the end-products are the same. In the case of the pancreas the following products have been obtained: ammonia, leucin, tyrosin, aspartic acid, glutaminic acid, arginin, lysin, histidin, cadaverin, oxy-phenyl-ethylamin, skatosin, uracil, guanin, adenin, xauthin, hypoxanthin, cholin, etc. Especially interesting is the formation of oxy-phenyl-ethylamin, which is derived from tyrosin through loss of $\mathrm{CO}_{2}$, as it demonstrates the possibility of a fermentative 
splitting off of $\mathrm{CO}_{2}$ in the body without the simultaneous intervention of oxygen and water.

The skatosin which Baum isolated from the products of pancreatic autolysis has the composition $\mathrm{C}_{10} \mathrm{H}_{16} \mathrm{~N}_{2} \mathrm{O}_{2}$. It contains two amino and two hydroxyl groups, and is probably identical with a body which Langstein found among the final products of peptic digestion in the case of serum-albumin.

The occurrence of xanthin bases among the products of autolysis shows that a nuclease also may have been active.

Autolysis of lymph-glands gives rise to ammonia, leucin, tyrosin, thymin, and uracil. Purin bases were here not obtained (Reh).

That tryptophan can also be formed during the autolysis of the gastric mucous membrane has been shown by Glässner; during the process both pepsin and chymosin are destroyed. 


\section{CHAPTER IX.}

\section{ANALYSIS OF THE PRODUCTS OF ALBUMINOUS DIGESTION.}

To demonstrate the formation of the various products of albuminous digestion and to separate the individual substances from each other, the following plan of work may be adopted:

\section{THE PRODUCTS OF PEPTIC DIGESTION.}

One hundred grammes of moist fibrin that has been thoroughly washed in running water are placed in 1000 c.c. of an 0.3 per cent. solution of hydrochloric acid, to which a few grammes of pepsin have been added. The mixture is kept at a temperature of $40^{\circ} \mathrm{C}$. From time to time it is necessary to ascertain whether free hydrochloric acid is still present, and to add an additional amount whenever it is wanting, so that the acidity referable to free acid remains about the same during the entire period of digestion. The occasional addition of a little pepsin is also advisable. The first specimen for examination is taken after two hours. The liquid is filtered and neutralized with a dilute solution of sodium hydrate. 'The precipitate which is thus formed consists of syntonin and is filtered off. The filtrate is rendered feebly acid with very dilute acetic acid, treated with an equal volume of a saturated solution of common salt, and boiled. Any coagulable albumin that may be present is thus precipitated and is filtered off on cooling. The solution is again made neutral and treated with an equal volume of a saturated solution of ammonium sulphate. In this manner the primary albumoses of Kühne are precipitated, and are filtered off after standing for about one-half hour. To separate the proto-albumose from the hetero-albumose, the precipitate is dissolved in hot water and treated with an equal volume of 95 per cent. alcohol. On standing, the hetero-albumose separates out, while the proto-albumose is found in the alcoholic filtrate. It is purified by repeated solution in hot water and precipitation with alcohol. To isolate the proto-albumose, the alcoholic filtrate is evaporated to dryness on a water-bath, or the alcohol is distilled off in the vacuum. The remaining material is repeatedly dissolved in water and treated with alcohol until the alcoholic solution remains clear on standing. To this end it is usually necessary to repeat the solution in water and the treatment with alcohol five or six times.

The further steps in the digestion of fibrin may be studied in a specimen which has been kept at a temperature of $40^{\circ} \mathrm{C}$. for two to three weeks. Syntonin or native soluble albumin that may still 
be present, as well as primary albumoses, are removed as just described. The neutral solution is then treated with one-half its volume of a saturated solution of ammonium sulphate. In this manner a two-thirds saturation is effected, and on standing the deutero-fraction A separates out; this is filtered off and the solution saturated with ammonium sulphate in substance. As a result the deutero-fraction B is thrown down, and on acidifying the filtrate with one-tenth its volume of a solution of sulphuric acid that has been saturated with ammonium sulphate, and of which 10 c.c. correspond in strength to 17 c.c. of a one-tenth normal solution of sodium hydrate, the deutero-albumose-C finally separates out on standing. The resulting filtrate is free from albumoses and should contain the amphopeptone of Kühne. Two additional fractions can be obtained from this final solution. To this end, a solution of iodopotassic iodide (containing 2 parts of the iodide to 1 part of iodine) which has been saturated with ammonium sulphate is added until precipitation is complete. The material which is thus thrown down is placed in 96 per cent. alcohol. Peptone-B (Pick) passes into solution, while peptone-A (Pick) remains undissolved. This portion is dissolved in a little warm water, the solution saturated with ammonium sulphate, and reprecipitated with the iodine solution. The peptone is then redissolved in warm water, reprecipitated with alcohol, and freed from any remaining iodine by shaking with ether. Peptone-B, on the other hand, is obtained by evaporating its alcoholic solution to dryness, when the residue is dissolved in water and freed from iodine by shaking with ether.

\section{THE PRODUCTS OF TRYPTIC DIGESTION.}

One hundred grammes of moist fibrin, as in the above experiment, are placed in a liter of an 0.25 per cent. solution of sodium carbonate, to which a few grammes of commercial pancreatin have been added. Putrefaction is guarded against by the addition of chloroform and thymol. The mixture is kept at a temperature of $40^{\circ} \mathrm{C}$., and can be examined after twenty-four to thirty-six hours. For the preparation of antipeptone, however, in amounts which can be utilized to demonstrate the presence of the diamino-acid, it is necessary to take a much larger quantity of fibrin and to extend the period of digestion over several weeks. From 1410 grammes Kutscher claims to have obtained 200 grammes.

The filtered fluid is first neutralized with dilute sulphuric acid, which causes the separation of any alkaline albuminate that may be present. Coagulable albumins are removed by acidifying the solution with acetic acid and boiling. The solution is then treated with one and one-half times its volume of a saturated solution of ammonium sulphate. On standing, the deutero-fraction A separates out. On complete saturation with the salt in substance the deuterofraction $\mathrm{B}$ is obtained; a C-albumose is not formed on tryptic diges- 
tion. On acidifying with sulphuric acid, however, as in the study of peptic digestion, it may happen that a turbidity appears, which is probably due to the presence of Neumeister's antideutero-albumose, resulting from anti-albumid. The final filtrate contains polypeptid and amino-acids in the free state.

\section{Reactions of the Individual Albumoses.}

Whether or not the different albumoses which are formed during the process of digestion are qualitatively the same, irrespective of their origin, is not known, but it is likely that certain differences exist. Quantitative differences also undoubtedly occur, as is suggested a priori by the varying amounts of the individual end-products which can be obtained on hydrolysis. The following account of the individual albumoses is largely based on a study of the fibrinoses.

Hetero-albumose.-Hetero-albumose is more closely related to the original albuminous molecule than any other albumose. It is but little soluble in water and does not dialyze in neutral solution. On heating a fairly concentrated solution in the presence of a moderate amount of salt, partial coagulation occurs between $55^{\circ}$ and $60^{\circ} \mathrm{C}$. ; on further heating, partial solution takes place. On standing hetero-albumose becomes insoluble in part (Kühne's dysalbumose); but upon the addition of a small amount of soda redissolution occurs and hetero-albumose again results. The same occurs, when the heat coagulum is dissolved in dilute hydrochloric acid. A denaturization thus does not occur. In acid solution hetero-albumose is precipitated in toto on half-saturation with sodium chloride. Its limits of precipitation for ammonium sulphate in neutral solution are 2.6 and 4.4. Alcohol precipitates it very readily ; it is insoluble in the presence of 32 per cent. With the usual albumin precipitants hetero-albumose shows a typical albumose reaction (page 63). Its elementary composition is $\mathrm{C}=55.12, \mathrm{H}=6.61, \mathrm{~N}=17.98$, $\mathrm{S}=1.22, \mathrm{O}=19.07$. It contains no earbohydrate group, anu possibly also no tyrosin or tryptophan radicle; it accordingly does not give the Molisch reaction or that of Adamkiewicz. The substance is an anti-body in the sense of Kühne, and is accordingly related to Kühne's anti-albumid and the antipeptone group. On further digestion it yields deutero-albumoses of the $\mathrm{A}$ and $\mathrm{B}$ group, traces of deutero-albumose $\mathrm{C}$, and a considerable amount of Pick's peptone B. On hydrolysis it yields much leucin and glycocoll and 39 per cent. of the total nitrogen in basic form; it also contains the phenyl-alanin complex.

Proto-albumose.-Proto-albumose in its physical behavior shows that it stands further removed from the original albuminous molecule than hetero-albumose. It is quite readily soluble in water and diffuses to some extent through vegetable parchment. It is not coagulated by heat. With nitric acid only partial precipitation occurs. The alkaloidal reagents cause the precipitation of proto- 


\begin{tabular}{|c|c|c|c|c|c|c|c|c|c|}
\hline & 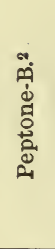 & 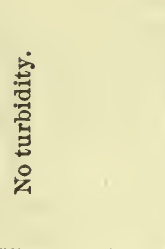 & 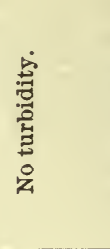 & 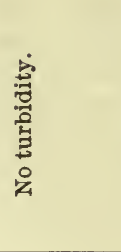 & 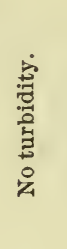 & 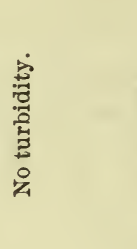 & 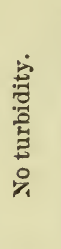 & 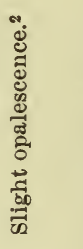 & 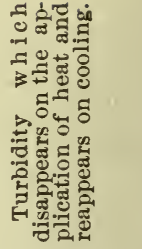 \\
\hline 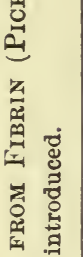 & 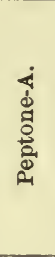 & 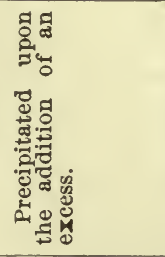 & 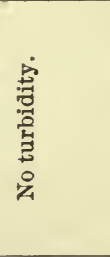 & 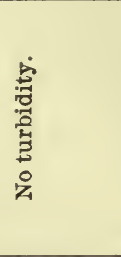 & 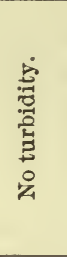 & 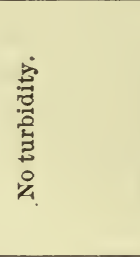 & 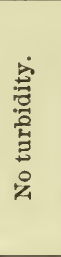 & 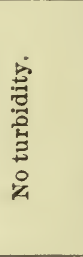 & 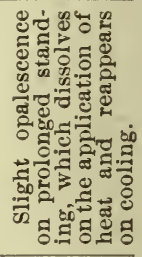 \\
\hline 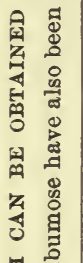 & 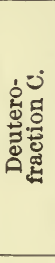 & 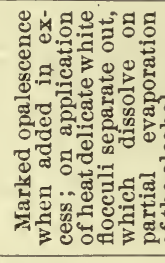 & 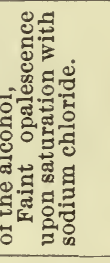 & 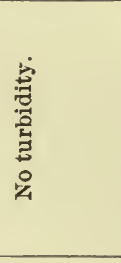 & 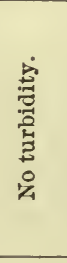 & 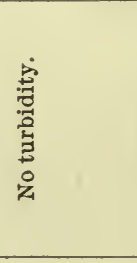 & 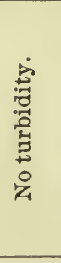 & 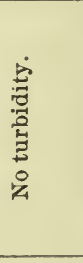 & 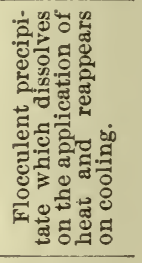 \\
\hline 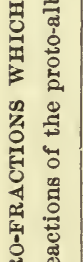 & 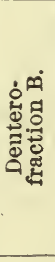 & 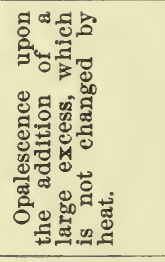 & 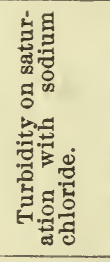 & 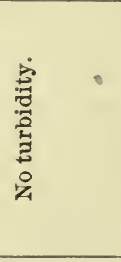 & 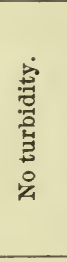 & 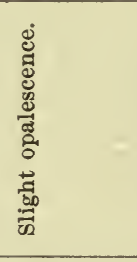 & 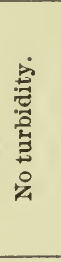 & 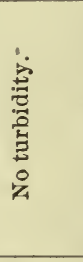 & 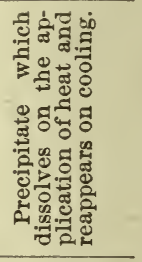 \\
\hline 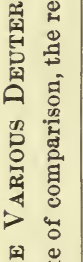 & 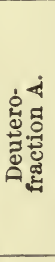 & 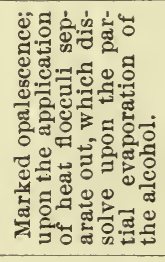 & 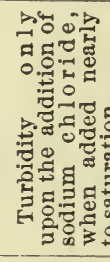 & 兽 & 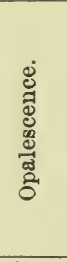 & 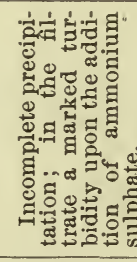 & 苞 & 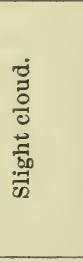 & 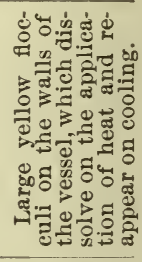 \\
\hline 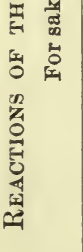 & 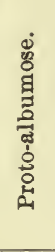 & 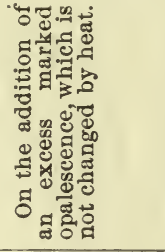 & 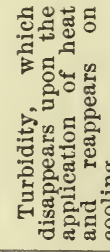 & 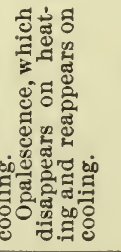 & 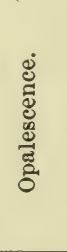 & 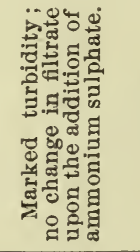 & 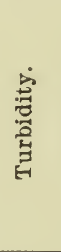 & 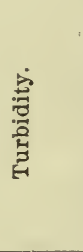 & 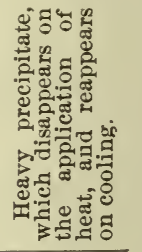 \\
\hline & 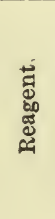 & 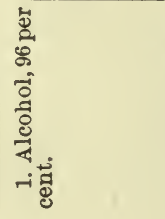 & 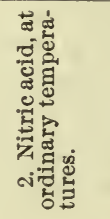 & 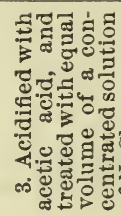 & 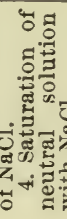 & 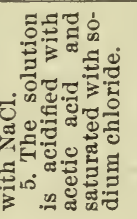 & 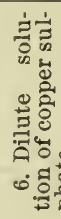 & 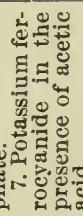 & 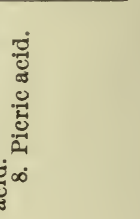 \\
\hline
\end{tabular}




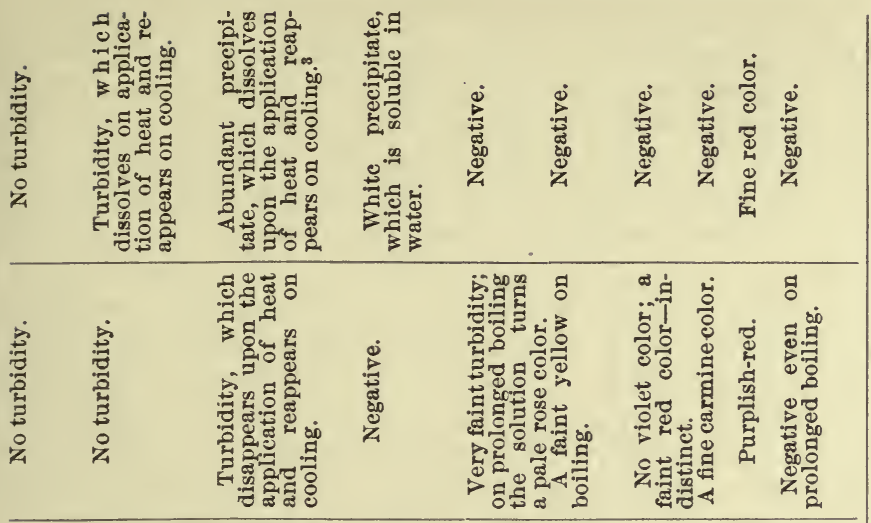

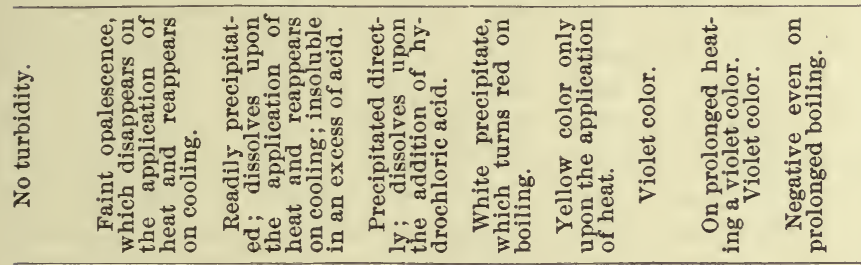

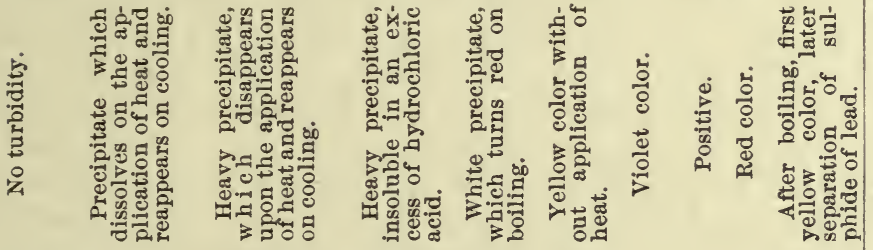

\begin{tabular}{|c|c|c|c|c|}
\hline 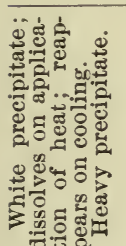 & 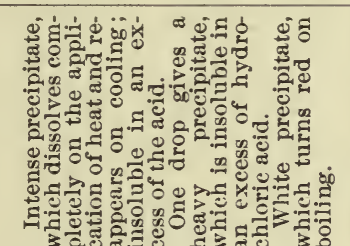 & 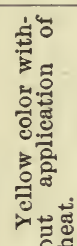 & 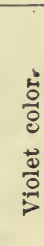 & 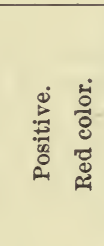 \\
\hline
\end{tabular}

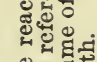

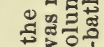

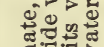

름롤

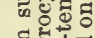

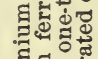

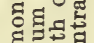

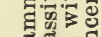

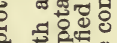

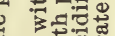

듀:

再范

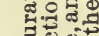

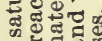

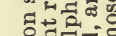

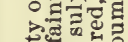

च

달

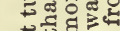

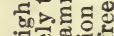

ज记

0.0 .

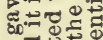

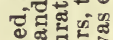

$4{ }^{2}$

때응 땓ㄷㅇ

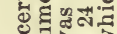

泮司

ธี ह

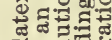

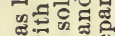
इ三丨

영

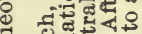

듬

纯

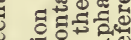

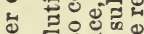

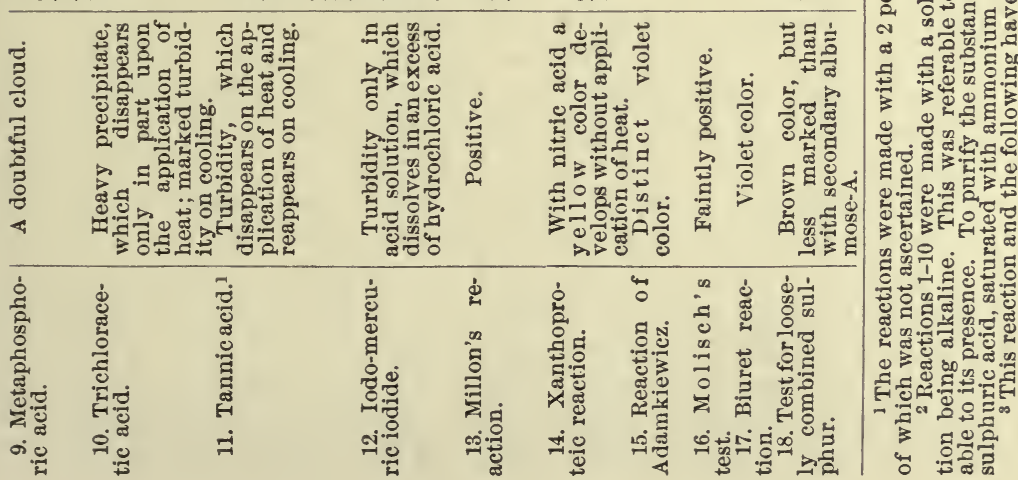


albumose, but the precipitate redissolves in an excess of the reagents. Sodium chloride brings about complete precipitation only in feebly acid solution, on complete saturation. With ammonium sulphate the limits of precipitation are the same as for the hetero-albumose. In dilute alcohol proto-albumose dissolves with ease; precipitation begins in the presence of 80 per cent. (separation from hetero-albumose), and only becomes complete when a mixture of alcohol and ether is added.

The elementary composition is $\mathrm{C}=55.64, \mathrm{H}=6.8, \mathrm{~N}=17.66$, $\mathrm{S}=1.21, \mathrm{O}=18.69$. It is a hemi-body and accordingly readily digested further; it yields much deutero-albumose of the $A$ fraction, some deutero-albumose $B$ and peptone $B$, but no deutero-albumose $\mathrm{C}$ or peptone A. Like hetero-albumose, it contains no carbohydrate group and hence does not give the Molisch reaction. On hydrolysis it yields 25 per cent. of its nitrogen in basic form (diamino-acids), much tyrosin and tryptophan, but little leuein and glycocoll.

The remaining albumoses are not known so well as the two that have been described; the most noteworthy data follow :

Gluco-albumose.-The gluco-albumose (synalbumose) is obtained together with the deutero-fraction $\mathrm{B}$ on complete saturation with ammonium sulphate, the reaction being neutral. It can be separated from the deutero-fraction B by careful treatment with alcohol (see below). Its elementary composition is $\mathrm{C}=48.72, \mathrm{H}=7.03$, $\mathrm{N}=13.61-14.77, \mathrm{~S}+\mathrm{O}=30.49$. There is but little loosely combined sulphur. The substance gives an intense Molisch reaction and is the only primary albumose containing a carbohydrate group. This can be split off by means of acids and yields an osazon, which in appearance resembles glucosazon and melts between $182^{\circ}$ and $184^{\circ} \mathrm{C}$. On further digestion it yields Pick's peptone A; the intermediate products have not been isolated.

Deutero-fraction A. - The deutero-fraction A is precipitated on 62 per cent. saturation with ammonium sulphate. By treating this fraction with 60-70 per cent. alcohol it can be further resolved into two fractions, of which the one (thio-albumose) is precipitated, while the other, deutero-albumose $\mathbf{A}$, passes into solution. The thioalbumose derives its name from the large amount of sulphur which it contains, viz., 2.97 per cent., and which is present almost entirely in loosely combined form (cystin). The elementary composition of this portion is: $\mathrm{C}=48.96, \mathrm{H}=6.9, \mathrm{~N}=16.02, \mathrm{~S}=2.97$, $\mathrm{O}=25.15$.

The deutero-albumose A contains much less sulphur, which is also present in loosely combined form. Its composition is $\mathrm{C}=53.11$, $\mathrm{H}=7.16, \mathrm{~N}=17.86, \mathrm{~S}=0.8$, and $\mathrm{O}=21.07$.

Neither fraction probably contains the carbohydrate group, while the Millon reaction, the xanthoproteic and biuret reactions are positive. On fusion with eaustic alkali indol is obtained.

Deutero-fraction B.-This fraction is obtained together with the gluco-albumose on complete saturation with ammonium sulphate in 
neutral solution. It consists of two fractions, which can be differentiated from each other and the gluco-albumose by means of alcohol. 35 per cent. alcohol will precipitate the deutero-albumose $B$; the gluco-albumose is then thrown down in the presence of 60 to 70 per cent.; while the $\mathrm{B}$ fraction remains soluble even with 80 per cent. Deutero-albumose $\mathrm{B}$, in the case of fibrin at least, is formed only in traces, and may indeed be absent. It does not give the reaction of Molisch ; the biuret reaction is intense and the sulphur reaction marked. It contains 16.94 per cent. of nitrogen.

Deutero-albumose B.-This usually represents the greater portion of the secondary albumoses. It is not absolutely insoluble in alcohol, but a large excess of strong alcohol is necessary to precipitate it from a concentrated solution. The substance does not give Molisch's reaction, nor does it contain loosely combined sulphur; the biuret, xanthoproteic, and Millon's reactions, however, are present and the indol reaction is very pronounced. Elementary analysis of this portion has not given constant results, which suggests that the substance as obtained by Pick was not pure. He found as a matter of fact that his material, which was obtained from Witte peptone, was contaminated by a melanin-like body, which he termed peptomelanin. The analytical figures follow : $\mathrm{C}=43.99-52.32$, $\mathrm{H}=6.91-7.32, \mathrm{~N}=14.25-15.38, \mathrm{~S}=1.63-1.21, \mathrm{O}=33.23-23.79$.

Deutero-fraction C.-This is precipitated on complete saturation with ammonium sulphate, and carefully acidifying with sulphuric acid saturated with ammonium sulphate. It is soluble in 76-80 per cent. alcohol. In the case of fibrin dentero-albumose $\mathrm{C}$ is formed only in small amount. It contains no loosely combined sulphur and does not give the reaction of Molisch nor that of Millon, or to so slight a degree only as to suggest contamination with other fractions. On fusion with caustic alkali it yields neither indol nor skatol. Elementary analysis has given the following results: $\mathrm{C}=34.52, \mathrm{H}=5.35, \mathrm{~N}=17.24$.

The fraction apparently represents an end-product of peptic digestion; it yields no peptone (Pick). It is not formed during tryptic digestion. The specific reactions of the individual fractions are given in the accompanying tables (pages 202 and 203).

\section{THE END-PRODUCTS OF ALBUMINOUS DIGESTION.}

To study the end-products of albuminous digestion it is well to digest fibrin for several weeks with trypsin as just described. Any deutero-albumoses still remaining are removed and the antipeptone fraction isolated as follows :

Antipeptone Fraction.- The mixture is filtered, slightly acidified with acetic acid, boiled, again filtered, and concentrated to about 1000 c.c. On cooling, a good deal of tyrosin separates out and is filtered off. The filtrate is diluted with water to about 2000 c.e., neutralized, heated to near the boiling-point, and saturated with 
ammonium sulphate in substance. On cooling, any albumoses that may have separated out, together with a large quantity of the salt, are filtered off. The filtrate is heated, and, while boiling, rendered strongly alkaline with ammonia and ammonium carbonate, and again saturated with ammonium sulphate. On cooling, a second fraction of albumoses is filtered off. The solution is then leated until the odor of ammonia has disappeared; ammonium sulphate is again added to saturation, and the liquid rendered distinctly acid with acetic acid, when on cooling a third fraction of albumoses separates out and is filtered off. The filtrate is concentrated to about one liter and freed from a large amount of ammonium sulphate, which separates out on cooling. It is then diluted with water to about 3000 c.c., and treated at a temperature of $30^{\circ} \mathrm{C}$. with barium hydrate in substance, to remove the remaining salt. A slight excess of the barium is removed with carbonic acid, and is filtered off after boiling for a moment. The filtrate is evaporated to about 1000 c.c., when the barium peptone is decomposed with dilute sulphuric acid, care being taken that the acid is not added in excess. The resulting barium sulphate is filtered off and the filtrate concentrated to a thin syrup. On cooling, absolute alcohol is added until the turbidity that first appears no longer disappears on stirring. After filtering with the aid of a suction pump, the solution is poured into absolute alcohol while stirring. The antipeptone is then precipitated and allowed to settle, when the supernatant fluid is siphoned off and the antipeptone collected on a filter with the aid of a suction-pump. It is finally washed with absolute alcohol, then with ether, and rapidly placed in a desiccator over sulphuric acid.

From this material arginin, lysin, and histidin may be isolated, as described by Kutscher (Zeit. f. phys. Chem., 1898, vol. xxvi., p. 110).

The Mono-amino-acids. - To study the mono-amino-acids the antipeptone fraction need not be removed. ${ }^{1}$

Leucin.-Aside from its formation during the process of pancreatic digestion or on artificial decomposition of albumin with dilute mineral acids and alkalies, leucin has been demonstrated in the spleen, in the lymph-glands, in the thyroid, the kidneys, the liver, and the brain, though mostly under pathological conditions, when it may also appear in the urine. It is further found in sheep's wool, in decomposing epithelial structures, as in the desquamated material which is found between the toes, etc. It is also formed as the result of bacterial action upon albuminous material.

In pure form leucin crystallizes in extremely thin white lustrous platelets ; but more commonly it is seen in the form of spherules of variable size, which closely resemble globules of fat. In these, concentric striations, as well as very fine radiating lines, can at times be made out on careful examination.

1 For the isolation of the various mono-amino-acids, according to E. Fischer's method, see Zeit. $f$. phys. Chem., 1901, vol. $\mathrm{x} \times \mathbf{x i i i . , ~ p . ~} 151$. 
Several leucins apparently exist. One form, which can be produced synthetically from hydrocyanic acid and ammonium-isovalerianic aldehyde, is optically inactive. The common leucin, on the other hand, which is formed during tryptic digestion or on decomposition of the native albumins with hydrochloric acid, is dextrorotatory. A third form results from the action of Penicilium glaucum upon the inactive substance, and is said to be lævorotatory. An isoleucin has been obtained as a by-product in the production of sugar from beets, and also is a component of various vegetable and animal proteins. It corresponds to an $a$-amino-methyl-ethylpropionic acid :

$$
\underset{\mathrm{C}_{2} \mathrm{H}_{5}}{\mathrm{CH}_{3}}>\mathrm{CH} \cdot \mathrm{CHNH}_{2} \cdot \mathrm{COOH} \text {. }
$$

The common form is easily soluble in water, in alkalies and acids, as also in hot alcohol; in ether it is insoluble. It combines with acids, alkalies, and the oxides of some of the heavy metals to form salts. On boiling a solution of leucin with subacetate of lead the eorresponding compound of lead oxide can be obtained if ammonia is carefully added to the cooled solution. A copper salt is similarly formed if leucin in aqueous solution and containing a small amount of alkali is treated with a solution of cupric sulphate, care being taken not to add an excess. On standing, the compound separates out in the form of elusters of blue needles, which are characterized by their pronounced insolubility.

When carefully heated to a temperature of $170^{\circ} \mathrm{C}$. leucin melts and sublimes in the form of white flakes, which are deposited on the cooler portion of the tube. At the same time the odor of amylamin develops.

On evaporating a small amount of leucin upon platinum foil with nitric acid a colorless residue is formed. If to this a drop of sodium hydrate solution is added and heat is carefully applied, a yellowish or brownish color develops, and on further heating an oil-like droplet is obtained, which rolls about upon the platinum without adhering (Scherer's test).

On decomposition with an alkali or during the process of putrefaction leucin yields ammonia and valerianic acid. On oxidation leucinic acid results.

As has been indicated, leucin is an amino-capronic acid of the formula $\left(\mathrm{CH}_{3}\right)_{2} \cdot \mathrm{CH} \cdot \mathrm{CH}_{2} \cdot \mathrm{CH}\left(\mathrm{NH}_{2}\right) \cdot \mathrm{COOH}$, and may hence be regarded as $\alpha$-amino-isobutyl-acetic acid.

Tyrosin.-Tyrosin can be obtained on tryptic digestion from all those albumins in which aromatic groups exist. Collagen, in which this is absent, accordingly yields no tyrosin, and very small amounts only are obtained from elastin. In the animal body it is practically found as such only under pathological conditions if we disregard the minute quantity which is found in the intestinal canal. Like leucin, it is also formed during the process of albuminous putrefaction, and 
can be obtained artificially by decomposing albuminous substances with dilute mineral acids or alkalies.

While impure tyrosin may occur in the form of spherules similar to those of leucin, the pure substance crystallizes in delicate, silky needles, which are often grouped in sheaves and rosettes. According to its mode of formation, the substance is optically inactive, as when formed synthetically or by decomposition with baryta-water, or it is lævorotatory when derived from albumins on boiling with acids. In cold water it is only slightly soluble, while in boiling water it dissolves in the proportion of 1 to 154 . Its solubility is increased in the presence of alkalies or mineral acids. In alcohol and ether it is insoluble.

Tyrosin may be regarded as para-oxy-phenyl-propionic acid, and has the formula $\mathrm{C}_{6} \mathrm{H}_{4}(\mathrm{OH}) \cdot \mathrm{CH}_{2} \cdot \mathrm{CH}\left(\mathrm{NH}_{2}\right) \cdot \mathrm{COOH}$. It may be formed synthetically from ethylene oxide and para-amino-benzoic acid, and can also be obtained from para-amino-phenyl alanin and para-nitrophenyl alanin. On bacterial decomposition it yields hydroparacumaric acid (para-oxy-phenyl-propionic acid), which can be further transformed into para-oxy-phenyl-acetic acid, and this into paracresol, as has been shown (page 85). On fusion with caustic alkali, in the other hand, it gives rise to the formation of para-oxy-benzoic ucid, acetic acid, and ammonia, as is shown in the equation :

$\mathrm{C}_{6} \mathrm{H}_{4} \succ_{\mathrm{CH}_{2} \cdot \mathrm{CH}\left(\mathrm{NH}_{2}\right) \cdot \mathrm{COOH}}^{\mathrm{OH}}+\mathrm{H}_{2} \mathrm{O}+\mathrm{O}=\mathrm{C}_{6} \mathrm{H}_{4}<{ }_{\mathrm{COOH}}^{\mathrm{OH}}+\mathrm{CH}_{3} \cdot \mathrm{COOH}+\mathrm{NH}_{3}$.

On oxidation with potassium bichromate and sulphuric acid hydrocyanic acid, benzoic acid, acetic acid, and formic acid result.

With acids and alkalies, as also with certain salts of the heavy metals, tyrosin combines with difficulty to form salt-like bodies.

Tests for Tyrosin.- Hoffmann's Test.-With Millon's reagent ty rosin gives the well-known reaction of those albumins in which a:fomatic groups are present, but, as would be expected, in a degree $\mathrm{n}$; uch more intense. The reaction is due primarily to the formation of oxy-benzoic acid (salicylic acid).

Piria's Test. - A few crystals of tyrosin are dissolved in concentrated sulphuric acid and heated to about $100^{\circ} \mathrm{C}$, , when the substance dissolves. Tyrosin-sulphuric acid is thus formed, and gives rise to a red color. On cooling, the liquid is diluted, and treated with barium carbonate while heating until the reaction becomes just alkaline. Tyrosin-sulphonate of barium thus results, which gives rise to a dark-violet color on treating with a very dilute solution of sesquichloride of iron. An excess of iron, however, must be carefully avoided. Oxy-benzoic acid gives the same reaction.

SCHERER's TEST.-On evaporating tyrosin with a few drops of nitric acid on platinum foil a yellow, transparent residue is obtained, which turns red on moistening the substance with a drop of sodium hydrate solution, and becomes brown on further evaporation. The 
reaction is due to the formation of nitro-tyrosin nitrate, but is not characteristic, as other bodies behave in a similar manner.

Mörner's Test.-A small amount of tyrosin, in substance or in solution, is added to a few c.c. of a special reagent which has the composition : formalin, 1 part; distilled water, 45 parts ; and concentrated sulphuric acid, 55 parts. The mixture is boiled, when either at once or a few minutes after boiling has begun a fine green color develops which persists for a long time. Other compounds which are closely related to tyrosin, as also native albumins, albumoses, and albuminoids, do not give the reaction. With chemically pure $p$-oxyphenyl propionic acid and the corresponding acetic acid Mörner had not been able to test his reaction.

Isolation of Leucin and Tyrosin.-To isolate leucin and tyrosin among the final products of tryptic digestion, and to separate the two from each other, the digestive mixture is first freed from alkaline. albuminate, coagulable albumins, and the albumoses, as already described (page 196). The final filtrate is concentrated to a syrupy consistence, when on cooling leucin and tyrosin crystallize out. The mass of crystals is then boiled with a large quantity of water, to which a sufficient amount of ammonia is added to insure solution of the substances. The boiling solution is treated with subacetate of lead until the resulting precipitate appears almost white. The filtrate is bronght to the boiling-point, nentralized with sulphuric acid, and filtered while boiling hot. On cooling, the tyrosin crystallizes out, while the leucin remains in solution. The former can then be purified by recrystallization from boiling water or from very dilute ammonia. The solution which contains the leucin is freed from lead with hydrogen sulphide, and the filtrate is concentrated and boiled with an excess of freshly precipitated cupric hydrate. A portion of the leucin is thus precipitated, while the rest remains in solution, but partly crystallizes out on cooling as the corresponding copper compound. The precipitate is placed in the copper-containing solution, and is freed from copper with hydrogen sulphide; the filtrate is then decolorized with animal charcoal, strongly concentrated, and set aside for crystallization.

Aspartic Acid.-While aspartic acid is apparently formed from all albuminous substances on digestion with trypsin, the largest amounts are obtained from certain vegetable albumins (edestin from hemp-seed, legumin, conglutin of lupinus seed). Like leucin and tyrosin, it likewise results on artificial decomposition of the albumins with dilute mineral acids and alkalies, and is also formed during the process of albuminous putrefaction. Outside the intestinal canal aspartic acid has not been found in the animal body. In the form of its amide asparagin it occurs widely distributed in the vegetable world, and supposedly plays an important rôle in the synthesis of the vegetable albumins.

The substance crystallizes in rhombic prisms, which are soluble with difficulty in cold water, but are quite soluble in hot water. 
In absolute alcohol it is insoluble. Its aqueous solutions are lævorotatory, while in the presence of nitric acid dextrorotation is observed.

As has been shown (page 81), aspartic acid is a dibasic acid of the fatty series. It is amido-succinic acid, and is represented by the formula $\mathrm{CH}_{2} \cdot \mathrm{CH}\left(\mathrm{NH}_{2}\right) \cdot(\mathrm{COOH})_{2}$. It can be obtained from asparagin on boiling with hydrochloric acid, as shown in the equation :

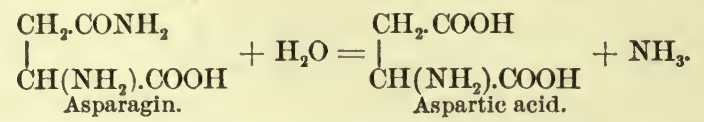

It has also been produced synthetically. On reduction it yields succinic acid.

With cupric oxide aspartic acid forms a crystalline compound which is almost insoluble in cold water, but dissolves in boiling water with comparative ease. This property is utilized for the purpose of isolating the substance from the mixture of digestive products.

Glutaminic Acid.-Glutaminic acid is one of the most constant decomposition-products of the proteins and found in especially large amounts in the vegetable albumins, where its amide glutamin also plays a very important rôle. The largest quantities have been obtained from the gliadin of wheaten flour (31.5 per cent.), zein, and edestin. In the albuminoids the amount is relatively small and in silk fibrin it seems to be absent. Kutscher claims to have found it in the so-called antipeptone of Kühne, which was obtained from fibrin. It is noteworthy that much larger quantities are found if the decomposition of the albumins is effected with hydrochloric acid than with sulphuric acid. Kutscher thus found only 1.8 per cent. among the decomposition-products of casein when using sulphuric acid, while Hlasiwez and Habermann obtained as much as 29 per cent. when hydrochloric acid was used.

Glutaminic acid crystallizes in small glistening crystals, which are soluble with difficulty in cold water, while in boiling water they dissolve with greater ease, but separate out on cooling. With acids and alkalies it combines to form salt-like products, among which the hydrochlorate is conveniently utilized for the purpose of identifying the substance. The melting-point of this compound is $193^{\circ} \mathrm{C}$.

The composition of glutaminic acid is expressed by the formula $\mathrm{CH}_{2} \cdot \mathrm{CH}_{2} \cdot \mathrm{CH}\left(\mathrm{NH}_{2}\right) \cdot(\mathrm{COOH})_{2}$. It is thus amino-glutaric acid, and bears the same relation to glutamin as that which exists between aspartic acid and asparagin. This is represented in the equation :

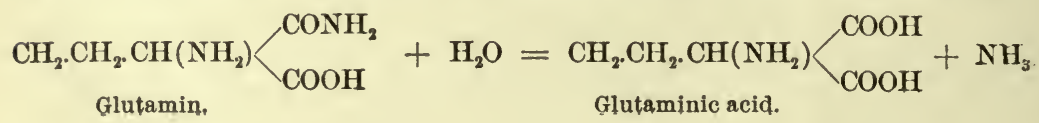


Isolation of Aspartic Acid and Glutaminic Acid.-To isolate the two acids in question among the products of tryptic digestion, the mixture must first be freed from albumins and albumoses, as has been described. 'The remaining solution is acidified with sulphuric acid and precipitated with phosphotungstic acid. The filtrate is freed from sulphuric acid and any excess of the phosphotungstic acid by means of barium hydrate. From the resulting filtrate leucin and tyrosin are then removed by concentration. The motherliquor contains the glutaminic acid and aspartic acid. These are now separated from each other in the following manner: the diluted solution is brought to the boiling-point and digested with carbonate of copper. It is filtered while still hot, and precipitated with subacetate of lead, care being taken to avoid an excess. This precipitate is decomposed with hydrogen sulphide, and the filtrate concentrated to a small volume. On standing a crystalline mass is obtained, which is then dissolved in boiling water and digested with an excess of carbonate of copper, as before. The hot filtrate is again concentrated, when on stanuing the copper salt of aspartic acid separates out in characteristic groups of needles. The filtrate is freed from copper by means of hydrogen sulphide, concentrated, and set aside, when the glutaminic acid crystallizes out.

Glycocoll.-While it is generally known that glycocoll plays an important part in the nitrogenous metabolism of the animal body, and is intimately concerned in the formation of urea, hippuric acid, phenaceturic acid, certain biliary acids, and in birds and reptiles of uric acid, it is of interest to note that the substance has thus far not been met with among the products of pancreatic digestion, although its radicle has been found present in all proteins examined, with the exception of casein and globin, and the so-called BenceJones albumin (which see), when hydrolysis was brought about by means of mineral acids. It is especially abundant in silk fibrin, elastin, and collagen. The hetero-albumose of fibrin, according to Spiro, yields a considerable amount of glycocoll, while from the proto-albumose it cannot be obtained.

Heretofore the isolation of glycocoll and its recognition as such were attended with great difficulties. A somewhat simpler procedure has recently been suggested by Baum. The method is based upon the observation that glycocoll can be transformed into hippuric acid in the test-tube by treating with benzoyl chloride in the presence of sodium hydrate, and that the formation of the resulting hippuric acid can be readily demonstrated by condensing this with benzaldehyde in the presence of sodium acetate and acetic anhydride. The lactimide of benzoyl-amino-cinnamic acid is thus formed. On decomposition with sodium hydrate this yields phenvl-pyro-racemic acid, which in ethereal solution gives a green color on treating with chloride of iron. With phenyl-hydrazin, moreover, it forms an osazon which melts at $161^{\circ} \mathrm{C}$. These changes may be represented by the equations : 
(1)

$$
\underset{\text { Glycocoll. }}{\mathrm{CH}_{2} \cdot\left(\mathrm{NH}_{2}\right) \cdot \mathrm{COOH}}+\underset{\text { Benzoyl chloride. }}{\mathrm{C}_{6} \mathrm{H}_{5} \cdot \mathrm{COCl}}=\underset{\text { Hippuric acid. }}{\mathrm{CH}_{2}} \cdot \underset{\mathrm{NH}\left(\mathrm{C}_{6} \mathrm{H}_{5} \cdot \mathrm{CO}\right)}{\mathrm{N}} \cdot \mathrm{COOH}+\mathrm{HCl}
$$

(2)

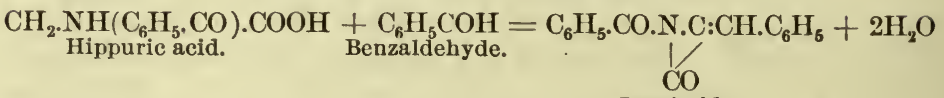
Lactimide.

(3) $\mathrm{C}_{6} \mathrm{H}_{5} \cdot$ CO.N.C:CH.C. $\mathrm{C}_{6} \mathrm{H}_{6}$

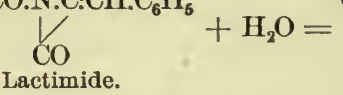<smiles>O=C(O)C(=Cc1ccccc1)C(=O)c1ccccc1</smiles>

(4)

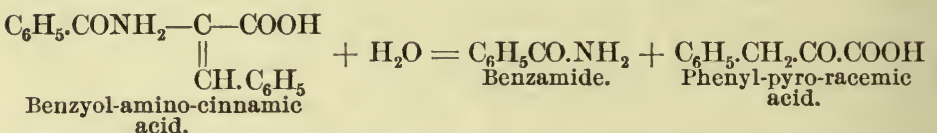

Method.-The decomposition of the albumins (gelatin) is effected by prolonged boiling with dilute sulphuric acid -25 per cent. solution. The excess of acid is removed with plumbic carbonate. The filtrate is concentrated, freed from any tyrosin that may have separated out, and then benzoylated with benzoyl chloride in the presence of sodium hydrate. Care should be had that the reaction of the solution is constantly alkaline during this process. The hippuric acid is then extracted with acetic ether. The dried substance is now treated with three molecules of acetic anhydride, one molecule of sodium acetate, and one molecule of benzaldehyde. The mixture is heated on a water-bath for half an hour. The condensation-product is then treated with water and gently warmed. The oil that separates out is dissolved in hot alcohol and allowed to cool. The lactimide then crystallizes out, and can be recognized as follows: the substance is heated with a strong solution of sodium hydrate until a distinct odor of ammonia is noticed. This is due to the decomposition of the benzamide. On acidifying the solution the phenyl-pyro-racemic acid separates out and can be readily extracted by shaking with ether. One portion of the ethereal extract is treated with a dilute solution of the sesquichloride of iron, when on agitation the watery layer assumes a dark-green color, which gradually changes to a characteristic yellow. The other portion is treated with an ethereal solution of phenylhydrazin, which leads to the separation of the hydrazon of phenyl-pyro-racemic acid. After washing with ether this may be identified by its melting-point $-161^{\circ} \mathrm{C}$.

As regards the general properties of glycocoll and its preparation as such, see pages 87 and 278 ).

Tryptophan.-This substance is apparently always formed when the tryptic digestion of the albumins has extended beyond the formation of albumoses. As its presence among the various digestive products is easily recognized, it is thus possible to ascertain whether the destruction of the albuminous molecule has extended to the formation of amino-acids, without testing for these directly. Like the amino-acids, it is also formed during the hydrolytic decomposition of the albumins with baryta-water, and likewise results during 
the process of intestinal putrefaction. Of special interest is the fact that while the primary albumoses of fibrin, as also the secondary albumose- $A$, on further digestion with trypsin, give rise to the formation of tryptophan, the secondary albumose-B apparently does not contain the chromogenic group.

Hopkins and Cole have shown that tryptophan is skatol-aminoacetic acid, and according to Ellinger it has either the formula:

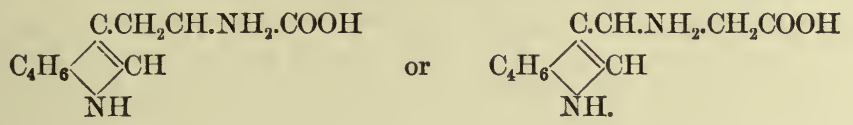

To the presence of this complex in the albuminous molecule the reaction of Adamkiewicz is due.

On bacterial decomposition it yields indol, skatol, skatol carbonic acid, and skatol acetic acid.

With chlorine and bromine it yields at least three colored products, the so-called proteinochromes. Of the bromine products, one is a bluish-violet substance, and contains about 35 per cent. of bromine; the second is a red body, with 27 per cent.; and the third a brown pigment, with the same amount of bromine.

According to Nencki, a certain similarity exists in the percentage composition of the red pigment with hæmatoporphyrin, viz., bilirubin, and of the brown pigment with the so-called melanins. Tryptophan, moreover, like hæmatin and hæmatoporphyrin, yields pyrrol, hydrogen sulphide, methyl mercaptan, indol, and skatol on fusion with caustic alkali.

Test.-The test for tryptophan and the isolation of the three known pigments is conducted as follows: the digestive mixture is acidified with acetic acid and treated with two and one-lialf times its volume of saturated bromine-water. A beautiful reddish-violet precipitate is thus formed, which increases on standing. After twenty-four hours this is filtered off. On the further addition of bromine-water the brown pigment separates out on standing. The red pigment will be found in the violet precipitate, and can be isolated as follows: the precipitate is first washed with water and then extracted with dilute ammonia; this extract is precipitated with acetic acid. The precipitate is separated from the brown filtrate, redissolved in very dilute ammonia, again precipitated with acetic acid, and washed with water. It is then extracted with amyl alcohol; this dissolves the red body. The alcohol is evaporated off at $40^{\circ} \mathrm{C}$., the residue dried at $106^{\circ} \mathrm{C}$., and finally washed with ether. The violet pigment is obtained on further extraction of the violet precipitate with a little stronger solution of ammonia than in the first instance. The substance is precipitated with acetic acid, well washed with water, and extracted with 95 per cent. alcohol. The alcoholic extract is evaporated to dryness at $40^{\circ} \mathrm{C}$., the residue dried at $106^{\circ} \mathrm{C}$. and washed with petroleum ether. 
To isolate the brown pigment, finally, the second bromine precipitate is filtered off, washed with water, dissolved in very dilute ammonia, reprecipitated with acetic acid and washed with water, and briefly with 95 per cent. alcohol, both of which dissolve a portion of the pigment. It is then dried and washed with ether. 'The resulting product is almost black.

For the isolation of the other amino-acids which result on hydrolytic decomposition of the albumins the reader is referred to the journalistic literature upon the subject, and notably the papers by Emil Fischer and his pupils (Zeit. $f$. Physiol. Chem., vol. xxxiii. et seq.). 


\section{CHAPTER X.}

\section{BACTERIAL ACTION IN THE INTESTINAL TRACT.}

I HAVE pointed out in a preceding chapter that the gastric juice possesses marked germicidal and antiseptic properties, so that a large number of bacteria which are constantly swallowed with the saliva and the food are subsequently destroyed in the stomach. A perfect barrier to the invasion of micro-organisms, however, does not exist, and after having passed the pylorus they are placed in surroundings which are in all respects most favorable to their development. Here they take an active part in the decomposition of the various food-stuff's which have escaped digestion in the stomach, and further modify the digestive products which have already been formed, as also those which result from the action of the various intestinal ferments. The greater portion of the products of normal digestion, however, escapes the specific activity of the bacteria, and is absorbed in a form which can be utilized by the body for purposes of nutrition. Formerly it was supposed that the biliary acids played an important part in preventing undue activity on the part of the bacteria, but this view has now been largely abandoned, and we are totally ignorant as to the manner in which the body here protects itself against excessive bacterial action. It has been argued that an accumulation of the decomposition-products which result from the action of bacteria upon the various food-stuffs in itself inhibits the further activity of the organisms, but we can hardly regard such an explanation as valid in view of the fact that in the intestines these decomposition-products are to a large extent absorbed, and it seems more probable that a vital activity of the epithelial cells is here of prime importance. In the small intestine at least, where peristalsis is extremely active, and where the intestinal contents are churned in such a manner that the individual particles are almost constantly in contact with the intestinal walls, we accordingly find that bacterial action is not nearly so extensive as in the large intestine, where the opposite conditions prevail. In the clinical laboratory we find, as a matter of fact, that the degree of intestinal putrefaction increases at once when the peristalsis of the small intestine is impeded, and reaches its greatest height if the secretion of hydrochloric acid becomes arrested at the same time.

In former years a tendency existed among physiologists to regard bacterial action in the intestine as serving a useful purpose, and it was even supposed that, as in the case of plants, animal life could not go on in the absence of micro-organisms from the alimentary 
canal. This view has now been abandoned, however, especially since Thierfelder and Nuttall were able to demonstrate that guineapigs, after removal from the uterus of the mother by Cæsarean section, can be maintained in perfect condition as to health and body-weight when fed on sterile food and when furnished with sterile air exclusively. On subsequent examination it was shown that the intestinal contents of these animals were also sterile. We may thus conclude that the presence of bacteria in the intestinal contents is at best unnecessary, and it is doubtful, indeed, whether they serve a useful purpose at any time.

The action of bacteria upon the food-stuffs is in certain respects quite analogous to that of the digestive ferments which are furnished by the digestive glands of the animal body. The primary digestion of the original material, however, does not cease with the production of substances which the animal can subsequently utilize for anabolic purposes, but is, on the whole, far more extensive. Polysaccharides and disaccharides are thus not only inverted to monosaccharides, but the latter are further decomposed into material which has no nutritive value whatever. Albumins are similarly decomposed, with the ultimate formation of substances which in part at least are distinctly toxic; and the fats are divided into their components, which are then further broken down, with the final formation of fatty acids of the lowest order, etc. A great variety of decomposition-products thus results from the normal foodstuffs, which are further increased by those arising from material which the ferments of the animal itself are incapable of digesting (cellulose derivatives, etc.). To these are added the decompositionproducts of the various biliary constituents and of the albuminons secretions which are poured into the intestinal canal by the digestive glands themselves.

As has been pointed out, the most intense degree of bacterial action is observed in the large intestine, and it is interesting to note that while albuminous putrefaction here prevails, the fermentative processes in the more restricted sense of the term, viz., the decomposition of carbohydrates and fats, occur almost exclusively in the small intestine. This difference may be dependent to a certain degree upon the difference in the reaction of the intestinal contents in the two sections of the gut-that of the small intestine in its lower portion at least being acid, while the reaction of the contents of the large intestine is usually alkaline. But it is also possible that other and still unknown factors determine this difference, and that the varying reaction is primarily due to the decomposition-products directly which result from the action of the bacteria. Among these factors the relative amount of water may be of importance.

Nencki, MacFadyen, and Sieber, who had occasion to study the chemical composition of the intestinal contents in a patient in whom an artificial anus had been established at the distal end of the ileum, give the following account of their observations: The reaction was 
quite constantly acid, owing to the presence of organic acids, and notably of acetic acid. Other acids that were present were lactic acid, paralactic acid, various volatile fatty acids, succinic acid, and the biliary acids. The odor but rarely suggested the existence of putrefactive changes. Indol, skatol, and phenol could not be demonstrated as such, although the urine contained indican on several occasions. Leucin and tyrosin were not found. Alcohol could always be demonstrated. Of gases, carbon dioxide was observed, as also faint traces of hydrogen sulphide, while methylmercaptan was absent.

Carbohydrate fermentation thus manifestly stands in the foreground, and is exemplified in various types by the equations :

(1) $\mathrm{C}_{6} \mathrm{H}_{12} \mathrm{O}_{6}=2 \mathrm{C}_{2} \mathrm{H}_{5} \cdot \mathrm{OH}+2 \mathrm{CO}_{2}$, alcoholic fermentation.

(2) $\mathrm{C}_{2} \mathrm{H}_{5} \cdot \mathrm{OH}+2 \mathrm{O}=\mathrm{CH}_{3} \cdot \mathrm{COOH}+\mathrm{H}_{2} \mathrm{O}$, acetic acid fermentation.

(3) $\mathrm{C}_{6} \mathrm{H}_{12} \mathrm{O}_{6}=2 \mathrm{CH}_{3} \cdot \mathrm{CH}(\mathrm{OH}) \mathrm{COOH}$, lactic acid fermentation.

(4) $2 \mathrm{C}_{3} \mathrm{H}_{6} \mathrm{O}_{3}=\mathrm{C}_{3} \mathrm{H}_{7} \cdot \mathrm{COOH}+2 \mathrm{CO}_{2}+4 \mathrm{H}$, butyric acid fermentation.

The products of albuminous putrefaction, on the other hand, are almost exclusively formed in the large intestine. Primarily they are in part at least the same as those which result from the action of trypsin on albumins, and in experiments in vitro we thus find albumoses, peptone-like bodies, tryptophan, leucin, tyrosin, aspartic acid, and glutaminic acid. In the contents of the large intestine, however, these substances are found only in traces, so that we are forced to the conclusion that they are either absorbed as soon as formed or that they are further decomposed. Both, no doubt, occurs, and related bodies are, as a matter of fact, encountered in the feces. As a result of bacterial activity still other substances are formed, however, which are apparently not derived from the final products of digestion, but which are formed from the more or less intact albuminous molecule directly.

The more important decomposition-products which result from the action of bacteria upon the products of albuminous digestion are here considered.

Indol.-Indol is a derivative of the tryptophan complex, viz., of skatol-amino-acetic acid (which see). Structurally it is closely related to indigo, and according to Nencki, this transformation can be effected through the action of ozone. It is represented by the equation :

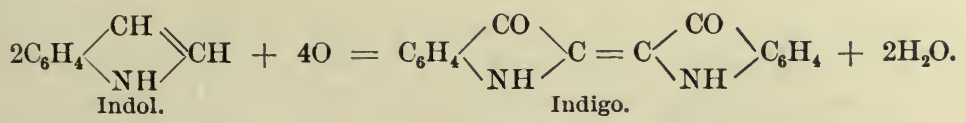

Conversely, indigo can be transformed into indol on reduction.

From the albumins the substance can also be obtained on fusion with potassium hydroxide. 
The greater portion of the indol that is formed in the large intestine is no doubt eliminated in the feces. A certain amount, however, is absorbed, and after oxidation to indoxyl appears in the urine in combination with sulphuric acid as so-called indican (which see). If larger quantities are formed, a variable fraction is further eliminated in the urine as an indoxyl compound of glucuronic acid.

Indol crystallizes in small platelets, which melt at $52^{\circ} \mathrm{C}$, and are soluble in hot water, ether, alcohol, and benzol. Its odor is feculent; it is quite volatile, and when boiled with water passes over into the distillate. With picric acid it forms a beantifully red crystalline compound, which is readily decomposed on boiling with dilute ammonia; the liberated indol is then found in the distillate. On distilling in the presence of sodium hydrate, on the other hand, the indol is decomposed.

Tests.-When treated in aqueous solution with nitric acid and a trace of sodium nitrite a red precipitate of the nitrate of nitrosoindol is formed. This is soluble in alcohol and crystallizes out upon the addition of ether.

If a small piece of pine wood is moistened with strong hydrochloric acid and then placed in a watery solution of indol, it gradually assumes a cherry-red color.

An aqueous solution of indol is treated with a small amount of a solution of sodium nitroprusside until a brownish-yellow color develops. If now a dilute solution of sodium hydrate is added drop by drop, the color changes to violet. Upon the further addition of a little dilute hydrochloric acid this becomes a deep blue, while an excess of the acid destroys the blue color.

On shaking a small quantity of an aqueous solution of indol with a few drops of a 2 per cent. solution of dimethyl-p-aminobenzaldehyde in equal parts of water and concentrated hydrochloric acid, a cherry-red color develops either at once or upon the application of heat.

For the isolation of indol, see page 221.

Skatol.-Skatol, like indol, is a direct derivative of tryptophan, and is likewise formed during the process of albuminous putrefaction. It is a methylated indol, and may be represented by the formula :<smiles>CC(C)(C)Nc1ccccc1</smiles>

By combining with carbon dioxide it gives rise to the formation of skatol-carbonic acid, which is also found in the contents of the large intestine, and belongs to the ortho-series. Its formula is<smiles>CC(C)(C)[C@H](Nc1ccccc1)C(=O)O</smiles> 
Like indol, skatol is also formed on fusing albumins with caustic soda, and can be obtained from indigo on reduction with tin and hydrochloric acid. When passed through a red-hot tube it yields indol. On absorption, it is oxidized to skatoxyl and is eliminated in the urine in combination with sulphuric acid and glucuronic acid, as in the case of indol (see Urine). Skatol-carbonic acid, on the other hand, appears in the urine as such.

Another derivative of skatol is Baum's skatosin- $-\mathrm{C}_{10} \mathrm{H}_{16} \mathrm{~N}_{2} \mathrm{O}_{2}$.

Skatol crystallizes in fine platelets, which melt at $95^{\circ} \mathrm{C}$. and are readily soluble in ether, alcohol, and benzol; in hot water it is soluble with greater difficulty than indol. Its odor is exceedingly offensive. Like indol, it is volatile, and combines with picric acid to form a red crystalline compound. On distilling this in ammoniacal solution or in the presence of sodium hydrate the skatol passes over as such, while indol in the latter instance is decomposed. On distilling a mixture of indol and skatol in aqueous solution the skatol passes over first, and it is thus possible to separate the two substances from each other.

Tests.-From its aqueous solutions skatol is precipitated by yellow nitric acid as a white substance-skatol nitrate.

If a small piece of pine wood is moistened with an alcoholic solution of skatol and then placed in strong hydrochloric acid, it assumes a red color. If, on the other hand, the test is conducted as with indol, no reaction is obtained.

With nitric acid of a specific gravity of 1.2 skatol gives a marked xanthoproteic reaction on boiling-i.e., a yellow color which changes to orange when ammonia is added in excess.

The substance does not give the reaction with sodium nitroprusside.

Isolation (see page 221).

Phenol. - The phenol which is formed during the process of intestinal putrefaction is derived from tyrosin. As has been shown, this is first reduced to hydroparacumaric acid. This in turn is oxidized to para-oxy-phenyl-acetic acid. Paracresol then is formed through a splitting off of carbon dioxide, and on subsequent oxidation phenol results (see page 89). To a certain extent this is eliminated in the feces, but a variable amount is always absorbed, and subsequently oxidized in part to hydroquinon or pyrocatechin. These three bodies then combine with sulphuric acid and are eliminated through the urine in this form. A certain amount of paracresol, moreover, is absorbed as such, and likewise appears in the urine as a conjugate sulphate. According to some observers, indeed, a larger quantity of paracresol is here encountered than of phenol.

Tests. - An aqueous solution of phenol when treated with a few drops of a solution of the sesquichloride of iron assumes an amethyst color, which becomes especially apparent on further dilution with water if much phenol is present.

With bromine-water a crystalline precipitate of tribromophenol is obtained. 
With Millon's reagent a red color develops, which, however, is common to other bodies of this series as well.

Isolation (see page 221).

In addition to phenol, indol, skatol, and skatol-carbonic acid, as also the two hydroxylated benzol-derivatives of tyrosin, viz., paraoxy-phenyl-propionic acid (hydroparacumaric acid) and para-oxyphenyl-acetic acid, we further meet with two non-hydroxylated aromatic acids, which are homologous with benzoic acid, viz., phenylpropionic or hydrocinnamic acid and phenyl-acetic acid. According to Salkowski, these may develop directly from the albuminous molecule, but may also result from tyrosin (see page 85 ).

The non-nitrogenous aromatic acids are in part eliminated in the feces. To some extent, however, they are also absorbed. The hydroxylated acids are then eliminated in the urine either as such, or, like phenol, indol, and skatoxyl, in combination with sulphuric acid, while the non-hydroxylated acids combine with glycocoll, and are eliminated as hippuric acid and phenaceturic acid, as already described.

As regards the fate of the small amounts of leucin, aspartic acid, and glutaminic acid which are also formed during the process of albuminous putrefaction, it seems probable that they are in part absorbed, while a portion no doubt is decomposed in the intestinal canal, with the production of succinic acid, glutaric acid, capronic acid, valerianic acid, butyric acid, and acetic acid. The sulphur of the albuminous molecule is usually set free in the form of hydrogen sulphide, but traces of methyl-mercaptan are also observed, and still further contribute to the offensive odor of the feces.

Of the gases which are constantly present in the contents of the large intestine, methane further deserves especial mention. It is to a great extent, no doubt, referable to the peculiar form of fermentation to which the celluloses are subject. But in part at least it probably also results from the decomposition of the fatty acids and of cholin.

Ptomains are normally not found in the intestinal contents, but may be encountered under certain pathological conditions. In Asiatic cholera and in cases of cystinuria putrescin and cadaverin have thus been isolated, and in other diseases, no doubt, they also occur.

The methods which are employed for the purpose of isolating the more important products of albuminous putrefaction are described in the chapter on the Feces.

\section{BACTERIAL DECOMPOSITION OF THE FATS.}

As in the case of the carbohydrates and albumins, a comparatively small portion of the fats only undergoes bacterial decomposition, and it appears that this principally occurs in the lower portion of the small intestine. As in the case of the lipase of the pancreatic juice, the neutral fats are first decomposed into glycerin and the 
corresponding fatty acids, but the process extends further and as a result a gradual reduction of the higher acids to the lowest forms takes place. To a certain extent these are then absorbed and further decomposed in the body, but a not inconsiderable portion is directly eliminated in the feces, and we accordingly find here representatives of the group, from palmitic, stearic, and oleic acids down to butyric acid and acetic acid. The glycerin is absorbed, and is to a certain extent no doubt utilized in the synthesis of fats.

The lecithins are decomposed in the same manner as under the influence of steapsin, with the formation of glycerin-phosphoric acid, fatty acids, and cholin. Whether or not the latter may then be transformed into neurin is not known, but under normal conditions this probably does not occur. The glycerin-phosphoric acid is subsequently no doubt absorbed together with some of the fatty acids, and appears in the urine as such. The cholin, on the other hand, is further decomposed, with the formation of ammonia, carbon dioxide, and methane.

\section{BACTERIAL DECOMPOSITION OF THE BILIARY CON- STITUENTS.}

In former years it was supposed that the biliary acids after their elimination into the intestinal canal were there largely absorbed and returned to the liver, while a smaller portion was decomposed and eliminated in the feces.

Within more recent years there has been a tendency among physiologists to deny the existence of a circulation of the bile acids, on the basis principally that bile acids could normally not be demonstrated in the blood or in the urine. Croftan, however, has shown that after all such a circulation may exist, for he succeeded in demonstrating the presence of biliary acids in the leucocytes. Occurring in this form, however, it suggests itself that they may play the rôle of foreign matter and do not enter into account physiologically.

That portion of the bile acids which is not resorbed is decomposed in the intestinal canal, and in the human being dyslysins only are encountered in the feces while the amino-radicles have apparently been further broken down. In other animals glycocholic acid has been found, but taurocholic acid apparently always succumbs to the action of the bacteria. Of the fate of the amino-radicles we know little, but it is possible that both are in part further decomposed and in part absorbed. Taurin may then appear in the urine either as such or as tauro-carbaminic acid; but it may, on the other hand, again combine with cholalic acid and reappear in the bile. The glycocoll similarly may in part be transformed into urea ; or it may combine with the non-hydroxylated aromatic acids which are also formed during the process of intestinal putrefaction, and appear in the 
urine as hippuric acid and phenaceturic acid; or it may find its way to the liver and be re-eliminated into the intestinal tract as glycocholic acid.

Bilirubin is reduced to hydrobilirubin during the process of intestinal putrefaction and largely eliminated in the feces as such. This reduction, according to Nencki, MacFadyen, and Sieber, occurs in man, in the large intestine. A portion, however, is probably absorbed and eliminated in the urine as urobilin. 


\section{CHAPTER XI.}

\section{THE FECES.}

I HAVE shown in the preceding chapters that the greater portion of the ingested food is transformed in the gastro-intestinal canal into material which can be utilized by the body for purposes of nutrition, and is there absorbed. A certain proportion, however, invariably escapes digestion, and is partly decomposed by the bacteria of the intestinal canal into the various substances which have been considered in the preceding chapter. These substances in turn are in part absorbed, and are partly eliminated in the feces, together with particles of undigested food and undigestible material which have passed through the digestive tract as such. In addition we find here the various native and decomposition-products of the bile, the pancreatic juice, the enteric juice, in so far as they have not been absorbed, together with intestinal mucus, desquamated epithelial cells, and bacteria.

Consistence and Form.-The consistence and form of the feces are principally dependent upon the amount of water that is present, and vary in different animals. Generally speaking, they are softer in the herbivorous animals than in the carnivora. In man they usually occur in the characteristic plastic, cylindrical form, but they may at times be mushy, or round and hard, even in health.

Amount.- The amount of fecal material which is eliminated in the twenty-four hours depends primarily upon the amount and the character of the food that has been ingested. In man it normally varies between 100 and 200 grammes, but may diminish to 60 grammes or rise to 250 grammes, even in health, according to the preponderance of animal food or of vegetable material, which has entered into the composition of the diet.

Odor.-The disagreeable odor of the feces is largely due to indol and skatol, but may be further increased by the presence of hydrogen sulphide, methane, and methyl-mercaptan.

Color.-The color varies with the character of the food ingested, and is usually but little influenced by the decomposition-products of the biliary pigments. In carnivorous animals the feces are almost black, owing to the presence of hæmatin and sulphide of iron. In adult man the color normally varies from a light to a dark brown. In infants in which the bile-pigments appear as such the feces are of a bright-yellow or a greenish-yellow color.

At times and apparently under normal conditions stools are also passed which are grayish white in color and closely resemble the so-called acholic stools which are observed in cases of biliary ob- 
struction. Fats, however, are not necessarily present in increased amounts, and there is no reason to assume that the biliary passages are not patent or that no bile is being secreted. Possibly the lack of color in such stools is referable to the formation of colorless decomposition-products of bilirubin, such as the leuko-urobilin of Nencki, and in the last instance to the presence in the intestinal canal of micro-organisms which are usually absent. Nothing definite is, however, as yet known of the conditions which favor the formation of such products.

Macroscopic Constituents. - On macroscopic examination of the feces we frequently find undigested particles of food, such as skins of berries, large pieces of connective tissue, woody vegetable fibres, undigested pieces of apples, pears, potatoes, grains of corn, flakes of caseïn, etc.

Microscopic Constituents.-On microscopic examination we usually find undigested bits of muscle-fibre, connective-tissue of the white fibrous variety, fragments of the framework of vegetable matter, often still enclosing cells with starch-granules, flakes of caseïn, globules of fat, fatty acid needles, crystals of calcium oxalate, neutral calcium phosphate, ammonio-magnesium phosphate, calcium lactate (these are seen especially in children on a milk diet), and more rarely of calcium carbonate, calcium sulphate, and cholesterin. So-called Charcot-Leyden crystals, which consist of the phosphate of spermin, are in my experience only found under pathological conditions. We further meet with more or less disintegrated epithelial cells, a few leucocytes, bits of mucus, and, above all, with innumerable micro-organisms. Often, indeed, it appears as though the stools consist of these exclusively. Their number, even in health, is enormous. Sucksdorff thus found in his own person that on an average $53,124,000,000$ were eliminated in the twenty-four hours.

Reaction.-In adult man the reaction of the stools is usually alkaline, sometimes neutral, and but rarely acid. Acid stools, on the other hand, are the rule in infants.

General Chemical Composition.-A general idea of the average composition of the human feces may be formed from the following analyses, which are taken from Gautier, and have reference to 1000 parts by weight of the fresh material:

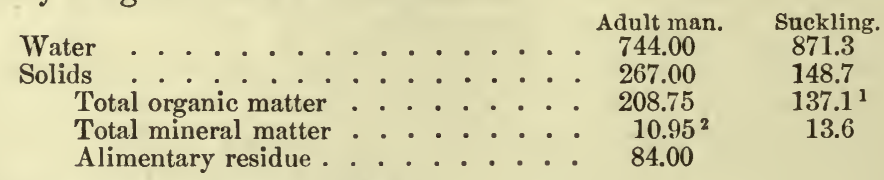

The organic material yielded:

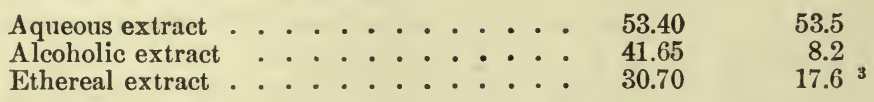

Including 54 parts of mucus, epithelium, and calcareous salts.

2 Not comprising earthy phosphates.

3 Of this, 3.2 parts of cholesterin. 
The individual constituents of the feces may be grouped as follows :

1. Food-material which has escaped the process of digestion, and of bacterial decomposition, such as starches, muscle-tissue, connectivetissue, fats, etc.

2. Undigestible material, which has been ingested as such, or which has resulted from the decomposition of complex substances which are partly digestible, such as gums, pectins, resins, chitin, chlorophyl, hæmatin, and insoluble silicates, sulphates, phosphates, etc.

3. Derivatives of the bile, such as the dyslysins, cholesterin, and exceptionally the native biliary acids as such, and further hydrobilirubin, stercobilin, etc.

4. Intestinal mucus.

5. Products of albuminous digestion, such as albumoses, peptonelike bodies, leucin, tyrosin, aspartic acid, and glutaminic acid.

6. Products of bacterial action. These comprise the entire series of fatty acids from acetic acid to palmitic acid; further, lactic acid, succinic acid, glutaric acid, leucin, tyrosin, hydroparacumaric acid, para-oxy-phenyl-acetic acid, phenyl-propionic acid, phenyl-acetic acid, phenol, paracresol, indol, skatol, skatol-carbonic acid, ammonium carbonate, ammonium sulphide, and conjugate glucuronates.

7. Products of metabolism, which are in part eliminated through the intestines, such as uric acid, urea, xanthin bases, etc.

8. Water.

9. Gases, which are in part referable to the various fermentative and putrefactive processes which take place in the intestinal canal, such as carbon dioxide, methane, hydrogen, hydrogen sulphide, methyl-mercaptan, and phosphin. The nitrogen, on the other hand, which is also constantly met with, is probably derived from the blood, and has in part been swallowed.

Many of these substances have already been considered in detail, and it will suffice at this place to indicate the manner in which the most important products of albuminous putrefaction can be isolated from the feces.

\section{ANALYSIS OF THE PRODUCTS OF ALBUIMINOUS PUTREFACTION.}

The feces are diluted with water, passed through a muslin filter to remove particles of food-material, and distilled until about fourfifths of the entire volume have passed over. The distillate B contains indol, skatol, phenol, paracresol, and the volatile acids which are present in the free state, while the remaining products of putrefaction are found in the residual solution $A$. The distillate $B$ is neutralized with sodium carbonate and redistilled. This second distillate, C, contains indol, skatol, phenol, and paracresol, while the volatile acids remain behind as sodium salts, and can be sepa- 
rated from each other according to usual analytical methods. Distillate $\mathrm{C}$ is now rendered alkaline with sodium hydrate and extracted with ether by shaking. This takes up the indol and skatol, which may be obtained in crystalline form on evaporation of the ether, and can be separated from each other by fractional distillation with steam, when the skatol passes into the distillate first. The residual solution of $\mathrm{C}$ contains phenol and paracresol as sodium compounds. This is now acidified with sulphuric acid and distilled, when the phenols pass over, and may then be separated from each other as described elsewhere.

The residual solution $\mathbf{A}$ is now concentrated and treated with a large excess of alcohol, in order to precipitate any albumins and mineral salts that may be present. 'The alcoholic filtrate is then transformed into an aqueous solution and acidified with sulphuric acid. The aromatic acids are thus set free and are extracted with ether by shaking. The ethereal solution is evaporated to dryness, the residue dissolved in a small amount of a dilute solution of sodium hydrate, and precipitated with barium chloride. The fatty acids are thus obtained as barium soaps, and are filtered off. They are placed in water, which dissolves the salts of the aromatic acids. In this solution the acids are then set free by acidifying with sulphuric acid, and are extracted with ether. The ethereal extract is now evaporated to dryness and the free acids dissolved in water. On distillation in a current of steam, phenyl-propionic acid and phenyl-acetic acid pass over, and can be subsequently separated from each other by fractional distillation. The hydroxylated oxyacids and skatol-carbonic acid remain behind. The latter separates out, on further concentration of the solution and cooling, in the form of white wart-like granules, while the oxy-acids remain behind, and can be separated from each other by means of their varying solubility in benzol. They can be recognized by applying Millon's test. Skatol-carbonic acid, on the other hand, reacts in very much the same manner with yellow nitric acid as does indol, but the red color is in this case referable to a different pigment (see also page 273 ).

Hydrobilirubin.- It has been stated that bilirubin under the influence of bacterial action supposedly undergoes a process of reduction in the intestinal canal and is transformed into hydrobilirubin. It is assumed that as such it is then in part absorbed and possibly appears in the urine, while the remaining portion is eliminated in the feces. According to some observers, it is identical with the stercobilin of Vanlair and Masius. The spectrum of the two bodies is very similar, but while solutions of hydrobilirubin on treatment with chloride of zinc and ammonia show three bands of absorption, stercobilin is said to give rise to four bands. Garrod claims that stercobilin is identical with the urobilin of the urine, and differs from hydrobilirubin in containing a much smaller percentage of nitrogen, viz., 4.11, as compared with 9.22. According to the same observer, 
lyclrobilirubin is a laboratory product, and is met with neither in the feces nor the urine.

Excretin.-This is a substance which was first isolated by Marcet from the feces of herbivorous animals, but is said to occur also in human stools. According to Hinterberger, it has the formula $\mathrm{C}_{20} \mathrm{H}_{36} \mathrm{O}$, and is thus closely related to cholesterin.

Stercorin.-Stercorin, or serolin, as it has been called, is a substance which Flint obtained from the feces of man, but which is probably an impure form of cholesterin, both having the same general reactions.

\section{MECONIUM.}

The term meconium has been applied to the material which accumulates in the intestinal tract during foetal life, and which is expelled soon after birth. Food-products are here, of course, wanting, and as the intestinal tract of the foetus is free from bacteria the meconium consists essentially of mucus, desquamated epithelial cells, and the normal biliary constituents which are present in the intestinal tract before birth. We accordingly find bilirubin and biliverdin, the former often in crystalline form, the native biliary acids, a small amount of fatty acids, cholesterin, and mineral salts, while hydrobilirubin, the dyslysins, leucin, tyrosin, indol, skatol, lactic acid, albumoses, etc., are absent. According to Zweifel, it contains from 79.8 to 80.5 per cent. of water and from 19.5 to 20.2 per cent. of solids, of which 0.978 is referable to mineral ash, 0.797 to cholesterin, and 0.772 to fatty acids.

Its color is a dark brownish-green, and the reaction usually acid. In general appearance it resembles pitch, and is hence also spoken of by the Germans as Kindspech (infant pitch). 


\section{CHAPTER XII.}

\section{THE URINE.}

THE urine is by far the most important excretory product of the animal body, and the medium through which the end-products of nitrogenous metabolism and soluble mineral salts are almost exclusively eliminated under normal conditions. Abnormal products of metabolism also, and many substances which have found their way into the circulation from without, and are foreign to the body, are likewise removed in this manner, either as such or in a more or less modified form. All these substances are found in the urine in aqueous solution, and it is to be noted that of the total amount of water which is daily excreted at least 50 per cent. appears in this form.

Formerly, it was supposed that the various elements which are found in the urine, and notably the mineral salts and water, were eliminated by a simple process of osmosis. Later it was shown, however, that in the elimination of the organic constituents at least the renal epithelium of the uriniferous tubules plays an active part, and it now appears, indeed, that all the substances which occur in the urine, including a certain amount of water even, are removed from the blood, viz., the lymph, through the active intervention of the epithelial cells. It is supposed, moreover, that these structures possess certain selective properties, and we can accorlingly understand why the composition of the blood remains constant.

The kidneys cannot be regarded as simple excretory organs, however, for we know that important synthetic processes also take place in them, the object of which is to transform certain substances which may occur in the circulating blood into compounds that can be more readily eliminated. The most important synthesis of this kind is that of glycocoll and benzoic acid, which results in the formation of hippuric acid (which see).

\section{GENERAL CHARACTERISTICS OF THE URINE.}

The general appearance of the urine varies in different animals. In man it is perfectly transparent when recently passed, but soon becomes turbid, and on standing deposits a light, flocculent sediment, which consists of a mucinous body and a few epithelial cells and leucocytes that are derived from the urinary passages.

In addition, a small number of crystals of uric acid or of oxalate of calcium may be seen. The supernatant fluid is then per- 
fectly clear, and remains so if care is taken to prevent the access of micro-organisms. If left exposed to the air, however, bacterial decomposition soon takes place. Ammonia appears in the free state, and as a consequence of the change in reaction certain constituents of the urine are precipitated and render the liquid turbid. Sooner or later they settle to the bottom, but owing to the presence of innumerable micro-organisms the supernatant fluid remains cloudy. Such urine is said to have undergone ammoniacal decomposition.

A formation of sediments, aside from the light cloud which develops in every urine on standing for a short while, may, however, also occur in the absence of micro-organisms. In the winter-time it is a common experience to see the entire volume of urine become turbid when kept in a cold room. This is owing to the fact that the urates of the urine are very much less soluble in cold than in warm water, and are hence thrown down. On standing, they soon settle to the bottom, and the supernatant liquid remains clear so long as bacterial decomposition does not occur. A similar formation of sediments is observed if the reaction of the urine is alkaline, owing to the presence of fixed alkali in contradistinction to free ammonia. This may at times be observed after a large meal, or after the administration of sufficiently large amounts of alkalies as such, or of substances which are oxidized to alkaline carbonates within the body. In such an event the urine may be clear when first passed, but after standing a short time it becomes turbid, and deposits a sediment of phosphates and carbonates of the alkaline earths. The change is, no doubt, due to an escape of the carbon dioxide which was present in solution. But here also the supernatant liquid is clear.

In herbivorous animals, by which an alkaline urine is passed habitually, the liquid is turbid when discharged. In man the passage of a turbid urine is always abnormal, excepting during the first days of life, when cloudy urine is the rule. This is largely referable to desquamated epithelial cells and relatively large amounts of urates.

While the urine of all mammalian animals is liquid, the lower animals excrete a urine that is more or less solid. In birds and reptiles, for example, in which the ureters end in a common cloaca with the rectum, the excrements appear in the form of a whitish pasty material. A gelatinous urine is observed in turtles.

The color of the urine in man normally varies from light yellow to dark amber, and is largely influenced by the concentration of the secretion and its reaction. Acid urine is thus always darker than an alkaline urine, and the color is naturally lighter when the secretion is abundant than when scanty. A gradual darkening of the urine is observed when the material is kept for some time and access of micro-organisms is prevented.

Deviation from the normal color is notably observed in disease, or following the administration of various drugs, but may also occur in 
apparently healthy individuals in consequence of certain abnormalities of metabolism (see page 274). The urine may then be of a normal color when recently passed, but soon darkens on standing, and finally appears almost black. 'In diabetes a light color may be associated with a high specific gravity.

The odor of recently passed urine is peculiarly aromatic, and is probably referable to the presence of several volatile acids. Decomposing urine has a characteristic odor, which is in part due to ammonia.

Amount.-The amount of urine eliminated in the twenty-four hours is quite variable even under normal conditions. It is, of course, primarily dependent upon the amount of water ingested, but is also influenced by the character and the quantity of the food, the process of digestion, the blood-pressure, the surrounding temperature, the emotions, sleep, exercise, body-weight, sex, age, etc. It must hence differ in different countries, according to the habits of the people, the climate, etc., and we accordingly find that different observers give different figures. In Germany and Austria, where much beer is consumed, from 1500 to 2000 c.c. are regarded as average amounts. In England 1000 to 1500 c.c. are regarded as normal; in France, 1250 to 1300 c.c.

In this country I have found that the average daily amount is somewhat lower, and am inclined to regard an elimination of from 1000 to 1200 c.c. as normal for men, while in women a somewhat smaller quantity is normally passed. Children pass absolutely less but relatively more urine, as compared with their body-weight, than adults.

In the summer-time, when the sweat-glands are especially active, and when larger amounts of water are eliminated through the lungs and the skin, the secretion of urine is proportionately less, but rarely falls below 800 c.c. unless active exercise is indulged in at the same time.

During repose, moreover, much less urine is voided than when exercise is taken, and we hence find a smaller secretion of urine during the night than during the day. The maximum secretion is usually observed a few hours after the midday meal.

Artificially, the secretion can be increased by the ingestion of coffee, tea, and alcohol. Many drugs also bring about the same effect. The most important medicinal diuretics are digitalis, squill, broom, juniper, nitrous ether, urea, etc. Distilled water also has distinct diuretic properties.

In disease, and notably in diabetes mellitus, diabetes insipidus, and chronic interstitial nephritis, the amount of urine may far surpass the usual quantity, and may indeed exceed 10,000 c.c. in the twenty-four hours (polyuria). Abnormally small amounts, on the other hand (oliguria), are observed in the acute febrile diseases, in various diseases of the circulatory apparatus, in certain diseases of the kidneys and liver, etc. Complete anuria may indeed occur. 
Specific Gravity.-The specific gravity of the total amount passed in twenty-four hours normally varies between 1.015 and 1.025. Generally speaking, it increases with the solids, the amount of water remaining the same, and diminishes as the amount of fluid increases while the solids remain constant. Under pathological conditions, however, deviations from this rule are not uncommon. The specific gravity may then fall as low as 1.000 and 1.002 , or may be increased to 1.050 and even higher.

Reaction.-The reaction of the twenty-four hours' urine is, in man, normally acid, sometimes amphoteric, and more rarely alkaline. The normal acidity is due in part to acid phosphates and in part to free organic acids. An alkaline urine results when the alkalies exceed the acid equivalents in amount. This may occur under normal conditions, and is then due to a preponderance of monacid over diacid phosphates. An amphoteric urine is the outcome when the acid equivalents of disodic phosphate equal the basic equivalents of the monophosphate, and essentially an accidental event.

The acidity of the urine is primarily due to the character of the diet. A vegetable diet, owing to the large amount of organic acids which are broken down to alkaline carbonates, tends to produce an alkaline reaction, while an animal diet leads to the secretion of an acid urine ; as in the latter case acid phosphates and certain organic acids predominate, which are not transformed to carbonates (hippuric acid, uric acid, oxalic acid, aromatic oxy-acids, etc.).

In the herbivorous animals in which a superabundance of alkaline salts is either directly ingested or is formed within the body from salts of organic acids which have been taken with the food, an alkaline urine is thus normally eliminated. Similar conditions at times occur in man, and the elimination of an alkaline urine, the alkalinity being due to fixed alkali, cannot hence be regarded as pathological. During the process of digestion, indeed, when an additional amount of alkaline salts finds its way into the blood in consequence of the formation of hydrochloric acid, an increased alkalinity of the blood would result. This, however, is prevented by the excretion of a urine which, if not alkaline, is at least less acid.

Generally speaking, the ammonium salts which are formed within the body appear in the urine as urea, but aside from their importance in this respect they represent a reserve of alkali which is capable of preventing an undue diminution in the alkalinity of the blood by vicariously taking the place of the fixed alkalies. This vicarious action is normally also at work, but is then comparatively insignificant. If, however, a specially large demand is made upon the alkalies of the body, as when mineral acids are ingested for experimental purposes, the vicarious action of the ammonium salts at once enters into play. Unless carried to extremes, the alkalinity 
of the blood, in the carnivorous animals at least, remains constant, but the elimination of urea is proportionately less, and the deficit of nitrogen in this form appears as ammonia in combination with acids (lactic acid).

By gradually increasing the amount of acid it is thus possible to bring about the almost complete disappearance of urea from the urine. A point, however, is finally reached where the animal succumbs to acid intoxication. Death in such cases results from tissucsuffocation, as there is not sufficient alkali left in the lymph and plasma to combine with the carbon dioxide in the tissues.

Conversely, it is possible to cause the ammonia to disappear from the urine by the administration of a sufficiently large quantity of alkali, and as a consequence an increase in the amount of urea occurs which is directly proportionate to the amount of ammonia formerly present. In herbivorous animals, in which such a vicarious action is never necessary under normal conditions, it is accordingly but little developed, and they hence soon die even after the administration of comparatively small amounts of mineral acids.

When allowed to stand exposed to the air, every urine undergoes ammoniacal decomposition. This is owing to the action of certain micro-organisms upon urea, which is decomposed, with the formation of ammonia, water, and carbon dioxide, as shown in the equations :

$$
\begin{aligned}
& \text { (1) } \mathrm{CO}\left(\mathrm{NH}_{2}\right)_{2}+2 \mathrm{H}_{2} \mathrm{O}=\left(\mathrm{NH}_{4}\right)_{2} \mathrm{CO}_{3} \text {. } \\
& \text { (2) }\left(\mathrm{NH}_{4}\right)_{2} \mathrm{CO}_{3}=2 \mathrm{NH}_{3}+\mathrm{H}_{2} \mathrm{O}+\mathrm{CO}_{2} \text {. }
\end{aligned}
$$

As a result of the presence of free ammonia, the soluble phosphates of the alkaline earths are then precipitated as tricalcium phosphate and as ammonio-magnesium phosphate, and the soluble urates are at the same time transformed into the insoluble ammonium salt.

At times an increase in the acidity of the urine is observed on standing, and is generally ascribed to a peculiar acid fermentation of contained alcohol, traces of carbohydrates, and the like. More often, however, a decrease in the acidity occurs, even though microorganisms are absent. This is owing to a decomposition of neutral urates by the acid phosphate of sodium. Acid urates thus result, and may be further decomposed, with the liberation of uric acid. Both urates and uric acid are then thrown down in consequence of the diminished acidity of the fluid, and they are hence no longer capable of influencing the reaction. The changes which here take place may be represented by the equations :

(1) $\mathrm{NaH}_{2} \mathrm{PO}_{4}+\mathrm{C}_{5} \mathrm{H}_{2} \mathrm{Na}_{2} \mathrm{~N}_{4} \mathrm{O}_{3}=\mathrm{Na}_{2} \mathrm{HPO}_{4}+\mathrm{C}_{5} \mathrm{H}_{3} \mathrm{NaN}_{4} \mathrm{O}_{3}$.

(2) $\mathrm{C}_{5} \mathrm{H}_{3} \mathrm{NaN}_{4} \mathrm{O}_{3}+\mathrm{NaH}_{2} \mathrm{PO}_{4}=\mathrm{Na}_{2} \mathrm{HPO}_{4}+\mathrm{C}_{5} \mathrm{H}_{4} \mathrm{~N}_{4} \mathrm{O}_{3}$.

As the reaction of the urine is dependent in the first instance upon the character and the quantity of the food ingested, viz., the amount 
of albumins and alkaline salts present, or of salts which can be transformed within the body into alkaline carbonates, it follows that a highly acid urine must also result when an increased destruction of tissue-albumins is taking place from whatever cause. We accordingly find a very acid urine in various pathological conditions, notably in fevers.

An alkaline urine will similarly result when, as in pneumonia and in diseases in which large accumulations of fluid occur in the serous cavities of the body, and where a certain amount of alkaline salts has thus been withdrawn from the circulation, absorption stibsequently occurs. Alkaline salts are, however, retained from the ingested food, and an increased elimination occurs when the additional supply finds its way into the plasma from these various sources. A notable change in the normal alkalinity of the blood can hence scarcely occur so long as a sufficient amount of alkali is furnished in the food.

In order to decide whether the alkaline reaction of a specimen of urine is due to the presence of fixed or volatile alkali, a strip of red litmus-paper is clamped in the cork of the bottle, and so arranged as not to touch the liquid. If free ammonia is present, the red color changes to blue, while fixed alkali is indicated only when the paper comes into contact with the urine.

Determination of the Acidity of the Urine.-The total acidity which indicates the acidity due to diacid phosphates and free organic acids is first obtained as follows : 25 c.c. of urine are treated with 1 , or at most 2 drops of an 0.5 per cent. alcoholic soltition of phenolphthalein and 15-20 grammes of powdered potassium oxalate. The solution is shaken for a minute and titrated at once with decinormal sodium hydrate solution until a faint, yet distinct pink color, is obtained. The flask should be shaken during the titration so as to keep the solution as strong as possible in oxalate. The acidity is expressed in terms of decinormal sodium hydrate solution, for the total amount of urine of twenty-four hours. The total acidity is termed T.

In a second specimen the total phosphates are then determinedP. (see p. 233). The result is expressed in terms of decinormal acid, viz., alkali as above ( 1 c.c. $\frac{n}{10}=7.1$ milligrammes of $\mathrm{P}_{2} \mathrm{O}_{5}$ ). T.-P. then indicates the acidity due to uncombined organic acids (O. A.), and the difference the mineral acidity (M. A.)

It may happen that the acidity calculated from the total phosphates is greater than the titrated acidity; in that case practically no free organic acids are present and the titrated acidity represents the amount of phosphates present in the diacid form. Urines of this kind are turbid, unless they are also free from calcium (Folin).

As average normal value for the acidities of the total bulk of the twenty-four-hour urine Folin obtained 617 (c.c $\frac{1}{10}$ n. acid, viz., alkali), of which 304 was referable to mineral and 313 to organic 
acidity. The corresponding minimal and maximal values were ' $\mathrm{I}$ '. 554, viz., 669 ; M. A. 204, viz., 417 ; O. A. 252, viz., 378.

Chemical Composition of the Urine.-A general idea of the chemical composition of the urine and the quantitative variations of the individual components may be formed from the accompanying table, which I have constructed from numerous analyses made in my laboratory. The individuals from which the urine was obtained were all adults, and in their general mode of life, as regards diet, exercise, etc., followed the common habits of the average American city-dweller.

ANALYSis OF URINe.

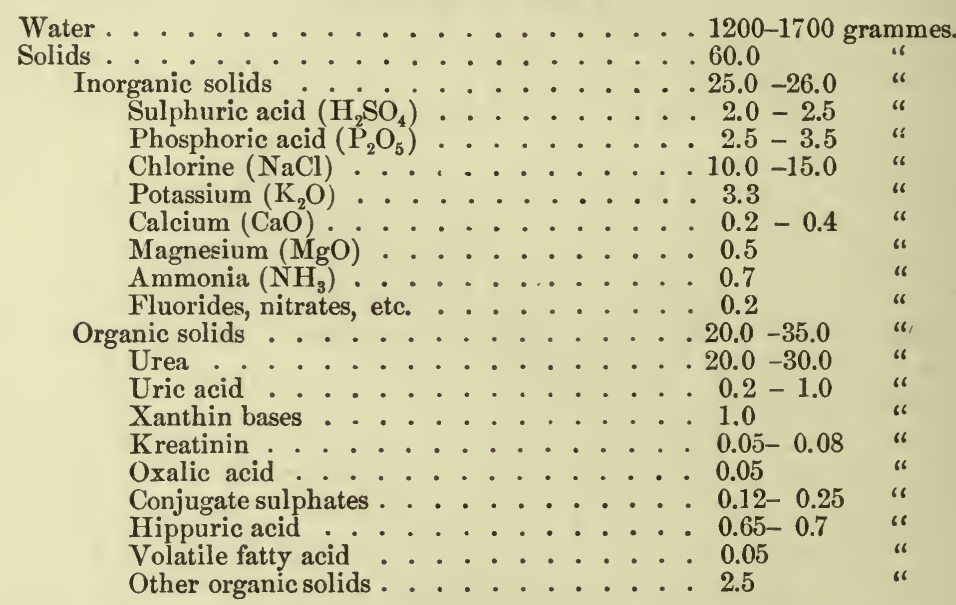

\section{THE INORGANIC CONSTITUENTS OF THE URINE.}

The inorganic constituents of the urine represent the excess of mineral salts which find their way into the blood from the digestive tract, or which develop within the body during the decomposition of the albumins. As has been indicated, they are eliminated through the specific activity of the renal epithelial cells, so that the composition of the blood always remains constant. We accordingly find that the ingestion of large amounts of food invariably leads to an increased elimination of salts, and that conversely smaller amounts are excreted when smaller amounts are ingested. This is true more especially of the chlorides and the phosphates, while the sulphates are largely referable to albuminous destruction, and are only ingested as such in minimal quantities. As the chlorides, moreover, are far more abundant in food-stuffs than the phosphates, any variations from the normal will affect these particularly. Under various pathological conditions, where a deficient amount of food and of inorganic salts is ingested, or where a considerable amount of the salts is removed from the circulation, as in consequence of hemorrhages, the formation of exudates and transudates, 
etc., smaller amounts are accordingly eliminated, and it may happen, indeed, that the chlorides disappear from the urine altogether. It is noteworthy, moreover, that an arrest in the elimination of the chlorides may then also occur even though a fair amount of salt is introduced with the food. In such an event we must assume that a retention is taking place in the body, in consequence of the fact that the lost fluid, with its various inorganic constituents, is gradually being replaced. Subsequently, when absorption of an exudate or a transudate takes place, the inorganic solids find their way into the general circulation, but are at once eliminated, as they are present in excess.

The phosphates and sulphates are likewise diminished under the conditions just mentioned, but do not disappear entirely, as they are in part derived from the albumins during the nitrogenous metabolism of the body. The diminution in the amount of the phosphates, however, exceeds that of the sulphates, as a small fraction only of the former is due to this source, while the latter are largely derived from the disintegrated albumins.

The tenacity with which the body maintains the normal composition of the blood is also well shown if the chlorides are gradually diminished in the food, and if their elimination from the blood is stimulated by the copious ingestion of diuretics. A point is soon reached when the salt in question no longer appears in the urine, beyond traces. If at this stage the blood is examined, it will be found that the amount of chlorides is practically the same as under normal conditions. There is a limit to this power of retaining the mineral salts, however, and if the chlorides are withheld for a length of time and diuresis remains active, a gradual loss occurs nevertheless, and in time results in the death of the animal. It appears, however, that it is not the loss of chlorine which the body tends to prevent, but that the sodium is the component which is of prime importance. This becomes apparent when the potassium salt is substituted for the sodium compound, when the same retention of sodium chloride occurs, while the potassium salt is eliminated in the urine. In this case, also, death ultimately results from what is very improperly termed "chlorine-hunger."

If at the stage when the chlorides have practically disappeared from the urine salt is added to the diet, a partial retention of this occurs until the original equilibrium has been restored. After that a normal elimination is again observed, and the amount then excreted practically corresponds to the quantity ingested.

These remarks also hold good for the phosphates and sulphates of the body, though with certain restrictions.

The bases which are found in the urine in combination with hydrochloric acid, sulphuric acid, and phosphoric acid are sodium, potassium, calcium, magnesium, and ammonium. The latter, however, occurs only in the urine of man and carnivorous animals. Calcium and magnesium occur almost exclusively as phosphates, either of the monacid or the diacid type. Traces, however, no doubt exist in 
combination with hydrochloric acid and sulphuric acid as well, but the greater portion of these two acids, as also of the phosphoric acid, is found in the form of sodium and potassium salts. The ratio between the two latter is usually placed at $3: 5$, in favor of sodium.

The alkaline phosphates normally exceed the earthy phosphates by one-third, and it is to be noted that the latter are, in part at least, also eliminated through the intestine.

While the greater portion of the sulphuric acid which results from the destruction of albumins within the tissues of the body is found in the urine in combination with inorganic bases only, a variable fraction also occurs united with certain aromatic substances which are formed during intestinal putrefaction. The resulting bodies are spoken of as conjugate or ethereal sulphates, and normally represent about one-tenth of the total amount of sulphuric acid that appears in the urine. They comprise the alkaline salts of phenol, indoxyl, and skatoxyl, and will be considered later.

The mineral and conjugate sulphates together are spoken of as the "acid" sulphur of the urine, in contradistinction to the so-called neutral sulphur, which represents a variable fraction that escapes oxidation in the body and finds its way into the urine as such. This comprises such substances as thiosulphuric acid, tauro-carbaminic acid, sulphocyanic acid, eystin, cysteïn, ethyl sulphide, uroferric acid, alloxyproteinic acid, etc. They are described in detail at another place.

In addition to the salts mentioned, a variable amount of carbonates may be found in the urine. In man and the carnivorous animals this is usually small; but in the herbivorous animals large quantities are normally found, and the alkaline reaction of such urines is indeed largely referable to this source. The acid occurs in combination with the alkalies and the alkaline earths, and owing to the presence of the latter especially the urine of such animals is normally turbid.

Of other inorganic constituents, every urine also contains iron (partly in organic combination), silicates, fluorides, hydrogen peroxide, and nitrates, all of which, however, are present only in traces. The nitrates are probably introduced with vegetable food, and disappear from the urine during starvation. During ammoniacal fermentation they are reduced to nitrites, and later disappear.

The quantitative variations of the inorganic constituents of human urine are shown in the following table:

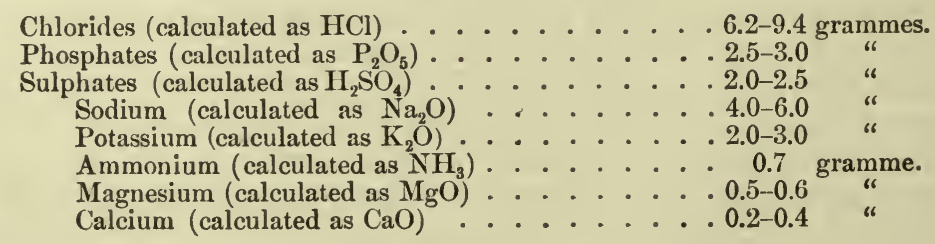




\section{Quantitative Estimation of the Mineral Ash.}

Ten c.c. of urine are placed in a weighed crucible and evaporated at a temperature of about $100^{\circ} \mathrm{C}$. The crucible is then covered with its lid and carefully heated over a small flame until the organic matter has been carbonized and fumes are no longer evolved. On cooling, the residue is extracted with boiling water. The washings are passed through a small filter, the weight of the ash of which is known, and the filter, together with the carbonaceous residue, is incinerated until a white ash is obtained. This process may be aided, if necessary, by moistening the material with a little alcohol or water. The washings are then placed in the crucible and evaporated at $100^{\circ} \mathrm{C}$. The residue is finally dried in a hot-air bath, heated until the bottom of the erucible just turns red, and is then allowed to cool over sulphuric acid and weighed. The weight of the mineral ash of the 10 c.c. of urine is then ascertained by deducting that of the crucible and the ash of the filter.

\section{Quantitative Estimation of the Chlorides.}

The chlorides of the urine are most conveniently estimated according to the method of Salkowski-Volhard. To this end, 10 c.c. of urine are diluted with 50 c.c. of distilled water, and treated with 4 c.c. of concentrated nitric acid and 15 c.c. of a standard solution of silver nitrate (such that 1 c.c. equals .01 gramme of sodium chloride). The mixture is further diluted to 100 c.c., thoroughly agitated, and passed through a dry filter. In a carefully measured portion of the filtrate the excess of silver is then titrated with a solution of potassium sulphocyanide of such strength that 25 c.c. correspond to 10 c.c. of the silver solution. A few drops of a saturated solution of ammonio-ferric alum serve as indicator. The amount of silver solution used in the precipitation of the chlorides in the 10 c.c. of urine is then calculated. The number of cubic centimeters which was necessary for this purpose, multiplied by 0.01 , indicates the amount of chlorides present in the 10 c.c. of urine, calculated as sodium salt.

The presence of albumins and sugar does not interfere with the method.

\section{Quantitative Estimation of the Phosphates.}

To determine the amount of the alkaline and earthy phosphates together, 50 c.c. of urine are treated with 5 c.c. of a solution containing about 100 grammes of sodium acetate and 100 c.c. of a 30 per cent. solution of acetic acid to the liter. In this manner any monacid phosphates that may be present are transformed into diacid phosphates. A few drops of tincture of cochineal are then added, and the mixture heated to the boiling-point and titrated with a 
standard solution of uranyl acetate or nitrate until a greenish color is noticed in the resulting precipitate of uranyl phosphate which does not disappear on stirring. From the number of cubic centimeters employed the corresponding amount of phosphates is then determined in terms of $\mathrm{P}_{2} \mathrm{O}_{5}$. The uranium solution is of such strength that 20 c.c. represent 0.1 gramme of $\mathrm{P}_{2} \mathrm{O}_{5}$.

The presence of sugar and albunins does not interfere with the method.

\section{Separate Estimation of the Earthy and Alkaline Phosphates.}

Two hundred c.c. of urine are rendered strongly alkaline with ammonia and set aside for several hours. The earthy phosphates are thus precipitated, and are collected on a small filter, washed with dilute ammonia $(1: 3)$, transferred to a beaker, and dissolved with as little acetic acid as possible. Distilled water is added so as to make the volume of the liquid about 50 c.c., when the solution is boiled and titrated as above. In a second portion of the urine the total amount of phosphates is then determined. The difference between the two results indicates the amount of phosphates which is present in combination with alkalies.

If it is desired to remove the total phosphates from a specimen of urine preliminary to some further step in analysis, the fluid is rendered alkaline with the hydrate of an alkaline earth and precipitated with a soluble calcium or barium salt. Or we may preeipitate directly with neutral or basic acetate of lead. In the first instance, the excess of calcium or barium, and in the second, that of lead, must then be removed.

\section{Quantitative Estimation of the Sulphates (Folin).}

In order to estimate the amount of mineral and conjugate sulphates it is best to determine the total sulphates in one portion and the conjugate sulphates in another, the difference between the two giving the mineral sulphates.

\section{Quantitative Estimation of the Total Sulphates.}

50 c.c. of clear filtered urine are treated with 5 c.c. of concentrated hydrochloric acid and 5 c.c. of a 4 per cent. solution of potassium chloride. The mixture is boiled until it is colorless (5-10 minutes), and then treated while still boiling with 25 c.c. of a 10 per cent. solution of barium chloride, drop by drop. It is then kept on a lot water-bath or an asbestos plate, hot (but not boiling) for from onehalf to one hour. The precipitate is now collected on a Schleicher and Schüll filter, the weight of the ash of which is known (No. 589). Care should be taken never to allow the filter to run dry, and small amounts of hot water must be added to the last cubic centimeters remaining, the final traces being placed upon the filter with the aid 
of a rubber-tipped glass rod. The precipitate is washed with hot water for half an hour, and at intervals of a few minutes hot ammonium chloride solution ( 5 per cent.) is substituted for the water, so that in all five or six additions of ammonium chloride take place in the course of the first twenty minutes' washing. In the end a specinien of the washings must no longer be rendered cloudy, even on standing a few minutes, after the addition of a drop of dilute sulphuric acid.

The paper filter is partially dried by folding and pressing gently between filter paper. It is then placed in a weighed crucible covered with 3 to 4 c.c. of alcohol and the alcohol ignited. The ash is heated, at first moderately, and almost completely covered with the lid, then only half covered, for from five to seven minutes, until the contents of the crucible are white. 'The crucible, when cooled, is placed in a desiccator and weighed, the difference between the first and second weighing giving the weight of the barium sulphate obtained from 50 c.c. of urine.

\section{Quantitative Estimation of the Conjugate Sulphates} (Folin). - 200 c.c. of urine (diluted to a liter if necessary) are treated with 100 c.c. of a 10 per cent. solution of barium chloride, at ordinary temperature. The mixture is set aside for twentyfour hours and the clear supernatant fluid poured into a dry beaker by decanting. This preliminary decanting is necessary, as the barium sulphate precipitate will otherwise go through the paper. The decanted liquid is filtered, 150 c.c. of the clear filtrate representing 100 c.c. of urine, measured into an Erlenmeyer flask, treated with 10 to 15 c.c. of concentrated hydrochloric acid and 10 to 15 c.c. of a 4 per cent. solution of potassium chlorate. The mixture is then heated to boiling and kept upon a boiling water-bath until the barium sulphate has settled and the supernatant fluid is clear. The precipitate is filtered off, washed, dried, and weighed, as described above. The weight thus obtained and deducted from the amount found according to the first method, indicates the amount referable to the mineral sulphates. The molecular weight of $\mathrm{BaSO}_{4}$ being 232.82, that of $\mathrm{SO}_{3} 79.86$, of $\mathrm{H}_{2} \mathrm{SO}_{4} 97.82$, and of $\mathrm{S} \mathrm{32,} \mathrm{the} \mathrm{figure} \mathrm{express-}$ ing the amount of $\mathrm{H}_{2} \mathrm{SO}_{4}, \mathrm{SO}_{3}$, or $\mathrm{S}$, corresponding to 1 granime of $\mathrm{BaSO}_{4}$, is found according to the following equations :

232.82: $79.86:: 1: x$; and $x=0.34301$. $\therefore 1$ gramme of $\mathrm{BaSO}$, $=0.34301$ gramme of $\mathrm{SO}_{3}$.

$232.82: 97.82:: 1: x$; and $x=0.420 .15$. $\therefore 1$ gramme of $\mathrm{BaSO}_{4}$ $=0.42015$ gramme of $\mathrm{H}_{2} \mathrm{SO}_{4}$.

$232.82: 32:: 1: x$; and $x=0.13744$. $\therefore 1$ gramme of $\mathrm{BaSO}_{4}=$ 0.13744 gramme of $\mathrm{S}$.

To calculate results, it is only necessary to multiply the weight of the $\mathrm{BaSO}_{4}$ by $0.34301,0.42015$, or 0.13744 , in order to ascertain the amount of sulphuric acid contained in 50 c.c. of urine, in terms of $\mathrm{SO}_{3}, \mathrm{H}_{2} \mathrm{SO}_{4}$, or $\mathrm{S}$, respectively. 
Test for Nitrates.-To demonstrate the presence of nitrates, 200 c.c. of urine are treated with 30 to 40 c.c. of chemically pure, concentrated sulphuric acid or hydrochloric acid, and distilled upon a sand-bath. The distillate is received into a dilute solution of caustic alkali. Owing to the presence of reducing substances in the urine, the nitric acid is thus transformed into nitrous acid, and passes over as such. The presence of nitrites may then be demonstrated as usual (see Saliva).

\section{THE ORGANIC CONSTITUENTS OF THE URINE.}

The organic constituents of the urine comprise the normal endproducts of the nitrogenous metabolism of the body, various products of albuminous putrefaction which have found their way into the general circulation from the intestinal canal, and certain pigments which are more or less intimately related to the normal blood-pigment. In addition, traces of various other substances may be encountered, the origin of which is obscure. Under pathological conditions we meet with certain normal constituents of the blood which generally do not appear in the urine as such, or occur in infinitesimally small amounts, and also with various abnormal products of metabolism, all of which will be considered in detail.

\section{The Nitrogenous Constituents of the Urine.}

\section{UREA.}

While in birds and reptiles the greater portion of the urinary nitrogen is excreted in the form of uric acid, urea constitutes the most important end-product of the nitrogenous metabolism of the remaining groups of vertebrate animals. In man, 86 per cent. of the total nitrogen eliminated in the urine appears in this form.

Origin.-Formerly it was supposed that urea resulted from uric acid through a process of oxidation, and that this was its only source. We have seen that the formation of urea from uric acid is possible, and we cannot deny that a certain proportion of the substance may be derived in this manner. Modern research, however, has shown that in man and the mammalian animals uric acid is derived from a destruction of the nucleins within the body, and results from oxidation of the xanthin bases which are thus set free. In birds and reptiles, on the other hand, the greater portion of the uric acid is formed synthetically from simpler substances, and is hence not directly comparable to the form which is found in the higher animals. In these a synthetic formation is also possible, but probably does not occur under normal conditions. As we can therefore recognize one origin of uric acid only in the mammal, and as this source of the nitrogen is insignificant when compared with the large amount of urea actually found, we are 
forced to the conclusion that the greater portion of the urea must originate in a different way.

It has been repeatedly shown that during the decomposition of the albumins by means of acids and alkalies, as also during the process of tryptic digestion and albuminous putrefaction, monoamino-acids result in large quantity. It has hence been supposed that these bodies may also represent intermediary products in the transformation of the tissue nitrogen into urea, which latter has generally been regarded as the end-product of the nitrogenous metabolism of the tissues. It has been shown as a matter of fact that in mammals - and to these I shall confine my remarks for the present-the administration of such acids in the food is followed by a corresponding increase in the amount of urea. Under certain pathological conditions, moreover, they appear in the urine as such, and it is then noted that the elimination of urea is much diminished. In health, however, this does not occur, and on examination of the different tissues and organs of the body amino-acids are found only in traces. We must hence assume, supposing them to occur as primary products of albuminous decomposition within the body, that they are transformed at once into other substances, which in turn give rise to urea. As all the amino-acids on oxidation yield ammonium carbonate, this substance would suggest itself as a possible antecedent of urea. We find, as a matter of fact, that ammonium carbonate when ingested by the mouth, or otherwise introduced into the body, appears in the urine as urea. This transformation of mono-amino-acids into urea may be-represented by the following equations :

(1) $\mathrm{CH}_{2}\left(\underset{\text { Glycocoll. }}{\left.\mathrm{NH}_{2}\right) \cdot \mathrm{COOH}}+2 \mathrm{O}=\underset{\text { Ammonium }}{\mathrm{H} \cdot \mathrm{COONH}}+\mathrm{CO}_{2}\right.$

(2) $2 \mathrm{H} . \mathrm{COONH}_{4}$

$$
+2 \mathrm{O}=\left(\mathrm{NH}_{4}\right)_{2} \cdot \mathrm{CO}_{3}+\mathrm{H}_{2} \mathrm{O}+\mathrm{CO}_{2}
$$

(3) $\left(\mathrm{NH}_{4}\right)_{2} \cdot \mathrm{CO}_{3}$<smiles>C=C(N)CN</smiles>

Drechsel has further shown that the amido-acids yield carbamic acid on oxidation, and that through alternate oxidation and reduction urea can result from the ammonium salt, as shown in the equation:<smiles>NOC(N)=O</smiles>

That carbamic acid is present in the normal acid urine of man and the dog has been proved. Nencki and Hahn, moreover, observed that in dogs in which the liver was temporarily excluded from the general circulation larger amounts of carbamic acid appeared in the urine than under normal conditions, and that the animals showed symptoms of intoxication identical with those observed when carbamates are directly introduced into the blood- 
current. These symptoms were also present when carbamates were introduced into the stumach, in which ease normal dogs show no signs of poisoning.

While it has been assumed above that urea is largely referable to a transformation of mono-amino-acids into ammonium carbonate or carbamate, as the ease may be, and while it has been shown that such a transformation can actually occur, we must yet remember that only traces of amino-acids are normally found in the tissues. The question hence arises: In what form does the waste nitrogen leave the tissues? The prevailing idea has been that the greater portion is normally set free from the various organs of the body in the form of the ammonium salt of paralactic acid, and the tendency has been to regard this salt as the antecedent of urea. It has been demonstrated, as a matter of fact, that urea results when ammonium lactate is passed through the isolated liver of dogs, and clinically, also, we observe that both ammonia and lactic acid appear in the urine in increased amounts when the liver is extensively diseased. Similar results are obtained in birds, in which uric acid represents the principal end-product of nitrogenous metabolism. In geese it is thus noted that after extirpation of the liver the greater portion of the urinary nitrogen appears in the form of ammonium lactate.

Under normal conditions it is then assumed that the lactate is transformed into ammonium carbonate, which in turn yields the earbamate, and that the urea finally results through a synthetic process, which is supposedly effected through the agency of a certain ferment. We may further imagine that the paralactic acid in the last instance may result from a decomposition of the mono-amino-radicles of the albuminous molecules, and in this form the theory would embrace the two outlined above. The various changes may be represented by the equations :

(1) $2 \mathrm{NH}_{4} \cdot \mathrm{C}_{3} \mathrm{H}_{5} \mathrm{O}_{3}+12 \mathrm{O}=\left(\mathrm{NH}_{4}\right)_{2} \mathrm{CO}_{3}+5 \mathrm{CO}_{2}+5 \mathrm{H}_{2} \mathrm{O}$

Ammonium lactate.

(2)

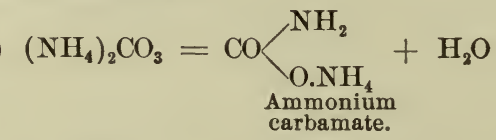

(3)

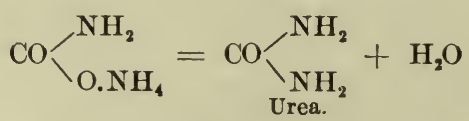

The ultimate formation of urea takes place in the liver. Of this we have abundant proof. It has thus been shown that in diseases of this organ which are associated with extensive destruction of the glandular elements a diminished amount of urea is found in the urine, while ammonia and lactic acid are present in increased quantity, and mono-amino-acids may indeed appear as such. In cases of this kind as much as 37 per cent. of the total amount of urinary nitrogen has been found in the form of ammonia. In the mammal, 
nıoreover, symptoms of carbamic-acid poisoning are observed when the liver is excluded from the general circulation, and, as has been shown, the formation of urea from ammonium lactate or carbonate may be demonstrated in the isolated livers of dogs.

IIore recently Folin has advanced another hypothesis regarding the origin of urea and the form in which the muscle nitrogen at least leaves the tissues, which seems fair to replace the older views. According to the hypothesis of Voit, albumin exists in the body in two forms, viz., as organized albumin, which is built up into tissues, and as so-called circulating albumin, which is present in excess of what is actually required. Voit further taught that the circulating albumin is broken down in the tissues at large through the special activity of the living protoplasm, but without becoming an integral part of the cells before its destruction. Pflüger, on the other hand, took the contrary view, according to which the circulating albumin must become part and parcel of the cell before it can undergo katabolic disintegration. In either event, however, it was assumed that the resulting nitrogenous product appeared in the urine in the form of urea. Folin distinguishes sharply between tissue or endogenous metabolism, which tends to be constant according to his investigations, and exogenous or intermediate metabolism, which is variable. He regards kreatinin as the essential nitrogenous end-product in the first instance, and finds that its elimination is practically constant for one and the same individual. Urea, on the other hand, according to Folin's concept, is the principal nitrogenous end-product of the exogenous metabolism. It results in such manner that the amino-acids which are formed during pancreatic digestion, in so far as they are not needed to make up for tissue loss of nitrogen, are at once desamidized in the liver; the non-nitrogenous remainder is then utilized in the formation of fats and carbohydrates, while the amino group gives rise to the formation of urea. In this manner the presence of the large amounts of ammonium compounds which are found in the portal blood during digestion is also well explained. But as Howell remarks: While a portion and perhaps even a larger portion of the urea arises from this early hydrolysis of the proteins of the food, we must admit also that ammonium compounds may be formed in the tissues of the body generally, probably by a similar process of hydrolysis, followed by oxidation. This would suggest itself especially under pathological conditions, where the amount of urea nitrogen may be in excess of that corresponding to the ingested food.

The total urinary nitrogen is under normal conditions practically equivalent to the quantity ingested, barring the small fraction which escapes digestion in the feces. Such a condition is spoken of as the nitrogenous equilibrium of the body. Of this different levels may exist, which may vary in the same individual. If the amount of nitrogenous food is thus diminished, the amount of urinary nitrogen 
will also decrease; and if the amount of food then remains constant, the nitrogenous output will likewise remain the same. If, on the other hand, more nitrogen is now ingested, an increased elimination will result; but a certain fraction is retained by the body and gradually a higher level of equilibrium becomes established.

There are natural limits to this power of accommodation, however, and we finally reach a point which varies in different individuals, where a further increase in the amount of nitrogen that is ingested does not lead to a higher level of equilibrium, and where consequently a further retention of nitrogen does not occur.

From the fact that the level of nitrogenous equilibrium is different in different people and may vary in one and the same individual, it follows that the amount of urea also must vary. Any figures indicating the amount of urea that is eliminated in the urine can therefore be of little value unless we are acquainted with the actual state of health of the individual, his body-weight, his habits of life as regards exercise, the amount of nitrogenous food ingested, etc. Having a knowledge of all these factors, however, we may be able to say whether the amount of urea is normal or not. Certain figures have been given by physiologists to indicate the amount of nitrogenous food which should enter into the composition of the diet, and from these we can approximately calculate the amount of urea that should appear in the urine. By estimating this in turn, or still better, of course, the total amount of nitrogen, we can accordingly decide whether or not the individual is consuming a sufficient amount of nitrogen in his food. The older figures, however, have been constructed without due regard to the factors above indicated, and are, in my opinion at least, too high as averages. This is now realized more generally, and the figures given by Chittenden and Folin, for example, are decidedly low. The latter thus gives about 120 grammes of albumin, 150 grammes of fat, and 225 grammes of carbohydrate.

I am willing to admit that an elimination of 40 to 50 grammes of urea may be normal in certain cases, as in soldiers on forced marches, among the laboring classes, etc., but I should certainly look upon the average merchant or student who leads a sedentary life as an overfed individual if his daily elimination of urea should exceed 30 grammes in the twenty-four hours. Among the well-to-do classes I find that an elimination of from 20 to 25 grammes is probably normal, taking the body-weight of the person into consideration. A smaller amount even is not infrequently met with in individuals of sedentary habits who are in perfect health, but I should scarcely regard such a quantity as normal for the average laboring-man. While extensive variations in the amount of urea are thus observed in health, still greater deviations from average figures are noted in disease, but here as there we must always take into account the amount of nitrogen that is ingested and the body weight. 
Properties of Urea.-Urea crystallizes in two forms, viz., in long, fine white needles if rapidly formed, or in long, colorless rhombic prisms when allowed to crystallize more slowly from its solutions.

It melts at $130^{\circ}$ to $132^{\circ} \mathrm{C}$, but is probably decomposed already at a temperature of $100^{\circ} \mathrm{C}$. It is readily soluble in water and alcohol, but is insoluble in anhydrous ether, chloroform, and benzol. As the substance is an acid amide, its solutions present a neutral reaction.

In accordance with its character as an unsaturated amide of carbonic acid, however, it combines with acids to form crystalline, salt-like compounds. The most important of these are the nitrate and the oxalate.

Urea nitrate, $\mathrm{CO}\left(\mathrm{NH}_{2}\right)_{2} \cdot \mathrm{HNO}_{3}$, crystallizes in two forms, viz., in delicate rhombic, horizontal platelets, which are commonly arranged overlapping in a shingle-like manner when rapidly formed, or as thicker rhombic columns or plates when allowed to crystallize more slowly.

Urea nitrate is readily soluble in distilled water, but dissolves with difficulty if this is acidulated with nitric acid, and also in alcohol. Its formation is frequently observed when urine containing much urea is examined for albumin in the cold with nitric acid. On standing, the nitrate may then separate out in crystalline form. On heating, the substance is decomposed without leaving a residue.

Urea oxalate, $\mathrm{CO}\left(\mathrm{NH}_{2}\right)_{2} \cdot \mathrm{C}_{2} \mathrm{H}_{2} \mathrm{O}_{4}$, crystallizes in rhombic plates, or hexagonal prisms, and is less soluble in water than the nitrate; in alcohol and in dilute solutions of oxalic acid it is nearly insoluble. The substance is obtained in crystalline form on adding a saturated solution of oxalic acid to a concentrated solution of urea.

Urea also combines with various neutral salts, such as sodium chloride and ammonium chloride, and also with the nitrates of sodium and the oxides of silver and mercury, to form double salts. With mercuric nitrate three different compounds result, according to the concentration of the two solutions, viz., $\mathrm{CO}\left(\mathrm{NH}_{2}\right)_{2} . \mathrm{Hg}_{2}\left(\mathrm{NO}_{3}\right)_{4}$, $\mathrm{CO}\left(\mathrm{NH}_{2}\right)_{2} \cdot \mathrm{Hg}_{3}\left(\mathrm{NO}_{3}\right)_{6}$, and $\left[\mathrm{CO}\left(\mathrm{NH}_{2}\right)_{2}\right]_{2} \cdot \mathrm{Hg}\left(\mathrm{NO}_{3}\right)_{2}+3 \mathrm{HgO}$. The latter compound is of special interest, as Liebig's quantitative estimation of urea, which was formerly much employed, was based upon its formation. It results when a 2 per cent. solution of urea is treated with a feebly acid solution of mercuric nitrate, and the mixture is subsequently neutralized.

Mercuric chloride precipitates urea in alkaline, but not in neutral solutions.

Very important, further, is the behavior of urea toward sodium hypochlorite or hypobromite, as the most usual method of estimating urea in the clinical laboratory is based upon the reaction which here takes place. This reaction may be represented by the equation : 


$$
\mathrm{CO}\left(\mathrm{NH}_{2}\right)_{2}+3 \mathrm{NaOBr}=3 \mathrm{NaBr}+\mathrm{CO}_{2}+2 \mathrm{H}_{2} \mathrm{O}+2 \mathrm{~N} \text {. }
$$

In practical work an alkaline solution of the hypobromite is employed; so that the carbon dioxide which is liberated is at once absorbed, while the nitrogen remains. It is to be noted, however, that while 1 gramme of urea should theoretically give rise to the formation of 372.7 c.c. of nitrogen, 354.3 c.c. are at best obtained at $0^{\circ} \mathrm{C}$. and a pressure of $760 \mathrm{Hgmm}$. In clinical work this difference is unimportant, and it is in a measure equalized by the evolution of a small amount of nitrogen from some of the other nitrogenous constituents which are at the same time present. On hydrolysis urea is transformed into ammonium carbonate:

$$
\mathrm{CO}\left(\mathrm{NH}_{2}\right)_{2}+2 \mathrm{H}_{2} \mathrm{O}=\left(\mathrm{NH}_{4}\right)_{2} \mathrm{CO}_{3} \text {. }
$$

This occurs during the process of ammoniacal fermentation, which results when urine is allowed to stand exposed to the air. 'The carbonate in turn is decomposed with the liberation of ammonia and carbon dioxide. On boiling with acids or alkalies the same result is obtained.

On heating the substance in aqueous solution in a sealed tube to a temperature of $100^{\circ} \mathrm{C}$. ammonia and carbon dioxide likewise result.

Nitrous acid when added in excess decomposes urea, with the formation of nitrogen, carbon dioxide, and water, but the acid is at the same time decomposed, as is seen in the equation :

$$
\mathrm{CO}\left(\mathrm{NH}_{2}\right)_{2}+2 \mathrm{HNO}_{2}=\mathrm{CO}_{2}+4 \mathrm{~N}+3 \mathrm{H}_{2} \mathrm{O} \text {. }
$$

This reaction is utilized when it is desired to remove nitrous acid from a solution.

On heating the dry substance in a test-tube to a temperature of about $150^{\circ}-170^{\circ} \mathrm{C}$., fumes of ammonia are freely evolved, owing to the decomposition of the urea with the formation of biuret, as shown in the equation :

$$
2 \mathrm{CO}\left(\mathrm{NH}_{2}\right)_{2}=\mathrm{NH}_{3}+\sum_{\mathrm{CO}}^{\mathrm{CO}} \sum_{\substack{\mathrm{NH}_{2} \\ \mathrm{NH}}}^{\mathrm{NH}_{2}}
$$

On further heating, more ammonia is given off; the melted mass finally solidifies, and may be shown to contain both biuret and cyanuric acid. The reaction which takes place may be represented as follows :

(1) $3 \mathrm{CO}\left(\mathrm{NH}_{2}\right)_{2}=\underset{\text { Cyanic acid. }}{3 \mathrm{CONH}}+3 \mathrm{NH}_{3}$

(2) $3 \mathrm{CONH}=\underset{\text { Cyanuric adid. }}{\mathrm{C}_{3} \mathrm{~N}_{3}(\mathrm{OH})_{3}}$

To demonstrate the presence of the biuret, the residue is dis- 
solved in a dilute solution of sodium hydrate, when upon the careful addition of a dilute solution of copper sulphate a beautiful, purple-red color develops.

A very delicate test also is the following: 2 c.c. of a concentrated solution of furfurol are treated with 4-6 drops of strong hydrochloric acid. If to this mixture, which should not present a red color, a small crystal of urea is then added, a deep violet develops in the course of a few minutes.

Synthetic Formation.-As has been mentioned, urea was the first organic substance formed in the animal body which was made synthetically in the chemical laboratory. Wöhler in 1828 produced the substance artificially by evaporating an aqueous solution of ammonium cyanate, when a rearrangement of atoms occurs and urea results :

$$
\left(\mathrm{NH}_{4}\right) \mathrm{CNO}=\mathrm{CO} / \frac{\mathrm{NH}_{2}}{<\mathrm{NH}_{2}}
$$

Other methods now exist by which urea can also be made synthetically, but they are all more or less modifications of the one just described.

Isolation from the Urine.-To isolate urea on a small scale, $50-100$ c.c. of urine are evaporated to a syrup on a water-bath and extracted with 150 c.c. of strong alcohol by rubbing in a mortar. The alcoholic extract is filtered, the alcohol distilled off, and the syrupy residue treated with concentrated nitric acid in the cold. The urea nitrate which crystallizes out on standing is filtered off with a suction-pump, dissolved in hot water, and the aqueous solution decolorized by gently heating with animal charcoal. The colorless filtrate is then treated with barium carbonate in substance so long as carbon dioxide is evolved, and finally rendered alkaline with barium hydrate solution. The urea is thus liberated according to the equation :

$$
2 \mathrm{CO}\left(\mathrm{NH}_{2}\right)_{2} \cdot \mathrm{HNO}_{3}+\mathrm{BaCO}_{3}=\mathrm{Ba}\left(\mathrm{NO}_{3}\right)_{2}+2 \mathrm{CO}\left(\mathrm{NH}_{2}\right)_{2}+\mathrm{H}_{2} \mathrm{O}+\mathrm{CO}_{2} \text {. }
$$

The solution is now evaporated to dryness and the residue extracted with absolute alcohol. On concentrating this extract the urea crystallizes out in colorless prisms, which may then be treated as above indicated.

Quantitative Estimation.-In the clinical laboratory the old method of Knop and Hüfner is almost exclusively employed. This is based upon the decomposition of urea with sodium hypobromite in alkaline solution, as already described. The nitrogen which is thus liberated is measured and the corresponding amount of urea determined by calculation.

For scientific purposes, however, this method in any one of its numerous modifications is not sufficiently accurate, as the actual volume of nitrogen which is obtained always falls somewhat short of the theoretical amount. The sodium hypobromite, moreover, also causes a partial decomposition of other nitrogenous constituents 
of the urine, and as the resulting amount of nitrogen is not constant, a further error is incurred. In scientific research we are hence forced to resort to some other procedure, such as that proposed by Folin.

METHOD of Folin.-This is based upon the following considerations: crystallized magnesium chloride, $\mathrm{MgCl}_{2} \cdot 6 \mathrm{H}_{2} \mathrm{O}$ boils in its water of crystallization at a temperature of about $160^{\circ} \mathrm{C}$. Urea is quantitatively decomposed in such a solution into ammonia and carbon dioxide within one-half hour. If the process is carried ont in acid solution, the ammonia can subsequently be distilled off after rendering the mixture alkaline, and is then titrated. The corresponding amount of urea is ascertained by calculation. At the same time, however, the preformed ammonia is obtained, and it is hence necessary to eliminate this source of error by a separate estimation of this form. This is conveniently done according to the method which has likewise been suggested by Folin (see below).

Method.-Three c.c. of urine are placed in an Erlenmeyer flask of 200 c.c. capacity, together with 20 grammes of magnesium chloride and 2 c.c. of concentrated hydrochloric acid. It is necessary to measure the urine very accurately with a pipette graduated in one-twentieths c.c. (The magnesium chloride usually contains a small amount of ammonia, which must be separately determined.) The flask is closed with a perforated stopper through which a specially constructed safety tube passes. ${ }^{1}$. The mixture is now boiled until the drops flowing back through the tube produce a hissing sound on coming in contact with the solution. After this point has been reached, the boiling is continued more moderately for about forty-five minutes. In order to obviate immoderate foaming a piece of paraffin about twice as large as a coffee bean is added. The solution while still hot is carefully diluted to about 500 c.c.-at first by allowing the water to flow drop by drop through the tube: it is then transferred to a 1000 c.c. retort, treated with about 7 or 8 c.c. of a 20 per cent. solution of sodium hydrate, and the ammonia distilled off into a measured amount of a decinormal solution of sulphuric acid. The distillation may be interrupted when about 350 c.c. have passed over (viz., after about sixty minutes). The distillate is boiled for a moment to remove any carbon dioxide which may be present in solution, and on cooling is titrated to determine the excess of acid. Each cubic centimeter of the decinormal ammonia present in the distillate corresponds to 0.003 gramme, viz., to 0.1 per cent of urea.

From this result the amount of preformed ammonia and that present in the 20 grammes of magnesium chloride must be deducted.

If desired, the estimation can also be made with the urea-containing filtrate obtained wịth Mörner and Sjöquist's method, but Folin states that the previous isolation of the urea in such manner is probably not necessary.

Estimation of the Preformed Ammonia (according to Folin).-

* The tube can be procured from Eimer \& Amend's, of New York. 
To 25 or 50 c.c. of urine, placed in an arëometer cylinder about 45 $\mathrm{cm}$. high and $5 \mathrm{~cm}$. in diameter, there are added 8 or 16 grammes of sodium chloride, according to the amount of urine, 5 to 10 c.c. of petroleum or toluol, and 1 or 2 grammes of sodium carbonate. The sodium chloride is added to prevent decomposition. A current of air is driven through the urine, carrying off the ammonia set free by the sodium carbonate; this is absorbed by passing through a definite amount of $\frac{1}{10}$ normal acid, the excess of which is afterward titrated and the ammonia calculated ( 1 c.c. of $\frac{\mathrm{n}}{10}$ sulphuric acid corresponds to .0017 gramme of ammonia. The length of time necessary for the completion of the operation will depend upon the amount of air passing through the liquid, as well as upon the temperature. At $22^{\circ}-25^{\circ} \mathrm{C}$. and with an air current of approximately 700 liters per hour, the ammonia will be completely driven off from 25 c.c. of urine in an hour and a quarter, or from 50 c.c. in an hour and a half. With a larger volume of urine, a smaller air current, or lower temperatures of the urine, a longer time will be necessary. The requisite time must be learned for each air current. This can easily be done by continuing the operation, until in an hour there is no ammonia given off. It is necessary to pass the air through a tuft of absorbent cotton, or closely packed glass wool, after it leaves the urine and before it passes through the acid, in order to prevent alkali from being carried over. The air current should be uniform and constant. Folin uses a specially constructed tube, for the absorption of the ammonia by the acid. Where this is not done, it will usually be found necessary to pass the air through two successive portions of acid to prevent loss of ammonia.

Folin employs alizarin red as indicator; 2 drops of a 1 per cent. solution suffice. The titration is carried to the red point, not to violet. The method as described is also applicable in the case of blood.

Estimation of the Total Urinary Nitrogen.-KJELDAHL'S METHOD. - The method is based upon the observation that on treating urine with a mixture of two parts of concentrated sulphuric acid and one part of fuming sulphuric acid and boiling, the entire amount of nitrogen can be transformed into ammonium sulphate. This is then decomposed with an excess of sodium hydrate and the liberated ammonia estimated by distilling into a known amount of dilute acid, and retitrating the excess of acid.

Five c.c. of urine are treated in a Kjeldahl digesting flask with a pinch of yellow oxide of mercury (about 0.3 gramme) and 20 c.c. of the sulphuric acid solution. The mixture is boiled until a perfectly colorless solution is obtained. Vigorous ebullition, however, must be avoided, and the flask should be placed at an angle of about 45 degrees, so as to prevent loss from spurting. The milder the ebullition the better. On cooling, the contents of the flask are transferred to a Kjeldahl retort, with the aid of a little distilled water. 
Sodium hydrate solution (27 per cent.) is then added until the greater portion of the acid is neutralized. The fluid is allowed to cool again, and a few pieces of granulated zinc or a little talcum is thrown in, when a mixture of the hydrate solution and of a 4 per cent. solution of potassium sulphide is further added in excess. ${ }^{1}$ Of either solution, 40 c.c. are added in all. The addition of the latter is necessary, as the mixture not only contains ammonium sulphate, but also aminocompounds of mercury, which latter would not give up their entire amount of nitrogen if sodium hydrate alone were present. The talcum or zinc merely prevents an unduly violent bumping when boiling. The retort is immediately connected with a condenser through the intervention of a Kjeldahl distilling tube. The condensing tube dips into a nitrogen bulb, which contains a carefully measured amount of a one-fourth normal solution of sulphuric acid; 30 c.c. are usually sufficient. The mixture is now distilled until about two-thirds have passed over. The condenser is rinsed with a little distilled water, which is added to the distillate. After the addition of a few drops of tincture of cochineal the excess of acid is retitrated with a one-fourth normal solution of sodium hydrate. The difference indicates the amount of acid which was consumed in uniting with the liberated ammonia. As 1 c.c. of the one-fourth normal solution represents 0.0035 gramme of nitrogen, the amount contained in the 5 c.c. of urine is ascertained by multiplying the number of cubic centimeters employed by this figure, from which the total amount of twenty-four hours is then readily calculated. The corresponding amount of albumin is obtained by multiplying this figure by 6.25 .

\section{Uric Acid.}

Whereas in mammals, the amphibia, and fishes urea is the most important end-product of nitrogenous metabolism, the greater portion of the urinary nitrogen in birds and reptiles is eliminated as uric acid.

Origin.-The formation of uric acid in birds and reptiles is analogous to the formation of urea in the mammal. This is true at least of the greater portion, while a variable fraction originates from the nucleins, viz., the xanthin bases. Organic ammonium salts, aminoacids, urea, and ammonium carbonate, when given to birds in their food, appear in the urine as uric acid and are transformed into uric acid in the liver. After extirpation of the liver almost all the urinary nitrogen appears in the form of ammonia, in association with

${ }^{1}$ Neuberg has suggested that it is more convenient to add about 1 gramme of sodium thiosulphate to the caustic alkali solution for every 0.4 gramme of oxide of mercury, instead of the sulphide, as the latter must always be prepared anew, and us the addition of the corresponding amount of liquid prolongs the distillation. In the alkaline solution the aminomercuric sulphate is then decomposed according to the equation:

$$
\mathrm{Hg}\langle\overbrace{\mathrm{NH}_{3}}^{\mathrm{NH}_{3} \cdot}\rangle \mathrm{SO}_{4}+\mathrm{Na}_{2} \mathrm{~S}_{2} \mathrm{O}_{3}+\mathrm{H}_{2} \mathrm{O}=\mathrm{HgS}+\left(\mathrm{NH}_{4}\right)_{2} \mathrm{SO}_{4}+\mathrm{Na}_{2} \mathrm{SO}_{4}
$$


lactic acid, and ammonium carbonate, when given by the mouth, is eliminated as such. Of the manner in which the synthesis of uric acid is effected in the liver, however, we know but little. Urea or ammonium carbonate cannot, of course, give rise to its formation alone, as the available amount of carbon is too small. We must hence assume that some other substance enters into the reaction. This substance is as yet unknown, but we may imagine that ammonium lactate may be formed as an intermediary product and that one portion is first transformed into ammonium carbonate and that the uric acid is then formed through the union of this with another molecule of lactic acid. Horbaczewski, indeed, has shown that artificially uric acid may be formed from lactic acid, ammonia, and carbon dioxide, by heating trichloro-lactic amide together with urea. The reaction which takes place may be represented by the equation :

$$
2 \mathrm{CO}\left(\mathrm{NH}_{2}\right)_{2}+\underset{\substack{\text { Trichloro-lactic } \\ \text { amide. }}}{\mathrm{C}_{3} \mathrm{Cl}_{3} \mathrm{O}_{2} \mathrm{H}_{2} \cdot \mathrm{NH}_{2}}=\mathrm{NH}_{4} \mathrm{Cl}+2 \mathrm{HCl}+\mathrm{H}_{2} \mathrm{O}+\underset{\text { Uric acid. }}{\mathrm{C}_{5} \mathrm{H}_{4} \mathrm{~N}_{4} \mathrm{O}_{3}}
$$

On the other hand, it is possible that uric acid may result through the union of glycocoll and urea, and artificially this synthesis can indeed be effected. We have seen, moreover, that on hydrolytic decomposition uric acid yields ammonia, carbon dioxide, and glycocoll. In this case the resulting reaction could be expressed by the equation:

$$
3 \mathrm{CO}\left(\mathrm{NH}_{2}\right)_{2}+\mathrm{CH}_{2}\left(\mathrm{NH}_{2}\right) \cdot \mathrm{COOH}=3 \mathrm{NH}_{3}+2 \mathrm{H}_{2} \mathrm{O}+\mathrm{C}_{5} \mathrm{H}_{4} \mathrm{~N}_{4} \mathrm{O}_{3} .
$$

Wiener has suggested that uric acid may be formed synthetically through the union of tartronic acid with two urea radicles, and the intermediate formation of dialuric acid, the tartronic acid being itself derived from lactic acid by oxidation. This transformation is represented by the following equations:
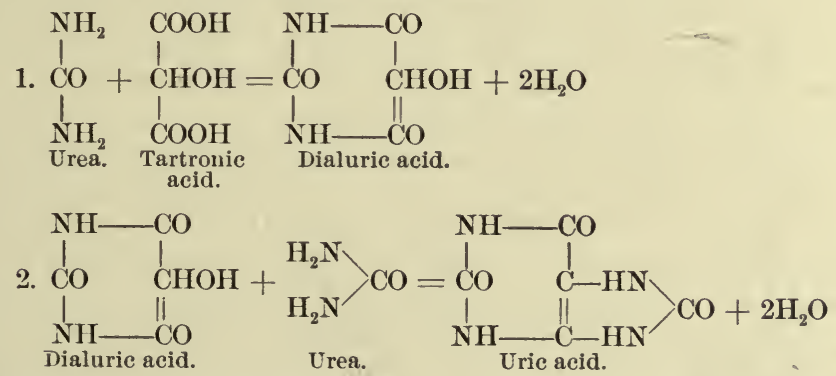

Wiener's experimental basis of the synthetic formation of uric acid in birds is quite convincing and accords well with other observed facts.

While the greater portion of the uric acid is thus formed synthetically in the liver of birds and reptiles, a variable but much smaller amount results directly from the xanthin bases through oxidation. 
These are in part derived from disintegrating cells of the body (endogenous uric acid) and in part from the ingested food (exogenous uric acid). Through the researches of Horbaczewski we know that in the mammalian organism uric acid is formed together with the xanthin bases in all organs of the body, and is most abundantly produced in those which are especially rich in nuclei, such as the spleen and the lymph-glands. Burian further has demonstrated that the muscle tissue also must be viewed as one of the most important sources of uric acid and that hypoxanthin is there its antecedent. The very interesting observation was further made that larger amounts of uric acid could be obtained from these parts when the blood used in the transfusion experiments contained much oxygen, while with venous blood xanthin bases only were obtained. In the amphibia and fish in which the oxidation-processes are especially sluggish, we accordingly find xanthin bases and little or no uric acid. The interesting question now suggests itself, Why is it that in mammals uric acid appears in the urine at all in view of the fact that uric acid which is introduced into the stomach is eliminated as urea? A final answer to this question cannot be given, but there is reason to suppose that the uric acid is here first carried to the liver, and is probably oxidized to urea by the oxidizing ferments of this organ. We find, as a matter of fact, that an increased elimination of uric acid results at once when the blood of the portal vein is prevented from flowing through the liver by establishing a so-called Eck fistula between this and the inferior cava, and when the hepatic artery is at the same time ligated. In this manner the blood of the spleen and the extensive lymphatic districts of the intestinal tract is carried directly into the general circulation, and the contained xanthin bases and uric acid hence find their way into the urine without being subjected to the action of the oxydases of the liver. We may hence conclude that the appearance of these bodies in the urine is under normal conditions, owing to the fact that not all the blood of the body reaches the liver before being carried to the kidneys.

In birds and reptiles we have also seen that a certain amount of urea appears in the urine, and, as I have already explained, this is no doubt produced directly in the tissues. As ingested urea is transformed into uric acid, we must assume that the portion which is eliminated in the urine has reached the kidneys without having previously passed through the liver, and the process is thus quite analogous to what we observe in mammals in the case of uric acid.

Under normal conditions the elimination of uric acid rarely exceeds 1 gramme, of which 0.3 to 0.6 gramme is represented by the endogenous form. Increased amounts are met with under various pathological conditions.

In leukæmia especially, a greatly increased elimination is commonly observed, and referable to the increased destruction of leucocytes. But excessive amounts of uric acid may also occur in conditions in which there is no direct evidence of increased nuclear 
destruction. In many cases of this kind the increased elimination is apparently dependent upon the amount of animal food that is ingested, and it would appear that in such cases the liver has lost to a greater or less degree its power of oxidizing the uric acid, which reaches it from this source. Were this true, we should then also expect that relatively larger amounts of xanthin bases should find their way into the urine, and this indeed may actually occur. But, on the other hand, an increased elimination of uric acid and xanthin bases may also be observed although the patient has been placed on a diet which is practically free from nucleins. An adequate explanation of such an occurrence is as yet wanting. We may suppose that the liver has lost its power of oxidation so far as the xanthin bases are concerned. But we must bear in mind that uric acid is formed in all the tissues of the body, and that the relative amount which thus originates, as compared with the xanthin bases, is largely influenced by the intensity of the process of oxidation (absence of uricolytic ferments?). It is hence also conceivable that in such cases these may be deficient, while the liver may function in a normal manner. The possibility of a synthetic production of uric acid, finally, may also enter into consideration.

The question of the nature of the so-called uric-acid diathesis is thus still in statu quo, and the same may be said of the formation of uratic deposits in the joints and tendons in gout. It appears, however, that an increased production of uric acid, contrary to what was formerly supposed, plays no rôle in the causation of the latter disease and that the essential abnormality consists in the inability of the body to break down the uric acid to simpler and more readily soluble products.

Properties of Uric Acid.-Pure uric acid crystallizes in transparent, colorless rhombic platelets, the angles of which are often rounded off. Such crystals are at times seen in urinary sediments, but more commonly the substance is here found in the form of brownish-yellow whetstone-like crystals, which may occur singly, but are frequently arranged in groups. These are quite characteristic, and cannot be confounded with crystals of any other substance that may occur in the urine.

A great many other forms may, however, also be encountered, such as dumb-bells, somewhat irregular hexagonal platelets, paddleshaped crystals, etc., the nature of which is not at once apparent.

Uric acid is almost insoluble in cold water $(1: 40,000)$, with difficulty also in boiling water $(1: 1800)$, and insoluble in alcohol and ether.

In concentrated sulphuric acid and boiling glycerin it dissolves with comparative ease and without undergoing decomposition. It is a dibasic acid, and accordingly combines with bases to form neutral and acid salts. Of these, the neutral salts of potassium and lithium are the most soluble, while the acid salts, and notably acid ammonium urate, are quite insoluble. Its compounds with the 
alkaline earths are likewise soluble only with great difficulty. In the urine uric acid is said to be present as a quadriurate, viz., as a hyperacid compound, in which one molecule of sodium (viz., potassium or ammonium) is in combination with two molecules of uric acid. Its solubility in the urine is largely dependent upon the amount of water, the reaction, and the presence of mineral salts and possibly of pigments. On standing, however, in the absence of micro-organisms, the quadriurate is decomposed, with the liberation of free uric acid and acid biurates, which latter are then again transformed into quadriurates through the agency of the diacid phosphates, and through a repetition of this process all the uric acid finally separates out, as is shown in the equations:

$$
\begin{aligned}
& \mathrm{HNa} \cdot \mathrm{C}_{5} \mathrm{H}_{2} \mathrm{~N}_{4} \mathrm{O}_{3} \cdot \mathrm{C}_{5} \mathrm{H}_{4} \mathrm{~N}_{4} \mathrm{O}_{3}+\mathrm{H}_{2} \mathrm{O}=\mathrm{C}_{5} \mathrm{H}_{4} \mathrm{~N}_{4} \mathrm{O}_{3}+\mathrm{HNa} \mathrm{C}_{5} \mathrm{H}_{2} \mathrm{~N}_{4} \mathrm{O}_{3} \\
& \begin{array}{l}
\text { Sodium quadriurate. } \\
\text { Uric acid. Acid sodium } \\
\text { biurate. }
\end{array} \\
& \text { (2) } 2 \mathrm{HNa}_{\text {Acid }} \mathrm{C}_{5} \mathrm{H}_{2} \mathrm{~N}_{4} \mathrm{O}_{3}+\mathrm{NaH}_{2} \mathrm{PO}_{4}=\mathrm{HNa} \cdot \mathrm{C}_{5} \mathrm{H}_{2} \mathrm{~N}_{4} \mathrm{O}_{3} \cdot \mathrm{C}_{5} \mathrm{H}_{4} \mathrm{~N}_{4} \mathrm{O}_{3}+\mathrm{Na}_{2} \mathrm{HPO}_{4}
\end{aligned}
$$

In the urine of birds and reptiles the uric acid is said to occur exclusively in the form of quadriurates. Neutral urates are not found in the urine. Of the compounds which uric acid forms with the salts of the heavy metals, the silver and copper salts deserve especial mention, as some of the methods which are employed in the quantitative estimation of the substance are dependent upon their formation. The salts of uric acid are readily decomposed by hydrochloric acid, and on standing the free substance crystallizes out from the solution. The intimate relation which exists between uric acid and the xanthin bases, as also its character as a diureid, has already been considered (pages 104 and 105).

Tests for Uric Acid.-Murexid Test.-If a few erystals of uric acid are evaporated with a few drops of concentrated nitric acid on a porcelain plate, a yellow or brick-red residue remains. On cooling, a drop or two of ammonia are added, when a beautiful purplered color develops, owing to the formation of ammonium purpurate (murexid). If now an excess of sodium hydrate solution is added, the ammonium salt is transformed into the corresponding sodium salt, and the purple red passes into a bluish violet. This disappears on heating and does not return on cooling (compare with the similar reaction of xanthin and guanin).

Copper Test.-A few crystals of uric acid are dissolved in sodium hydrate solution and treated with a few drops of Fehling's solution. On heating, white urate of copper separates out. If more copper solution is added, a partial reduction of the cupric oxide occurs, owing to the formation of allantoin.

Denniges' 'Test.-If uric acid is transformed into alloxan by means of nitric acid, and the excess of acid is carefully evaporated, a blue color results on treating the residue with a few drops of concentrated sulphuric acid and commercial benzol containing thiophen. 
Schiff's Test.-If a piece of filter-paper is moistened with a solution of nitrate of silver, and a drop of a solution of uric acid in sodium carbonate is added, a brownish-black color develops, owing to reduction of the oxide of silver. In the presence of only 0.002 milligramme of uric acid a yellow color is obtained.

Isolation of Uric Acid.-Uric acid is most conveniently prepared from the excrements of snakes, in which, as has been stated, it exists in the form of the quadriurate. To this end, the material is boiled with a dilute solution of sodium hydrate so long as ammonia is evolved, when carbon dioxide is passed through the solution until the alkaline reaction has largely disappeared. The acid biurate of sodium which separates out is then washed with cold water and dissolved in a dilute sodium hydrate solution. On adding an excess of concentrated hydrochloric acid the uric acid crystallizes out on standing.

From human urine the substance can be obtained by adding concentrated hydrochloric acid in the proportion of $50: 1000$, and keeping the mixture at a low temperature for about forty-eight hours. The crystals which then separate out are treated with water, dissolved in dilute sodium hydrate solution, decolorized with animal charcoal, and reprecipitated with hydrochloric acid. This method was formerly employed for estimating the amount of uric acid in the urine, but has been abandoned, as it does not furnish reliable results and in its place the method of Folin may be recommended.

Folin's Method.-This is based upon the precipitation of uric acid by means of ammonium sulphate as ammonium urate, the decomposition of the latter by means of sulphuric acid and its titration with potassium permanganate. To precipitate the uric acid, and also to remove the small amount of mucoid substance which is found in every urine, the following reagent is employed: 500 grammes of ammonium sulphate, 5 grammes of uranium acetate, and 60 c.c. of a 10 per cent. solution of acetic acid are dissolved in 650 c.c. of water. The resulting solution measures about 1000 c.c. Seventy-five c.c. of the reagent are added to 300 c.c. of urine in a flask holding 500 c.c. After standing for five minutes the mixture is filtered through two folded filters, and thus freed from the mucoid body, which is carried down with the uranium phosphate in acid solution. The filtrate is divided into two portions of 125 c.c. each, which are placed in beakers and treated with 5 c.c. of concentrated ammonia. After stirring a little the solutions are set aside until the next day. The supernatant fluid is then carefully poured off through a filter (Schleicher and Schüll No. 597); the precipitated ammonium urate is collected with the aid of a small amount of a 10 per cent. solution of ammonium sulphate and washed with the same reagent. Traces of chlorides do not interfere with the subsequent titration, and the process of filtration and washing can be completed in from twenty to thirty minutes. The ammonium urate is then 
washed into a beaker, after opening the filter, using about 100 c.c. of water. Fifteen c.c. of concentrated sulphuric acid are then added and the solution is titrated at once with a one-twentieth normal solution of potassium permanganate. Toward the end of the titration Folin suggests to add the permanganate in portions of two drops at a time, until the first trace of rose is apparent throughout the entire fluid. Each cubic centimeter of the reagent corresponds to 0.00375 gramme of uric acid. A final correction of 0.003 gramme for every 100 c.c. of urine employed is necessary, owing to the slight degree to which ammonium urate is soluble.

\section{The Xanthin Bases.}

The xanthin bases which have been found in the urine of man are xanthin, hypoxanthin, guanin, carnin, paraxanthin, heteroxanthin, episarcin, and under certain pathological conditions adenin. Their amount is always small, and normally constitutes about 10 per cent. of the quantity of uric acid, viz., from 0.02 to 0.06 gramme. Of this amount 0.02 to 0.03 gramme is represented by xanthin. Hypoxanthin and guanin probably stand next in order, while paraxanthin and heteroxanthin are found only in traces. From 10,000 liters of urine Krüger and Salomon obtained only 7.5 grammes of the latter.

Origin.-It has been shown that the xanthin bases are derived from the nucleins, and are probably formed in all tissues of the body (endogenous form). In addition a certain amount is introduced from without (exogenous form). Under normal conditions the greater portion of the xanthin bases is no doubt oxidized to uric acid, but a variable fraction escapes as such. To a certain extent the oxidation to uric acid occurs in the liver, but, as I have shown, this takes place also in the other organs of the body (notably the muscle tissue), as both xanthin bases and uric acid are obtained in transfusion experiments. At the same time it was noted that the relative amount of the two was largely influenced by the degree of oxygenation of the blood, so that xanthin bases only were obtained if venous blood was used, while both were found when arterial blood was employed. We can thus understand that, as a general rule at least, a certain relation exists in the elimination of uric acid and the xanthin bases. A diminished elimination of the latter is thus quite frequently associated with a corresponding increase of the former, or vice versa, and both may, of course, be increased or diminished together. The most notable increase in their elimination is observed in leukæmia, and here adenin also appears in the urine.

Theobromin (dimethyl-xanthin) and caffein (trimethyl-xanthin) are partly eliminated in the urine as such, and partly appear as a methyl-xanthin which is apparently identical with heteroxanthin.

Xanthin has once been found in crystalline form in a urinary sediment, and has in several instances been encountered in vesical calculi. 
As the isolation of the individual substances from the urine in amounts sufficient for purposes of study is a very complicated process, and requires facilities which are not generally found in university laboratories, it will suffice at this place to describe a method by which they can be collectively estimated. For a consideration of the chemical properties of the more important members of the group and their isolation from other sources, as also of their relation to uric acid and the nucleinic bases, the reader is referred to other sections.

Quantitative Estimation.-The xanthin bases are best isolated from the urine according to Salkowski's method, which is based upon their precipitation as silver compounds, the separation of the latter, and the determination of the amount of silver in combination with the bases.

600 c.c. of urine are precipitated with 200 c.c. of magnesia mixture, ${ }^{1}$ when a 3 per cent. ammoniacal solution of silver nitrate is added to from 700 to 750 c.c. of the filtrate. The proportion should be 6 c.c. for each 100 c.c. of urine. After standing for one hour the mixture is filtered and the precipitate washed with water until all the free silver has been removed. The filter is then perforated, the precipitate washed into a flask with from 600 to 800 c.c. of water, acidified with hydrochloric acid, and decomposed with hydrogen sulphide. The excess of hydrogen sulphide is removed by lieating on a water-bath, when the silver sulphide is filtered off and the filtrate evaporated to dryness. The residue is treated with from 25 to 30 c.c. of dilute sulphuric acid $(1: 100)$. This solution is brought to the boiling-point and is allowed to stand over night. The uric acid which has then separated out is filtered off, washed with a small amount of dilute sulphuric acid (not more than 50 c.c.), then with alcohol and ether, and weighed. To the resulting weight 0.0005 gramme is added for each 10 c.c. of the acid filtrate, to allow for the trace of uric acid which is thus lost.

After having filtered off the uric acid the filtrate is again treated with ammonia and silver solution, and the xanthin bases thus precipitated. The precipitate is collected on a small filter, washed with water, dried, and incinerated. The ash is dissolved in nitric acid, and the silver estimated by titration with a solution of potassium sulphocyanide, using ammonio-ferric alum as an indicator. The solution of potassium sulphocyanide employed in the estimation of the chlorides may be used, and is of such strength that 1 c.c. corresponds to 0.00734 gramme of silver. As 1 atom of silver in a mixture of the silver compounds of guanin, xanthin, hypoxanthin, etc., represents 0.277 gramme of nitrogen or 0.7381 gramme of the alloxur bases, it is apparent that 1 c.c. of the potassium sulphocyanide solution will represent 0.002 gramme of nitro-

\footnotetext{
${ }^{1} 1$ part of crystallized $\mathrm{MgSO}_{4}, 2$ parts of $\mathrm{NH}_{4} \mathrm{Cl}, 4$ parts of $\mathrm{NH}_{4} \mathrm{OH}$, and 8 parts of distilled water.
} 
gen and 0.00542 gramme of alloxur bases. In every case an accurate record must, of course, be kept of the amount of urine and filtrate used.

\section{Oxalic Acid and Oxaluric Acid.}

Of the origin of oxalic acid and oxaluric acid, both of which may be regarded as normal constituents of the urine, but little is known. The former is supposedly present as a calcium salt, which is held in solution owing to the presence of diacid sodium phosphate, but readily separates out on standing and is then frequently encountered in urinary sediments. Here it generally occurs in the very characteristic envelope or dumb-bell forms, and can be readily distinguished from other constituents by its insolubility in acetic acid, and its solubility in hydrochloric acid. Its amount normally varies between 0.02 and 0.05 gramme. Oxaluric acid, on the other hand, exists in the urine as an ammonium salt and is not found in sediments. Its amount is even smaller than that of oxalic acid.

As many articles of food, such as asparagus, spinach, grapes, apples, etc., contain oxalic acid in not inconsiderable amounts, it is supposed that a certain fraction of the oxalic acid of the urine is referable to this source. We find, as a matter of fact, that in the asparagus season larger amounts are eliminated than at any other time of the year. But it has also been noted that oxalic acid does not disappear from the urine when the diet consists exclusively of albumins and fats, and that during starvation also oxalic acid can still be found. We are consequently forced to the conclusion that a certain amount of the substance must originate in the tissues of the body. We know, indeed, that oxaluric acid is closely related to uric acid, and can be decomposed into urea and oxalic acid, as is shown by the equations:

(1) $\underset{\text { Uric acld. }}{\mathrm{C}_{5} \mathrm{H}_{4} \mathrm{~N}_{\mathrm{O}_{3}}}+\mathrm{O}+\mathrm{H}_{2} \mathrm{O}$<smiles>NC(=O)C1CNOC(=O)N1</smiles>

(2)<smiles>O=C1NOOC2NC1OO2</smiles>

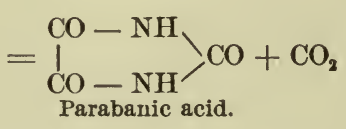

(3)<smiles>O=c1[nH]c(=O)oc(=O)[nH]1</smiles>

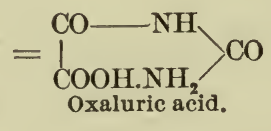

(4)

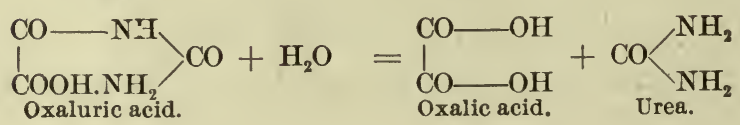


As oxalic acid on further oxidation is decomposed into water and carbon dioxide, it would thus appear that both oxaluric acid and oxalic acid may be regarded as complete oxidation-products of uric acid. We find, as a matter of fact, that oxalic acid is increased in various diseases in which the oxidation-processes are manifestly at fault, such as diabetes mellitus, various diseases of the circulatory apparatus when associated with deficient oxygenation of the blood, in obesity, etc. Whether or not oxalic acid may be derived from carbohydrates is unknown, but rather improbable. Aside from its occurrence in solution and in urinary sediments, oxalic acid is also not infrequently found as the principal constituent of renal and vesical calculi.

Quantitative Estimation of Oralic Acid.-DUNLOP's METHOD (modified by Baldwin).-Five hundred c.c. of a well-mixed specimen of the twenty-four hours' urine are treated with 150 c.c. of over 90 per cent. alcohol, to precipitate the oxalate of calcium. After fortyeight hours the crystals are collected on a filter, thoroughly washed with hot and cold water and with dilute acetic acid ( 1 per cent.). The filter is placed in a small beaker and soaked in a small amount of dilute hydrochloric acid. It is then washed with hot water until there is no further acid reaction. The washings are filtered and evaporated to about 20 c.c. A very little calcium chloride solution is added to insure an excess of calcium. The hydrochloric acid is neutralized with ammonia and the solution then rendered slightly acid with acetic acid. Strong alcohol is now added to the amount of 50 per cent. of the volume of the fluid and the solution set aside for forty-eight hours. The sediment is collected on a filter that is free from ash, and washed with cold water and dilute acetic acid until free from chlorides. (Hot water should here be avoided, as it carries the finely divided precipitate through the pores of the filter.) The filter is incinerated over a Bunsen burner, and afterward heated in the blowpipe-flame. The residue is allowed to cool over sulphuric acid and weighed. The ash is calcium oxide, each gramme of which corresponds to 1.6 grammes of oxalic acid.

The urine in every case should be thymolized as soon as possible, to prevent fermentation and the precipitation of phosphates. If the specimen is alkaline, it is rendered slightly acid with acetic acid.

The method is applicable in the case of human urine, but in that of dogs with a high specific gravity it is very difficult to remove the phosphates. In such an event Salkowski's method is best employed.

SALKowski's Method.-If the urine is concentrated (sp. gr. $1.040-1.050$ ), it is treated with 20 c.c. of hydrochloric acid (sp. gr. 1.12) for 200-250 c.c., and extracted in a separating funnel three times with alcoholic ether (5-10 per cent. alcohol). The ethereal extract is filtered through a dry filter, the ether is distilled off, the remaining fluid evaporated to 20 c.c., and filtered on cooling. The filtrate is rendered alkaline with ammonia, and is then treated with 
1-2 c.c. of a 10 per cent. solution of calcium chloride and acetic acid. The process is continued as described.

With human urine larger quantities, such as 500 c.c., are employed, which are first concentrated to about one-third of their original volume.

\section{Allantoin.}

Allantoin is a normal constituent of the urine of man, as also of various animals, but is usually present only in traces in the adult, while during the first weeks of life it is more abundant. Larger amounts also occur during pregnancy. Aside from the urine, it is found in the amniotic fluid and in the allantoic fluid of cows. Like oxalurie acid, it is an oxidation-product of uric acid, and Salkowski demonstrated that an increased elimination occurred in dogs when uric acid was ingested. An artificial increase is similarly observed in poisoning with hydrazin, and we can readily understand that in consequence of the degenerative changes which are thus produced in the liver the oxidation-processes are accordingly diminished, and allantoin results.

The formation of allantoin from uric acid may be represented by the equation :

$$
\begin{aligned}
& \mathrm{C}_{5} \mathrm{H}_{4} \mathrm{~N}_{4} \mathrm{O}_{3} \\
& \text { Uric acid. }
\end{aligned}
$$

It is a glyoxyl-diureid, and may be produced artificially by heating glyoxylic acid and urea together at a temperature of $100^{\circ} \mathrm{C}$. The reaction then takes place according to the equation :

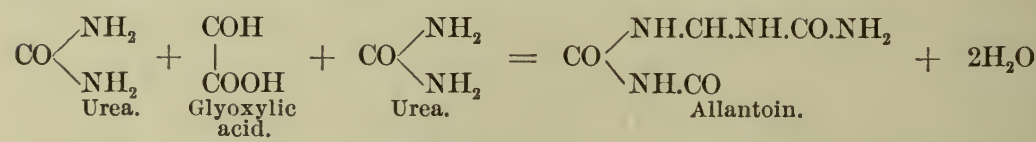

The substance crystallizes in colorless rhombic prisms, which are frequently grouped in the form of stars and rosettes. It is soluble with some difficulty in cold water, more readily in hot water and hot alcohol, while in cold alcohol and ether it is insoluble. On prolonged boiling, it reduces Fehling's solution, and it is owing to the formation of this substance, as already indicated, that uric acid can reduce cupric oxide when boiled in alkaline solution (see page 256). With silver nitrate allantoin forms a crystalline precipitate of allantoin silver, if ammonia is cautiously added to the solution, but it is soluble in an excess of the alkalies.

Isolation.-Allantoin is most conveniently obtained from the urine of calves. To this end, the urine is evaporated on a waterbath to a thick syrup and allowed to stand for several days in the cold. The crystalline constituents which have formed are then separated mechanically from the mother-liquor and the gelatinous material which is present, and dissolved in a small amount of hot 
water. The solution is decolorized with animal charcoal, filtered while hot, and the filtrate acidified slightly with hydrochloric acid. On standing, the allantoin separates out.

\section{Kreatin and Kreatinin.}

According to older concepts kreatin is a waste product of the protein metabolism and transformed in the body into its " anhydride" kreatinin and is thus eliminated in the urine. Folin and Klercker have shown, however, that this view is erroneous. Folin has pointed out that the transformation of the one substance into the other is not at all so easily accomplished as was formerly supposed and that kreatin fed by the mouth is not eliminated in the urine as kreatinin, while the introduction of the latter gives rise to a corresponding increase of the normal kreatinin in the urine. With the experimental subject on a diet poor in nitrogen Folin could show that the ingested kreatin is not eliminated either in the feces or the urine and is apparently largely retained in the body. To a certain degree this occurs also when the subject is placed on a diet rich in proteins. It accordingly appears that kreatin may be an important factor in the nitrogenous metabolism and can in a certain sense be viewed as a foodstuff. Further research, however, is necessary to clear the situation. When administered in excess a fraction appears in the urine as such. It then gives rise to the formation of neither kreatinin nor of urea.

Kreatinin, according to Folin, is the essential end-product of the endogenous nitrogenous katabolism, in so far at least as the muscle tissue is concerned. He has demonstrated the interesting fact that its absolute quantity eliminated on a meat-free diet is a constant quantity, which is different for different individuals, but wholly independent of quantitative changes in the total amount of nitrogen eliminated. Its relative amount is increased when the urea nitrogen falls. On a diet rich in proteins the kreatinin nitrogen represents $3.2-4.5$ per cent. of the total, while on one free from proteins (starch and cream) the amount may rise to 17.4 per cent. 'The absolute amount seems to depend to a certain extent upon the body weight. Fat or corpulent persons yield less kreatinin per unit of body weight, namely 20 mgrms. per kilo, while lean persons yield about $25 \mathrm{mgrms}$; 1.15-1.6 grammes may thus be regarded as average values.

The study of pathological variations in the amount of kreatinin has been greatly facilitated through the introduction of Folin's method (see below). The older data are of little importance, unless the diet of the individual has been carefully considered. A diet rich in meat, it should be borne in mind, greatly increases the amount.

Properties.-Kreatin crystallizes in colorless, highly refractive monoclinic prisms, and is quite soluble in hot and cold water, less 
readily so in alcohol, and is insoluble in ether. In aqueous solution it is gradually transformed into kreatin. The same transformation results more rapidly when the substance is heated in alkaline solution. It combines with acids and various salts to form crystalline compounds, some of which are characteristic. This is true especially of the chlorozincate- $\left(\mathrm{C}_{4} \mathrm{H}_{7} \mathrm{~N}_{3} \mathrm{O}_{2}\right)_{2} . \mathrm{ZnCl}_{2}$ - which results when a concentrated alcoholic solution of kreatinin is treated with a solution of zinc chloride, which should be as little acid as possible. The crystalline form of this compound depends very much upon its purity. As first obtained from the urine, it occurs in the form of varicose conglomerations, which usually adhere firmly to the walls of the vessel. In pure form it crystallizes in fine needles, which are commonly grouped in sheaves and stars. The salt is almost insoluble in alcohol, and nearly so in water, while free mineral acids dissolve it.

Kreatinin is further precipitated by mercuric nitrate, notably in the presence of sodium carbonate, by silver nitrate, platinum chloride, stannous chloride, mercuric chloride, picric acid, phosphotungstic acid, etc. With all these substances it forms well-characterized crystalline salts. In alkaline solution it reduces cupric hydroxide, and it is for this reason that urines which contain much kreatinin but no sugar give a positive reaction with Fehling's solution. The separation of the cupric oxide, however, only occurs on prolonged boiling. An alkaline solution of bismuth, on the other hand, is not affected.

Tests.-WEYL's TEST.-A few cubic centimeters of urine are treated with a few drops of a freshly prepared, very dilute solution of sodium nitroprusside, and then drop by drop with a solution of sodium hydrate, when in the presence of kreatinin a ruby-red color develops, which is especially pronounced in the lower portion of the tube. After a few minutes the color disappears. If now acetic acid is added in excess and the mixture heated, it assumes a greenish color, then turns blue, and on standing deposits a sediment of Prussian blue. Acetone and diacetic acid give a similar reaction if ammonia is added instead of sodium hydrate; but with kreatinin no red color is thus obtained.

JAFFE'S TEST.-If a few cubic centimeters of urine are treated with a dilute aqueous solution of picric acid, the corresponding compound of kreatinin is precipitated. On adding a few drops of a dilute solution of sodium hydrate a red color develops, which persists for several hours, and is changed to yellow upon the addition of an acid. With glucose a red color also is obtained, but only on heating, while the reaction in the case of kreatinin takes place only at ordinary temperatures. Acetone gives a reddish-orange color.

Synthesis of Kreatinin.-Kreatinin can be formed synthetically from methyl-glycocoll and cyanamide. Kreatin is first produced, and then yields the anhydride, as shown in the equations: 
(1)

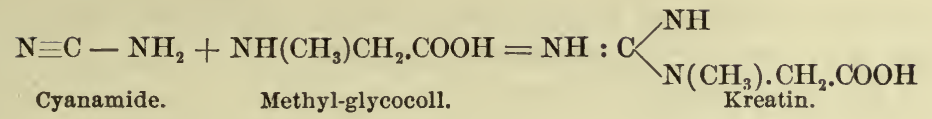

(2)
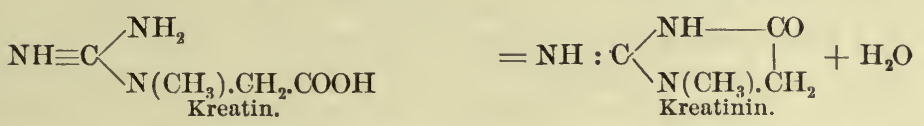

Isolation.-The isolation of kreatinin from the urine is based upon the formation of the chlorozincate, which is almost insoluble in alcohol : 240 c.c. of urine, which should be free from albumin and sugar, are rendered alkaline with milk of lime, and treated with a solution of calcium chloride so long as a precipitate forms. Water is then added to the 300 c.c. mark. After standing for a while the phosphates are filtered off. The precipitate is washed with a little water. Filtrate and washings are rendered slightly acid with acetic acid, and are then evaporated to a syrup. This is stirred while still warm with about 25 to 30 c.c. of absolute alcohol, transferred to a glass-stoppered flask, and diluted with absolute alcohol to 100 c.c. After twenty-four hours the mixture is filtered. The filtrate is treated with a small amount of sodium acetate in solution, and is concentrated to about 50 c.c. To this is added 0.5 c.c of a concentrated alcoholic solution of zinc clloride, which is prepared by dissolving a small amount of the salt in 80 per cent. alcohol, and diluting with 95 per cent. alcohol to a specific gravity of 1.2 . The mixture is then well stirred and set aside in a cool place for several days. Crystals of the chlorozincate of kreatinin are thus obtained.

To isolate the kreatinin from the chlorozincate, the latter is dissolved in a small amount of hot water and boiled for ten minutes with well-washed plumbic hydrate. After filtering off the insoluble oxide of zinc and the chloride of lead the filtrate is evaporated to dryness and extracted with cold absolute alcohol. This takes up the kreatinin, while a small amount of kreatin formed during the process of boiling remains. On evaporation the kreatinin is obtained in erystalline form, and can be further purified by recrystallization from water.

Quantitative Estimation.-FoLIN's METHOD.-This method is based on Jaffe's reaction of kreatinin with alkaline picric acid solution. The red colored solution produced in this reaction has in proper concentration and when viewed by transmitted light exactly the same shade as a potassium bichromate solution. Half-normal potassium bichromate solution (containing 24.55 grammes per liter) is therefore used as a standard for comparison.

A high-grade colorimeter, by means of which the depths both of the unknown solution and of the bichromate can be adjusted to tenths of millimeters, is necessary for the comparison. ${ }^{1}$

The following solutions are also necessary: The half-normal po-

1 The French instrument of Duboscq, which can be obtained through Eimer \& Amend, is admirably suited for the purpose. 
tassium bichromate solution, 10 per cent. sodic hydrate, and a saturated (1.2 per cent.) picric acid solution.

If to $10 \mathrm{mgrms}$. of chemically pure kreatinin dissolved in 10 c.c. of water in a 500 c.c. volumetric flask are added 15 c.c. of picric acid solution and 5 c.c. of sodic hydrate, the maximum color is obtained at the end of five minutes. If at the end of this time the solution be diluted to the 500 c.c. mark and at once compared with the standard bichromate solution, it will be found that $8.1 \mathrm{~mm}$. of the kreatinin-picrate solution have in the colorimeter exactly the same shade and depth of color as $8 \mathrm{~mm}$. of the bichromate solution.

The actual determination in urine is carried out in exactly the same way, substituting 10 c.c. of urine for the kreatinin solution. The more kreatinin that is present in the 10 c.c. of urine the deeper will, of course, be the color of the solution obtained. Supposing the colorimetric observation shows that $7.1 \mathrm{~mm}$. of the urine picrate solution are equal in color to $8 \mathrm{~mm}$. of the standard. The 10 c.c. of urine would then contain $10 \times \frac{8.1}{7.1}=11.4 \mathrm{mgrms}$. of kreatinin.

The following precautions are to be observed in the determination:

1. Make first a preliminary colorimetric observation, using halfnormal potassium bichromate solution in both cylinders of the colorimeter, adjusting first one to the $8 \mathrm{~mm}$. mark. The average of three or four readings of the other cylinder should also be $8 \mathrm{~mm}$., and after the first observation no two should differ by more than 0.2 $\mathrm{mm}$. This preliminary observation takes only two or three minutes, and is exceedingly useful in making the eye sure of the correct point to be ascertained.

2. Exactly $8 \mathrm{~mm}$. of the half-normal potassium bichromate solution must be used as the standard for comparison; 16 or $24 \mathrm{~mm}$., for example, cannot be substituted on the basis of the calculation given above because the kreatinin picrate solution absorbs light at an entirely different rate from that of the bichromate solution.

3 . For the reason given in the preceding paragraph it is necessary to make each deternination with a quantity of urine containing not less than 5 or more than 15 mgrms. of kreatinin. Within these limits the determination as described is correct within $0.2 \mathrm{mgrm}$.

4. Sugar and albumin do not interfere with the determination. Acetone, diacetic acid, and hydrogen sulphide do interfere. Where these are present the urine should be measured into a porcelain evaporating-dish and heated on a water-bath with 10 c.c. of 10 per cent. hydrochloric acid for about half an hour. When the dish is again cooled, the reagents are added directly into the dish, and finally rinsed into the volumetric flask after five minutes.

5. The color due to the urine is ordinarily of no appreciable consequence because of the great dilution. Urines containing bile-pigments can, however, first be cleared by the addition of egg-albumin and then removing this by coagulation (heat). 
The whole operation can be finished in less than fifteen minutes; indeed, it should be finished at once, as the colored product obtained by the interaction of kreatinin and picric acid is not very stable.

\section{THE AROMATIC CONSTITUENTS OF THE ORINE.}

It has been pointed out in a preceding chapter that during the process of intestinal putrefaction various aromatic bodies are formed from the albumins of the food or their products of digestion, and are then absorbed and eliminated in the urine, either as such or in combination with sulphuric acid, glucuronic acid, or glycocoll. Some of these bodies, such as indol and skatol, may be regarded as specific products of putrefaction; while others or closely related substances represent radicles which exist as such in the albuminous molecule. We consequently recognize two sources of the aromatic bodies which are found in the urine, viz., the aromatic bodies which enter into the composition of our diet as such, and those which result from the destruction of albumins through the activity of micro-organisms. Under certain pathological conditions, further, substances of this character may also be formed in the body proper, owing to degenerative changes, which may or may not be the result of bacterial action. Under normal conditions, however, this source scarcely enters into consideration.

\section{The Conjugate Sulphates.}

Paracresol, phenol, hydroquinon, pyrocatechin, indol, and skatol are largely eliminated in the urine in combination with sulphuric acid as sodium or potassium salts. But while paracresol and its derivatives combine with sulphuric acid directly, indol and skatol are previously oxidized to indoxyl and skatoxyl, as has been shown. Conjointly the resulting compounds are spoken of as the conjugate or ethereal sulphates of the urine. Their daily excretion in man corresponds to about one-tenth of the mineral sulphates, viz., from 0.094 to 0.620 gramme, under normal conditions. Increased amounts are observed when from whatever cause the intestinal putrefaction is increased. It is to be noted, however, that the ratio between the individual substances is even normally not constant, and it seems that the relative preponderance of the one over the other is primarily referable to the extent to which individual groups of micro-organisms are active. In some instances we may thus find that the increase in the conjugate sulphates is referable to an increased production of indol and skatol, while in others phenols are largely formed.

Aside from an increase in the degree of intestinal putrefaction, larger amounts of the conjugate sulphates may also be observed if putrefactive processes are taking place within the body proper, providing that active resorption occurs from the diseased area. An increased elimination is also noted when any of the aromatic sub- 
stances mentioned are ingested as such or otherwise introduced into the circulation from without. A notable increase is thus observed in poisoning with carbolic acid or its congeners, and is then, of course, principally owing to an increased formation of phenol sulphates. The ingestion of ortho-nitro-phenyl-propiolic acid, which is reduced to indoxyl within the body, similarly leads to an increased elimination of indoxyl sulphate.

The synthesis of the various conjugate sulphates is largely effected in the liver, but may also occur in other organs of the body, such as the kidneys and lungs. In some experiments positive results were also obtained in the case of the muscle-tissue and the intestinal wall, but these organs play only a secondary role as compared to the liver. Their quantitative estimation in toto has been described.

The Phenols.-Of the phenols which occur in the urine, paracresol is the most abundant; next in order comes phenol, while pyrocatechin and hydroquinon are found only in traces. Besides paracresol, the normal urine of man is said also to contain minute amounts of meta- and ortho-cresols. The total elimination of cresols and phenols, however, normally corresponds to only about 0.03 gramme in the twenty-four hours.

Urine which contains much hydroquinon or pyrocatechin gradually assumes a dark-brown color on standing if the reaction is alkaline, and it is noted that this change in color begins in the upper layers and gradually extends downward. Ultimately the urine becomes almost black. This change is referable to oxidation of the dioxybenzols, but of the resulting compounds nothing is known. Urines of this character are mostly observed in poisoning with carbolic acid, following the administration of benzol, of phenol sulphates, etc.

To demonstrate the presence of phenol or of paracresol, 1000 c.c. of urine are treated with 70 c.c. of concentrated hydrochloric acid, and distilled until about one-fourth of the total amount has passed over. The conjugate sulphates are thus decomposed, and phenol and cresol are found in the distillate. Their presence can here be demonstrated by testing with Millon's reagent or by adding bromine-water, when tribromophenol crystallizes out on standing. If much phenol is present, it may further be possible to obtain a positive reaction with ferric chloride solution if this is added drop by drop in very dilute solution (an amethystblue color resulting). Hydroquinon and pyrocatechin remain in the acid solution. To demonstrate their presence, this is evaporated to about 100 c.c., and on cooling extracted with an equal volume of ether. Hydroquinon and pyrocatechin together with the aromatic oxy-acids are thus removed. On adding a dilute solution of sodium carbonate to the ethereal solution the aromatic oxy-acids are transformed into the corresponding sodium salts. The ethereal extract, which now contains only the dioxy-benzols, is evaporated to dryness; the residue is dissolved in 
a little water and examined as follows: one portion is treated drep by drop with a dilute solution of ferric chloride, when in the presence of pyrocatechin a green color develops, which turns to violet upon the addition of a small amount of tartaric acid and ammonia. The remainder of the solution is precipitated with lead acetate and filtered. The filtrate contains the hydroquinon, while the pyrocatechin is in the precipitate. The hydroquinon can then be isolated by acidifying and extracting with ether, when the substance crystallizes out on evaporation. It is dissolved in a little water, and treated drop by drop with the dilute iron solution. Quinon, $\mathrm{C}_{6} \mathrm{H}_{4} \mathrm{O}_{2}$, results, and may be recognized by its penetrating odor.

Quantitative Estimation.-Method of Kossler and Penny, MODIFIED BY NeuberG.-This method is based upon the precipitation of phenol and paracresol, by means of iodine, as tri-iodophenol. From the amount of iodine which is thus used the corresponding amount of monoxy-benzols can be calculated, and is expressed either in terms of phenol or cresol, as the method does not indicate the separate amount of the individual bodies that are present. The reaction which takes place may be represented by the equation :

$$
\mathrm{C}_{6} \mathrm{H}_{5} \cdot \mathrm{OH}+6 \mathrm{I}=\mathrm{C}_{6} \mathrm{H}_{2} \cdot \mathrm{I}_{3} \mathrm{OH}+3 \mathrm{HI} .
$$

Five hundred c.c. of urine are rendered feebly alkaline and evaporated to about 100 c.c. Any acetone which may have been present is thus removed. The residual fluid is acidified with sulphuric acid, so as to contain 5 per cent. of the original volume, and is then repeatedly distilled. The individual portions thus obtained are shaken with calcium carbonate until the acid reaction has disappeared, so as to remove any nitrous or formic acid that may be present. The fluid is now again distilled, and the distillate treated with a solution of 1 gramme of caustic soda and 6 grammes of lead acetate in substance. The mixture is kept on a boiling water-bath for about fifteen minutes. A portion of the lead oxide is thus dissolved by the phenol to form basic phenolates, while any aldehydes or ketones that may have been formed from the small amount of carbohydrates that are present in every urine escape. To remove these entirely, the mixture is heated over a free flame connected with a condenser until a few cubic centimeters of the distillate no longer reduce an alkaline solution of silver nitrate. After five minutes this point is usually reached. The fluid is then acidified with sulphuric acid as before, and is distilled, water being added from time to time. The distillate is placed in a glass-stoppered bottle, treated with a decinormal solution of sodium hydrate until the reaction is markedly alkaline, and immersed in hot water. 'To the hot fluid a decinormal solution of iodine is added in an amount which should exceed that of the alkali solution by 15 to 25 c.c. The bottle is now closed at once, shaken, and set aside until cool. The solution is then acidified with dilute sulphuric acid, and the excess of iodine, 
which was not used in the formation of tri-iodophenol, retitrated with a decinormal solution of sodium thiosulphate. One c.c. of the iodine solution represents 1.567 milligramme of phenol, or 1.8018 milligramme of cresol. As the latter predominates in the urine, it is best to express the results in terms of cresol. Thus modified, the method is also applicable in the presence of sugar. The older gravimetric method, by which the phenols were isolated as bromine substitutionproducts, has now been largely abandoned, as it has been shown that the resulting precipitate is not of constant composition, and contains variable amounts of dibromocresol, besides tribromophenol and bromo-tribromophenol.

Indoxyl Sulphate.-The indoxyl sulphate which occurs in the urine in combination with potassium and sodium is usually spoken of as indican, but should not be confounded with vegetable indican, which is a glucoside of the composition $\mathrm{C}_{26} \mathrm{H}_{31} \mathrm{NO}_{17}$. The amount which is daily excreted by man is normally small, and corresponds to about 0.0066 gramme. Larger quantities are observed when from any cause intestinal putrefaction is increased, or in cases in which putrefactive changes are taking place in the body proper, as in empyema, providing that active resorption can occur.

In herbivorous animals larger amounts are found than in the carnivora. Artificially an increased elimination can be effected by feeding animals with ortho-nitro-phenyl-propiolic acid, which is reduced in the body to indoxyl, according to the equation:

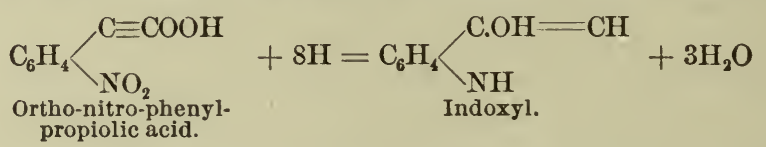

Indican crystallizes in colorless platelets, which are readily soluble in water and hot alcohol, while in cold alcohol they dissolve with great difficulty. On decomposition with hydrochloric acid the indoxyl is obtained in the form of oily droplets of an exceedingly offensive, feculent odor. On oxidation this is then transformed into indigo-blue, as is shown in the equation :

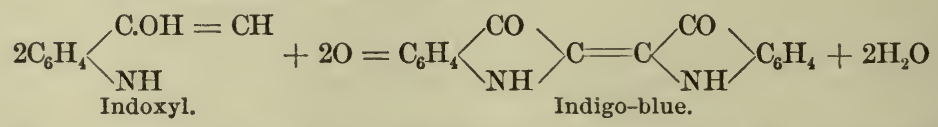

On heating an aqueous solution of indoxyl to a temperature of $130^{\circ} \mathrm{C}$. indoxyl-red results. This is a brown amorphous substance, which is insoluble in water, but dissolves with ease in alcohol, ether, and chloroform, with a beautiful red color. When Jaffe's test for indican is applied to the urine, or when this is boiled and treated drop by drop with concentrated nitric acid (Rosenbach's reaction), a mixture of a blue and a red pigment is not infrequently obtained, and it is quite likely that the latter is in part at least referable to the formation of the indoxyl-red. According to some observ- 
ers, the chromogen of this substance is identical with the so-called urohæmatin, and the pigment is probably the same as the red pigment of Scherer, the urrhodin of Heller, the urorubin of Plosz, the indirubin of Schunk, the indigo-purpurin of Bayer, the pigment of Giacosa and others.

The blue pigment which is found together with the red pigment when urine is treated with a strong mineral acid and an oxidizing agent is, as has been indicated, indigo-blue, and is identical with urocyanin, cyanurin, Harnblau, uroglaucin, etc., of former observers. As a general rule, its amount is far greater than that of the red pigment, and is at times the only one that is obtained. In other cases, however, the red seems to prevail, and in still others both are apparently present in about equal proportion. The cause of these variations is not understood, but probably depends upon variations in bacterial action in the intestinal tract. As a general rule, indeed, notable quantities of the red pigment are observed only under pathological conditions.

Tests for Indican.-All the tests employed for the purpose of demonstrating the presence of indican in the urine are essentially based upon the decomposition of the substance, with the liberation of indoxyl and its oxidation to indigo-blue.

JAFFe's 'Test, As Modrfied By STOKVIS.-A few cubic centimeters of urine are treated with an equal volume of concentrated hydrochloric acid, and two or three drops of a strong solution of sodium hypochlorite. The indigo-blue which thus results is then extracted by shaking with a little chloroform. If red pigment has been formed at the same time, the color varies from a violet to a purplish red.

If it is desired to separate the two pigments, the chloroform extract is evaporated to dryness and the residue washed with a mixture of equal parts of 96 per cent. alcohol, ether, and water. This dissolves the red pigment and leaves the indigo-blue behind. Care must be had, however, not to add too much of the hypochlorite solution, as otherwise the indigo-blue is oxidized to isatin, and no color at all is obtained. Should this happen after the addition of only one or two drops, the following test had better be employed, as a further oxidation is here not effected :

OBERMA YER's TEST.-A few cubic centimeters of urine are treated with an equal volume of a 2 pro mille solution of ferric chloride in concentrated hydrochloric acid. The indigo-blue is extracted, as above, by shaking with a little chloroform. As in the above test, indoxyl-red may thus also be obtained, and is separated from the blue pigment as just described.

Test for Urohæmatin (so-called).-A small amount of urine is thoroughly agitated with chloroform and allowed to stand for a few days. The chromogen of indoxyl-red is thus extracted, for on adding a drop of concentrated hydrochloric acid to the chloroform extract a beautiful rose-color appears, which varies in intensity with the 
amount of the chromogen present. In normal urine a faint reaction only is usually seen; but in disease, and notably in ileus, peritonitis, and cancer of the stomach, I have repeatedly met with more indigored than indigo-blue.

Rosenbach's REACTION.-This reaction is mostly obtained under pathological conditions, and indicates the existence of greatly increased intestinal putrefaction. It is referable to the simultaneous formation of indigo-red and indigo-blue.

While boiling, a few cubic centimeters of urine are treated drop by drop with concentrated nitric acid containing a little nitrous acid, when in the presence of much indigo-red, viz., its chromogen, the urine assumes a dark Burgundy color, and usually shows a bluish tint when held to the light. On standing, the red pigment is precipitated. If much indigo-blue is present at the same time, as is usual, the foam of the liquid is colored blue. On adding an excess of the acid the color often disappears and the urine turns yellow.

The isolation of indican from urine as such is a rather complicated process, and need not be described at this place.

Quantitative Estimation.-W ANG'S METHOD(modified by Ellinger). - This method is based upon the decomposition of the indican by strong hydrochloric acid, and the oxidation of the resulting indoxyl to indigo-blue. This is then transformed into indigo-sulphuric acid, and estimated as such by titration with a solution of potassium permanganate of known strength. To this end, a stock solution of the salt is kept on hand, which contains about 3 grammes to the liter. Five c.c. of this solution are diluted with 195 c.c. of water, when the titre is ascertained before each titration by comparing it with a dilute solution of oxalic acid. The amount of indigo-blue which each cubic centimeter represents is ascertained by multiplying the corresponding amount of oxalic acid by 1.04 .

The amount of urine which is necessary varies with the amount of indican present. If a preliminary test gives an intense reaction, from 25 to 50 c.c. are sufficient; otherwise it is better to use larger amounts, as from 200 to 250 c.c. The urine, which should be acid, is then precipitated with a 20 per cent. solution of basic lead acetate, care being taken to avoid an excess. A large portion of the filtrate, representing a known amount of urine, is then treated with an equal volume of Obermayer's reagent, and extracted with chloroform by shaking. This is continued with portions of 30 c.c. until the chloroform takes up no more coloring-matter. The combined extracts are freed from chloroform by distillation. The residue is dried for a few minutes on a water-bath, and is then washed with hot water. The solution is passed through a small filter, so as to collect any particles of the blue pigment which may be present in suspension. The filter is dried, extracted with boiling chloroform, and the resulting solution filtered into the flask containing the residual indigo-blue. The chloroform is distilled off, and the resi- 
due treated with 3 or 4 c.c. of concentrated sulphuric acid, while still warm. This solution is kept on the water-bath for five to ten minutes. It is then poured into 100 c.c. of water, the bottle is washed out with a little more water, when the solution and washings are filtered and titrated with the permanganate solution. The color at first changes to green, and finally the solution becomes yellowish or colorless. The calculation is conducted as outlined above.

Skatoxyl Sulphate.-Skatoxyl sulphate, like indoxyl sulphate, occurs in the urine in combination with potassium and sodium. Its amount, however, is normally small, and it may at times be absent altogether. Larger quantities are found under pathological conditions associated with an increased degree of intestinal putrefaction, and it may then happen that more skatoxyl sulphate is found than ipdican. This, however, is uncommon, and in disease also more indican is usually present. Like indican, it is decomposed on treating with concentrated hydrochloric acid, and on subsequent oxidation the liberated skatoxyl yields pigments which are for the most part of a red color. Of their chemical nature, however, nothing is known. One of these may possibly be identical with Rosin's urorosein. Rosin, to be sure, claims that the chromogen of urorosein is not a conjugate sulphate but we know that a portion of the skatol also appears in combination with glucuronic acid in the urine, and it is hence possible that his pigment may be derived from this source. Urines containing notable quantities of skatoxyl become darker on exposure to the air, and may gradually turn a reddish-violet or almost a black color. This change, as in the phenol urines, begins at the surface and gradually extends downward.

Tests.-To demonstrate the presence of skatoxyl in urine, this is strongly acidified with hydrochloric acid and extracted with amyl alcohol, which takes up the coloring-matter. Chloroform and ether do not dissolve this in acid solution, but do so in neutral or alkaline solution, providing that the pigment has been freshly formed.

Test for Urorosein (so-called).-A few cubic centimeters of urine are treated with an equal amount of concentrated hydrochloric acid and one or two drops of a strong solution of calcium hypochlorite. The indigo-blue is extracted with chloroform, and it will now be olserved that the supernatant fluid presents a red color. On shaking with amyl alcohol this takes up the red pigment, which is thus manifestly not identical with indigo-red. Upon the addition of sodium hydrate to the alcoholic solution the color disappears, but reappears upon the subsequent addition of hydrochloric acid. On standing, the color gradually disappears. Under normal conditions this reaction is not well marked, but becomes quite distinct in cases in which intestinal putrefaction is much increased.

A portion of the skatol, as I have already stated, appears in the urine as skatol-carbonic acid: 
<smiles>CC(C)(C)[C@H](Nc1ccccc1)C(=O)O</smiles>

The substance is usually present in exceedingly small amounts, however, and has not been isolated in substance.

In addition to the aromatic bodies which have thus far been considered, traces of the aromatic oxy-acids may also appear in the urine in combination with sulphuric acid, but the amount is exceedingly small and may well be ignored.

\section{The Conjugate Glucuronates.}

Glucuronic acid as such does not occur in the urine. The substance can combine with various aromatic bodies, however, and may in this manner escape further oxidation. Normally it is found only in traces (approximately 0.004 per cent.), in combination with indoxyl, skatoxyl, and especially with phenol, while the greater portion of these bodies is eliminated in the form of conjugate sulphates, as already described. Larger, amounts of conjugate glucuronates are found in the urine after the administration of chloral, camphor, naphthol, terpene, borneol, menthol, toluol, euxanthin, morphin, antipyrin, and numerous alcohols and ketones. The resulting compounds are closely related to the glucosides. So far as the hydroxylated compounds are concerned, these are apparently capable of uniting with glucuronic acid (or sulphuric acid) to form conjugate glucuronates (or sulphates) without previous oxidation, while this is not the case with compounds of the same composition, in which, however, the oxygen is present in ketone form. At the same time it is an open question whether the union of such alcohols with the acids occurs directly, or, as is possibly the case with glucuronic acid, with antecedents of the same, and that the resulting compounds are subsequently oxidized to glucuronates during their passage through the organism.

In part at least the synthesis of the conjugate glucuronates occurs in the liver, and in some cases their appearance is manifestly the expression of a poison-destruction on the part of the organism. This explanation does not hold good in all cases, however. Following the ingestion of glucose in large amounts or in diabetes the appearance of glucuronates is evidence primarily of deficient oxidation. We may here imagine that the oxidation of glucose to glucuronic acid (see below) pursues a normal course, but that its further destruction is impeded. The larger amount of the circulating glucuronic acid then combines with phenol, indoxyl, and skatoxyl, which would otherwise have united with sulphuric acid, and as a result we find a diminished excretion of conjugate sulphates. Any glucuronic acid remaining is probably oxidized to oxalic acid. This explains satisfactorily the oxaluria which is frequently observed in diabetes and which follows the ingestion of large amounts of glucose. Ac- 
cording to the character of the aromatic component uniting with glucuronic acid, the resulting compounds have been termed camphorglucuronic acid, urochloralic acid, menthol-glucuronic acid, phenylindoxyl-, skatoxyl-glucuronic acid, etc.

Of the origin of glucuronic acid little is known. That it is formed in the tissues of the body is apparent from the fact that even in a starving animal the administration of camphor, chloral, etc., leads to elimination of these substances in combination with the acid in question. As glucuronic acid is a derivative of glucose, we may imagine that during starvation it is derived from glycogen, or even from the albumins. It has been demonstrated, as a matter of fact, that the formation of glycogen in the liver can be artificially increased by introducing glucuronic acid with the food. The chemical relation of glucuronic acid to glucose has already been considered. On oxidation glucose thus first yields the monobasic gluconic acid, and then the dibasic saccharinic acid. The latter in turn may be transformed into saccharo-lactonic acid, which on reduction yields glucuronic acid, so that this stands midway between gluconic acid and saccharinic acid. These relations are shown by the formulæ:

$$
\begin{aligned}
& \mathrm{CH}_{2} \cdot \mathrm{OH}-(\mathrm{CH} . \mathrm{OH})_{4}-\mathrm{COH} \text {, glucose. } \\
& \mathrm{CH}_{2} \cdot \mathrm{OH}-(\mathrm{CH} . \mathrm{OH})_{4}-\mathrm{COOH} \text {, gluconic acid. } \\
& \mathrm{COOH}-(\mathrm{CH} . \mathrm{OH})_{4}-\mathrm{COH} \text {, glucuronic acid. } \\
& \mathrm{COOH}-(\mathrm{CH} . \mathrm{OH})_{4}-\mathrm{COOH} \text {, saccharinic acid. }
\end{aligned}
$$

On boiling with water glucuronic acid is, in part at least, transformed into its anhydride, glucuron, $\mathrm{C}_{6} \mathrm{H}_{8} \mathrm{O}_{6}$.

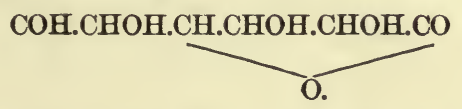

Formerly glucuronic acid was also thought to be derived from chondroitin-sulphuric acid; this view, however, has now been abandoned.

Glucuronic acid does not occur in crystalline form; it is a syrupy substance which is readily soluble in water and alcohol. Its anhydride, glucuron, however, is a crystalline body, and is likewise soluble in water, but insoluble in alcohol. The free acid and its alkaline salts are dextrorotatory, while the conjugate glucuronates turn the polarized light to the left. The free acid, moreover, as well as its salts and most of its compound ethers, reduce the oxides of copper, bismuth, and silver in alkaline solution, and it is thus possible to confound them with glucose if reliance is placed upon the corresponding tests alone. With phenyl-hydrazin the free acid is said to form a crystalline compound with a melting-point of $114^{\circ}-$ $115^{\circ} \mathrm{C}$. Unlike glucose, it is non-fermentable. It gives the furfurol reaction and simulates the pentoses in reacting with phloroglucin hydrochlorate, but not with orcin (see Pentoses). The amount of furfurol which may be obtained from glucuronic acid on 
distillation with hydrochloric acid is quite considerable. According to Günther, Chalmot, and Tollens, it yields 46 per cent., and its derivatives, euxanthinic acid and urochloralic acid, 12.5 and 17 per cent. respectively.

The demonstration of the presence of glucuronic acid in the urine and other fluids of the body directly is most conveniently conducted by decomposing the conjugate glucuronates with 1 per cent. sulphuric acid in the autoclave, and preparing its $p$-bromphenylhydrazin compound (COH. (CH.OH) $)_{4} \cdot \mathrm{COOH} . \mathrm{NH}_{2} \cdot \mathrm{NH}_{6} \mathrm{C}_{6} \mathrm{Br}$ ). This is characterized by its high melting-point, $236^{\circ} \mathrm{C} .\left(200^{\circ}-216^{\circ} \mathrm{C}\right.$.) in impure form) its insolubility in absolute acohol, and its high degree of lævorotation in a pyridin-alcoholic solution, viz., $7^{\circ} 25^{\prime}$. The same method may be used for its quantitative estimation (Zeit. $f$. phys. Chem., 1900, vol. xxix., p. 256).

For the clinical recognition of glucuronic acid compounds the following data suffice: lævorotation of the urine after fermentation, which diminishes on boiling with acids or changes to dextrorotation; an increased reduction after boiling with dilute sulphuric acid, and a positive orcin reaction (see Pentoses), which was negative before boiling.

\section{The Compound Glycocolls.}

As has been pointed out, phenyl-propionic acid and phenyl-acetic acid, which are both formed from albuminous material during the process of intestinal putrefaction, are in part absorbed in the intestinal tract, and are eliminated in the urine in combination with glycocoll as hippuric acid and phenaceturic acid, respectively. But while phenyl-acetic acid unites with glycocoll directly, phenyl-propionic acid is usually first oxidized to benzoic acid (see page 95).

Hippuric Acid.-While a certain amount of the benzoic acid which enters into the construction of the hippuric acid molecule is derived from the phenyl-propionic acid which results during the process of intestinal putrefaction, another portion is ingested as such, or in the form of other aromatic substances which can be transformed into benzoic acid in the animal body. We can thus understand why larger amounts of hippuric acid are encountered in the urine of herbivorous animals than in that of the carnivora, as the food of the former always contains very considerable amounts of toluol, cinnamic acid, quinic acid, etc., all of which may give rise to benzoic acid. In man the daily elimination corresponds to about 0.7 gramme, but may be increased by the ingestion of such articles of food as cranberries, prunes, reine-claudes, etc.

In a few instances the substance has been found in urinary sediments.

While the source of the aromatic component of hippuric acid is thus quite well understood, we know but little of the origin of glycocoll. To a certain extent this may also be formed during the process of pancreatic digestion, as we know that all albumins con- 
tain a glycocoll radicle, but there is reason to believe that it may likewise originate within the tissues of the body.

Through the interesting researches of Schmiedeberg we know that the synthesis of glycocoll and benzoic acid is in dogs effected in the kidneys exclusively. In other animals, however, this process may occur in other organs as well, for it has been shown that after removal of the kidneys hippuric acid ean be isolated from the liver and the muscles, at least, if benzoic acid has been previously administered.

Properties.-Hippuric acid crystallizes in long rhombic prisms when allowed to separate from its solutions slowly, while when rapidly formed, and especially if the amount is small, it occurs in long needles which are frequently grouped in stars and rosettes. The melting-point of the substance is $187.5^{\circ} \mathrm{C}$. It is soluble with great difficulty in cold water and ether, while in hot water and alcohol it dissolves with comparative ease. In aqueous solutions of the alkaline hydrates and carbonates it dissolves with the formation of the corresponding salts, from which the free acid may again be obtained by acidifying with a mineral acid.

On boiling with dilute mineral acids or alkalies hippuric acid is decomposed into its components. The same result is reached if ammoniacal decomposition is allowed to occur, and in such urines benzoic acid only is found. In traces, benzoic acid is said to occur in every urine together with hippuric acid, and it is thought that its presence under normal conditions may be due to the action of a ferment, the so-called histenzyme of Schmiedeberg, which has been found in the kidneys, and which is known to be capable of effecting the decomposition of hippuric acid as outlined.

On heating hippuric acid in a dry test-tube it melts, and is then decomposed with the formation of benzoic acid, which sublimes in the upper portion of the tube. The liquid mass at the same time assumes a red color, and develops an odor which at first is suggestive of hay, but subsequently resembles that of hydrocyanic acid. This reaction, together with the form of the crystals and their insolubility in petroleum-ether, serves to distinguish hippuric acid from benzoic acid. But like this, it develops a marked odor of bitter almonds when it is evaporated with nitric acid and the residue is then heated. The reaction is due to the formation of nitrobenzol. In the urine hippuric acid is, of course, not present in the free state, but in combination with alkalies, and notably potassium and sodium.

Synthesis of Hippuric Acid.-Hippuric acid can be formed synthetically in vitro also from benzoic acid and glycocoll by heating the two substances together at a temperature of $160^{\circ} \mathrm{C}$. in a sealed tube. In a similar manner it is obtained from benzamide and monochlor-acetic acid. The reactions which take place may be represented by the equations: 


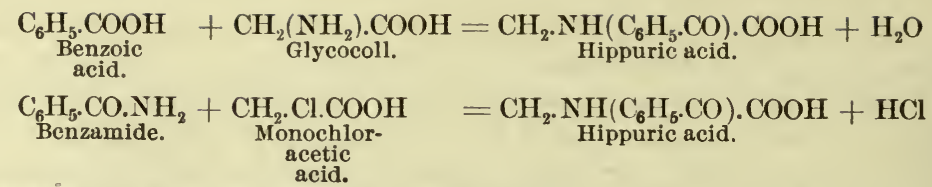

Isolation and Quantitative Estimation. -500 to 1000 c.c. of urine are rendered slightly alkaline with sodium carbonate, and are evaporoted to a thick syrup, taking care that the reaction remains alkaline. This is then extracted with cold strong alcohol (90 to 95 per cent.) by adding about one-half the volume of the original solution, and allowing the mixture to stand for twentyfour hours. It is then filtered and the alcohol distilled off. The remaining aqueous solution is acidified with dilute sulphuric acid, and the liberated hippuric acid extracted with several portions of acetic ether. The ethereal solution is evaporated to dryness, when the remaining impurities, such as phenols, aromatic oxy-acids, benzoic acid, and fat, are removed by washing with cold petroleumether, in which hippuric acid is insoluble. It is then dissolved in warm water and the solution evaporated at a temperature of from $50^{\circ}$ to $60^{\circ} \mathrm{C}$. until crystallization occurs. On cooling, the crystals are filtered off and weighed. The mother-liquor is extracted with acetic ether, the ethereal solution is evaporated to dryness, and the weight of the residue added to that of the crystals.

Isolation of Glycocoll and Benzoic Acid from Hippuric Acid.-As stated before, glycocoll is most conveniently obtained from hippuric acid. To effect the decomposition, this is boiled for ten to twelve hours with 4 parts of dilute sulphuric acid. On cooling, the benzoic acid which has separated out is filtered off. The filtrate is concentrated and extracted with ether, which takes up the remaining benzoic acid. The sulphuric acid is now removed by means of barium hydrate and the excess of barium precipitated with carbon dioxide. The filtrate is concentrated, when on cooling the glycocoll is obtained in crystalline form.

Phenaceturic Acid.-In small amounts phenaceturic acid has been repeatedly obtained from the urine of man, but is principally met with in that of herbivorous animals, in which the putrefactive processes, owing to the greater length of the intestinal tract and the character of the food, are more extensive. On boiling with dilute mineral acids it is decomposed into its components, as shown in the equation :

$\mathrm{CH}_{2} \cdot \underset{\text { Phenaceturic acid. }}{\mathrm{NH}}\left(\mathrm{CH}_{2} \mathrm{C}_{6} \mathrm{H}_{5} \cdot \mathrm{CO}\right) \cdot \mathrm{COOH}+\mathrm{H}_{2} \mathrm{O}=\underset{\text { Phenyl-acetic acid. }}{\mathrm{CH}_{2}\left(\mathrm{C}_{6} \mathrm{H}_{5}\right) \cdot \mathrm{COOH}}+\underset{\mathrm{CH}_{2}}{\left.\mathrm{NH}_{2}\right) \cdot \mathrm{COOH}}$

Properties.-Phenaceturic acid crystallizes in small rhombic plates with rounded angles, which are very similar to the corresponding crystals of uric acid.

Isolation.-Phenaceturic acid may be isolated from the urine of the horse after separation of the hippuric acid, as shown above. 
The mother-liquor is then extracted with acetic ether, which takes up the acid. On evaporation the residue is dissolved in a dilute solution of sodium hydrate. From this solution the substance is again extracted with ether, after acidifying with hydrochloric acid, and thus purified, and finally allowed to crystallize from its aqueous solution.

In this connection brief reference may be made to ornithuric acid, which may be obtained from the urine of birds when these are fed with benzoic acid, and which probably also represents a normal constituent of such urine. Its formation is analogous to that of hippuric acid in mammals, but the benzoic acid here combines with ornithin, which is $\alpha, \delta$-diamino-valerianic acid, as has been shown before.

The relation of ornithin to arginin has already been considered.

\section{THE ARCMATIC OXY-ACIDS.}

The aromatic oxy-acids which may be found in the urine under normal conditions are para-oxyphenyl-propionic acid or hydroparacumaric acid, and para-oxyphenyl-acetic acid, which results from the former on oxidation. Both are derivatives of tyrosin, and are formed during the process of intestinal putrefaction, as has been shown. Para-oxyphenyl-lactic acid may further be encountered when tyrosin has been administered to animals in large amounts. Under pathological conditions, as in acute yellow atrophy, where leucin and tyrosin may appear in the urine as such, para-oxyphenyl-glycolic acid or oxy-amygdalic acid has also been found. Of special interest also is the fact that in some instances dioxyphenyl-acetic acid (homogentisinic acid) and trixophenyl-propionic acid or uroleucinic acid may occur in the urine. The chemical relationship which exists between these various substances and tyrosin is apparent from their formulæ :
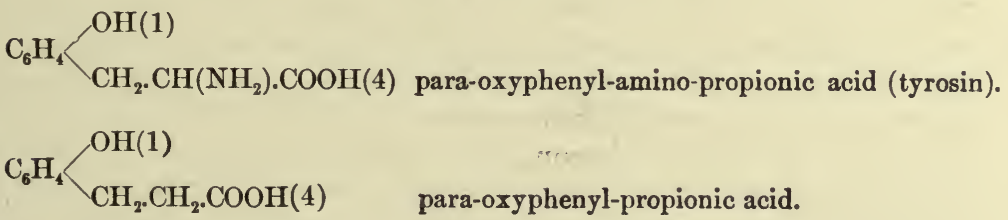

para-oxyphenyl-propionic acid.

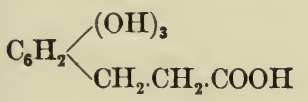

trioxyphenyl-propionic acid.<smiles>O=C(O)C(O)C(O)Cc1ccccc1</smiles>

para-oxyphenyl-lactic acid。 


$$
\mathrm{C}_{6} \mathrm{H}_{4} \backslash \underset{\mathrm{CH}_{2} \text {. } \mathrm{COOH}}{\mathrm{OH}}
$$<smiles>O=C(O)Cc1ccccc1</smiles><smiles>O=C(O)C(O)C(O)c1ccccc1</smiles>

para-oxyphenyl-acetic acid.

dioxyphenyl-acetic acid.

para-oxyphenyl-glycolic acid.

A certain fraction of these bodies appears in the urine in combination with sulphuric acid, but the greater portion is eliminated as such, viz., as potassium and sodium salts. The amount of the common oxy-acids, however, is always small, and rarely exceeds 0.03 gramme in the twenty-four hours.

To demonstrate the presence of the common oxy-acids in the urine, we proceed as follows : 500 c.c. of urine are strongly acidified with hydrochloric acid and distilled until the phenols, viz., phenol and paracresol, have passed over. This can be recognized by testing the distillate from time to time with Millon's reagent. On cooling, the remaining fluid is thoroughly extracted with ether, which takes up the oxy-acids as well as pyrocatechin and hydroquinon. To separate the acids from the latter, the ethereal extract is shaken with a dilute solution of sodium carbonate. The acids are thus transformed into the corresponding salts and are found in the alkaline solution. After separation from the ether this is acidified with dilute sulphuric acid and extracted with ether. The ethereal solution contains the free oxy-acids. Their presence can be demonstrated by evaporating to dryness, when the residue is dissolved in a little water and tested with Millon's reagent.

To isolate the individual acids, much larger quantities of urine are necessary. We may then proceed as described in the section on the Feces.

Homogentisinic Acid.-The presence of homogentisinic acid may be suspected if a urine, on being rendered alkaline, turns a dari reddish-brown on standing, and ultimately becomes black. At the same time a positive reaction with Fehling's solution is obtained, while polarimetric examination shows that the urine is optically inactive. Nylander's solution is not reduced. Upon the addition of a small amount of a dilute solution of ferric chloride a greenishblue color develops, which is only of momentary occurrence, however.

Boedeker was the first to describe a urine of this kind, and termed the substance giving rise to the above reaction alkapton. Subsequently, however, he expressed the opinion that his alkapton may have been pyrocatechin. Other investigators have isolated substances from such urines, which have been variously termed pyrocatechuic acid, urrhodinic acid, glycosuric acid, uroxanthinic acid, and uroleucinic acid, but there is reason to suppose that, with the 
possible exception of the last mentioned, all these substances are identical with homogentisinic acid. This was first isolated from an "alkapton" urine by Baumann and Wolkow, and has since been found in every case that has been examined in this direction.

Alkaptonuria, though it may occur in disease, has been regarded as the expression of an unusual form of intestinal putrefaction which in no way affects the health of the individual. Some observers, on the other hand, look upon it as a metabolic abnormality, and it must be confessed that micro-organisms have thus far not been isolated from the intestinal contents of such cases which are capable of effecting the transformation of tyrosin to homogentisinic acid in vitro. That tyrosin can be its source is undoubted, for it has been shown that following the administration of this substance homogentisinic acid appears in the urine in greatly increased amount. Baumann thus noted that while the average elimination in one of his cases amounted to 4.6 grammes, 14 grammes were once extracted in the twenty-four hours after tyrosin had been ingested. It is to be noted, however, that tyrosin is a member of the para-series, while homogentisinic acid belongs to the ortho-series:
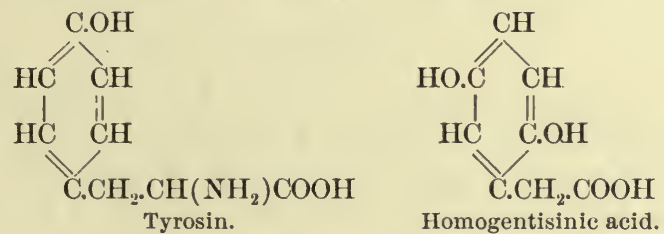

A direct transformation of the one into the other can accordingly not occur. But we may imagine that the hydroxyl group of the tyrosin is first removed by reduction, and that the benzol radicle is then oxidized again in two para-positions.

Phenyl-alanin, like tyrosin, will also increase the elimination of homogentisinic acid (in one case 89.32 per cent. of the ingested phenyl-alanin reappeared as homogentisinic acid).

The opinion has been expressed of late that homogentisinic acid may be a normal intermediary product in the destruction of tyrosin and phenyl-alanin and that its formation represents an oxidation which precedes the breaking up of the benzene ring. Its appearance in the urine, according to this idea, would be the expression of a metabolic insufficiency which renders the destruction of the benzene ring impossible. As a matter of fact, there are certain observations which render this view quite probable. It is thus known that homogentisinic acid is formed in the rootlets of certain plants from tyrosin, but is normally rapidly destroyed. It appears that both tyrosin and phenyl-alanin are first desamidized, $p$-oxyphenyl-propionic and $p$-oxy-phenyl-acetic acid resulting, from which homogentisinic acid is formed through oxidation with coincident reduction or transposition of the para-oxy group.

Isolation.-Homogentisinic acid may be conveniently isolated 
from the urine according to the method suggested by Garrod. The collected urine of twenty-four hours is heated nearly to the boilingpoint, and then treated with 5 or 6 grammes of neutral lead acetate in substance for every 100 c.c. of the urine. As soon as the salt is dissolved, the resulting precipitate is filtered off and the filtrate set aside in the cold for twenty-four hours. The crystals of lead homogentisinate are then collected on a filter and dissolved in hot water. This solution gives the various reactions described above. To isolate the free acid, the lead compound is decomposed with hydrogen sulphide and the filtrate carefully evaporated on a water-bath until the fluid begins to darken, when it is further concentrated in a vacuum to the point of crystallization. The resulting crystals are soluble in water, alcohol, and ether, but are insoluble in chloroform, benzol, and toluol. They melt at $146.5^{\circ}-147^{\circ} \mathrm{C}$.

In rare cases uroleucinic acid-dioxy-phenyl- $\alpha$-lactic acid-

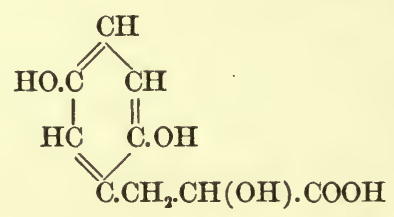

also can appear in the urine. In its general reactions it resembles homogentisinic acid, but does not give the iron reaction described above. Unlike the latter, it reduces Nylander's solution when present to the extent of 0.5 per cent. or more. It is manifestly an antecedent of homogentisinic acid.

Inosit.-The origin and chemical constitution of inosit will be considered elsewhere. According to Hoppe-Seyler, it may occur in the urine under normal conditions, but more commonly it is found in diseases which are associated with a high grade of polyuria, such as diabetes insipidus, diabetes mellitus, in chronic interstitial nephritis, etc.

To demonstrate its presence in the urine large amounts are concentrated to a syrup, which is then extracted with four times its volume of alcohol by boiling. On cooling, this extract is treated with an excess of ether, when the inosit gradually crystallizes out and may be recognized by its special tests (see Muscle Tissue).

The remaining aromatic substances which have been found in the urine are the kynurenic acid and urocaninic acid of the urine of dogs, and the so-called lithuric acid, which has been obtained from the urine of the ox. The two latter have thus far been found in only one instance and need not be considered at this place. Their formulæ are given as :

$\mathrm{C}_{12} \mathrm{H}_{12} \mathrm{~N}_{4} \mathrm{O}_{4}$, urocaninic acid.

$\mathrm{C}_{15} \mathrm{H}_{19} \mathrm{NO}_{9}$, lithuric acid. 
The so-called damalic acid and damaluric acid, which are obtained from the urine of the horse and the cow, probably represent a mixture of benzoic acid and volatile fatty acids.

Kynurenic Acid.-Kynurenic acid is said to be a constant constituent of the urine of dogs. Its mother-substance is manifestly an albuminous derivative, as the amount which appears in the urine is largely dependent upon the quantity of albuminous food ingested. Its elimination, moreover, continues during starvation, although the amount is then reduced to a minimum. Its formation is apparently not influenced by the degree of intestinal putrefaction, as the same amounts are excreted when putrefactive processes have been reduced to the lowest level by the administration of calomel or iodoform.

Recent investigations have shown that the direct antecedent is tryptophan (Ellinger). When this is fed to dogs a large increase of kynurenic acid occurs. The same is seen in rabbits, even though kynurenic acid does not normally occur in the urine of these animals. In man, on the other hand, no kynurenic acid production occurs. Of the manner in which the transformation is effected nothing is known.

Kynurenic acid is now regarded as $\gamma$-oxy- $\beta$-quinolin-carbonic acid :

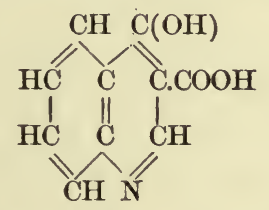

It is decomposed by heat, with the formation of carbon dioxide and a basic substance, kynurin, viz. $\gamma$-oxyquinolin. On reduction the latter is transformed into quinolin: The changes are represented by the equations:

$$
\begin{aligned}
& \text { (1) } \underset{\text { Kynurenic acid. }}{\mathrm{C}_{9} \mathrm{H}_{5} \mathrm{~N}(\mathrm{OH}) \cdot \mathrm{COOH}}=\underset{\text { Kynurin. }}{\mathrm{C}_{9} \mathrm{H}_{6} \mathrm{~N}(\mathrm{OH})}+\mathrm{CO}_{2} \\
& \text { (2) } \mathrm{C}_{9} \mathrm{H}_{6} \mathrm{~N}(\mathrm{OH})+2 \mathrm{H}=\underset{\substack{\mathrm{K}_{9} \mathrm{H}_{7} \mathrm{~N} \\
\text { Quinolin. }}}{\mathrm{K}_{\text {gurin. }}}+\mathrm{H}_{2} \mathrm{O}
\end{aligned}
$$

On oxidation both kynurenic acid and kynurin yield kynuric or oxalyl-anthranilic acid :

$$
\mathrm{C}_{6} \mathrm{H}_{4} \overbrace{\text { NH.CO.COOH }}^{\mathrm{COOH}}
$$

The synthesis of kynurenic acid has been accomplished by $R$. Camps.

Isolation.-To isolate kynurenic acid from the urine, 500 c.c. are treated with hydrochloric acid in the proportion of 4 c.c. for every 100 c.c. of the urine. On standing for forty-eight hours the substance in question crystallizes out together with uric acid. To separate it from the latter, dilute ammonia is added to the crystalline 
precipitate. The uric acid remains, and from its ammoniacal solution the kynurenic acid is then precipitated by acidifying with hydrochloric acid.

The crystals are soluble in alcohol and melt at $253^{\circ} \mathrm{C}$. On evaporating a bit of the material with hydrochloric acid and potassium chlorate on a porcelain plate a reddish residue is obtained, which principally consists of tetrachloro-oxykynurin. When this is moistened with ammonia a brownish-green color develops, and on standing this soon passes into a fine emerald green.

\section{The Fatty Acids.}

The Volatile Fatty Acids. - The volatile fatty acids which may be isolated from any urine, and which are especially abundant in that of herbivorous animals, are normally derived from the intestinal tract, where they are principally formed during the process of carbohydrate fermentation; a certain fraction, however, is also referable to albuminous putrefaction. These acids are acetic acid, formic acid, propionic acid, and butyric acid.

The non-volatile acids, capric aciō and caprylic acid, have further been found in the urine of herbivorous animals.

In man about 0.05 gramme of volatile fatty acids is excreted in twenty-four hours. Especially large amounts, such as 3 granmes pro die, have been found in the urine of the goat. In various diseases larger amounts have also been encountered in man, but it is still an open question whether they are then derived from the intestinal tract exclusively. From decomposing urine a larger quantity can be obtained than from fresh urine. This is no doubt owing to the fact that every urine contains a small amount of carbohydrates, which yield fatty acids on bacterial decomposition. Old diabetic urine is hence especially rich in such acids. The higher fatty acids are normally not observed in the urine, but traces may appear under certain pathological conditions.

Isolation and Quantitative Estimation.-To isolate the volatile fatty acids the collected urine of twenty-four hours is acidified with phosphoric acid in the proportion of $10: 100$, and distilled in a current of steam so long as the distillate shows an acid reaction. This is then neutralized, evaporated to dryness, and the residue extracted with alcohol. Traces of sodium chloride, which are formed on the addition of the alkali, owing to the presence of a little hydrochloric acid that has passed over, remain behind. The alcoholic solution is then evaporated to dryness, the residue is dissolved in a little water, acidified with sulphuric acid, and set aside in the cold, when traces of benzoic acid are precipitated. The filtrate is neutralized with a solution of sodium carbonate and extracted with ether, which removes the phenols. The solution is now acidified with sulphuric acid and is again distilled in a current of steam, when the volatile fatty acids pass over. Their presence can be established according 
to the common methods of analysis. To estimate the amount, the final distillate is neutralized with barium hydrate, evaporated to dryness, and the residue weighed. This weight less that of the barium, which is in combination, and which can be determined as barium sulphate, after incineration and extraction with dilute hydrochloric acid, indicates the amount of the fatty acids in general.

\section{$\beta$-Oxybutyric Acid.}

This acid is never found in the urine under normal conditions. It is principally met with in the severer forms of diabetes, when it is associated with the presence of diacetic acid and acetone. It may, however, also be found in other diseases, as in the continued fevers, in cachectic conditions, inanition, etc. As a general rule it is found in combination with the common alkalies of the blood, but when it is produced in especially large amounts a corresponding quantity of ammonia is furnished by the body to effect its neutralization. It may happen, however, that the acid formation exceeds that of ammonia, and in such cases the free acid occurs in the urine, and can also be demonstrated in the blood as such. Symptoms of acid intoxication then exist, and it is noteworthy that in such cases the amount of carbonic acid in the blood has been found markedly diminished, showing that the alkaline salts are not present in sufficient amount to remove the carbonic acid from the tissues.

As regards the origin of the $\beta$-oxybutyric acid, there is evidence to show that it may originate from the albumins, but it is questionable whether this is its only source. Some writers claim that it may also originate from the fats, and they adduce as proof the observation, that the amount of acetone which, as seen below, is a derivative of $\beta$-oxybutyric acid, is increased by a diet rich in fats, even though there may be no increased destruction of tissue-albumins. On the other hand, it may be said that no proof has been offered to show that an increased destruction of tissue-fat increases the production of acetone, and it is quite likely that the increased elimination on a diet rich in fats is referable to an increased production in the alimentary canal as the result of bacterial action, and resultant absorption and elimination in the urine. More plausible is the supposed origin of $\beta$-oxybutyric acid from the carbohydrates. During the katalysis of the latter, and notably the hexoses, according to Magnus-Levy, lactic acid is formed, which is readily decomposed into formic acid and acetic aldehyde. Through a condensation of two molecules of the latter $\beta$-oxybutyric aldehyde would then be formed, which on oxidation yields $\beta$-oxybutyric acid, as shown by the equations :
(1) $\mathrm{C}_{6} \mathrm{H}_{12} \mathrm{O}_{6}=2 \mathrm{CH}_{3}$. CHOH.COOH
(2) $\mathrm{CH}_{3} \cdot \mathrm{CHOH} \cdot \mathrm{COOH}=\mathrm{H} \cdot \mathrm{COOH}+\mathrm{CH}_{3} \cdot \mathrm{CHO}$
(3) $\mathrm{CH}_{3} \cdot \mathrm{CHO}+\mathrm{CH}_{3} \cdot \mathrm{CHO}=\mathrm{CH}_{3} \cdot \mathrm{CHOH} . \mathrm{CH}_{2} \cdot \mathrm{CHO}$
(4) $\mathrm{CH}_{3} \cdot \mathrm{CHOH} \cdot \mathrm{CH}_{2} \cdot \mathrm{CHO}+\mathrm{O}=\mathrm{CH}_{3} \cdot \mathrm{CHOH} \cdot \mathrm{CH}_{2} \cdot \mathrm{COOH}$ 
On the other hand, it is known that an existing acetonuria can be diminished or indeed suppressed by the administration of carbohydrates and that the elimination of carbohydrates from the diabetic's diet often leads to acetonuria. A liberal administration of albumins has in some cases the same effect upon the acetonuria as the carbohydrates. These facts in turn could be interpreted as supporting the view regarding the derivation of oxybutyric acid from the fats, both carbohydrates and albumins preventing the destruction of fats. But, on the other hand, as has just been stated, the acetonuria may be increased by the administration of fats in the absence of carbohydrates. The entire question is thus still sub judice.

The amount of oxybutyric acid which may occur in the urine is extremely variable. In the milder cases of diabetes it is usually absent; in the severer forms, however, large quantities may be found, and $\mathrm{Külz}$ reports that in three cases a daily elimination of 67,100 , and 226 grammes, respectively, was observed.

The chemical relation which exists between $\beta$-oxybutyric acid, diacetic acid, and acetone is seen from the equations :

$$
\begin{aligned}
& \text { (1) } \mathrm{CH}_{3} \cdot \mathrm{CH}(\mathrm{OH}) \cdot \mathrm{CH}_{2} \cdot \mathrm{COOH}+\mathrm{O}=\left(\mathrm{CH}_{3} \cdot \mathrm{CO}\right) \cdot \mathrm{CH}_{2} \cdot \mathrm{COOH}+\mathrm{H}_{2} \mathrm{O} \text {. } \\
& \text { (2) } \underset{\text { Diacetic acid. }}{\left(\mathrm{CH}_{3} \cdot \mathrm{CO}\right) \mathrm{CH}_{2} \cdot \mathrm{COOH}}=\underset{\text { Acetone. }}{\mathrm{CO}\left(\mathrm{CH}_{3}\right)_{2}}+\mathrm{CO}_{2} \text {. }
\end{aligned}
$$

We can thus readily understand that in certain conditions acetone may be found in the urine alone, while in others diacetic acid, and in still others $\beta$-oxybutyric acid may be present as well.

On boiling $\beta$-oxybutyric acid in aqueous solution with dilute mineral acids, $\alpha$-crotonic acid results, and it is thus apparent that this acid is also found in the distillate, when urine containing the first is distilled with sulphuric acid. Otherwise, however, it does not occur. The reaction which takes place may be represented by the equation:

$$
\underset{\beta}{\mathrm{CH}_{3} \cdot \mathrm{CH}(\mathrm{OH}) \cdot \mathrm{CH}_{2} \cdot \mathrm{COOH}=\mathrm{CH}_{3} \cdot \underset{\alpha-\text { crotonic acid. }}{\mathrm{CH}} \mathrm{CH} \text { acid. }} \mathrm{COOH}+\mathrm{H}_{2} \mathrm{O}
$$

Test.-As the presence of oxybutyric acid presupposes that of diacetic acid, and as the presence of the latter can much more readily be demonstrated than that of oxybutyric acid, a test in this direction should always precede a more detailed examination (see below). If a positive reaction is thus obtained, any sugar that may be present is removed by fermentation. The liquid is cleared by adding neutral acetate of lead, when the filtrate is examined with the polarimeter. Should lævorotation now be observed, the presence of oxybutyric acid is rendered very probable. To demonstrate this beyond a doubt, the liquid is evaporated to a syrup, treated with an equal volume of concentrated sulphuric acid and distilled, without cooling. In this manner the oxybutyric acid is decomposed with the formation of $\alpha$-crotonic acid, which is accordingly found in the distillate. If this is present in larger amounts, it crystallizes out in the distillate, when this is strongly cooled, and may be identified by 
its melting-point, $72^{\circ} \mathrm{C}$. Should smaller amounts, however, be present, crystallization does not occur. In this case the distillate is extracted with ether by shaking. Traces of benzoic acid and the phenols are thus likewise extracted, but if then the residue of the ethereal solution is washed with water other impurities are removed, and the crotonic acid remains.

Quantitative Estimation (according to Darmstaedter).-The method is based on the decomposition of the $\beta$-oxybutyric acid with the formation of $a$-crotonic acid and the estimation of the latter. 100 c.c. of urine are rendered feebly alkaline with sodium carbonate and evaporated on a water-bath almost to dryness. With the aid of 150-200 e.c. of sulphuric acid (50-55 per cent.) the residue is transferred to a liter flask, which is closed with a doubly perforated stopper. Through the one aperture a drip-tube passes, while a bent glass tube passes through the other to a condenser. Heat is applied, at first mildly, so as to avoid foaming; then vigorously. Water is allowed to enter through the drip-tube as fast as the distillate passes over. The distillation is interrupted when from 300 to 350 c.c. have been obtained, which usually takes from two to two and one-half hours. The distillate is extracted two or three times with ether. The ether is distilled off, the residue heated for a few minutes on a sand-bath to $160^{\circ} \mathrm{C}$. in order to drive off any fatty acids that may be present, and then dissolved on cooling with 50 c.c. of water. The solution is filtered, and the filter washed with a little water. The aqueous solution of the crotonic acid is now titrated with a decinormal sodium hydrate solution, using phenolphthalein as an indicator. 1 c.c. of the soda solution corresponds to 0.0086 gramme of crotonic acid. The corresponding amount of oxybutyric acid is obtained by multiplying by 1.21. Sugar does not interfere with the process.

From what has been said above, it is clear that every urine which contains $\beta$-oxybutyric acid will probably also contain diacetic acid. On the other hand, it will also be understood that diacetic acid may occur in the urine in the absence of $\beta$-oxybutyric acid, and this is indeed more common.

\section{Diacetic Acid.}

Tests.-As diacetic acid is rapidly decomposed on standing, it is necessary that the urine should be as fresh as possible, when it is to be examined in this direction. To this end, several direct tests are available.

Arnold's Test (as modified by Lipliawski).-Two solutions are employed, viz., a 1 per cent. solution of para-amino-acetophenon and a 1 per cent. solution of potassium nitrite; 6 c.c. of the first solution and 3 c.c. of the second are added to an equal volume of urine, together with a drop of concentrated am- 
monia. The mixture is shaken until it assumes a brick-red color. From 10 drops to 2 e.c., according to the amount of diacetic acid prescnt, are treated with 15-20 c.c. of concentrated hydrochloric acid (sp. gr. 1.19), 3 c.c. of chloroform, and 2-4 drops of an aqueous solution of ferric chloride. The tube is closed with a. cork and gently agitated (so as to avoid emulsification), when after one-half to one minute a beautiful and very characteristic violet tinge results if diacetic acid is present. In its absence the color is yellowish or slightly reddish. The violet color persists for a long time. Salicylic acid, phenacetin, antipyrin, phenol, and other drugs are without disturbing influence upon the reaction.

Allard states that both Arnold's test and that of Lipliawski give a positive result also with acetone, when this is present to the extent of more than 1 per cent.

GERHARDT'S TEST.-In its original form this test also reacts with the common antipyretics, and it is hence necessary to isolate the diacetic acid to a certain degree. To this end, a few cubic centimeters of the urine are strongly acidified with sulphuric acid and extracted with ether, which takes up the acid. The extract is then shaken with a few cubic centimeters of a dilute solution of the chloride of iron, when in the presence of diacetic acid the aqueous layer assumes a violet or Bordeaux-red color. This, however, is not permanent, and soon fades on boiling the solution.

\section{Acetone.}

The origin of acetone has been discussed above (see Oxybutyric Acid).

Traces varying between 0.008 and 0.027 gramme in the twentyfour hours are normally found in the urine.

Acetonuria is essentially a pathological phenomenon, and is observed in most pronounced form in severe cases of diabetes, in which, as I have stated, it is frequently met with in association with $\beta$-oxybutyric acid and diacetic acid. Like diacetic acid, however, it may occur in the absence of oxybutyric acid, and in the milder forms of diabetes, as also under normal conditions it may be present alone.

Tests.-Should diacetic acid be demonstrated in the urine, the simultaneous presence of acetone may be directly inferred. If this is not the case, it is best to distill from 250 to 500 c.c. of the urine, after the addition of a small amount of phosphoric acid, and to apply the following tests to the first 15 or 30 c.c. of the distillate that has passed over.

Legal's Test.-A few cubic centimeters of the distillate are rendered strongly alkaline with caustic soda solution and then treated with several drops of a freshly prepared, concentrated solution of sodium nitroprusside. In the presence of acetone a red color develops, which rapidly fades, however, but is replaced by a beautiful 
carmin or purple red if the solution is treated with acetic acid in excess; on standing, this turns to a bluish violet.

Lieben's Test.-On adding a few drops of a dilute solution of iodopotassic iodide to a small amount of the distillate that has been rendered alkaline with sodium hydrate solution, a precipitate of iodoform develops in the presence of acetone, which may be recognized by its odor on warming the mixture, as also by the form of the crystals, which occur as hexagonal or stellar platelets. Alcohol and acetic aldehyde give the same reaction. For this reason Gunning's modification, although not so delicate, is sometimes preferred:

Gunning's Test. - A small amount of Lugol's solution is added to the distillate and a sufficient amount of ammonia to produce a black precipitate of nitrogen iodide. This disappears on standing and in the presence of acetone is replaced by iodoform.

Gunning's test, like that of Legal, may also be tried directly with the native urine.

Dennigès' TEsT (as modified by Oppenheimer).-The reagent is prepared as follows : 20 grammes of concentrated sulphuric acid are poured into 100 c.c. of distilled water, when 5 grammes of freshly prepared yellow mercuric oxide (see Reynold's test) are added. 'The mixture is allowed to stand for twenty-four hours and is then ready for use.

This reagent is added to about 3 c.c. of urine, drop by drop, until the precipitate which is thus formed no longer disappears on stirring. When this point is reached a few more drops are added. After two to three minutes the precipitate is filtered off. The clear filtrate is further treated with about 2 c.c. of the reagent and 3 to 4 c.c. of a 30 per cent. solution of sulphuric acid and boiled for a minute or two or, still better, placed in a vessel with boiling water. In the presence of an abundant amount of acetone a copious white precipitate forms immediately; while in the presence of traces only (less than $1: 50,000)$, a slight cloud develops on standing for several minutes. The precipitate is almost entirely soluble in an excess of hydrochloric acid.

If albumin is present, the urine becomes turbid at once when the reagent is added. In that case the test is continued as described, attention being directed to the coarser precipitate which occurs later. To such urines large amounts of the reagent must be added, the idea being to precipitate everything that can be precipitated with the reagent before heating.

Oppenheimer claims that the test is as delicate as that of Lieben, giving a well-pronounced reaction with a dilution of $1: 20,000$, and being still discernible with a dilution of $1: 60,000$. As diacetic acid yields acetone when treated with mineral acids, a positive result is always obtained when this is present. But as diacetic acid is usually found only in association with acetone, this fact does not lessen the value of the test, and is an error, moreover, which is common to the other tests as well. 
Quantitative Estimation.-The quantitative estimation of acetone is best conducted according to the method of Messinger, as modified by Huppert. It is based upon the principle underlying Lieben's test, viz., the formation of iodoform when a dilute solution of indopotassic iodide is added to an alkaline solution of acetone. By determining the amount of iodine which is consumed in this reaction the corresponding amount of acetone can then be calculated.

One hundred c.c. of urine, or less if much acetone is present, as determined by Legal's test applied directly to the urine, are treated with 2 c.c. of a 50 per cent. solution of acetic acid and distilled until all the acetone has passed over. The distillate is received in a bulb-tube containing water. The solution which thus results is treated with 1 c.c. of a 12 per cent. solution of sulphuric acid and redistilled. The second distillate is free from phenols. To it a carefully measured quantity of a one-tenth normal solution of iodine is added (10 c.c. for every 100 c.c. of urine), together with a 50 per cent. solution of sodium hydrate, until the iodoform separates out. After shaking, the mixture is set aside for a few minutes, and then acidified with concentrated hydrochloric acid. If iodine is present in excess, a brown color thus develops. This excess is then titrated with a decinormal solution of sodium thiosulphate, using starch solution as a final indicator. The number of cubic centimeters emyloyed in this titration is deducted from the amount of the iodine solution added. The difference multiplied by 0.967 then indicates the amount of acetone in the 100 c.c. of urine, in milligrammes.

\section{Lactic Acid.}

Normally lactic acid is not found in the urine. It is met with in various diseases of the liver which are associated with an extensive destruction of the hepatic parenchyma, as also in conditions in which the oxidation-processes of the body are impaired in general. It is notably seen in acute yellow atrophy, in poisoning with phosphorus and carbon monoxide, in long-continued anæmic conditions, etc. Smaller amounts have been found in soldiers after marches and in epileptic patients after severe seizures.

Isolation.-To isolate the substance from the urine the following method may be employed, as suggested by Araki :

The collected urine of twenty-four hours is evaporated to about 50 or 60 c.c., treated with ten times as much of 95 per cent. alcohol, and set aside for twelve hours. It is then filtered and freed from the alcohol by distillation. The residual fluid is acidified with phosphoric acid, and repeatedly extracted with five times its volume of ether. The ethereal extract is evaporated and the remaining yellow syrup dissolved in a little water. Any hippuric acid which may be present thus separates out and is filtered off. The filtrate is now treated with pure lead carbonate in substance, heated on a water-bath for thirty minutes, and filtered on cooling. From the 
filtrate the lead is removed by means of hydrogen sulphide, and the excess of the latter by gently warming on a water-bath. The fluid is then concentrated to a thick syrup and extracted with ether. The ethereal solution is evaporated and the residue boiled for some time with water and an excess of zinc earbonate. The mixture is filtered while hot, concentrated to a small volume, and then set aside in the cold after adding a little alcohol. The zine salts of both paralactic acid and the common optically inactive lactic acid which may also be present in traces, then crystallize out. They can be separated from each other by treating with absolute alcohol, in which the latter is insoluble. It must be noted that the solubility of the paralactate is also slight $(1: 1100)$, so that it is necessary to add a large amount of alcohol. In order to prevent confusion with the aromatic oxy-acids of the urine, the lactate crystals should now be further identified, which is most conveniently done by estimating the water of crystallization. The paralactate contains two molecules of this, which escapes at $105^{\circ} \mathrm{C}$., and at this temperature the weight of the crystals should therefore diminish $\mathbf{1 2 . 9}$ per cent. The salt, moreover, like its acid, is lævorotatory, while the common lactate is optically inactive.

\section{Mono-amino Acids.}

Tyrosin, leucin, and glycocoll have long been known to occur in the urine in acute yellow atrophy and phosphorus poisoning, but aside from these conditions nothing further was known of the presence of amino-acids under other pathological conditions (barring eystinuria). Within recent years, however, and with more exact methods it has been possible to show that bodies of this order may occur under the most divers conditions. Phenyl-alanin, alanin, and arginin have been found in phosphorus poisoning, besides tyrosin, leucin, and glycocoll. Glycocoll, indeed, according to a recent announcement by v. Noorden, is a normal constituent of the urine and may amount to 1 per cent. of the total nitrogen sulphate. (This is in marked eontrast to the statement of Ignatowski that normal human urine only contains traces of amino-acids at best, and that even after the subcutaneous injection of 6 grammes of glycocoll none are demonstrable.)

Abderhalden found tyrosin in a patient dying with pneumonia who had been suffering from arteriosclerosis, myocarditis, and diabetes. In a second case of diabetes he likewise found tyrosin and obtained a marked Millon reaction. In a third case with coma tyrosin was present also during the attack, but absent in the interim. In a case of severe hepatic cirrhosis a marked $\beta$-naphthalin-sulphochloride reaction occurred, but it was impossible to isolate amino-acids in pure form. The same observer obtained ty rosin in a case of severe icterus referable to complete occlusion of the common duct and in a patient who had undergone prolonged narcosis; both urines gave a marked Millon reaction. 
Ignatowski found glycocoll constantly in the urine of 7 gouty patients; in 3 of these also amino-acids, probably leucin and asparaginic acid. In pneumonia, especially about the time of the crisis, and in leukæmia he likewise obtained positive results.

In this connection the observations of Herter, Wakeman, and Baldwin are of special interest. Using the method of MagnusLevy of balancing the total bases against the total known acids, they found that in certain conditions, notably dilatation of the stomach, rheumatoid arthritis, and cirrhosis of the liver, there was a notable excess of bases over known acid equivalents. This leads to the inference that in the diseases mentioned there must have been present some other organic acids. Magnus-Levy had in this manner previously established the presence of such acids in starvation, in intestinal disturbances, phosphorus poisoning, acute yellow atrophy, and fever.

I append a few of Baldwin's results :

APPARENT EXCESS OF ACIDS OVER BASES,

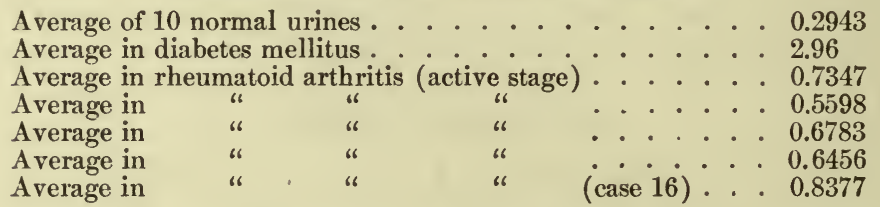

Isolation.-If leucin and tyrosin are present in the urine in small amounts, they are held in solution. In the presence of larger amounts the tyrosin may separate out, and can then be isolated from the sediment and identified as described. Leucin, however, is rarely found in this manner, and remains in solution even though very large quantities are eliminated.

To demonstrate both when they are held in solution, it is sometimes only necessary to concentrate a small amount of urine on a water-bath and to examine the residual syrup with the microscope. Otherwise it is advisable to precipitate the collected urine of twenty-four hours, after removing any albumin that may be present, with basic lead acetate. The filtrate is then freed from lead with hydrogen sulphide, evaporated to a thick syrup, and set aside for crystallization. Tyrosin and leucin can then be demonstrated by a microscopical examination and identified in the usual manner (see page 207).

\section{THE NEUTRAL SULPHUR BODIES OF THE URINE.}

In the section on the mineral constituents of the urine I pointed out that the greater portion of the sulphur which is set free during the metabolism of the nitrogenous constituents of the body is eliminated in the urine in a completely oxidized form. A much smaller fraction, however, escapes oxidation, and appears in the urine as 
so-called neutral sulphur. Normally this constitutes about 12-15 per cent. of the total amount.

The neutral sulphur bodies which have been described in the urine of man under normal conditions are certain sulphocyanides, the socalled oxyproteïnic acid, alloxyproteïnic acid, and antoxyproteïnic acid of Bondzynski and Gottlieb and Panek respectively, the uroproteic acid of Cloëtta, and the uroferric acid of Thiel. The sulphocyanides in question are probably derived from the saliva and the gastric juice, where they are normally found in traces. Oxyproteïnic acid contains 1.12 per cent. of sulphur, the alloxy compound 2.19 per cent. and the antoxy acid 0.61 per cent. The uroferric acid, according to Thiele, contains 3.46 per cent. of sulphur, of which about one-half can be split off on prolonged boiling with hydrochloric acid or sulphuric acid. In this respect, therefore, it shows the behavior of a conjugate sulphate. In addition, however, it contains sulphur, which cannot be split off even on prolonged boiling with an alkaline solution of acetate of lead. Its amount is quite small.

In the urine of cats, and less constantly of dogs, traces of thiosulphates are found, while in man they are normally absent. They have been once found in a case of typhoid fever. Cysteïn and ethyl sulphide are constant constituents of the urine of dogs.

Whether or not taurocarbaminic acid is constantly present in human urine has not been ascertained. I have shown, however, that to a certain extent at least taurin is eliminated in this form when given by the mouth. In cases of obstructive jaundice, moreover, or after ligation of the common duct in dogs, the neutral sulphur may increase to 40 per cent. of the total amount, and it is known that in such cases taurocarbaminic acid is constantly present. Its formation may be represented by the equation :

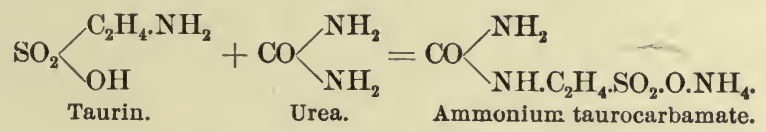

In all probability this synthesis is effected in the kidneys. In rabbits, on the other hand, taurin is largely oxidized to sulphuric acid, while a small portion appears as thiosulphuric acid.

Cystein.-On feeding dogs with halogen benzols, peculiar products appear in the urine, which contain both sulphur and nitrogen, and which are apparently united with glucuronic acid. Baumann and Preusse have termed these mercapturic acids. Following the administration of monobrom benzol they could isolate a brom-phenyl mercapturic acid of the composition $\mathrm{C}_{11} \mathrm{H}_{12} \mathrm{BrSNO}_{3}$, which on hydrolysis yielded acetic acid and a substance of the formula $\mathrm{C}_{9} \mathrm{H}_{10} \mathrm{BrNSO}_{2}$ according to the equation :

$$
\mathrm{C}_{11} \mathrm{H}_{12} \mathrm{BrSNO}_{3}+\mathrm{H}_{2} \mathrm{O}=\mathrm{CH}_{3} \cdot \mathrm{COOH}+\mathrm{C}_{9} \mathrm{H}_{10} \mathrm{BrNSO}_{2}
$$


This product they subsequently identified as brom-phenyl cysteïn, and the proof had thus been furnished that a cystin complex is normally produced in the sulphur metabolism of the dog, at any rate. It is identical with the cysteïn which can be obtained from the albuminous cystin on reduction, and the elimination of mercapturic acid can hence be regarded as an experimental cystinuria.

The amount of cystein which is normally present in the urine probably does not exceed 0.015 gramme in the twenty-four hours. Larger quantities are found under pathological conditions, as in phosphorus poisoning, but on the whole its elimination in disease has received little attention.

Cystin.-As has been shown, cystin is a constant decompositionproduct of all albumins and represents one of the primary radicles of the original molecule. Under normal conditions it is not found in the urine. As in the case of alkapton, its presence represents the existence of a distinct metabolic anomaly, of the true nature of which, however, nothing is known. Very curiously its presence in the urine may be associated with the simultaneous presence of certain diamins, viz., cadaverin and putrescin. As these were formerly regarded as specific products of bacterial activity, their presence was brought into a supposed causal relationship to that of cystinuria. In the light of more modern investigations, however, we may assume that the diaminuria in these cases is in all likelihood also the expression of a metabolic anomaly, and like the cystinuria of histogenic origin.

Outside of the urine cystin has been encountered, as such, in a few instances only. Cloëtta claims to have found it in the kidneys of the ox, Scherer in the liver of a patient dead with typhoid fever, and Drechsel isolated the body from the liver of a horse and a porpoise. Abderhalden has described the case of a child which died at the age of twenty-one months and a half, and in which post mortem the various organs, and notably the liver and spleen, were found incrustated with cystin crystals.

Clinically cystin is of interest as its elimination in the urine favors the formation of cystin concretions.

The amount which may be met with is extremely variable. On some days traces only are found, while on others the elimination may amount to a gramme or more. The neutral sulphur is correspondingly increased (to 60 per cent. of the total and even higher). Very commonly the cystin separates out in crystals shortly after being voided, but sometimes it is necessary to acidify the urine strongly with an excess of acetic acid. On decomposition such urines develop a strong odor of hydrogen sulphide.

Properties.-Structurally cystin is $\alpha$-diamido- $\beta$-dithio-dilactic acid; it is the disulphide of $\beta$-cysteïn and results from this on oxidation according to the equation : 


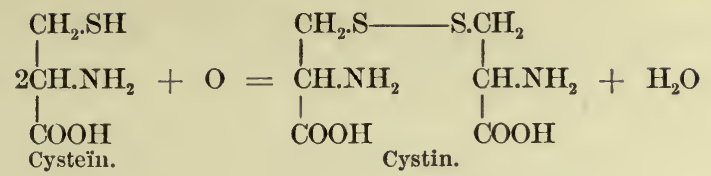

On oxidation with bromine it is possible to transform the loosely combined sulphur into oxidized sulphur with the production of cysteïnic acid, which apparently represents the sulpho-acid of cysteïn. Its probable formula is<smiles>NC(CS(=O)(=O)O)C(=O)O</smiles>

Throngh loss of $\mathrm{CO}_{2}$ this then gives rise to taurin (which see).

Two varieties of cystin exist, of which one is lævorotatory and the other dextrorotatory. The variety which is found in the urine is lævorotatory. The substance usually crystallizes in colorless hexagonal platelets which are very characteristic in appearance. But it may also separate out in needles, which differ from those of tyrosin in the fact that they appear more highly refractive under the microscope and present obliquely cut ends. On recrystallization from a 10 per cent. solution of ammonia these needle-like crystals disappear and are replaced by hexagonal platelets.

Cystin is soluble in solutions of the alkaline hydrates, in ammonia, and the mineral acids. In water, alcohol, ether, and acetic acid it is insoluble, as also in solutions of ammonium carbonate; for this reason the cystin is apt to crystallize out from decomposing urines, when previously it has been present in solution only.

On heating cystin on platinum foil it does not melt, but ignites and burns with a bluish-green flame; at the same time a peculiar, penetrating odor develops. It does not give the murexid reaction. When boiled with caustic alkali it is decomposed and the sulphur liberated as a sulphide. With benzoyl chloride, in the presence of an excess of caustic alkali, it forms benzoyl-cystin, and is thus precipitated as a sodium salt in the form of fine lustrous platelets, which are readily soluble in water, but insoluble in solutions of the caustic alkalies. Upon the addition of an acid to such a solution, benzoyl-cystin separates out as such. It is soluble in alcohol and alcohol-ether, slightly so in pure ether, and almost insoluble in water. Its neerle-like crystals melt at $156^{\circ}-158^{\circ} \mathrm{C}$. The formation of benzoyl-cystin may be expressed by the equation :

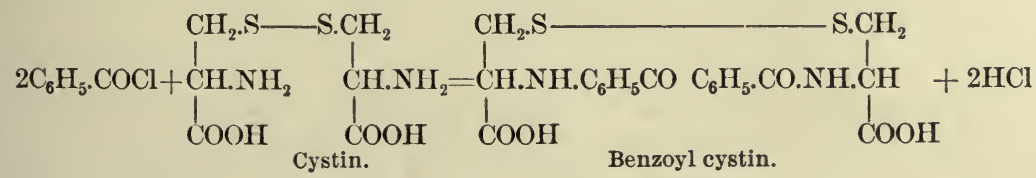


On boiling with concentrated hydrochloric acid benzoyl-cystin is decomposed with the formation of benzoic acid and cystin.

On shaking cystin in alkaline solution with $\beta$-naphthalin-sulphochloride (dissolved in ether) and subsequently acidifying with hydrochloric acid (after removal of the ether) a precipitate of $\beta$-naphthalin-sulphocvstin is formed, of the composition $\mathrm{C}_{26} \mathrm{H}_{24} \mathrm{~S}_{4} \mathrm{~N}_{2} \mathrm{O}_{8}$. This dissolves with difficulty in water and cold absolute alcohol, but readily in hot absolute alcohol; from the latter the substance crystallizes out in flat, partly bent needles, which at $215^{\circ} \mathrm{C}$. (uncorrected) decompose with the formation of a brown oily material.

Isolation bf Cystin from the Urine and Quantitative Estimation.To this end Abderhalden has recommended the following method, based on the principle just outlined. The entire amount of urine of twenty-four hours is filtered and the residue washed with ammonia. The filtered urine and the ammonia washings are united. 500 c.c. (best after previous concentration in the vacuum at $40^{\circ} \mathrm{C}$.) are then treated with 4 c.c. of normal sodium hydrate solution and then shaken for six to eight hours with an ethereal solution of 4 grammes of $\beta$-naphthalin-sulphochloride. At intervals of one and a half hours 3 c.c. of the alkali solution are further added. At the expiration of the shaking the ether layer is removed and the aqueous solution oversaturated with hydrochloric acid. The resulting precipitate is filtered off and after decolorization with animal charcoal crystallized from hot absolute alcohol. The crystals are dried at $100^{\circ} \mathrm{C}$. and weighed. 1 gramme of the compound corresponds to 0.35 gramme of cystin.

Normal urine gives no precipitate with $\beta$-naphthalin-sulphochloride, or a slight turbidity only.

Preparation of Cystin from Albumins.-For purposes of study cystin is most conveniently obtained from human hair as follows: 500 grammes of hair are boiled for four hours with 1500 c.c. of hydrochloric acid (specific gravity 1.19). On cooling, concentrated sodium hydrate solution is carefully added until the reaction is nearly neutral (not alkaline). Animal charcoal is added in excess; the mixture is boiled for about three-quarters of an hour and filtered while hot. On cooling, impure cystin separates out. This is collected on a filter, dissolved in hot dilute ammonia, and reprecipitated by the addition of glacial acetic acid. The resulting precipitate is again dissolved in a small amount of hot dilute ammonia, the solution is filtered, when on spontaneous evaporation of the ammonia the cystin separates out in rosettes composed of the typical hexagonal platelets. These can be further purified by a repetition of the precipitation of the ammoniacal solution with glacial acetic acid. The crystals are finally washed with water, alcohol, and ether.

Quantitative Estimation of the Neutral Sulphur.-In one portion of the urine the oxidized sulphur, viz., the mineral and the conjugate sulphur, is estimated as previously described (page 238). In a 
second portion the total sulphur is determined as follows (the difference between the two results indicates the amount of neutral sulphur):

Method of Höhnei, Glaser, v. Asboth (modified by Modrakowski).-1-2 grammes of sodium peroxide are placed in a nickel dish, and covered with 50 c.c. of urine, which is added from a pipette drop by drop. The fluid is evaporated on a waterbath to a syrup, and is further treated with 2-3 grammes of the veroxide, which is added slowly and carefully, while stirring. As soon as the reaction, which at first is fairly vigorous, becomes calmer, the dish is removed from the water-bath and heated with a small alcohol lamp, if necessary adding from 1 to 3 grammes of peroxide more. The mass now appears as a brown syrup and finally becomes thick. This ends the reaction. On cooling the fusion is dissolved in hot water; the solution is filtered and feebly acidified with hydrochloric acid. Barium chloride is then added and the process continued as described elsewhere (page 238).

\section{THE CARBOHYDRATES.}

The carbohydrates which may be found in the urine comprise glucose, lævulose, laiose, maltose, lactose, dextrin, and certain pentoses. Of these, traces of glucose, dextrin, animal gum, and possibly also pentoses, may be found at all times. Their amount, however, is normally so small that their presence cannot be recognized by the common tests. Larger amounts of carbohydrates are found in health only during the puerperal state and in the course of lactation, when lactose is commonly present. Otherwise the elimination of sugar in amounts which can be demonstrated by the ordinary tests must be regarded as abnormal.

Glucose.-As I have indicated, glucose appears in the urine whenever its amount in the blood exceeds 3 pro mille. This, however, occurs only under abnormal conditions, and in the presence of small amounts the kidneys are manifestly capable of preventing its passage into the urine. Under certain conditions, however, this power is apparently lost, and we find, as a matter of fact, that following the administration of phlorhizin glucosuria occurs, although the percentage of sugar is not increased in the blood. Whether or not such an insufficiency on the part of the kidneys may also occur spontaneously we do not know. As a general rule, however, glucosuria is associated with a hypergluchæmia. This may result if unduly large amounts of sugar reach the liver, so that the organ is incapable of transforming the entire quantity into glycogen, and I have pointed out that the functional capacity of the liver in this respect is of a much lower order than the ability of the intestinal epithelium to transform polysaccharides and disaccharides into glucose. The extent to which the liver can normally transform glucose into glycogen seems to vary 
with different individuals. Generally, glucosuria occurs when the amount of sugar ingested at one time exceeds 200 grammics. There are many individuals, however, in which this occurs following the administration of only 150 grammes, and there are others in which the ingestion of 250 grammes does not cause glucose to appear in the urine. Glucosuria following the ingestion of 100 grammes of grape-sugar is now regarded as abnormal, and there is reason to believe that the hepatic insufficiency thus manifested may be referable to a mild form of diabetes. The amount of sugar which then appears in the urine rarely exceeds 3 per cent. The glucosuria, moreover, is only temporary, and disappears as soon as the ingestion of sugar (viz., starches) is diminished. Between this form of glucosuria and the comman form of diabetes, in which practically no sugar can be utilized by the body, but in which the elimination ceases as soon as carbohydrates are withdrawn from the diet, all gradations may occur. These forms are now generally regarded as referable to a hepatic insufficiency. Quite different from diabetes of this character, on the other hand, is the type in which the glucosuria continues although no sugars are ingested. In such cases a hepatic insufficiency need not necessarily exist, and there is evidence to show that in these forms the formation of glycogen may still occur. We must therefore assume that other organs are primarily involved, and there is every reason to suppose that the metabolism of muscle-tissue is here principally at fault, and that the tissue has lost the power of decomposing the sugar which reaches it from the liver. As a consequence, increased destruction of muscle-tissue occurs, as the inability on the part of these structures to decompose sugar amounts to the same as though no sugar were present at all. The body therefore furnishes glucose from its albumins to supply the apparent deficit, and thus further increases the hyperglucamia and the resulting glucosuria. We accordingly find that even though the carbohydrates have been withdrawn from the food sugar still appears in the urine. The increased destruction of the tissue-albumins is in such cases sufficiently apparent from the progressive loss of flesh which is so constantly observed. That certain nervous influences may here be at work is probable, and we know, as a matter of fact, that injury to a certain region in the floor of the fourth ventricle is invariably followed by the appearance of glucose in the urine. But, on the other hand, we may also imagine that the normal decomposition of the sugar is prevented owing to the absence of a glucolytic ferment or its corresponding kinase. In support of this view is the fact that after extirpation of the pancreas death invariably results with symptoms which are practically identical with those seen in the gravest types of diabetes. Ligation of the duct does not produce this effect; and it is noteworthy, moreover, that the glucosuria disappears when pieces of the pancreas are transplanted under the skin or when fresh raw pancreas is given 
with the food. Within the past ten years it has been found that in a not inconsiderable number of cases of diabetes degenerative lesions can be demonstrated in the pancreas, and there can be no doubt at the present time that a certain percentage of cases are directly referable to such origin. That the islands of Langerhans are here primarily concerned has been demonstrated by Opie. In this connection Cohnheim's researches are very significant, in which he could show that while neither pancreas nor muscle-tissue contain ferments which are capable of splitting glucose by themselves, extensive glucolysis is effected if an extract of pancreas and muscleplasma conjointly are allowed to act upon glucose. A condition thus exists which seems quite analogous to the relation between trypsin and enterokinase.

In the milder forms of diabetes an insufficiency on the part of the muscle-tissue manifestly does not exist, as it is possible to prevent the occurrence of glucosuria, temporarily at least, if the demand for sugar is increased by abundant muscular exercise. That a hepatogenic diabetes may coexist with a myogenic form, however, cannot be doubted.

The amount of sugar which may be present in the urine under pathological conditions is exceedingly variable. On the one hand, traces only may be found, which may be normal ; while, on the other hand, the daily excretion may exceed 1000 grammes. In diabetes an elimination of from 3 to 6 per cent. in an amount of urine varying between 3000 and 6000 c.c. may be regarded as moderate.

Tests for Sugar.-Simple tests by means of which glucose can be demonstrated directly in the urine as such are, unfortunately, not available. Other sugars, it is true, enter into consideration only under exceptional conditions, but if it is desired to prove that the substance which gives the common sugar reactions is actually glucose, a more detailed examination is necessary. Some of these reactions, moreover, may be simulated by substances which are not carbohydrates, and deductions as to the presence or absence of sugar are hence only warrantable when these can be excluded. If albumins are present, they must first be removed.

Nylander's TEST.-This test is to be preferred to the more common one of Trommer, as the reagent does not react with uric acid, kreatinin, or homogentisinic acid. With many of the conjugate glucuronates, however, a reduction is observed, and it is hence necessary to eliminate this source of error when a positive reaction is obtained, or to apply additional tests in which this possibility does not enter into consideration.

The reagent is prepared as follows : 4 grammes of the tartrate of potassium and sodium, together with 2 grammes of subnitrate of kismuth and 10 grammes of sodium hydrate are placed in 90 c.c. of water. The solution is heated to the boiling-point, filtered on cooling, and is then ready for use. It is kept in a dark-colored bottle.

A few cubic centimeters of the urine are treated with the reagent, 
in the proportion of $11: 1$, and boiled, when in the presence of sugar a reduction of the subnitrate of bismuth to bismuthous oxide, or even to the metallic form, occurs. As a consequence the mixture assumes a grayish, dark-brown, or black color, and on standing the precipitated oxide or metal settles to the bottom together with the earthy phosphates.

Fenuma's Test.-This is merely a modification of the older Trommer's test. The reagent consists of two solutions, viz., one containing 34.64 grammes of copper sulphate in 500 c.c. of water, while the other is prepared by dissolving 173 grammes of tartrate of potassium and sodium and 125 grammes of caustic soda in a like amount of water. Before using, equal parts of the two solutions are mixed and diluted with four times as much water. A few cubic centimeters of the resulting reagent are boiled and treated with a small amount of the urine, when in the presence of sugar yellow cuprous hydroxide or red cuprous oxide separates out, and on standing settles to the bottom. After the addition of the urine the solutions should no longer be boiled, but may be held near the flame for a few moments. Unless this precaution is taken, fallacious results are often obtained, as uric acid, and kreatinin more especially, may cause a partial reduction of the copper solution on prolonged boiling. Conjugate glucuronates and homogentisinic acid likewise give a positive reaction, and ammonia, if present beyond traces, may hold in solution any cuprous oxide that may be referable to very small amounts of sugar.

Fermentation Test. - This test, when controlled by Nylander's test, is the most satisfactory one. To this end, a little compressed yeast is shaken with about 20 c.c. of urine, and the mixture is placed in a saccharimetric tube, such as that devised by Lohnstein or Einhorn. On standing at the ordinary temperature of the room, or, still better, at $37^{\circ} \mathrm{C}$., fermentation occurs if glucose is present, and the liberated carbon dioxide collects at the top of the tube. In any case, however, two controls should be made, viz., one to determine that the yeast is active, and another with normal urine. If a small amount of sugar is present, it may happen that the resulting carbon dioxide is absorbed. If in such a case Nylander's test first gave a positive reaction, but no longer reacts after fermentation is complete (in twelve to twenty-four hours), the presence of sugar may be inferred. If, on the other hand, no fermentation occurs, and Nylander's test still gives a positive result, we may conclude that the reaction is due to the presence of a non-fermentable reducing substance.

Phenylhydrazin Test.-As has been pointed out, all monosaccharides and some of the disaccharides, such as maltose, isomaltose, and lactose, form compounds with phenylhydrazin which are known as osazons (see page 65). The resulting bodies are all very similar, but may be distinguished from each other by the meltingpoint of their crystals, and to some extent also by their microscopical 
appearance. With free glucuronic acid a similar compound may be obtained, according to Thierfelder, which may also be recognized by its melting-point, while the conjugate glucuronates are inactive in this respect. Pentoses likewise give rise to the formation of osazons, but the melting-point of the resulting crystals serves to distinguish these also from the osazons of the hexoses. As a general rule, however, neither the pentoses nor glucuronic acid interferes with the reliability of the test. If doubt should arise, a special examination should be made to ascertain whether pentoses or glucuronates are present in amounts sufficient to react with the reagent. A further objection to the phenylhydrazin test has been urged on the basis that its delicacy is such that a positive reaction is obtained even under normal conditions. This, however, I must deny.

The test is conveniently conducted as follows: 5 drops of pure phenylhydrazin are mixed in a test-tube with 10 drops of glacial acetic acid and 1 c.c. of a saturated solution of common salt. To this are added 3 c.c. of urine, when the mixture is boiled for two minutes and is then set aside to cool. In the presence of more than 0.5 per cent. of glucose, crystals of phenyl-glucosazon begin to separate out after one or two minutes. Should smaller amounts be present, it is necessary to wait. The sediment is then examined microscopically. As we are generally only dealing with glucose in the urine, a further examination is usually not necessary, especially if the substance srystallizes out in large needles, which are often collected in stars and sheaves. To identify these further, however, their melting-point must be determined. As has been stated, this differs in the different osazons, with the exception of lævulose and glucose, which have the same melting-point. Lævulose, however, is found only under exceptional conditions. Its presence may be established as shown below. The melting-points of the various usazons which may be encountered are as follows:

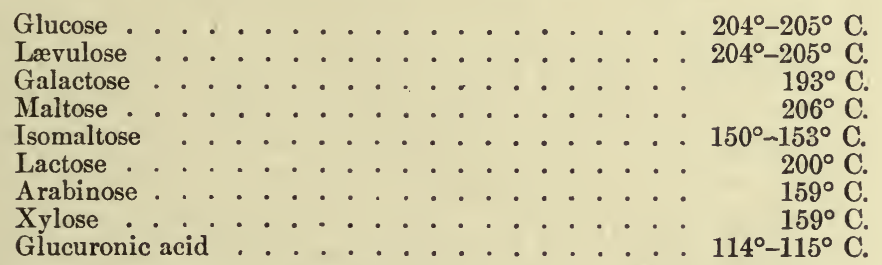

The glucosazon is insoluble in water, but dissolves with ease in hot alcohol, from which it can be precipitated on cooling in crystalline form, by diluting with water. The crystals are then collected on a filter, dried over sulphuric acid, and further examined if desired.

Polarimetric Examination.-The polarimetric examination for the presence of sugar should always be controlled by one or more of the tests that have just been described. Dextrorotation, 
unless biliary acids are present, can be directly referred to the presence of sugar, and usually to glucose. Lævorotation, however, may be referable to other reducing substances besides lævulose, such as the conjugate glucuronates, $\beta$-oxybutyric acid, and others. If such substances, moreover, are present in larger amounts, traces of dextrose may be overlookod. It is hence advisable to examine the urine both before and after treatment with yeast, and in doubtful cases to control the quantitative results, which are obtained by the polarimeter, by some other method. For a detailed description of this method I must refer the reader to special works. In every case the urine must be perfectly clear and free from albumin. If highly colored, it should be treated with lead acetate solution and then filtered, in which case allowance must be made for the degree of dilution if quantitative results are desired.

Quantitative Estimation.-KNAPP's METHOD.-The method is based upon the observation that mercuric cyanide in alkaline solution is reduced by sugar to metallic mercury. If urine is then added to a solution containing a known amount of the cyanide until this is entirely reduced, the corresponding amount of sugar can be directly ascertained.

The solution which is generally employed for this purpose contains 10 grammes of the chemically pure cyanide, and 100 c.c. of a solution of sodium hydrate (sp. gr. 1.145) in the liter : 20 c.c. correspond to 0.05 gramme of glucose.

The urine must be free from albumin and should contain not more than 0.5 to 1 per cent. of sugar. This should first be ascertained by a preliminary test. If more is present, the urine should be correspondingly diluted.

Twenty c.c. of the reagent are diluted with 80 c.c. of distilled water, or with less if a smaller amount of sugar than 0.5 per cent. is present. The solution is heated to the boiling-point, and then titrated with the diluted urine, boiling for one-half minute after the addition of every 2 c.c. or less of the urine. As the end-reaction is approached, the mercury together with the phosphates settles to the bottom and the supernatant fluid becomes clear. The final point is reached when a drop of the liquid, placed upon filter-paper, and successively held over the mouth of a bottle containing funing hydrochloric acid and over that of one containing a strong solution of hydrogen sulphide, is no longer colored yellow. The results are then calculated on the basis outlined above.

Fehling's Method.-Two solutions are employed, which must be kept in separate bottles; the one contains 34.64 grammes of crystallized cupric sulphate, dissolved in 500 c.c. of distilled water, and the other 173 grammes of potassium and sodium tartrate and 125 grammes of potassium hydrate, dissolved in an equal volume of water. This solution is of such strength that the copper contained in 10 c.c. ( 5 c.c. of each) will be completely reduced by 0.05 gramme of glucose. If then urine is carefully added to this quantity until 
complete reduction takes place, the amount of sugar contained in a given specimen of urine can be readily calculated according to the following equation :

$$
y: 0.05:: 100: x ; \text { and } x=\frac{5}{y^{\prime}}
$$

in which $y$ indicates the number of cubic centimeters of urine required to reduce the 10 c.c. of Fehling's solution, and $x$ the amount of sugar contained in 100 c.c. of urine.

As the best results are obtained only if from 5 to 10 c.c. of urine are used in one titration, it is usually necessary to dilute the urine to the required degree; in the determination of this point the specific gravity may serve as a guide. As a general rule, urines of a specific gravity of 1.030 should be diluted five times, and if the density is still higher ten times. To be certain that the proper degree of dilution has been reached, 5 c.c. of Fehling's solution are treated with 1 c.c. of the diluted urine, a little caustic soda solution and distilled water being added to make in all about 25 c.c. This mixture is. thoroughly boiled; if the fluid still remains blue, another 1 c.c. of diluted urine is added, and so on, until the last two tests differ by 1 c.c. of urine, the last cubic centimeter added causing a separation of cuprous oxide. In this manner the percentage of sugar may be approximately determined. Albumin, if present, must first be removed by boiling.

10 c.c. of Fehling's solution, diluted with 40 c.c. of water, are placed in a porcelain dish and boiled. While boiling, the diluted urine is added from a burette, 0.5 c.c. at a time, when, as a rule, the precipitated cuprous oxide will rapidly settle, so that gradually a white bottom may be seen through the blue field, the color of which becomes less and less intense upon the further addition of urine until finally the solution is almost colorless. - When this point is reached, the urine is added drop by drop until decolorization is complete. The degree of dilution multiplied by 5 and the result divided by the number of cubic centimeters of diluted urine employed will then indicate the percentage-amount of sugar.

Unfortunately, it is at times difficult to determine exactly the point when all the copper has been reduced-i. e., the point at which the blue color has entirely disappeared. As this is approached droplets of the hot mixture may be transferred by means of a glass rod to a piece of filter-paper that has been soaked with a solution of potassium ferrocyanide. So long as unreduced copper is present reddish brownish rings result. The solution is carried to the point where this no longer occurs. The immediate result, however, counts; on prolonged exposure to the air some of the copper in suspension becomes oxidized and gives rise to the color also.

To obviate this difficulty it has also been suggested to double the amount of alkali in the Fehling's solution, in which case no separation of cuprons oxide occurs; the solution is then titrated to the point of decolorization, but remains clear. 
Differential Density Method.-The specific gravity is first determined in the fresh urine, after adling 2 grammes of tartrate of potassium and sodium, and 2 grammes of diacid sodium phosphate to every 100 c.c. To about 200 c.c. which have thus been prepared, from 5 to 10 grammes of fresh yeast are added, and the mixture is set aside at a temperature of from $20^{\circ}$ to $25^{\circ} \mathrm{C}$. until fermentation is completed. If but little sugar is present, two or three hours will suffice; otherwise the mixture is allowed to stand for twelve hours. Evaporation is guarded against by closing the bottle with a perforated stopper through which a finely drawn out tube passes, which is open at the distal end. The specific gravity is then again determined at the same time as before, and the difference multiplied by 0.230 . The result indicates the amount of sugar in per cent.

The method yields good results unless very small amounts of sugar are present, viz., less than 0.5 per cent. In such an event, the reducing power of the urine is first ascertained according to Fehling's or Knapp's method. It is then fermented, when the remaining reducing substances are again determined. The difference may be referred to sugar.

Fermentation Method.-In the clinical laboratory especially constructed saccharimetric tubes are used, of which Lohnstein's is probably the best. These are provided with a scale which enables the percentage of sugar to be read off directly from the amount of carbonic acid that has gathered in the upper end of the tube. The instruments are accompanied by printed instructions, which need not be considered at this place.

Lactose.-The presence of lactose in the urine is a normal occurrence in nursing women, and it is at times found also shortly before confinement. Its appearance in the urine is undoubtedly referable to absorption, owing to the fact that a superabundance of milk is being produced, and we accordingly also find the substance in the urine when for any reason lactation is suppressed. Once it has found its way into the circulation, its elimination through the kidneys necessarily follows, as the body is incapable of inverting the disaccharides to monosaccharides.

Aside from its occurrence in connection with lactation, lactose is found in the urine only if abnormally large amounts have been ingested. In such an event, as has been stated, a certain proportion of the sugar escapes inversion, and on entering the general circulation it is eliminated as such. The amount of lactose which may be Iiound in the urine of nursing women varies between 0.013 and 0.438 per cent. Its presence in the urine may be suspected if the reduction test and the phenylhydrazin test yield a positive result, while the fermentation test, as usually conducted, is negative. Like glucose, the substance is dextrorotatory. To identify the sugar positively as lactose, it is necessary to isolate it as such. 
Isolation.-The collected urine of twenty-four hours is precipitated with lead subacetate and filtered. After washing with water the filtrate and washings are mixed and treated with ammonia. The resulting precipitate is filtered off and the filtrate again precipitated with lead subacetate and ammonia, and so on until the final filtrate is optically inactive. The precipitates, with the exception of the first, are then mixed, washed with water, decomposed with hydrogen sulphide, and filtered. In the filtrate the excess of hydrogen sulphide is removed by a current of air, and freed from any acids that have been liberated by shaking with argentic oxide. The mixture is filtered, freed from soluble silver with hydrogen sulphide, treated with barium carbonate, and concentrated to a small volume; 90 per cent. alcohol is then added, which causes the formation of a flocculent precipitate. This is filtered off. The filtrate is placed in the desiccator, when on standing crystals of lactose gradually separate out. These may be purified by recrystallization, decolorization with animal charcoal, and extraction with 60-70 per cent. alcohol.

Lævulose.-The occurrence of a lævorotatory sugar has been at times, though rarely, observed in the urine of diabetic patients, where it was present either alone or in association with glucose. Like dextrose, the substance reduced Fehling's and Nylander's solution, and formed an osazon with phenylhydrazin, with a meltingpoint of $205^{\circ} \mathrm{C}$. It was fermentable, but, unlike true lævulose, could be precipitated with basic lead acetate.

Leo's laiose, which was also obtained from a diabetic urine on one occasion, was very similar to the body just described, but, unlike this, could not be fermented. With phenylhydrazin, moreover, it formed a yellowish-brown non-crystallizable oil.

Of the nature of these bodies nothing further is known.

The presence of a lævorotatory sugar can, of course, readily be established by the common tests, supplemented by a polarimetric examination, if it is present alone. If glucose, however, also is contained in the urine in amounts sufficient to counteract the lævorotation, the matter is more difficult. In such an event, however, it will be observed that higher values are obtained in estimating the sugar by titration, or according to the differential density method, than with the polarimeter, for reasons which are self-evident.

According to Neuberg and Strauss, the presence of lævulose can be definitely established in the urine, the blood-serum, ascitic fluid, or in pleural effusions by its transformation into the corresponding methyl-phenyl-osazon, which can be obtained in crystalline form.

Lævulose also gives Seliwanoff's reaction, viz., it gives rise to the formation of a red pigment on boiling with resorcin and hydrochloric acid, which is soluble in alcohol. The reaction in question is common to all the ketoses of the hexose series (fructose and

${ }^{1}$ The method is described in my Clinical Diagnosis, 6th edition. 
sorbinose), but is also obtaine? with those polysaccharides which yield fructose on hydrolysis, viz., cane-sugar, raffinose, inulin, etc.

Maltose.-Maltose together with glucose was found on one oceasion in the urine of a patient supposedly the subject of pancreatic disease. Its recognition is essentially dependent upon the formation of its osazon, and the identification of the latter by its meltingpoint.

Dextrin.-That traces of dextrin are found in the urine under normal conditions has been pointed out. Larger amounts have been observed in the case of a diabetic patient, where the substance apparently took the place of glucose. Of its origin nothing definite is known, but it is likely that in health the substance gains entrance to the circulation in a more or less accidental way, and is then, of course, eliminated at once. It is now regarded as identical with the animal gum of Landwehr.

Pentoses.-That traces of pentoses may occur in the urine under normal conditions has been stated. As has been shown, the pancreas contains a nucleoproteid which yields a very large amount of pentose on decomposition, and one might imagine that the normal pentose of the urine might possibly be referable to this source. Neuberg, however, has shown that the two are not identical. The urine pentose (arabinose) is optically inactive, while the pentose of the pancreas is l-xylose. The normal pentoses, no doubt, are referable to the ingestion of such articles of food as prunes, cherries, grapes, beer, wine, etc., in which arabinose occurs preformed. As in the case of glucose, the power to assimi.. late pentoses seems to vary with different individuals, and here, as there, a digestive pentosuria can be artificially produced. In diabetes the power to oxidize the pentoses may be much impaired, and, very curiously, the largest quantities have been observed in morphin habitués.

The individual pentoses which have thus far been encountered in the urine are arabinose, xylose, and rhamnose. They all reduce Fehling's solution and give rise to osazons with phenylhydrazin. The melting-points of the resulting compounds, however, are different from those of the common hexosazons (see page 295). In the amounts, however, in which they are usually present no reactions are obtained in this manner. The fermentation test is negative. Xylose and rhamnose turn the plane of polarization to the right, while arabinose is optically inactive.

To demonstrate the presence of pentoses in the urine the following test of Tollens is employed :

Orcin Test (as deseribed by Bial).-4-5 c.c. of the reagent (500 c.c. of 30 per cent. hydrochloric acid, 1 gramme of orcin, and 25 drops of liquor ferri sesquichloridi) are heated to boiling; the tube is removed from the flame and a few drops to 1 c.c. of urine added. With pentose urine there is immediately a fine green coloration. Normal and diabetic urines do not give the reaction, nor do urines 
containing conjugate glucuronates. The reagent keeps for at least a year.

With Tollens' phloroglucin test, which is conducted in the same manner, a deep-red color develops instead; but this reaction is also common to the glucuronates. (The reagent is prepared in the same manner as in the case of the orcin reagent, phloroglucin being simply substituted for the orcin.)

\section{THE ALBUMINS.}

As every urine contains a small number of cellular elements which are derived from the urinary tract, it can readily be understood that even under normal conditions albumin can be demonstrated with suitable methods. The amount, however, is exceedingly small, and with the common tests a positive reaction cannot be obtained unless the substance in question has been previously isolated from a large quantity of urine. In such an event a trace of a nucleo-albumin can be demonstrated. The occurrence of the common albumins of the blood, on the other hand, is always a pathological phenomenon. Some writers, it is true, speak of a physiological albuminuria, which may be observed after severe muscular exercise, following cold baths, during pregnancy, etc., and it is even claimed that the elimination of albumin in young persons, which is so commonly observed in neurotic and anæmic individuals, in association with an increased amount of uric acid and oxalic acid, belongs to this order. There is an increasing tendency among pathologists, however, to doubt the physiological character of such forms of albuminuria, and personally I maintain that every albuminuria of a hæmatogenic type is a pathological phenomenon. We may, in fact, go further, and assume that the appearance of nucleo-albumin also in amounts which can be demonstrated by ordinary tests is abnormal, as such an occurrence must of necessity be associated with an increased desquamation of epithelial cells, which in itself is evidence of a pathological process. This, however, is hardly the place to enter upon a detailed consideration of the various morbid conditions in which albuminuria may occur, and it will suffice to state that any disturbance in the nutrition of the glandular elements of the kidney, from whatever cause, will at once find expression in the appearance of albumin in the urine. The albumins which are then eliminated are the common albumins of the blood, and notably serum-albumin and serumglobulin. Fibrinogen, on the other hand, is usually not found. In cases of hæmaturia and chyluria, however, its presence may be inferred from the formation of coagula of fibrin. This may occur in the urinary passages already, but more commonly it is observed after the urine has been voided.

Other albumins besides those which are normally found in the blood are encountered only exceptionally in the urine; but it may 
be stated that whenever such substances find their way into the general circulation their elimination at once follows. Formerly it was taught that peptones could thus appear, and for many years various types of peptonuria were described. More recent investigations, however, have shown that the substances in question were in reality no peptones in the sense of Kühne, but albumoses. Some of these are, no doubt, identical with the common digestive albumoses, and find their way into the blood, when their further transformation into native albumins does not occur in the epithelial cells of the digestive tract. Others again are probably formed in the body proper in diseases which are associated with suppurative processes, and in which the formation of albumoses occurs at the expense of the tissue albumins under the influence of various microorganisms. Under still other conditions, as in the various nonseptic fevers, in phosphorus poisoning, etc., the albumosuria may be the expression of a metabolic abnormality per se, and is possibly dependent upon the action of the various tissue ferments.

Of special interest, further, is the appearance in the urine of the so-called albumin of Bence Jones, which has been repeatedly observed in association with multiple myelomata of the bones. Of its chemical nature, however, little is known. By some it is regarded as an albumose, but, according to Neumeister, it is not identical with any of the known digestive albumoses. I shall revert to it later.

In diseases, finally, in which an increased destruction of leucocytes is taking place, both histon and nucleohiston have been found.

Of other albumins which are foreign to the blood, only eggalbumin has been encountered, following the ingestion of excessive amounts of the substance.

Tests for the Common Albumins of the Blood.-The Nitric AcID TesT.-A small amount of urine is placed in a conical glass and is underlaid with a few cubic centimeters of concentrated nitric acid, when in the presence of serum-albumin and serum-globulin a white, opaque disk of coagulated albumin is formed at the zone of contact, which varies in intensity and extent with the amount of albumin present. Immediately below this variously colored rings also are observed, which are in part referable to the decomposition of indoxyl and skatoxyl sulphate, and the oxidation of the liberated indoxyl and skatoxyl to blue and red pigments. In the presence of bile-pigment a green color will then also be noted. If much urea be present at the same time, it may happen that after a few minutes a dense disk of urea nitrate crystals separates out in the lower pigmented layer, but more commonly these are formed throughout the mixture on standing, and gradually settle to the bottom. In every urine a more or less well-marked white ring appears in the upper strata of the specimen and separated from the albumin ring (if present) by a layer of clear urine. This was formerly regarded as being referable to uric acid, but Mörner has shown that it 
is probably of albuminous character. Nothing further, however, is known of the substance. In rare instances I have found it present in large amounts.

As nucleo-albumins, when present beyond traces, can simulate the true albumin reaction, it is well to dilute the urine with water and to examine again when its presence is suspected. If then the reaction is more pronounced than before, the precipitate may, in part at least, be referable to this source. This possibility should be considered if the urine contains an increased number of morphological elements, and if the reaction is slight. Other tests should then also be employed.

Albumoses, if present beyond traces, also react with nitric acid, but it is to be noted that in such cases the precipitate disappears on heating and reappears on cooling, while the liquid at the same time assumes an intensely yellow color. Should a mixed albuminuria exist-i. e., should albumoses and albumin be present simultaneously - the clearing of the urine is only partial.

As nitric acid also precipitates certain resins which may have been administered for medicinal purposes, it is at times necessary to eliminate this possibility of error. Their presence is indicated if the precipitate disappears on shaking the mixture with ether.

The Boiling Test.-The test is best conducted in the following modification: A few cubic centimeters of urine are tested with onesixth of its volume of a saturated solution of common salt, after having rendered it distinctly acid by the addition of a drop or two of a 25 per cent. solution of acetic acid. The mixture is then boiled, when in the presence of coagulable albumins the liquid becomes turbid, and on standing a flocculent precipitate gathers at the bottom of the tube. The turbidity may, however, at times be due to a precipitation of earthy phosphates. To distinguish between the two, one or two drops of a 25 per cent. solution of nitric acid are now added for every 1 c.c. of the urine. The earthy phosphates are thus dissolved, while the precipitate of albumin remains unaffected. The test in this modification is very satisfactory and delicate.

If the albumin of Bence Jones should be present, coagulation occurs at a temperature of $50^{\circ}$ C. already, but it will be noted that the precipitate disappears on subsequent boiling and reappears on cooling.

The common albumoses, as well as nucleo-albumin, are not thrown down. The presence of the former may be inferred if after the addition of the acid and subsequent cooling a white precipitate is formed, which dissolves upon the application of heat and reappears on cooling.

The Potassium Ferrocyanide Test.-A few cubic centimeters of urine are strongly acidified with acetic acid, and treated with a 10 per cent. solution of potassium ferrocyanide drop by drop, when in the presence of albumin a precipitation occurs which varies 
in intensity with the amount present. Concentrated urines should first be diluted. Albumoses are also thrown down, but are redissolved on boiling and reappear on cooling. Should nucleo-albumins be present beyond traces, a precipitate develops upon the addition of the acetic acid. This may also occur if urates are present in large amounts. But in this event the precipitate clears upon warming the solution; and if the urine, moreover, is previously diluted, it does not occur at all, while the separation of nucleo-albumin takes place more rapidly in the latter case than before.

Still other tests exist which are equally good, but for practical purposes the three just described will suffice. In every case, however, it is necessary that the urine should be perfectly clear. If turbid, owing to precipitation of any of the normal constituents of the urine, simple filtration generally suffices; but if referable to bacteria, it is best shaken with powdered talcum and then filtered.

Special Test for Serum-albumin.-If it is desired to demonstrate the presence of serum-albumin by itself, the urine is rendered amphoteric or faintly alkaline with sodium hydrate, and saturated with magnesium sulphate to remove any globulins that may be present. The filtrate is then acidified with acetic acid and boiled, when in the presence of serum-albumin precipitation occurs.

Special Test for Serum-globulin.-This is conducted as above, or by adding an equal volume of a saturated solution of ammonium sulphate to the amphoteric urine, when the globulins are thrown down. Urates may then also separate out, but this always occurs later. The precipitated globulins are soluble in acetic acid. As I have stated before, there is one instance of globulinuria on record in which the substance was found in the sediment in crystalline form.

Test for Nucleo-albumin.-A small amount of urine is diluted with water and then treated drop by drop with strong acetic acid. In this manner the precipitation of urates is prevented, while the nucleo-albumin separates out. To identify it as such, however, it is necessary to isolate the substance in larger amounts. To this end, the collected urine of twenty-four hours is carefully neutralized and concentrated to about 1000 c.c. at a temperature of $60^{\circ}-70^{\circ} \mathrm{C}$. On filtering, it is saturated with ammonium sulphate in substance and the precipitate collected on a filter. This is dissolved in a little water and freed from salts by dialysis. Should hetero-albumose be present, this separates out and is removed by filtration. A portion of the remaining solution is then tested with acetic acid, as described; the precipitate should be soluble in mineral acids. In the remaining solution the body is completely thrown down with acetic acid. The precipitate is filtered off, washed with dilute acetic acid, and dried. On fusion with caustic alkali and potassium nitrate, phosphoric acid should then be liberated if. the substance is a nucleo-albumin. If this reaction is not obtained, but if on boiling with dilute mineral 
acids a reducing substance is set free, it may be assumed that the body in question is mucin.

Test for Albumoses.-To test for albumoses in general, a small amount of the urine is acidified with acetic acid and treated with an equal volume of a saturated solution of common salt. The solution is then boiled and filtered while still hot, so as to remove any coagulable albumins that may be present. On cooling, the albumoses separate out, but redissolve on boiling. In such an event, the solution also gives the biuret reaction and that of Millon.

More exact is the following method, which should always be employed when there is reason to believe that albumoses are present only in traces. 'The collected twenty-four-hours' urine is carefully neutralized, concentrated to about 1000 c.c. at $60^{\circ}-70^{\circ} \mathrm{C}$., filtered, and saturated with ammonium sulphate in substance. The precipitate is collected on a filter, and dissolved in a little water, when a small portion is treated with an equal volume of a saturated solution of common salt, and with acetic acid or nitric acid drop by drop so long as any precipitate that has formed is thus increased. The solution is then boiled. If coagulable albumins are present, these are precipitated, and are filtered off from the hot solution. If the filtrate becomes turbid again on cooling, and clears upon subsequent boiling, the presence of albumoses may be inferred. - To determine the character of the albumoses in question, the remaining liquid is dialyzed (see above), freed from nucleo-albumin by means of acetic acid, neutralized, concentrated on a water-bath, and treated with an equal volume of a saturated solution of ammonium sulphate. On standing, the primary albumoses are precipitated. The filtrate is then saturated with the salt in substance, when, on further standing, the secondary albumoses separate out.

True peptones, in the sense of Kühne, do not occur in the urine, and it is hence unnecessary to describe the older and more complicated methods which formerly were employed in their search.

Bence Jones' Albumin.-This body, as has been pointed out, has repeatedly been encountered in the urine in association with the existence of multiple myelomata of the bones. Of its nature, however, little is known. Most observers have regarded it as an albumose, but it is admitted that it is not identical with any of the known digestive albumoses. Like the globulin described by Paton, it has been found in crystalline form in the urinary sediment, and can be made to crystallize after its isolation in amorphous form. Magnus-Levy, while likewise unable to identify it with any of the known albumins or albumoses, points out that it has in reality only one property in common with the albumoses, viz., the solubility of its precipitate on boiling. He has shown, however, that this is only apparent, and that under suitable conditions the body is coagulated on heating to $100^{\circ} \mathrm{C}$., like the native albumins. He further noted that, like the true albumins, the substance yields the common digestive products of these bodies, viz., primary and secondary 
albumoses; but, as in the case of casein, no hetero-group could be demonstrated. These results I have confirmed, and it is thus conclusively established that the body cannot be an albumose. Pending further investigations, it is hence advisable to term the substance the albumin of Bence Jones. Of its origin nothing definite is known. The amount which is often found is so large that the conclusion suggests itself that the substance may be formed as the result of a definite metabolic anomaly in the place of some one of the normal albumins (possibly globulin). The presence of the substance may be suspected if a urine gives the usual albumose reaction to a marked degree, as the disease in question is in reality the only one in which larger amounts of an "albumose"-like body are obtained. It can then be isolated by treating the neutralizerl urine with double its volume of a saturated solution of ammonium sulphate. It coagulates at a relatively low temperature $\left(50^{\circ}-55^{\circ} \mathrm{C}\right.$.), and dissolves more or less completely before the boiling point is reached, to reappear again on cooling. Bence Jones' albuminuria is generally unaccompanied by common albuminuia. To identify the substance, it is advisable to digest the body with pepsin, to demonstrate the formation of proto-albumose, and to show that no hetero-albumose is produced.

Test for Fibrin.-When fibrin is present in the urine, it usually occurs in the form of distinct clots, the nature of which is commonly apparent without chemical examination. If it is to be identified in this manner, however, the clots are washed with water until free from blood-pigments. They are then placed in a 5 per cent. solution of sodiun chloride containing an excess of thymol, to guard against putrefactive changes. It will be observed that the substance does not dissolve, while in a 0.3 per cent. solution of hydrochloric acid it rapidly swells and is digested after the addition of a little pepsin.

Test for Histon.-The twenty-four hours' urine is first freed from coagulable albumins by boiling. It is then precipitated with a large excess of 94 per cent. alcohol. The precipitate is washed with hot alcohol and dissolved in boiling water. On cooling, the solution is acidified with hydrochloric acid and allowed to stand for a number of hours. Any uric acid that has separated out is removed by filtration, when the filtrate is precipitated with ammonia. The collected material is washed with ammoniacal water until the washings no longer give the biuret reaction. It is then dissolved in dilute acetic acid. If histon is present, the solution coagulates on boiling and gives the biuret reaction. The coagulated material dissolves in mineral acids.

Quantitative Estimation of the Coagulable Albumins.-In the clinical laboratory the so-called albuminimeters of Esbach are conveniently employed for this purpose. The method is exceedingly simple, and gives results which are sufficiently accurate for ordinary purposes. To this end, the tube is filled with urine to the mark $U$. 
Esbach's reagent, which consists of an aqueous solution containing 10 grammes of picric acid and 20 grammes of citric acid to the liter, is then added to the mark $R$. The tube is closed, inverted a number of times, and set aside for twenty-four hours. The number of the scale which corresponds to the height of the precipitated albumins indicates the amount in grammes. in 1000 c.c. of urine. Care must be had, however, that the urine is acid, that the density does not exceed 1.006-1.008, and that the temperature remains at about $15^{\circ} \mathrm{C}$.

If more accurate results are desired, a known volume of urine, feebly acidified with nitric acid if necessary, is heated, first on a water-bath and then over a free flame, until coagulation is complete. The precipitate is collected on a small filter, and washed with water, alcohol, and ether. The contained nitrogen is now estimated according to Kjeldahl's method, when the result multiplied by 6.3 will indicate the corresponding amount of albumin.

If it is desired to estimate the amount of the individual albumins separately, they are first isolated, as has been described, and are then subjected to Kjeldahl's process. In this case, however, ammonium sulphate cannot be used for purposes of salting.

\section{THE PIGMENTS OF THE URINE.}

Of the chemical nature of the pigments of normal urine little is known that is definite. According to some observers, the yellow color is due, in part at least, to the presence of so-called urochrome, which in turn is regarded as identical with the normal urobilin of MacMunn. Others, again, claim that there is no reason to suppose that a difference exists between this normal urobilin and the urobilin of Jaffe, which is mostly observed under pathological conditions, but which may occur also in health. Jaffe's urobilin, further, is held by some to be identical with the hydrobilirubin which results from bilirubin on reduction. That the urobilin which is notably observed under pathological conditions can be formed within the body in the absence of micro-organisms is now a well-established fact. We thus find that in diseases in which the elimination of bile through the usual channels is prevented, urobilin may occur in the urine, nevertheless; and it has further been noted that both at the beginning and at the disappearance of jaundice increased amounts are found. Similar results have been obtained when from any cause an increased destruction of blood-pigment occurs. We may thus imagine that in such eases the urobilin results from bilirubin through oxidation to choletelin. This view of the origin of urobilin, of course, does not necessarily preclude the possibility that a certain amount of the pigment, which, as I have said, may normally also occur in the urine, may be derived from bilirubin through a process of reduction in the intestinal tract. But, as is apparent from the considerations just related, we are scarcely in a position to speak authoritatively of the 
origin of the normal urinary pigments. The chemical position of the colorless mother-substance of urobilin, moreover, which is spoken of as urobilinogen, and which can usually be demonstrated whenever urobilin also is present, is thus far not clear.

According to Garrod, the urobilin of the urine is identical with the stercobilin of the feces, both in composition and properties, but differs conspicuously from hydrobilirubin, especially in the much smaller percentage of nitrogen which it contains, viz., 4.11 per cent., as compared with 9.22. The elementary composition of urobilin is given by Garrod and Hopkins as : C, 63.58 per cent.; H, 7.84; $\mathrm{N}, 4.11$, and $\mathrm{O}, 24.47$. Garrod further states that by acting upon urochrome with acids he never succeded in obtaining any product showing the urobilin band, or yielding the well-known fluorescence with zinc chloride and ammonia, as Thudichum claimed. But he found that a substance having both these properties is readily obtained by the action of aldehyde upon an alcoholic solution of urochrome. In a short time-shorter still when the liquid is warmed-an absorption-band appears like that of urobilin, and the tint of the solution deepens to a rich orange-yellow. With zinc chloride and ammonia a brilliant green fluorescence occurs, and the band is shifted toward red, as that of urobilin is under like conditions.

Isolation of Urochrome.-To demonstrate the presence of so-called urochrome in normal urine, the fluid is acidulated with 1 or 2 c.c. of dilute sulphuric acid pro liter. On filtering, it is saturated with ammonium sulphate. The resulting precipitate is dried and extracted with warm and slightly ammoniacal alcohol. The pigment passes into solution, and is obtained on evaporation of the alcohol as an amorphous reddish-brown substance, which is readily soluble in acidulated water, chloroform, and common alcohol, but is practically insoluble in ether and benzol. According to Garrod, its solutions do not give rise to any bands of absorption, and do not fluoresce upon the addition of ammonia and zinc chloride. Gautier, on the other hand, states that its acidulated solutions show a band of absorption about $\mathrm{F}$, and that the remainder of the spectrum from about $\mathrm{G}$ on to the right is obscured. He adds that in this respect urochrome and choletelin are alike.

Garrod regards the action of aldehyde upon an alcoholic solution of urochrome, outlined above, as a very delicate test for the pigment. The process can be stopped then by simple dilution with water, as aldehyde has no such action upon aqueous solutions of urochrome. If, however, the action is allowed to continue, a further change ensues. The liquid reddens and a second band appears in the violet. The fluorescence can still be obtained with zinc chloride and ammonia, and both bands are shifted toward red and are closer together than before.

The tern uroerythrin has been applied to the pigment which imparts the salmon-red color to sediments which are composed of 
urates or uric acid. Of its chemical nature, however, nothing definite is known, but there is evidence to show that it also is a derivative of the normal coloring-matter of the blood. It contains 62.51 per cent. of carbon and 5.79 per cent. of hydrogen. Its amount is noticeably increased in extensive disease of the liver, as also in conditions associated with an increased destruction of red corpuscles.

Isolation of Uroerythrin.-As has been stated, the salmon color of sediments of urates and uric acid is due to this pigment. In their absence the urine is precipitated with neutral acetate of lead or barium chloride. If uroerythrin is present beyond traces, it is thrown down, and colors the resulting precipitate a more or less intense salmon red. The pigment is soluble in boiling alcohol, and may thus be extracted. Its solutions are said to give rise to two bands of absorption to the left of F.

Urobilin.-Urines which contain much urobilin, viz., the pathological urobilin of Jaffé, present a dark-yellow color, which may be imparted to the foam on shaking. They are thus quite similar to icteric urines.

Tests.-To identify the substance, the urine is precipitated with a mixture of barium hydrate and barium chloride. If notable quantities of urobilin are present, the precipitate is thus colored a more or less intense brownish-red. On boiling with acidulated alcohol the pigment is then extracted, and imparts a brownish or pomegranatered color to the alcoholic solution (v. Jaksch).

Gerhardt's test also is very serviceable. To this end, 10-20 c.c. of urine are extracted with chloroform by shaking. A few drops of a dilute solution of iodopotassic iodide are added to the extract, when, upon the further addition of a dilute solution of sodium hydrate, the solution is colored yellow or yellowish brown and exhibits a beautiful greenish fluorescence.

If the substance cannot be demonstrated with these tests, the urine is acidulated with hydrochloric acid and allowed to stand exposed to the air, so that any urobilinogen that may be present is transformed into the free pigment. The fluid is then examined with the spectroscope, when in the presence of urobilin a distinct band of absorption is obtained between $\mathrm{b}$ and $\mathrm{F}$, extending beyond $\mathrm{F}$ to the right. A similar band is also obtained in alkaline solution, but is not so intense and does not extend beyond F.

Isolation.-To isolate the pigment, if present in large amounts, the urine is directly precipitated with ammonia and chloride of zinc. The precipitate is thoroughly washed with water, extracted with alcohol by boiling, dried, and then dissolved in anmonia. The resulting solution is precipitated with subacetate of lead, the precipitate washed with water, and extracted with boiling alcohol as before, and decomposed with acid alcohol. The filtered alcoholic solution is treated with one-half its volume of chloroform and diluted with water; the urobilin passes into the chloroform on moderate agita- 
tion. The chloroform solution is then washed with water. On final distillation the pigment remains as an amorphous reddish material, which can be further purified by washing with ether, which takes up contaminating red pigments. The substance is readily soluble in alcohol, amyl alcohol, and chloroform, less readily so in water and ether.

Ehrlich's Reaction.-When normal urine is treated with an equal volume of a saturated solution of sulphanilic acid in 5 per cent. hydrochloric acid to which a 0.5 per cent. solution of sodium nitrite has been added in the proportion of $1: 40$, and the mixture is then rendered strongly alkaline with ammonia, a more or less wellmarked orange color develops. Under pathological conditions, on the other hand, and notably in typhoid fever, a red color results which varies in intensity from a light carmin to a deep garnet-red. This reaction is known as Ehrlich's diazo-reaction, as it is dependent upon the presence of a diazo- compound of the nature of diazo-benzene-sulphonic acid, in the reagent. This results through an interaction between the sodium nitrite and the sulphanilic acid, as represented in the equations :

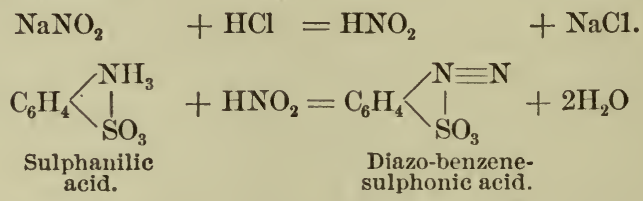

The action is supposedly referable to alloxy-proteinic acid.

Under pathological conditions still other pigments may be found in the urine. These comprise hæmoglobin and its derivatives, hæmatin, methæmoglobin, and hæmatoporphyrin ; further, also urorubrohæmatin and urofuscohæmatin, which are also undoubtedly derived from hæmoglobin, but which have thus far been found in the urine on only one oceasion; further, the common pigments of the bile; and, finally, substances which belong to the class of the so-called melanins. For a consideration of the various pathological conditions under which the bodies may be met with, the reader is referred to special works on diagnosis. At this place I shall merely describe the more common tests by which their presence can be demonstrated.

The Blood-pigments.-If the microscopical examination of the urine shows the presence of red blood-corpuscles in the sediment, further chemical examination is, of course, unnecessary. Cases of simple hæmoglobinuria, in contradistinction to hæmaturia, may occur, however, in which dissolution of the hæmoglobin has taken place in the circulation already, and in which blood-corpuscles do not appear in the urine. In such an event the demonstration of blood-pigment can be made only by chemical methods. Its presence, it is 
true, is usually indicated by the color of the urine, but this may be simulated by other substances as well.

Heller's Test.- This is the most convenient test for demonstrating the presence of blood-pigment in the urine, and, in the modification here given, exceedingly. sensitive. It is based upon the decomposition of the pigment in question by means of caustic alkali and the resulting formation of hæmochromogen. To this end, a small amount of the urine, or, still better, of the sediment, is rendered strongly alkaline with caustic alkali and boiled. On standing, the precipitated earthy phosphates settle to the bottom, and are colored a more or less intense carmin by the hæmochromogen, which has likewise separated out. That the pigment is in reality hæmochromogen can be readily demonstrated on spectroscopic examination. When controlled in this manner, the test is exceedingly sensitive, and may still yield a positive result even when the chemical test by itself does not give a well-pronounced reaction.

Spectroscopic Examination.-On direct spectroscopic examination the spectrum of methæmoglobin is usually obtained. The urine should first be acid, and if necessary a little acetic acid is added. On the addition of a little ammonia and ammonium sulphide and subsequent filtration the broad band of hæmoglobin is then obtained. With oxyhæmoglobin, on the other hand, the two bands between $\mathrm{D}$ and $\mathrm{E}$ are observed; and upon the subsequent addition of ammonia and ammonium sulphide and filtration the spectrum of reduced hæmoglobin results. If this does not appear distinctly, the solution is treated with an excess of sodium hydrate solution, and will then give the spectrum of hæmochromogen.

Hæmatin.-Hæmatin is very rarely found in the urine. Its presence as such can be determined only by spectroscopic examination. Like hæmoglobin and methæmoglobin, it gives Heller's reaction.

Hæmatoporphyrin.-According to Garrod, traces of hæmatoporphyrin may be found in every urine. Larger quantities are observed in a number of diseases, but even in these the amount is usually so small that its presence will scarcely be suspected from simple inspection. Typical hæmatoporphyrinuria, on the other hand, may be observed following the prolonged administration of sulphonal, trional, and tetronal, or in cases of poisoning with the substances in question. The urine then appears dark red in color, and on standing may turn almost black. As Hammarsten has pointed out, this change in color is only in part due to hæmatoporphyrin, and is largely referable to other red and reddish-brown pigments of unknown character. Whether or not different hæmatoporphyrins exist has not been definitely determined, but is probable. In freshly voided urines hæmatoporphyrin probably exists in combination with some other, still unknown body, with which it forms a colorless chromogen. From this the free pigment then develops on exposure to the air. 
Like the common blood-pigments, and hæmatin, hæmatoporphyrin also reacts with Heller's test. To prove its presence, however, as such, a spectroscopic examination is necessary. To this end, the urine is precipitated with barium hydrate and bariun chloride. The precipitate is washed and allowed to stand in contact with acidulated alcohol, which extracts the pigment. After filtering, the solution is examined with the spectroscope; if subsequently the solution is rendered alkaline with ammonia, the spectrum of hæmatoporphyrin in alkaline solution is obtained. To isolate the substance as such, the acid solution is mixed with a little chloroform and diluted with water. On gentle agitation the chloroform takes up the greater portion of the hæmatoporphyrin, while a small fraction and other pigments remain in the diluted alcoholic solution. On evaporating the chloroform extract the substance is obtained in comparatively pure form.

Neumeister states that besides hæmatoporphyrin another derivative of the blood-pigment may be observed in cases of poisoning with sulphonal, which, in contradistinction to the first, contains iron. This does not react with Heller's test, however, while the color of the urine is the same as in typical hæmatoporphyrinuria. The pigment is precipitated by an alkaline barium chloride solution, and can be subsequently dissolved in acid alcohol. This solution presents a reddish-violet color, and shows one broad band of absorption in the blue portion of the spectrum immediately bordering on the green.

On rendering the solution alkaline with ammonia the pigment is thrown down. On adding an excess of sodium hydrate solution, on the other hand, it is dissolved, while the liquid assumes a yellow color. This solution then shows a sharp, narrow line in the green, near the blue portion of the spectrum, but disappears after the solution has stood for some time.

Urorubrohæmatin and Urofuscohæmatin.-These pigments were isolated by Baumstark from the urine of a leprosy patient, but have not been encountered since. Their relation to hæmatin is apparent from the formulæ:

$$
\begin{aligned}
& \mathrm{C}_{32} \mathrm{H}_{32} \mathrm{~N}_{4} \mathrm{O}_{4} \mathrm{Fe} \text {, hæmatin (Nencki and Sieber). } \\
& \mathrm{C}_{34} \mathrm{H}_{35} \mathrm{~N}_{4} \mathrm{O}_{5} \mathrm{Fe} \text {, hæmatin (Hoppe-Seyler). } \\
& \mathrm{C}_{68} \mathrm{H}_{94} \mathrm{~N}_{8} \mathrm{O}_{30} \mathrm{Fe} \text {, urorubrohæmatin. } \\
& \mathrm{C}_{68} \mathrm{H}_{106} \mathrm{~N}_{8} \mathrm{O}_{26} \text {, urofuscohæmatin. }
\end{aligned}
$$

Whether or not any relation exists between these two bodies and hæmatoporphyrin in impure form, as Hammarsten suggests, must remain an open question.

Melanins. - Notably in association with the existence of melanotic tumors, but at times also in other diseases which are associated with an increased destruction of red blood-corpuscles, urines are met with which gradually turn a dark brown or black on standing. When 
freshly voided, however, they commonly present a normal color. The pigment or pigments which are thus formed belong to the class of melanins, and are identical with those which can be obtained from the pigmented growths. They are probably eliminated in combination with some other substance which is as yet unknown, as colorless melanogens and from which the free pigments are obtained on oxidation. They are unquestionably derived from the common pigments of the blood, but are individually little known.

To prove that the change in the color of the urine is referable to melanins, a fresh specimen should be procured, and treated with bromine-water. If the chromogens in question are present, the resulting precipitate, which is yellow at first, turns black on standing. On the addition of a few drops of a strong solution of ferric chloride a similar reaction is obtained.

To isolate the pigments from the urine, the fluid is first precipitated with an alkaline solution of barium chloride. From the resulting precipitate the pigments are extracted with a concentrated solution of sodium carbonate, and are then precipitated by adding an excess of sulphuric acid. By redissolution in a dilute solution of sodium hydrate and reprecipitation with acetic acid they can be obtained in comparatively pure form. But it will be noted that a certain fraction remains in the acetic acid solution, which indicates the existence of at least two different pigments. The soluble form has been termed phymatorhusin, and, according to Nencki and Sieber, contains no iron, while Mörner claims that this is present. Elementary analysis of this pigment has given the following results (Mörner):

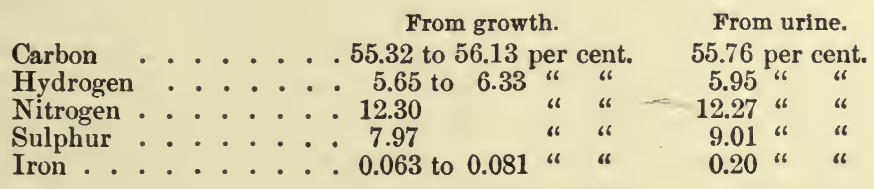

From melanotic growths in horses a hippomelanin has been obtained, which, in contradistinction to the first, is soluble in solutions of the alkalies with difficulty.

The Bile-pigments.-Bile-pigments are never found in the urine under normal conditions. As a rule, freshly voided urine contains only bilirubin. If a complicating cystitis, however, exists, the common derivatives of bilirubin, viz., biliverdin, bilifuscin, biliprasin, and bilihumin, may also be encountered.

Bile-containing urines present a very characteristic color, which may vary from a bright golden-yellow to a greenish brown, and on microscopical examination it is common to find the morphological elements stained an intense yellow. This color is further imparted to the foam on shaking. But as urobilin when present in large amounts may impart a similar color to the urine, it is always 
better to resort to ehemical tests. These have been described in detail in the section on the Bile, and are directly applicable also to the urine (see page 175).

Other pigments also may occur in the urine after the ingestion of various drugs, but as the products thus formed are of no special interest from the standpoint of animal chemistry, they are not considered at this place.

The Bile-acids.-The occurrence of bile-acids in the urine is solely a pathological phenomenon. In normal urines they are never found, and even in complete obstruction of the common duct their amount is quite small. To demonstrate their presence, they must first be isolated as Platner's bile, and can then be identified by polarimetric examination, their action upon the frog's heart, etc. (see page 163).

\section{Fats, Cholesterin, and Lecithins.}

Fats.-Traces of fat may be observed in the urine under normal conditions when excessive amounts have been ingested. During pregnancy also a lipuria has been noted, and is probably associated with the development of the function of lactation. Otherwise the condition is essentially a pathological phenomenon, and is notably observed in acute yellow atrophy, in phosphorus poisoning, following fracture of the long bones, etc. The largest amounts, however, are found in cases of so-called chyluria. Owing to the existence of the fats in fine emulsions, such urines may resemble milk in their general appearance, and on standing a layer of "cream" forms at the top.

To establish the presence of fats, the surface layer of the urine is extracted with ether, the ether is evaporated, and the residue brought in contact with a piece of paper, when characteristic stains result.

Cholesterin.-Cholesterin is very rarely found in the urine, and has thus far been encountered only under pathological conditions. It probably always occurs in crystalline form, and is thus readily recognized. If any doubt exists, the substance in question is examined as has been described (page 180).

Lecithins.-Lecithins as such have been observed in the urine only in chyluria, where they are commonly present in association with cholesterin and fat. One of the derivatives of lecithin, however, is found also in the urine under normal conditions, though in very small amounts. This is glycerin-phosphoric acid. It is no doubt referable to decomposition of the lecithins of the food in the intestinal canal, but may at times also be derived from the lecithins of the tissues. To demonstrate its presence, several liters of urine are freed from the common phosphates by rendering the urine alkaline with barium hydrate and precipitating the heated mixture 
with barium chloride. The excess of barium is removed with carbonic acid and the filtrate evaporated to a syrup. This is extracted with absolute alcohol, when the remaining material is dissolved in a little water and boiled with hydrochloric acid. The glycerin-phosphoric acid is thus decomposed, with the liberation of glycerin and phosphoric acid. On evaporating to dryness, the residue is extracted with water, and the presence of phosphoric acid demonstrated in the aqueous solution by the usual tests.

\section{Ferments.}

Every urine contains ferments which were formerly regarded as identical with the pepsin, ptyalin, and chymosin of the digestive fluids. It can be shown, as a matter of fact, that substances are present which are capable of digesting fibrin in acid solution, of inverting starch to maltose, and of coagulating milk. There is no proof, however, that these bodies are derived from the digestive glands, as Neumeister and others claim.

In certain diseases of the pancreas lipase has been found.

\section{Gases.}

In normal urine a certain amount of oxygen, nitrogen, and notably of carbon dioxide is found in solution, and can be withdrawn by the air-pump. Under pathological conditions, a variable amount of hydrogen sulphide may be encountered. This notably occurs in cases of cystitis, in which the decomposition of albumin and sulphur bodies may already take place within the bladder, owing to the activity of various micro-organisms. But in a few isolated cases the gas was apparently derived from the intestinal tract, and absorbed either directly from the rectum or indirectly from the blood.

All urines when exposed to the air sooner or later contain hydrogen sulphide in the free state, which is referable, as stated above, to the action of certain micro-organisms. Especially large amounts are observed when cystin-containing urines are thus allowed to undergo decomposition. To test for hydrogen sulphide, a strip of filter-paper is moistened with a few drops of a solution of sodium hydrate and one of lead acetate, and is then clamped in the neck of the bottle containing the urine. If the gas in question is present, the paper is colored a grayish brown or black, owing to the formation of lead sulphide. When present in large amounts it is detected also by its odor.

\section{Ptomains.}

So far as known, ptomains are not found in the urine under normal conditions. In disease, however, various basic substances have been encountered which supposedly belong to this class. But 
with the exception of eadaverin and putrescin, which, as has been stated, may occur in association with cystinuria, these bodies have been isolated in amounts scarcely sufficient to establish their chemical nature. This holds good more especially of the bodies which Griffith claims to have isolated from the urine of patients suffering from scarlatina, measles, mumps, carcinoma, etc., and which no one else has seen.

As regards the origin of putrescin and cadaverin in cystinuria, the opinion formerly prevailed that they are due to a specific form of intestinal putrefaction. This is, however, not likely, and there is good evidence to show that diaminuria, like cystinuria, is the expression of a distinct metabolic disturbance. I have pointed out that both diamins can be derived from arginin and lysin in the laboratory, and there is every reason to suppose that the same transformation can also occur in the living organism. That arginin actually occurs in the tissues of the body has been demonstrated by Gulewitch, who found the substance in the spleen.

The quantity of the diamins which may be eliminated in the urine in cases of cystinuria is quite variable. On some days traces only or none at all is found, while at other times very considerable amounts may be obtained, In one of my cases I was able to isolate 1.6 grammes of the benzoylated cadaverin from the collected urine of twenty-four hours.

To demonstrate the presence of diamins, the method of Baumann and v. Udranszky has been almost exclusively employed. To this end, the collected urine of twenty-four hours or more is benzoylated by shaking with benzoyl chloride in the presence of sodium hydrate. As a general rule, 25 c.c. of the chloride and 200 c.c. of a 10 per cent. solution of sodium hydrate are used for 1500 c.c. of the urine. The resulting precipitate, which contains the earthy phosphates, the benzoylated carbohydrates which are normally present in every urine, and the greater portion of the benzoylated diamins, is then filtered off, extracted with boiling alcohol, filtered, and the alcoholic extract concentrated on a water-bath. This solution is then poured into thirty times its volume of water. On standing, the benzoylated diamins separate out in crystalline form, and are then freed from adhering carbohydrates by repeated solution in alcohol and precipitation in water. The process is continued until the desired degree of purity is obtained. The resulting crystals are finally filtered off, dried over sulphuric acid, and identified by their melting-point and the contained amount of nitrogen. If both diamins are present, the crystals lose their water of crystallization at $120^{\circ} \mathrm{C}$, and melt at $140^{\circ} \mathrm{C}$. To separate them from each other, they are dissolved in a little warm alcohol, and are treated with twenty times as much ether. Benzoyl putrescin is thus thrown down, while the cadaverin compound remains in solution. The crystals of the former melt at $175^{\circ}-176^{\circ} \mathrm{C}$., while the melting-point of the latter lies between $129^{\circ}$ and $130^{\circ} \mathrm{C}$. 
A small portion of the diamins remains in the first filtrate. To isolate these, the liquid is acidified with sulphuric acid and extracted with ether. The ethereal extract is evaporated, and the final solution, before congealing, placed in as much of a 12 per cent. solution of sodium hydrate as is required for its neutralization. From three to four times as much of the alkali solution is then added. On standing in the cold, sodium benzoyl-cystin separates out, together with the benzoylated diamins. The crystals are filtered off and placed in cold water. This dissolves the cystin compound, while the diamins remain undissolved. They are soluble in warm alcohol, and can then be separated from each other, as has been described.

More recently, Löwy and Neuberg have advised another method which is based upon the isolation of diamins as phenyl cyanates, and which has the advantage that phenyl isocyanate in aqueous solution does not unite with carbohydrates, and that the cystin derivative does not separate out if the reaction is kept alkaline. For a detailed account the reader is referred to Löwy and Neuberg's paper (Zeitschr. f. physiol. Chem., 1904, vol. 43, p. 338). 


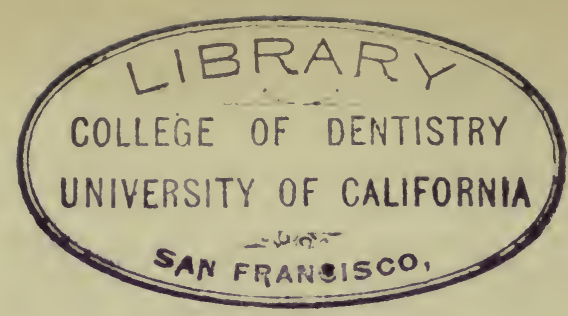

CHAPTER XIII.

\section{THE ANIMAL CELL.}

THE cell constitutes the morphological unit of all animal and vegetable life, and as such is capable of manifesting those peculiar activities which we regard as characteristic of living matter. In its simplest form it represents a tiny bit of a more or less granular, gelatinous substance-the so-called protoplasm-in the interior of which a somewhat more solid-looking body can be made out, which is termed the nucleus. Such simple cells exist in nature, either as such or as conglomerations of many cells which represent the higher forms of animal and vegetable life. All living matter, however, whether simple or complex, has for its origin the single cell. But while in the lowest forms of life the single cell is capable of performing all those functions which are characteristic of living matter by itself, we find, as we ascend in the scale of animal and vegetable life, that certain groups of cells are here set aside for the purpose of executing separate functions. Such groups of cells we speak of as tissues, and we accordingly find in the highly organized mammal a differentiation of the entire body into tissues, which according to their functions may be grouped as tissues of locomotion, of reproduction, of digestion, of excretion, etc. With such a differentiation of cells into tissues, however, the original aspect of the cell is more or less changed. The highly differentiated voluntary musclecell would thus at first sight scarcely be recognized as being in any way related to the oval cell from which it is primarily derived. On careful examination, however, we find that, no matter how unlike its ancestral cell such a specialized cell may appear, the difference is only apparent. The striated portion of the muscle-cell is thus nothing more than the protoplasm of the original cell, differentiated and modified in accordance with the function which the cell is to perform. In some cells, however, such as those of the adipose tissue, the original differentiation into protoplasm and nucleus is apparently lost, and on ordinary microscopical examination it appears that such cells are nothing but large globules of fat. But with special methods of staining we can demonstrate even here that there are a nucleus and protoplasm. The only cells, in fact, in which a nucleus cannot always be demonstrated are the red corpuseles of the circulating blood of the mammalia. We find, however, that even in adult man all red corpuscles at one period of their existence, viz., in their juvenile form, are nucleated, and that under certain condi- 
tions, as after copious hemorrhages, such nucleated corpuscles may occur in the circulating blood in large numbers. In the bonemarrow, where they are apparently formed, they are always present.

As all manifestations of life are intimately associated with chemical changes which bring about a transformation of potential into kinetic energy, such changes must of necessity occur in every living cell. These changes, moreover, must vary with the function which the cell is to perform, and will hence differ, to a certain extent at least, with the different tissues of the complex organism. In the monocellular organisms, where all the various functions are performed by the single cell, all these varying changes must hence be represented. But it is to be inferred that in accordance with the greater simplicity in structure the chemical changes also must be of a simpler character. We should hence expect that a study of the chemical processes which take place in such low forms of life would furnish us with a better insight into the metabolism of the complex organism than could be attained from an investigation of the higher forms. Unfortunately, however, this is almost an impossibility with the usual chemical and physical methods, for in attempting such a study we are met with a most serious obstacle, viz., our inability to maintain the life of the individual cell during such an investigation. We would consequently have no proof that those products which we could isolate from the dead cells were present as such in the living organism. The technical difficulties, moreover, which stand in the way of such a study are almost insurmountable. With microchemical methods, it is true, something more definite might be accomplished, and although this branch of investigation is still in its infancy, it has already furnished us with a certain amount of valuable information. The great advances which have thus been made in our knowledge of the structure of cells have largely been accomplished in this manner. Upon the chemical processes themselves, however, which take place in the cell, not much light has as yet been thrown in this manner. We are consequently dependent for our knowledge of the metabolic processes which take place in the living body upon a study of the individual tissues as such, and the changes which result in certain substances when introduced from without. With some tissues this is more difficult than with others. The most satisfactory results, on the whole, regarding the chemical structure of the individual cell, have thus far been obtained from an investigation of those organs which are especially rich in cells, and in which the cells can be more or less completely separated from the underlying matrix and from other components which may be present at the same time. This is especially true of the leucocytes of the blood. As these bodies, moreover, are but little differentiated, they may well serve as types of primitive cells. They are all nucleated, and contain a varying amount of protoplasm, which in some is capable of progressive movement. A limiting membrane, 
as in most animal cells, does not exist. But it is generally supposed that a meshwork of fine fibrils pervades the protoplasm, and that in the meshes a more liquid portion is contained. This is termed the hyaloplasm, in contradistinction to the more solid spongioplasm. In some forms the protoplasm is apparently perfectly homogeneous, while in others it is studded with numerous granules of variable size, which execute more or less active oscillatory movements, which are spoken of as the molecular movements of Brown.

The reaction of the protoplasm is alkaline, while the nucleus apparently contains no free alkali. This may be shown by staining dried blood films with a solution of acid erythrosin in chloroform, when it will be seen that the body of the cell is colored a bright red, while the nucleus is not stained. The most intense reaction is obtained with the protoplasm of the so-called lymphocytes.

The granules which are found in certain forms of leucocytes are apparently of an albuminous nature. According to their affinity for acid, basic, or neutral dyes, they are termed oxyphilic, basophilic, and neutrophilic, respectively. Fatty, mineral, or pigment granules, which may be found in other animal cells, are usually not seen in leucocytes. In the eosinophilic leucocytes, however, the presence of iron can readily be demonstrated by microchemical methods. In another form it seems to be present in all varieties of cells, and is especially abundant in the nuclei.

In the mineral ash we find potassium, sodium, calcium, magnesium, phosphorus, and chlorine, and it is to be noted that, in contradistinction to the animal fluids, the cell contains a relatively larger amount of potassium and phosphorus, while sodium and chlorine are more abundant in the fluids. That the phosphates are of prime importance in the life of the cells is now definitely established, and Loew showed that in the spirogyra, for example, growth and cellular division are greatly interfered with by their absence. The importance of the phosphates is without doubt connected with the presence of the nucleins in the nuclei-i. e., of albuminous substances-which, as we have seen, contain a relatively large amount of phosphorus in organic combination.

The protoplasm of the cell is very rich in water, and, in addition to small amounts of mineral salts, consists essentially of albumins. Some of these are albumins proper, but the greater portion is represented by substances which belong to the nucleoproteid group. It appears, moreover, that the traces of serum-albumin and globulin which are present do not represent integral constituents of the living protoplasm, but are merely to be regarded as food-stuffs, or possibly even as decomposition-products of the protoplasmic molecule.

Of other. constituents of the protoplasm, lecithin is the most constant. In addition, we find protagon, glycogen, cholesterin, and in dead cells also paralactic acid, to which the acid reaction of dead protoplasm is due. 
The total amount of solids, including the mineral salts, which are thus found in protoplasm, is always small, and probably never exceeds $15-20$ per cent., while the remaining weight is referable to water. In some cells almost the entire body is taken up by the nucleus, and in the lymphocytes, for example, only 1.76 per cent. of the total 79.21 per cent. of albumins, as calculated for the dry material, is present in the protoplasm.

The nucleus may be regarded as the essential living part of all animal and vegetable cells, and from it, no doubt, the various functions of the cell as a whole are directed. It is intimately connected with the process of reproduction, and during this process it undergoes a series of most remarkable changes, which are collectively termed the karyokinesis or karyomitosis of the nucleus. Microscopically the quiescent nucleus represents a round or oval little body, which usually occupies an excentric position within the cell. It is surrounded by a nuclear membrane, and contains a meshwork of extremely fine fibrils, and one or more nucleoli. Both fibrils and nucleoli possess a marked affinity for anilin dyes, while the nuclear membrane and the more liquid hyaloplasm within the nuclear meshes are scarcely stained at all. We therefore recognize in the nucleus the existence of chromatic and achromatic substances, which are usually spoken of as the nuclear chromatins and achromatins. During the process of division a peculiar spindle-like body is also observed in the nucleus, which, like the nuclear hyaloplasm, is achromatic.

In contradistinction to the cellular protoplasm, the nucleus contains a much larger quantity of solids, but here as there the albumins stand in the foreground. Whether or not native albumins also occur in the nucleus is not definitely known, but it is generally assumed that this is not the case. The proteids, on the other hand, are abundant and largely represented by nucleoproteids. They are thought to constitute the greater portion of the chromatic constituents of the nucleus. Especially abundant is a nucleoproteid, which Kossel and Lilienfeld first obtained from the thymus gland of the calf. This they termed nucleohiston, from the fact that on treatment with hydrochloric acid it is supposedly decomposed into a nuclein-leuconuclein and histon. Bang, however, denies that this nucleohiston is a true histon. He regards it as an albuminate, and states that he could not demonstrate a nucleinate of histon in the leucocytes.

Whether lecithins, protagon, and glycogen, which are constantly found in the cellular protoplasm, likewise occur in the nucleus, is not known.

Of mineral constituents, iron is constantly present, and apparently occurs in combination with the nucleins in organic form.

Justus has further shown that every nucleus normally contains iodine. 


\section{CHAPTER XIV.}

\section{THE BLOOD.}

General Considerations.-The blood of man and of almost all vertebrate animals is a slightly viscid, somewhat opaque-looking fluid, which, according to its origin from an artery or a vein, presents a color that varies from a bright scarlet to a dark bluish-red. On microscopical examination it is seen to contain a large number of cellular elements, which are partly colored and partly colorless. The former, which greatly predominate, are the red corpuscles, or erythrocytes of the blood. In man they are homogeneous, normally non-nucleated, circular disks, measuring on an average $7.5 \mu$ in liameter, which were usually regarded as biconcave, but are by some also described as bell shaped. When viewed through the microscope they are of a faint greenish-yellow color, while en masse they present the ordinary color of the blood. The colorless bodies, or leucocytes, on the other hand, are all nucleated and in part capable of executing amoboid movements. Some of them are of about the same size as the red corpuscles, while others are larger. The nucleus may be single or multiple, and it will be noted that in the mononuclear forms the surrounding protoplasm is more or less homogeneous, while in the polynuclear varieties it is distinctly granular. The total number of the leucocytes per cubic millimeter varies under normal conditions between 3000 and 10,000, and is thus much smaller than the number of the red corpuscles, which is generally placed at between 4,000,000 and 5,000,000 in the same volume of blood.

In addition to the red corpuscles and the leucocytes, we further find a large number of minute colorless disks, measuring less than one-half the diameter of a red corpuscle, and usually occurring in bunches of from six to a dozen or more. They are termed the plaques or blood-plates of Bizzozero. On an average, about 635,000 are found in the cbmm. Other morphological elements are not found in the blood under normal conditions, while in disease nucleated red corpuscles, both of the adult and the embryonic type, as well as other forms of leucocytes, may be encountered.

When blood is drawn from the living body and is allowed to stand, it will be noted that after a variable length of time the entire mass is transformed into a semisolid, jelly-like material, which is termed the placenta sanguinis or blood-clot. On microscopical examination this will be seen to consist of a dense network of fibres, in the meshes of which the corpuscles of the blood are found. If the clot is now carefully separated from the walls of the vessel. it 
undergoes shrinkage, and presses out from its meshes a clear, strawcolored fluid, which is termed the blood-serum. This gradually increases in amount, while the size of the clot diminishes. The fibrous material which is formed during the process of clotting is termed fibrin. Its formation is intimately associated with the death of the organism, either local or general, and is dependent in the first instance upon the presence of a specific ferment-the so-called fibrin ferment, or thrombin of Alexander Schmidt. In the circulating blood fibrin is not found, but we here meet with its mother-substance, fibrinogen, which is not present in the blood-serum. The bloodplasma, viz., the fluid, non-cellular portion of the circulating blood, thus differs from the blood-serum in containing fibrinogenic material, but not the fibrin ferment, which is found in the serum. The ferment itself results from decomposition of the cellular elements of the blood, notably of the blood-plates. This may be seen when the process of coagulation is observed through a microscope. After a variable length of time, more rapidly when the blood-drop is not too small and when the surface of the glass is a little uneven, fine filaments of fibrin thus begin to appear, which usually have for their starting-point those bunch-like conglomerations of the plaques which have already been describer. In these bodies the pro-enzyme of the ferment, the so-called prothrombin of Alexander Schmidt, is probably contained. It should be stated, however, that the fibrin ferment is not only derived from the plaques, but may also be formed during the decomposition of the remaining cellular elements of the blood, and, to judge from recent observations, from protoplasmic material in general.

\section{PHYSICAL CHARACTERISTICS OF THE BLOOD.}

Color.-The color of normal blood is referable to the presence of a peculiar albuminous substance in the red corpuscles belonging to the class of proteids which is termed hæmoglobin. In arterial blood this is principally found in combination with oxygen as oxyhæmoglobin, while in venous blood a mixture of both occurs. With a preponderance of oxyhæmoglobin over hæmoglobin the color of the blood tends toward a bright scarlet-red. In its absence it assumes a dark-bluish color, and we accordingly find all gradations in shade between the two. When venous blood is exposed to the air the hæmoglobin immediately absorbs oxygen, and is transformed into oxyhæmoglobin. This actually takes place in the alveoli of the lungs, and explains the difference in color between the blood of the right and the left heart.

Under pathological conditions we may find still other colors than those which have been described. In coal-gas poisoning the blood is thus of a bright cherry-red; in poisoning with potassium chlorate, anilin, hydrocyanic acid, nitrobenzol, etc., it is of a brownish-red or a chocolate color. 'These changes are, as we shall presently see, due 
to certain chemical compounds of hæmoglobin which are not normally found in the blood. In leukæmia, in which a most remarkable increase in the leucocytes may occur, the blood at times presents a milky appearance. This is not referable to any change of the normal coloring-matter, however, but to increase of the leucocytes as such.

The Odor.-The odor of the blood is characteristic, but is different in different species of animals. It can be intensified by treating the blood with a small amount of fairly concentrated sulphuric acid. In part it is owing to the presence of odorous salts of certain fatty acids, and to a slight degree also to trimethylamin. Other substances, however, are also concerned in its production, but of their nature nothing is known.

The taste of blood is salty and insipid.

The Specific Gravity.-The specific gravity of normal blood seems to vary with the amount of hæmoglobin. It is influenced by the age and sex of the individual, the process of digestion, the amount of exercise taken, pregnancy, etc. It is dependent, moreover, upon the bloodvessel from which it is drawn, and differs somewhat in different animals. Generally speaking, it varies in healthy adults between 1.058 and 1.062. It is higher, as a rule, in men (1.059) than in women (1.065) and in children (1.050-1.052). Under pathological conditions the variations are much greater. It may thus fall to 1.025 and rise to 1.068 . It is to be noted, moreover, that the specific gravity does not necessarily vary with the amount of hæmoglobin, and in nephritis, various circulatory disturbances, in leukæmia, and in the anæmias following profuse hemorrhages or inanition, eare should be had not to draw inferences as to the amount of blood coloring-matter from a determination of the specific gravity.

Determination of the Specific Gravity.-Hammerschlag's Method.-A cylinder measuring about $10 \mathrm{~cm}$. in height is partly filled with a mixture of benzol (sp. gr. 0.889) and chloroform (sp. gr. 1.526), so that the specific gravity lies between 1.050 and 1.060. Into this solution a drop of blood is allowed to fall directly from the finger, care being taken that it does not come in contact with the walls of the vessel. The drop, moreover, should not be too large, as it will otherwise separate into several droplets, and thus give rise to inaccurate results. It is then brought to suspension in the middle of the fluid, by adding a little chloroform or ether, according to its tendency to sink to the bottom or to rise to the surface. As soon as it remains in the middle the mixture is filtered through a layer of linen, and its specific gravity determined by means of an accurate hydrometer, which is graduated to the fourth decimal. The figure obtained represents the specific gravity of the blood.

Spanje recommends a mixture of 3 parts of chloroform and 1 of olive oil, which has a specific gravity of 1.056 . 
The Amount.-The total amount of blood in the body of man corresponds to $\frac{1}{16}$ to $\frac{1}{30}$ of the body weight, in dogs to $7-9$ per cent., and in rabbits to 5-9 per cent. of the weight. It can be approximately determined according to the following method:

Method of Welcker.-From the animal to be investigated 10 to 30 c.c. of blood are first withdrawn and carefully defibrinated by whipping. This amount is weighed together with the fibrin and set aside. The animal is then bled to death and the blood defibrinated as before. After removal of the feces, the intestinal contents, and the gall-bladder, the entire body is finely minced and repeatedly extracted with water; the washings are added to the large mass of blood. The total volume is now ascertained and the eolor of the bloody fluid compared with that of the first 10 to 30 c.c., by diluting this portion with water until the color of both portions is the same. From the degree of dilution, the amount of blood which is present in the larger volume of fluid can then be determined.

\section{CHEMICAL EXAMINATION OF THE BLOOD.}

Reaction.-The reaction of the blood, owing to the presence of sodium carbonate and disodium phosphate, is slightly alkaline. This may be demonstrated by repeatedly drawing a strip of neutral litmus-paper thoroughly moistened with a concentrated solution of common salt through the blood, and rapidly washing off the corpuscles with the same solution. In man the degree of alkalinity under normal conditions corresponds to from 300 to 325 milligrammes of sodium hydrate for every 100 c.c. of blood. These figures were obtained with Löwy's method (see below), and are higher than those usually given in text-books, but probably more nearly correct.

Owing to the formation of certain acids the alkalinity of the blood rapidly diminishes after being shed, and for this reason its determination is a somewhat difficult matter. Generally speaking, it is a little lower in women and children than in men, and is influenced to a certain degree by the process of digestion, the amount of exercise taken, etc. At the beginning of digestion, when hydrochloric acid is being secreted in large amounts, it is thus increased, while later on, when resorption is actively going on, it is diminished. On the whole, however, these normal variations are slight. Greater deviations have been observed under pathological conditions, and are especially noted in leukæmia, pernicious anæmia, nephritis, and diabetes when accompanied by coma, in connection with high fever, during the algid state of Asiatic cholera, etc. It is interesting to note, however, that according to v. Limbeck, these observations may be referable to faulty technique, as with his method (see below) no difference could be shown to exist between normal and pathological conditions. It is conceivable, of course, that in disease the 
alkalinity of the blood may diminish more rapidly after being shed than under normal conditions, and this may account for the different results which have been reached with other methods. But, on the other hand, v. Limbeck's method may likewise not be free from error.

The tenacity with which the living organism tends to maintain the normal composition of the bodily fluid is, of course, well known, but that it is not always able to do so is also an established fact. Herbivorous animals thus rapidly die when given large amounts of mineral acids, and it may be shown that the alkalinity of their blood is then markedly diminished. In cases of poisoning with strychnin, arsenious acid, carbon monoxide, and amyl nitrite, moreover, where a marked albuminous decomposition occurs and lactic acid appears in the urine, the same result is obtained. Carnivorous animals, on the other hand, are more resistant in this respect, and will stand much larger amounts. The result, however, is the same. In diabetic coma, owing to the presence of large amounts of oxybutyric acid, the alkalinity may not only be much diminished, but the blood may actually show an acid reaction.

Löwy's Method.-Five c.c. of blood, obtained from one of the superficial veins of the arm, are allowed to flow into a small flask, which is provided with a long and partially graduated neck, and contains 45 c.c. of a 0.25 per cent. solution of ammonium oxalate. Coagulation is thus prevented, and the blood made lake-color-i. e., the hæmoglobin is dissolved from the stroma of the red corpuseles. The mixture is then titrated with a one-twenty-fifth normal solution of tartaric acid, using as an indicator lacmoid paper which has been soaked in a concentrated solution of magnesium sulphate. The number of cubic centimeters employed to neutralize the 5 c.c. of blood, multiplied by 0.0016 , will then indicate the degree of alkalinity in terms of sodium hydrate. The percentage is obtained by multiplying the resulting figure by 20 .

v. Limbeck's Method.-Ten c.c. of blood are allowed to flow into 200 c.c. of boiling water, to which 5 c.c. of a one-tenth normal solution of hydrochloric acid have been added. The resulting solution, which is clear and of a brownish color, is now retitrated with a one-tenth normal solution of sodium hydrate, using as indicator the syntonin precipitate which occurs on neutralization. The difference between the 10 c.c. of the hydrochloric acid and the sodium hydrate solution is multiplied by 0.004 . The result indicates the alkalinity of the 5 c.c. of blood, and to obtain the percentage this is multiplied by 20 .

Dare's Method.- This method is based upon the fact that the characteristic spectrum of oxyhæmoglobin disappears at the point of exact neutralization when the blood is titrated with a dilute solution of tartaric acid.

The examination is made with the aid of a special instrument, the hcemoalkalimeter. 
To neutralize the blood, a $\frac{1}{200}$ normal solution of tartaric acid is used, which should contain an amount of alcohol sufficient to prevent the growth of bacteria, but insufficient to precipitate the albumins of the blood. The reagent may be prepared by dissolving 0.075 gramme of tartaric acid (Merck's crystals ; guaranteed reagent) in a small amount of distilled water, adding 20 c.c. of alcohol (93-94 per cent.), and diluting to 200 c.c. with water.

For the spectroscopic examination a Browning instrument will suffice. A detailed description accompanies the instrument.

\section{The Chemical Composition of the Blood as a Whole.}

As the blood constitutes the most important channel through which the food-stuffs reach the various tissues of the body, and through which waste matter is carried away, we may expect to find here representatives of both classes of substances. This is actually the case; but as the waste matter is rapidly eliminated, representatives of this group are normally present in only very small amounts. Certain food-stuffs, moreover, which are not immediately required by the body in large quantities, such as fats and carbohydrates, are likewise present only in traces. They are stored in various tissues of the body until needed, but even then only small quantities appear in the blood at one time. The only foodstuffs, in fact, which are always present in the blood in large quantities, are the albumins. These, however, must be sharply separated into two classes, viz., into those which are normally present as integral constituents of the cellular elements of the blood, and into the circulating albumins of the plasma. In addition to these elements, we also meet with mineral salts, a very large amount of water, and with certain gases.

A general idea of the chemical composition of human blood may be had from the following table, which is calculated for 1000 parts by weight:

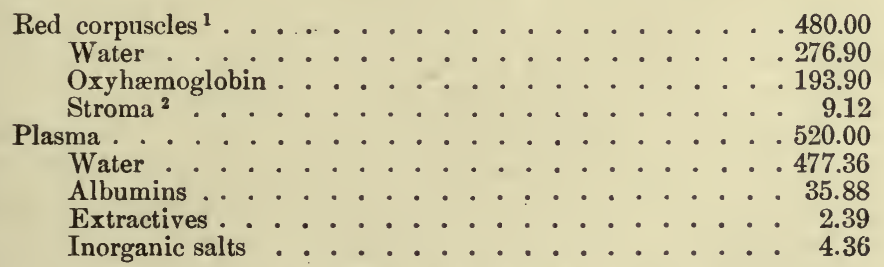

From this analysis it will be seen that almost one-half of the total weight of the blood is referable to cellular elements, and that in the liquid portion proper there is not more than 8.2 per cent. of solids, of which 6.9 per cent. is represented by albumins, 0.84 per cent. by mineral salts, and 0.46 per cent. by extractives.

1 The white corpuscles, because insignificant in amount, have been ignored.

2 This includes mineral salts. 
The predominating solid substance in the blood is the oxyhæmoglobin; it represents about 19 per cent. of the total weight of the blood, 40 per cent. of the weight of the blood-corpuscles, and 95 per cent. of all organic material present.

The native albumins, which are found in the circulating blood, are serum-albumin, serum-globulin, and fibrinogen. The extractives comprise traces of fats, soaps of the higher fatty acids, lecithin, glucose, animal gum, glycogen, sarcolactic acid, urea, kreatin, uric acid, and possibly also minimal amounts of the xanthin bases. Nucleo-albumins, albumoses, and some of the lower fatty acids, oxybutyric acid, acetone, bilirubin, melanin, and other less well-known bodies have further been found under pathological conditions, but are not seen in normal blood.

The mineral constituents comprise sodium, potassium, calcium, magnesium, and iron. With the exception of the last mentioned, they are present as chlorides, phosphates, carbonates, and to a slight extent also as fluorides. Some of these occur in the blood as such, while others form more or less intimate combinations with the albumins. The iron largely occurs as an integral constituent of the hæmoglobin molecule, of which it forms from 0.39 to 0.47 per cent. Traces are also present in certain leucocytes, and notably those of the oxyphilic variety. In the plasma itself it is at times met with in infinitesimally small amounts, and is then referable to the destruction of leucocytes.

In addition to these constituents of the normal blood, we further meet with certain gases, viz., oxygen, carbon dioxide, and nitrogen. Of these, oxygen and carbon dioxide occur partly in solution and partly in combination with hæmoglobin, while nitrogen is found only in solution. The carbon dioxide, moreover, is in part present as a soluble bicarbonate, and to a certain extent also in combination with the albumins of the plasma. These gases may be extracted from the blood in their entirety by exposure to a vacuum. As the nitrogen is simply held in solution, its volume is constant, and corresponds to 2 per cent. by volume no matter whether the blood is obtained from an artery or a vein. The relative amount of oxygen and carbon dioxide, on the other hand, is subject to great variations. From the arterial blood of dogs it is thus possible to obtain 21 per cent. of oxygen by volume, and 38 per cent. of carbon dioxide, while venous blood contains as much as 46 per cent. of carbon dioxide and only 12 per cent. of oxygen.

As these gases can be obtained by exposure to a vacuum, it follows that their combination with oxyhæmoglobin cannot be very strong; it is surprising, however, to note that in this manner not only that portion of the carbon dioxide is obtained which is in combination with albuminous material, but also the carbon dioxide of the carbonates. This phenomenon is owing to the fact that in consequence of the vacuum the red corpuscles are broken down, and that the hæmoglobin which is thus set free is then capable of exercising its acid properties, and causes decomposition of the salts. 


\section{The Plasma.}

In order to obtain blood-plasma it is necessary to prevent coagulation of the blood. This may be accomplished in various ways. It has thus been found that following the intravenous injection of certain albumoses (peptones), or of an infusion of the mouth parts of the officinal leech (hirudin), as also after ligation of the bloodvessels of the liver and intestines, the blood remains liquid after being shed. On allowing it to stand at a low temperature the bloodcorpuscles settle to the bottom, when the supernatant fluid may be siphoned off; or the blood may be centrifugalized at once and separation of the cellular elements effected in this way. Bloodplasma that has been obtained after the injection of albumoses is termed albumose-plasma, or, less correctly, peptone-plasma, in contradistinction to salt-plasma, which results when blood is received in a solution of a neutral salt, whereby coagulation is also prevented. To this end it is best to employ a saturated solution of sodium sulphate or a 10 per cent. solution of sodium chloride, to which the blood is added in an equal amount. A saturated solution of magnesium sulphate may likewise be used, in the proportion of one part for three parts of blood, but is not so satisfactory, as it causes precipitation of certain albumins which are essential to coagulation. After standing for twenty-four hours the plasma may be siphoned off, or may be separated from the corpuscles at once by centrifugation.

As coagulation of the blood is dependent upon the presence of soluble calcium salts, coagulation may also be prevented by the addition of ammonium oxalate ( 0.1 per cent.) or sodium citrate (1.0 per cent.). Plasma obtained in this manner is spoken of as oxalate or citrate plasma.

Of especial value in these examinations is the blood of the horse, in which coagulation occurs much more slowly than in that of mammals. If this is available, it is only necessary to receive it in a narrow cylinder surrounded with a freezing-mixture. Kept in this manner it will remain liquid for several days.

Separated from the corpuscles, the plasma is a clear, straw-colored, slightly viscid fluid, of alkaline reaction, and a specific gravity varying between 1.026 and 1.029 in man. It is capable of undergoing coagulation, like the native blood, and is thus converted into blood-serum. Its general chemical composition has already been considered. It contains but 8.2 per cent. of solids, of which 6.9 per cent. is represented by albumins. These are serum-albumin, serum-globulin, and fibrinogen. The relation between these bodies is subject to cousiderable variations. In all animals, however, the globulins predominate, and in some indeed, as in snakes, serumalbumin is apparently absent. In the horse the globulins constitute about 64.6 per cent. of the total amount of albumins. In 1000 parts by weight Hammarsten thus found 38.4 parts of serumglobulin, 6.5 parts of fibrinogen, and 24.6 parts of serum-albumin. 
Of these albumins, fibrinogen is of especial interest, as it represents the mother-substance of fibrin, and is thus intimately connected with the process of coagulation.

Fibrinogen.-Isolation.-Fibrinogen is most conveniently obtained from the plasma by half-saturation with sodium chloride$i$. e., by treating one volume of the plasma with an equal volume of a saturated solution of common salt. The resulting precipitate of fibrinogen is filtered off, washed with a half-saturated solution of sodium chloride, and dissolved in an 8 per cent. solution of the salt. To further purify the substance, this solution is reprecipitated, redissolved, and the process repeated twice. The final precipitate is pressed between filter-paper and suspended in water, in which it readily dissolves owing to the small amount of salt that still remains. This may be removed by dialysis. The purified substance, in a moist state, appears in the form of white floceuli, which readily coalesce to form a tough elastic mass.

The isolation of the fibrinogen must be performed rapidly, as prolonged exposure to the half-saturated salt solution tends to render the substance insoluble.

Properties.-Fibrinogen belongs to the class of globulins. It is insoluble in distilled water, but soluble in dilute solutions of the neutral salts. From these solutions it may be precipitated by dialysis, by increasing the amount of the salt, and by passing a stream of carbon dioxide through the solution. When kept under water for a comparatively short time it is rendered insoluble. When heated to $56^{\circ}$ C. coagulation occurs, but it appears that the fibrinogen is at the same time decomposed into two other globulins, one of which coagulates at the temperature just mentioned, while the other remains in solution until the temperature reaches $65^{\circ} \mathrm{C}$. The latter has been termed fibrinoglobulin and, according to Hammarsten, is also formed during ferment coagulation. This, however, is apparently not necessarily the case. Fibrinogen turns the plane of polarized light to the left; its rotation for the yellow D line corresponds to - 52.5 degrees. It consists of carbon, hydrogen, nitrogen, sulphur, and oxygen, in the proportion of $52.93,6.9,16.6,1.25$; and 22.26 respectively. Its most characteristic property is its tendency to undergo coagulation under the action of a specific ferment, and upon this its specific test and quantitative estimation are based. This transformation will be considered in detail later (see Coagulation).

In addition to the blood-plasma, fibrinogen has been found in the chyle, the lymph, and various exudates and transudates.

Through the researches of Doyon, Morel, Kareff, and Nolf it has been rendered probable that fibrinogen is formed in the liver, as its quantity in the blood rapidly diminishes after extirpation of the organ, or when fatty degeneration has been caused by the administration of phosphorus.

Serum-globulin.-On half-saturation of the blood-plasma with 
ammonium sulphate, or on complete saturation with magnesium sulphate, an albuminous precipitate is obtained, which was formerly regarded as a unity and termed serum-globulin (sive paraglobulin, serum-casein, Schmidt's fibrinoplastic substance). Later researches have shown that this consists of several distinct bodies, and for this reason it would be better to discard the term serum-globulin as such, and to speak of a serum-globulin fraction. The most notable components of this fraction are euglobulin and pseudoglobulin. The former is precipitated in the presence of 28-34 per cent. of ammonium sulphate, while the latter is thrown down with 36-44 per cent. These two fractions further differ from each other in the behavior of their saline solutions on dialysis. The euglobulin is thus thrown down while the pseudoglobulin remains in solution.

Fuld and Spiro have shown that the coagulating effect which blood produces on milk is referable to the euglobulin fraction, while the anti-action is a property of the pseudoglobulin. Pick has demonstrated still a further difference; in horses immunized to diphtheria the antitoxic properties of the serum are connected with the pseudoglobulin while the other fraction is inert. In the case of the opsonins I have shown that they are associated with the euglobulin fraction.

As the two fractions in their general properties are otherwise apparently identical, it has been suggested that the difference in their behavior to ammonium sulphate and on dialysis, etc., might possibly be due to a third factor. Mörner thus thinks that contamination of the globulin precipitate by soaps, fatty acids, lecithins, ete., is the determining factor in this respect. The majority of physiological chemists, inclusive of Hammarsten, who has been especially active in this field, however, incline to the view that serum-globulin represents a mixture of at least two or more bodies.

Freund and Joachim have further pointed out that from both euglobulin and pseudoglobulin they conld obtain one fraction which was preeipitated on dialysis and a second one which remained in solution. The water-insoluble portion of each fraction they designate as para-euglobulin and para-pseudoglobulin respeetively, while the terms euglobulin and pseudoglobulin are retained for the watersoluble portion of each fraction.

Serum-globulin, using the term in the older sense of the word, is found not only in the plasma of the blood, but also in the lymph, in various exudates and transudates, in the serum, in the white and red corpuscles of the blood, and in traces at least in all the cellular elements of the body (cell-globulins). In the urine serum-globulin usually accompanies serum-albumin.

Properties. - As a class the serum-globulins represent a snowywhite, finely flocculent material, which is not tough and elastic like fibrinogen. They have not been obtained in crystalline form. In water the euglobulins are practically insoluble, while the pseudoglobulins will dissolve. The insolubility in water was formerly 
regarded as one of the characteristic features of the globulins. In dilute saline solution they are all soluble with comparative readiness, but on standing the substance gradually becomes insoluble.

From their solutions the globulins are precipitated by half-saturation with ammonium sulphate, or by complete saturation with magnesium sulphate. Sodium chloride causes only an incomplete separation when added to saturation, and with half-saturation no effect at all is produced. It is thus an easy matter to isolate serum-globulin, even though fibrinogen be present at the same time. An incomplete precipitation occurs when its neutral or feebly acid solutions (using acetic acid) are diluted from 10 to 20 times with distilled water, or on passing a stream of carbon dioxide through such dilute solutions. In the presence of 5 to 10 per cent. of sodium chloride the globulin fraction coagulates at $75^{\circ} \mathrm{C}$.

Elementary analysis of the entire fraction, as may be expected, has not given rise to constant results. An analysis by Hammarsten gave the following figures: $\mathrm{C}=52.71 ; \mathrm{H}=7.01 ; \mathrm{N}=15.85$; $\mathrm{S}=1.11 ; \mathrm{O}=23.32$.

Isolation.-Serum-globulin is most conveniently obtained from blood-serum by half-saturation with ammonium sulphate-i. $e$, by treating a given volume of the serum with the same amount of a saturated solution of the salt. Saturation with magnesium sulphate in substance may also be employed. In either case the precipitated serum-globulin is filtered off, washed with the corresponding salt solution, dried at $115^{\circ} \mathrm{C}$., then washed with boiling water to remove the remaining salts, extracted with alcohol, then with ether, and finally dried and weighed. In any case the original solution should be nearly neutral in reaction.

Serum-albumin.-Serum-albumin is found in the plasma, the serum, the lymph, in exudates and transudates, and under certain pathological conditions also in the urine, where it usually occurs in association with serum-globulin.

In the dry state serum-albumin is a transparent, gum-like, brittle, hygroscopic mass, or a white powder, which can be heated to $100^{\circ} \mathrm{C}$. without undergoing decomposition. Solutions of the pure substance in distilled water coagulate at $50^{\circ} \mathrm{C}$, while in the presence of salts a higher temperature is necessary. This varies with the amount of salt present, as also with the concentration of the albumin. A 1 to 2 per cent. solution containing 5 per cent. of sodium chloride coagulates between $75^{\circ}$ and $90^{\circ} \mathrm{C}$. From its salt solutions serumalbumin may be obtained in crystalline form. Its specific rotation in distilled water varies between $62.6^{\circ}$ and $64.6^{\circ}$.

One of the most remarkable properties of serum-albumin is its pronounced tendency to form a sulphate. It is manifestly capable of abstracting sulphuric acid (not sulphates) from the sulphates used in its isolation, and this sulphur cannot be removed by washing with water (Mörner).

According to Halliburton, the serum-albumin of mammalian 
blood-serum is not a single substance, but consists of three distinct albumins, which he terms $\alpha_{-}, \beta_{-}$, and $\gamma_{\text {-serum-albumin. They are }}$ said to coagulate at $73^{\circ} \mathrm{C}$., $77^{\circ} \mathrm{C}$, and $84^{\circ} \mathrm{C}$, respectively. In cold-blooded animals, $\alpha$-serum-albumin only is said to occur.

More recently Oppenheimer has shown that by salting with ammonium sulphate serum-albumin can be divided into two fractions, one of which is thrown down at $66 \frac{2}{3}$ per cent. saturation, while the second fraction is precipitated on 82 per cent. saturation.

Isolation.-Serum-albumin is most conveniently obtained from blood-serum after removal of the serum-globulin by saturation with magnesium sulphate at a temperature of $30^{\circ} \mathrm{C}$. The filtrate is saturated with sodium sulphate or ammonium sulphate at $40^{\circ} \mathrm{C}$, or treated with acetic acid, so that the solution contains about 1 per cent. In either case the precipitated serum-albumin is filtered off, pressed between layers of filter-paper, dissolved in water (the reaction should be neutral), and separated from the remaining salt by dialysis. From its aqueous solutions it is finally obtained by evaporation at a low temperature or by precipitation with alcohol, which must be rapidly removed, however, as otherwise it will cause coagulation of the albumin.

Separation of the Albumins of the Blood-plasma from Each Other.-To isolate the fibrinogen, the plasma is treated with an equal volume of a saturated solution of sodium chloride. The resulting precipitate is filtered off and purified as described. The filtrate contains serum-globulin and serum-albumin. The globulin is precipitated by saturation with magnesium sulphate, filtered off, and likewise purified. The filtrate contains only the serum-albumin, which may be obtained, as just described, by saturation with sodium or ammonium sulphate.

Quantitative Estimation of the Total Albumin of the Plasma. -The albumins are most conveniently estimated by treating a carefully measured and weighed amount of the plasma, after neutralization with acetic acid, with five times its volume of alcohol. After standing for twenty-four hours the solution is boiled for several minutes, and the resulting precipitate collected on a weighed filter, washed with hot alcohol, then with ether, dried at $115^{\circ} \mathrm{C}$., weighed, and incinerated. The weight of the ash is deducted from the weight of the precipitate.

More accurate is the following method: a carefully measured and weighed amount of the plasma is treated with one-half its volume of a saturated solution of sodium chloride and a slight excess of tannic acid. In the resulting precipitate the nitrogen is then estimated according to Kjeldahl's method. When multiplied by 6.37 the corresponding amount of albumin is obtained.

To remove all albumins from the blood, Cavazzani's method may be employed. To this end, 20-30 c.c. of blood are added to 200 c.c. of distilled water, and treated with five or six drops of a solution consisting of ten parts of acetic acid (sp. gr. 1.040) and one part of 
lactic acid. The mixture is boiled for about ten minutes, filtered, and the precipitate washed separately with hot water, and finally pressed in a piece of muslin. The filtrate and washings, which are practically colorless, are then concentrated to a small volume. Any traces of albumin which may still be present thus separate out and are filtered off. If too much of the acid solution has been added, the mixture may not clear on boiling. In that event a few crystals of sodium carbonate are added, when coagulation promptly occurs. On the other hand, it may at times be necessary to add a few drops more of the acid solution.

The remaining constituents of the plasma are also found in the serum, and will be considered in that connection.

\section{The Serum.}

The serum results from the blood-plasma during the process of coagulation. It is most conveniently obtained by whipping blood immediately after being shed, whereby the greater portion of the fibrin is removed and the formation of large clots prevented. The corpuscles and smaller pieces of fibrin are separated by centrifugation or by allowing the fluid to stand in the cold until sedimentation has occurred. The serum is then siphoned off and filtered. It thus appears as a slightly viscid, fairly transparent fluid of a light straw color, which presents a feebly alkaline reaction and a specific gravity varying in man between 1.026 and 1.029. In its chemical composition serum differs from plasma principally in the presence of the fibrin ferment and in the absence of fibrinogen. In its place, however, traces of two other globulins, which are not present in the plasma, are found. One of these is termed fibrinoglobulin, and is thought to result during the formation of fibrin from fibrinogen. The other is the so-called cell-globulin, and is supposedly referable to the decomposition of leucocytes during the process of coagulation. The remaining constituents are qualitatively the same in both fluids. Slight quantitative differences, however, exist. A portion of the calcium, magnesium, and phosphoric acid is thus eliminated together with the fibrin, and accordingly lower values are found in the serum than in the plasma.

An idea of the mineral constituents of the serum, and their quantitative relations, may be had from the accompanying table:

Man.

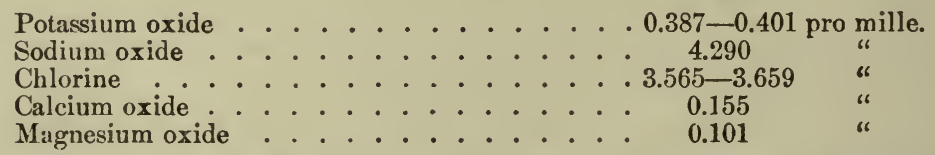

From this it will be seen that sodium in the form of the chloride largely predominates in the serum, while potassium occurs only in 
small amounts. This is exactly the reverse of what is seen in the morphólogical elements of the body, in which potassium compounds are the principal salts present. It is noteworthy, moreover, that the amount of sodium chloride is practically constant in the blood, no matter whether large quantities are ingested or the salt is given in only small amounts. During starvation even, or when the potassium salt is artificially substituted, the amount present in the blood remains practically constant. Apparently it occurs only in solution, and does not form an integral part of the albuminous molecule, as is the case with the phosphates of the blood. Of these, traces only are present in solution, while the greater portion is more or less intimately combined with the albumins. As the lecithins which are found in the blood also contain phosphorus, it follows that these figures, which are obtained by incinerating a given amount of serum or plasma and determining the phosphoric acid in the ash, are too high. In serum which had been freed from lecithin Sertoli and Mroczkowski found amounts varying between 0.02 and 0.09 pro mille, calculated as disodium phosphate. In the estimation of the sulphates we meet with still greater difficulties, as the sulphur of the albumins is included in the determination. The amount which is present in solution, however, is certainly very small. The iron which is at times met with in the serum is unquestionably derived from the leucocytes, and is an accidental constituent. The amount which may be obtained is always exceedingly small.

Of other elements, traces of silicon, fluorine, copper, and manganese have at times been observed.

The coloring-matter of the serum and plasma is supposedly due to a substance belonging to the class of lipocnromes or luteïns.

The albumins of the serum which also occur in the plasma, viz., serum-albumin and serum-globulin, have been considered. Of the fibrinoglobulin which is formed during coagulation of the blood, and which is thought to result from decomposition of fibrinogen, comparatively little is known. It coagulates at $64^{\circ} \mathrm{C}$, and apparently represents about one-third of the fibrinogen molecule. Of the so-called cell-globulin, still less is known, and both are found only in traces.

\section{The Coagulation of the Blood.}

The Fibrin-ferment.-Through the researches of A. Schmidt, Buchanan, Hammarsten, Arthus, and Morawitz more particularly it has been ascertained that the coagulation of the blood is referable to the action of a special ferment-the fibrin-ferment or thrombin of Schmidt-upon fibrinogen. As a result this is transformed into the insoluble fibrin. Of the chemical nature of this process practieally nothing is known. Hammarsten expressed the opinion that the transformation was essentially a hydrolytic process and taught that during this process a small amount of a water-soluble globulin -fibrinoglobulin-is split off, while the remainder of the molecule 
appears as insoluble fibrin. At present this view has lost support through the fact that fibrinogen solutions can be prepared from which no fibrinoglobulin is split off either during clotting or upon heat coagulation. Nevertheless it is possible that the process is a hydrolytic one and we may imagine, as Abderhalden suggests, that fibrinogen is composed of several fibrin molecules which are set free as the result of the action of the fibrin ferment, with a coincident taking up of water.

The fibrin-ferment, according to our present concept, does not exist in the blood as such, but as an inactive zymogen-prothrombin -which must first be activated before it can manifest its activity. It was formerly supposed that the transformation of the zymogen into the enzyme was brought about by soluble calcium salts, as it could be shown that coagulation only occurs when such are present. There is evidence, however, to show that the activation occurs through a special kinase (cytozyme, zymoplastic substance), as in the case of trypsin, which is now generally spoken of as thrombokinase, and which itself must first be activated; this is probably brought about through the calcium salts.

As regards the origin of the kinase we know that it is derived from the tissues in general. Under suitable conditions (blood of birds and terrapins) no coagulation of the blood thus occurs if precautions are taken to prevent its coming in contact with the wound and if the corpuscles are then at once thrown down by centrifugation (Howell, Delezenne). The zymogen, on the other hand, is supposedly furnished by the blood-platelets and leucocytes. It is known, however, that other cells of the body also contain substances which can induce or, at least, hasten coagulation.

The ferment nature of the thrombin is shown by the facts that a very small amount is necessary to effect the coagulation of a very large amount of blood and that no coagulation occurs on heating the ferment to $100^{\circ} \mathrm{C}$. Its optimum temperature lies at $37^{\circ} \mathrm{C}$.

Of interest in this connection is the fact that the ferment (according to Schmidt) cannot be kept in solution and retain its activity. It becomes inactive, but can under certain conditions be activated again by the addition of an alkali, to become inactive again on neutralization, etc. Of the chemical nature of the ferment nothing is known. It is generally regarded as a nucleoproteid (Pekelharing) and is said to yield a nuclein on peptic digestion.

Isolation.-The isolation of impure fibrin-ferment is most conveniently accomplished in the following manner: Taking the serum of the ox, the globulins are first precipitated by saturation with magnesium sulphate. The filtrate is then diluted with water, and treated while stirring with a very dilute solution of sodium hydrate until an abundant and flocculent precipitation of magnesium hydroxide has been brought about. This precipitate, which contains a large proportion of the ferment, is washed with water, pressed between filter-paper, and dissolved in water by neutralizing the 
solution with diluted acetic acid. The salts are then removed by dialysis, when the ferment can be precipitated by a suitable addition of acetic acid.

To test for the fibrin-ferment, Arthus has suggested the use of fluoride-plasma of dogs. In such plasma, as also in the corresponding product of horses (Fuld), coagulation cannot be brought about without the ferment; neither the addition of a calcium salt nor attempts at activation by treating a portion with alkali and adding it to the rest will succeed. If then coagulation does occur after adding a solution to be tested, we may infer that the fibrin-ferment was present in the latter.

Fibrin.-Fibrin is formed during the spontaneous coagulation of all albuminous solutions which contain fibrinogen, soluble calcium salts, and cellular elements that can give rise to the fibrin-ferment and its kinase. It is most conveniently obtained by whipping freshly-shed blood with a suitable instrument, when the fibrin is deposited as an elastic, stringy material, which may be freed from adhering corpuscles by thorough washing and kneading in running water. Such fibrin, however, is still contaminated with serumglobulin and certain phosphorus-containing substances which have resulted from the decomposition of leucocytes. The serum-globulin may be removed by separate washing and kneading in a 5 per cent. solution of common salt; but the other products, as well as the remains of the corpuscles of the blood, can scarcely be removed. To obtain pure fibrin, therefore, it is necessary to start with filtered plasma or with filtered transudates, which are beaten with a piece of whalebone, after adding a little serum, if the fluid is not spontaneously coagulable. The resulting material is washed with water, then with a 5 per cent. solution of sodium chloride, and finally extracted with alcohol and ether.

The fibrin then appears as a white stringy substance, which is somewhat elastic, but is easily rendered brittle on contact with alcohol or on warming the substance in water to a temperature of $75^{\circ} \mathrm{C}$. It is closely related to the coagulated albumins, and accordingly is soluble only with difficulty. It is questionable, moreover, whether solution of the substance can be accomplished without causing its decomposition. If fibrin is thus placed in a 5 to 10 per cent. solution of sodium chloride, or a 6 per cent. solution of sodium nitrate, and kept at a temperature of $40^{\circ} \mathrm{C}$., it first swells up and gradually disappears as such. What takes place under those conditions is not known. In dilute alkalies and acids it likewise dissolves. Stronger acids, as also the proteolytic ferments, dissolve the fibrin, but at the same time cause its transformation into acid albumin and albumoses. In water, alcohol, and ether it is entirely insoluble.

The elementary analysis of fibrin gives 52.68 parts of carbon, 6.83 of hydrogen, 16.41 of nitrogen, 1.1 of sulphur, and 22.48 of oxygen. It is to be noted, further, that in addition to these 
elements calcium is constantly present, and, as has been seen, its formation is largely dependent upon the presence of a soluble calcium salt.

The amount of fibrin which may be obtained from the blood, notwithstanding its bulk, does not exceed $0.1-0.4$ per cent.

Estimation.-In order to determine the amount of fibrin in a given volume of blood, from 30 to 40 c.c. are placed in a previously weighed beaker, which is elosed with an India-rubber cap. Through the centre of this passes a piece of whalebone that is firmly fixed and provided with a rudder-like end, which dips into the blood. This is now defibrinated by beating with the whalebone paddle, when the beaker is again weighed with its contents. The difference, as compared with the first weight, indicates the weight of the blood. The beaker is then filled with water and the mixture again beaten. The fibrin is allowed to settle, and after being washed by decantation with normal salt solution it is collected on a filter of known weight. On this it is further washed with normal salt solution until free from coloring-matter; it is then extracted with boiling alcohol, then with ether, and finally dried at $115^{\circ} \mathrm{C}$. and weighed.

The question, why the blood does not coagulate within the vessels of the body where fibrinogenic material is available, and where leucocytes no doubt undergo degeneration, has been variously answered. On the one hand, it is stated that the integrity of the endothelial lining is here of prime importance, and that coagulation will occur whenever this is impaired. As a matter of fact, we find that coagulation takes place after ligation of an artery, and that the coagulum invariably extends as far as the next collateral vessel. That the nutrition of the intima is here seriously interfered with cannot be doubted. Similarly we find a more or less extensive thrombosis in atheromatous vessels, not to speak of the process of clotting in association with wounds. In such cases it appears that owing to the lesion of the endothelial coat an aggregation of leucocytes occurs in the affected parts, which in turn results in the death and dissolution of many of the cells at these places. Contact with a foreign substance, and as such diseased or dying endothelial cells must be viewed, in some manner brings about the early dissolution of the leucocytes, and we find accordingly that on introducing a silk thread into the bloodvessel of a living animal coagulation takes place around the foreign body. Similarly, it may be observed that when blood is received in a vessel, the walls of which have been carefully lubricated with vaselin, coagulation is greatly delayed, but may be brought about at once on introducing bits of foreign material, such as dust or ashes and the like.

While the integrity of the bloodvessel walls is thus unquestionably of moment in preventing coagulation in the living organism, we may also imagine that an antiferment-antithrombin-may be present in the blood which prevents the activation of the ferment. Pugliese has shown, as a matter of fact, that such substances are formed in the liver and muscle tissue. 
Rapidity of Coagulation.-The rapidity with which coagulation of the blood occurs after being shed varies with different animals, with the districts from which the blood is taken, etc. In birds it thus occurs after one and a half minutes; in man after from three to four minutes; while in cold-blooded animals it begins only after a quarter of an hour. In the horse, in which coagulation is likewise delayed, the corpuscles of the blood have time to settle and form two distinct layers-the red at the bottom and the white on top, where they appear as a grayish-white zone, and constitute the so-called crusta phlogistica or inflammatoria. Above this we then see the plasma, which has undergone coagulation, and on top of it the serum that has been squeezed out from the clot. The same phenomenon may be observed in human blood when cooled to about $0^{\circ} \mathrm{C}$., and from the extent of the crusta phlogistica (leucocytosis) in such blood the older physicians were wont to draw prognostic conclusions as to the course of the disease.

The rapidity of coagulation can be artificially increased and diminished. By beating the blood, by increasing its temperature a little beyond that of the body, and by diluting with water it is increased; the same occurs after the administration of calcium salts and of gelatine, while exposure to a low temperature, the presence of much carbon dioxide in the blood, the careful lubrication of the vessel with vaselin or similar unguents, cause a retardation of coagulation. Its prevention finally may be brought about through influences already mentioned, viz., by salting with the neutral salts, following the previous injection into the body of albumoses or of histon, of diastatic ferments, of extracts of the mouth-parts of the leech, after elimination of the intestinal bloodvessels by ligation, etc. It has been known for a long time also that after the bite of certain snakes the blood does not coagulate. Cobra poison is said to contain a substance which acts upon the thrombokinase directly.

In addition to the albumins already considered, viz., fibrinogen, the serum-albumin, and serum-globulin fraction, and Pekelharing's nucleoproteid (fibrin-ferment), the blood also contains a peculiar albuminous substance which is termed glutolin. It was discovered by E. Faust in the globulin fraction obtained on half-saturation of the blood-serum with ammonium sulphate, and supposedly occupies a position intermediate between the albumins and collagen. Its significance is not known, and it is possible that the substance represents no preformed body, but is a denaturized globulin.

Glässner and Langstein believe to have shown that albumoses also can occur in the blood under normal conditions; that this is possible in disease has long been known. The occurrence of still other albuminous substances has from time to time been announced, but has not been satisfactorily established (Eichholz's mucoid, Zanetti's ovomucoid-like body, etc.).

Ferments. - In addition to the fibrin-ferment the blood-plasma 
apparently contains a number of other ferments. N. Sieber could thus demonstrate three different oxidizing ferments, which are capable not only of decomposing glucose (Lepine's glucolytic ferment), but also disaccharides and polysaccharides. Schumm has demonstrated a proteolytic ferment, others a lipase, etc. Opie mentions an antileucoprotease (antitrypsin). On immunization with various foreign cells and cell products, moreover, corresponding antibodies are encountered in the blood, which in part at least may be related to ferments. The list includes the antitoxins, the agglutinins, the cytolysins (bacteriolysins, hæmolysins, spermatolysins, etc.), precipitins, coagulins, antiferments, anti-aggressins, etc.

The remaining solids which are found in both plasma and serum are, as has been pointed out, present in only very small amounts. The most important of these is glucose. Its amount varies between 1 and 1.5 pro mille, and is but little influenced by the character of the food unless a large excess of earbohydrates has been ingested. In such an event the amount may increase to 3 pro mille, or even higher, but it then appears also in the urine, whereby a further increase is prevented. Larger amounts, such as 9 pro mille, are found only under pathological conditions.

In addition to glucose, another reducing substance is found in the blood, which to a certain degree is fermentable and is soluble in ether. From the researches of P. Mayer, it appears that this substance is a conjugate glucuronate.

Neuberg and Strauss have shown that at times traces of lævulose can also be demonstrated in the blood-serum, and that artificially a lævulosæmia can be produced in certain individuals following the ingestion of 100 grammes. The presence of jecorin, which has repeatedly been reported, is doubtful.

Glycogen is constantly present in normal blood. Its amount, however, is subject to great variations. As a rule, traces only are found, but it may increase at times to 1.56 per cent., as calculated for the blood as a whole. Larger amounts are seen under pathological conditions.

Fat is normally found to the extent of from 0.2 to 0.3 per cent., but may be greatly increased by the ingestion of much fatty food, as also in various pathological conditions.

Urea is likewise found in only very small amounts under normal conditions ( 0.016 to 0.020 per cent.), while in disease much greater quantities may be encountered. Ammonia is said to be present in normal blood to the amount of 0.001 per cent.

The further occurrence in the blood of soaps, cholesterin, and lecithins, as also of uric acid, kreatin, carbaminic acid, paralactic acid, hippuric acid, etc., has been mentioned. In addition Tanella claims to have found small amounts of phosphocarnic acid in the blood. All these bodies are found in only extremely small amounts, and need not be considered at this place. The pathological constit- 
uents of blood, such as leucin, tyrosin, acetone, bilirubin, etc., have been considered in preceding chapters.

Of gases, finally, we find in the plasma and serum small amounts of nitrogen and oxygen, which are present simply in solution, and somewhat larger amounts of carbon dioxide, which in part at least is more or less firmly combined with albumins.

\section{The Leucocytes.}

The morphological characteristics and general chemical composition of the leucocytes have already been considered (see pages 326 . and 329). At this place I wish merely to draw attention to one substance which, according to Lilienfeld, is found in special abundance in the nuclei of these bodies, and which has been termed nucleohiston.

Nucleohiston.-This substance was first isolated by Kossel and Lilienfeld from the thymus gland of the calf, but has since been obtained from the leucocytes of the lymph-glands, as also from the splenic cells, the testicular cells, the spermatozoa, and from the epithelial lining of the small intestine. In all probability it represents an important constituent of all cellular nuclei.

Isolation.-Nucleohiston is most conveniently obtained from the leucocytes of the thymus gland. To this end, the gland is carefully dissected free from fat and all larger bloodvessels, and finely hashed. This mass is extracted with cold water, passed through muslin, and centrifugalized. The aqueous extract is further filtered, and the nucleohiston precipitated by the careful addition of dilute acetic acid. It is filtered off, dissolved in water with the aid of a small amount of a dilute solution of sodium carbonate, reprecipitated with acetic acid, and purified by a repetition of this process. It is then washed with acetic water, extracted with alcohol and ether, and finally dried at a temperature of from $110^{\circ}$ to $115^{\circ} \mathrm{C}$. Gamgee and Jones have pointed out that solutions of Lilienfeld's nucleohiston prepared in this manner are very opalescent, but that this objection to the polarimetric examination can be removed by extracting the nucleohiston with a 5 per cent. solution of ammonium acetate and filtering. The liquid filters very slowly, but steadily. The proteid is then precipitated by pouring the solution into 95 per cent. alcohol, after which it is washed and dried with alcohol and ether.

Properties.-Thus obtained, nucleohiston represents a snowywhite fine powder, which is insoluble in benzol, alcohol, chloroform, methyl alcohol, ether, and acetic acid, but is soluble in water, glacial acetic acid, concentrated nitric acid, and hydrochloric acid, in solutions of sodium carbonate, sodium hydrate, ammonia, and, when freshly precipitated, also in solutions of sodium chloride and magnesium sulphate, especially in the presence of a little acetic acid. It has the properties of an acid salt, and on boiling with water or on 
treating with baryta water or very dilute hydrochloric acid, it is decomposed into a nuclein, the so-called leuconuclein, and histon. It may therefore be regarded as a nucleoproteid, but differs from most of the other representatives of this group in the large amount of phosphorus-3.025 per cent.-which it zontains. The histon radicle possesses marked basic properties, and readily combines with acids. From its acid solutions it is precipitated by ammonia, and it is insoluble in an excess of the reagent. The leuconuclein, on the other hand, has markedly acid properties. On treating with an alcoholic alkali solution it is decomposed into an albuminous substance and thymonucleinic acid. The nucleohiston, according to Gamgee and Jones, is dextrorotatory $(\alpha) \mathrm{D}=+37.5^{\circ}$.

Elementary analysis of nucleohiston has given the following results : carbon, 48.46 ; hydrogen, 7 ; nitrogen, 16.86 ; phosphorus, 3.025 ; sulphur, 0.701 ; and oxygen, 23.95 per cent.

Bang has lately denied the existence of a leuconuclein in the sense of Kossel. According to his researches, Lilienfeld's preparation represents a combination of histon nucleinate with parahiston nucleinate, which is analogous to a double salt ( 54 per cent. of nucleinic acirl, 30.7 per cent. of histon, and 15.3 per cent. of parahiston). It has a constant composition, viz., it is a hexanormal acid (adeninguaninic acid $)=$ histon + triadenylic acid $=$ parahiston.

Bang has pointed out, moreover, that the results obtained in the case of the thymus cells are not directly applicable to the leucocytes in general. While the thymus gland and lymph-glands contain about 20 per cent. of histon nucleinate, no substance of this character occurs in the bone-marrow. The assumption is that a different nucleoproteid is here represented.

The chemical analyses which Lilienfeld made of the leucocytes of the thymus gland gave the following results :

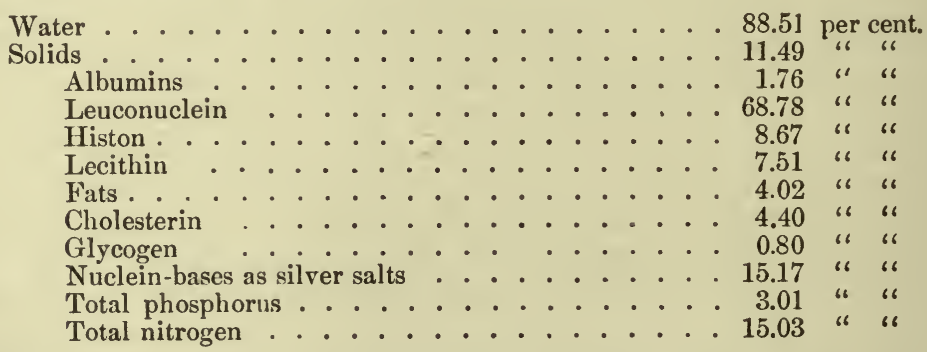

Of albumins proper, there are said to be present in the leucocytes the so-called cell-globulins, of which Halliburton recognizes twoone coagulating at $50^{\circ} \mathrm{C}$., the other at $73^{\circ} \mathrm{C}$. - and one of which is by some thought to be identical with the fibrin ferment; then serum-albumin, and a mucinous body, the so-called hyalin substance of Rovida. These bodies, however, represent only a very small portion of the solids of the leucocytes, as is seen from Lilien- 
feld's table, and it is doubtful indeed whether their true chemical nature has been sufficiently established.

\section{The Plaques.}

Of the chemical composition of the plaques little is known. According to Lilienfeld, they contain an albuminous substance and a nuclein ; for on treatment with artificial gastric juice they can be differentiated into a homogeneous portion, which is subsequently dissolved, and an insoluble granular portion, which gives the various reactions of the nucleins, and may be shown to contain phosphorus. In the plaques the albumin is probably combined with the nuclein to form a nucleoproteid, which may be identical with the nucleohiston just described. According to Lilienfeld, indeed the plaques must be regarded as nuclear derivatives, and he has accordingly termed them the nuclein platelets of the blood.

\section{The Red Corpuscles.}

The red corpuscles of the blood, as has been mentioned, owe their color to the presence of hæmoglobin or its oxygen compound, oxyhæmoglobin. This may be extracted by diluting with water, by alternate freezing and thawing, by shaking with ether, chloroform, etc. The blood is thereby rendered lake-colored, and on microscopical examination it will be observed that instead of the original corpuscles, so-called blood-shadows are now found. These are colorless ring-like bodies, and constitute the stroma of the red corpuscles. In the circulating blood the dissolution of the hæmoglobin is prevented by the presence of large amounts of sodium chloride. Such blood is said to be hyperisotonic-i. e., it contains more sodium chloride than is necessary to prevent the dissolution of the coloringmatter from its corpuscles. Within the corpuscles the hæmoglobin is supposedly not present in the free state, but in combination with some other substance, such as lecithin; and Hoppe-Seyler accordingly distinguishes between the so-called arterin and phlebin, which represent the lecithin compound of oxyhæmoglobin and hæmoglobin respectively.

As has been mentioned, the red corpuscles represent nearly onehalf of the liquid blood. They contain about 57.7 per cent. of water and $40 . \overline{5}$ per cent. of oxyhæmoglobin, while the constituents of the stroma inclusive of mineral salts amount only to about 1.9 per cent. Among these constituents Halliburton's cell-globulin is said to be most abundant; in addition we find traces of lecithin, cholesterin, and nucleo-albumin, while serum-albumin and albumoses are apparently absent. In the nucleated red corpuscles of birds we further meet with the integral constituents of the nuclei, among which nucleohiston probably prevails. Its basic constituent, histon, was first discovered by Kossel in the red corpuscles of the goose. 
An idea of the mineral constituents of the red corpuscles, viz., their stroma, may be had from the following table, which is taken from C. Schmidt, and calculated for 100 parts of the moist corpuscles.

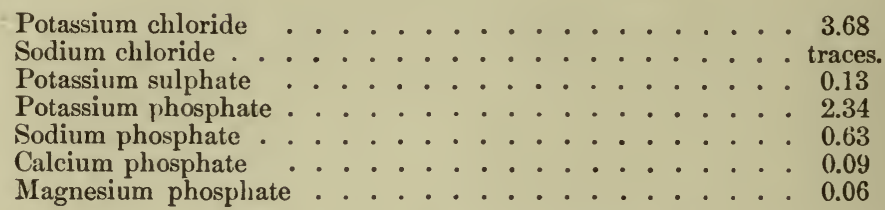

The iron of the hæmoglobin is not included in this table; in man it varies between 0.506 and 0.537 pro mille. In addition traces of copper are not infrequently met with, and, as will be seen later, this element in some of the invertebrate animals apparently takes the place of iron in the coloring-matter of the blood.

Isolation of the Red Corpuscles of the Blood.-To isolate the red corpuscles, the blood is defibrinated by beating, diluted with ten times its volume of a 1 per cent. solution of sodium chloride, and passed through a muslin filter. On subsequent centrifugation and repeated washing with the salt solution, they are then freed from the serum, and may now be collected on a paper filter, after the previous addition of a large amount of alcohol. Any fats, lecithins, or cholesterins that may be present are extracted with warm alcohol and ether, when the corpuscles can be dried and weighed. To isolate the stromata, the mass of corpuscles, when freed from serum, is shaken with five or six times its volume of water and a small amount of ether. The mixture is then centrifugalized, and is thus separated from the leucocytes. The stromata are now precipitated by adding a few drops of a 1 per cent. solution of acid sodium phosphate, until the liquid has almost assumed the consistence of the original blood. They are then collected on a filter, quickly washed with water, and may now be dissolved in a 5 per cent. solution of magnesium sulphate.

\section{Hæmoglobin and Its Derivatives.}

Hæmoglobin.-The hæmoglobin is the coloring-matter of the red corpuscles, and is supposedly present in these in combination with another body, which may be a lecithin, as the phlebin of HoppeSeyler, while the corresponding compound of oxyhæmoglobin is termed arterin. Of the nature of these compounds, however, but little is known, and it has not even been definitely ascertained that the pairling of the coloring-matter is really a lecithin.

Hæmoglobin or its oxy-compound occurs widely distributed in the animal world, and is found not only in the vertebrates, but also in many of the invertebrates. But while in the former it is contained in definite cellular elements of the blood, it may also occur as such among certain invertebrate animals. Closely related to it is 
the so-called oxyhomocyanin, which is found in certain arthropods and molluses, and in which the iron is apparently replaced by copper. Then again we find among invertebrate animals various violet and purplish-red pigments, the so-called floridins, which are likewise to be classed with hæmoglobin, and as we have previously seen, a genetic relationship apparently also exists between hæmoglobin and the chlorophyl of plants. These various pigments are collectively spoken of as respiratory pigments, as they are intimately concerned in the transportation of the oxygen of the air to the various tissues of the body, and in the removal of the carbon dioxide which results as a product of cellular metabolism.

Outside of the blood hæmoglobin is found also in striped and unstriped muscle-tissues, and under pathological conditions it may appear in the urine as such. Different hæmoglobins apparently exist. It is hence impossible to give a definite formula which expresses the constitution of all. An idea of their quantitative elementary composition may be had from the accompanying table:

\begin{tabular}{|c|c|c|c|c|c|c|c|c|c|}
\hline & & & Carbon. & Hydrogen. & Nitrogen. & Oxygen. & Sulphur. & Phosphorus. & Iron \\
\hline Hors & & & .54 .87 & 6.97 & 17.31 & 19.730 & 0.650 & & 0.4 \\
\hline & & & .54. & 7.22 & 16.38 & & & & \\
\hline & . & & . 54.71 & 7.38 & 17.43 & & & & \\
\hline Guinea-pig & & & . 54.12 & 7.3 & 16. & & 0.580 & & \\
\hline rrel. & & & . 54.09 & 7.3 & 16.09 & 21.4 & 0.400 & & \\
\hline & & & .54 & & & & & 0.770 & \\
\hline ken & & & .52 .47 & 7.19 & 16.45 & 22.500 & 0.857 & 0.197 & \\
\hline
\end{tabular}

The size of the hæmoglobin molecule is, like that of all albuminous substances, very large. For that of dog's blood Hüfner obtained the figure 14,129, which would correspond to the formula $\mathrm{C}_{636} \mathrm{H}_{1025} \mathrm{~N}_{164} \mathrm{FeS}_{3} \mathrm{O}_{181}$. It thus contains three atoms of sulphur for one atom of iron, while the hæmoglobin of the horse and pig has only two atoms of sulphur for one atom of iron. Of interest further is the presence of phosphorus in the hæmoglobin of the goose and chicken. Whether this forms an integral component of the hæmoglobin molecule, however, is questionable, and it is quite possible that its presence is owing to a contamination of the coloringmatter with nucleinic acid derived from the nuclei of the red corpuscles.

Structurally, hæmoglobin must be regarded as a proteid, viz., as a compound of an albuminous radicle with another complex organic radicle. This other radicle is here an iron-containing pigment, which may be separated from its albuminous pairling, and is termed hamochromogen (see below), while the albuminous component is known as globin. These two substances are apparently united in the hæmoglobin molecule, through an additional radicle, which is as yet unknown. Hæmoglobin, like the nucleoproteids, is dextrorotatory.

Globin is a histon, and accordingly presents the following characteristic reactions: it is precipitated by ammonia from its solutions in 
dilute hydrochloric acid, and is insoluble in an excess of the reagent. With concentrated nitric acid it is thrown down in the cold, but not from its heated solutions. Under certain conditions it can be coagulated on boiling, but, unlike the other coagulable albumins, its coagulate is readily soluble in acids. It contains 54.97 per cent. of carbon, 7.2 per cent. of hydrogen, 16.89 per cent. of nitrogen, and 0.42 per cent. of sulphur. In its behavior toward polarized light globin behaves like a true albumin-i.e., it is lævorotatory. The amount of globin which can be obtained from the hæmoglobin molecule is quite large, and according to Schultz amounts to 86.5 per cent., while 4.2 per cent. only is represented by the pigment itself.

Isolation.-A solution of oxyhæmoglobin in water, prepared at a temperature of $40^{\circ} \mathrm{C}$., is treated with dilute hydrochloric acid until the red color changes to brown. This mixture is extracted with 80 per cent. alcohol (one-fifth volume) and ether (one-half volume) until the ether takes up no more coloring-matter. The resulting aqueous-alcoholic solution is precipitated with ammonia, filtered, and the precipitate dissolved in very dilute acetic acid. On filtering, the globin is again precipitated with ammonia and collected on a silk filter. After washing with absolute alcohol, then with water, again with alcohol, and finally with ether, the substance is dried first in the air and then at a temperature of $100^{\circ} \mathrm{C}$. The resulting material constitutes pure globin, as a yellowish loose powder, which is not especially hygroscopic.

Hæmochromogen.-The isolation of hæmochromogen is rather difficult, owing to the avidity with which it combines with oxygen to form hoomatin in alkaline solution. Hoppe-Seyler, however, succeeded in obtaining the substance in crystalline form, by heating hæmoglobin with sodium hydrate solution in an atmosphere of hydrogen. In acid solutions hæmochromogen gradually loses its iron and is converted into hoematoporphyrin. In alkaline solution it presents a beautiful cherry-red color, and on spectroscopic examination gives two bands of absorption. One of these is very intense, and located between $\mathrm{D}$ and $\mathrm{E}$, nearer $\mathrm{D}$, while the other is not so dark, but wider, and found about $\mathrm{E}$ and extending beyond $\mathrm{b}$. To demonstrate the spectrum of hæmochromogen, bloody fluid is mixed with a solution of sodium hydrate, when the resulting hæmatin is reduced with ammonium sulphide, or Stokes' reagent, viz., an ammoniacal solution of ferrous tartrate, or stannous chloride.

The hæmochromogen radicle, as has been stated, represents the pigmented group of the hæmoglobin molecule, and to its presence the power of hæmoglobin to combine with oxygen, carbon dioxide, and other gases, is unquestionably due.

Homoglobin itself can be obtained in crystalline form, and is characterized by the great resistance which it offers to putrefaction and tryptic decomposition. It is stated that even after the lapse of years decomposed blood contains its hæmoglobin as such, and that on shaking with air it may again be transformed into pure oxy- 
hæmoglobin. Its solutions present a beautiful purplish-red color, and on spectroscopic examination give rise to a single band of absorption, which lies between $\mathrm{D}$ and $\mathrm{E}$ and extends slightly to the left beyond $\mathrm{D}$. This is most conveniently shown by taking a solution of oxyhæmoglobin, and reducing this with ammonium sulphide or Stokes' fluid, as directed above.

Most important is the avidity with which hæmoglobin combines with various gases, and upon this characteristic indeed its chief function, as a respiratory pigment, is based. This property, as has been stated, is referable to the chromogen radicle, and more particularly to the iron, which it contains. Every atom of this is capable of combining with one molecule of oxygen or of carbon dioxide, and in this form largely the oxygen of the air is carried to the various tissues of the body, and the carbon dioxide removed. In the circulating blood we accordingly find only relatively small amounts of free hæmoglobin, and in arterial blood its oxy-compound is almost exclusively encountered.

The amount of hæmoglobin which is contained in human blood, either as such or in combination with oxygen or carbon dioxide, is about 14 per cent., but subject to certain variations, even in health, while in disease still greater deviations from the average normal amount are observed. A great diminution may here occur, and is most marked in chlorosis and pernicious anæmia, in which the percentage may fall as low as 2.35 .

As the isolation of the hæmoglobin from the blood resolves itself into the isolation of its oxy-compound, this will be considered together with its quantitative estimation under that heading.

Oxyhæmoglobin.-Oxyhæmoglobin is the oxy-compound of hæmoglobin, and differs from its mother-substance in containing two atoms more of oxygen, which are bound to the one atom of iron, than are present in the hæmochromogen radicle. In this manner, however, the hæmochromogen is converted into hæmatin, and we may therefore say that oxyhæmoglobin, in contradistinction to hæmoglobin, consists of the globin radicle united by some unknown group to a hæmatin radicle.

Hæmatin.-In accordance with the above considerations, we find that on decomposition of oxyhæmoglobin hæmatin is obtained instead of hæmochromogen. This latter, indeed, is at once transformed into hæmatin on exposure to oxygen, and, as we have seen, the hæmatin is correspondingly reconverted into hæmochromogen by treating with reducing agents. The decomposition of oxyhæmoglobin with the formation of hæmatin can be readily effected by heating its solutions to a temperature of $80^{\circ} \mathrm{C}$, by treating with dilute mineral acids, or with stronger solutions of the alkaline hydrates, as also by peptic or tryptic digestion. 'To obtain the substance in a pure state, however, it is best to start with hicemin (see below), which can be readily obtained in crystalline form. To this end, oxyhæmoglobin is treated with a trace of sodium 
chloride and dissolved in glacial acetic acid. On heating, the hæmin is precipitated in the form of very characteristic, drawnout, rhombic platelets, which are insoluble in water, alcohol, and ether, with difficulty so in glacial acetic acid and in dilute mineral acids, but are readily soluble in dilute alkaline solutions and acid alcohol. The crystals are collected on a filter, washed with alcohol and ether, and are thus obtained in pure form. To prepare the hæmatin, the hæmin is dissolved in a dilute solution of sodium hydrate and supersaturated with dilute hydrochloric acid. The substance is thus precipitated in the form of brownish flakes, which are washed free from chlorides and dried at $120^{\circ} \mathrm{C}$. Hæmatin, in contradistinction to oxyhæmoglobin and hæmin, is non-crystallizable. Its solubility is essentially the same as that of hæmin. In acid solution both hæmatin and hæmin show a well-defined spectral band between $\mathrm{C}$ and $\mathrm{D}$. Between $\mathrm{D}$ and $\mathrm{F}$ a second band is seen, which is much wider but less sharply defined than the first. By diluting the solution this band may be resolved into three bands, of which one is located between $\mathrm{b}$ and $\mathrm{F}$, near $\mathrm{F}$; another, between $\mathrm{D}$ and $\mathrm{E}$, nearer $\mathrm{E}$; and a third faint band between $\mathrm{D}$ and $\mathrm{E}$, nearer $\mathrm{D}$. As a rule, however, only two bands are seen. The alkaline solutions of hæmatin are distinctly dichrotic and give only one band of absorption, the greater portion of which lies between $\mathrm{C}$ and $\mathrm{D}$, and extends slightly beyond $\mathrm{D}$.

The basis of the hæmatin molecule is supposedly a methyl-propylpyrrol (hæmopyrrol) :<smiles>CCCCCc1c[nH]cc1C</smiles>

This is probably the mother-substance of the hæmatinic acids which Küster obtained from hæmatin on careful oxidation. One of these is the dibasic acid, $\mathrm{C}_{8} \mathrm{H}_{9} \mathrm{NO}_{4}$, while the other may be regarded as the anhydride of the tribasic acid, $\mathrm{C}_{8} \mathrm{H}_{8} \mathrm{O}_{5}$, which in turn through loss of $\mathrm{CO}_{2}$ can be transformed into the anhydride of methylethyl maleic acid, $\mathrm{C}_{7} \mathrm{H}_{8} \mathrm{O}_{3}$. The structural formulæ of these acids, according to Küster, are the following:

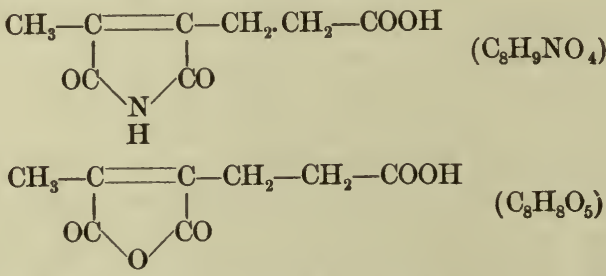

On treating with concentrated sulphuric acid, with hydrochloric acid, or with glacial acetic and hydrobromic acids, hæmatin loses its iron, and on the subsequent addition of water is transformed into 
hematoporphyrin, which is isomeric with bilirubin. This change is expressed in the equation :

$$
\underset{\text { Hæmatin }}{\mathrm{C}_{32} \mathrm{H}_{32} \mathrm{~N}_{4} \mathrm{O}_{4} \mathrm{Fe}}+2 \mathrm{H}_{2} \mathrm{O}-\mathrm{Fe}=\underset{\substack{\text { Bilirubin } \\ \text { (hæmatoporphyrin). }}}{\mathrm{C}_{32} \mathrm{H}_{36} \mathrm{~N}_{4} \mathrm{O}_{6}}
$$

Bilirubin, according to Küster, yields the same acids on oxidation as hæmatin or at least isomeric bodies. The intimate connection existing between the blood-pigment and bile-pigment, and through this with the urinary pigment, is thus satisfactorily established.

The various data further permit an insight into the more intimate structure of the hæmatin molecule. It may thus be inferred that hæmatin consists of two symmetrical halves which are united by iron. Since both hæmatin and hæmatoporphyrin yield the same quantity of the same hæmatinic acid, and since the latter each contain 8 carbon atoms it suggests itself that hæmatoporphyrin in turn consists of two symmetrical halves. Accordingly the structure of hæmin could be viewed as shown in the formula :

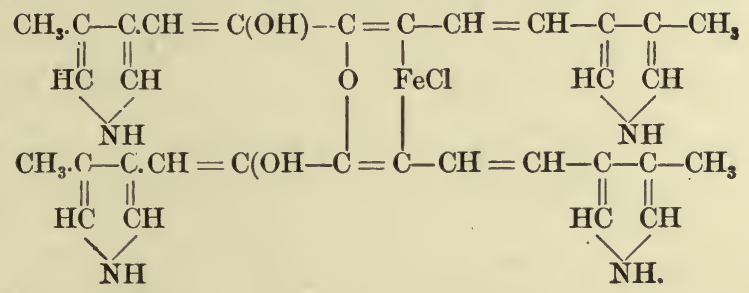

On reduction with tin and hydrochloric acid in alcoholic solution and the subsequent addition of caustic alkali in excess hæmatin gives rise to skatol. On intense oxidation with ammonium persulphate cyanic acid and succinic acid result.

As hæmatin is a decomposition-product of oxyhæmoglobin it is not found as such in the circulating blond. It is said to occur in the urine, however, in cases of poisoning with arsenious hydride. In the stools it is found after hemorrhages into the stomach or the upper portion of the small intestine, and also after the ingestion of large amounts of red meats. In such cases, of course, its origin is referable to the decomposition of hæmoglobin through the agency of the gastric and pancreatic juices.

Hæmin.-Hæmin has been generally regarded as hæmatin hydrochlorate and formed through the direct apposition of hydrochloric acid. But as Nencki already suggested it is more likely that the $\mathrm{HCl}$ replaces an $\mathrm{OH}$ group :

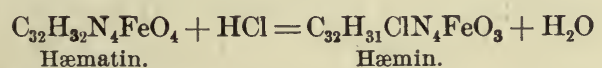

Very curiously elementary analysis of the substance has given the same results to the same observer, but different results to different 
observers. Nencki has shown that this is owing to the fact that hæmin contains two hydroxyl groups, and that the substance not only forms ethers with acid and alkyl radicles with great readiness, but also addition-products with indifferent compounds. Its preparation has been described above.

Isolation of Oxyhæmoglobin.-Oxyhæmoglobin in crystalline form is best obtained from the red corpuscles of the horse or dog, according to the following method: the blood is first rendered uncoagulable by treating with ammonium oxalate $(0.06-0.1$ per cent.), and is then centrifugalized to effect the separation of the corpuscles. This mass, when freed from plasma by siphonage, is treated with twice its volume of water and placed on ice. The resulting fluid is now mixed with an equal volume of a saturated solution of ammonium sulphate that has likewise been cooled to a low temperature. 'This mixture is also placed on ice until the precipitate of globulins, which is referable to remaining plasma, has settled. On filtering in the refrigerator a perfectly clear dark-red filtrate is obtained, which contains the greater portion of the coloring-matter. If now the solution is brought to the temperature of the room, the separation of crystalline oxyhæmoglobin begins, and may be hastened, if necessary, by the further addition of a small amount of the ammonium sulphate solution. After a few days the process is completed, when the crystals are filtered off through a Büchner filter by the aid of a suction pump, and are partially freed from the mother-liquor by pressing between filter-paper. The substance is then purified by recrystallization. To this end, the crystalline mass is dissolved in water, reprecipitated by the addition of an equal volume of the ammonium sulphate solution, and so on, until the required degree of purity has been attained. Adhering ammonium sulphate is finally removed mechanically. To preserve the substance, it is dried in a vacuum or at a low temperature over sulphuric acid, and is then quite stable.

The ease with which oxyhæmoglobin can be brought to crystallization differs with different animals. In guinea-pigs, squirrels, and rats it is most pronounced; and it is here only necessary to mix a few drops of the blood with an equal amount of water, when the process may be directly observed with the microscope. Human blood, as also that of the pig and the ox, is much more refractory, and is not well adapted for the preparation of the pigment in its crystalline state.

The form of the crystals varies in different animals. We thus find hexagonal platelets in the squirrel, rhombic tetrahedra in the guinea-pig and various birds, rhombic needles in man, etc.

In its chemical behavior oxyhæmoglobin manifests its albuminous nature. It is thus coagulated on boiling, and on treating with absolute alcohol and most of the salts of the heavy metals. It is to be noted, however, that the process of coagulation is associated with the decomposition of the compound into hæmatin and globin, which 
latter is precipitated in its coagulated state. From its solutions the substance is thrown down by half-saturation with ammonium sulphate. On reduction with ammonium sulphide, with Stokes' reagent, or during the process of putrefaction, it is transformed into hæmoglobin. Other reducing agents, such as sodium hydrosulphite, give rise to the formation of so-called pseudohæmoglobin, which apparently stands midway between oxyhæmoglobin and hæmoglobin in containing less oxygen than the former, but more than the latter. Its spectrum, however, is the same as that of the completely reduced hænıglobin.

In sufficiently dilute solution oxyhæmoglobin shows two bands of absorption between $\mathrm{D}$ and $\mathrm{E}$. The one to the left, which is not so wide as the other, but darker and more sharply defined, borders on $\mathrm{D}$, while the second lies at $\mathrm{E}$.

The Quantitative Estimation of Oxyhæmoglobin.-This is best accomplished by Hoppe-Seyler's method, which is based upon the comparison of a given amount of diluted blood with a standard solution of crystallized oxyhæmoglobin. This solution is prepared by dissolving 2 grammes of the pure coloring-matter in 50 c.c. of distilled water. The oxyhæmoglobin is then transformed into carbon monoxide hæmoglobin by passing a current of the gas through the solution to saturation. It is then stored in drawnout and sealed glass tubes, such that each tube contains about 6 c.c. The contents of each tube, when diluted with ten times its volume of water, will then represent a 0.2 per cent. solution of the oxyhæmoglobin.

A carefully measured or weighed amount of blood, not exceeding 0.5 c.c., is now diluted with water that has been saturated with carbon monoxide to exactly 5 c.c. A small drop of a very dilute solution of sodium hydrate is added if necessary to remove any turbidity that may exist. 'This solution is further saturated with carbon monoxide, and freed from fibrin by filtration. The filtrate should measure exactly 4 c.c. The comparison of the two solutions, and the further dilution of the blood with carbon monoxide water then takes place in the so-called double pipette of Hoppe-Seyler. The color of the two solutions is here equalized, and the amount of hæmoglobin present in the specimen of blood calculated from the degree of dilution.

Example.-Suppose that we started with 0.5 gramme of blood, and that the standard solution contained 0.002 gramme of oxyhæmoglobin in the cubic centimeter. The 4 c.c. of the diluted and filtered blood are further diluted in the pipette to 22 c.c, which corresponds to a total solution of 27.5 c.c. for the total 5 c.c. of the first dilution. In these 27.5 c.c., which represent the original 0.5 gramme of blood, there will consequently be $27.5 \times 0.002=0.0550$ gramme of hæmoglobin. The percentage will accordingly be 11 per cent.

In the clinical laboratory other forms of apparatus are in use, such as the homometers of Dare and Fleischl, and the homoglobin- 
ometer of Sahli. ${ }^{1}$ In the first the color of the blood is compared with that of a glass wedge that has been stained with the golden purple of Cassius. The same principle holds good for the second. In the third a standard solution of hæmatin hydrochlorate is employed.

The spectro-photometric determination of the blood coloringmatter is not described at this place. It is undoubtedly the most exact, but necessitates the use of costly apparatus, which will be found in only few laboratories.

It has been pointed out that hæmoglobin is characterized by the readiness with which it combines with certain gases, and we have just considered the most important of these compounds. Others are carbohæmoglobin, carbon monoxide hæmoglobin, nitric oxide hæmoglobin, sulphohæmoglobin, and cyanhæmoglobin.

Carbohæmoglobin.- Three different forms are said to exist, which have been respectively termed $\alpha, \beta$, and $\gamma$ carbohomoglobin, but they are comparatively little known. According to Bohr, the carbon dioxide in these compounds is united with the albuminous radicle of the hæmoglobin, while the oxygen of oxyhæmoglobin is combined with the pigmented group. He accordingly finds that when a solution of hæmoglobin is shaken with a mixture of oxygen and carbon dioxide both of these gases are taken up independently of each other.

Carbon Monoxide Hæmoglobin.-This compound results from the union of one molecule hæmoglobin with one molecule of carbon monoxide, and is characterized by its greater stability as compared with oxyhæmoglobin. The carbon monoxide is in this case united with the pigment radicle of the hæmoglobin at the same point where oxygen usually enters, and may be split off in this combination as carbon monoxide hæmochromogen. Like the native hæmochromogen, the carbon monoxide compound can be obtained in crystalline form, and on exposure to the air is likewise transformed into hæmatin. Under the same conditions the hæmoglobin compound is gradually reconverted into oxyhæmoglobin.

Blood containing carbon monoxide hæmoglobin is characterized by its cherry-red color, its resistance to putrefactive changes in the absence of oxygen, and by its spectrum. This is similar to that of oxyhæmoglobin, but its two bands of absorption, between $\mathrm{D}$ and $\mathrm{E}$, are placed rather nearer the violet end of the spectrum. Unlike the spectrum of oxyhæmoglobin, however, that of the carbon monoxide compound is not changed to the hæmoglobin spectrum on treating with reducing agents. Should oxyhæmoglobin be simultaneously present, a mixed spectrum of the two substances is obtained.

Such blood, moreover, when treated with double its volume of a solution of sodium hydrate (sp. gr. 1.3), is not changed to a dirty brownish mass, with a tint of green, as with normal blood, but presents a beautiful red color, which changes to brown only on standing.

1 For a description of these, see Simon's Clinical Diagnosis, Lea Bros., 6th edition. 
In its crystalline state carbon monoxide hæmoglobin may be obtained by saturating a sufficiently concentrated solution with carbon monoxide and cooling the mixture to $0^{\circ} \mathrm{C}$., when one-fourth of its volume of cooled alcohol is added. On standing in the refrigerator the substance separates out in the form of bluish-red crystals, which are isomorphous with those of oxyhæmoglobin, but much more stable.

Nitric Oxide Hæmoglobin.-This compound is more stable even than carbon monoxide hæmoglobin, and, like this, may be obtained in crystalline form. Its spectrum is similar to that of carbon monoxide hæmoglobin. The bands, however, are less sharply defined and paler than those of that compound, and, like these, do not disappear on the addition of a reducing agent. The substance is met with in poisoning by the gas in question.

Cyanhæmoglobin.-This has been obtained in crystalline form by v. Zeynek; it contains 1 molecule of hydrocyanic acid or the univalent cyanogen radicle in firm combination. Its toxicity is comparatively slight.

Sulphohæmoglobin.-This is met with in poisoning with hydrogen sulphide. The blood then becomes dark and of a dull-greenish tint and on spectroscopic examination it shows one band in the green portion and a second one in the orange between $\mathrm{C}$ and $\mathrm{D}$.

Kathæmoglobin.-This term has been applied by Van Klaveren to a decomposition-product of hæmoglobin which results on boiling a mixture of defibrinated blood with an alcoholic solution of sodium hydrate. Unlike hæmatin, it is not a decomposition-product that is free from albumin, but is a proteid which is still quite closely related to hæmoglobin. Arnold first described this substance as neutral hæmatin. It contains somewhat less iron than hæmoglobin, a portion being split off during its formation as a water-soluble organic compound which escapes on dialysis.

Methæmoglobin.-Methæmoglobin is a pigment which normally does not occur in the blood, but is found after the ingestion of large amounts of potassium chlorate, antifebrin, potassium permanganate, turpentine, kairin, thallin, following the inhalation of nitrite of amyl, ether, etc. It is encountered also in hemorrhagic transudates and cystic fluids, and may occur in the urine when methæmoglobinæmia exists.

The elementary composition of methæmoglobin is the same as that of oxyhæmoglobin, but its molecular structure is manifestly different, as in a vacuum it does not give up its oxygen. On treating with reducing agents or on exposure to putrefactive organisms, in the absence of oxygen, it is converted into hæmoglobin. When oxyhæmoglobin is decomposed with dilute acids or alkalies, methæmoglobin is formed at some stage of the process, and precedes the formation of hæmatin. During the preservation of oxyhæmoglobin in the dry state, moreover, a partial transformation into methæmoglobin is very likely to occur. The substance is crystal- 
lizable, and may be obtained in this form by treating a concentrated solution of oxyhæmoglobin with a saturated solution of potassium ferricyanide until the color has changed to a port-brown. The mixture is cooled to $0^{\circ} \mathrm{C}$., and treated with one-quarter of its volume of cooled alcohol. When kept in the refrigerator crystallization takes place in the course of a few days. The crystals are of a brown color, and occur as needles, prisms, or hexagonal platelets. They may be purified by recrystallization from water in the presence of alcohol. An aqueous solution of the substance is brown, while its alkaline solutions are beautifully red. On exposure to sunlight its neutral and dilute solutions gradually assume a dark-red color, which is thought to be referable to a transformation of methæmoglobin into photomethcemoglobin. On spectroscopic examination such solutions show one broad band of absorption in the green portion of the spectrum no matter whether the reaction is alkaline, neutral, or acid. Methæmoglobin proper under the same conditions gives a fairly broad band of absorption between C and D, nearer $C$, which is characteristic, and disappears on the addition of sodium hydrate solution. In addition, two different bands may at times be seen between $\mathrm{D}$ and $\mathrm{E}$, which are thought to be referable, however, to a contamination of the substance with hæmoglobin. Some observers further speak of an additional band near $\mathrm{F}$, but this is not characteristic. On reduction of the alkalinized solution with ammonium sulphide the spectrum of hæmochromogen results.

According to v. Zeynek, photomethomoglobin is in reality cyanhæmoglobin, and is formed through the action upon the hæmoglobin of hydrocyanic acid which results from the ferricyanide in consequence of the effect of sunlight. Pure methæmoglobin is not affected by sunlight.

Like oxyhæmoglobin, methæmoglobin is capable of combining with certain gases to form molecular compounds. Of these, a carbon dioxide methoemoglobin, a methomoglobin sulphide, and a cyanmethoemoglobin have been described.

Hæmatoporphyrin.-This substance results from hæmatin when this is treated with concentrated sulphuric acid saturated with hydrobromic acid :

$$
\underset{\substack{32 \\ \text { Hæmatin. }} \mathrm{C}_{32} \mathrm{~N}_{4} \mathrm{FeO}_{4}}{\mathrm{C}_{2}}+2 \mathrm{H}_{2} \mathrm{O}+2 \mathrm{HBr}=\underset{\text { Hæmatoporphyrin. }}{2 \mathrm{C}_{16} \mathrm{H}_{18} \mathrm{~N}_{2} \mathrm{O}_{3}}+\underset{\mathrm{FeBr}_{2}}{\mathrm{Fen}}+2 \mathrm{H}
$$

During this process the iron of the hæmatin is split off, and a new pigment, hæmatoporphyrin, is formed. In the circulating blood of vertebrate animals it is not found under normal conditions, but is apparently formed in certain diseases, and during the long-continued administration of sulphonal and related bodies, as also in leadpoisoning and following intestinal hemorrhages, when it may also be found in the urine. Among invertebrates it is said to occur in the integument of the star-fish, in certain snails, in the earth-worm, in various sponges, etc. 
Hæmatoporphyrin is isomeric with bilirubin. On reduction it yields a pigment which is apparently identical with Maly's hydrobilirubin and Jaffe's urobilin. It may be obtained in erystalline form as a hydrochlorate, while the pigment itself is amorphous. Its solutions in acid alcohol present a beautiful purple color, which is changed to a violet blue on adding an excess of the acid. It is most conveniently obtained by starting with hæmin and decomposing this with glacial acetic acid that has been saturated with hydrobromic acid. Its solutions in acid alcohol give two bands of absorption. One of these is located between $\mathrm{C}$ and $\mathrm{D}$, while the second band, which is much darker and more strongly defined, occupies a position midway between $\mathrm{D}$ and $\mathrm{E}$, and extends as a shadow toward D. In dilute alkaline solutions, on the other hand, we find four bands: one between $\mathrm{C}$ and $\mathrm{D}$; a second one, which is broader than the first, between $\mathrm{D}$ and $\mathrm{E}$ and about $\mathrm{D}$; a third band, between $\mathrm{D}$ and $\mathrm{E}$, near $\mathrm{E}$; and finally a further band between $\mathrm{b}$ and $\mathrm{F}$, which is the widest and much darker than the rest. On treating with an alkaline solution of zinc chloride this spectrum gradually passes into a new spectrum with only two bands, of which one is seen about $\mathrm{D}$, and the other between $\mathrm{D}$ and $\mathrm{E}$.

The transformation of acid hæmatoporphy rin to the alkaline form can be readily effected by oversaturating a solution of hæmatoporphyrin in sulphuric acid with pyridin; the garnet color changes to a chestnut, while the solution remains perfectly clear.

On oxidation hæmatoporphyrin yields the same hæmatinic acids as hæmatin (see above). The resulting tribasic acid, $\mathrm{C}_{8} \mathrm{H}_{8} \mathrm{O}_{5}$, on reduction with hydriodic acid is transformed into the tribasic hæmotricarbonic acid, $\mathrm{C}_{8} \mathrm{H}_{12} \mathrm{O}_{6}$; this is possibly identical with ethyltricarballylic acid, which has been obtained synthetically.

Closely related to hæmatoporphyrin, apparently, is the phylloporphyrin which may be obtained from the chlorophyl of plants. From its formula, $\mathrm{C}_{16} \mathrm{H}_{18} \mathrm{~N}_{2} \mathrm{O}$, and that of hæmatoporphyrin, $\mathrm{C}_{16} \mathrm{H}_{18^{-}}$ $\mathrm{N}_{2} \mathrm{O}_{3}$, it is suggested that both are different oxidation-products of one and the same substance. On reduction with phosphonium iodıde in glacial acetic acid hæmatoporphyrin yields a product of the composition $\mathrm{C}_{16} \mathrm{H}_{18} \mathrm{~N}_{2} \mathrm{O}_{2}$, which Nencki and Saleski designate as mesoporphyrin. This manifestly occupies a position intermediate between hæmatoporphyrin and phylloporphyrın. On still further reduction methyl-propyl-pyrrol is obtained (see Hæmatin), and it is noteworthy that the same body has also been obtained in the same manner from phylloporphyrin. We see that the principal animal pigment is thus intimately related to the principal pigment of the higher plants. The spectra of hæmatoporphyrin, mesoporphyrin, and phylloporphyrin are very similar; they differ from one another by only a slight displacement of all bands in one direction.

Hæmatoidin.-This pigment, which was first observed by Virchow in old extravasations of blood, in which it may occur in crystalline form, is now known to be identical with bilirubin. As a separate substance it therefore no longer meritș consideration, 
I have pointed our that in some of the lower animals hæmoglobin is also found, and may occur in the blood either as such or bound to certain cellular elements which may be compared to the red corpuscles of the vertebrates. In other invertebrate animals we find no hæmoglobin, but related respiratory pigments, which are partly violet or purplish red in color, and partly blue. The former comprise the so-called floridins, of which little is known, while the latter group is represented by the oxy-compound of hæmocyanin.

Hæmocyanin is of special interest, as it is apparently closely related to hæmoglobin, but contains copper in its molecule in the place of iron. Unlike hæmoglobin, however, hæmocyanin is itself colorless, while its oxy-compound, oxyhæmocyanin, presents a beautiful blue color. According to Frederique, oxyhæmocyanin yields an albuminous substance on decomposition with hydrochloric acid as also with nitric acid, which may be compared to globin, and a copper-containing pigment which corresponds to hæmatin. Henzel however, was unable to confirm this observation. He obtained an albuminous body of the character of an acid albumin, but no histon. The copper of the hæmocyanin is not present in firm organic combination, but in a loosely combined form, analogous to a copper albuminate.

Henze has recently succeeded in crystallizing the hæmocyanin, and on elementary analysis obtained the following values: $\mathrm{C}=53.66$; $\mathrm{H}=7.33 ; \mathrm{N}=16.09 ; \mathrm{S}=0.86 ; \mathrm{Cu}=0.38$, and $\mathrm{O}=21.67$.

On reduction with ammonium sulphide or on exposure to an atmosphere of an indifferent gas like $\mathrm{H}, \mathrm{CO}_{2}, \mathrm{~N}$, etc., the oxyhæmocyanin yields the colorless hæmocyanin. On spectroscopic examination hæmocyanin and oxyhæmocyanin show a shadow at both ends of the spectrum, which is more marked in the latter ; true absorptionbands, however, are not observed.

Other invertebrate animals contain only lipochromic pigments in their hæmolymph, which probably do not possess a respiratory function however, and in the lowest forms of life, of course, special oxygen-carriers are not required. 


\section{CHAPTER XV.}

\section{THE LYMPH.}

IN its course through the blood-capillaries a portion of the plasma passes out through the vessel-walls and enters a system of irregular interfascicular clefts, which are bounded by bundles of fibrous tissue and constitute the radicles of the lymphatic system. Through these clefts the plasma reaches the individual cells of the various tissues and organs of the body, and supplies these with the requisite nourishment, while at the same time it takes up the waste matter that is formed in the metabolism of the cells, and through the lymph-vessels carries these into the venous current of the blood. This fluid, which thus contains the various constituents of the blood-plasma and the decomposition-products of the cells, is termed the lymph.

In addition to the lymph-vessels proper and their radicles, the lymph-clefts, this fluid is also found in the so-called serous cavities of the body, viz., the pleura, the peritoneal and pericardial cavities, in the ventricles of the brain and the spinal cord, in the communicating subarachnoid space, and also in the anterior chamber of the eye. In health, however, these cavities contain but little fluid, and quantities sufficient for analytical purposes can normally be obtained only from the pericardial sac, and at times from the subarachnoid space. Under pathological conditions, however, large accumulations of fluid may be observed, and not only in the serous cavities of the body, but also in the areolar connective tissue, beneath the skin, and beneath the muscles. When due to circulatory disturbances, a hydræmic condition of the blood, or an insufficient elimination of water through the kidneys, such accumulations of fluid are spoken of as transudates, while the term exudates is applied to similar accumulations of inflammatory origin.

Formerly it was supposed that the lymph resulted from the bloodplasma through a simple process of filtration or transudation only, and in accordance with this view we find that in the various accumutions of lymph the salts and extractives are present in about the same amount as in the blood-plasma. Heidenhain, however, has shown that the flow of the lymph-current is far too sluggish to supply the various organs of the body with the proper amount of nourishment, supposing its composition to be everywhere the same as that of the blood-plasma. We are hence forced to the conclusion that the endothelial cells of the capillaries possess a selective secretory power similar to that of the renal epithelium, and are thus 
capable of furnishing to each tissue its proper amount and kind of food. This view, however, does not preclude the possibility that some of the constituents of the plasma nuay pass over into the lymph by a simple process of filtration, and it is likely that this actually occurs with the water and most of the mineral salts.

According to its origin, then, we may expect to find certain differences in the chemical composition of the lymph, and we find, as a matter of fact, that such differences exist. These are, however, essentially of a quantitative kind, and qualitatively we find the same constituents in the lymph from the various districts as compared with each other and with the blood-plasma.

Like the blood-plasma, the lymph consists of a liquid portion, the lymph-plasma, and cellular elements, the lymph-corpuscles. These latter are essentially mononuclear leucocytes, and are largely derived from the lymphoid tissue which abounds in the course of the lymphvessels. Red corpuscles are either lacking entirely or they are present in very small numbers. They are of much darker color than those of the blood, but on exposure to the air they take on the bright-red color of oxyhæmoglobin. According to some observers, they represent transition-forms between the leucocytes and the normal red corpuscles of the blood.

In various inflammatory diseases of the serous cavities the leucocytes may be present in very large numbers, and in extreme cases, indeed, they predominate to such an extent that the liquid character of the lymph may be almost entirely lost.

The appearance of the lymph is dependent upon the number of the leucocytes and the amount of fat present. As obtained from fasting animals, it represents a slightly viscid, straw- or rose-colored, transparent fluid. During the process of digestion, on the other hand, and especially after the ingestion of much fatty food, it becomes more or less opaque, owing to the admixture of the fat, which is carried into the general lymph-current through the chyle, viz., the lymph coming from the intestinal canal.

The odor of the lymph, like that of the blood, is different in different animals. Its taste is salty, and the reaction slightly alkaline. The specific gravity varies between 1.015 and 1.021 .

The amount of lymph which is produced in the twenty-four hours is largely influenced by the process of digestion. During starvation a smaller amount is thus found than after the ingestion of focd, and it appears, moreover, that an albuminous diet causes a much greater increase than one of carbohydrates. Active muscular exercise has a similar stimulating effect upon its formation. Artificially the amount of lymph can be increased by the intravenous injection of so-called lymphagogues, of which Heidenhain recognizes two classes, viz., those which merely increase the amount of water in the lymph and those which also bring about an increase of the organic solids. The former include such crystalline substances like sugar, urea, sodium chloride, etc.; and Heidenhain supposes that their action is dependent upon their passage into the lymph, where they exert 
a stimulating effect upon the cells of the tissues and cause an absorption of cellular water. The latter, on the other hand, are in part unknown substances which can be extracted from the muscles of the crab, from the head and body of leeches, from the bodies of anodonts, from the liver and intestines of the dog, and also comprise the peptones and egg-albumin. The influence of these substances is apparently exerted upon the endothelial cells of the blood-capillaries, whereby the secretory power is increased, and we accordingly find more albumin in the lymph than in the remaining blood-plasma. Such lymph then, in contradistinction to the cellular lymph which is found in the first instance, may be termed blood-lymph. The state of the blood-pressure, according to Heidenhain, is of no moment in bringing about these changes, and he found, as a matter of fact, that variations between 10 and $20 \mathrm{Hgmm}$. on the one hand, and 150 to $200 \mathrm{Hgmm}$. on the other hand, are of little influence upon the amount of lymph that is produced. This view, however, is in all probability not final.

According to Bunge, the amount of lymph that is formed in the twenty-four hours by the human being amounts to about 4000 c.c.

A general idea of the chemical composition of lymph may be formed from the following analysis of Munck and Rosenstein. The material was obtained from a fistula in the thigh of a young woman. Accompanying this is an analysis of the blood-plasma (taken from Hammarsten) for comparison:

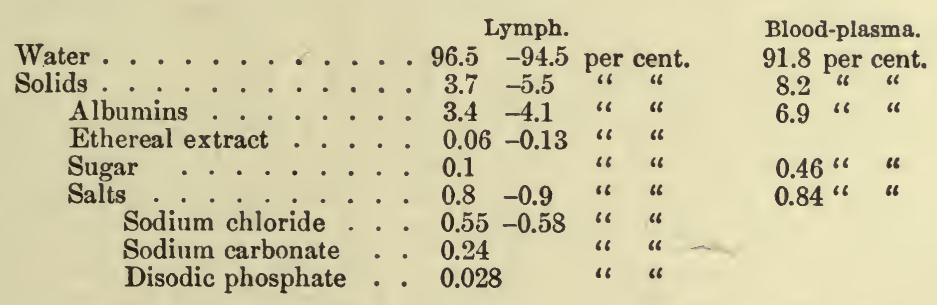

Of these constituents, the fat is subject to the greatest variations, and is, of course, always more abundant during the process of digestion in the chyle than in any other lymphatic district. In Munck's case the amount rose to 4.7 per cent. after the ingestion of a large amount of fatty food, and decreased to $0.06-0.26$ per cent. when food was withheld for twenty-four hours.

The fat is present in the lymph to the greatest extent as neutral fat, and it is to be noted that it here exists in a state of emulsion, so that upon microscopical examination the chyle more especially will be seen to contain innumerable fat droplets, which vary but little in size and have no tendency to flow together, as in the case of milk. Why this is we do not know, but it has been suggested that each fat droplet is surrounded by a delicate albuminous envelope, which is derived from the normal albumins of the lymph. On the other hand, it is conceivable that the surface layer of each droplet con- 
sists of modified fat or of a denser layer of the fluid in which it is suspended.

The fats which are found in the lymph are always identical with those of the food, and can be recovered for analytical purposes by extracting with ether. In its course through tissues which are rich in fat no fat is absorbed.

Soaps are present in the lymph in only very small amounts.

The albumins which are found in the lymph are the same as those of the blood, and are present in the same ratio to each other. The amount varies somewhat with the character of the lymph, but is normally always smaller than that of the blood-plasma. Like the blood-plasma, so also does the lymph coagulate on standing. The coagula, however, are very delicate and tend to separate out in fractions. Some transudates, indeed, such as pericardial effusions and hydrocele fluid, do not coagulate spontaneously at all, while coagulation occurs at once if leucocytes or blood is added. The peculiar behavior of such lymph is no doubt due to the absence of cellular elements. It is to be noted also that following the injection of those substances which prevent coagulation of the blood coagulation of the lymph similarly does not occur.

Other albumins besides serum-albumin, serum-globulin, and fibrinogen are not found in normal lymph, as we obtain it from the thoracic duct, for example. Under pathological conditions, however, the exudates more particularly may contain the various albuminous derivatives of the leucocytes, such as nucleins, nucleoalbumins, etc. In cysts of the ovaries and their appendages metalbumin or paralbumin may further be found.

The amount of sugar which is found in normal lymph is fairly constant, and is derived from the hepatic lymph. It can be increased artificially by ligating the ureters and then injecting glucose into the blood. It is noteworthy that under such conditions the lymph may contain a larger percentage of sugar than the blood itself. This further shows that the formation of lymph cannot be explained upon the basis of filtration and osmosis only, and demonstrates the specific activity of the endothelial lining of the capillaries. Under pathological conditions, it is claimed, sugar may be altogether absent.

The extractives of the lymph are essentially the same as those of the plasma. In certain districts, however, the one or the other will be found to preponderate, and in some localities we further meet with extractives which are peculiar to that particular region. In the cerebrospinal fluid, for example, pyrocatechin has been found; allantoin is present in the allantoic fluid and in ascitic accumulations; succinic acid and inosit may be obtained from hydrocele fluid in which cholesterin may also be present in very considerable amount. Of interest also is the fact that in the lymph of the thoracic duct bile acids can be demonstrated, and it appears from the researches of Croftan that the acids in question are present in the leucocytes. They supposedly represent that portion of the bile acids 
which has escaped decomposition in the intestinal canal and has been resorbed. They subsequently enter the blood-current, carried by the leucocytes, and are again eliminated in the bile.

Traces of urea, uric acid, lecithin, xanthin, kreatin, and lactic acid are commonly found.

Of ferments, a diastatic ferment and a glucolytic ferment have been described.

The gases of the lymph differ from those of the blood in the presence of larger amounts of carbon dioxide- 35 to 45 per cent.as compared with arterial blond, and smaller amounts than are found in venous blood. The tension of the carbon dioxide, as compared with venous blood, is lower, which suggests that a portion of the gas enters the blood from the lymph through a reverse secretory activity of the capillary endothelium. Oxygen is present only in traces, while the amount of nitrogen is the same in both, viz., 1.6 per cent.

For purposes of comparison and reference, a few analyses of some of the more important normal and pathological varieties of lymph are appended. Some of these will be considered in greater detail in subsequent chapters.

Analysis of Human Pericardiat, Fluid (Hammarsten).

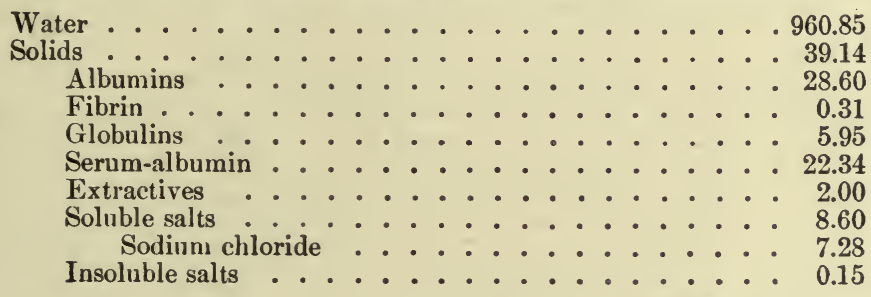

Analysis of Dog's Chyle (Hoppe-Seyler).

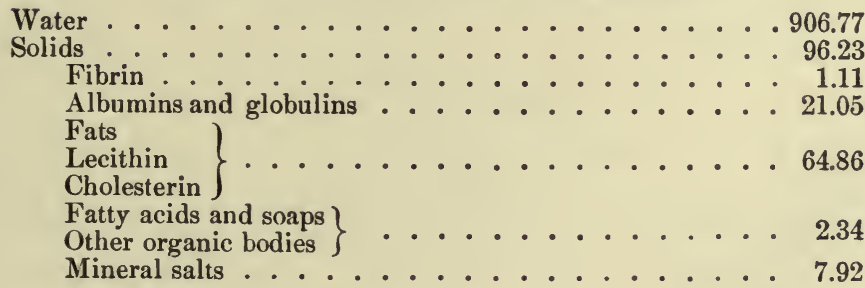

Analysis of Aqueous Humor of Calf (Halliburton).

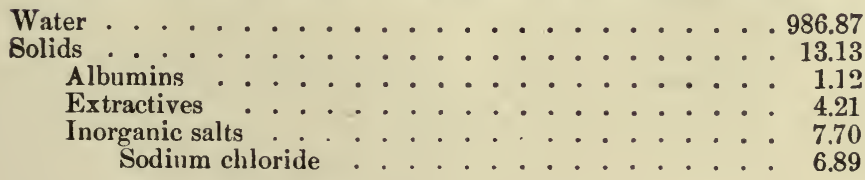


Analysis of Cerebrospinal Fluid (Gautier).

Water .................. . . 987.00

Solids . . . . . . . . . . . . . 10.59

Albumins ............... 1.10

Fats . . . . . . . . . . . . . 0.09

Cholesterin . . . . . . . . . . . 0.21

Alcoholic and aqueous extracts, minus salts, but including sodium lactate . . . . . . . . . . . . 2.75

Salts . . . . . . . . . . . . 6.44

Chlorides . . . . . . . . . . . . 6.14

Sulphates . . . . . . . . . . 0.20

Earthy phosphates . . . . . . . . 0.10 Ammonia . . . . . . . . . . . traces.

Analyses of Pleural Effusions (Gautier).

Water pleurisy.

Acute
pleurisy
937.60
54.40

Organic matter $\ldots 0.09$
Fibrin

Mineral matter . . . . . 8.00

$\begin{array}{cc}\begin{array}{c}\text { Chronic } \\ \text { pleurisy. }\end{array} & \begin{array}{c}\text { Hydrothorax } \\ \text { (cardiac). }\end{array} \\ 933.80 & 958.70 \\ 58.20 & 32.30 \\ 0.00 & 0.19 \\ 8.00 & 9.00\end{array}$

Analyses of Pertoneal Effusions (Drivon and Scherer).

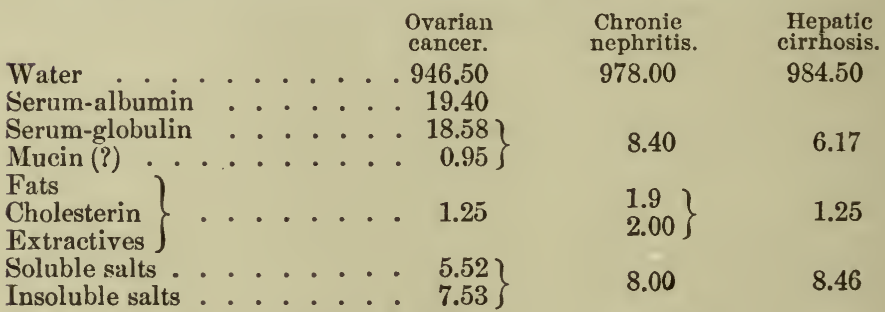

ANalysis of Hydrocele Fluid (Hammarsten).

Water . . . . . . . . . . . . 938.85

Solids

Fibrin .................. 0.59

Globulins . . . . . . . . . . 13.25

Serum-albumin . . . . . . . . . . . 35.94

Ethereal extract . . . . . . . . . . 4.02

Soluble salts . . . . . . . . . 8.60

Insoluble salts . . . . . . . . . . . 0.66

Analysis of Amniotic Fluid (Labroche).

Human.

Water ...................... 987.300

Solids . . . . . . . . . . . . 16.700

Serum-albumin . . . . . . . . . 2.590

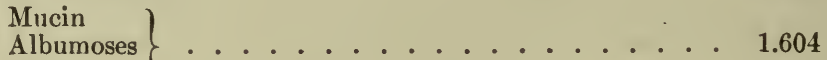

Glucose

Urea .................. 0.450

Fats ................ . . 0.356

Mineral salts 7.695

Sodium chloride . . . . . . . . . . 6.071

Disodium phosphate . . . . . . . . . 1.621

Sulphates . . . . . . . . . traces.

Salts of calcium and potassium . . . . . none. 
Analysis of lymph from Cellular Tissue (Edema).

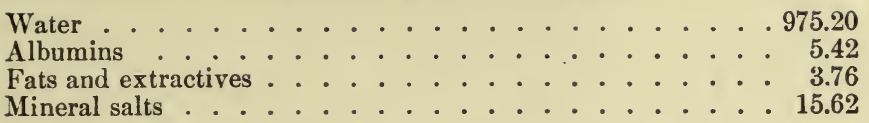

Analysis of Pus.-The composition of the leucocytes which enter into the formation of pus has been considered (pages 324 and 342). An analysis of pus-serum is here given, which is taken from Robin :

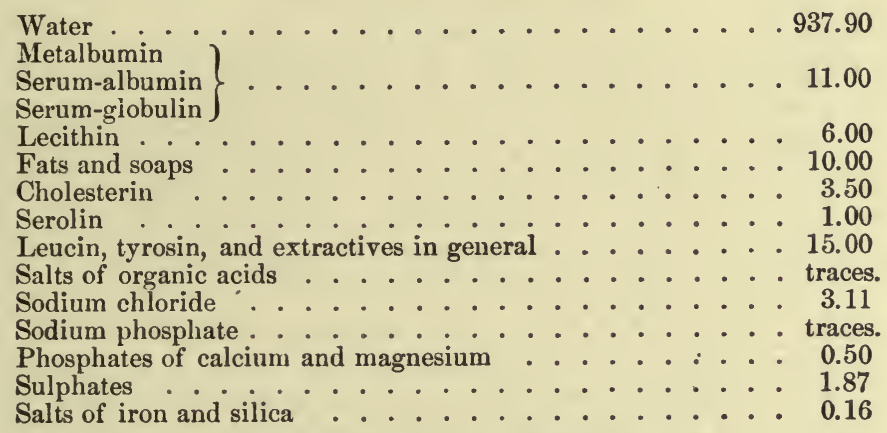

\section{THE SYNOVIAL FLUID.}

The synovial fluid, though not a lymph in the narrower sense of the term, is for convenience' sake briefly described at this place. It is the specific secretion of the synovial membrane of the joints, and constitutes a strongly alkaline, viscid, yellowish, somewhat cloudy fluid. In addition to the common albumins, fats, salts, and extractives of the lymph, it contains also a peculiar mucinous body, which is termed synovin, and apparently belongs neither to the nucleo-albumins nor the mucins or mucoids. It can be precipitated with acetic acid and coagulates on the application of heat. Of its nature, however, nothing further is known. In addition, another mucin-like body is found which is rich in phosphorus, and probably belongs to the nucleo-albumins.

Quantitatively the composition of the synovial fluid varies with exercise and rest in such a manner that on motion the mucinous body, as also the albumins and extractives, increase, while the salts diminish. This is shown in the appended analyses, which are taken from Frerichs :

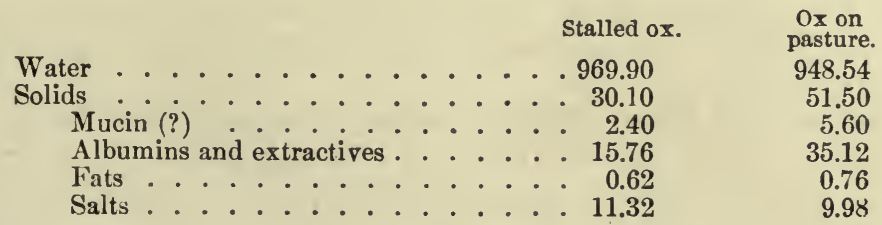




\section{CHAPTER XVI.}

\section{THE MUSCLE-TISSUE.}

I HAVE pointed out that while in the monocellular organisms the various functions of the body are carried on by the single cell, a gradual division of labor occurs as we ascend in the scale of both animal and vegetable life, where groups of cells are set aside for the performance of certain special functions. Structurally this division of labor finds its expression in a more or less well-marked deviation from the original type, as has been shown. At first sight, it is thus difficult to connect the highly differentiated muscle-cell with the apparently much more simple ovum from which it has originated. The element of reproduction and secretion is here manifestly placed in the background, while in its co-ordinate and rapid contraction on stimulation we have abundant evidence of its highly specialized function. That this should further be expressed in the chemical composition of the cells suggests itself at once. As a matter of fact, we here find substances which may be regarded as specific muscle components, and it seems warrantable to assume that a definite connection exists between these bodies and the special function of the cell. On chemical examination we may then further expect to meet with the various products of katabolism, so far as these are found in the muscle-tissue proper, and have not as yet been removed by the blood or the lymph.

Before proceeding to a study of these various substances in detail, a few analyses of muscle-tissue are here introduced, which will furnish a general idea of its chemical composition. Qualitatively this is fairly constant, but quantitative variations occur which are often very marked.

In preparing the tissue for analytical purposes, the blood should first be washed out entirely with dilute saline solution ( 0.6 per cent.). Fibrous tissue and fat must be dissected away as far as possible and all larger bloodvessels removed. The material is then further scraped, so as to get rid of as much of the connective tissue as possible which binds the individual fibres together, and is now ready for examination.

Analyses of Fresh Muscle-tissue (Neumeister).-The figures represent average values, which have been collected from various sources, and have reference to mammalian muscle-tissue in general, unless otherwise stated. 
Per cent.

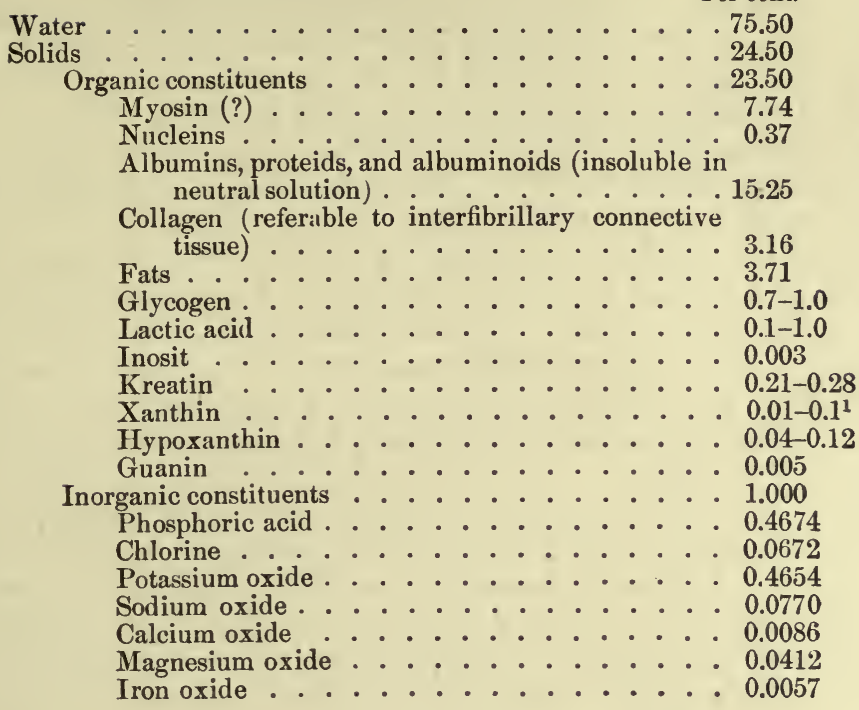

In studying this analysis we observe that, aside from the mineral constituents, various bodies are encountered here which represent distinct products of katabolism, and which are hence most likely not concerned in the specific function of the muscle-tissue. These comprise the common extractives, viz., xanthin, hypoxanthin, guanin, kreatin, lactic acid, and possibly also inosit. They are no doubt formed as a consequence of cellular activity, but play no róle in the function of the muscle proper. On the other hand, we meet with food-stuffs proper, viz., albumins, carbohydrates, and fats, and we may a priori expect that all these substances, conjointly or individually, are directly concerned in the contractile function of the cell.

\section{THE MUSCLE-ALBUMINS.}

As in the case of all tissues of the body, the muscle-tissue also consists of a liquid portion, the so-called muscle-plasma, and a more solid portion, which may be termed the muscle-stroma.

Muscle-plasma may be obtained in the following manner: while the animal is still living the blood is thoroughly washed from the large skeletal muscles by injecting into the larger arteries a dilute saline solution that has been warmed to the temperature of the body, and allowing the fluid to escape from the corresponding veins. This is continued after death until the outflowing water is colorless. The muscles are then rapidly dissected off, ground to a pulp together with pumice-stone, and passed through a filter-press. The resulting liquid is the muscle-plasma. 
While this procedure is applicable in the case of mammalian muscle-tissue in general, special precautions are necessary if the muscles of the lower animals are to be studied. In the frog, for example, the tissue, after removal of the blood, must be frozen in a gradual manner, after which the entire process is continued at a temperature below $-3^{\circ} \mathrm{C}$. A snow-like mass is finally obtained, which melts at $-3^{\circ} \mathrm{C}$.

The color of the muscle-plasma varies with the color of the muscles from which it has been obtained. In the case of the dog it is of a brownish color, in rabbits it is yellowish red, and in frogs a light yellow. 'The particular shade of color, as will be seen later, is a direct expression of the degree of functional activity of the individual muscles, and is in part due to hæmoglobin and in part to certain lipochromes.

The reaction of the plasma is neutral or slightly alkaline.

On standing for a length of time mammalian muscle-plasma gradually undergoes a process of coagulation, but it is to be noted that the coagulum which separates out is slight in amount. In the case of the frog, on the other hand, the entire bulk of the plasma becomes gelatinous, and, in contradistinction to the mammalian plasma, this process begins at a temperature of $0^{\circ} \mathrm{C}$. As in the case of the blood-plasma, the coagulum gradually contracts, and the liquid which remains is spoken of as muscle-serum. The reaction is then acid. The substance which composes the clot is termed myogen-fibrin. The behavior of muscle-plasma is thus quite similar to that of blood-plasma, and here, as there, the resulting fibrin is derived from an albuminous substance which was previonsly present in solution. This substance is termed myogen. In addition the muscle-plasma contains another albuminous body, myosin; and it appears from the researches of v. Fürth that these two substances are the only soluble albumins which are contained in muscle-tissue, if we disregard a variable amount of a soluble myogen-fibrin, which is itself a derivative of myogen.

Myogen.-Isolation.-Myogen is most conveniently obtained from muscle-plasma after the myosin has been removed by the previous addition of ammonium sulphate to the extent of 28 per cent. The resulting precipitate is filtered off and the filtrate saturated with the same salt in substance. The myogen is thus thrown down together with the soluble myogen-fibrin. It is washed with a saturated solution of ammonium sulphate, dissolved in water, and freed from the soluble myogen-fibrin by heating to $40^{\circ} \mathrm{C}$, , when this is transformed into the insoluble form and is filtered off. The remaining solution contains the myogen in pure form.

In its general properties it resembles the albumins proper, in contradistinction to the globulins. It is soluble in water, and can be precipitated by alcohol, by salting with ammonium sulphate, sodium chloride, and magnesium sulphate. The two latter salts, however, do not cause a complete precipitation. Alcohol (92 per cent.) 
renders the substance insoluble to a slight extent, but the greater portion is refractory in this respect.

By acetic acid myogen is precipitated only in the presence of a neutral salt, but redissolves in an excess of the acid, with the formation of syntonin. The tendency to the transformation into albuminates is indeed more marked in the case of the soluble musclealbumins, in general, than with any other forms. Mineral acids are in this respect still more active than acetic acid, and as a consequence a precipitation of myogen is observed only when such acids are present in certain proportion.

Carbonic acid and the salts of the heavy metals precipitate myogen only in the presence of a neutral salt.

Myogen coagulates at a temperature of from $55^{\circ}$ to $65^{\circ} \mathrm{C}$. It is not precipitated on dialysis.

Elementary analysis of a myogen preparation obtained from the muscles of a rabbit gave the following average results: $\mathrm{C}=52.69$; $\mathrm{H}=6.93 ; \mathrm{N}=16.20$.

When solutions of myogen are kept at a certain temperature, and in the presence of a definite amount of a neutral salt, the substance is gradually transformed into a soluble form of myogen-fibrin, which differs from myogen in the fact that it is thrown down on dialysis, and in its point of coagulation, which lies at $40^{\circ} \mathrm{C}$. As has been stated, a certain amount of soluble myogen-fibrin seems to occur preformed in the muscle-tissue, and separates out gradually on standing. At $40^{\circ} \mathrm{C}$, , however, this occurs instantaneously. By coagulation the soluble myogen-fibrin is transformed into the insoluble form, the myogen-fibrin proper.

The relative amount of myogen, as compared with myosin (see below), which is found in muscle-tissue varies in all probability with different animals. In rabbits, v. Fürth observed that myogen represented about 80 per cent. of the total amount of soluble albumins.

The amount of soluble myogen-fibrin which is included in the above figures is in mammals apparently very small, as only slight coagula are formed when the plasma is heated to $40^{\circ} \mathrm{C}$. But in the frog large amounts are manifestly present. In some animals, on the other hand, it is apparently absent.

Myosin.-Myosin is conveniently isolated from muscle-plasma bv salting with ammonium sulphate to the extent of 28 per cent. Sodium chloride and magnesium sulphate may also be employed, but it is then necessary to add the salt to saturation.

The substance is a globulin, and, curiously, contains a considerable amount of calcium. It is soluble in dilute saline solutions, and is precipitated from these solutions by salting, as just indicated, by passing a.stream of carbon dioxide through its solutions, by diluting with water, and on dialysis. It is characterized by its pronounced tendency to coagulate, and, unlike myogen, is rendered almost entirely insoluble on precipitation with alcohol. Like this, it is also readily transformed into syntonin or alkaline albuminate on treating 
with acids or alkalies; and here, as there, a precipitation results only if very dilute acids are used. In an excess the precipitate rapidly dissolves. On heating solutions of myosin to $35^{\circ} \mathrm{C}$. the substance is gradually coagulated, while this occurs at once at a temperature of $50^{\circ} \mathrm{C}$.

In its insoluble form myosin is termed myosin-fibrin, which, like the insoluble myogen-fibrin, belongs to the class of the coagulated albumins.

Of special interest, further, is the fact that on evaporating a few drops of a solution of myosin in soda solution on a slide, at a low temperature, a jelly-like material is obtained, which on polariscopic examination is seen to be doubly refracting. In this respect it behaves exactly as the anisotropic material which is found in the dark bands of the voluntary muscle-fibres.

The amount of myosin found in the muscle-tissue of the rabbit is much less than that of myogen, and, according to v. Fürth, corresponds to only 20 per cent. of the total amount of soluble albumins.

Significance of the Common Muscle-albumins.-Of the part which the common muscle-albumins take in the function of the cell little is known that is definite. From the researches of some observers, it appears that the nitrogenous components of the albumins, at least, do not furnish the energy which is here required. Pettenkofer and Voit have thus shown that an increase in the amount of muscular work does not lead to an increased elimination of nitrogen or canses an increase which is insignificant. This view is now generally held; but it must be admitted that evidence is not lacking which suggests that an increased albuminous destruction may occur nevertheless when the amount of work is increased. It has been shown, as a matter of fact, that the total elimination of sulphur, which usually follows that of the nitrogen quite closely, is increased by muscular exercise and diminished thereafter. But while we may admit that the nitrogenous components of albumin may furnish a certain fraction of the energy which is required in muscular work, this is, after all, but slight, and there is abundant evidence to show that by far the greater amount of energy must be referable to the decomposition of non-nitrogenous material.

The question, of course, suggests itself, Do the soluble albumins of the muscle-plasma represent the contractile element of the muscle-tissue? but to this question no answer can as yet be given. We might imagine that in some manner a transformation of the soluble albumins into the fibrin form occurs, and vice versa; but of this we have no evidence in the living tissue. On the other hand, it is supposed that rigor mortis, as well as the rigor which results from exposure of muscle-tissue to a temperature of $47^{\circ} \mathrm{C}$., is owing to such a change, and it is possible that in either event both myosin and myogen pass over into the coagulated state. Folin in a recent 1 dper, however, has again thrown doubt on the correctness of the coagulation theory in the explanation of rigor mortis, and has 
offered evidence which goes to show that, after all, the process may be a physical one, as the muscle-albumins, according to his experiments, take no part in the process, viz., there is no coagulation of the muscle-albumins.

Other Albumins.-Besides myosin and myogen, which latter was formerly termed myosinogen, muscle-plasma was also supposed to contain traces of serum-albumin, myoglobulin, and myo-albumose. v. Fürth, however, has shown that any trace of serum-albumin that may be found is referable to the presence of small amounts of lymph or blood that have not been removed by washing, and that if this is done with special care no serum-albumin can be demonstrated. Halliburton's myoglobulin he regards as identical with myogen, while the existence of a myo-albumose in muscle-plasma has been disproved by more recent investigations. That substances belonging to the albumoses may be found in muscle-tissue after death, when syntonin also is found, is, of course, likely, but in the living tissue their presence can hardly be expected under normal conditions.

Myoproteid is a substance which v. Fürth obtained from the muscle-plasma of fish. Of its chemical nature nothing further is known than the fact that it apparently does not belong to the commonly recognized classes of albumins.

Nucleoproteids are not found in the muscle-plasma, but can be isolated from the muscle-tissue as a whole or from the insoluble material which remains in the filter-press after separation from the plasma. Their amount is small, and in accordance with the slight degree to which the nuclei enter into the structural composition of the musclecell. Larger amounts are obtained from embryonic muscle, where cellular reproduction is, of course, more active. From the tissue of an adult dog Pekelharing obtained about 0.37 per cent. These bodies must be regarded as the material from which the xanthinhases that can always be demonstrated in the musele-tissue are derived. These will be considered in detail later.

Phospho-carnic Acid. - Some years ago Siegfrid announced that after removing the phosphates from extracts of muscle-tissue, and treating with ferric chloride, under the application of heat, a phosphorus-containing iron compound is obtained, which is insoluble in water, but easily soluble in solutions of the alkalies. This substance he regards as the iron salt of an organic acid, which he terms phosphor-carnic acid; the salt he speaks of as carniferrin. On decomposition with barium hydrate he obtained the barium salt of a crystallizable acid carnic acid, to which he gives the formula $\mathrm{C}_{10} \mathrm{H}_{15} \mathrm{~N}_{3} \mathrm{O}_{5}$. In addition, phosphoric acid, carbonic acid, paralactic acid, succinic acid, and a substance which apparently belongs to the carbohydrate group are found.

Panella has recently pointed out that phospho-carnic acid occurs in much larger amounts in the muscles of rabbits than in those of dogs ; that the amount diminishes after death in proportion to the appear- 
ance of rigor mortis; and that it increases again with the disappearance of rigor mortis and beginning putrefaction.

As regards the significance of his phospho-carnic acid, Siegfried expresses the opinion that it may serve as one of the sources of muscular energy, and he points out that in the working muscle carbonic acid must of necessity be formed on hydrolysis of phosphorcarnic acid even though oxygen be absent. In this manner the observation of Hermann would be explained, viz., that a bloodless muscle can still work for a while in the absence of oxygen and give off carbon dioxide. The lactic acid and phosphoric acid which are also known to be set free during muscular activity, Siegfried likewise refers, in part at least, to a hydrolytic decomposition of his phosphor-carnic acid. The question, however, whether the carbohydrate and phosphoric acid group only are liberated, he leaves undecided.

Of the chemical nature of phosphor-carnic acid little is known; but it is manifestly closely related to the nucleins, and is termed a nucleon.

Ferments.-Of late, Cohnheim has pointed out the remarkable fact that, while the muscle-tissue itself does not contain a ferment which is capable of decomposing the large amounts of glucose which are daily used in the metabolism of the muscles, and while in the pancreas also no such ferment has been demonstrated, extensive glucolysis can be effected by a mixture of fresh pancreatic extract and the muscle-plasma. This suggests a relation between two possible ferments analogous to that existing between trypsin and enterokinase.

Of other ferments the muscle-tissue manifestly contains a proteolytic ferment, as the tissue readily undergoes extensive autolysis after removal from the body. Rosell claims to have isolated a trypsinoid ferment of this order with the uranyl-acetate method. In addition to these ferments we find evidence of a ptyalin, a maltase, and a lactic-acid-producing enzyme. A myosin-ferment, which might be responsible for the development of rigor mortis, according to older concepts, does not seem to exist (v. Fürth).

Muscle-stroma.-Of the chemical nature of the so-called musclestroma, which remains after the extraction of the soluble albumins with a 5 per cent. solution of ammonium chloride, we know only that the material in question consists of an albuminous substance. It is apparently a native albumin, and, like the soluble musclealbumins, characterized by the ease with which it is transformed into alkaline albuminate on treating with dilute solutions of alkalies.

According to Danilewski and Holmgren, the structure of the muscle-fibre is in no ways altered by dissolving out the soluble albumins, and it would thus appear that the stroma represents the actual contractile substance of the tissue. Whether or not this is actually the case, however, is as yet unknown.

The sarcolemma apparently consists of a substance which belongs to the albuminoids, and resembles elastin in its general properties. 


\section{THE MUSCLE-PIGMENTS.}

As I have already indicated, the color of the muscle-plasma is different in different animals, and practically coincides with the color of the muscle-tissue itself. In some animals, and notably the mammals, this is dark red, while the muscles of others are almost colorless. But even in those vertebrate animals in which no color is observed in the skeletal muscles as a whole the heart-muscle and the diaphragm always appear dark red. This difference is thought to depend upon the degree of activity of the different muscles, but apparently has nothing to do with the velocity of contraction of which a muscle is capable.

The red-muscle pigment proper is now known to be identical with the hæmoglobin of the blood, and probably serves the same purpose, as a carrier of oxygen, in the internal respiration of the tissue. That it actually occurs within the cells is now undoubted. Curiously enough, the same pigment is found in the red muscles of certain insects, in which no hæmoglobin otherwise occurs.

In addition to hæmoglobin various lipochromes may also be encountered in muscle-tissue, and are especially abundant in certain fishes, such as the salmon and the sea trout. Of their origin and significance nothing is known.

\section{GLYCOGEN.}

The glycogen which is found in muscle-tissue does not occur in the cells proper, but is distributed between the individual fibres in the form of fine threads, which are apparently connected with the connective-tissue corpuscles.

The substance is formed synthetically in the muscle-tissue through a polymerization of the anhydride radicle of glucose, which is carried to the tissue either directly from the intestinal tract, or which results from the hepatic glycogen through a process of depolymerization. That the muscle-tissue is in fact capable of effecting this synthesis is now undoubted. It has thus been shown that in frogs a deposition of glycogen occurs following the subcutaneous injection of a solution of glucose, even after removal of the liver. The amount of glycogen which is deposited in the muscle-tissue probably represents about one-half of the total amount that is found in the entire body, and in man corresponds to 150 grammes. It represents the most important source of energy which is at the disposal of the tissue, and is constantly consumed, even when the muscle is at rest. This is apparent from the fact that after section of the nerves more glycogen is found in a given muscle than in the corresponding muscle of the other side, while ordinarily this is the same. While at work the consumption of glycogen increases, and after a comparatively short time already the substance has entirely disappeared. If now a period of rest follows, glycogen is again stored in the muscle, and so on. It is to be noted, however, that 
the working muscle is constantly taking up sugar from the blood, which in turn is derived from the glycogen of the liver, and that its function may continue even though a deposition of glycogen, as such, does not occur. This shows that the muscle glycogen, like that of the liver, is in reality a reserve food, and is here deposited for immediate use. In starving animals it gradually disappears, but, in contradistinction to the glycogen of the liver, its supply is not exhausted until the liver itself is free from glycogen. In this connection it is interesting to note that the heart-muscle still has glycogen at its disposal under conditions when the skeletal muscles are already free from glycogen, viz., during starvation or in cases in which the glycogen is rapidly consumed as the result of excessive work.

The chemical changes which are involved in the transformation of glycogen into glucose are probably the same as those which occur during the process of digestion. Erythrodextrin thus first results, and is then transformed into achroodextrin, and this into maltose, which in turn is inverted to glucose. This is then supposedly decomposed through the agency of a specific muscle-ferment, activated by a pancreatic kinase. Recent research has shown that this decomposition must be effected with the intermediate formation of acetic aldehyde and formic acid, as shown in the equation :

$$
\mathrm{C}_{6} \mathrm{H}_{12} \mathrm{O}_{6}=2 \mathrm{CH}_{3} \cdot \mathrm{COH}+2 \mathrm{H} . \mathrm{COOH} \text {. }
$$

The aldehyde may then be reduced to ethyl alcohol, carbon dioxide and water ultimately resulting. Lactic acid, it will be noted, is not formed during this process, but may result under certain circumstances instead, from antecedents which will stand intermediary between glucose and acetic aldehyde and formic acid. The details of the entire process, however, are very little understood. The amount of earbon dioxide that is eliminated during a period of exercise, as compared with one of rest, may serve as an index of the amount of muscular work done. I have already pointed out that the nitrogenous constituents of the muscle-tissue cannot be regarded as a source of muscular energy, and that this must be sought in its non-nitrogenous components. This fact was well shown during the ascent of the Faulhorn mountain by Fick and Wislicenus, in which it was calculated that the total amount of work done by the latter amounted to at least 368,000 kilogrammeters. The amount of nitrogen which he eliminated during the ascent and the six hours following corresponded to 37 grammes of albumin. Translated into calories, this would represent about 106,000 kilogrammeters of work. Deducting this from 368,000 , there would remain 262,000 kilogrammeters, which could not be accounted for by a decomposition of nitrogenous material, and which must hence be referable to the destruction in the muscles of other bodies which are free from nitrogen. Of these, the glycogen which is referable to ingested carbohydrates is no doubt the most important. While normally the muscle glycogen is prob- 
ably derived from this source exclusively, there is evidence to show that it may be formed from the albumins as well. If animals are allowed to starve until the entire reserve of glycogen has been consumed, and they are then fed on albumins exclusively, it will be observed that a gradual deposition of glycogen occurs nevertheless, which can be referable only to the ingested albumins. In the severer forms of diabetes, moreover, as will be shown later, sugar appears in the urine although all carbohydrates are excluded from the diet. If then in such cases the nitrogenous metabolism is diminished as much as possible by reducing the albumins of the food to a minimum, and a day of fasting is further introduced into the regime, or when diarrhœa occurs, the amount of sugar that appears in the urine is notably reduced. If, on the other hand, the amount of albumin in the food is increased, an increased elimination of sugar occurs; the parallelism between the elimination of nitrogen and sugar may then be quite remarkable. At times the ratio of nitrogen to sugar has been found to be $1: 3$ to $1: 4$. Similar conditions prevail in cases of experimental diabetes following extirpation of the pancreas or poisoning by phloridzin.

The manner in which the glycogen, viz., the glucose, is formed under such conditions has long been a matter of speculation. That the carbohydrate group of the albumins does not play an important role in this connection is apparent at once, as the amount is far too small to account for the large amount of glucose, which may still be eliminated in the urine in severe diabetes at a time when no carbohydrates are administered in the food. Benedix, noreover, has shown that in dogs which are fed on caseïn (in which a carbohydrate group does not exist) glycogen is nevertheless stored in the liver in notable amounts. Under such circumstances the leucin complex may be the source of the glucose, with the intermediate formation of oxy-capronic acid. This possibility has been suggested by Müller, Seeman, and Cohn. Opposed to this view is the fact that the leucin of the tissues contains a divided chain of carbon atoms, being the isobutyl-amido-capronic acid. But, on the other hand, Müller has pointed ont the readiness with which glucose, with its uninterrupted chain, will pass over into tetraoxy-capronic acid on standing in contact with calcium hydrate. This latter has a divided chain, and we can thus imagine perfectly well that the reverse also can happen.

This view accords well with Folin's teaching that the aminogroup of the amino-acids which are liberated during pancreatic digestion is split off in the liver, and that the remaining acid radicles are stored as glycogen or as fat.

Whether or not the fats also can give rise to the formation of glycogen has not been established beyond doubt. It seems, however, that this does not occur. Lüttje has thus shown in a severe case of diabetes that while the patient eliminated 60 grammes of nitrogen and 112 grammes of sugar while receiving 400 grammes of nutrose, the nitrogen fell to 9.9 grammes and the sugar disappeared alto- 
gether, when three days later the albuminous metabolism was diminished as much as possible by giving but little albumin, but furnishing much fat. To a certain extent the fats may supply the energy, however, which is necessary for the functioning of muscle-tissue when a sufficient supply of glycogen is not available.

Of the role which the pancreas plays in the sugar metabolism of muscle-tissue mention has already been made. In the formation of the glycogen and its inversion to glucose ferments are also no doubt active.

Isolation.-If it is desired to isolate the glycogen from muscletissue, it is necessary to place the material in boiling water immediately after the death of the animal, so as to prevent its transformation into glucose and the resulting products of decomposition. Otherwise this will occur, as the death of the individual cells does not coincide in point of time with the death of the animal as a whole, and there is danger, moreover, that the inverting ferments of the tissue remain active. That this actually occurs can be readily demonstrated by treating one portion of the muscle-tissue as described, while a second portion is allowed to remain exposed to the air for a few hours. Both portions are then examined for glycogen, when it will be seen that only the first gives a positive reaction. (As regards the details of the method, see page 435.)

Quantitative Estimation (according to Pflüger). -100 grammes of finely hashed, perfectly fresh muscle-tissue and 100 c.c. of a 60 per cent. solution of potassium hydrate (Merck Ia) are shaken in a 200 c.c. flask, so that the meat is evenly distributed. The mixture is heated for two hours on a boiling water-bath, then transferred to a 400 c.c. flask, diluted to the 400 c.c. mark, and filtered through glass wool. The filtrate should be clear or but faintly opalescent.

A carefully measured portion of 100 c.c. of the filtrate is treated with an equal quantity of 96 per cent. alcohol. After standing for twenty-four hours the precipitate is collected on a 15 c.c. filter (Swedish Munktell's), first washed with a mixture of 1 volume of 15 per cent. potassium hydrate solution and 2 volumes of 96 per cent. alcohol and then with the alcohol alone. It is now dissolved in water, the solution accurately neutralized with hydrochloric acid, transferred to a 500 c.c. flask, treated with 25 c.c. of hydrochloric acid (specific gravity 1.19) and almost diluted to the mark. Should the amount of glycogen be very small, as much as 300 e.c. of the original filtrate must be used. The flask is closed and heated for three hours 'on a boiling water-bath. Water is then added to the 500 c.c. mark and the sugar estimated according to the method which Pflüger describes. For a consideration of this method the reader is referred to the original (Pflüger's Arch., 1902, vol. 93, p. 163). 


\section{GLUCOSE.}

That traces of glucose and maltose may be found in fresh muscletissue is, of course, not surprising in view of the above considerations. To demonstrate their presence, the fresh material is finely hashed, placed in boiling water, and boiled for a few minutes. On cooling, the mixture is filtered, the filtrate concentrated to a small volume and examined in the usual manner. Larger quantities may be obtained if the finely minced tissue is placed in chloroform-water and autogestion is allowed to proceed for several weeks.

\section{LACTIC ACID.}

The reaction of living muscle-tissue while at rest is neutral or slightly alkaline. After death, however, it becomes acid, and it can then be demonstrated that the acidity is in part, at least, referable to paralactic acid. Through the action of the latter upon dipotassium phosphate monopotassium phosphate then results, and a second factor thus appears, to which the acid reaction is due.

Formerly it was supposed that rigor mortis was the result of the formation of lactic acid, but we now know that this is not the ease, and that the coagulation of the muscle-tissue precedes the appearance of the acid reaction. To use the words of Salkowski, the muscle does not form lactic acid because it dies, but because it lives, and only as long as it lives. With the occurrence of its death the formation ceases. This is, therefore, a vital or ultravital process, and there is abundant evidence to show that this view, which is now quite generally accepted, is correct. Under ordinary conditions it is difficult to show that acid material is produced while the muscle is at work, as it is then removed by the circulation as rapidly as formed; but if this is prevented, the fact can readily be demonstrated. To this end, one sciatic nerve of a rabbit is divided and the animal poisoned with strychnin. If then the muscles of both legs are removed during the final convulsions of the animal, it will be noted that the reaction of those groups which had remained in connection with their nervesupply is distinctly acid, while the others, the nerve of which was severed, show a neutral reaction. That the acid reaction in such cases is, in part at least, actually due to lactic acid, can be shown by extracting the rested and the tetanized groups with water and then with alcohol. On evaporating the resulting extracts and weighing the corresponding residue it will be noted that the weight of the alcoholic fraction is greater in the case of the worked muscle than of those that have rested, while the reverse holds good for the aqueous portions.

Lactic acid is, however, produced by the muscle not only when at work, but also while resting. This has been shown by Zillessen and v. Frey. These observers found that on transfusing the muscles of the hindquarters of a dog, during three hours, an increase in the amount of lactic acid resulted, which, calculated for the entire amount of blood, corresponded to as much as 1.48 grammes of zinc lactate. 
The amount of lactic acid that may be isolated from dead muscles while still rigid varies between 0.1 and 1.0 per cent., and it is noteworthy that for definite groups of muscles this amount is constant, no matter whether the formation of the acid is allowed to proceed rapidly or slowly. This, however, holds good only for corresponding muscles, and is different in different groups. In rabbits larger amounts can thus always be obtained from the muscles of the trunk than from those of the extremities.

To the general rule that the acidity of corresponding muscles is always the same, there is one exception, viz., the heart, which is the only muscle of the body, moreover, that normally presents an acid reaction. Larger amounts of lactic acid are here always found in the left than in the right side.

As regards the origin of lactic acid in muscle-tissue, it was long thought that the glycogen probably represented its principal source. There are a number of facts indeed which favor such an assumption. I have pointed out already that after death the glycogen gradually disappears, and we have just seen that lactic acid is then found. Glycogen is similarly decomposed during muscular activity in the living animal, where lactic acid is also constantly produced, and, as I have shown, the same also occurs in the muscle while at rest. Then again there is evidence to show that during the decomposition of glucose in the muscle-tissue lactic acid may be one of the resulting products. But, on the other hand, observations exist which go to show that the amount of lactic acid that is produced during rigor mortis bears no relation to the amount of glycogen which was present at the time, and it has further been noted that lactic acid is still formed in muscles from which all glycogen has previously been removed by starvation. The conclusion hence suggests itself that while a certain amount of lactic acid may be derived from glycogen, this does not represent its only source, and we must admit that to some extent the albumins of muscle-tissue also contribute toward its formation. There is a tendency among physiological chemists at the present time to regard this source indeed as the most important. We have seen that Siegfried's phospho-carnic acid gives rise to the formation of lactic acid on hydrolytic decomposition, and it is thus possible that this substance may be its immediate antecedent. Further researches, however, are necessary before the formation of lactic acid from the muscle-albumins can be satisfactorily explained. Whether or not enzymotic influences are here at work we do not know. Salkowski denies this possibility on the basis that lactic acid is not found among the products of autodigestion when perfectly fresh muscle-tissue is allowed to stand in contact with chloroformwater, as the chloroform, according to this observer, does not prevent the action of enzymes. If this property holds for all ferments, the conclusion would also follow that the formation of lactic acid can neither be referable to the action of living protoplasm, as the chloroform represents a strong protoplasmatic poison. We have seen, as a matter of fact, that muscle-plasma also becomes acid after 
the occurrence of coagulation, and protoplasmatic activity here manifestly does not enter into consideration. We are hence forced to the conclusion that the formation of lactic acid is either referable to the action of a ferment which is destroyed by chloroform, or that it results from a spontaneous decomposition of certain substances which are especially unstable.

Besides paralactic acid, traces of common lactic acid also are said to occur in muscle-tissue. To isolate the bodies in question, the following procedure may be employed.

Isolation and Quantitative Estimation.-A carefully weighed amount of muscle-tissue is finely hashed, repeatedly extracted with cold water, and the mixture passed through a muslin filter. The resulting fluid is feebly acidified with sulphuric acid and boiled, so as to remove the coagulable albumins. Baryta-water is now added so long as a precipitate is formed; this is filtered off. The filtrate is freed from the barium that was added in excess by passing carbon dioxide into the solution, when it is boiled, filtered, and concentrated to a thin syrup. Care should be taken, however, that the temperature does not exceed $70^{\circ} \mathrm{C}$. toward the end. The resulting material is treated with ten times its volume of absolute alcohol, set aside for a while, and filtered. The alcoholic solution is evaporated on a water-bath, and the remaining thick syrup treated with about an equal amount of a moderately dilute solution of phosphoric acid. This liberates the lactic acid from its salts, while the chlorides and sulphates remain unaffected. The lactic acid is then extracted with ether. The ether is distilled off, the residue boiled with water and an excess of carbonate of zinc, and filtered while still hot. The filtrate is concentrated to a small volume, when on standing, and especially after the addition of a small amount of alcohol, the zinc lactate crystallizes out. To separate the paralactate from the common lactate, which, as I have said, is also found in traces in the muscle-tissue, the crystals are placed in absolute alcohol, which dissolves the paralactate (solubility $1: 1100$ ), while the common form is insoluble. They are then finally dried and weighed.

To obtain the lactic acid as such the lactates are decomposed with hydrogen sulphide. The resulting zinc sulphide is filtered off, washed with water, when filtrate and washings are evaporated at $70^{\circ} \mathrm{C}$. to a small volume.

Both acids are amorphous, and are obtained in the form of a thick syrup, which is soluble in water, alcohol, and ether. Of their salts, the zine salts are especially characteristic and serve to distinguish the two forms from each other. As I have indicated, the common lactate is insoluble in absolute alcohol. It crystallizes with three molecules of water, which escape at a temperature of $105^{\circ} \mathrm{C}$., so that the loss of weight will then correspond to 18.18 per cent. The paralactate, on the other hand, is soluble in absolute alcohol, though with difficulty, and crystallizes out with only two molecules of water, which likewise escapes at $105^{\circ} \mathrm{C}$. In this case the loss of weight amounts to 12 per cent. Both the free acid and the paralactate are 
lævorotatory, while the common form and its salts are optically inactive.

\section{INOSIT.}

Of the origin of inosit, which is apparently a constant constituent of muscle-tissue, but which is found also in other organs of the body, and appears in the urine when polyuria is either artificially produced or results from some morbid process, nothing is known. It is not peculiar to the animal world, however, but occurs widely distributed in the vegetable kingdom also, and is identical with the so-called phaseo-mannite, which is especially abundant in certain beans. In muscle-tissue it is found only in traces.

The substance is not a carbohydrate, as was once supposed, but belongs to the aromatic series, and is commonly regarded as hexahydroxybenzol :

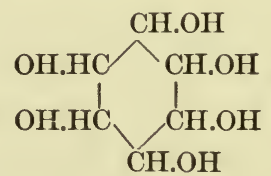

In pure form it crystallizes in colorless, monoclinic prisms, which are often grouped in rosettes. It melts at $217^{\circ} \mathrm{C}$. It is soluble in water and dilute alcohol, but is insoluble in absolute alcohol and ether. The substance does not reduce the metallic oxides in alkaline solution, and is optically inactive. It is not fermentable with common yeast, but is decomposed by the Bacterium lactis with the formation of lactic acid, and subsequently yields butyric acid.

Tests.-Scherer's Test. - If a few crystals of inosit are evaporated on platinum foil with a little nitric acid, and the residue is treated with ammonia and a drop of dilute solution of calcium chloride, a rose-red spot remains on further evaporation. The reaction is due to the formation of rhodizonic acid.

Gallois' Test.-On evaporating a small amount of a solution of inosit and adding a few drops of a dilute solution of mercuric nitrate before the residue has become dry, a yellow spot develops on the further application of heat, which ultimately turns red. On cooling, the red color disappears, but reappears on heating.

Isolation.-To demonstrate the presence of inosit in muscletissue, this is finely hashed, and extracted with hot water, when the albumins are removed by boiling. The filtrate is precipitated with barium hydrate, so as to remove the phosphates that are present. After filtering the liquid is then concentrated, until most of the kreatin has separated out. This is removed by filtration; the filtrate is boiled with four times its volume of alcohol; the solution is allowed to cool, freed from the mineral constituents that have separated out, and shaken with ether. The inosit then separates out in the form of fine platelets, which can be further purified by dissolution in alcohol and reprecipitation with ether. 
The scyllit which is found in cartilaginous fish, where inosit is absent, is closely related to the latter and, like this, gives the reaction of Gallois.

\section{THE NITROGENOUS EXTRACTIVES.}

The nitrogenous extractives of muscle-tissue comprise the common derivatives of the nuclear nucleins, viz., xanthin, hypoxanthin, guanin, and carnin; further, traces of taurin, glycocoll, urea, and uric acid; more abundantly kreatin and kreatinin, and finally a basic substance which has been isolated from beef extract, and which Gulewitsch has termed carnosin.

Kreatin and Kreatinin.-Kreatin is a constant constituent of the muscle-tissue of the vertebrate animals, while in the invertebrates it has not as yet been found. Its amount is often quite considerable, and it has been calculated that in adult man as much as 90 grammes could be extracted from the muscles of the entire body. Its anhydride, kreatinin, on the other hard, is usually found only in traces, but may occur in larger amounts, and notably in certain fishes.

Of the origin of kreatin little is known, and it is noteworthy that the substance has thus far not been obtained from the animal tissues directly by artificial means. It has been found in the brain, in the thyroid gland, in the blood, in transudates, in the amniotic fluid, and is also a constant constituent of the urine. In the vegetable world it does not occur.

From the observation of St. Johnson that the urinary kreatinin is not identical with the substance, which can be isolated from muscle-tissue, it has been concluded that the former may not be derived from the muscles at all, but may possibly be referable to the kreatin, which is found in other organs of the body and notably in the thyroid gland. Its identity with these kreatinins, however, has not yet been established, nor is there reason to suppose that these forms differ from the common kreatinin of the muscles. However this may be, the kreatins, viz., kreatinins, are essentially specific decomposition-products of ' muscle-tissue, and are unquestionably derived from the common muscle-albumins. This is suggested by the observation that larger amounts of kreatin and kreatinin can be isolated from muscles that have previously been worked, than from muscles that have been at rest. I have shown, it is true, that the nitrogenous components of the muscle-tissue enter into consideration only in a secondary manner, as a source of muscular energy, but this does not preclude the possibility, that during work the metabolism of the muscle-albumins is increased. That such an increase will of necessity become more apparent during starvation is, of course, self-evident, and we find, as a matter of fact, that under such conditions a corresponding increase in the formation of kreatin occurs. 
According to Folin kreatinin represents the most important nitrogenous end-product of the muscle katabolism and is not further converted into urea. As I have shown at another place, the latter is the essential end-product of the exogenous metabolism, while kreatinin represents the same principle for the endogenous metabolism. Folin has demonstrated conclusively that on a kreatinin-free diet the daily kreatinin elimination is for any given person practically a constant quantity and independent of how much or how little protein is contained in the food. (See also Urine.)

How kreatin is transformed into kreatinin within the body is not known. Folin has shown that this is artificially effected only with difficulty and it is possible that in the living body it may be brought about through a ferment.

Properties.-Kreatin crystallizes in rhombic prisms, with one molecule of water, which escapes at $100^{\circ} \mathrm{C}$. It is readily soluble in warm water, less so in cold water, and is insoluble in alcohol and ether.

As has been indicated before, it can be formed synthetically from cyanamide and methyl-glycocoll, according to the equation :

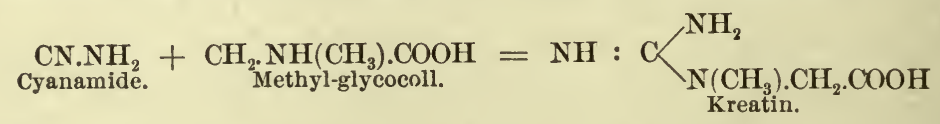

The relation existing between kreatin and kreatinin can be represented by the equation:

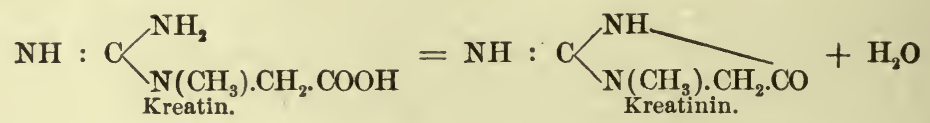

The same relation thus exists between kreatin and kreatinin as between glucocyamin and glucocyamidin, and, as a matter of fact, both are methyl-substitution-products of the two latter, and are accordingly also termed methyl-glucocyamin and methyl-glucocyamidin.

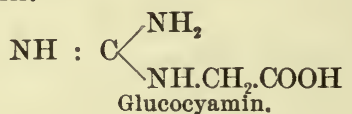$$
\mathrm{NH}: \mathrm{C}<\frac{\mathrm{NH} .}{\mathrm{CH}_{2}} \text {. }
$$

Glucocyamidin.

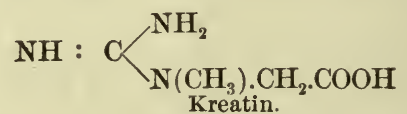

$\mathrm{NH}: \mathrm{C}_{\mathrm{N}\left(\mathrm{CH}_{3}\right) \cdot \mathrm{CH}_{2} \cdot \mathrm{CO}}^{\mathrm{NH}}$

Kreatinin crystallizes in prisms, without water of crystallization, and is soluble in water and alcohol (see also pages 108 and 263).

Isolation of Kreatin.-To isolate kreatin from muscle-tissue, this is finely hashed and repeatedly extracted with an equal weight of water at a temperature of from $55^{\circ}$ to $60^{\circ} \mathrm{C}$. The extracts are boiled so as to remove coagulable albumins. The filtrate is precipitated with subacetate of lead, care being taken to avoid an 
excess. The resulting filtrate is freed from lead by hydrogen sulphide and is concentrated to a small volume at as low a temperature as possible. On standing, the kreatin crystallizes out and can then be purified as desired. To identify the substance, it is best transformed into kreatinin by prolonged boiling (one-half to one hour) with dilute hydrochloric acid. The resulting material is then examined as described in the section on the Urine.

In addition to the common kreatins which have just been considered, Gautier has described a xanthokreatinin, crusokreatinin, and amphikreatin from muscle-tissue. The substances are, however, found only in traces and have not been studied in detail.

\section{THE XANTHIN-BASES.}

The xanthin-bases which have been isolated from muscle-tissue comprise xanthin, hypoxanthin, and guanin. In addition, another basic substance has been obtained, which is termed carnin. This is manifestly closely related to the xanthins proper, as on oxidation it is transformed into hypoxanthin. These bodies are formed during the metabolism of the muscle nuclei, and are in part eliminated in the urine as such. A variable fraction, however, is directly oxidized to uric acid, which in turn may contribute to the formation of urea, but is in part also eliminated directly (see Urine).

Isolation.-To demonstrate the presence of the xanthin-bases in muscle-tissue it is necessary to work with large amounts of material, as the quantity present is always small. On the whole it is more convenient to start with some extract of beef, such as that of Liebig, and to proceed as follows :

The material in question is taken up with an amount of water which is just sufficient for its solution, at a temperature of about $50^{\circ} \mathrm{C}$. The liquid is then precipitated with a solution of subacetate of lead. The resulting filtrate we term A. The precipitate contains the carnin in combination with lead. To isolate the substance the mass is suspended in water and boiled. The filtrate while still hot is saturated with hydrogen sulphide, which decomposes the lead compound of carnin with the liberation of the free base. The lead sulphide is filtered off, the filtrate concentrated to a small volume and precipitated with a concentrated solution of silver nitrate. Ammonia is added to dissolve any precipitated chlorides when the insoluble silver-carnin is placed in a small amount of hot water and is decomposed with hydrogen sulphide. The solution is filtered while hot, decolorized with animal charcoal, and precipitated with alcohol. The carnin is thus thrown down.

Filtrate $\mathrm{A}$ is decomposed with hydrogen sulphide, filtered and strongly concentrated, when on standing kreatin separates out. The filtrate is rendered alkaline with ammonia and is precipitated with ammoniacal silver nitrate solution. The xanthin bases are thus 
thrown down as double silver salts. They are filtered off and dissolved in a small amount of hot nitric acid. The solution is placed in the refrigerator when the salts of hypoxanthin and guanin crystallize out. The filtrate is termed B.

To separate the guanin from the hypoxanthin, the material is suspended in water; the liquid is brought to the boiling-point and treated with a solution of ammonium sulphide, drop by drop. The silver salts are thus decomposed. The liquid is filtered while hot. The filtrate $\mathrm{C}$ contains a portion of the guanin and all of the hypoxanthin, while a fraction of the guanin remains in the precipitate. This can be extracted by boiling with a very dilute solution of hydrochloric acid, when the free base is precipitated by adding an excess of ammonia to the acid solution. Filtrate $\mathrm{C}$ is placed on a water-bath and treated with ammonia, when the remaining portion of the guanin is thrown down. The hypoxanthin is then obtained after filtration on evaporating the ammoniacal solution.

Filtrate B contains the xanthin salt of silver nitrate. To isolate the free base the double salt is first precipitated from its acid solution by ammonia. It is suspended in water, decomposed with hydrogen sulphide, and extracted with ammonia. On evaporation the xanthin crystallizes out.

This method is well adapted for extracting the xanthin bases from any organs of the body, and also serves for the isolation of adenin. Should this be present, it is found in filtrate C. On adding ammonia the adenin together with the hypoxanthin remains in solution. On cooling the adenin separates out, especially after the ammonia has been evaporated off. Hypoxanthin remains in solution and is obtained on final evaporation.

As the general chemical relations of the xanthin-bases lave already been considered (page 101), it will suffice to give a brief account of the more important properties of the individual substances at this place.

Xanthin.-Pure xanthin is either amorphous or occurs in the form of fine platelets which are gathered into little clumps so that the material often presents a granular aspect. In cold water it is almost insoluble, and in hot water also it dissolves with difficulty. In alcohol and ether it is insoluble. In acids and alkalies it dissolves with comparative ease, but at the same time it combines with these to form compounds, which are for the most part readily crystallizable. From its ammoniacal solution it is obtained as such on evaporation of the ammonia. From this solution it is thrown down by silver nitrate as a gelatinous precipitate of the composition $\mathrm{C}_{5} \mathrm{H}_{4} \mathrm{~N}_{4} \mathrm{O}_{2} \cdot \mathrm{Ag}_{2} \mathrm{O}$. If this is dissolved in nitric acid, a double salt results, which crystallizes out on standing. Unlike hypoxanthin, xanthin is precipitated by an ammoniacal solution of lead subacetate. From its aqueous solution it is precipitated as a greenishyellow material by means of cupric acetate on boiling.

Tests.-Nitric ACID Test.-On evaporating a few crystals of 
xanthin on platinum foil with nitric acid a yellow spot remains, which turns red when moistened with a drop of sodium hydrate solution. On further heating, it becames a beautiful purplish violet. The reaction is thus similar to the murexid test for uric acid, but it will be noted that in this case a red color develops on the addition of the alkali, while with uric acid a blue color is obtained.

Hoppe-Seyler's Test.-A few crystals of xanthin are placed in a watch-crystal containing a mixture of a few drops of a solution of sodium hydrate and of calcium hypochlorite. A dark-green zone then appears about the xanthin, which subsequently turns brown and ultimately disappears.

WEIDEL'S TEST. - A small amount of xanthin is covered with freshly prepared chlorine-water containing a trace of nitric acid. The mixture is evaporated to dryness on a water-bath, and the residue exposed to the fumes of ammonia, when a beautiful red or purplishviolet color develops.

Hypoxanthin (Sarcin).-Hypoxanthin crystallizes in small white needles. It is soluble in hot water, less readily so in cold water, while in cold alcohol it is almost insoluble. Like xanthin, it dissolves with comparative ease in dilute solutions of the alkaline hydrates and acids, forming salts, which are decomposed by distilled water, with the liberation of the free base. Of these, the chlorhydrate is fairly characteristic, as on rapid evaporation it crystallizes out in distinct whetstone crystals similar to those of uric acid. On treating the substance in ammoniacal solution with an ammoniacal solution of silver nitrate, a double salt of hypoxanthin with silver is precipitated, which is soluble with difficulty in boiling nitric acid. On cooling, it separates out in the form of curiously bent prisms, which are quite characteristic. On boiling with a solution of acetate of copper, hypoxanthin is thrown down as a cupric salt.

Test.-The substance does not give the common reactions of xanthin. When treated with zinc and hydrochloric acid, however, hypoxanthin gives rise to a ruby-red color, which later changes to a brownish red, when sodium hydrate solution is added in excess.

Guanin.-Guanin is usually obtained in amorphous form, but can be brought to crystallize out from its solutions in strong ammonia, on spontaneous evaporation. It is insoluble in water, alcohol, and ether. In mineral acids it dissolves with comparative ease, at the same time forming salt-like products, which are crystallizable, but quite unstable, so that in the case of some of them at least the free base is liberated by water. With the common alkalies it likewise combines to form compounds which are somewhat soluble in warm water, but more readily so if a little fixed alkali is present. In ammonia it dissolves with great difficulty, so that it is possible to precipitate the substance from its acid solutions by the addition of ammonia. Silver nitrate precipitates the substance from its solutions in nitric acid as a double salt, which dissolves in boiling nitric acid, and crystallizes out on cooling in the form of fine needles. On 
boiling a solution of guanin (as calcium compound) with acetate of copper the corresponding salt is thrown down.

Tests.-Like xanthin, guanin gives the nitric acid reaction, but with a somewhat more bluish-violet color, while Hoppe-Seyler's test and that of Weidel are negative. With picric acid it combines to form a yellow crystalline precipitate when a saturated solution of the acid is added to a warm solution of the hydrochlorate.

Adenin.-Adenin crystallizes with three molecules of water in the form of long hexagonal needles. If these are placed in an amount of water which is insufficient for their solution and heat is now applied to the temperature of $53^{\circ} \mathrm{C}$, they suddenly lose their transparency and become opaque. This peculiar behavior may aid in the identification of the substance. It is soluble with difficulty in cold water, more readily so in hot water, but is insoluble in ether. In hot alcohol it dissolves to a slight extent. In acids and alkalies it is soluble with ease. In ammonia it is less readily soluble than hypoxanthin, but more readily so than guanin. From its alkaline solutions the free base is precipitated by the addition of very dilute acids, care being taken to avoid an excess. With silver nitrate it forms a compound which is soluble with difficulty in boiling nitric acid, and crystallizes out on cooling. Like the xanthin bases, that have already been considered, it is precipitated from its solutions on boiling with acetate of copper as a double salt.

Tests.-Like hypoxanthin, it does not give the common xanthin reactions, nor does it react with zine and hydrochloric acid. It is most readily identified by the behavior of its crystals, as has just been described, or by adding a solution of auric chloride to its solution as a hydrochlorate, when a double salt is formed, which crystallizes in part in octahedral or prismatic crystals, the angles of which are often rounded off.

Carnin.-Carnin, as I have stated before, is not a true xanthinbase, but is manifestly closely related to this group, as on oxidation with bromine-water or with nitric acid it is transformed into hypoxanthin.

It is obtained in indistinctly erystalline form. In hot water it is readily soluble, while in cold water it dissolves with great difficulty, and is insoluble in alcohol and ether. It is neutral in reaction, and combines with acids and alkalies to form salts. These salts are not decomposed by water. Its hydrochlorate, which results when adenin is dissolved in warm hydrochloric acid, crystallizes out on cooling, and combines with platinum chloride to form a double salt. Silver nitrate precipitates it from its aqueous solutions as a silver salt, and it is to be noted that this compound is insoluble both in ammonia and nitric acid. Subacetate of lead precipitates adenin as a lead salt; this is soluble in boiling water. Acetate of copper produces no precipitate. The substance gives none of the common reactions of the xanthin-bases, and is best identified by the behavior of its lead and silver salts. 
Still other nitrogenous extractives may be obtained from muscletissue, but, with the exception of taurin, inosinic acid, and carnosin they are scarcely known. For a description of taurin see page 170 .

Inosinic Acid.-Inosinic acid is apparently a constant constituent of muscle-tissue, but is most abundantly encountered in the muscles of ducks, from which Creite was able to isolate as much as 0.26 per cent., calculated as barium salt.

The substance has the composition $\mathrm{C}_{10} \mathrm{H}_{13} \mathrm{~N}_{4} \mathrm{PO}_{8}$, and is commonly regarded as a nucleinic acid. On decomposition with boiling water it is said to yield hypoxanthin, trioxy-valerianic acid, and phosphoric acid. Whether or not a relationship exists between inosinic acid and phospho-carnic acid is unknown.

Carnosin.-Carnosin is a basic substance which has been found in beef extract, and which possibly also exists as such in the fresh muscle-tissue. Its formula is given by Gulewitsch as $\mathrm{C}_{9} \mathrm{H}_{14} \mathrm{~N}_{4} \mathrm{O}_{3}$. In its general properties the substance resembles arginin. On decomposition it yields histidin and alanin. It is identical with Kutscher's ignotin.

\section{GASES.}

Both, when at rest as also during its activity, the muscle-tissue is constantly taking up oxygen from the blood and the lymph. This is stored in the cells proper, and is extensively utilized in the oxidation-processes which are constantly going on, but which occur with increased intensity when the muscle is at work. Carbon dioxide is similarly given off, and it can be readily proved that the oxygen which is utilized in its formation is in part at least stored within the tissue. Carbon dioxide is thus still given off, even when a muscle is removed from the body and worked in an atmosphere which is free from oxygen. It has been noted, moreover, that the amount which is then set free is the same as that which results when the muscle is worked in the presence of an abundance of oxygen. Of the form, however, in which the gas exists in the muscle we know nothing, but it is manifestly not present in the free state, as no oxygen at all, or very small amounts only, can be extracted by a vacuum pump. With increasing activity larger amounts of oxygen are taken up, while larger amounts of carbon dioxide are being given off. This difference is well shown in the following table, which is taken from Gautier. The figures have reference to 100 volumes of blood, calculated at $0^{\circ} \mathrm{C}$. and $1000 \mathrm{Hgmm}$. pressure:

\begin{tabular}{|c|c|c|}
\hline & Oxygen. & $\begin{array}{l}\text { Carbon } \\
\text { dioxide. }\end{array}$ \\
\hline Arterial blood from muscle-tissue $\ldots \ldots$ & 15.25 & 26.71 \\
\hline Venous blood from muscle-tissue while at rest & 6.70 & 33.20 \\
\hline Venous blood from muscle-tissue while at work & 2.97 & 36.38 \\
\hline
\end{tabular}

In addition to carbonic acid, small amounts of nitrogen can further be obtained from muscle-tissue, which are manifestly absorbed from the blood and apparently exist in a state of solution. As in 
other tissues and fluids of the body where nitrogen is also found, its presence is probably of no significance. The quantity that can be obtained by the vacuum pump is essentially the same as that which is found in the lymph and in the blood.

\section{FAT.}

The amount of fat which is found in muscle-tissue varies not only with different animals but also in one and the same individual at different periods of life. Some of the analytical results which have been obtained are shown below :

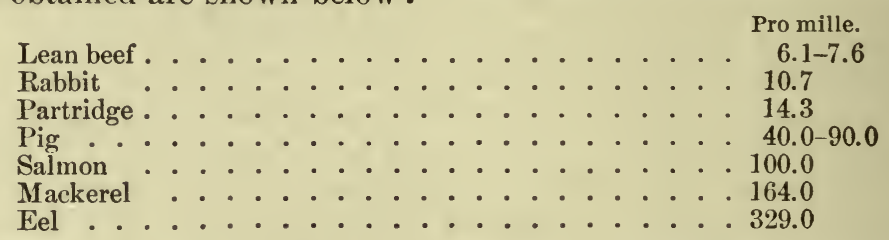

The fat is deposited not only in the interfibrillary connective tissue, but also in the sarcoplasm proper, and is apparently more abundant in the red meat, which contains more sarcoplasm than in white meat.

Like glycogen, it here represents a reserve source of muscular energy, but is apparently utilized more especially when a sufficient supply of the former or of grape-sugar, as such, is not available.

While it is ordinarily derived from the ingested fats or from carbohydrates, there can be no doubt that under certain pathological conditions, which are associated with an increased destruction of tissue albumins, it can also originate from these. This question, however, we shall not consider in detail at this place, but shall revert to it in a future section.

In addition to fats, muscle-tissue also contains a small amount of cholesterin, fats, and fatty acids, and at times considerable quantities of lecithins ( 0.69 per cent.).

The chemical composition of involuntary muscle-tissue is apparently similar to that of the striped variety. From the muscles of the stomach of the pig and goose Velichi obtained a spontaneously coagulating plasma, from which one albumin was precipitated on dialysis, while a second remained in solution. The first was soluble in dilute solutions of the neutral salts and was precipitated by acetic acid; it coagulated between $54^{\circ}$ and $60^{\circ}$ C. The second coagulated between $46^{\circ}$ and $50^{\circ} \mathrm{C}$.

In the holothurians, which probably represent one of the lowest forms of animal life in which an actual differentiation of protoplasm to inuscle-tissue has occurred, the tissue is apparently of the unstriped variety. In stictopus, which belongs to this order, v. Fürth was unable to isolate an albumin coagulating below $50^{\circ} \mathrm{C}$, , while Krukenberg states that in the case of Holothuria tubulosa his extraction liquid coagulated at $45^{\circ} \mathrm{C}$. 


\section{CHAPTER XVII.}

\section{THE NERVE-TISSUE.}

OwING to the difficulty which attends the separation of the various morphological components of nerve-tissue, and the peculiar properties of some of its most important chemical constituents, it is as yet impossible to give an account of its chemical composition which is at all satisfactory. Here, as in the other organs of the body, we meet with certain substances which are generally spoken of as extractives, and which are manifestly katabolic products that result during the functional activity of the tissue in question. These are in no sense specific of nerve-tissue, however. They comprise kreatin, uric acid, urea, xanthin, hypoxanthin, guanin, adenin, inosit, and lactic acid-viz., substances which, as we have just seen, are also found in muscle-tissue. In addition, we find certain albumins which in part belong to the native albumins and in part to the globulins; further, nucleins and a special keratin-neurokeratin-which largely enters into the composition of the supporting structure of the nerve-tissue, and is characteristic of this. Besides these various substances nerve-tissue contains especially large amounts of so-called myelins-viz., lecithin, cholesterin, and protagon. A certain amount of mineral salts and a large quantity of water constitute the remaining known components of the tissue.

As regards the distribution of these substances among the ganglionic cells and nerve-fibres more especially, our knowledge is as yet quite incomplete; but it appears from the analyses which are available that the gray substance of the brain in the dried state consists to the extent of one-half at least of albumins, while the substances which are soluble in ether amount to only about one-quarter of the total quantity. Protagon is here present in only very small quantity. In the white substance of the brain, on the other hand, it is found in considerable amount, while the albumins constitute only onequarter of the dry material. In embryonic brains, in which the medullary sheaths are not as yet developed, much smaller amounts of lecithins are found than in the adult brain ; and it is noteworthy, moreover, that protagon and neurokeratin are here both absent. It may thus be concluded that these substances are essentially components of the medullary nerve-fibres. According to Hoppe-Seyler, indeed, the small amount of protagon which is found in the gray substance of the brain is entirely referable to this source. The 
presence of smaller amounts of lecithins in the embryonic brain, as 'compared with the adult brain, and notably the gray matter, is now generally explained upon the assumption that the material in question is in some manner intimately concerned in the growth of the cellular elements proper.

\begin{tabular}{|c|c|c|}
\hline Analysis of Brain-tissue & $\begin{array}{l}\text { (Baumstark). } \\
\text { White matter.1 }\end{array}$ & \\
\hline Water & . . 69.53 & 77.00 \\
\hline Solids . . . . . . & . 30.47 & 23.00 \\
\hline Insoluble albumins and connective-tissu & 5.00 & 6.08 \\
\hline Neurokeratin . . . . . . & 1.89 & 1.04 \\
\hline Nucleins . . & 0.29 & 0.20 \\
\hline Protagon . . . . & 2.51 & 1.08 \\
\hline Cholesterins, free .... & 1.82 & 0.63 \\
\hline Cholesterins, combined . . . . . . . & 2.69 & 1.75 \\
\hline Mineral salts . . . . . . . . & 0.52 & 0.56 \\
\hline Other substances, soluble in ether . & 30.47 & 23.00 \\
\hline
\end{tabular}

Analysis of the Mineral Salts (Geoghegan).

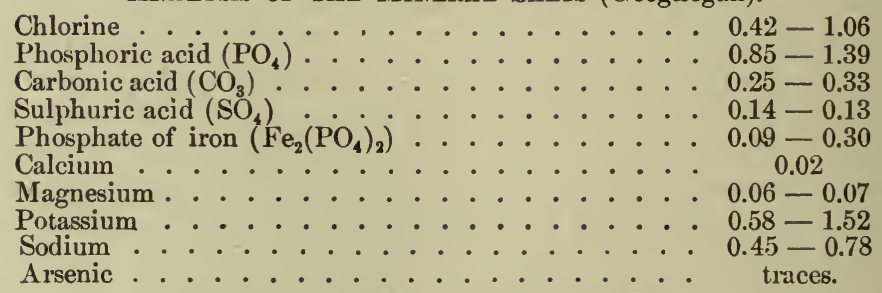

Albumins.-Of the character of the individual albumins which occur in nerve-tissue very little is known. Baumstark states that they are essentially the same as those of muscle-tissue, and, to judge from the researches of Petrowski, it appears that one of these may be identical with myosin, as it is soluble in dilute saline solution, and can be precipitated by diluting with water or by salting with sodium chloride. The coagulation-point of the substance has, however, not been determined. It is said to occur both in the gray and the white matter.

More recently Halliburton claims to have isolated two neuroglobulins, both from the white and the gray matter, with a coagulation-point of $47^{\circ}$ and $75^{\circ} \mathrm{C}$., respectively. In addition he found a nucleo-albumin in the gray substance, with 0.5 per cent. of phosphorus, which coagulated between $55^{\circ}$ and $60^{\circ} \mathrm{C}$. v. Jaksch further claims to have isolated a nuclein from the gray matter which contains but little phosphorus, and yields hypoxanthin, xanthin, phosphoric acid, and an albuminous body on decomposition.

The albumins, as I have indicated, are principally found in the gray matter of the brain, and constitute about one-half of the dried substance. In the white matter, however, they are also found, though in much smaller amount, and it is thought that the cylinder

1 The complete separation into gray and white matter is, of course, impossible. 
of the nerve-fibre is essentially of an albuminous nature. They are without doubt intimately concerned in the specific function of the nerve-tissue, but of the part which they take in such function nothing whatever is known. It is interesting to note that the gray matter of the brain, as also of the spinal cord and groups of ganglionic cells outside of the central nervous system, always present an acid reaction, while the white matter of the brain and cord and the peripheral nerves is always neutral or slightly alkaline. The substance which produces the acid reaction of the gray matter is apparently the common, optically inactive lactic acid, and it is noteworthy that in the nerve-tissue also a lactic acid is encountered in those portions which are especially rich in albumins. But while the acid reaction of muscle-tissue becomes manifest only after death, it can be readily shown that in the case of the brain and spinal cord this is normal during life. Whether or not other substances besides lactic acid contribute to the acid reaction of the gray matter has not been definitely established. But it is quite likely that this is the case, as in the presence of lactic acid a transformation of diphosphates to monophosphates would of necessity occur. Bibra and Müller, moreover, claim to have obtained traces of formic acid from the aqueous extract of the gray matter. Paralactic acid has not been found in nerve-tissue.

Neurokeratin.-This substance, which was first isolated by Kühne, forms the greater portion of the supporting tissue of the central nervous system, and is likewise found in the medullary fibres, where it constitutes the axilemma and outer sheath of the medullary substance. According to some observers, moreover, it forms a fine reticulated network in the latter.

Neurokeratin belongs to the group of the keratins, which are found widely distributed among the tissues of epiblastic origin. In the invertebrate animals, in which medullary fibres are not found and chitinous substances largely enter into the composition of the outer skeleton of the body, it is accordingly represented by a neurochitin.

Neurokeratin is insoluble in water, ether, alcohol, in dilute solutions of the alkaline hydrates, in gastric juice and pancreatic juice. To isolate the substance from nerve-tissue, this is accordingly extracted with alcohol and ether, to remove the myelin substances. The remaining material is freed from albumins and other albuminoids by digestion with gastric juice, and is then treated with a dilute solution of sodium hydrate, which dissolves the nucleins. The keratin then remains. From the other keratins, which may be obtained from hair, nails, horns, etc., neurokeratin differs especially in its relatively small amount of sulphur, and the large amount of carbon and hydrogen and the smaller quantity of nitrogen which it contains. This is shown in the following table, which is taken from Hammarsten : 


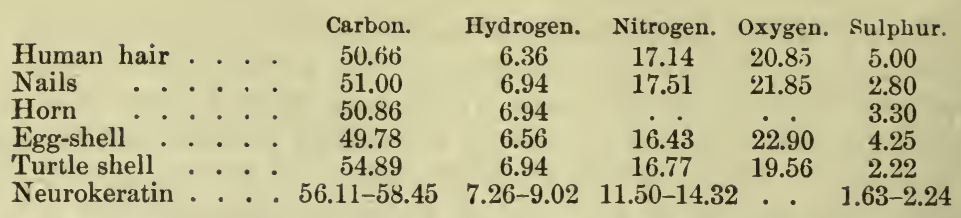

Its properties are the same as those of the keratins in general (which see).

\section{THE MYELIN BODIES.}

In former years it was supposed that the medullary substance of nerve-fibres consisted of a single substance, myelin, which was characterized by the fact that on treating with water it formed double-contoured droplets, which can readily be seen on microscopical examination. According to Gad and Heymans, this myelin is in reality lecithin in the free state or in loose combination, and we know as a matter of fact that the peculiar reaction is due to decompositionproducts of such complex substances as protagon and certain compound cholesterins. In speaking of myelin substances at the present time we have reference to protagons, lecithins, and cholesterins.

Protagon.- While there is evidence to show that different protagons exist, we are not as yet in a position to characterize such forms individually, and for convenience' sake we shall speak of protagons as a chemical unity at this place. The substance is not strictly characteristic of nerve-tissue, as it has also been found in other organs of the body, such as the spleen, in the stroma of the red corpuscles, in pus, and in spermatozoa. But while it is here present in only small amounts, it enters into the composition of nerve-tissue to a considerable extent, and is thus quantitatively at least peculiar to these structures. Whether or not the substance occurs also in the gray matter appears doubtful, but in the white matter and in the peripheral medullated nerve-fibres it is abundant. ${ }^{1}$

According to Liebreich, who was the first to isolate protagon from brain-tissue, the substance has the composition $\mathrm{C}_{116} \mathrm{H}_{241} \mathrm{~N}_{4} \mathrm{PO}_{2}$. It is to be noted, however, that the elementary analysis of different preparations has given rise to different results, which in itself suggests the probability that different forms exist. According to some observers, it also contains sulphur in molecular combination, but recent investigations have shown that this is probably not the case. Wörner and Thierfelder have recently succeeded in obtaining a substance from a solution of so-called protagon, which they term cerebron. On hydrolytic decomposition it yields galactose, sphingosin, and cerebronic acid, as shown in the equation :

$$
\underset{\text { Cerebron. }}{\mathrm{C}_{48} \mathrm{~N}_{93} \mathrm{NO}_{9}}+2 \mathrm{H}_{2} \mathrm{O} \underset{\text { Cerebronic acid. }}{=} \underset{\mathrm{C}_{25} \mathrm{H}_{50} \mathrm{O}_{3}}{\mathrm{C}_{17} \mathrm{H}_{35} \mathrm{NO}_{2}}+\underset{\text { Galactose. }}{\mathrm{C}_{6} \mathrm{H}_{12} \mathrm{O}_{6}}
$$

On decomposition with boiling baryta-water protagon yields fatty

1 For an account of the methods for the quantitative chemical analysis of the brain and cord, the reader is referred to the paper of Waldemar Koch (Amer. Journ. of Physiol., vol. ix., 1904, No. iii. 
acids, glycerin-phosphoric acid, cholin, and in addition cerebrosides, of which three are now recognized. These are known as cerebrin, kerasin, or homocerebrin, and encephalin. Others also may possibly exist, and it is likely that the pyosin and pyogenin, which Kossel and Freitag obtained from pus, belong to this order.

On boiling with dilute mineral acids protagon also yields a reducing substance, which is commonly regarded as galactose, and is referable to the decomposition of the cerebrosides just mentioned.

Protagon is easily soluble in warm alcohol and ether, while in cold alcohol and cold ether it dissolves with difficulty. On cooling the substance crystallizes out in fine needles or in waxy masses, which can readily be broken up into a fine powder. On heating its alcoholic solutions to a temperature of $48^{\circ} \mathrm{C}$. or on boiling its ethereal solutions the substance is readily decomposed into its components, as indicated above. In the dry state it can be heated to a higher temperature, but it is then also decomposed before $100^{\circ} \mathrm{C}$. is reached. The resulting products melt between $200^{\circ}$ and $203^{\circ} \mathrm{C}$, and begin to volatilize at $220^{\circ}$. When moistened with water, the substance swells and is partly decomposed, with the formation of . so-called "myelin" droplets. If much water is added, an opaque fluid is obtained.

Isolation.-To isolate protagon from brain-tissue this should be as fresh as possible, as otherwise partial decomposition occurs spontaneously. The material is freed from its membranes and adhering blood, and is then stirred to a pulp, and extracted with 85 per cent. alcohol, at a temperature of $45^{\circ} \mathrm{C}$., using fresh portions of alcohol from time to time until a specimen no longer deposits a sediment when cooled to $0^{\circ} \mathrm{C}$. The extracts are filtered at $45^{\circ} \mathrm{C}$., and subsequently kept at $0^{\circ} \mathrm{C}$. The resulting precipitates are extracted with cold ether to remove cholesterins and lecithins, when the remaining material is pressed between filter paper and dried over sulphuric acid. It is finally pulverized, again extracted with alcohol at $45^{\circ} \mathrm{C}$., when the solution is filtered and cooled to $0^{\circ} \mathrm{C}$. To purify the substance it is recrystallized from warm alcohol or ether.

Cerebrin.-Cerebrin, as I have stated, is a normal decompositionproduct of protagon, but probably does not occur in the living nerve-tissue as such. Associated with lecithin, it is also found in the stroma of the red corpuscles of the blood, in leucocytes, in spermatozoa, in the spleen, in the yolk of birds' eggs, etc. It is questionable, however, whether it actually exists in the free state, and the fact of its constant association with lecithin rather suggests that here also it is primarily present in the protagon molecule.

Cerebrin is said to have the formula $\mathrm{C}_{70} \mathrm{H}_{140} \mathrm{~N}_{2} \mathrm{O}_{13}$. Elementary analysis has given the following results: C, 69.08 per cent.; H, 11.47; N, 2.13; O, 17.32. On decomposition with boiling mineral acids it yields a reducing substance which is commonly regarded as galactose. On oxidation with nitric acid or on fusion with caustic alkali palmitic acid or stearic acid is obtained. If 
the substance is dissolved in concentrated sulphuric acid, the solution gradually assumes a purplish-red color, which changes to violet, and finally to brown. On adding an equal amount of water and boiling, a flocculent precipitate appears, which is known as cetylid, and is said to have the composition $\mathrm{C}_{64} \mathrm{H}_{120} \mathrm{O}_{75}$ or $\left(\mathrm{C}_{16} \mathrm{H}_{31} \mathrm{O}_{2}\right)_{3} \cdot\left[\mathrm{C}_{16} \mathrm{H}_{18}(\mathrm{OH})_{3}\right]$. This substance supposedly represents about 85 per cent. of the entire weight of the cerebrin. It is soluble in water, in hot alcohol, and especially in ether and chloroform. On fusion with caustic alkali, cetylid yields methane, hydrogen, and palmitic acid.

Pure cerebrin is a colorless substance which occurs in the form of a crystalline powder, consisting of microscopical globulites. It is soluble in warm acetone, acetic ether, benzene, and boiling alcohol, but is insoluble in sulphuric ether, even at its boiling-point; with chloroform it forms an emulsion. In cold water it is likewise insoluble. In boiling water it swells to a certain extent, like starch paste. It melts at $177^{\circ}$ C., but is decomposed long before with the development of a yellow or brownish color. Its reaction is -neutral.

Isolation.-Cerebrin can be obtained either from protagon after the separation of the latter or directly from the brain by decomposing its antecedents with baryta-water. To this end, the material after being freed from its membranes and blood is stirred with baryta-water, and brought to the boil. The insoluble portion is then pressed out and repeatedly extracted with alcohol by boiling. The extract is filtered while still hot, when on cooling to $0^{\circ} \mathrm{C}$., the cerebrin separates out in impure form. It is freed from cholesterin and fats by skaking with ether, and purified by repeated solution in hot alcohol and subsequent cooling, until jelly-like precipitates, which are referable to homocerebrin or encephalin, are no longer obtained.

With this method, however, a considerable portion of the cerebrin is decomposed, and Kossel has accordingly suggested that the protagon be first extracted and subsequently decomposed. To this end, the substance is dissolved in methyl alcohol, and is treated with a hot methyl alcoholic solution of barium hydrate. The mixture is warmed for a few minutes on a water-bath, and then allowed to cool. The resulting precipitate contains the entire amount of cerebrin which can be obtained, and represents 50 per cent. of the original quantity of protagon. It is filtered off, suspended in water, and freed from barium by a current of carbon dioxide. The insoluble residue contains the cerebrosides, which are now extracted with hot alcohol and isolated by fractional crystallization.

Homocerebrin (Kerasin).- The formula of homocerebrin is given as $\mathrm{C}_{70} \mathrm{H}_{138} \mathrm{~N}_{2} \mathrm{O}_{12}$, and the substance could hence be regarded as an anhydride of cerebrin. In the dry state it occurs as a wax-like mass, which can be pulverized only with difficulty. From its solution in alcohol and boiling ether it separates out in aggregates of 
exceedingly fine needles. At $130^{\circ} \mathrm{C}$. it is decomposed with the appearance of a yellow color, and melts at $150^{\circ} \mathrm{C}$. Toward consentrated sulphuric acid and water it behaves like cerebrin. Like this, it yields a reducing substance of the character of galactose when boiled with dilute mineral acids, and gives rise to the formation of cetylid, like cerebrin.

The amount of homocerebrin which may be obtained from protagon is about one-fourth that of cerebrin. It is isolated in association with the latter, as described, and can be separated from the cerebrin by fractional crystallization from the alcoholic solution, in which it is more readily soluble than cerebrin.

Encephalin.-Encephalin is regarded as a transformation-product of cerebrin, and, like this, yields galactose on boiling with dilute mineral acids. Cetylid is also obtained on treating with concentrated sulphuric acid. It is soluble in hot alcohol, but tends to separate out on cooling as a jelly-like material. On slow evaporation it crystallizes in platelets, which melt at $150^{\circ} \mathrm{C}$., but are already decomposed at $125^{\circ} \mathrm{C}$. In hot water it swells to form a thick paste, which remains on cooling. Like homocerebrin, the substance is found in the alcoholic solution after the cerebrin has separated out (see above), and can be isolated by fractional crystallization from the mother-liquor, or from a solution of acetone, in which the homocerebrin is likewise soluble.

Lecithins.-The lecithins, which may be isolated from nervetissue, where they largely exist in combination with the cerebrosides, in the form of protagon, but undoubtedly also occur as such in the free state, are as yet but little known. On decomposition they yield palmitic acid, stearic acid, oleic acid, glycerin-phosphoric acid, and cholin. Of their significance nothing definite is known, but it appears that they are in some manner intimately concerned in the development of the cells, and it is for this reason probably that much smaller amounts can be obtained from the embryonic brain than from that of the adult.

(For the general description of the lecithins, see p. 78).

Isolation.-To isolate the lecithins of the brain, the following method, which has been suggested by Zülzer, is conveniently employed. The brain, which must be perfectly fresh, is freed from membranes, cut into thin pieces, and placed in a jar with ether. The material should rest on a layer of cotton. After standing for several days the ethereal extract is poured off, and separated from the lower layer of blood. The extraction is continued with new portions of ether so long as anything passes into solution. If desired, the remaining material can then be extracted with 80 per cent. alcohol at $45^{\circ} \mathrm{C}$., which takes up the protagon, as has been described.

The ethereal extracts are united and concentrated in the vacuum. Any protagon that has passed into solution is thus thrown down and filtered off. The clear solution is now treated with an excess of acetone so long as a precipitate is formed. This is filtered off 
and thoroughly washed with acetone. The acetone-ethereal solution we term $\mathrm{A}$, and the precipitate $\mathrm{B}$. A, contains the entire quantity of cholesterin. To recover this, the acetone-ether is distilled off, the residue is boiled with alcohol, the alcoholic solution is filtered while still hot, when, on cooling, the substance crystallizes out. Its melting-point is $145^{\circ} \mathrm{C}$.

The precipitate $\mathrm{B}$ is placed in ether. This dissolves the greater portion, while a smaller amount remains undissolved. 'The latter consists of protagon, which was previously held in solution, owing to the presence of cholesterin. The soluble portion is treated with alcohol so long as a precipitate forms. This precipitate we term C, and the alcoholic filtrate $\mathrm{D}$. If a specimen of $\mathrm{D}$ is treated with an alcoholic solution of platinum chloride, a precipitate results; this is not abundant, however, and consists of a chloroplatinate of lecithin. To isolate the lecithins as such, the alcoholic solution is precipitated with acetone, or the ether-alcohol is distilled off, when the lecithins remain as a tough, wax-like mass.

Of the nature of the substance or substances which are contained in the precipitate $\mathrm{C}$, nothing definite is known. Zülzer apparently was able to isolate one of these, however, and found it to contain both nitrogen and phosphorus. He suggests that it may possibly belong to the so-called cephalins of Thudichum. But of the nature of these also our knowledge is as yet insufficient to warrant their description at this place.

The Cholesterins.-Cholesterins are found in nerve-tissue, both in the free state, and as so-called combined cholesterins, but of the chemical character of the latter we are as yet in ignorance (see p. 80).

The isolation of free cholesterin has been described above.

The Extractives.-The extractives of nerve-tissue, as I have already stated, are essentially the same as those which can be isolated from other organs of the body. They comprise traces of kreatin, uric acid, xanthin, hypoxanthin, guanin, adenin, inosit, volatile fatty acids (acetic acid and formic acid), lactic acid, glycogen, leucin, tyrosin (both probably referable to post-mortem autolysis), and urea. In addition, jecorin, cholin, and neuridin have been found; neurin, on the other hand, does not occur in the brain under normal conditions. Very recently Panella claims to have isolated phospho-carnic acid from the normal brain of dogs, rabbits, and calves.

Neuridin is of special interest, as the substance is constantly formed during the putrefaction of meat and gelatin, and has also leen obtained from cultures of the typhoid organism. According to Brieger, who first isolated the body, it is also present in traces in the yolk of birds' eggs. It is a diamin of the composition $\mathrm{C}_{5} \mathrm{H}_{14} \mathrm{~N}_{2}$. With the chlorides of gold and platinum it forms well-defined 
crystalline salts. On boiling with caustic alkalies it is decomposed into trimethyl-amin and dimethyl-amin. It is not toxic.

Jecorin is a substance of unknown composition, but apparently contains both sulphur and phosphorus. It is not exclusively encountered in nerve-tissue, but has also been found in traces in the liver, in the muscles, and, according to some observers, in the blood. It reduces cupric oxide in alkaline solution on boiling, and on cooling separates out in the form of a thick jelly. It is soluble in ether, and can be precipitated from its solutions by alcohol. 


\title{
CHAPTER XVIII.
}

\author{
THE EYE AND THE EAR.
}

THE EYE.

Is studying the chemical composition of the eye, we shall consider the most important parts of the organ in succession. It should be pointed out in advance, however, that the subject has thus far received but little attention, and our account must hence of necessity be very imperfect.

The Cornea.-An analysis of the cornea of the ox has given the following results:

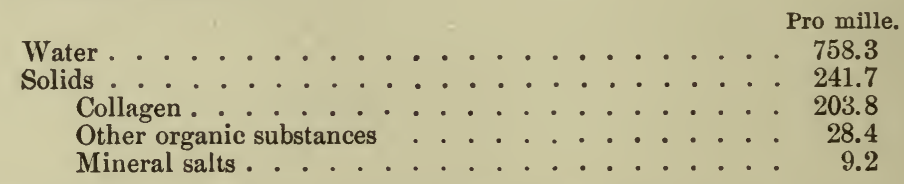

The collagen, which forms the greater portion of the fibrous network of the cornea, is probably identical with the common form which can be isolated from cartilage, and, according to Mörner, contains 16.95 per cent. of nitrogen.

The semiliquid interfibrillary substance consists of a mucoid, which yields a reducing substance on boiling with dilute mineral acids. It contains about 2 per cent. of sulphur, and seems to be characteristic of corneal tissue. In addition, we find two globulins, which, according to Mörner, do not belong to the cornea proper, however, but are contained in the epithelial layer. Nucleins have not been found.

Descemet's membrane principally consists of a membranin, which contains 14.77 per cent. of nitrogen, and 0.90 per cent. of sulphur. The substance is a glucoproteid, and belongs to the group of hyalogens (which see). On boiling with dilute hydrochloric acid it yields a reducing substance. In ordinary boiling water it is insoluble, but dissolves under the action of superheated steam. It is digested by trypsin, while the gastric juice is without effect.

The Sclerotic.-The composition of the sclerotic coat of the eye is very much the same as that of the cornea, but it appears that the quantity of the mucoid is here much less, while collagen represents about seven-eighths of the entire amount of solids.

The Aqueous Humor.-The aqueous humor is a clear fluid of an alkaline reaction and a specific gravity varying between 1.003 and 
1.009. Its quantitative composition has already been given (page 343). According to Grünhagen, it contains traces of paralactic acid, a dextrorotatory body, and a reducing substance, which is not sugar. Both the latter are unknown. The albumins in question are serumalbumin, serum-globulin, and traces of fibrinogen.

The Crystalline Lens.- The capsule of the crystalline lens, like Descemet's membrane, consists essentially of a membranin, which is not identical with that found in the latter, however, as it is less resistant to the action of boiling water and of acids and alkalies. According to Mörner, it contains 14.10 per cent. of nitrogen and 0.83 per cent. of sulphur.

A general idea of the chemical composition of the lens itself may be formed from the following analysis, which I have taken from Neumeister :

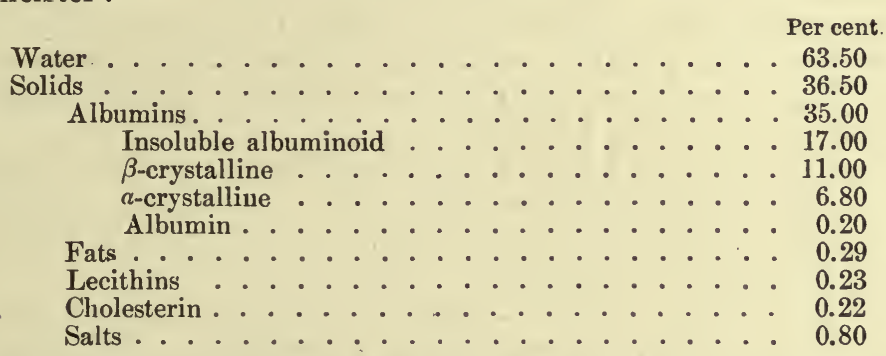

The albumins of the lens can be divided into two groups, viz., those which are soluble in dilute saline solution, and those which are insoluble. The latter group is represented by a substance which is spoken of as albumoid. It is manifestly a true albumin, as it is entirely dissolved by the gastric juice, and does not yield a reducing substance on boiling with mineral acids. It gives all the common color reactions of the true albumins, and has the same elementary composition. In dilute mineral acids and alkalies it dissolves with ease, and is reprecipitated on neutralization. Unlike the alkaline albuminates, however, its solution in dilute alkalies coagulates at $50^{\circ} \mathrm{C}$, in the presence of 8 per cent. of sodium chloride. The substance manifestly constitutes the greater portion of the lens-fibres, as the nitrogen and sulphur values of the two are practically the same, viz., N, 16.62 and S, 0.79 per cent. in the case of the albumoid, as compared with $\mathrm{N}, 16.61$ and $\mathrm{S}, 0.77$ of the fibres. It can be shown, moreover, that after extraction of the soluble constituents of the lens the fibrous framework remains and gives the same reactions as the isolated albumoid. Its amount increases from without inward, in accordance with the increasing age of the fibres.

- Aside from a very small amount of serum-albumin, the remaining soluble albumins of the lens are represented by two vitellins, which are termed $\alpha$-crystalline and $\beta$-crystalline, respectively. Of these, the $\alpha$-body is notably found in the outer portion of the lens, while the $\beta$-substance occurs in the inner portion more particularly, 
and is apparently the only one that is found in the centre of the lens.

The two substances can be isolated from an aqueous extract of the lens by saturating the solution with magnesium sulphate at a temperature of $30^{\circ} \mathrm{C}$. The precipitate is then dissolved in water, dialyzed, and the resulting solution precipitated with acetic acid, which throws down the $\alpha$-body, while the $\beta$-crystalline remains in solution. A small amount of the $\beta$-substance, it is true, is also precipitated by the acetic acid, but can be separated from the $\alpha$-body by a repetition of the process.

Both substances are precipitated from their neutral solutions by carbon dioxide, but in the case of the $\beta$-crystalline this precipitation is never complete. The latter coagulates at $63^{\circ} \mathrm{C}$., and the $\alpha$-crystalline at $72^{\circ} \mathrm{C}$. The $\beta$-substance further differs from the $\alpha$-body in containing much more sulphur, 1.27 per cent., as compared with 0.56 per cent., which is, moreover, in part at least, present in a loosely combined form, while the entire quantity that is found in the $\alpha$-crystalline is firmly combined.

That these bodies are intimately concerned in the concentration of the light cannot be doubted. The refractive index of the inner layer of the lens, in man, is given as 1.407 , while that of the central portion is 1.456 .

Of the significance of the fats, lecithins and cholesterins in the lens, nothing is known, but it appears that the amount of the two latter, at least, is much increased in senile cataract, while the quantity of the albumins, as a whole, is diminished. The albumoid, however, is then possibly increased.

The Vitreous Body.-The vitreous body of the eye is a jellylike material, which consists of a fine framework of collagen, enclosing the liquid portion of the body proper. This presents an alkaline reaction, and contains only a very small amount of solids. Its general composition is seen below :

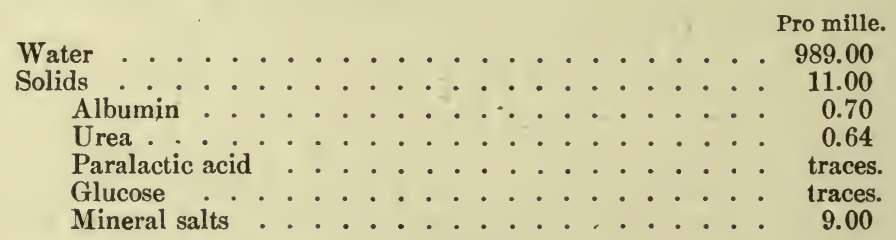

Among the albumins present Mörner claims to have found a hyalomucoid, which is closely related to the corneal mucoid, but contains 12.27 per cent. of nitrogen and 1.19 per cent. of sulphur, as compared with 12.79 per cent. of nitrogen and 2.07 per cent. of sulphur in the case of the latter.

The Retina.-A general idea of the chemical composition of the retina may be formed from the following analyses, which are taken from Cahn : 


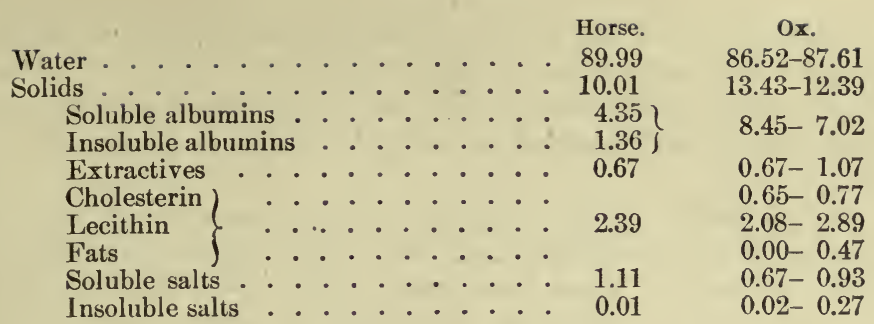

Like the gray matter of the brain, from which the retina is essentially derived, the membrane presents an acid reaction when perfectly fresh, but becomes alkaline soon after death.

The albumins which are found in the retina appear to be identical with those of the brain substance, and here, as there, we also meet with neurokeratin. This apparently forms the sheath of the outer portion of the rods. In their interior we meet with protagon, lecith-albumins, and in many animals with a peculiar red pigment, which has been termed rhodopsin.

Rhodopsin.-Of the significance of this pigment nothing is known. It occurs in the outer portion of the rods and is absent in the cones. As the macula lutea, viz., the point of clearest vision, is composed only of cones, we may conclude that its presence is not essential to sight, and, as I have just said, the pigment is not found in all animals. It is absent in chickens, pigeons, in certain reptiles, bats, etc., but is present in owls and deep-sea fishes. On exposure to daylight the pigment fades, and, to isolate the substance, it is necessary to work with sodium light. If the living retina, after having been kept in the dark for some time, is suddenly exposed to an intense light, which is broken in part through the interposition of some dark object, such as the framework of a window, and if the remaining pigment is then fixed with a 4 per cent. solution of alum, red pictures of the interposed object can be obtained on the retina, while the remaining portion has become decolorized. Such pictures are termed optograms.

The regeneration of the pigment, which is constantly going on in the living animal, is apparently dependent upon the integral union of the layer of rods and cones with the pigmented epithelial layer of the retina; but of the manner in which this restitution takes place, we know nothing. It appears, however, that its formation is preceded by the development of a yellow pigment, which is termed xanthopsin.

Of the chemical nature of rhodopsin nothing is known. Besides daylight, it is decomposed by acids, alcohol, ether, chloroform, and solutions of the alkaline hydrates, by heating to a temperature of from $52^{\circ}$ to $53^{\circ} \mathrm{C}$. for several hours, or instantaneously at $76^{\circ} \mathrm{C}$. Toward ammonia and a solution of alum it is refractory.

It is easily soluble in water, containing from 2 to 5 per cent. of Platner's bile. From such a sulution it is precipitated on dialysis 
or by salting with ammonium sulphate or magnesium sulphate. The substance then appears as a violet amorphous material. On spectroscopic examination no specific bands are observed, but merely a general absorption between $\mathrm{D}$ and $\mathrm{C}$, which is especially marked about E.

Chromophanes.-Chromophanes are pigments which apparently belong to the lipochromes, and are found in the retinal cones of reptiles and birds. They here occur in the form of red, green, and yellow oil globules, which are quite distinct, and are situated at the inner ends of the cones. The pigments in question are termed rhodophane, chlorophane, and xanthophane, respectively. Unlike the pigment of the rods, these chromophanes are apparently not affected by light, unless exposed for several days. Of their significance, nothing is known.

The epithelial layer of the retina, which adjoins the choroid, contains a black pigment, which is probably identical with that of the choroid. This is termed fuscin, and belongs to the class of the melanins, which comprise the black pigments that are found in the hair, in the negro-skin, in melanotic tumors, etc. According to Landolt, this particular form contains $\mathrm{C}, 54.48$ per cent.; $\mathrm{H}, 5.36$; $\mathrm{N}, 12.65 ; \mathrm{O}, 27.52$. In addition iron is also found, and the opinion has been expressed that the substance may be derived from the coloring-matter of the blood. This supposition is strengthened by the observation that the first appearance of the pigment in the embryo coincides in point of time with the development of the choroidal bloodvessels.

Besides fuscin, a pigment of a yellow color has also been found in the pigmented epithelial lining of the retina, which is termed lipochrin. It is apparently a yellow lipochrome.

The Choroid.-Aside from the common components of connective tissue, the choroid, as has just been mentioned, contains a black pigment, fuscin, which is probably identical with that found in the pigmented epithelial lining of the retina (see above).

\section{THE EAR.}

The chemical composition of the organic portion of the middle and the internal ear has not as yet been studied. The perilymph and endolymph present an alkaline reaction, and, in addition to the common mineral salts of the lymph, contain traces of albumin, and in some animals a mucinous body of unknown character. 


\section{CHA PTER XIX.}

\section{THE SUPPORTING TISSUES}

In contradistinction to those tissues of the animal body which are essentially composed of cells, and in which the albumins proper constitute the greater portion of the organic solids, we find that in the so-called supporting tissues which comprise the common connective tissues, cartilage, and bone, the albuminoids stand in the foreground. Their preponderance here coincides with the extensive development of the matrix, while the cellular elements enter into the histological picture to a more or less insignificant extent. This statement, however, holds good only for the higher animals, and more specifically for the fully developed animals. In lower forms of life, and during the embryonic stage of the development of the higher forms, these structures are rich in cells, and we find then an underlying matrix in which a differentiation into supporting tissue proper has not as yet occurred or exists to only a limited extent. Such tissue is termed embryonic connective tissue, and is also known as mucous tissue. In the adult animal it is found only in the vitreous humor of the eye. In typical form it is seen in the umbilical cord, in which it constitutes the so-called jelly of Wharton. The matrix is here very rich in water, and contains a mucinous substance, which is soluble in a 0.5 pro mille solution of hydrochloric acid. In addition, traces of albumin are met with, while collagen is usually absent. Of the composition of the cells nothing specific is known, but it is quite likely that their processes consist of collagen.

White Fibrous Tissue.-The fibrils of white fibrous tissue consist of collagen, and are bound together by a cement-substance, which represents the original undifferentiated matrix. As in the case of the embryonic connective tissue, this contains traces of the common albumins of the plasma, and a mucinous substance, which, in contradistinction to that of the umbilical cord, is insoluble in a 0.5 pro mille solution of hydrochloric acid. Analysis of this substance has given the following results: $\mathrm{C}, 48.30 ; \mathrm{H}, 6.44 ; \mathrm{N}$, 11.75 ; S, 0.81 ; and $\mathrm{O}, 32.70$ per cent. According to Löbisch, its formula is $\mathrm{C}_{160} \mathrm{H}_{256} \mathrm{~N}_{32} \mathrm{SO}_{80}$. To isolate the body in question, ligaments, such as the tendo Achillis, are cut into small pieces and first extracted with cold water, which dissolves the albumins and a small fraction of the mucin. The remaining material is then placed in a half-saturated solution of lime-water, in which the mucin is readily soluble. After filtering, the substance is precipitated by adding an excess of acetic acid (see page 124). The residual sub- 
stance, after removal of the mucin, consists of the collagen-fibrils, a few cellular elements, and quite commonly also contains isolated fibrils of the yellow or elastic variety, which can be readily recognized on microscopical examination by their higher power of refraction. When placed in water, or, still better, in a dilute solution of acetic acid or caustie alkali, the white fibres swell, while solutions of some of the metallic salts, such as ferric sulphate and mercuric chloride, cause them to shrink. Tannic acid acts in a similar manner. Owing to the great stability of the compound of the latter with collagen, tannic acid is extensively utilized in the preparation of leather.

On boiling white fibrous tissue in water the collagen dissolves, with the formation of gelatin, which latter separates as a jelly-like mass on cooling.

Yellow or Elastic Tissue.-In the yellow elastic fibres, elastin takes the place of the collagen of the white fibrous variety. For purposes of study, the substance is most conveniently obtained from the ligamentum nuchæ of the $o x$, in which such fibres are almost exclusively found.

Reticulated Tissue.-In the reticulated tissue, which constitutes the fibrous framework of the lymph-glands of the body, but which is also found in the alveoli of the lungs, in the liver, the kidneys, and the intestinal mucous membrane, the fibres consist of reticulin.

Reticulin is said to have the composition $\mathrm{C}, 52.88 ; \mathrm{H}, 6.97$; N, $15.63 ; \mathrm{S}, 1.88 ; \mathrm{P}, 0.34$. It is insoluble in water, alcohol, ether, dilute mineral acids, lime-water, and solutions of sodium carbonate. It resists the action of pepsin and trypsin, and is dissolved only in cold sodium hydrate solution on standing for several weeks. It does not give Millon's reaction, and accordingly yields no tyrosin on hydrolytic decomposition. On prolonged boiling with water or dilute alkalies, its phosphorus is split off; the residual material is then soluble in water, and can be precipitated from its solutions by means of acetic acid.

\section{CARTILAGE.}

Histologically considered, cartilage consists of a more or less hyalin matrix, in which a variable number of cartilage-cells are found imbedded. In certain localities, further, a differentiation of the matrix into fibres, both of the white and the yellow elastic variety, is observed. Such fibres, as in the case of the corresponding connective tissue, consist of collagen and elastin, respectively.

Of the composition of the cells nothing specific is known. Apparently they contain a small amount of glycogen, which disappears during starvation. Traces of fat are also found. During embryonic life they are quite numerous, but later they diminish in number, and in the adult animal the matrix largely predominates. Embryonic cartilage does not yield gelatin on boiling with water, and it is quite likely that as in the case of the matrix of embryonic connective 
tissue the matrix here also consists essentially of water and some mucinous substance. Whether or not this is identical with the socalled chondromucoid, which can be obtained from the cartilage of the adult animal, is not known.

A general idea of the chemical composition of cartilage may be formed from the following analyses, which are taken from $\mathrm{His}$ :

$\begin{array}{ccc} & \begin{array}{c}\text { Costal cartilage } \\ \text { (human). }\end{array} & \begin{array}{c}\text { Articular cartilage } \\ \text { from knee-joint (human). }\end{array} \\ \text { Water . . . . . . . . } & 67.67 \text { per cent. } & 73.59 \text { per cent. } \\ \text { Solids . . . . . . . } & 32.33 \text { " " } & 26.41 \text { " " } \\ \text { Organic material . . . . } & 30.13 \text { " " } & 24.87 \text { " " } \\ \text { Mineral salts . . . . . } & 2.20 \text { " " } & 1.54 \text { " " }\end{array}$

Analysis of the mineral salts has given the following results (calculated for 100 parts of the mineral ash):

\begin{tabular}{|c|c|c|c|c|}
\hline Sodium chloride & . . & 6.11 & per cent. & 22.48 per cent. \\
\hline Sodium sulphate & . & 44.81 & " " " & 55.17 " " \\
\hline Potassium sulphate & & 26.66 & “ & \\
\hline Sodium phosphate & & 8.42 & " & 7.39 “ \\
\hline $\begin{array}{l}\text { Calcium phosphate } \\
\text { Magnesium phosphate }\end{array}$ & & $\left.\begin{array}{l}7.88 \\
4.55\end{array}\right\}$ & “ & 15.51 “ \\
\hline
\end{tabular}

The organic constituents of the cartilaginous matrix are essentially represented by chondroitin-sulphuric acid as such, and its compounds with collagen and albumins. In addition, a small amount of soluble albumins is found, as also a peculiar insoluble albuminous substance, which has been termed albumoid. Händel further found 0.2168 per cent. of glycogen, viz., much larger announts than are obtained from any of the other skeletal parts.

Chondroitin-sulphuric Acid.-This substance is a conjugate sulphate, and, according to Schmiedeberg, has the composition $\mathrm{C}_{18} \mathrm{H}_{27} \mathrm{NO}_{14} \cdot \mathrm{SO}_{3}$. On hydrolytic decomposition it yields a hyalin, chondroitin, which in turn gives rise to the formation of chondrosin. The chondrosin, according to Schmiedeberg, can further give rise to glucuronic acid and glucosamin. But this has been disproved (Neubauer, Orgler-Neuberg). Neuberg obtained a substance on hydrolysis of chondrosin, which was shown to be tetra-oxyaminocapronic acid $\left[\mathrm{C}_{6} \mathrm{H}_{7} \mathrm{O}_{2}(\mathrm{OH})_{4}\left(\mathrm{NH}_{2}\right)\right]$. 'This in turn is combined in the chondrosin witl a carbohydrate-like substance of unknown composition.

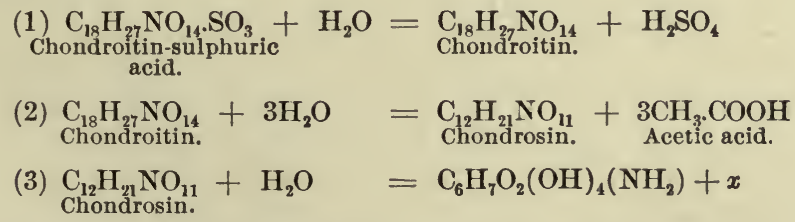

Both chondroitin and chondrosin are monobasic acids. The latter reduces Fehling's solution directly, while in the case of chondroitin this occurs only after the substance has been decomposed.

Chondroitin-sulphuric acid is an amorphous substance, and is 
soluble in water. A concentrated solution resembles mucilage in appearance and consistence. Its salts are also for the most part soluble in water. The sodium and potassium salts can be precipitated by means of ferric chloride, lead subacetate, and alcohol, while silver nitrate, zinc chloride, tannic acid, and potassium ferrocyanide, the latter in the presence of acetic acid, are without effect.

In the cartilage its potassium and sodium salts occur both as such and in combination with collagen and albumins. A mixture of these compounds, according to Schmiedeberg, constitutes the so-called chondromucoid of Mörner. If a solution of gelatin is mixed with an acidified solution of the potassium or sodium salts of the acid, a precipitate occurs. This also results if cartilage is boiled with water and the resulting impure solution of gelatin, which was formerly termed chondrin, is acidified with a dilute mineral acid. The precipitate consists of the free chondroitin-sulphuric acid, and is soluble in an excess of the acid.

Chondroitin-sulphuric acid, while essentially a constituent of hyaline cartilage, has also been found in elastic and fibrous cartilage, in bone, in the inner coats of the larger arteries, in the chondromas, in the amyloid substance obtained from liver, in the mucosa of the pig's stomach, and in the ligamentum nuchæ. Traces are likewise found in the urine.

Isolation.-To isolate chondroitin-sulphuric acid from cartilage shavings, the material is boiled with a 5 per cent. solution of caustic alkali. The solution is neutralized, and freed by filtration from the alkaline albuminates which have been formed during the process of boiling. Albumoses are removed by means of tannic acid, the excess of the latter by means of lead subacetate, and the excess of lead with hydrogen sulphide. The acid is then precipitated with alcohol. To purify the substance, it is dissolved in water, and the solution dialyzed and reprecipitated with alcohol. The solution in water and precipitation with alcohol is repeated several times, when the acid is finally washed with alcoholic ether.

Isolation of Chondromucoid.-To isolate the chondromucoid, viz., the compounds of chondroitin-sulphuric acid with collagen and albumins, the cartilage shavings are first extracted with water, which dissolves the free chondroitin-sulphuric acid and a small amount of the chondromucoid. On acidulating this solution with a 3 pro mille solution of hydrochloric acid and heating on a waterbath, the chondromucoid is gradually precipitated, while the free acid remains in solution. The cartilaginous residue is then extracted with a 2 to 3 pro mille solution of hydrochloric acid at a temperature of $35^{\circ}$ to $40^{\circ} \mathrm{C}$., which dissolves any collagen that may be present as such. After washing with water the remaining material is extracted with a 5 pro mille solution of caustic alkali. The chondromucoid is thus dissolved, and is then precipitated with an acid. After repeated solution in an alkali and precipitation with an acid it is finally washed with alcohol and ether. 
Albumoid.-The albumoid which is found in the cartilage of adult animals is apparently closely related to elastin and keratin, but differs from the latter in containing sulphur, and from the former in its digestibility by gastric juice. It gives the common color-reactions of the albumins, but is insoluble in all neutral solvents, and dissolves in acids and alkalies only with great difficulty.

Isolation.-To isolate the substance, cartilage shavings are first extracted with a 0.5 per cent. solution of caustic alkali, to remove the chondromucoid, and the free chondroitin-sulphuric acid. The remaining material is then washed with water and boiled with water in a Papin digester. Any collagen that may be present is thus dissolved, while the albuminoid together with the cartilage-cells remains behind.

Mineral Constituents.-Among the mineral constituents of cartilage, the very large amount of alkaline sulphates is especially noteworthy. These are supposedly not present in the free state, however, beyond traces perhaps, but result from the chondroitinsulphate on incineration. In the cartilage of the shark, very curiously, sodium chloride constitutes as much as 94.2 per cent. of the total amount of mineral ash. As this represents 17.7 per cent. of the moist material, the amount of sodium chloride would be sufficient to form a concentrated solution in the cartilage, which, of course, is scarcely conceivable as occurring in living tissue. It is hence assumed that the salt is present in organic combination, but of its pairling nothing definite is known. According to Bunge, such large amounts of sodium chloride are also found in mammals during the period of intra-uterine life and shortly after birth, and he believes that this is in accordance with the biogenetic law which underlies the development of the higher forms of life from those of a lower order.

With the appearance of old age a gradual deposition of calcium salts occurs in the matrix of the cartilage, so that partial ossification takes place. This, of course, also occurs during the development of normal bone, but it is to be noted that, in contradistinction to true bone, the matrix of senile, ossified cartilage retains its original characteristics.

\section{BONE.}

The matrix of bone-tissue, like its contained fibrils, is composed of collagen, which is here termed ossein, and is supposedly identical with the common form that is obtained from connective tissue. Of the composition of the cells, viz., the so-called bonecorpuscles, nothing is known. With their processes they occupy the lacunæ and canaliculi, and are separated from the bony structure proper by a layer of a very resistant albuminous substance of unknown character.

In contradistinction to the other supporting tissues of the body 
which have thus far been considered, bone-tissue apparently contains no glucoproteids.

The function of the bone-tissue, as the principal supporting tissue of the body, finds its expression in the preponderance of the mineral constituents over the organic solids, and it is interesting to note that the ratio between the two is fairly constant, not only in different bones, but also in different animals. These salts are largely represented by calcium phosphate and carbonate, which impregnate the entire matrix. In addition, we find magnesium phosphate and small amounts of calcium chloride, calcium fluoride, potassium and sodium salts, and a little iron. Of the mañner in which the different salts are combined with each other, nothing definite is known, but we may possibly assume, with Gabriel, that the composition of the bone-ash, as well as the tooth-ash, can be represented by the formula $\left[\mathrm{Ca}_{3}\left(\mathrm{PO}_{4}\right)_{2}+\mathrm{Ca}_{5} \mathrm{HP}_{3} \mathrm{O}_{13}+\mathrm{H}_{2} \mathrm{O}\right]$, in which 2 to 3 per cent. of calcium is replaced by magnesium, potassium, and sodium, and 4 to 6 per cent. of the phosphoric acid by carbonic acid, chlorine, and fluorine.

Whether or not the mineral constituents of the bone exist in combination with the organic components of the tissue has not as yet been definitely ascertained, but does not appear improbable.

An idea of the quantitative distribution of the different salts in different animals and bones may be formed from the accompanying analyses. The figures have reference to 100 parts of bone-ash (Zalefsky):

\begin{tabular}{|c|c|c|c|c|}
\hline & Human. & ox. & Turtle. & $\begin{array}{l}\text { Guinea- } \\
\text { pig. }\end{array}$ \\
\hline Calcium phosphate $\left(\mathrm{Ca}_{3}\left(\mathrm{PO}_{4}\right)_{2}\right)$ & 83.89 & 86.09 & 85.98 & 87.38 \\
\hline $\begin{array}{l}\text { Magnesium phosphate }\left(\mathrm{Mg}_{3}\left(\mathrm{PO}_{4}\right)_{2}\right) \text {. } \\
\text { Calcium in combination with carbon }\end{array}$ & 1.04 & 1.02 & 1.36 & 1.05 \\
\hline $\begin{array}{l}\text { dioxide, chlorine, and fluorine } \\
\text { dion }\end{array}$ & 7.65 & 7.36 & 6.32 & 7.03 \\
\hline Carbon dioxide $^{1}$. . . . . & 5.73 & 6.20 & 5.27 & 1.00 \\
\hline Chlorine..... & 1.80 & 2.00 & & \\
\hline Fluorine $^{2}$ & 2.30 & 3.00 & 2.00 & \\
\hline & $\begin{array}{l}\text { Calcium } \\
\text { oxide } \\
\text { (CaO). }\end{array}$ & \multicolumn{2}{|c|}{$\begin{array}{c}\text { Phosphoric } \\
\text { acid } \\
\left(\mathrm{P}_{2} \mathrm{O}_{5}\right) \text {. }\end{array}$} & $\begin{array}{l}\text { Magnesium } \\
\text { oxide } \\
\text { (Mgo). }\end{array}$ \\
\hline Small flounder (ash in general). . & 53.13 & \multicolumn{2}{|c|}{42.72} & 0.91 \\
\hline Man (ash in general) $\ldots$ & 52.83 & \multicolumn{2}{|c|}{38.73} & 0.48 \\
\hline Man (humerus) . . & 51.31 & \multicolumn{2}{|c|}{36.65} & 0.77 \\
\hline $\mathrm{Ox}$ (femur) $\ldots$ & 51.28 & \multicolumn{2}{|c|}{37.46} & 1.05 \\
\hline Goose (ash in general) . . . . & 51.01 & \multicolumn{2}{|c|}{38.19} & 1.27 \\
\hline $\begin{array}{l}\text { Rabbits, varying in age between } \\
\text { one day and four years (general } \\
\text { ash) }\end{array}$ & $91-52.89$ & & & -1.38 \\
\hline
\end{tabular}

The variations in the amount of bone-ash, as a whole, in different bones of the same animal, are seen in the following table (Frémy):

1 These figures are somewhat too low, as a certain amount of carbon dioxide escapes during the incineration of the bone.

2 According to Gubriel, the amount of fluorine does not exceed 0.1 percent., and is usually less than 0.5 per cent. 


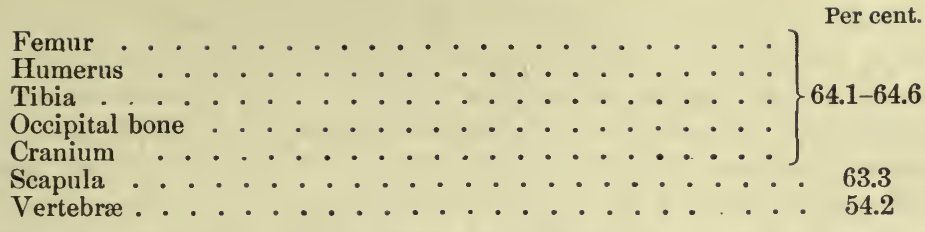

The amount of water which is found in bones varies between 13.8 and 44.3 per cent. It is greater in the spongy bones than in those of the compact variety, and gradually diminishes with age.

The bone-marrow is pervaded by a network of connective tissue, which is partly of the white fibrous variety and partly reticulated. In its meshes we find the cellular elements of the marrow, viz., the so-called myeloplaxes, the juvenile forms of the polynuclear neutrophilic and eosinophilic leucocytes, viz., the myelocytes, red corpuscles in various stages of development, and a variable number of fat cells. These latter are especially numerous in the so-called yellow marrow, where the amount of fat may represent as much as 96 per cent. of the entire substance. It consists of olein, palmitin, and stearin. The red marrow, on the other hand, contains a much smaller amount of fat, and owes its color to large numbers of red corpuscles. It contains albumins, of which one is regarded as a globulin, and is said to coagulate at $50^{\circ} \mathrm{C}$. But especially interesting is the presence of peculiar iron compounds which are as yet but little known, but probably belong to the nucleo-albumins and iron-containing albuminates. Their presence is no doubt intimately associated with the formation of red corpuscles. Among the extractives of bonemarrow we notably find lactic acid and hypoxanthin.

\section{THE TEETH.}

The dentin of the teeth is a peculiarly modified form of bonetissue, and is likewise composed of an organic matrix, which consists of collagen and is impregnated with mineral salts. The latter are here even more abundant than in true bone, and represent from 64 to 68 per cent. of the fresh tissue, while of organic matter we find between 26 and 28 per cent., thus leaving 10 per cent. for water. The relative distribution of the individual salts is about the same as in common bone.

The dentinal tubules, like the lacunæ and canaliculi, are apparently lined by the same albuminous substance.

The cement which surrounds the dentin of the root as far as the neck of the tooth consists of true bone.

The enamel, in accordance with its epithelial origin, contains no collagen. It is very rich in lime salts, and its mineral constituents represent as much as 96 per cent. of the total substance. Its organic components correspond to about 3.6 per cent., but are as yet unknown. Water is practically absent.

Other tissues which are closely related to bone are ivory, tortoise- 
shell, and the scales of fishes. In the two former the mineral constituents predominate over the organic matter, as in bone, while in the latter more organic material is found. Ivory is especially rich in magnesium phosphate, of which it contains about 15.72 per cent. The outer surface of tortoise-shell is covered with a layer of keratinized epidermis. In fish-scales mineral material, collagen, and a peculiar albuminoid body, which is termed ichthylepidin, are found. According to Mörner, this gives an intense Millon reaction and contains a large amount of loosely combined sulphur. Green and Tower were able to demonstrate its presence in a large number of different fishes. It is apparently found in most of the teleosts, but is absent in the elasmobranchs.

In the invertebrate animals, with the exception of the cephalopods, and possibly alsc the branchiopods, collagen is not found The internal supporting structures are here represented by ingrowths of the cuticular formations, which are derived from the epidermal cells, and consist largely of skeletins and hyalogens which have become impregnated with lime salts. Closely related to the latter is chitin, which enters largely into the composition of the outer skeleton of the arthropods and their nerve-sheaths. Associated with it we find the so-called tunicin, which is regarded as a cellulose, and which is found in especial abundance in the mantle of the tunicates.

\section{ADIPOSE TISSUE.}

Adipose tissue may be regarded as a special form of connective tissue in which the cellular elements enclose globules of fat. When fully developed, the individual cells appear as greatly distended vesicles, which are covered by a cell-membrane. The original protoplasm has been almost entirely replaced by fat, and occurs merely as a thin layer beneath the membrane. The nucleus also has been displaced to the periphery, and can scarcely be discerned without special methods of staining. Such fat-cells usually occur in groups, and are held together by delicate fibres of connective tissue, in the meshes of which a network of blood-capillaries is found surrounding each cell. When large numbers of fat-cells occur, the individual groups are gathered into lobules, and these into lobes. In the living tissue the contained fat exists in a liquid form, but congeals after death, and is then more or less solid according to the character of the individual fats. Stearin and palmitin often separate out in crystalline form. Of the chemical composition of the original cells, before their invasion with fat-globules, nothing definite is known. They contain albumin and are apparently rich in water. The cell-membrane is exceedingly resistant to solvents, but is digested by the gastric juice, and possibly consists of an elastin-like substance.

The relative amount of water and fat which is found in adipose tissue varies primarily with the state of nutrition, and differs in different animals. 
The fats in question are principally the triglycerides of stearic acid, palmitic acid, and oleic acid. Others, such as the glycerides of capronic acid and valerianic acid, are not constant constituents of adipose tissue, but are met with only exceptionally, and always in very small amounts. In man, a comparatively large amount of olein is found, but it is not so abundant as in certain cold-blooded animals, in which it may form the greater portion of the fat. The quantitative relation between the three forms is by no means constant in all parts of the body, so that the melting-point of the fats from different regions may be quite different. It differs, moreover, in different animals. This is shown in the following table, which is taken from Gautier :

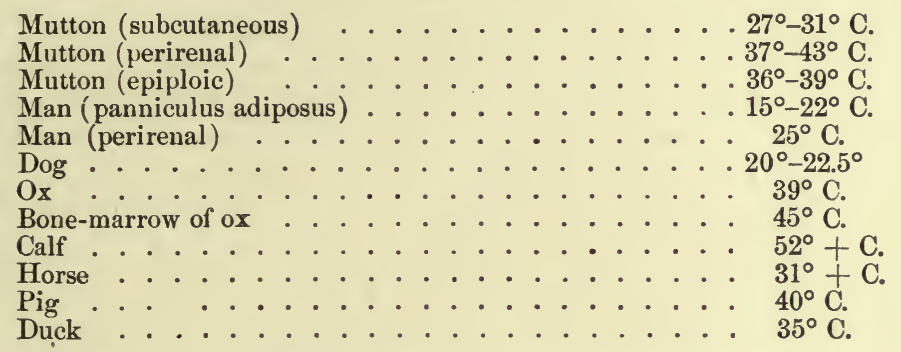

Of special interest is the fact that it is possible to replace the common fats of one animal by those of another, and even by fats which are not found normally in the animal world. If dogs, in which the fats have been removed by starvation, are thus fed with vegetable fats, such as rape-oil, this is subsequently found in the tissues of the animal, and may be recognized by its low melting-point $\left(23^{\circ} \mathrm{C}\right.$.) and the presence of the glyceride of erucic acid. In a similar manner a deposition of mutton tallow may be effected, which begins to melt at about $40^{\circ} \mathrm{C}$., while the common fat of dogs melts at $20^{\circ} \mathrm{C}$.

In addition to the fats, small amounts of lecithin, cholesterin, and free fatty acids may also be isolated from adipose tissue. We further find a yellow lipochrome, to which the color of the fat is due. General analysis of human fat has given the following results (Jaeckli) :

Fat from subcutaneous Fat from child tissue. three days old.

Olein 72.1-85.3 per cent. 55.0 per cent.

Fat from lipoma.

Corresponding amount of

oleic acid . . . 69.6-81.6 "6

Palmitic acid . . . 16.9-21.1 "

\begin{tabular}{|c|c|c|}
\hline & " & $65.7-86.4$ \\
\hline & & $1.5-5.9$ \\
\hline
\end{tabular}

Free acid (calculated as oleic acid) . . . . 0.19-0.52 "

Phosphoric acid $\left(\mathrm{P}_{2} \mathrm{O}_{5}\right) \quad 0.006-0.007$ "

Lecithin . . . . 0 0.07-0.08 "

Pure cholesterin . . . . 0.24

Non-saponifiable material exclusive of cholesterin 0.08

$\begin{array}{lll}0.36 \text { " } & 0.15-0.34 \text { " } \\ \cdot . & 0.001-0.63 \text { " } \\ . . & 0.01-7.21 \text { " } \\ . . & 0.34-1.69 \text { " }\end{array}$


As compared with that of other higher mammals human fat shows no special points of difference in its composition.

Analysis of Adipose Tissue.-The material in question is first dried, ground with a little sand, and extracted successively with ether and alcohol. The alcoholic extract is evaporated to dryness, the residue washed with water to remove the soluble salts, and is then extracted with ether. The ethereal extracts are united, and the ether is distilled off, when the fats, lecithins, cholesterins, free fatty acids, and the lipochromes remain. The fatty acids are transformed into their salts by adding a slight excess of sodium carbonate, and heating to a temperature of $100^{\circ} \mathrm{C}$. The resulting soaps are extracted with water. The insoluble portion is dissolved in ether, the ether is distilled off, and the residue is heated on a water-bath with an alcoholic solution of sodium hydrate, which saponifies the fat's. The resulting material is extracted with ether, which dissolves the cholesterin. The insoluble residue is dissolved in water, and the solution is saturated with carbon dioxide and extracted with strong alcohol. This takes up the soaps and the glycerin. The alcoholic solution is transformed into an aqueous solution, in which the soaps are decomposed with a dilute acid. The free fatty acids are thus precipitated, and can then be separated from each other according to the usual methods.

Aside from adipose tissue, fats are met with in all the organs of the body, but, with the exception of the mammary glands during their functional activity, they are found normally only in traces. Under pathological conditions, however, notable quantities of fat may be met with. We then speak of a fatty degeneration of the organs. This is especially observed in the liver in cases of acute yellow atrophy, and can also be brought about artificially by poisoning with phosphorus, antimony, arsenic, etc.

Among the fluids of the body, large quantities are normally found only in the milk, and in the chyle during the process of digestion.

Origin of the Fats.- That a portion of the fat that is found in the animal body is directly referable to the fats which have been ingested as such cannot be doubted. This is proved not only by the observation that it is possible to replace the fats which are peculiar to a certain animal by those of another, or even by vegetable fats, as has been shown above, but also by the fact that a gradual deposition of fats occurs in dogs which have been starved anl are then fed on very little albuminous material, but with much fat. In such cases it can easily be proved that the amount of albumins ingested is far too small to be the source of the fat that has been stored.

The ingested fats, however, are not the only source of the fats found in the tissues, and there is evidence to show that they may also be derived from the albumins and the carbohydrates. Their 
origin from the former is suggested by many observations. It is thus well known that the albuninous constituents of human bodies when buried in moist ground, and notably the muscle-tissue, may undergo a peculiar transformation, which is characterized by the disappearance of the albumins and their replacement by the free fatty acids and the calcium and ammonium soaps of palmitic acid and stearic acid, which constitute the so-called adipocire, or Leichenwax of the Germans. This transformation is probably brought about through the activity of micro-organisms, and does not prove in itself that in the living animal an actual formation of neutral fats can occur from the albumins. But it shows, at all events, that forces which are at work in the living world can bring about the formation of two of the higher fatty acids at least which enter into the composition of the common fat from albuminous material. In the laboratory such a transformation has not as yet been accomplished, if we disregard the observations of E. Voit, who claims to have noted the appearance of higher fatty acids when albumin was kept in milk of lime for twelve months.

Proof of the possible origin of fats from albumins seems further to be afforded by the phenomena of fatty degeneration, where an actual deposition of large amounts of fat can be demonstrated in the cells of organs in which only traces are normally found. It has been urged, however, that the fat which is here encountered has not developed $i n$ situ, but has been carried to the organs in question from the adipose tissue proper. That such a transportation of fats may occur is possible, and has indeed been proved for the fatty degeneration of the liver which results from poisoning with phloridzin; Leick and Winckler could also demonstrate that in dogs which had first been starved and then fed with mutton tallow, and finally poisoned with phosphorus, the fatty degeneration of the heart muscle also was referable to transported fat. On the other hand, there can be no doubt that under normal conditions fats can originate from albumins. Large quantities can thus be isolated from the livers of dogs which have been poisoned with phosphorus after having previously fasted for twelve days. In such an event it scarcely seems admissible to attempt to account for the presence of the large amounts of fat in the liver on the theory of transportation. Bauer, moreover, has pointed out that while in such cases a largely increased elimination of nitrogen occurs, there is evidence to show that a non-nitrogenous portion of the albuminous molecule is retained, as the absorption of oxygen and the elimination of carbon dioxide are decreased by one-half.

A further proof of the possible origin of fats from albumins has been furnished by Hoffmann. Experimenting with maggots of flies, he determined the amount of fat in one portion directly, and then permitted a second portion of the same weight to develop in defibrinated blood containing a known amount of fat. These were 
then killed and analyzed. The result showed that they contained an amount of fat which was from seven to eleven times as large as the total amount of fat in the blood, plus the amount which they originally contained.

Of the manner in which the fat originates from albuminous material our knowledge is still imperfect. That an actual liberation of fatty radicles can occur directly appears unlikely, as we have no evidence whatever to show that the albuminous molecule contains radicles with more than six or nine atoms of carbon. We have shown, however, that glucose and glycogen can both be derived from this source. The question hence suggests itself, Is it possible that the formation of fats from albumins takes place with the intermediate formation of carbohydrates? As a matter of fact, this possibility is now well established. This transformation represents one of the most important synthetic phenomena which occur in the animal world, and is of the nature of a synthetic reduction in which the $\mathrm{CHOH}$ groups of the carbohydrates are transformed into $\mathrm{CH}_{2}$ groups.

Magnus-Levy has recently suggested that the natural synthesis of fats from carbohydrates takes place by way of lactic acid and acetic aldehyde in such manner that the higher fatty acids result through repeated condensation of acetic aldehyde or croton aldehyde groups and subsequent reduction.

The possible origin of fats from carbohydrates can be demonstrated in various ways. Two animals from the same litter and approximately of equal weight are starved until the stored fat has mostly disappeared. The one is then killed so as to ascertain the amount of albumins and fats which still remain. The other animal is fed with a definite quantity of some cereal, the contained amounts of albumins, starch, and fat of which are known. The feces are carefully collected and the amount of non-resorbed fat and albumins ascertained. After a variable period of time this animal also is killed, and the amount of albumins and fat estimated. The increase in the amount of albumins must, of course, be referable to that ingested, while the increase in the fat may be in part due to the ingested fat, in part to albumins, and in part to carbohydrates. In such cases it has been found that the amount of both albumins and fat which were contained in the food are by no means sufficient to account for all the accumulated fat, so that the conclusion is unavoidable that a certain proportion of this must be referable to the ingested carbohydrates. Calculation has, indeed, shown that as much as 86.7 per cent. of the fat is of such origin.

Significance of the Fats.-As regards the function of fat in the animal body our knowledge is very imperfect. Owing to its property as a poor conductor of heat, it is probably of moment in preventing undue irradiation. Its principal significance, how 
ever, is undoubtedly connected with its manifest value as a food-stuff and as a source of energy. But we do not know whether this is expressed in any specific function of the body beyond the production of heat in general. As the fat disappears during starvation before the albumins are attacked, we may assume that its presence under normal conditions prevents an undue destruction of those elements which essentially represent the living tissue. In this respect, however, the fats are inferior to the carbohydrates, as is apparent from the fact that in starving animals the administration of fats does not lead to so marked a diminution in the elimination of nitrogen as is effected by a corresponding amount of carbohydrates. Upon this basis also Voit has explained the well-known phenomenon that herbivorous animals are more likely to accumulate albumins than the carnivora, as the latter receive scarcely any carbohydrates in their food, while that of the former contains comparatively large amounts. 


\section{CHAPTER XX.}

\section{THE SKIN AND ITS APPENDAGES.}

In considering the chemical composition of the skin and its appendages, we shall deal more exclusively with those substances which are more or less peculiar to its epidermal structures. The remaining components have already been studied in detail and require no further consideration at this place.

'The epidermal structures in the case of the vertebrate animals comprise the epithelial lining of the skin, together with its sweat glands, the sebaceous glands and allied glands, the hair, the nails, the hoofs, the feathers, etc.

On section of the epidermis we discern different layers of cells. The lowest of these, which is known as the Malpighian layer, is composed of the youngest cells, from which all others are derived. These are distinctly protoplasmic in character, but with increasing age they become dry and scaly, and are finally represented by fine lamellæ of keratin, which are constantly thrown off and regenerated from below. Keratin, itself, however, is not found in the lower layers of the epidermis, as has been shown by Ernst, but appears only above the so-called stratum granulosum. In the latter we find peculiar granules, which are seattered about the nuclei of the cells, and which Ernst regards as derivatives of the nuclei. They are known as eleiddin granules, and, according to some observers, represent intermediary products in the formation of keratin. Of their chemical nature, however, nothing is known.

The transition of the soluble albumins of the lower strata of cells into the insoluble keratin also finds its expression in the greater resistance which the upper layers offer to the action of the caustic alkalies. For, while the lower cells are dissolved with comparative ease the upper strata are scarcely affected by the reagent. Pancreatic juice and gastric juice behave in the same manner, and it is thus possible to separate the insoluble keratin from the soluble albumins which may be present at the same time. Like the horny layer of the skin, so also are other epidermal structures, such as nails, hair, horn, feathers, etc., largely composed of keratins, and it is noteworthy that these keratins are especially rich in sulphur. That of human hair, according to Suter, contains 2.52 per cent., of which 2.34 per cent. is present in loosely combined form, which, as we have seen, is most likely represented by a cystin group. Horn 
shavings contain even more, viz., 3.42 , of which 2.53 is loosely combined.

In addition, we find a variable amount of salts, among which the insoluble forms are especially important. Their presence manifestly serves the purpose of increasing the rigidity of these structures. Especially noteworthy is the large amount of silicic acid which is found in hair and in feathers. Besides this, we meet with variable amounts of phosphates and sulphates of the alkalies and alkaline earths, and very curiously also with iron salts. The fact that larger amounts of the latter are found in dark-colored hair than in hair of a lighter color suggests that their presence may be dependent upon these pigments. Of interest is the fact that Gautier was able to demonstrate the presence of traces of arsenic as a constant constituent of the skin and its appendages, viz., the hair, the wool of sheep, etc.

The black and brown pigments which are found in the hair and in the skin of the negro belong to the group of the melanins. Individually these bodies are but little known, and it is an open question whether the iron that is found in the ash is present in these structures in molecular combination with the pigments. Unlike the fuscin of the choroid and the hippomelanin that has been obtained from melanotic tumors in horses, the melanins of the skin and the hair are easily soluble in solutions of the alkaline hydrates. They contain sulphur ( 2 to 4 per cent.), but not in so large amounts as the phymatorhusin that has been isolated from melanotic growths of man and from the urine ( 8 to 10 per cent.). Bodies belonging to this order apparently have also been encountered among the products of hydrolytic decomposition in the case of the common albumins, and Pick has described a similar substance which he found in Witte's peptone, and which he designates as peptomelanin. Conjointly the melanins, which can be obtained from the albumins, are termed melanoidins (Schmiedeberg). On fusion with potash skatol and indol result; heated in the dry state with powdered zinc they give an intense pyrrol reaction with pine wood and hydrochloric acid. On reduction of the melanoidin obtained from serum-albumin with zine in a current of hydrogen, as also from the melanin of the choroid, and that of melanotic growths, pyridin has been obtained. In addition Samuely found a supposedly aromatic body with a benzaldehyde-like odor.

As regards the origin of the melanoidins, we may imagine that the various chromogenic groups which occur in the albumins, and which contain or form aromatic (tyrosin) and mainly heterocyclic radicles (pyrrol, pyridin, skatol), are condensed to dark-colored products on boiling with acids, with coincident loss of water and taking up of oxygen, and that the mixtures of these products represent the melanoids.

E. Spiegler seems to have demonstrated conclusively that the 
pigment of black hair is not a derivative of blood-pigment, as it is impossible to obtain either hæmopyrrol or a hæmatinic acid on appropriate treatment of the isolated product, viz., substances which must be regarded as characteristic reduction-, viz., oxidation-, products of the blood-pigment. On oxidation of the black pigment of horse-hair he obtained a substance of the composition $\mathrm{C}_{11} \mathrm{H}_{22} \mathrm{O}_{2}$, which seems to be identical with Butlerow's methy-dibutylacetic acid. The elementary composition of the black pigment obtained from different sources differs somewhat; that derived from black horse-hair had the composition $\mathrm{C}_{50} \mathrm{H}_{58} \mathrm{~N}_{8} \mathrm{SO}_{12}$, another from black sheep wool $\mathrm{C}_{46} \mathrm{H}_{68} \mathrm{~N}_{8} \mathrm{SO}_{20}$.

From white hair of horses and sheep he obtained white pigments of the composition $\mathrm{C}_{45} \mathrm{H}_{78} \mathrm{~N}_{10} \mathrm{SO}_{20}$ and $\mathrm{C}_{61} \mathrm{H}_{98} \mathrm{~N}_{10} \mathrm{SO}_{20}$. These white pigments apparently represent the chromogens of the corresponding black pigments.

Into the various pigments which have been found in the skin of reptiles, in the scales of fishes, in the feathers of birds, etc., it is scarcely necessary to enter at this place. They partly belong to the melanins, partly to the lipochromes; others are classified as melanoids, while still others are closely related to the hæmoglobins. To a certain extent, moreover, the colors of birds' feathers appear to be of a physical nature and referable to certain phenomena of interference.

In the invertebrate animals various pigments are also observed, but are for the most part unknown. The keratin, as I have already stated, is here represented by other tegumentary substances, such as chitin, tunicin, the hyalogens, and the skeletins (which see).

The Sweat.-The sweat is the specific, secretory product of the corresponding glands, which are found imbedded in the lower portion of the dermis. In man, these glands rank next in order to the kidneys in importance as excretory organs of water, and are capable, to a certain extent at least, of assuming a vicarious activity when the kidneys are diseased. In man their number is quite large, exceeding 2,000,000. Their distribution, however, is not uniform, and as a result there are certain portions of the body in which a more abundant secretion is noted than in others. Such regions are the forehead, the armpits, the palms of the hands, the soles of the feet, etc.

Among the mammalian animals, however, some exist in which a secretion of sweat does not occur. This is the case in many of the rodents and the goat. The sheep, the horse, and the apes, on the other hand, sweat over their entire body, while other animals, like the cat and the dog, sweat only from the balls of the toes.

The amount of sweat excreted in man is very variable. It differs in different individuals; and is dependent upon the surrounding temperature, the amount of water ingested, the temperature of the body, the amount of exercise taken, etc. It is manifestly under the control of the central nervous system, and is increased by painful 
sensations and emotions, by stimulation of the sciatic nerve, following the administration of pilocarpin, etc.

Ordinarily the secretion of sweat is scarcely noticeable, as the droplets evaporate almost as rapidly as they appear on the surface of the skin. But even so, from 700 to 900 c.c. of water are daily eliminated by the body. Artificially this amount can be greatly increased, and it is stated that from 6000 to 8000 c.c. may be excreted in the twenty-four hours if the body is kept at a temperature of from $40^{\circ}$ to $50^{\circ} \mathrm{C}$, and large amounts of fluid are ingested.

Sweat, recently secreted, is more or less turbid, owing to an admixture of desquamated epithelial cells, and droplets of fat, which are in part derived from the sebaceous glands, but are to some extent also referable to the sweat-glands proper. After filtration it appears as a clear, transparent, colorless fluid, of a salty, somewhat acrid taste, and a very characteristic odor, which differs somewhat according to the region of the body from which the sweat is derived. Its specific gravity varies between 1.004 and 1.005 .

At the beginning of its secretion the sweat presents an acid reaction, which is probably referable to an admixture of fatty acids derived from the sebaceous glands; later, however, it is alkaline.

Under normal conditions the sweat is essentially a very dilute aqueous solution of mineral salts, but in addition we also find small amounts of many urinary components, such as urea, uric acid, kreatinin, aromatic oxy-acids, volatile fatty acids, skatoxyl and phenol sulphate, besides fat, cholesterin, traces of albumin, salts of lactic acid, and so-called sudoric or hydratic acid, etc. Larger amounts of solids are principally met with in cases of renal insufficiency, and it may then happen that the elimination of urea through the sweat increases to 10 grammes, as compared with $0.043-1.55$ pro mille, which may be regarded as normal. In some cases of this kind the urea may actually be found in the form of a fine crystalline powder deposited all over the skin. Glucose has been observed in diabetes. Cystin has been noted in cases of cystinuria, and abnormally large amounts of uric acid have been found in gout. In jaundice bilirubin may color the sweat a bright yellow. A blue and red color, which is thought to be referable to the presence of indigo-blue and indigo-red, has also been observed (chromhidrosis or cyanhidrosis); and it is stated that in rare instances blood may appear in the sweat (hæmahidrosis). It is noteworthy, furthermore, that a number of foreign substances, when ingested by the mouth, such as quinin, the various salts of iodine, mercury, and arsenic, are in part eliminated in the sweat.

A general idea of the quantitative composition of the sweat may be formed from the accompanying analyses, which are taken from Favre, Schöttin, and Fünke. 


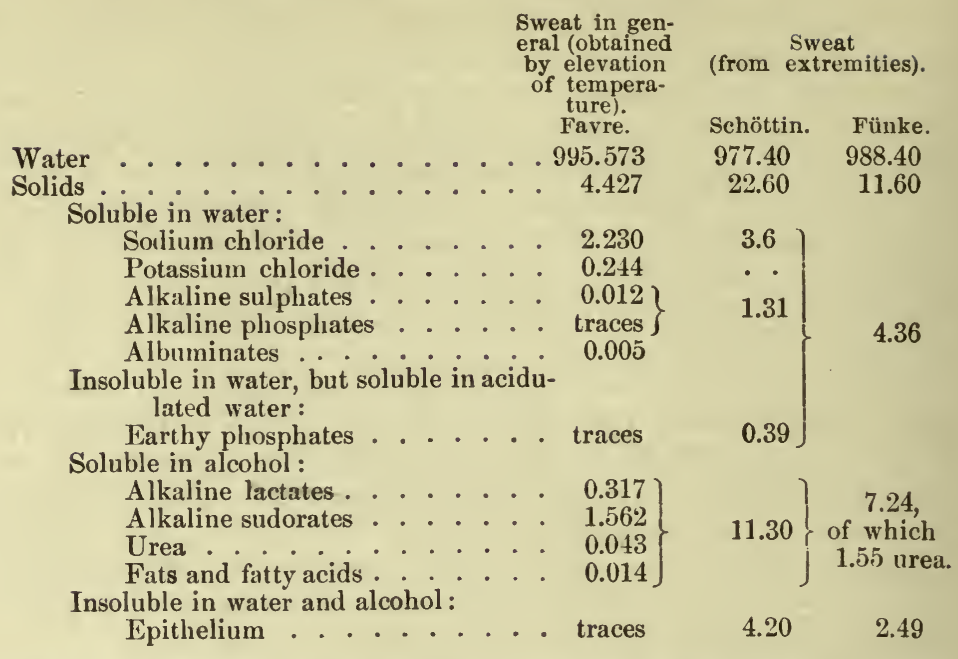

Gases.-While in mammals and birds the respiratory function of the skin is insignificant as compared with that of the lungs, we find that in the amphibia life may persist for quite a while after removal of the lungs, and that during this time oxygen is actively taken up from the air and carbon dioxide eliminated in turn. If, however, the exchange of gases is impeded or prevented by covering the skin with a thin layer of varnish, death rapidly takes place. This also occurs, it is true, in some of the smaller mammals which have a delicate skin, but it is now known that the fatal end is here not referable to the impairment of the cutaneous respiration, nor to a retention of waste products, as was formerly supposed, but to a paresis of the cutaneous vasomotor nerves and a resulting dilatation of the bloodvessels. As a result an abnormally increased irradiation of heat occurs, which constitutes the direct cause of death. If this is prevented by placing the animal in a warm chamber or by surrounding it with cotton and the like, recovery may take place. In the larger mammals, including man, in which a coarser skin exists, no deleterious effects are noted even after ten days.

The amount of carbon dioxide which is given off by man through the skin is principally dependent upon the surrounding temperature, and varies between 8.4 grammes at $29^{\circ}$ to $33^{\circ} \mathrm{C}$., and 28.8 grammes at $38.5^{\circ} \mathrm{C}$.

The Sebum.-The sebum is the specific secretory product of the sebaceous glands, and serves the purpose of a lubricant. Amounts sufficient for analytical purposes can be obtained from newly born children, in which the secretion constitutes the so-called vernix caseosa. In the fresh state it is a semiliquid, oily material, in which on microscopical examination can be discerned desquamated epithelial cells in various stages of degeneration, fat droplets, fatty acid needles, and quite constantly also plates of cholesterin. Almost 
immediately on exposure to the air it solidifies to a white, tallow-like material. Its reaction is alkaline.

The most important constituents of the sebum, as also of the related secretion of the uropygian gland of birds, are the compound cholesterins (see page 80). Their presence has been demonstrated on the feathers and bills of birds, in the wool of sheep, in the hair of mammals, on the spikes of porcupines, etc.; and as these bodies are remarkably resistant to the influence of putrefactive organisms, it has been supposed that their presence on the skin and its appendages serves the purposes of protecting the exposed portions of the body against bacterial invasion.

In addition to substances of this order, the sebum contains a variable amount of fats, fatty acids, soaps, lecithins, mineral salts, and at least two albumins, of which one is commonly regarded as caseïn.

Closely related to the common sebum of the sebaceous glands of the skin proper is the secretion of the preputial gland-the so-called smegma præputii and the cerumen of the ceruminous glands of the external ear. In addition to the common constituents of the sebum, the smegma also contains certain components of the urine and their decomposition-products, such as ammonium soaps, and in the horse hippuric acid, benzoic acid, and oxalate of lime. Its peculiar odor in man is no doubt due to the presence of certain fatty acids. In the secretion of the beaver-the castoreum of the shops-this is thought to be referable to a phenol-like body, while in the corresponding product of the musk-deer (musc) a volatile base is, according to Wöhler, the active odorous principle.

The cerumen differs from the common sebum in containing a very considerable proportion of potassium soaps. In addition, we also meet with a peculiar yellow pigment, which has an exceedingly bitter taste; but of the composition of this nothing is known.

In the skin glands of certain amphibia, finally, and notably the toar and the salamander, poisonous substances have been found which in their physiological effect closely resemble the action of digitalis and strychnin. They have been termed bufidin and salamandrin, respectively. In the secretion of the toad, moreover, methyl-carbylamin and isocyan-acetic acid have been found, of which the former is especially toxic. 


\section{CHA PTER XXI.}

\section{THE GLANDULAR ORGANS.}

\section{THE LIVER.}

THE functions of the liver, as is apparent from a survey of the foregoing chapters, are manifold. During embryonic life the organ is intimately concerned in the production of red corpuscles, and at this time already manifests its function as an excretory organ also in the production of bile. After birth its hæmopoietic activity ceases, but it continues important as an excretory organ through which the decomposition-products of hæmoglobin, in so far as they are not retained and utilized in the formation of new corpuscles, are eliminated from the body in association with taurin, glycocoll, cholesterin, and the cholalic acids. At the same time, however, the liver is the seat of some of the most important syntheses which occur in the animal body, and in which both anabolic and katabolic products of the metabolism are involved. We have thus seen that the greater portion of urea in mammals, and of uric acid in birds and reptiles, is here produced, and we have also pointed out that certain aromatic substances which are formed during the process of intestinal putrefaction or have been ingested as such are transformed in the liver into conjugate sulphates and glucuronates and are thus rendered innocuous. Still other substances, moreover, which are foreign to the body, such as various metallic salts and certain alkaloids, are here removed from the general circulation when artificially introduced, and it is for this reason also that the hypodermic injection of such substances is much more efficacious than their administration by the mouth. The subsequent elimination of the metallic salts then occurs in part through the bile, and to a great extent also through the intestinal epithelium. The alkaloids are similarly removed, and also appear in the urine in a more or less modified form.

Formerly it was supposed that the retransformation of peptones (in the older sense of the word) into native albumins occurred in the liver, but, as I have shown, this is not the case. On the other hand, we have seen that the carbohydrates after their transformation into monosaccharides, are carried to the liver, and are here stored in the form of glycogen, when an immediate demand for glucose does not exist on the part of the other organs and tissues of the body. This transformation of monosaccharides into glycogen represents one of the most important syntheses which occur in the animal body, and it is interesting to note that whereas glycogen on decomposition always gives rise to the formation of glucose, the liver is capable of transforming the other monosaccharides into glycogen as well. 
Of the forces which are at work in bringing about these various changes in the liver we know very little, but to judge from recent observations it appears certain that various tissue ferments are here active.

The reaction of the living liver-tissue is alkaline. After death, however, it becomes acid, and there is reason to believe that, as in the case of the muscle-tissue, this acid reaction is essentially referable to the formation of lactic acid. At the same time the tissue becomes opaque, owing to a coagulation of the liver albumins.

A general idea of the chemical composition of the liver may be formed from the following analyses, which are taken from v. Bibra:

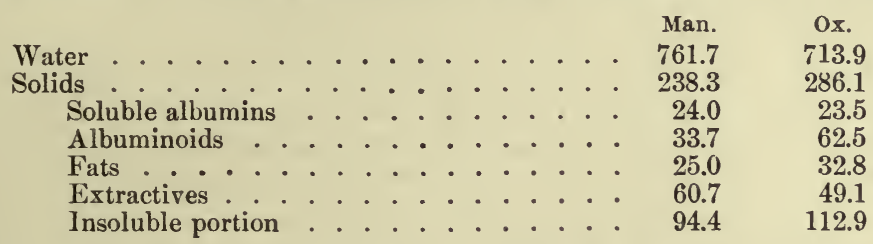

The mineral salts, according to $\mathrm{v}$. Bibra, constitute about 1 per cent. of the fresh tissue, and are essentially represented by the phosphates of potassium and sodium and a fairly large amount of iron. Traces of manganese, copper, and lead are also found.

The Albumins.-The albumins of the liver-tissue have been notably studied by Halliburton and Plosz. The gland was freed from blood and bile by transfusion with ice-water containing 0.75 per cent. of common salt. The tissue was then cut into small pieces with cooled knives, frozen and placed under pressure. On thawing, an alkaline fluid could thus be obtained, which represents the liverplasma. In this fluid a globulin exists which coagulates at $45^{\circ} \mathrm{C}$., and is regarded by Halliburton as being possibly identical with one of his cell-globulins. It can be digested by gastric juice. In addition a nucleoproteid was found, which coagulated at $70^{\circ} \mathrm{C}$., and which yielded an insoluble residue of nuclein on digestion. From the cells proper they extracted a globulin with a 10 per cent. solution of sodium chloride, which coagulated at $75^{\circ} \mathrm{C}$., and which may also be identical with one of Halliburton's cell-globulins; further, an albumin (coagulation-point $70^{\circ}-73^{\circ} \mathrm{C}$.) and an alkaline albuminate. In addition, a glucoproteid has also been demonstrated, which yields a reducing substance on boiling with dilute mineral acids, and which is probably of a mucinous character and derived from the connective tissue of the organ. According to Wohlgemuth, the nucleoproteid of the liver contains 2.98 per cent. of phosphorus, and a pentose, which he identified as l-xyluse.

The nuclei finally contain nucleoproteid, and it is of special interest to note that at least two of these contain iron. The one is apparently identical with, or at least closely related to, the homatogen of birds' eggs, while in the other, which Zalesky terms hepatin, the iron is even more firmly combined. The occurrence of these 
iron-containing products is important in view of the fact that the iron which is furnished in the food can apparently be utilized only by the body in the formation of hrmoglobin, when introduced in such form. It has hence been suggested that these nucleins after resorption are temporarily stored in the liver until required by the hæmopoietic organs.

The iron which is present in the liver in molecular combination with the nucleoproteids can be demonstrated only after the isolation of the nucleins in question and their incineration. But in addition we also find iron in combination with albumin as so-called ironalbuminate, from which the metal can be split off by treating with acid alcohol. The presence of this form can be directly demonstrated by moistening a slice of the liver-tissue with hydrochloric acid, and then with a solution of potassium ferrocyanide or potassium sulphocyanide, when a blue, viz., a red color develops. As regards the origin of these iron-albuminates, the opinion prevails that they are formed within the tissues of the body, and are referable to the disintegration of red corpuscles. They are accordingly also found in the spleen and the bone-marrow, and are met with in increased amounts in conditions which are associated with an increased destruction of red corpuscles. Large quantities are thus especially noted in cases of pernicious anæmia, in acute infantile gastritis, and in poisoning with arsenious hydride, where their presence constitutes the phenomenon of so-called siderosis of the liver. According to Vay, the average quantity of iron-albuminate which can be isolated from the fresh organ under normal conditions amounts to from 0.15 to 0.3 per cent., corresponding to from 0.01 to 0.018 per cent. of iron. The total amount of iron which is normally found in the liver-cells during adult life is subject to considerable variation, which is more extensive in men ( 0.048 to 0.367 per cent.) than in women ( 0.05 to 0.092 per cent.). On an average the cells of women contain less iron than the cells of men. The lowest values are found between the twentieth and the twenty-fifth year.

The occurrence of especially large amounts of iron in the liver of newly born animals is probably referable to the hæmapoietic activity of the organ during embryonic life.

Isolation of the Iron-containing Nucleins.-To prevent any contamination with hæmoglobin, it is necessary to remove all traces of blood from the liver. To this end, Bunge has suggested the following method: in the living animal which has been anæsthetized with morphin and chloroform a cannula is tied into the portal vein. Through this a stream of a 1 per cent. solution of sodium chloride heated to the body temperature is introduced under moderate pressure. As soon as the solution begins to flow the hepatic artery and the hepatic veins are divided and the abdomen closed. A minute later it is reopened, the liver is dissected out and placed in a porcelain bowl, while the transfusion is continued. The bowls 
are changed until perfectly clear saline solution flows from the veins. To attain this end, the transfusion need be carried on for only a few minutes. If successfully performed, the liver should present a uniformly light-brown color, and a portion of the minced organ when placed in distilled water should leave this entirely uncolored. The gall-bladder is now removed, the organ pressed between filter-paper, finely hashed, and enveloped in muslin. It is then thoroughly kneaded under water. The connective tissue and vessels are thus separated from the cellular elements and remain behind. The cells are thoroughly extracted with water and with dilute saline solution, by decantation, until all soluble substances have been removed. They are then digested with gastric juice. The non-digested residue is extracted with acidulated alcohol and subsequently with ether, to remove pigments, cholesterin, and fats. It is then treated with weak ammonia-water, which dissolves the iron-containing nucleins. From this solution they are precipitated with absolute alcohol when added in excess. The resulting material constitutes the hepatin of Zaleski. The other iron-containing nuclein is apparently present in the liver as a nucleo-albumin, and is found in this form in the saline extract of the cells. To demonstrate its presence, the previous extraction with saline solution is omitted. If the residue of nucleins, which remains after digestion with gastric juice, is then placed in a solution of ammonium sulphide, a greenish color gradually develops which ultimately turns black, owing to the formation of sulphide of iron. The hepatin itself does not give this reaction. Neither substance gives up its iron, even when treated with acidulated alcohol ${ }^{1}$ for days, thus differing from the iron albuminate, which behaves in this manner exactly like inorganic preparations of iron.

Isolation of the Iron-containing Albuminates.-In this case it is not necessary previously to wash out the blood. The organ is minced without further preparation, and is placed in from three to four times its volume of water. The mixture is slowly heated, boiled for about fifteen minutes, and filtered on cooling. The filtrate is carefully precipitated with a 10 per cent. solution of tartaric acid. The resulting flocculent material, which presents a brown color, is collected on a filter, washed with a weak solution of tartaric acid, then with 50 per cent. alcohol, and finally with absolute alcohol. It contains about 6 per cent. of iron. It is soluble in solutions of the alkalies, and does not react with ammonium sulphide at once. After a few minutes, however, the solution becomes darker and gradually turns black. On treating with acid alcohol (see above) the iron is split off, and can be directly demonstrated by testing with potassium ferrocyanide or potassium sulphocyanide. Schmiedeberg has termed the substance in question ferratin, and regards it as a ferri-albuminic acid.

${ }^{1}$ The acidulated alcohol contains 10 volumes of a 25 per cent. solution of hydrochloric acid and 90 volumes of 96 per cent. alcohol (Bunge's fluid). 
Ferments.-Thus far the following tissue-ferments have been demonstrated in the liver-cell : a maltase, a glucase, a proteolytic ferment, a nuclease, an aldehydase, a laccase, a ferment which is capable of transforming the firmly united nitrogen of the amino-acids into ammonia ; further, a fibrin-ferment, one which effects the transformation of glycogen to glucose, a powerful esterase, viz., a lipase which splits especially the lower esters, and finally a rennin-like ferment.

In addition the liver contains a substance, possibly a proferment, which when activated by a certain principle obtained from the pancreas (a kinase) is capable of causing extensive glucolysis. To be sure, the glucose of the liver disappears of itself when the organ is removed from the body, but we may well imagine that this is owing to the presence of a certain amount of the kinase. Livertissue to which pancreas is added will hydrolize a much greater amount of glucose than liver-tissue alone can do (Hirsch). The conditions here are thus quite similar to what takes place in the carbohydrate metabolism of muscle-tissue.

An antithrombin which inhibits the action of the fibrin-ferment has further been described (see Blood-cuagulation).

Glycogen.-Amount.-The amount of glycogen which occurs in the liver is primarily dependent upon the state of nutrition of the animal and the amount of exercise that is taken. This is apparent from the fact that it is constantly consumed during the activity of the muscle-tissue more especially, but is also utilized in the regeneration of all cellular elements of the body. During starvation it rapidly disappears, but it is also rapidly formed if carbohydrates are then ingested. Maximal amounts, according to Külz, are found after from fourteen to sixteen hours following the administration of foorl. It has been calculated that in the liver of man 150 grammes can be stored at one time. This would correspond to about 10 per cent. for an organ weighing 1500 grammes. In dogs which have been fed on potatoes and bread Pavy claims to have found as much as 17 per cent. After death the transformation of glycogen into glucose continues as in the case of muscle-tissue, and in order to ascertain the exact amount which was present during life it is hence necessary to remove the organ at once and to prevent the further inversion of the material, by the living protoplasm or the contained ferments, by placing the tissue in boiling water.

Properties.-The pure substance represents a white, amorphous powder, which is both odorless and tasteless. In water it forms an opalescent solution, from which it can be precipitated by the addition of alcohol, after adding a little sodium chloride, or by means of lead subacetate. The substance is dextrorotatory. The specific degree of rotation, however, seems to be influenced by various factors. In pure solution it is given as $+196.63^{\circ}$. It does not reduce Fehling's solution, but can maintain cupric hydroxide in solution. After the addition of a little sodium chloride its solutions are colored red by treating with iodine. With benzoyl chloride, in the presence of sodium hydrate, it gives a granular precipitate of 
benzoyl-glycogen. On boiling with dilute mineral acids it is transformed into glucose. Ferments invert it to maltose or glucose, according to the nature of the enzymes at work.

Isolation and Quantitative Estimation.-The perfectly fresh liver, immediately after removal from the animal, is placed in boiling water and divided into small pieces. After boiling for a few minutes these are removed, ground to a pulp with sand or pulverized glass, and then boiled in a 1 per cent. solution of sodium hydrate, using 400 c.c. for every 100 grammes of tissue. With liver-tissue two to three hours suffice, while with muscle-tissue it is best to boil for from four to eight hours. Care must be had during this process that the concentration of the alkali does not exceed 2 per cent.; to this end water is added from time to time. The alkaline extract after filtration is then united with the watery solution first obtained, and neutralized with hydrochloric acid. After concentrating the resulting solution, the remaining albumins, notably gelatin, are precipitated on cooling by alternate treatment with a solution of iodomercuric iodide and hydrochloric acid added drop by drop. In the filtrate the glycogen is precipitated with an excess of alcohol. It is collected on a filter, washed with 60 per cent. alcohol, then with absolute alcohol and ether, and is finally dried in a desiccator over sulphuric acid. From the weight thus obtained, that of the combined mineral salts must be deducted after incineration.

Glucose.-The amount of glucose in the perfectly fresh liver varies between 0.2 and 0.6 per cent., but rapidly increases at the expense of the glycogen after the removal of the organ from the body. To obtain results which represent the actual amount that is present during life, it is hence necessary to eliminate the inverting action of the living protoplasm and of ferments by placing the organ in boiling water immediately after the death of the animal. It is then finely minced, thoroughly extracted with boiling water, and the sugar determined in the filtrate according to the usual methods.

Fat.-The amount of fat which is found in the liver is quite large, as compared with the other organs of the body, and normally varies between 2 and 3.6 per cent. It is deposited in the cells, and beginning along the periphery of the acini increases in amount toward the centre. It is most abundant after meals, and to a certain degree is dependent upon the amount of fat ingested. Under suitable conditions the infiltration may become so marked as to simulate fatty degeneration; but, in contradistinction to fatty infiltration, we find that in fatty degeneration the amount of the solids is markedly diminished. The amount of water in fatty infiltration is diminished, while in degenerative changes it is perhaps slightly increased. These relations are exemplified by the following figures, which are taken from Hammarsten :

$$
\text { Water. Fat. } \quad \begin{gathered}
\text { Remaining } \\
\text { solids. }
\end{gathered}
$$

Normal liver . . 770 pro mille 20-35 pro mille 207-195 pro mille.

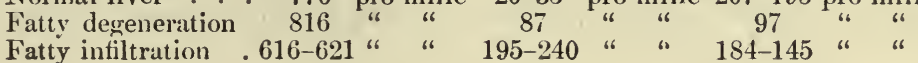


Extractives.-The extractives which are found in the liver, aside from glyeogen and glucose, are notably xanthin-bases, which are derived from the nuclei. They comprise xanthin, hypoxanthin, guanin, and adenin. Conjointly they represent about 4.52 pro mille of the dried tissue. They can be isolated, according to the method described on page 391. In addition we find small amounts of urea, uric acid, paralactic acid, and jecorin. Cystin also has been isolated from the normal liver of a horse and from that of the porpoise, but it is questionable whether the substance can actually be regarded as a normal constituent of the gland. It has once been obtained from the liver of a patient who during life had eliminated cystin in the urine. Under pathological conditions, and especially in acute yellow atrophy, large quantities of paralactic acid have been found, in addition to a notable amount of leucin and tyrosin. In amyloid degeneration of the organ chondroitin-sulphuric acid has been observed. Biliary pigments are normally not encountered in the liver-cells, but quite commonly they stain these an intense yellow in cases of obstructive jaundice. These various constituents have been studied in the foregoing chapters, and need not be reconsidered at this place.

\section{The Digestive Glands.}

Our knowledge of the chemical composition of the digestive glands, viz., the salivary glands, the glands of the stomach, the intestinal mucosa, and the pancreas, is largely expressed in what has been said regarding their specific secretions. With the exception of the pancreas, the cellular elements proper have not been studied in detail.

In the pancreas a highly complex nucleoproteid occurs, which on boiling with water yields a second body of the same order, which, however, is of simpler composition. The first is known as Hammarsten's nucleoproteid- $\alpha$, the second as the corresponding $-\beta$ product. Elementary analysis of this $\beta$ product has given the following results: $\mathrm{C}=34.0 ; \mathrm{H}=5.0 ; \mathrm{N}=0.7 ; \mathrm{P}=4.5$. In addition the substance contains a considerable amount of iron. Both products contain a pentose group ; from the $\alpha$ - body Grund obtained 6.25 per cent., and from the derived body amounts varying between 9.2 and 15.4 per cent.

On digestion with pepsin-hydrochloric acid a nuclein remains behind which is very rich in phosphorus. This yields a nucleinic acid, which Bang has termed guanylic acid from the fact that on decomposition only one purin base, guanin, is obtained.

Isolation of Guanylic Acid (Bang).-1000-1200 grammes of pancreas (from the ox) are finely hashed and suspended in two liters of 1 per cent. sodium hydrate solution. After standing for twentyfour hours the mixture is heated until it becomes a thin liquid; acetic acid is then added until the reaction is distinctly acid. The brownish-black precipitate which results is collected and boiled out 
with water. Filtrate and washings are filtered, rendered feebly alkaline with ammonia, concentrated to about 300 c.c., and while still hot treated with 3 volumes of alcohol. The resulting precipitate is filtered off, dissolved in 150 c.c. of water, and filtered while hot. On cooling, the solution is again precipitated with 3 volumes of alcohol. This process is repeated once more, when the guanylic acid is obtained in pure form. 1000 grammes of pancreas yield about 3 grammes of the acid.

The ferments of the pancreatic juice have already been, considered. In addition we find various autolytic ferments, and, as I have pointed out, the pancreas (possibly the cells composing the areas of Langerhans) also furnishes a body, which may belong to the group of kinases, and which activates certain glucolytic ferments of the liver and the muscle-tissue.

\section{The Lymph-glands.}

The lymph-glands comprise the lymph-glands proper, the thymus gland, and the spleen. Their fibrous network consists essentially of reticulin, but also contains fibres of collagen and elastin. The cellular elements have been studied especially in the case of the thymus. They contain small amounts of albumins, lecithins, fats, cholesterins, traces of glycogen, succinic acid, and, according to Kossel and Lilienfeld, large amounts of so-called nucleohiston. Lilienfeld's nucleohiston, according to the researches of Huiskamp, Malengreau and Bang is, however, no unity, but a mixture of two nucleoproteids, viz., nucleohiston and a nucleoproteid which contains no histon group. The nucleohiston further is a double compound of a nucleinate of histon and a paranucleinate of histon6 parts of what Bang terms normal acid-histon (the normal acid being an adenin-guanylic acid) and 3 parts of adenylic parahiston. The difference in Bang's concept of the nucleohiston, as compared with that of Kossel and Lilienfeld, is shown below :

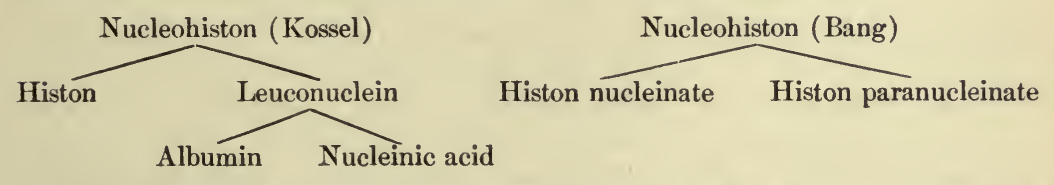

In the thymus the histon (viz., parahiston) nucleinate represents 20 per cent. of the total weight, as compared with 7 per cent. of the second nucleoproteid. In the lymph-glands the same amount of nucleoproteid approximately is present, but only 5 per cent. of the nucleinate of histon. In the spleen still less is found, and the bonemarrow probably contains none.

In the spleen uric acid has also been met with, and as in the liver iron-albuminates occur, which may be isolated as there described. In addition small amounts of inosit, jecorin, and cerebro- 
sides have been encountered. Gulewitsch further demonstrated that arginin is a normal constituent of the spleen.

Of ferments a proteolytic ferment has been discovered in the spleen and in the thymus gland by Kutscher, Conradi and Rowland.

\section{THE KIDNEYS.}

In addition to the common albuminoids which enter into the composition of the supporting tissue of the kidneys, we find the common extractives, viz., nucleinic bases, uric acid, urea, leucin, inosit, glycogen, fats, and at times taurin. All these substances, however, are present in only small amounts. On one occasion, in the ox, cystin has also been encountered, but it is questionable whether this is a constant constituent of the organs. Of albumins, Halliburton has isolated a globulin and a nucleoproteid, with coagulation-points of $52^{\circ} \mathrm{C}$. and $63^{\circ} \mathrm{C}$., respectively. In addition, a mucin-like body has been found, which does not yield a reducing substance, however, on boiling with mineral acids, and which is probably a nucleoproteid. It is notably found in the papillary portion of the kidneys, while the other nucleoproteid is principally met with in the cortical portion. Serum-albumin is said to be absent.

\section{THE MAMMARY GLANDS.}

The chemical composition of the mammary glands has not been studied in detail. We know, however, that the protoplasm of the functionally active glands is rich in albumins, and it appears that, as in the case of the pancreas, a very complex nucleo-glucoproteid is here also present, and is probably intimately concerned in the formation of two of the most important constituents of the milk, viz., the caseïn and lactose. It may be obtained in solution by first washing the gland thoroughly in water, so as to free it from milk; it is then extracted with a 0.5 pro mille solution of sodium hydrate at ordinary temperatures. Such solutions also corrtain the common albumins, and represent an exceedingly viscid, stringy fluid, from which the proteid in question can be precipitated by acidifying carefully with dilute acetic acid. On boiling with dilute acids the substance is decomposed into albumin, phosphoric acid, and a reducing substance of unknown composition. On digestion with gastric juice it yields a paranuclein.

As in the case of the pancreas, the substance is decomposed by boiling the gland with water. A coagulable albumin and a nucleoglucoproteid, which is somewhat less complex than the original substance, thus result. From this solution the proteid can be precipitated by the addition of a dilute acid. Like its mother-substance, it also yields a reducing substance on hydrolytic decomposition. Of the relation of the latter to lactose nothing is known, but it is noteworthy that this is formed on standing if a functionally active 
gland, while perfectly fresh, is ground to a pulp and kept in normal salt solution at the temperature of the body. An intermediary product is then also apparently formed, which is of a colloid nature, but not identical with glycogen. In view of recent researches, which tend to show that the reducing group which is present in the glucoproteids is, in the case of the mucins at least, not a true carbohydrate, but of the nature of chondroitin-sulphuric acid or an allied substance, it would be exceedingly interesting to ascertain whether the reducing substance in the case of the mammary nucleo-glucoproteid also may not be of this order.

Of other constituents of the gland, we find various xanthinbases, and in the functionally active organ also a certain amount of fat which is present in the form of globules of variable size, in the bodies of the cells.

The specific secretory product of the mammary glands is the milk.

The Milk.-The milk is the specific secretory product of the mammary glands, and constitutes the natural food of all mammals in the early stages of their extra-uterine existence. It contains all those food-stuffs which are necessary for the maintenance of life, viz., albumins, carbohydrates, and fats. The nutrient components of the milk, however, are more or less specific of the secretion in question, and are not found elsewhere in the body as such. They are produced in the gland itself from the common constituents of the blood. Among these the albumins are the most important, and there can be little doubt at the present time that the fats of the milk also are largely referable to this source. This is apparent from the fact that in the bitch, for example, the amount of fat increases with an increased ingestion of meat that is free from fats, while it is diminished when the animal is fed on fats only. The so-called milk-sugar also is apparently derived from albumins, as the substance continues to be formed although no carbohydrates are ingested. Its amount, however, is then somewhat smaller, and increases if cane-sugar or starch is added to the diet.

General Characteristics.-Fresh milk is an opaque, white, yellowish-white, or bluish-white liquid, of a somewhat creamy consistence, a more or less sweetish taste, and an insipid odor which is peculiar to the particular animal from which the milk has been obtained. The opacity is largely due to the presence of calcium caseinogen.

On microscopic examination innumerable fat globules are seen, which vary from 0.0024 to $0.0046 \mathrm{~mm}$. in diameter, and number from 200,000 to $5,000,000$ per cbmm., with an average of about $1,050,000$. The fat is thus present in a state of fine emulsion, but, in contradistinction to other emulsions, in feebly alkaline media, it cannot be extracted by shaking with ether directly, or at least only with much difficulty. If, on the other hand, an acid or a caustic alkali is previously added to the milk, this is readily accomplished. From this olsservation it has been concluded that each fat-globule is surrounded by an albuminous membrane, the haptogenic membrane of Ascherson, which is 
dissolved by acids and alkalies, but which normally prevents the solvent action of the ether upon the contained fat. Later investigations have rendered this view improbable, however, and, as a matter of fact, no one has ever succeeded in demonstrating the presence of a special membrane. The normal resistance to the action of ether is now explained upon the assumption that each globule is surrounded by a delicate layer of albumin, which does not constitute a true membrane, however, but is formed as a result of molecular attraction. It is possible, indeed, to prepare emulsions of fat artificially by shaking with albuminous solutions, which in their behavior to ether are quite similar to milk. As regards the character of the particular albumin which forms this layer, our knowledge is not complete. It has been supposed by some that it is formed by casein, but there are reasons for believing that the albumins of the milk in general may here be concerned.

On standing, the greater portion of the fat rises to the surface of the milk and forms its cream. On beating the milk for some time, the individual fat-globules are caused to coalesce, and separate out as a semisolid mass, which constitutes the butter. The remaining liquid is termed buttermilk, and still contains some fat which has remained in emulsion.

Besides the fat-globules the milk contains also innumerable granules of calcium phosphate (probably a mixture of diphosphates and triphosphates) in suspension, which are visible only on microscopical examination and are said to number about 4,000,000 per cbmm. On filtration through a Chamberlain filter, under pressure, these remain behind together with the fat. But we then also find that one of the most important albuminous constituents of the milk, viz., caseïn, which is found in combination with lime, is likewise not present in solution, and is thus obtained in the form of a thin, jellylike material. The filtrate constitutes the milk-serum and contains those components of the fluid which are present in a state of actual solution.

Upon the addition of chymosin to fresh milk, at the temperature of the body, it coagulates almost at once. The resulting clot, which constitutes cheese, then contracts and a yellowish fluid gradually appears, which is termed sweet whey. During this process the reaction of the milk is not changed. A similar coagulation is noted when fresh milk is allowed to stand exposed to the air. In this ease, however, the reaction of the whey is acid, owing to the formation of lactic acid from lactose in consequence of the activity of certain micro-organisms.

Perfectly fresh milk does not coagulate on boiling, but it will be noted that a skin forms on the surface of the milk, which is rapidly reformed when removed. This consists of coagulated caseïn in combination with mineral salts, and especially phosphates of calcium. Actual coagulation does not occur, even if a current of carbon dioxide has previously been passed through the liquid. If the milk has 
stool for some time, however, and lactic acid fermentation has begun, a tendency to coagulation soon becomes manifest, and at different stages this may then be effected by boiling after saturation with carbon dioxide, then by boiling alone, subsequently on treating with carbon dioxide without boiling; and finally, as I have stated, it occurs spontaneously. Sterilization of the milk, with the subsequent exclusion of micro-organisms, as also the addition of preservatives, such as boric acid, salicylic acid, thymol, etc., will prevent lactic acid fermentation, and consequently also coagulation referable to this source.

On exposure to the air, milk is said to absorb its own volume of oxygen within three days.

Amount.-The amount of milk furnished in the twenty-four hours is, of course, different in different animals. It is largely dependent upon the development of the glands, and accordingly is most abundant in those animals in which by artificial selection a marked hypertrophy of the organs has been produced. Some cows may thus yield 24 liters of milk in the twenty-four hours. The amount is further influenced by the age, as also by the character of the diet, the amount of liquid ingested, etc. Especially important is the character of the diet, and notably the amount of albuminous food that is ingested. Where this is deficient the amount of milk is diminished, while, coteris paribus, larger amounts are furnished if an abundance of albumins is ingested.

Women furnish from 900 to 1000 grammes on an average during the height of lactation ; 1500 grammes probably represent the maximum output. The amount seems to depend somewhat upon the demand. It is thus not rare in institutions where one woman nurses several babies that 4 liter's of milk are secreted in the twentyfour hours. Good cows commonly yield from 6 to 10 liters, goats and sheep about 1 liter, in the twenty-four hours. With the gradual cessation of lactation and the coincident atrophy of the manmary glands the amount decreases, until finally the secretion is arrested entirely. In women and cows the period of lactation usually lasts about ten months.

Specific Gravity.-The specific gravity of the milk is largely dependent upon the amount of fat present, and is much the same in different animals. Its normal variations are seen in the accompanying table:

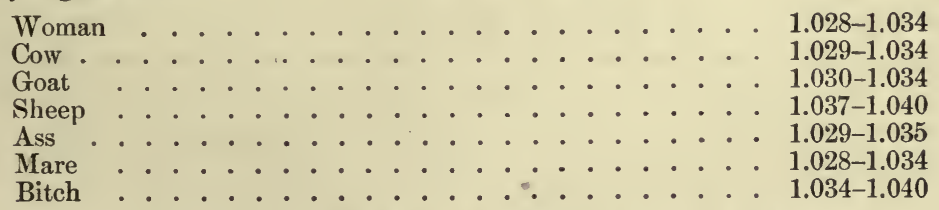

Skimmed milk is, of course, specifically heavier than full milk, and a higher specific gravity is accordingly also noted in milk which is poor in fat than in rich milk.

Reaction.-Woman's milk and that of most herbivorous animals owing to the presence of diacid and monacid phosphates in association 
with the calcium compound of caseinogen, is alkaline to lacmoid and acid to phenolphthalein. The relative values of the acid and basic components in cows' milk and human milk are given below in terms of decinormal sodium hydrate and sulphuric acid solution. The figures have reference to 100 c.c. of milk and are average values (Courant) :

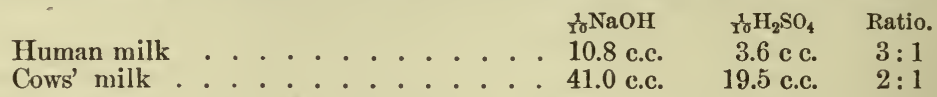

Human milk is thus relatively more alkaline than cows' milk, but is absolutely both less alkaline and less acid.

Mares' milk is alkaline and that of the carnivorous animals acid.

Chemical Composition.-A general idea of the chemical composition of the milk of different animals and of woman may be formed from the following analyses, which are taken from König, GorupBesanez, Hoppe-Seyler, and others :

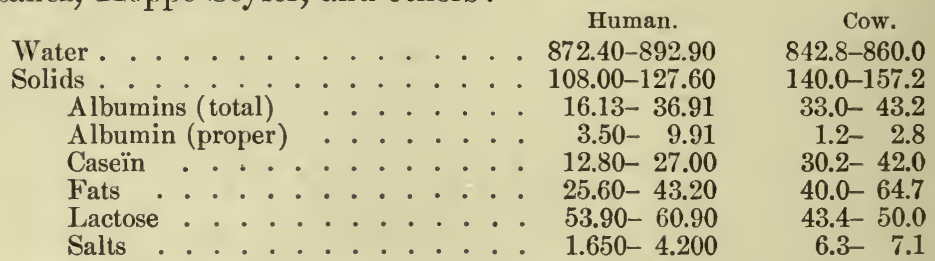

A survey of this table thus shows that human milk contains a smaller amount of albumins and fats but more lactose than cows' milk.

In addition to the above components the milk contains traces of urea, kreatin, kreatinin, hypoxanthin, cholesterin, animal gum, and, curiously enough, citric acid, which is present as a calcium salt to the extent of from 0.18 to 0.25 per cent. Besides these, we find a small amount of lecithins and a yellow lipochrome.

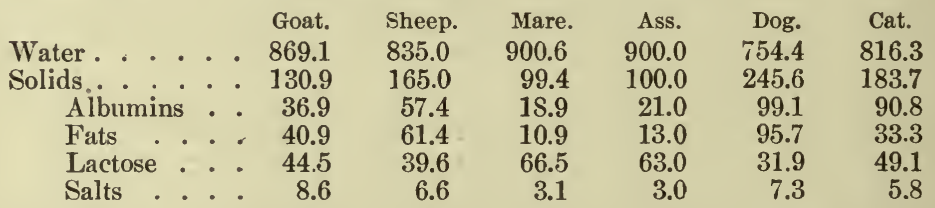

Analysis of the inorganic components of human milk has given the following results (Bunge): the figures of the first column were obtained at a time when but little sodium chloride was ingested, while those of the second column were gotten while the woman ingested 30 grammes a day (the total ash is calculated as 1000 parts by weight):

I.

Potassium $\left(\mathrm{K}_{2} \mathrm{O}\right) \ldots \ldots \ldots . \ldots . \ldots .780$

Sodium $\left(\mathrm{Na}_{2} \mathrm{O}\right)$

Calcium $(\mathrm{CaO})$. . . . . . . . . . 0.328

Magnesium $(\mathrm{MgO}) \ldots \ldots . . . . . .0064$

Iron $\left(\mathrm{Fe}_{2} \mathrm{O}_{3}\right)$. . . . . . . . 0.004

Phosphoric acid $\left(\mathrm{P}_{2} \mathrm{O}_{5}\right) \ldots . . . . .0 .473$

Chlorine $(\mathrm{Cl}) \ldots \ldots . \ldots . . \ldots 0.438$
II.

0.703

0.257

0.343

0.065

0.006

0.469

0.445 
The differences which exist in the composition of full milk, as compared with skimmed milk, cream, buttermilk, and whey, are shown below :

$\begin{array}{cccccr} & \begin{array}{c}\text { Full milk } \\ (\text { cows'). }\end{array} & \begin{array}{c}\text { Skimmed } \\ \text { milk. }\end{array} & \text { Cream. } & \text { Buttermilk. } & \text { Whey. } \\ \text { Water . . . . . } & 871.7 & 906.6 & 655.1 & 902.7 & 932.4 \\ \text { Solids . . . . } & 128.3 & 93.4 & 344.9 & 97.3 & 67.6 \\ \text { Albumins . . } & 35.5 & 31.1 & 35.5 & 35.5 & 8.5 \\ \text { Fats . . . } & 36.9 & 7.4 & 267.5 & 9.3 & 2.3 \\ \text { Lactose . . } & 48.8 & 47.5 & 35.2 & 37.3 & 47.0 \\ \text { Lactic acid . . } & \text { none } & \text { none } & \text { none } & 3.4 & 3.3 \\ \text { Salts . . . . . } & 7.4 & 7.4 & 6.1 & 6.7 & 6.5\end{array}$

Of gases, milk contains a small amount of oxygen and nitrogen, and from 5.8 to 7.5 per cent. of carbon dioxide, which can be removed with the exhaust pump.

The Albumins.-The albumins which are found in milk are caseinogen, lactalbumin, and so-called lactoglobulin, which is probably identical with the serum-globulin of the blood-plasma. Of these, caseinogen is the most abundant and the most important.

CASEINOGEN.-Caseinogen is a nucleo-albumin (phosphoglobulin) and probably a hexabasic acid. In the dry state it occurs as a white amorphous powder, which is almost insoluble in water, in dilute acids, and solutions of the neutral salts. In dilute solutions of the alkaline hydrates and in lime-water it dissolves with ease, at the same time forming salts. Such solutions are neutral or slightly acid in reaction, according to the amount of alkali that has been added, which is owing to the formation of neutral or acid salts respectively. When triturated in water with calcium or sodium carbonate, the carbonates are decomposed with the liberation of carbon dioxide; the same salts are then formed as in the case of the alkaline hydrates. Söldner has isolated two calcium salts of caseinogen, containing 1.55 and 2.36 per cent. of calcium oxide; according to Courant, these are dicalcium and tricalcium caseinogen respectively. The salts of caseinogen with the alkalies and alkaline earths are readily soluble in water, even in the absence of neutral salts, and are hence not precipitated on dialysis. On decomposition with dilute acids the free caseinogen is obtained again in insoluble form. Suspended in water, the substance is coagulated on boiling, and can then no longer be dissolved without undergoing denaturization, as on boiling with acids and alkalies. Solutions of the caseinogen salts, on the other hand, do not coagulate on boiling, but form a surface skin, as in the case of milk. The salts can be precipitated from their solutions by salting with sodium chloride or magnesium sulphate to saturation. Metallic salts, such as copper sulphate, also precipitate a neutral solution completely.

On filtering milk through a Chamberlain filter under pressure caseinogen remains behind, together with the fat and calcium phosphate, as a jelly-like material. On treating milk with a dilute acid the caseinogen is precipitated, as in the case of the aqueous solution of its salts. To a certain extent this may occur in the stomach, 
providing that a sufficient amount of free hydrochloric acid is present ; but, as we have seen, the gastric juice is further capable of effecting the coagulation of caseinogen even though hydrochloric acid is absent. This is brought about through the specific activity of the milkcurdling ferment; but it is to be noted that the coagulation of milk is in this case not directly comparable to the action of an acid. Our knowledge of the mechanism of rennin coagulation of milk has been materially advanced through the researches of Loevenhart. It has thus been ascertained that rennin first changes caseinogen to paracasein and renders the calcium salts available for the precipitation of the latter; subsequently the paracasein is precipitated by the calcium salts as casein. In this connection it is to be noted that the "rendering of calcium salts available" does not necessarily mean that they are soluble. Sodium citrate, for instance, prevents the coagulation of milk by rennin; the calcium present combines with the citric acid and is thus rendered unavailable (exactly as in the case of the coagulation of the blood). Nevertheless, calcium citrate is soluble to a sufficient degree, particularly in the cold.

In all the work previously done on rennin coagulation one phasc of the subject has in a measure been neglected. It has been claimed that the caseinogen exists in the milk in solution as a calcium salt, viz., as a so-called neutral calcium salt (neutral to litmus, but acid to phenolphthalein), and that in this form it becomes subject to the action of rennin. Paracasein then results, but to obtain coagulation it is still necessary to heat or to add a calcium salt. To produce rennin coagulation directly under such conditions phosphoric acid (or possibly some other acid) is necessary in certain concentration. 'The part which this plays, however, has not yet been determined.

The pepsin of the gastric juice plays no part whatever in the coagulation of the milk. But after this has taken place the actual digestion of the precipitated caseinogen begins. As I have pointed out, this is then decomposed, with the formation of a paranuclein and albumin, which latter is digested in the usual manner. A heteroalbumose, however, is not formed during the process.

From the above considerations it is clear that all those factors which tend to increase the amount of soluble lime salts in the milk will increase the tendency of the caseinogen to coagulate upon the subsequent addition of chymosin, while this is diminished if the soluble salts are transformed into the insoluble form or if their amount is diminished. It is for this reason also that boiled milk does not coagulate so rapidly as fresh milk, as the free carbonic acid, which holds a certain amount of calcium in solution, is thereby removed. The common addition of lime-water to milk similarly increases the tendency to coagulation, but does not render it more digestible, as is generally supposed.

The coagulation of the milk which occurs spontaneously on standing is analogous to that which results upon the addition of a mineral acid, and is referable to the formation of lactic acid from lactose as a result of bacterial action. 
From the fact that the coagulum which results in cows' milk upon the addition of chymosin is much tougher and denser than that which is obtained with human milk, it has been concluded that the caseinogen of the two is not identical. Soxhlet, however, has shown that the density of the coagulum is primarily dependent upon the concentration of the caseinogen solution and the amount of soluble calcium salts and acid phosphates present. As this is much greater in cows' milk than in human milk, it follow's that marked differences must thus exist. There is evidence to show, nevertheless, that different forms of caseinogen occur. Elementary analysis of human caseinogen (Hammarsten) and cows' caseinogen (W'róblewski) has given the following results :

$$
\begin{aligned}
& \text { Human, C, 52.96; H, 7.05; N, } 1 \dot{5} .65 ; \mathrm{S}, 0.75 ; \mathrm{P}, 0.84 ; \mathrm{O}, 22.78 \text { per cent. } \\
& \text { Cows', C, } 52.24 ; \mathrm{H}, 7.32 ; \mathrm{N}, 14.97 ; \mathrm{S}, 1.11 ; \mathrm{P}, 0.68 ; \mathrm{O}, 23.66 \text { “ “ }
\end{aligned}
$$

The difference is here especially noticeable in the amount of sulphur. Human caseinogen, moreover, is not so readily precipitated by salting or by the addition of acids, and does not always coagulate with chymosin. The gastric juice, it is true, can precipitate the substance, but it readily dissolves in an excess without leaving any residue of nuclein. From this observation Szontagh has concluded that human caseinogen is in reality no nucleo-albumin. But aside from these data we have abundant evidence that human caseinogen and cows' caseinogen are not identical, in the fact that no modification of cows' milk, however produced, is so readily digested by the infant as is human milk.

Like all albumins, caseinogen is optically active ; its specific rotation in neutral solution is -80 degrees.

The isolation of the caseinogen from milk will be described below, in association with the isolation of the soluble albumins. These, as I have already said, are lactalbumin and lactoglobulin.

LACTALBUMin.-Lactalbumin is found both in human milk and cows' milk, and is manifestly closely related to the common serumalbumin of the blood-plasma. Its specific rotation, however, is markedly less, viz., -37 degrees, as compared with -62.6 to -64.6 degrees. Its composition according to Sebelien is C, 52.19 per cent. ; H, 7.18 ; N. 15.77 ; S, 1.73 ; and O, 23.13 ; while that of serumalbumin is given as $\mathrm{C}, 52.25-53.06$ per cent.; $\mathrm{H}, 6.65-6.85 ; \mathrm{N}$, 15.88-16.04; S, 1.8-2.25; and O, 22.25-22.97 (Hammarsten).

LACTOGLOBULIN. - The lactoglobulin which has been isolated from cows' milk seems to be identical with the serum-globulin of the blood. It requires no further description.

That still other albuminous substances may occur in the milk is possible; but if so, they are present only in traces and have not as yet been identified. Albumoses and peptones are not found in fresh milk. According to Siegfried, a phosphor-carnic acid can be isolated from milk after removal of the caseïn and the coagulable albumins; this, however, is supposedly not identical with that found in muscle-plasma. 
Origin of the Albumins. - Caseinogen, as has been stated, is a specific product of the activity of the mammary glands, and is probably formed from the complex nucleo-glucoproteid which occurs in the functionally active organ. As this is not found in the milk, we may conclude that after its formation it is decomposed and probably yields caseïn, on the one hand; while its reducing radicle may be concerned in the production of lactose.

Of the origin of lactoglobulin and lactalbumin, nothing is known; but, as I have said, the former is probably identical with the serumglobulin of the blood, while in the case of the latter we may imagine that it has originated through a peculiar transformation of the serum-albumin.

Isolation of the Albumins of the Milk.-ISOLATION OF CASEINOGEN. - The milk is diluted with four times its volume of water and acidified with acetic acid to the extent of $0.75-1.0$ pro mille. On standing, the caseinogen separates out and is filtered off. It is purified by repeated solution in water with the aid of a little caustic alkali, filtration, and reprecipitation with acetic acid. It is then washed with water and freed from traces of fat by means of etheralcohol. The greater portion of fat remains on the first filter.

Isolation OF LACTOGLOBULIN. - The milk is saturated with common salt in substance, which precipitates the caseinogen together with a small portion of the globulin. If then the neutral filtrate is saturated with magnesium sulphate at $30^{\circ} \mathrm{C}$., the remaining portion of the lactoglobulin is obtained. This is purified as described in the case of the serum globulin of the blood.

Isolation of LACTALBUMIN.- The caseinogen and globulin are first precipitated by salting with magnesium sulphate in substance at $30^{\circ} \mathrm{C}$., and are filtered off. In the filtrate the lactalbumin can then be demonstrated by acidifying with acetic acid to the extent of a little less than 1 per cent. and boiling or by salting with ammonium sulphate or sodium sulphate in substance. The albumin is filtered off and purified as described on page 339.

Quantitative Estimation of the Total Albumins. - To this end, a few grammes of milk are diluted with water, treated with a small amount of sodium chloride solution, and precipitated with tannic acid or phosphotungstic acid in excess. In the precipitate, which is washed with water, the amount of nitrogen is then estimated by Kjeldahl's method. By multiplying the result by 6.37 in the case of cow's milk, or by 6.34 with human nilk, the corresponding amount of albumin is ascertained. The nitrogen of some of the extractives is included in the result, but may be ignored. In cows' milk it represents about one-sixteenth of the total amount of nitrogen, and in human milk about one-eleventh.

Separate Estimation of the Caseinogen and the Soluble Albumins.A few grammes of milk are diluted with two or three volumes of a saturated solution of magnesium sulphate, and are then saturated with the salt in substance. In the precipitate, which is washed with 
a saturated solution of the salt, the nitrogen is then determined as above. The result multiplied by 6.37 indicates the amount of caseinogen. The amount of lactalbumin can be ascertained by deducting the value found for caseinogen from the total amount of albumin, or by diluting the filtrate, after separation of the casein, precipitating with tannic acid, and determining the amount of nitrogen as before. In this case also we multiply by 6.37 .

The results for caseinogen thus obtained are not absolutely accurate, as the globulin is likewise precipitated by magnesium sulphate. Its amount, however, is so small that it may well be disregarded.

The Fats.-The fats which are found in the milk, viz., in butter, are essentially the same as those which occur elsewhere in the animal body, viz., stearin, palmitin, and olein. In addition, however, we also find small amounts of the triglycerides of myristinic acid, butyric acid, and capronic acid, and traces of caprylic acid, caprinic acid, laurinic acid, and arachinic acid.

Stearin, palmitin, and olein constitute about 98 per cent. of the total amount, and of these, olein represents about 29.4-39.2 per cent. As a consequence of the large quantity of olein which is thus present, the melting-point of butter is relatively low, viz., $31^{\circ}-34^{\circ} \mathrm{C}$., while it solidifies between $19^{\circ}$ and $24^{\circ} \mathrm{C}$.

In addition to the neutral fats, butter also contains about 7 per cent. of volatile fatty acids, of which 3.7-5.1 per cent. are represented by butyric acid and 2-3.3 per cent. by capronic acid.

Formic acid has been found in butter which had been exposed to sunlight.

Of the origin of the fats which are found in milk we know that they are to a large extent derived from albumins, and I have already pointed out that their amount increases with a diet that is rich in such material, even though no fat is ingested at all. They diminish materially if fat alone is ingested, and are not increased if much fat is administered, while the ingestion of albumin remains constant. They are probably formed in the gland directly, and on microscopical examination it is possible to demonstrate their presence in the cells, in the form of fine globules, which are soluble in ether, and are colored black on treating with osmic acid.

More recently Engel has shown that the sources of the milk fat may not be found in the breaking down of the gland elements, but that they are furnished by the body fat and the fat of the food. It has been shown conclusively that the fat of the food enters the milk fat and that feeding with fat increases the amount of fat in the milk, but whether this can be done for a prolonged period of time is still doubtful. Engel is of the opinion that the fat of the food enters regularly into the secretion of the mammary gland. The colostral fat seems to be, in the human species at least, constantly identical with the body fat.

To isolate the individual acids which enter into the composition 
of the neutral fats, the butter is first saponified, when the resulting soaps may be separated from each other according to the usual methods of analysis.

Quantitative Estimation.-The amount of fat in milk is most conveniently estimated densimetrically with Soxhlet's apparatus. To this end, a known amount of milk is mixed with a solution of sodium hydrate and the fat extracted with a definite quantity of ether. The ethereal solution is allowed to separate, and is then forced into a glass cylinder provided with an aërometer. From the specific gravity the percentage of fat is then read off from a table which accompanies the apparatus. The latter is so constructed that evaporation of the ether cannot occur.

In the absence of such an apparatus the amount of fat can be ascertained gravimetrically as follows : 20 c.c. of milk are treated with a small amount of sodium hydrate solution, and are extracted with 80 c.c. of ether which has been saturated with water. This is done by shaking in a tightly closed bottle. After the ethereal extract has entirely separated, 60 c.c. are placed in a weighed beaker; the ether is allowed to evaporate; the residue is dried and weighed. The result is calculated out for 80 c.c. of the ethereal extract, corresponding to 20 c.c. of milk.

Lactose.-In the animal body lactose is found only in the milk, if we disregard the small amounts that may appear in the urine of nursing females, and which must hence of necessity occur also in the blood. It is formed in the mammary glands, and may possibly be related to the reducing substance which results from the nucleoglucoproteid when this is boiled with mineral acids. On exposure to the air it undergoes a peculiar fermentation, with the formation of lactic acid. This, in turn, combines with the calcium of the lime-caseïn, and as a result the easeïn separates out, and constitutes what is popularly termed clabber. On further standing, this contracts, and finally floats in a clear, light-yellow fluid-the acid whey. The fermentation in question is produced by definite micro-organisms, of which fourteen varieties are now known.

On inversion, lactose is decomposed into glucose and galactose.

Isolation.-To isolate lactose from milk, this is first curdled by the addition of chymosin. The filtrate is slightly acidified with acetic acid and boiled, so as to remove the coaguilable albumins. The second filtrate is then concentrated to a small volume, when on cooling the lactose crystallizes out. To purify the substance, this is dissolved in water, decolorized with animal chareoal, and recrystallized by evaporation. It is thus obtained in the form of white rhombic prisms, which are soluble in water, but insoluble in absolute alcohol. The substance has a somewhat sweetish taste, and contains one molecule of water of erystallization, which rapidly cscapes at $130^{\circ} \mathrm{C}$.

Estimation.-To estimate the amount of lactose, the milk must 
first be freed from fats and albumins. To this end, it is most convenient to dilute with water and to remove the casein by the cautious addition of acetic acid. The resulting precipitate, which contains both the caseïn and the fat, is filtered off and the filtrate boiled. After the removal of the precipitated coagulable albumins, the sugar is then estimated in the filtrate by titrating with Fehling's solution, as described in the section on the Urine; 10 c.c. of the reagent correspond to 0.06 gramme of lactose, providing that the solution contains from 0.5 to 1 per cent. of sugar.

In addition to lactose, the milk contains also small amounts of a reducing substance, which is supposedly identical with Landwehr's animal gum (dextrin). It is possible, however, that, as in the case of the reducing substance of the mucins and mucoids, chondroitinsulphuric acid or an allied substance may be responsible for the reactions.

Extractives.-Among the extractives of the milk, which comprise traces of urea, kreatin, kreatinin, xanthin-bases, lecithins, cholesterin, and citric acid, the latter is of especial interest, as it is apparently also formed in the mammary glands, and is not referable to the ingestion of the substance as such. It has been found in human milk as well as in cows' milk, and is notably present in combination with calcium. Its amount in cows' milk is given as 0.25 per cent. Human milk contains a somewhat smaller amount of eitric acid; it varies between 0.024 and 0.07 per cent. (Sieber); the higher values are found between the sixth and the eleventh month of lactation.

To the presence of citric acid apparently the reaction of Umikoff is due. To make the test, about 5 c.c. of milk are treated with onehalf the amount of a 10 per cent. solution of ammonium hydrate and heated on a water-bath at $60^{\circ} \mathrm{C}$. for fifteen to twenty minutes. Human milk then assumes a violet-reddish color, which is the more intense the older the milk in reference to the time of lactation. Cows' milk treated in the same manner assumes a yellow, at mosta yellowishbrown color, so that it is thus possible to distinguish human milk from cows' milk. According to Sieber, it is also possible thus to distinguish the milk of the earlier months of lactation from that of the later months; after the eighth month, however, the reaction no longer gives uniform results, but is sometimes intense, and at others quite feeble. Sieber has ascertained that the difference between human and cows' milk in this respect is probably primarily dependent upon the differing amount of calcium salt that is present. On heating cows' milk with ammonia all the citric acid is precipitated as calcium citrate, while in the case of human milk, which contains only one-sixth the amount of calcium, a certain proportion of the eitric acid remains in solution.

The formula of the acid is $\mathrm{CH}_{2} \cdot \mathrm{COOH} \cdot \mathrm{C}(\mathrm{OH}) \cdot \mathrm{COOH} \cdot \mathrm{CH}_{2}$ $\mathrm{COOH}$, viz., $\mathrm{C}_{6} \mathrm{H}_{8} \mathrm{O}_{7}$; it is thus oxy-tricarballylic acid. 
Ferments._At least three ferments seem to occur in cows' milk, viz., a milk-trypsin, a milk-katalase, and a milk-peroxydase. In addition Babcock and Russell have described a galaktase. In human milk there is a diastase (absent in cows' milk), very little if any peroxydase, but more katalase than in cows' milk; and in addition a proteolytic ferment. Possibly still other ferments are present.

\section{Colostrum.}

The term colostrum is applied to the secretion of the mammary glands which is furnished by the female animal during the first days of lactation, and which may also be expressed from the glands during a variable period preceding parturition.

On microscopical examination such fluid is seen to contain innumerable fat-globules, and in addition a variable number of granular cells, which are capable of manifesting amœboid movements. These are termed colostrum-corpuscles, and are commonly regarded as leucocytes. This, however, is doubtful. According to Woodward, they have a small irregular, but much degenerated nucleus. Of the granules, a few are stained by osmic acid, while none of them takes up either acid, neutral, or basic dyes. In their reactions they show the characteristics of proteid material.

The secretion is a thick yellowish fluid, of an alkaline and sometimes acid reaction, and a specific gravity that is much higher than that of true milk. In the cow this varies between 1.046 and 1.080 , and in the human female between 1.040 and 1.060. This is principally owing to the presence of large amounts of lactalbumin and lactoglobulin. As a consequence, the colostrum coagulates on boiling, while true milk, as we have seen, is then covered merely by a skin, which is composed of caseïn and calcium phosphates. The total quantity of the coagulable albumins may reach 15 per cent., while in milk about 0.5 per cent. is the rule.

The amount of caseïn and of mineral salts in colostrum is also somewhat greater than in milk, and it is further stated that more lecithin and cholesterin is present. The quantity of fat is practically the same, while that of lactose is somewhat smaller. As a result of the increase in the amount of albumins and of mineral salts, the total solids are also proportionately increased, and may amount to 25.3 per cent. in cows' colostrum, as compared with 12.8 per cent. in the case of the milk.

The quantitative composition of the colostrum after parturition is rapidly altered, so that after a few days already the normal composition of true milk is approached. This is well shown in the following table, which is taken from Gautier. The results have reference to the human being and the cow, and are expressed in percentages: 


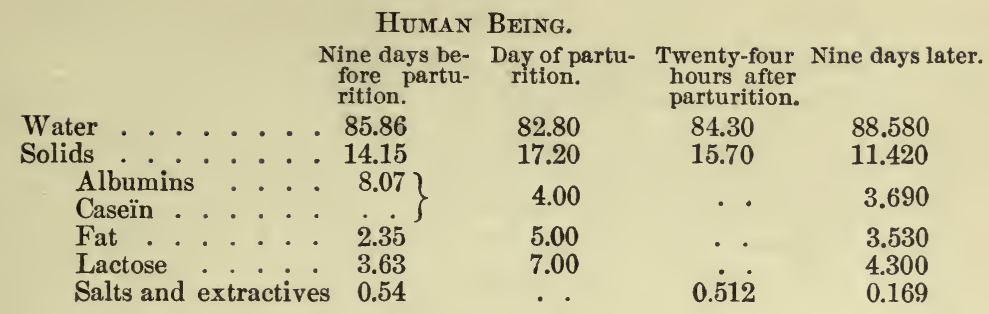

Cow.

\begin{tabular}{|c|c|c|c|c|}
\hline & $\begin{array}{l}\text { Immediately } \\
\text { after partu- } \\
\text { rition. }\end{array}$ & $\begin{array}{l}\text { Twenty-four } \\
\text { hours later. }\end{array}$ & $\begin{array}{l}\text { Three days } \\
\text { later. }\end{array}$ & $\begin{array}{l}\text { Average of } \\
30 \text { analyses. }\end{array}$ \\
\hline Water & . 73.07 & 82.38 & 78.70 & 74.05 \\
\hline lid & 26.93 & 17.62 & 21.30 & 25.95 \\
\hline Albumins & . 16.56 & 4.50 & 7.50 & 13.62 \\
\hline Caseïn . . & . 2.65 & 4.50 & 7.30 & 4.66 \\
\hline Fat . & . 3.54 & 4.75 & 4.00 & 3.43 \\
\hline Lactose . . . & 3.00 & 2.85 & 1.50 & 2.66 \\
\hline Salts and extractiv & 1.18 & 1.02 & 1.00 & 1.58 \\
\hline
\end{tabular}

I'also append a few analyses of cows' colostrum, which I have taken from $G$. Simon (the analysis were made on successive dogs):

\begin{tabular}{|c|c|c|c|c|c|}
\hline Specific gravity & 1.0705 & 1.0690 & 1.0366 & 1.035 & 9 per cent. \\
\hline & 3.00 & 3.00 & 3.03 & 3.54 & " \\
\hline Solids . . . . & .23 .85 & 23.60 & 13.30 & 13.20 & “ \\
\hline Total albumins & .17 .00 & 16.87 & 4.93 & 4.35 & “ \\
\hline Caseïn . . . . & . 5.50 & 4.47 & 3.02 & 3.06 & “ \\
\hline Albumin . . & .11 .93 & 11.79 & 1.91 & 1.15 & “ \\
\hline Extractives . & . 0.07 & 0.07 & 0.04 & & “ \\
\hline
\end{tabular}

As I have already indicated, probably all colostra color active tincture of guaiacum blue even in the absence of hydrogen peroxide, and also react with the Röhmann-Spitzer mixture. The reaction is due to a globulin-oxydase.

So-called witch's milk is the fluid which can be expressed from the mammary glands of both sexes immediately after birth. Its qualitative composition is the same as that of milk. Like the colostrum, it is said to contain colostrum-corpuscles. According to Schlossberger, Hauff, and others, it contains from 1.05 to 2.8 per cent. of albumin, 0.82 to 1.46 per cent. of fat, and 0.9 to 6 per cent. of lactose. It thus contains a smaller amount of water than the milk. The secretion ceases several weeks after birth.

Uterine milk is a fluid which can be obtained from the uterine glands of ruminants after careful separation of the chorion villi. It has the appearance of cream, and is morphologically and chemically quite similar to colostrum.

\section{THE REPRODUCTIVE GLANDS.}

The Testicles.-Of the chemical composition of the testicles as such, little is known. Aside from the albuminoids which enter into the construction of the supporting tissues of the glands, and the extrac- 
tives which are common to all organs of the body, we notably meet with albumins, among which the nucleins are especially abundant. In addition, serum-albumin, a globulin, and a substance which apparently belongs to the hyalins, have been encountered. Of mineral salts, we notably meet with the chlorides of sodium and potassium. The reaction of the glands is alkaline.

The Semen.-The specific product of the functional activity of the testicles is represented by the semen, and notably its morphological elements, the spermatozoa, which result from the spermatogenetic cells through a complicated process of metamorphosis, in which the cell-nuclei are especially concerned. On its passage to the outside the testicular fluid is mixed with the secretions of the seminal vesicles, the glands of Cowper, and notably with the secretion of the prostate gland.

Recently ejaculated semen is a markedly viscid, white or yellowish-white, opaque fluid of the appearance of milk, in which microscopical examination reveals the presence of innumerable spermatozoa, and a few hyalin globules, which are derived from the seminal vesicles; further, isolated testicular and urethral cells, prostatic corpuscles, and cellular bodies enclosing lecithin granules, besides a large number of free granules, which are apparently of an albuminous nature. In a fresh specimen the normal spermatozoa are actively motile, and continue so for a variable length of time if evaporation is prevented. The movements are in all likelihood analogous to those of the cilia of certain epithelial elements of the body, and are arrested by the addition of water, dilute acids, alcohol, ether, strongly alkaline solutions, etc. In dilute alkaline solutions, on the other hand, and those of the neutral salts they continue for a long time.

Semen is heavier than water, and falls to the bottom as a jellylike mass: at the same time a light flocculent precipitate develops, which consists of the so-called fibrin of Henle. On exposure to the air it is apparently coagulated, but later becomes liquid, as before. Its reaction is neutral or slightly alkaline; the alkalinity corresponds to about 0.148 per cent. of sodium hydrate. The specific gravity varies from 1.020 to 1.039 .

Testicular semen, in contradistinction to that which has been ejaculated, is said to be odorless. After emission, however, an odor develops which is suggestive of glutin, and is supposedly referable to the presence of an alkaloidal substance-spermin. In combination with phosphoric acid, this is found in the secretion of the prostate gland, as phosphate of spermin, and is partly decomposed on exposure to the air, with liberation of the free base (see below).

A quantitative analysis of human semen gave the following results (Slowtzoff); the figures have reference to 100 parts of the fresh material : 


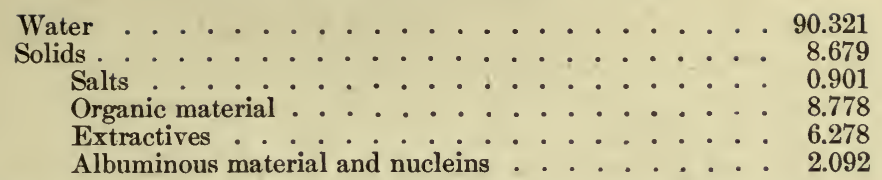

The Spermatic Liquid.-The liquid in which the spermatozoa are suspended is nearly transparent. It contains a small amount of mucin (?); a nucleo-albumin, which has been termed spermatin, and which is precipitated by acetic acid, but is readily soluble in an excess of the reagent; common albumin; an albumose-like body which can be precipitated on two-thirds saturation with ammonium sulphate ; cerebrin and lecithins, phosphate of spermin, and various mineral salts, among which sodium chloride and the phosphates of the alkaline earths predominate.

Spermin.-Spermin, as stated above, occurs in the spermatic liquid in combination with phosphoric acid, as phosphate of spermin ; it is viewed as ethylenimin, $\mathrm{C}_{2} \mathrm{H}_{5} \mathrm{~N}$, and is manifestly closely related to the diethylene diamin (piperazin) of Ladenburg and Abel.

To the free base the peculiar odor of the semen is, as I have said, supposedly due. This disappears after a short while, owing to a polymerization of the ethylenimin to diethylene diamin (piperazin) as shown in the equation:

$$
2 \mathrm{~N}\left\langle\underset{\mathrm{CH}_{2}}{\mathrm{H}}=\mathrm{C}_{2} \mathrm{H}_{4}\left\langle{ }_{\mathrm{NH}}^{\mathrm{NH}}\right\rangle_{2} \mathrm{H}_{4}\right. \text {. }
$$

The phosphate can be readily obtained in crystalline form on slow evaporation of the semen, but may also separate out spontaneously on standing for about twenty-four hours. It occurs in the form of hexagonal pyramids, which appear under the microscope as flat needles. They are soluble in dilute acids and alkalies, as also in ammonia, less readily so in hot water, and are insoluble in alcohol, ether, and chloroform. These crystals are known as Böttcher's spermin-crystals, and are probably identical with the so-called Charcot-Leyden crystals, which are commonly found in asthmatic sputa, and also occur in the blood and lymph-gland of lenkæmic patients. They have likewise been observed in dried egg-albumin and in anatomical specimens preserved in alcohol, and also develop in red bone-marrow that has been exposed to the air for a few days. Heated to $100^{\circ} \mathrm{C}$., the crystals turn yellow and melt near $170^{\circ} \mathrm{C}$., but are at the same time decomposed.

To isolate the free base, the semen is extracted with alcohol and then with dilute sulphuric acid. If the acid extract is then treated with baryta-water and evaporated at a low temperature, the free base is obtained. It can be precipitated from its solution by treating with auric chloride, platinum chloride, argentic nitrate, tannic acid, phosphotungstic acid, etc.

Spermin has attracted muck attention of late, owing to the 
stimulating effect which the substance is supposed to exert upon the oxidation processes of the body, the functions of the central nervous system, and the reproductive organs. It represents the active principle of Brown-Séquard's elixir.

The Spermatozoa.- Our knowledge of the chemical composition of the spermatozoa has been greatly extended within recent years through the researches of Kossel and his pupils, preceded by those of Miescher and Piccard. These observers were able to show that in certain fishes, such as the salmon, sturgeon, pike, shad, herring, mackerel, etc., protamins can be isolated in large amount. Their general characteristics have already been described (page 52). These protamins, of which several varieties are known, and which yield diamino-acids and in some cases also histidin on hydrolytic decomposition, are supposedly combined with nucleinic acids to form nucleoproteids. The individual nucleinic bases which enter into the construction of the nucleinic acids are the common forms, which are also found elsewhere in the animal body. But it appears that the spermatozoa of different animals do not contain all forms. In the case of the salmon, Miescher and Piccard thus found guanin and hypoxanthin, while from the semen of the carp Kossel obtained adenin and hypoxanthin, as also small amounts of xanthin, but no guanin. Inoko, on the other hand, claims to have found all forms in the semen of the salmon, boar, and ox, but states that the relative amounts of the individual forms are not constant. Immature spermatozoa apparently contain histons in the place of protamins.

Analysis of the spermatozoa of the salmon (Miescher):

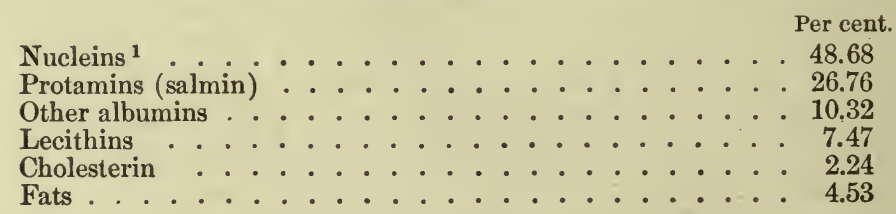

The albumins referred to in this table have not been studied in detail. One of them, according to Miescher, contains 4 per cent. of sulphur. In addition, the spermatozoa are said to contain a cerebroside, which is similar to cerebrin; also a very considerable proportion of inorganic salts, which are essentially represented by phosphates.

Detailed analyses of the spermatozoa of the higher animals and of man are not yet available.

As regards the composition of the separate parts of the spermatozoa very little is known, but it seems that the protamins, combined with nucleinic acids, are the most important components of the head. The tails are dissolved in gastric juice on prolonged

1 The nucleins, according to Kossel, are nucleinic acids. 
digestion, and hence probably consist of albumins. As a whole the spermatozoa are exceedingly resistant to ordinary solvents. They are soluble in boiling solutions of the caustic alkalies, while in concentrated sulphuric acid, nitric acid, acetic acid, and boiling solutions of sodium carbonate they dissolve only in part. They are likewise resistant to putrefactive changes, and can be obtained from dried semen, with the preservation of their natural form, by placing the material in a 1 per cent. solution of sodium chloride.

The Ovaries.-Thus far a study of the chemical composition of the ovaries has not revealed any special points of interest. In addition to collagen and mucins, which enter into the construction of the supporting tissue of the organs, nucleins and true albumins have also been found, and are probably derived from the contained ova and other cellular elements.

The most important constituents of the cortex of the gland, viz., the Graafian follicles, which enclose the specific product of the functional activity of the ovaries, viz., the ova, have for obvious reasons not been open to a detailed investigation. The contained fluid is apparently serous in character. After the discharge of the ova the remaining follicles are first filled with blood from the torn vessels of the vesicle, and are subsequently transformed into the so-called corpora lutea. The yellow color of these is owing to lipochromes, or luteins, of which an amorphous and a crystalline form may be isolated (see also page 462).

The Ovum.-Of the chemical composition of the ova of the human being and mammals in general, nothing definite is known, as it is impossible to collect them for purposes of analysis. The eggs of fishes, amphibia, reptiles, and especially of birds, on the other hand, can readily be obtained and have been studied in greater detail. In the following pages we shall confine our attention to the composition of birds' eggs, which is best understood. The egg proper is here surrounded by the so-called white of egg, which in turn is enclosed in a double membrane, and is covered by the shell. These additional structures, however, are not formed in the ovary, but are produced during the passage of the egg through the oviduct from material, which is here secreted by the lining cells.

The Shell.- The shell consists essentially of an organic matrix of the character of keratin, which is largely impregnated with lime salts. Of these, calcium carbonate is the most abundant, and constitutes about 90 per cent. of the weight of the entire shell. In addition, we find a small amount of magnesium carbonate, as also phosphates of both elements. Water is present to the extent of only about 1 per cent. 'The pigments met with in birds' eggs are closely related to the biliary pigments, and, like these, are derived from the common pigment of blood. The oörhodeïn, which presents a reddish or brownish-red color, is supposedly identical with hæmatoporphyrin; while the blue or green pigment, which is 
termed oöcyanin, is composed partly of biliverdin, and is in part a blue derivative of bilirubin.

The membranes of birds' eggs consist essentially of keratin, but contain also a small amount of mineral salts, of which calcium phosphate is the most abundant.

In fishes and amphibia the egg envelope is represented by a transparent gelatinous material, which seems to consist almost exclusively of mucin. In the invertebrates chitin and skeletins take the place of the keratin of birds' eggs, but in some the latter also is found.

'The weight of the shell and membranes in the case of hens' eggs represents about 9 to 11 per cent. of the total weight of the egg, while the albumen constitutes about 60.5 per cent. and the yolk, viz., the ovum proper, the remaining 29 per cent. The total weight of hens' eggs may vary between 40 and 70 grammes.

The Albumen.-The albumen or white of egg, as obtained directly from the raw egg, appears as a faintly yellow, exceedingly viscid, semiliquid material. On microscopical examination this can be shown to consist of compartments, which are limited by very delicate membranes, and enclose the albumen proper. These membranes are continuous with the so-called chalazæ and the membranes immediately beneath the shell, and are, like these, composed of keratin.

The albumen proper may be separated from its membranous constituents by pressing the material through a cloth, and then appears as an opalescent fluid, which is only slightly viscid, and can be filtered without much difficulty. Its reaction is distinctly alkaline, and the specific gravity about 1.045 . On boiling, it coagulates to a compact mass, which in the case of hens' eggs is entirely opaque. In some birds, however, such as the swallow, the crow, the finch, etc. - $i$. e., in true nesting birds-the albumen remains transparent, owing to the formation of alkaline albuminates. Such albumen has been termed tata-albumen. It may be produced artificially by placing hens' eggs in a 10 per cent. solution of sodium hydrate for two or three days, when a gradual diffusion of alkali occurs into the albumen. On subsequent boiling, this appears like true tata-albumen.

Analysis of the albumen of hens' eggs has given the following results :

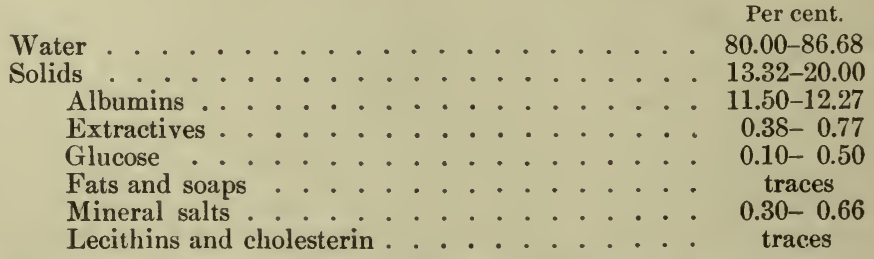

According to Poleck and Weber, the mineral ash has the following composition, calculated for 100 parts: 


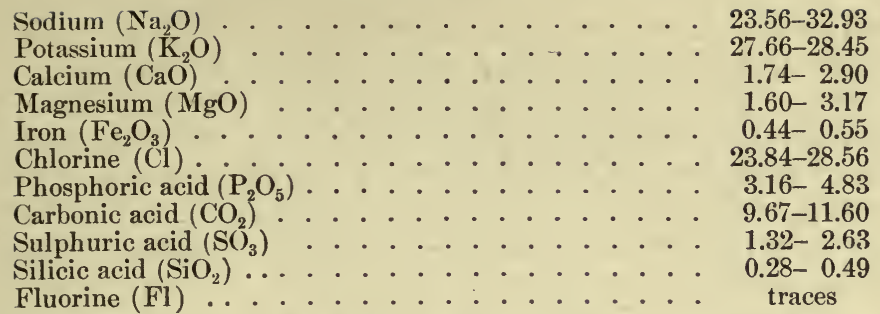

Of these constituents, the large amount of sodium chloride is especially noteworthy, and shows in itself that the albumen is in reality a secretory product, and does not represent a mere transudation from the blood-plasma.

One portion of the bases is in combination with the albumins of the albumen, while the remainder exists in the form of sulphates, phosphates, and notably carbonates.

The slightly yellow color of albumen is referable to the presence of a lipochrome, which can be demonstrated on spectroscopic examination.

The Albumins.-According to Gautier and some of the older observers, white of egg (albumen) contains a number of different albumins, which in part seem to belong to the true albumins and in part to the globulins. They have been designated as $\alpha-, \beta$-, and $\gamma$-ovalbumin, and $\alpha$ - and $\beta$-ovoglobulin. In so far as the globulin fraction is concerned, Langstein has recently shown that it consists of two portions, one of which after precipitation with ammonium sulphate is insoluble in dilute saline solution, while the other dissolves. This latter fraction was further subdivided by salting with potassium acetate into an euglobulin and a second fraction which can subsequently no longer be precipitated by half-saturation with ammonium sulphate. The euglobulin represents about two-thirds of the total globulin fraction.

The greater portion of the white of egg does not belong to the globulins, and can be obtained in crystalline form, viz., the ovalbumin proper. In addition we find a fraction which is not crystallizable - the so-called conalbumin of Osborne and Campbell, and finally the ovomucoid of Mörner.

Globulin Fraction.-The euglobulin above mentioned gives the biuret reaction, the xanthoproteic reaction, that of Millon, Adamkiewicz, and markedly also that of Molisch. It is precipitated, on dialysis, by a current of carbon dioxide, and on careful addition of dilute acetic acid. It is soluble in an excess of acids and in dilute saline solution, but readily passes over into an insoluble modification. In a 2 per cent. solution it coagulates between $64^{\circ}$ and $67^{\circ} \mathrm{C}$. Elementary analysis gave the following results: $\mathrm{C}=49.88 ; \mathrm{H}=$ $7.09 ; \mathrm{N}=14.36 ; \mathrm{S}=1.73 ; \mathrm{O}=26.92$ (Langstein). On hydrolysis with dilute hydrochloric acid it yields 11 per cent. of glucosamin. 
Ovalbumin.-The amount of crystallizable ovalbumin which can be obtained from white of egg varies between 30 and 40 grammes pro liter. The limits of precipitation of the substance, when purified carefully and brought into a 10 per cent. solution, containing the normal amount of alkali of white of egg, viz., 0.225 gramme of sodium carbonate for 100 grammes of albumin, were 62 and 68 . Elementary analysis of a carefully purified preparation gave the following results (Langstein) : $\mathrm{C}=52.46 ; \mathrm{H}=7.19 ; \mathrm{N}=15.29$; $\mathrm{S}=1.34$; and $\mathrm{O}=23.72$. On hydrolysis with baryta ovalbumin yields a polymeric nitrogenous carbohydrate, which S. Fränkel has termed albamin, and which appears to be an acetylated glucosamin. Seemann and Langstein could both demonstrate the formation of glucosamin on hydrolysis with dilute acids. The formula which Fränkel suggests for his albamin is $2\left(\mathrm{C}_{6} \mathrm{H}_{9} \mathrm{O}_{4} \mathrm{NH}_{2}\right)+\mathrm{H}_{2} \mathrm{O}$.

Conalbumin.-Whether the conalbumin is in reality a unity and not a mixture of two or more bodies is uncertain. Elementary analysis has given the following results (Osborne and Campbell): $\mathrm{C}=52.25 ; \mathrm{H}=6.99 ; \mathrm{N}=16.11 ; \mathrm{S}=1.7 ; \mathrm{O}=22.95$. According to Langstein, the substance is free from phosphorus. Like all the other albumins of white of egg, the conalbumin also yields glucosamin on hydrolytic decomposition. It appears, as Hofmeister suggests, that the glucosamin in the white of egg fulfils the same object as the milk-sugar in the case of milk.

Analysis of the Albumins of White of Egg.-The whites of a large number of eggs are freed from their membranes by beating; the material is filtered and the filtrate treated with an equal rolume of an accurately neutral solution of ammonium sulphate. (Care should be had that only such eggs are used which present an alkaline reaction with litmus.) After standing for one-half hour the precipitated globulins are filtered off. The clear filtrate, which usually presents a reddish color, is now treated with one-fifth normal solution of sulphuric acid until the fluid becomes opaque. If any crystals of ovalbumin are available, a few are added to hasten crystallization. This, however, is not necessary. The solution is allowed to stand, when gradually a separation of crystals of ovalbumin will occur. The best results are obtained if the temperature of the room is not less than $15^{\circ} \mathrm{C}$.; in a cold room no result is usually gotten. To purify the crystals, a feebly acid solution of ammonium sulphate is used, crystallization being hastened by inoculation with a few erystals of the substance. After standing until crystals cease to separate out, and after they have been removed, the remaining solution is dialyzed against running water until the sulphuric acid has been almost entirely removed. It is then heated on a water-bath until all coagulable material has separated out. This is filtered off and thoroughly washed with hot water until the filtrate is free from sulphuric acid, and no longer gives a cloud with phosphotungstic acid. The resulting substance is dried at $110^{\circ} \mathrm{C}$. and represents the conalbumin. 
To isolate the euglobulin from the total globulin fraction, this is repeatedly washerl with a one-half saturated solution of ammonium sulphate (by centrifugation) until the salt solution no longer gives the biuret reaction. The euglobulin is finally collected on a filter, coagulated in the drying-oven at $100^{\circ} \mathrm{C}$., and washed with hot distilled water until free from salts.

Ovomucoid.-The mucoid substance which can be isolated from the albumen of hens' eggs is present in considerable amount, constituting about 10 per cent. of the total solids. Elementary analysis of ovomucoid has given the following results: $\mathrm{C}=48.82, \overrightarrow{\mathrm{H}}=$ $6.96, \mathrm{~N}=12.51, \mathrm{~S}=2.19$. The greater part of the sulphur (not less than three-fourths) is present in loosely combined form. On boiling with dilute mineral acids it yields a reducing substanceglucosamin. A chondroitin-sulphuric acid complex is not present in the ovomucoid molecule.

According to most authors, the ovomucoid does not give the Adamkiewicz reaction, while Langstein states that in the case of his own preparations he always obtained a positive result. $\mathrm{He}$ suggests that the negative findings of others may have been referable to the possible absence of glyoxylic acid in the glacial acetic acid employed.

The substance cannot be precipitated with the common mineral acids, acetic acid, and potassium ferrocyanide, nor by salting with sodium chloride, magnesium sulphate, or sodium sulphate. Tannic acid, phosphotungstic acid, ammoniacal subacetate of lead solution, alcohol, and ammonium sulphate, when added to saturation, cause the substance to separate out. It is soluble in water, and is not coagulated by boiling. On evaporating its solutions to dryness it is rendered insoluble in cold water, but dissolves on boiling.

Isolation.- To isolate the ovomucoid, the albumen is diluted with water, as above, slightly acidified with acetic acid, and boiled. The coagulable albumins are thus coagulated and filtered off. The filtrate, which still gives the biuret reaction, owing to the presence of the mucoid, is concentrated and precipitated with alcohol, or saturated with ammonium sulphate. The mucoid is filtered off and can then be purified by repeated solution in water and reprecipitation with alcohol.

The Yolk.-The yolk of the egg represents the ovum proper. It is surrounded by a delicate membrane-the membrana pellucidawhich supposedly consists of keratin or a closely related substance. Owing to the extensive development of the protoplasmic portion of the cell proper, the germinal vesicle is found at the extreme periphery of the yolk, immediately beneath the limiting membrane. It occupies the centre of the discus proligerus or cicatricula, which rests upon a flask-like cavity with a long, narrow neck that extends to the centre of the yolk, and is occupied by the so-called white yolk. This surrounds the cicatricula and also forms a layer along the periphery of the yolk, immediately beneath the vitelline membrane. 
It contains albumins, nucleins, lecithins, potassium salts, and possibly also traces of glycogen, though this is doubtful.

When broken, the yolk constitutes a creamy, viscid material, of an orange-yellow color, which forms an emulsion with water, and is coagulated by alcohol and on boiling. Its reaction is feebly alkaline. On microscopical examination it is seen to consist of innumerable spherules, some of which are rich in fats and lipochromes, while others, which are smaller, are colorless, transparent, semicrystalline structures of an albuminous character. In the eggs of certain amphibia and fishes distinctly crystalline bodies are further met with, which are spoken of as yolk platelets, and are analogous to the aleuron granules of seeds. As has already been mentioned, they probably consist of a compound of albumins with lecithins and nucleins. The ichthidin, which is found in carp eggs, and which in amorphous form is known as ichthulin, belongs to this category. ${ }^{1}$ The same holds good of the ichthin of shark eggs and the emydin of tortoise eggs.

As I have stated, the yolk of hens' eggs represents about 29 per cent. of the entire weight. Its actual weight may thus vary between 8.7 and 20.3 grammes. The general composition of the yolk is seen in the following analyses, which are taken from Gautier:

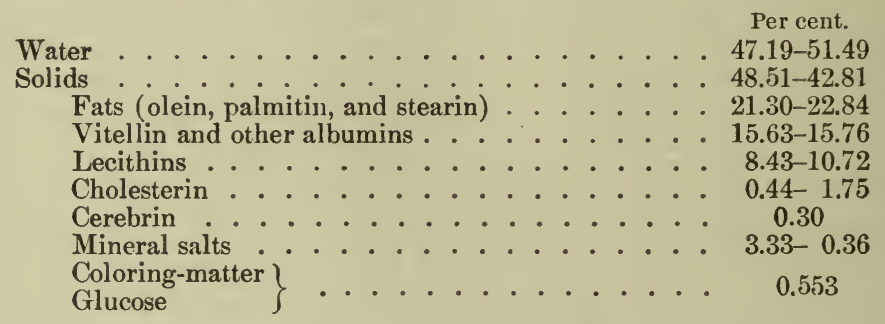

Analysis of the mineral salts, calculated for 100 parts of ash, has given the following results (Poleck and Weber):

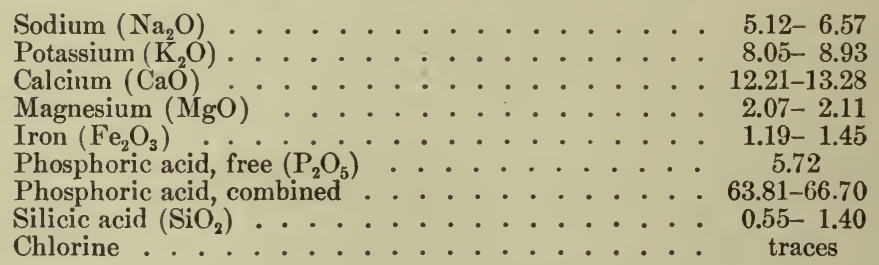

1 From recent researches of Levene it appears that different forms of ichthulin exist. The ichthulin of carp eggs thus yields a reducing substance on hydrolytic decomposition, while that of the cod apparently contains no carbohydrate radicle. The latter, on treating with alkalies, yields a paranucleinic acid, which is similar to vitellinic acid (see below). Elementary analysis of the two forms has given the following results : Ichthulin of carp eggs (Walter): C, 53.52; H, 7.6; N, 15.63; $\mathrm{S}, 0.41 ; \mathrm{P}, 0.43 ; \mathrm{Fe}, 0.10 ; \mathrm{O}, 22.19$ per cent. Ichthulin of codfish eggs : C, 52.44 ; $\mathrm{H}, 7.45 ; \mathrm{N}, 15.96 ; \mathrm{S}, 0.92 ; \mathrm{P}, 0.65 ; \mathrm{Fe}$ and $\mathrm{O}, 22.58$ per cent. 
Of the mineral constituents, the large amount of calcium and phosphoric acid is especially noteworthy. Soluble phosphates, however, are not found as such in the yolk. 'The amount of potassium and sodium, it will be observed, is much smaller than in the albumen.

The Albumins.-Our knowledge of the individual albumins which are found in the yolk is still very imperfect. But it appears from recent researches that they are represented notably by nucleo-albumins, which in turn may be combined with lecithins to form complex lecithalbumins. This, however, is not proved, and it is assumed by some that the lecithins which are obtained so commonly together with the albumins do not exist in chemical combination, but are to be regarded as contaminations. The best known representative of the nucleo-albumins of the yolk is the so-called ovovitellin. True nucleins do not occur in the yolk.

Ovovitellin.-Formerly this was regarded as a globulin, but it is now known to be a nucleo-albumin in which an albuminous radicle is combined with a paranuclein-i. e., a nuclein which does not yield nucleinic bases on decomposition with mineral acids. The substance has thus far not been obtained free from lecithins, and it is for this reason that the latter is thought by some to be present in chemical combination.

Of the character of the albuminous radicle which is present in combination with the paranuclein nothing is known. The paranuclein has recently been studied in detail by Levene and Alsberg. They term it avivitellinic acid, and give the following figures to express its elementary composition : C, 32.31; H, 5.58; $\mathrm{N}, 13.13 ; \mathrm{P}, 9.88 ; \mathrm{S}, 0.3266 ; \mathrm{O}, 38.28$ per cent. In addition they found 0.57 per cent. of iron, which is present in organic combination. This is especially interesting in view of the fact that Bunge also obtained a nuclein from the yolk of hens' eggs, which contained iron, and which he termed homatogen, as the product must of necessity be concerned in the formation of the blood-coloring matter of the developing animal. The elementary composition of Bunge's hæmatogen, however, is different from Levene's avivitellinic acid, viz., $\mathrm{C}, 42.11 ; \mathrm{H}, 6.08 ; \mathrm{N}, 14.73 ; \mathrm{S}, 0.55 ; \mathrm{P}, 5.19 ; \mathrm{Fe}, 0.29$; and $\mathrm{O}, 31.05$ per cent. Its relation to ovovitellin is at present not clear, but it is manifestly closely related to avivitellinic acid.

The albuminous radicle of avivitellinic acid manifestly contains the protamin group, as Levene was able to isolate both arginin and histidin from its decomposition-products, which resulted on boiling the substance for seventy-two hours with a 20 per cent. solution of hydrochloric acid. Whether or not lysin is also present remains to be seen. The amount of arginin and histidin obtained was so small, however, that it is scarcely warrantable to assume that a protamin constitutes the entire albuminous radicle, as in the case of the nucleins which can be obtained from certain fishes. The substance gives Millon's reaction, moreover, which is not obtained with pro- 
tamins. The biuret reaction was positive. For a more detailed account of Levene's most interesting work, and a description of the method which was employed for isolating the avivitellinic acid, I must refer the reader to his article. ${ }^{1}$

The ovivitellin as it is obtained from the yolk contains about 25 per cent. of lecithin. It is soluble in dilute solutions of the neutral salts, and in very dilute ( 1 pro mille) solutions of hydrochloric acid, and the hydrates and the carbonates of the alkalies. In water it is insoluble, and accordingly is precipitated from its solutions on copious dilution. On prolonged contact with water its properties are changed, and it is converted gradually into an albuminate-like substance. Sodium chloride when added to saturation causes only a partial precipitation. When slowly heated in its solutions of neutral salts it coagulates between $70^{\circ}$ and $75^{\circ} \mathrm{C}$; ; when rapidly heated, coagulation is retarded until $80^{\circ} \mathrm{C}$. is reached. On digestion with gastric juice ovivitellin yields a paranuclein-avivitellinic acid. From the ovivitellin of the eggs of the bony fishes a glucoparanuclein may be obtained.

Elementary analysis of the ichthulin of carp eggs has given the following results : $\mathrm{C}, 53.52 ; \mathrm{H}, 7.6 ; \mathrm{N}, 15.63 ; \mathrm{O}, 22.19 ; \mathrm{S}, 0.41$; $\mathrm{P}, 0.43$; and $\mathrm{Fe}, 0.1$ per cent. For the ichthulin of codfish eggs Levene found $\mathrm{C}, 52.44 ; \mathrm{H}, 7.45 ; \mathrm{N}, 15.96 ; \mathrm{S}, 0.92 ; \mathrm{P}, 0.65 ; \mathrm{Fe}$ and $\mathrm{O}, 22.58$ per cent. On treating with alkalies a substance is obtained from this latter form, which is quite similar in composition to avivitellinic acid, as is seen from the figures: $\mathrm{C}, 32.56$; $\mathrm{H}, 6 ; \mathrm{N}, 14.03 ; \mathrm{S}, 0.146 ; \mathrm{P}, 10.34$ per cent. It is termed $i \mathrm{ch}$ thulinic acid (see also page 461 ).

Isolation.-To isolate the ovivitellin from the yolk, it is well to employ a large number of eggs. The yolks are thoroughly mixed with an equal volume of a 10 per cent. solution of sodium chloride, and are completely extracted with ether, by shaking, viz., until no more coloring-matter can be removed, and the sodium chloride solution has become perfectly transparent. This is then diluted with twenty times its volume of water, and the ovivitellin thus precipitated. To purify the substance further, it is dissolved repeatedly in a 10 per cent. saline solution, and reprecipitated with water. It is washed finally with alcohol and ether, and dried over sulphuric acid.

The Fats.-The fat of the yolk consists almost entirely of olein, palmitin, and stearin. As a whole, it contains a somewhat smaller amount of carbon than ordinary fat, which may be due to the presence of mono- and diglycerides, or to the presence of a fatty acid which contains less carbon than usual. On saponification Liebermann obtained 40 per cent. of oleic acid, 38.04 per cent. of palmitic acid, and 15.21 per cent. of stearic acid.

The lipochromes or luteins of the yolk ean be isolated as follows: the fats of the yolk are saponified by boiling with an alcoholic

1 Zeit. f. physiol. Chem., vol. xxxi. pp. 543-556. 
solution of sodium hydrate. The alcohol is then evaporated. The remaining solution is treated with calcium chloride, which transforms the soluble sodium salts into the corresponding insoluble calcium salts. On cooling, the soaps are extracted with petrolenmether, which takes up the lipochromes. On evaporation they are then obtained in pure form. The entire process of isolation must be carried on in the absence of daylight, as otherwise the pigments are decomposed after being separated from the fats. In birds' eggs a yellow lipochrome, vitellolutein, is notably found, but, in addition, traces of a red pigment of the same order, which is termed vitellorubin, may also be encountered. This latter cannot well be obtained by extracting the soaps with petroleum-ether directly, but it is necessary previonsly to decompose these with a mineral acid.

Lecithins.- The general properties of the lecithins have been considered in a previous chapter.

Isolation.-To isolate the lecithins, the method of Zülzer may be conveniently employed. To this end, the yolks of a large number of eggs (fifty or more) are first extracted with ether by shaking, until the ethereal solution takes up no more pigment. The ethereal extracts are united, the ether is distilled off, and the oil filtered off at the temperature of the body. This is best accomplished in a thermostat. The yellow, somewhat frothy material which remains on the filter is dissolved in as little ether as possible, and precipitated with acetone. The precipitate is collected on a filter and washed with acetone until the wash-acetone dissolves no more cholesterin. The residue is again dissolved in a small amount of ether or benzol. To this solution an excess of absolute alcohol is added, when on standing a white amorphous substance separates out, which can be obtained in crystalline form by solution in hot alcohol and cooling; this apparently consists of tripalmitin. After filtration the pure lecithin can then be obtained from the etheralcoholic solution by precipitating with acetone, as before, or by distilling off the alcohol and ether. The resulting material is dried in the vacuum. Its phosphorus varies between 3.7 and 4.1 per cent. in amount.

Incubation.-Of the chemical changes which take place during the process of fertilization, and in which the nucleus of the ovum is primarily concerned, we know nothing. But there can be no doubt that, in fishes at least, the protamin radicle of the nucleins of the spermatozoa plays an important part. As a result, the reproductive function of the ovum, which previously has remained dormant, now manifests itself in the mysterious morphological changes which the cell undergoes, and which end in the production of an organism that is morphologically and chemically like its parents.

In mammals the food-stuffs which are required by the developing organism are constantly supplied through the blood of the mother-animal, but in the lower forms of life they are furnished 
directly in the egg itself. These products have been studied in some detail in the foregoing pages, and we have seen that they are in part, at least, specific of the egg, and do not occur elsewhere in the animal body. This holds good more especially of the albumins, and it follows that all those forms that enter into the composition of the various tissues must of necessity be produced from the pre-existing forms during the development of the young animal. The fats may, in part, be utilized directly in the construction of the fats of the embryo, but to a large extent, no doubt, they represent the prineipal form of energy which is placed at the disposal of the developing organism. Carbohydrates, as such, are practically lacking among the food-stuffs of the egg, and must hence be formed synthetically. That glycogen ean be demonstrated in the tissues of the embryo at a very early date, has already been stated, which proves in itself that the animal organism is not dependent upon the ingested carbohydrates for its glyeogen supply. As nucleoproteids, moreover, do not occur in the egg, it would appear that these also must be formed from other albuminous substances, and there can be little doubt that the nucleo-albumins are here of prime importance. In this connection it is interesting to note, however, that Mesernitzki found xanthin-bases in identical amounts in non-incubated eggs, as in early chick-embryos. The salts which are required by the developing organism are, as has been seen, present in the egg in abundance.

The essential factor, however, which is neeessary to development after fertilization, is an abundant supply of oxygen, and a temperature of about $40^{\circ} \mathrm{C}$. The requisite amount of oxygen is obtained from the air by a process of diffusion through the shell. In return carbon dioxide is eliminated, together with a small amount of nitrogen. These respiratory changes are but slight in the beginning, but gradually increase. Water also is given off, and, as a result, the weight of the egg diminishes. The increase of the solids in the developing animal is, of course, accompanied by a eorresponding diminution of those of the egg itself.

Systematic chemical examinations of the ovum in its various stages of development have thus far not been made. Liebermann appears to be the only one, indeed, who has attempted the problem. His prineipal results may be summarized as follows: during the first stage of development tissues are formed, which are very rich in water ; later, however, the amount of water decreases. The absolute amount of substances which are soluble in water steadily increases, while their relative amount diminishes as compared with the remaining solids. After the fourteenth day a large inerease in the amount of fat is noted, while previously this remains fairly constant. The amount of soluble albumins and albuminoids inereases steadily and in such a manner that the absolute quantity increases, while their relative amount remains nearly constant. Up to the tenth day no collagen is found, but after the fourteenth day a sub- 
stance is present which on boiling with water yields a material similar to cartilaginous glutin. A mucinous substance is found about the sixth day, but it subsequently disappears. The amount of hæmoglobin steadily increases in its relation to the body-weight.

Levene has recently shown that incubated eggs contain monoamido-acids, and he regards it as probable that the preparations which he obtained consisted of an equimolecular mixture of monoamido-butyric acid and mono-amido-valerianic acid. The results were obtained with eggs that were twenty-four hours and seven days old.

Interesting also are Levene's observations on the amount of albumins at different stages of development, which were marle on fish eggs. Non-incubated eggs contained 66 per cent. of albuminous nitrogen; after twenty-four hours' incubation, 53.57 per cent. was found; after ten days, 64.79 per cent; and at the expiration of nineteen days, 71.84 per cent. During this period there was also a steady increase in the amount of nitrogen referable to non-albuminous substances that could be precipitated with phosphotungstic acid, viz., from 12.07 to 28.25 per cent.

On the basis of Spitzer's observations on the nature of the oxidation-ferments as nucleoproteids Löb expressed the opinion that the nucleus represents the organ of oxidation of living matter, and that cellular matter devoid of nuclei is incapable of regenerating its kind for the reason that its power of oxidation is too insignificant. Jacoby, however, has shown that in pig embryos, at least at a time when the first indications of the formation of a bony skeleton exist, aldehydase at any rate cannot be demonstrated. Whether or not it is present as a zymogen, has not been investigated. Later the aldehydase appears, viz., in embryos which are $9 \mathrm{~cm}$. long and longer. A tangible basis for Löb's assumption hence does not exist.

The chemical composition of the allantoic fuid and the amniotic fluid has already been considered.

The placenta has not as yet been studied in detail, but it is likely that its greater portion consists of collagen, in accordance with its fibrous structure. In its marginal zone two pigments have been encountered which apparently are related closely to bilirubin and biliverdin, and are derivatives of hæmoglobin. The orange pigment may be obtained in erystalline form, while the green pigment, which has been termed homatochlorine, is amorphous. 


\title{
CHA P'TER XXII.
}

\author{
THE DUCTLESS GLANDS.
}

\section{THE THYROID GLAND.}

OF the function of the thyroid gland little is known. Removal of the organ gives rise to serious symptoms which may be of a chronic or an acute character. In the first instance there is evidence of marked impairment of the general metabolic functions and of heat-production, associated with impairment of muscular and mental power (myxœdema), and if occurring in children there is marked retardation in growth (cretinism). When of an acute character tetanic symptoms commonly develop. When removal is complete, death sooner or later results. If, however, the destruction or removal of the gland is partial, deleterious results do not necessarily follow. It is thus manifest that the function of the organ is a most important one, and it is interesting to note that the apparent antitoxic properties of the gland persist even after its removal from the body and subsequent desiccation. The various symptoms which have just been described as following extirpation of the organ may thus be prevented by administration of the dried gland, and in cases of atrophy a curative effect may similarly be obtained. It is noteworthy, moreover, that the administration of the substance has a marked effect upon the nitrogenous metabolism of the body, which is distinctly increased, and, if continued, emaciation results although an abundant amount of food is ingested, and digestion and resorption remain unimpaired. In some instances true diabetes develops. In addition, an increased pulse-rate and heightened blood-pressure are commonly observed, and even sudden death may occur during the use of the substance.

That these curious properties of the thyroid gland should also find expression in its chemical composition suggests itself at once. Numerous attempts have accordingly been made to isolate the "active principle" of the organ, and to a certain extent these attempts have been successful. Baumann and Roos thus succeeded in isolating from the gland a substance which has manifestly the same properties as the entire organ in preventing the deleterious results which follow its extirpation or atrophy, and which also has the same effect upon the circulation and the nitrogenous metabolism. This substance Baumann termed thyroiodine, from the fact that it contains iodine in organic combination. It is obtained by boiling the 
gland for several hours with a 10 per cent. solution of sulphuric acid, and by subsequently extracting the insoluble residue with 90 per cent. alcohol. It is insoluble in water and acids, but readily dissolves in dilute alkaline solutions, from which it is precipitated by adding an excess of an acid. The substance, as first obtained by Baumann, contained 9.3 per cent. of iodine and a small amount of phosphorus. This latter, however, he regarded as a contamination, and he expressed the opinion that future researches would show that the chemically pure body contained even more iodine than the crude product he obtained. Regarding the chemical nature of the thyroiodine, which itself does not give the biuret reaction, Baumann supposed that it existed in the gland in combination with an albumin, viz., as a thyro-iodoglobulin- or albumin. This has since been proved, through the researches of Oswald, who succeeded in extracting from the gland a globulin which contains the entire amount of iodine, and has all the physiological properties of the thyroiodine (iodothyrin). It yields Baumann's thyroiodine on decomposition with mineral acids. This substance is termed thyreoglobulin. Together with another albuminous substance belonging to the nucleoproteids the thyreoglobulin forms the colloid substance of the gland.

Thyreoglobulin.-The quantity of thyreoglobulin is directly dependent upon the amount of the colloid, and is thus subject to variation. Normal glands contain on an average from 1 to 8 grammes of the dried substance. Much larger amounts, viz., 50 to 100 grammes, have been observed in goitres that were especially rich in colloid. In sheep it has been found in the proportion of $1: 3$, or $2: 3$, as compared with the total amount of solids, and similar results have been obtained in the pig. Its general elementary composition in animals of the same species is quite constant, and varies but little indeed in animals of different species. The amount of iodine, however, which is present in organic combination is subject to fairly wide variations. This is shown in the following analyses, which are taken from Oswald:

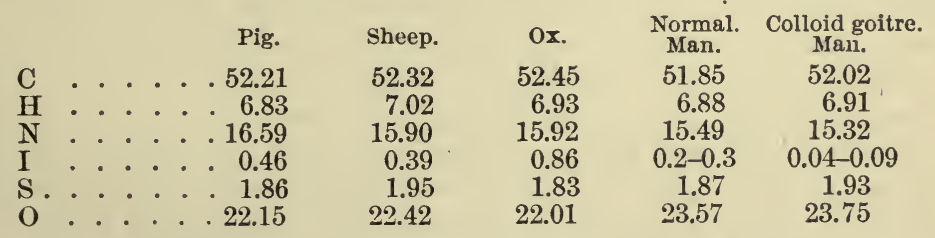

It is thus seen that in colloid goitres especially small amounts of iodine are apparently present. And it appears that the amount of iodine is the lower, the more advanced the colloid degeneration$i$. e., the larger the amount of thyrenglobulin. Oswald explains this observation by assuming the simultaneous existence in goitres of an iodized and a non-iodized globulin. The iodized form is found only in glands which contain colloid, while it is never found in 
thyroids that are free from colloid, such as parenchymatous goitres and the glands of the newborn. Oswald concludes that the thyreoglobulin is only iodized after it leaves the follicle-cells. At the same time it is noteworthy that non-iodized thyreoglobulin alone never leaves the cells, but only if iodothyreoglobulin is simultaneously secreted. The amount of iodine in a gland can be artificially increased by the ingestion of iodine or iodides as such, and it is to be noted that the physiological activity of the gland can thus be increased, while this is not possible by iodizing the thyreoglobulin in vitro.

The existence of a non-iodized thyreoglobulin is especially interesting in view of the fact that, whereas the iodized globulin possesses all the specific properties of the entire gland, the noniodized substance is inert. An adequate explanation of this curious phenomenon cannot at present be given, but we may imagine that in those instances in which the iodine is normally absent its place may be taken by some other halogen, or a compound halogen which has escaped observation. To conclude that the iodized substance is not the active principle of the gland, on the basis that the glands of some animals contain no iodine, and that its amount is more or less variable and can artificially be increased, is scarcely warrantable. It is conceivable that in sucklings, for example, in which iodine is commonly absent, a compound halogen takes its place, and is, for the time being at least, fully capable of preventing the development of the complex of symptoms which we term cachexia strumipriva.

In its general properties thyreoglobulin resembles the common globulin of the blood. It differs, from this, however, in the fact that it is precipitated from its saline solutions on the addition of dilute acids. In this respect it resembles the myosin of muscletissue. Its point of coagulation, in a 10 per cent. solution of magnesium sulphate, varies between $65^{\circ}$ and $67^{\circ} \mathrm{C}$. It is precipitated from its solutions by the addition of an equal volume of a saturated solution of ammonium sulphate.

On decomposition with dilute acids it yields the thyroiodine of Baumann, but contains more iodine, viz., 14.29 per cent., and, like its mother-substance, it is free from phosphorus. On further decomposition it loses its activity altogether.

Thyreo-nucleoproteid.-A nucleoproteid is found in association with thyreoglobulin in the colloid material of the gland, but is present in much smaller amounts ; it contains 0.16 per cent. of phosphorus. In a 10 per cent. solution of magnesium sulphate it coagulates at $73^{\circ} \mathrm{C}$. On digestion with gastric juice a nuclein is split off, which contains xanthin-bases. The substance is free from iodine, and is physiologically inert. It is precipitated from its solutions by salting with ammonium sulphate to saturation.

For a further description of the two albumins, and of the 
methods which may be employed for their isolation, the reader is referred to Oswald's paper. ${ }^{1}$

The extractives of the thyroid gland are represented by traces of xanthin, hypoxanthin, leucin, succinic acid, and paralactic acid. In addition, notable quantities of kreatinin may be obtained.

Among the mineral constituents of the thyroid gland the presence of traces of arsenic is of interest ; according to Gautier and Bertrand, it represents a normal and constant component of the gland.

\section{THE ADRENAL GLANDS.}

Of the function of the adrenal glands, nothing definite is knownTheir integrity, however, is essential to life, and, as in the case of the thyroid, their removal leads to the death of the animal. It has been noted, moreover, that the injection of blood from a dog which has died as a result of the operation, into the circulation of a second animal that has been operated in the same manner, will hasten the fatal end, while in normal dogs no deleterious results are observed. It has hence been concluded that the glands normally furnish a secretion which renders certain metabolic products innocuous, and that the fatal result which follows the removal of the organs is the result of an auto-intoxication.

In man, disease of the adrenal glands leads to the complex of symptoms which is commonly known as Addison's disease, and likewise results in death; but as in the case of the thyroid gland, it has been observed that the fatal issue may here also at least be retarded by the administration of an aqueous extract of the organs. Experiments with such extracts have further shown that the gland contains a substance which has a very marked effect upon the blood-pressure, raising this far beyond the normal. This substance is found in the medullary portion of the glands. Further investigations have then demonstrated the existence of a chromogen which on exposure to the air in aqueous solution yields a beautiful carmin-colored pigment, which, like its mother-substance, is soluble in water. The same result is reached at once on treating with chlorine-, bromine-, or iodine-water. This chromogen is present in the intracellular fluid of the medullary portion of the gland. When this is extracted with a dilute acid, a violet-red precipitate results on the addition of an excess of ammonia, which suggests that the pigment is of a basic nature.

With a solution of ferric chloride the juice that can be expressed from the glands gives a bright emerald-green color. This reaction has been referred to the supposed presence of pyrocatechin, but thus far this has never been isolated.

Modern researches lead to the conclusion that the blood-pressureraising constituent of the gland, as also the chromogen, which gives rise to the carmin color and the pyrocatechin reaction, are identical

1 Zeit. f. physiol. Chem., vol. xxvii. p. 14. 
bodies. Abel, who claims to have isolated the blood-pressure-raising principle of the glands, states that this must in all probability be classed with the pyrrol compounds or with the pyridin bases or alkaloids. He was unable, however, to obtain the free base, which he terms epinephrin, in crystalline form. Pyrocatechin could not be split off from this product on boiling with an acid, but he states that a carmin-red pigment can be separated from the sulphate of the active principle without destroying its power to raise the bloodpressure.

v. Fürth, who calls the active principle suprarenin, was likewise not able to obtain it in pure form.

Later, Takamine announced that he had succeeded in isolating the blood-pressure-raising constituent of the gland in a stable and crystalline form. This substance he terms adrenalin.

Adrenalin is a light, white crystalline substance, of a slightly bitter taste, leaving a numb feeling on the tongue where it has been applied. When dry, it is perfectly stable. On heating, it turns brown at $205^{\circ} \mathrm{C}$. At $207^{\circ} \mathrm{C}$. it melts, and is at the same time decomposed. Its reaction is slightly alkaline. In cold water it is soluble with difficulty, but more readily so in hot water. From its hot solution it crystallizes out on cooling. It is easily soluble in acids and alkalies, but not in ammonia or solutions of the alkaline carbonates. Upon the addition of ferric chloride its solutions are colored a fine emerald-green, which changes to a purple and then to a carmin red upon the careful addition of caustic alkali. Strong acids prevent the reaction, limiting the change of color to a dirty yellowish-green. It reduces silver salts and auric chloride very energetically, while the liquid at the same time turns red. This also occurs on treating with oxidizing agents, such as potassium ferricyanide and potassium bichromate. The usual alkaloidal reagents do not precipitate the substance. With acids it forms salts, but these have not been obtained in crystalline form.

Abel's most recent analysis of his epinephrin hydrate only differs from Takamine's adrenalin by one-half a molecule of water; its formula is $\mathrm{C}_{10} \mathrm{H}_{13} \mathrm{NO}_{3} \cdot \frac{1}{2} \mathrm{H}_{2} \mathrm{O}$. Its structural composition is still unknown.

The blood-pressure-raising power of adrenalin is very marked. The amount pro kilo of body-weight which is required to raise the blood-pressure $14 \mathrm{Hgmm}$. beyond the normal is one-millionth part of a gramme, and distinct physiological effects can be obtained by the administration of even one-fourteenth millionth part of a gramme.

Aside from the blood-pressure-raising properties the adrenal glands are also of interest from the fact that hypodermic injection of adrenal extract results in glucosuria, even though carbohydrates have been excluded from the diet of the animal (dogs). The amount of sugar which may appear is at times considerable, viz., 3.8 per cent. Analogous results are obtained during starvation and at a time when the liver is free from glycogen. The glucosuria 
persists for from one to three days, and is manifestly not dependent upon changes in the blood-plasma. It is noteworthy that in these cases the elimination of nitrogen is not increased, and that neither acetone nor diacetic acid is found in the urine. As explanation of the glucosuria in these cases Blum is inclined to assume some special action on the part of the adrenal extract upon the pancreas or possibly upon the liver. Similar results were obtained by Herter and Wakeman with adrenalin directly. These observers also found that painting the pancreas with adrenalin solution produced marked glucosuria, while this was only slight if the same was done in the case of the liver, the spleen, or the brain. Other reducing agents produced a similar effect, so that the authors concluded that a toxic effect is exerted by all these bodies upon the pancreatic cells leading to impairment of their oxidizing power, so that normal combustion of the sugar does not oceur.

In a number of instances in which glucosuria was produced by the injection of adrenal extract, bile-pigment also appeared in the urine, but there is no constant relation between the two conditions (Blum).

In addition to these bodies the adrenal glands contain collagen, which enters into the composition of the supporting tissue; albumins, which have not been studied in detail ; and, further, a substance which apparently is closely related to jecorin, and yields fatty acids, neurin, glycerin-phosphoric acid, and glucose on hydrolytic decomposition with baryta-water. Besides, lecithins and a small amount of inosit are met with. Benzoic acid, hippuric acid, and biliary acids are not present, as was formerly supposed.

Jones has isolated a nucleoproteid from the adrenal gland which seems to have the same composition as Hammarsten's nucleoproteid which he obtained from the pancreas. It is dextrorotatory $(\alpha) \mathrm{D}+43.1^{\circ}$.

Of ferments, the adrenal gland contains an aldehydase (Jacoby) which is principally found -in the cortex; further, a diastase (Croftan) which is capable of forming both maltose and dextrose, and finally an autolytic proteolytic ferment. 



\section{A PPENDIX.}

\section{LABORATORY EXERCISES.}

Exercise I.-Prepare a 1 per cent. (0.8 per cent.) solution of serum-albumin, serum-globulin, and egg-albumin in dilute saline solution. Determine the coagulation-point of each, after acidifying slightly with very dilute acetic acid. To this end, place the albuminous solution in a test-tube, of which it should fill about onethird to one-half; clamp this, immersed in a beaker with water, and fasten a thermometer in the albuminous solution so that the bulb nearly reaches the bottom. Heat with a small flame and note the temperature at which coagulation just begins ; continue to heat, and finally boil the solution; note the changing appearance of the precipitate. Collect some of this and attempt to dissolve it in water or dilute saline solution; then try concentrated acid and alkali with the application of heat.

Study the behavior of an albuminous solution on heating in the presence of an excess of dilute acid and alkali and in the presence and absence of salt (page 30).

Exercise II.-1. Prepare a dilute solution of serum-albumin; render it feebly acid with acetic acid; coagulate it by boiling; render the solution strongly acid with hydrochloric; acid, heat, and note that the coagulated albumin dissolves; on cooling add an alkali until the reaction is only faintly acid, and heat again : coagulation does not occur.

2. Render a solution of serum-albumin strongly alkaline with sodium hydrate and boil: coagulation does not occur; then add acetic acid to a feebly acid reaction and heat again : no coagulation occurs.

Exercise III.-Prepare solutions of serum-albumin, serumglobulin, easein, egg-albumin, and test the behavior of these solutions to sodium chloride, magnesium sulphate, and ammonium sulphate by salting the solutions to saturation; let the precipitates stand over night and examine the filtrates the next day ; test with the biuret test (page 35) to ascertain whether the precipitation is complete.

Determine the upper and lower limits of precipitation of the different albumins in nentral solutions with a saturated neutral aqueous solution of ammonium sulphate, as described (page 32).

Exercise IV. - Prepare dilute aqueous solutions of serum-albumin, 
egg-albumin, and albumoses (Witte's peptone), also a dilute saline solution of serum-globulin, and perform the various qualitative tests outlined on page 34 . Repeat the tests with an albuminous urine. Remove all albumins from one of the albuminous solutions with ferric chloride as described, filter, and demonstrate the absence of albumin in the filtrate with potassium ferrocyanide and acetic acid.

Exercise V.-Perform the albumin color-tests with various albumins (egg-albumin, serum-albumin, caseïn) either in solution or in substance, as directed on page 35 . Note the negative Molisch reaction in the case of caseïn, and the relative amount of sulphur in serum-albumin as compared with caseïn.

If no glyoxylic acid is available, use glacial acetic acid in the Adamkiewicz reaction; if the reaction is negative, add a small amount of a solution of oxalic acid which has been previously treated with sodium amalgam.

Exercise VI.-Examine solutions of different albumins with the polarimeter; note the degree of lævorotation.

Exercise VII.-Place 100 grammes of dry egg-albumin in a flask holding about 1 liter; add 500 c.c. of concentrated hydrochloric acid and boil for twelve hours. Let cool ; add strong caustic alkali solution until the reaction is feebly acid; boil with much animal charcoal for about one-half hour and filter. In the filtrate look for leucin, tyrosin, glutaminic acid, asparaginic acid, etc., as deseribed in Exercise XXIII.

Exercise VIII.-See exercises on serum-albumin and serumglobulin, Exercise XLVI.

Exercise IX.-Suspend a small amount of caseïn (0.5 gramme) in 25 c.c. of water and dissolve it by the careful addition of dilute caustic alkali solution $(1: 10)$, then precipitate the caseïn with acetic acid and wash with water; this is to remove any adherent phosphates. Now fuse the purified and dried casein with soda and saltpeter and test for alkali phosphate.

Exercise X.-See Exercise XXXVI.

Exercise XI.-Make a little starch paste; boil with 25 per cent. hydrochloric acid for ten minutes; filter; render the filtrate strongly alkaline with caustic soda solution and test for glucose. (See Exercise XXXVI.)

Exercise XII.-Prepare a 90 per cent. solution of alcohol (100 c.c.), add 25 c.c. of a concentrated solution of caustic soda, melt about 50 grammes of lard, and pour all into a half liter flask. Heat on a water-bath and shake the mixture frequently as soon as the alcohol begins to boil. When saponification is complete, which occurs very rapidly if the experiment is conducted as just outlined, a small amount of the solution on being poured into distilled water should give rise to no turbidity. The resulting solution contains soap and glycerin, which can be demonstrated according to the usual methods. 
Exercise XIII.-1. Collect about 50 c.c. of saliva by chewing some paraffin or rubber and spitting into a beaker. Note the appearance of the secretion and test the reaction.

2. Demonstrate the presence of ptyalin as described on page 122 .

3. Demonstrate the salivary mucin (page 124).

4. Demonstrate the presence of sulphocyanides (page 1.25).

5. Demonstrate the presence of nitrites (page 125).

Exercise XIV.-Procure gastric juice from a human being at the height of digestion; or prepare an artificial mixture containing 2 to 3 pro mille of hydrochloric acid, some essence of pepsin, and albumoses (liquid peptonoids or panopeptone).

1. Test the reaction with litmus; with congo red (using congo red paper on a very dilute solution).

2. Perform Günzburg's test, Töpfer's test, and Boas' test for free hydrochloric acid (pages 130 and 131).

3. Determine the total acidity (page 128).

4. Determine the amount of free and combined hydrochloric acid : $(a)$ according to Töpfer ; $(b)$ according to Mörner and Sjöqvist ; (c) according to Leo (pages 131 and 132).

Exercise XV.-1. With the artificial gastric juice used in Exercise XIV. test: (a) for pepsin ; $(b)$ for chymosin (pages 140, 143).

2. Estimate the amount of pepsin (page 140).

Exercise XVI.-Prepare a 3 pro mille solution of lactic acid.

1. Perform Uffelmann's test and Kelling's test (page 133).

2. Estimate the amount according to Boas' method (page 135).

3. With a 3 pro mille solution of acetic acid and butyric acid perform the tests described on page 134 .

Exercise XVII.-1. Prepare a solution of pancreatin ( 0.5 per cent.) in a 0.25 to 1 per cent. solution of sodium carbonate; add a small flake of fibrin and place in an incubator at $40^{\circ} \mathrm{C}$.

2. Prepare a neutral aqueous solution of pancreatin (10-15 c.c.) and add a small flake of fibrin; place in an incubator.

3. Prepare an acid solution of pancreatin in a similar manner (containing 2 to 3 pro mille of hydrochloric acid), add a small flake of fibrin, and place in an incubator. Observe the effect at the end of one hour.

4. Perform similar tests with pepsin.

5. Note that previous boiling will destroy the action of the ferment.

Exercise XVIII.-From a 10 per cent. solution of Witte's peptone (1000 c.c.) isolate the various albumose fractions as in Exercise III. Demonstrate the absence of a carbohydrate group in proto-albumose and hetero-albumose; its presence in the deutero-fraction $B$. Isolate gluco-albumose from the B-fraction, and also thio-albumose (test the sulphur reaction in this, page 38 ).

Exercise XIX.-1. Procure 250 c.c. of ox-bile and of sheep-bile. Note the general characteristics of each.

2. From the ox-bile prepare Platner's bile, and isolate from it glycocholic acid and taurocholic acid (page 160). 
3. With Platner's bile and the isolated acids perform Pettenkofer's test (page 161).

4. Isolate taurin from the sheep-bile and identify it (page 169).

5. With the fresh sheep-bile, somewhat diluted, perform Gmelin's test, Huppert's test, and Smith's test (page 173).

6. Isolate the biliary mucin from ox-bile (page 159).

7. Procure some human bile (at autopsy) and examine into the presence of true mucin (page 159).

8. Procure some gall-stones (preferably cholesterin stones); isolate the cholesterin in pure form and perform the Liebermann-Burckhard and Salkowski tests (page 177).

Exercise XX.-Carbohydrate digestion (see Exercise XIII.).

Exercise XXI.-1. Digest 100 grammes of fresh fibrin with pepsin-hydrochloric acid and isolate the varions fractions as described on page 195. If fibrin preserved in chloroform is only available, heat this in a 2 per cent. solution of hydrochloric acid until it swells and assumes a jelly-like appearance.

Exercise XXII.-Digest 100 grammes of fibrin with pancreatin and isolate the various albumoses as described on page 196 .

Exercise XXIII.-From a fibrin-pancreatin digestive mixture that has stood for a week well guarded against putrefactive changes with toluol ( $2 \mathrm{~cm}$. of toluol should stand above the mixture), isolate (a) the anti-peptone fraction. Demonstrate that this contains a fraction which can be precipitated with phosphotungstic acid and one which remains in solution (page 201). (b) Use this same mixture to demonstrate the presence of leucin and tyrosin (page 205), aspartic acid and glutaminic acid (page 207). (c) In the same mixture demonstrate the presence of tryptophan (page 209).

4. To demonstrate glycocoll as a decomposition-product of the albumins it is best to start with gelatin. This is conveniently hydrolyzed with 25 per cent. sulphuric acid (page 208). Use about 100 grammes of gelatin, 1000 c.c. of dilute acid, and boil for twentyfour hours.

Exercise XXIV.-Hash 100 grammes of liver-tissue; suspend in 1000 c.c. of water; add an abundance of toluol, shake well (the tolıol should form a layer $1 \mathrm{~cm}$. deep on the surface), and keep at a tenperature of $40^{\circ} \mathrm{C}$. for three to four weeks. Then examine for albumoses, tyrosin, leucin, etc., as above.

Exercise XXV.-Hash 100 grammes of pancreas; suspend in 1000 c.c. of water; place in an incubator at $40^{\circ} \mathrm{C}$. for a week, and then examine for indol, skatol, and phenol (page 221).

Exercise XXVI.-1. Collect the urine of twenty-four hours from an individual. Measure the amount, note the color, odor, reaction, and specific gravity (pages 224 to 229 ).

2. Determine the degree of acidity (page 229).

3. Estimate the mineral ash (page 233).

Exercise XXVII.-Estimate the chlorides of the urine (use the 
collected amount of twenty-four hours in all the quantitative work on the urine which is to follow) (page 233).

2. Estimate the phosphates: (a) total ; $(b)$ the earthy ; and (c) the alkaline (page 233).

3. Estimate: (a) the total sulphates; (b) the conjugate sulphates; (c) the neutral sulphur (pages 234, 235, and 290).

Exercise XXVIII. - 1. Prepare some urea from the urine (page 243 ) ; examine with a microscope the urea nitrate which is formed.

2. Dissolve a few crystals of urea in 1 c.c. of water and add a small amount of a strong solution of oxalic acid; urea oxalate is precipitated ; examine with a microscope.

3. Heat some urea in a test-tube as described on page 242 ; note the formation of biuret (page 36).

4. Estimate the urea with sodium hypobromite solution, using Doremus' ureometer; the gas evolved is nitrogen; the $\mathrm{CO}_{2}$ which is formed is taken up by the excess of sodium hydrate in the hypobromite solution (page 243).

5. Estimate the urea according to Folin's method (page 244).

Exercise XXIX. - Determine the total amount of nitrogen in the urine according to Kjeldahl's method (page 245).

Exercise XXX.-Isolate some uric acid from human urine (page 251).

2. Note the insolubility in water.

3. Perform the murexid test, the copper test, Dennigè's test, and Schiff's test (page 250).

4. Estimate the uric acid according to Folin's method (page 251).

Exercise XXXI.-1. Estimate the oxalic acid according to Dunlop's method (page 255).

2. Isolate kreatinin as kreatinin-zine chloride (page 259).

Exercise XXXII. - 1. Test the urine for indican, using Jaffe's test or Obermeyer's test (page 265).

2. Estimate the indican according to Wang's method (page 266).

Exercise XXXIII.-Feed a dog a couple of grammes of chloral and demonstrate glucuronates in the urine the next day (page 270).

Exercise XXXIV.-Prepare artificial diabetic urine, containing $\beta$-oxybutyric acid, diacetic acid, acetone, and glucose; let stand for several hours.

1. Polarize the urine and note the degree of dextrorotation.

2. Ferment the urine with yeast for twenty-four hours and reexamine (lævorotation due to oxybutyric acid).

3. Estimate the oxybutyric acid as a $a$-crotonic acid (page 281).

4. Test for diacetic acid, using both Arnold's and Gerhardt's test (pages 281 and 282).

5. Test for acetone in the distillate (see page 282); use Legal's, Lieben's, and Gunning's tests (page 282).

6 . Estimate the acetone (page 284).

Exercise XXXV.-1. Prepare some cystin from human hair as described on page 290 . 
2. Examine the resulting crystals with a microscope and test their solubility in ammonia, caustic soda, and hydrochloric acid.

3. Prove that the substance contains sulphur, as follows: place a small amount in a test-tube, dissolve in caustic alkali, add a few drops of acetate of lead solution, and boil ; note the brownish-black color which results owing to the formation of sulphide of lead.

4. Repeat the preceding experiment: $(a)$ with normal urine; (b) with urine to which a solution of cystin has been added.

5. Estimate the amount of neutral sulphur in the urine as described on page 290. (Be careful with the sodium peroxide.)

Exercise XXXVI. - 1. Prepare a solution of glucose in water (about 1 per cent.) or in urine, and perform: (a) Nylander's test; (b) Fehling's test; (c) the fermentation test; and (d) the phenylhydrazin test, as described on pages 293 and 294 .

2. Determine the melting-point of : (a) phenyl-glucosazon ; to this end, purify the erystals and dry them as described on page 295. Prepare a capillary tube about $1 \frac{1}{2}$ inches long and with a lumen of approximately $1 \mathrm{~mm}$., seal one end, place some crystals of the osazon in the interior; fasten the tube with platinum wire to the bulb of a thermometer registering about $250^{\circ} \mathrm{C}$.; suspend the thermometer in concentrated sulphuric acid in a beaker; heat gently and note the temperature at which the crystals melt. In a similar manner prepare the osazons of: $(b)$ lactose, $(c)$ lævulose, and $(d)$ maltose, and determine the melting-points as described.

3. Estimate the amount of glucose in solution: (a) with the polarimeter; (b) by the differential density method; (c) by Knapp's method; and $(d)$ by Fehling's method. (The solution of glucose should stand twenty-four hours before being polarized.)

Exercise XXXVII. - 1. Prepare a dilute solution of arabinose (0.1 per cent.); perform the orcin test as described by Bial (page 300).

2. Test with Fehling's solution.

3. Prepare the corresponding osazon and determine the meltingpoint (see preceding Exercise).

Exercise XXXVIII.-1. Procure some albuminous urine and perform: $(a)$ the nitric acid test; $(b)$ the boiling test; $(c)$ the potassium ferrocyanide test (pages 302 and 303).

2. Demonstrate the presence of two albumins in such urine, one of which coagulates between $50^{\circ}$ and $60^{\circ} \mathrm{C}$., and the other between $65^{\circ}$ and $75^{\circ} \mathrm{C}$.; note that the former is present in smaller amount. Make the examination by hanging a thermometer registering $100^{\circ} \mathrm{C}$. in a wide test-tube about one-third full of urine; immerse this well in a large beaker of water; heat very slowly. Prove that one of the two albumins is serum-albumin and the other serum-globulin (page 304).

3. Estimate the amount of albumin : (a) gravimetrically ; (b) by Esbach's method.

4. Procure urine from a case of acute cystitis. Test for nucleoalbumin as described on page 304 . 
5. Add a couple of grammes of Witte-peptone to a liter of urine; then examine the solution as described on page 305 .

Exercise XXXIX. - 1. Procure some febrile urine and examine this: (a) for urochrome; (b) for uroerythrin; (c) for urobilin.

2. Procure some typhoid urine between the seventh and the tenth day of the disease and apply Ehrlich's test. Use about 5 c.c. of urine and an equal amount of the sulphanilic acid mixture. After adding the ammonia to form a layer above the urine and acid mixture note the color-ring at the zone of contact. Shake the fluid and note the color of the foam; pour all into a white basin and dilute copiously with water; note the salmon pink. Repeat the experiment with normal urine.

3. Add a little blood to urine: (a) perform Heller's test; (b) examine the urine spectroscopically (page 311 ).

4. Procure some urine containing hæmatoporphyrin : (a) examine this spectroscopically; $(b)$ isolate the hæmatoporphyrin and examine its pure solution with a spectroscope (page 311).

5. With urine from a case of melanotic sarcoma perform: (a) the bromine test; $(b)$ the iron test (page 313 ).

6. Procure bile from a dog or from a human being (at autopsy), dilute with water, and perform the various tests described on page 173 :

Exercise XL.-1. Procure blood at a slaughter-house ; let a portion flow directly from the vessel into a saturated solution of sodium sulphate or a 10 per cent. solution of sodium chloride (equal parts); receive a second portion in a dry cylinder. Note that in the first portion coagulation does not occur. Observe the phenomenon of coagulation in the second. Prepare also some oxalate plasma and some albumose plasma (page 329). Keep some oxalate plasma on ice and note the separation of the corpuscles on standing.

2. Determine the specific gravity of human blood according to Hammerschlag's method (page 324).

3. Determine the reaction of fresh cat's or dog's blood according to Löwy's method, and that of human blood according to Dare (page 326).

4. Isolate the fibrinogen from about 50 c.c. of plasma by halfsaturation with sodium chloride (page 330). To the resulting solution of fibrinogen add a little serum. Note the occurrence of eoagulation.

5. Prepare blood-serum by allowing defibrinated blood (500 c.c.) to stand on ice or, still better, by separating the cellular elements by centrifugation.

6. From 100 c.c. of serum isolate the serum-albumin fraction and the globulin fraction by means of ammonium sulphate (pages 332 and 333$)$.

A. Note the behavior of the globulin fraction $(a)$ on dialysis (examine the remaining solution for globulins by saturation with magnesium sulphate), (b) on passing a stream of $\mathrm{CO}_{2}$ through a 
dilute solution of the globulin, $(c)$ on diluting 20 times with water after acidifying faintly with acetic acid (page 331 ). Note the coagulation-point of the serum-globulin in the presence of 5 per cent. of sodium chloride as described below.

B. Note the solubility of the serum-albumin in water; estimate the coagulation-point in the presence of 5 per cent. of sodium chloride. To this end, a test-tube is filled about one-half with the solution and immersed in a large beaker with water; a thermometer registering $100^{\circ} \mathrm{C}$. should dip nearly to the bottom of the test-tube; heat slowly.

7. Remove all albumins from 20 to 30 c.c. of serum, according to Cavazzani's method (page 333). In the resulting solution demonstrate the presence of glucose by means of the phenyl-hydrazin test.

Exercise XLI.-1. Examine fresh blood directly with a spectroscope.

2. Estimate the amount of hæmoglobin in human blood with Dare's hæmoglobinometer, or with v. Fleischl's hæmometer.

3. Prepare some crystalline oxyhæmoglobin from dog's blood (100 c.c.) (page 350$)$; examine the resulting product spectroscopically; note the change in the spectrum on the addition of caustic alkali and reduction with ammonium sulphide (page 346).

4. Prepare some hæmin from oxyhæmoglobin and from this, in turn, hæmatin (page 347); note the spectrum of the latter in acid and in alkaline solution.

5. Perform the hæmin test with a drop of blood. To this end, let a delicate film of sodium chloride form in the middle of a slide; place on this a drop of blood; let dry and add glacial acetic acid, a drop at a time, while heating very gently over a flame; examine from time to time with a microscope. Finally add a drop of glycerin, cover, and examine. Note the color, form, and varying size of the crystals.

6. Prepare some carbon monoxide hæmoglobin from oxyhæmoglobin (page 352). Note the spectrum.

7. Prepare a solution of hæmatoporphyrin in a test-tube by adding about 5 drops of blood, drop by drop, and shaking constantly, to 8 or 10 c.c. of concentrated sulphuric acid ; examine with a spectroscope (page 355).

Exercise XLII.-1. Hash 100 grammes of lean muscle-tissue (beef); suspend in 300 c.c. of water; stir well; allow to stand for a couple of hours; filter through muslin and then through filterpaper. (a) Examine the reaction of the filtrate. (b) Demonstrate the presence of three albumin fractions by fractional coagulation. (Arrange the apparatus as described above, XL., 6, B.) Heat very slowly and filter whenever one fraction has been coagulated. (c) Prepare myosin from the meat residue after thorough washing with water as follows: prepare a 15 per cent. solution of ammonium chloride, suspend the meat in this; stir well and allow to stand for 
twenty-four hours. The resulting solution contains the myosin. Note the readiness with which myosin coagulates by diluting a few c.c. of the solution with water, and by adding finely powdered salt to the solution and stirring. Boil a few c.c. of the solution, filter, and examine the filtrate for calcium salts by adding ammonium oxalate.

2. Prepare muscle-plasma and isolate the myogen from this as described on page 366. Examine the resulting product.

3. Demonstrate the presence of glycogen in fresh muscle-tissue (page 374).

4. Demonstrate the presence of glucose (page 375 ).

5. Demonstrate the presence of lactic acid (using about 100 grammes of meat) (page 377 ).

6. Demonstrate the presence of kreatin (page 380 ).

7. To demonstrate the xanthin bases it is most convenient to start with a meat extract, for example, with 100 grammes of Liebig's extract. Proceed with this as described on page 381 .

Exercise XLIII.-1. Isolate protagon from a fresh calf's brain (page 391).

2. Demonstrate the presence of lecithins in the brain (page 393).

Exercise XLIV.-1. Decalcify pieces of bone with 50 per cent. hydrochloric acid; let stand from twenty-four to forty-eight hours. Decant the acid solution which contains the mineral constituents. Wash the remaining ossein with water and with dilute soda solution, then place in a bowl with water and boil until the ossein has dissolved, neutralize or make faintly alkaline with soda, decant, and cool; note that the solution jellies (gelatin).

2. From cartilage-shavings isolate the chondromucoid (page 404).

Exercise XLV.-1. Demonstrate the presence of iron-containing albuminates in the liver (page 423). Use about 100 grammes of tissue.

2. Feed a rabbit about 25 grammes of glucose through a tube; kill it several hours later, and demonstrate the presence of glycogen in the liver (page 425); use a portion of the same liver for the following experiment :

3. The demonstration of glucose (page 425).

Exercise XLVI.-Prepare guanylic acid from pancreas as described on page 426.

Exercise XLVII.-1. Note the general physical properties of milk; examine a drop microscopically; note the reaction and the specific gravity ; boil some milk.

2. Isolate the different albumins of the milk (page 436).

3. Estimate the total albumins (page 436).

4. Add some essence of pepsin (rennin) to milk; place in the incubator at $40^{\circ} \mathrm{C}$. and note the occurrence of coagulation (page 434).

5. Let a specimen of milk stand exposed to the air for forty-eight hours; note the reaction. What has occurred? (Page 434.) 
6. Estimate the amount of fat (page 438).

7. Estimate the amount of lactose (page 438).

8. Perform the test of Umikoff for citric acid (page 439).

Exercise XLVIII.-1. Demonstrate the presence of the various albumins in white of egg (use the whites of about 20 eggs) (page 448).

2. Isolate the ovomucoid (page 449).

3. Demonstrate the lecithins in the yolk (page 453). 


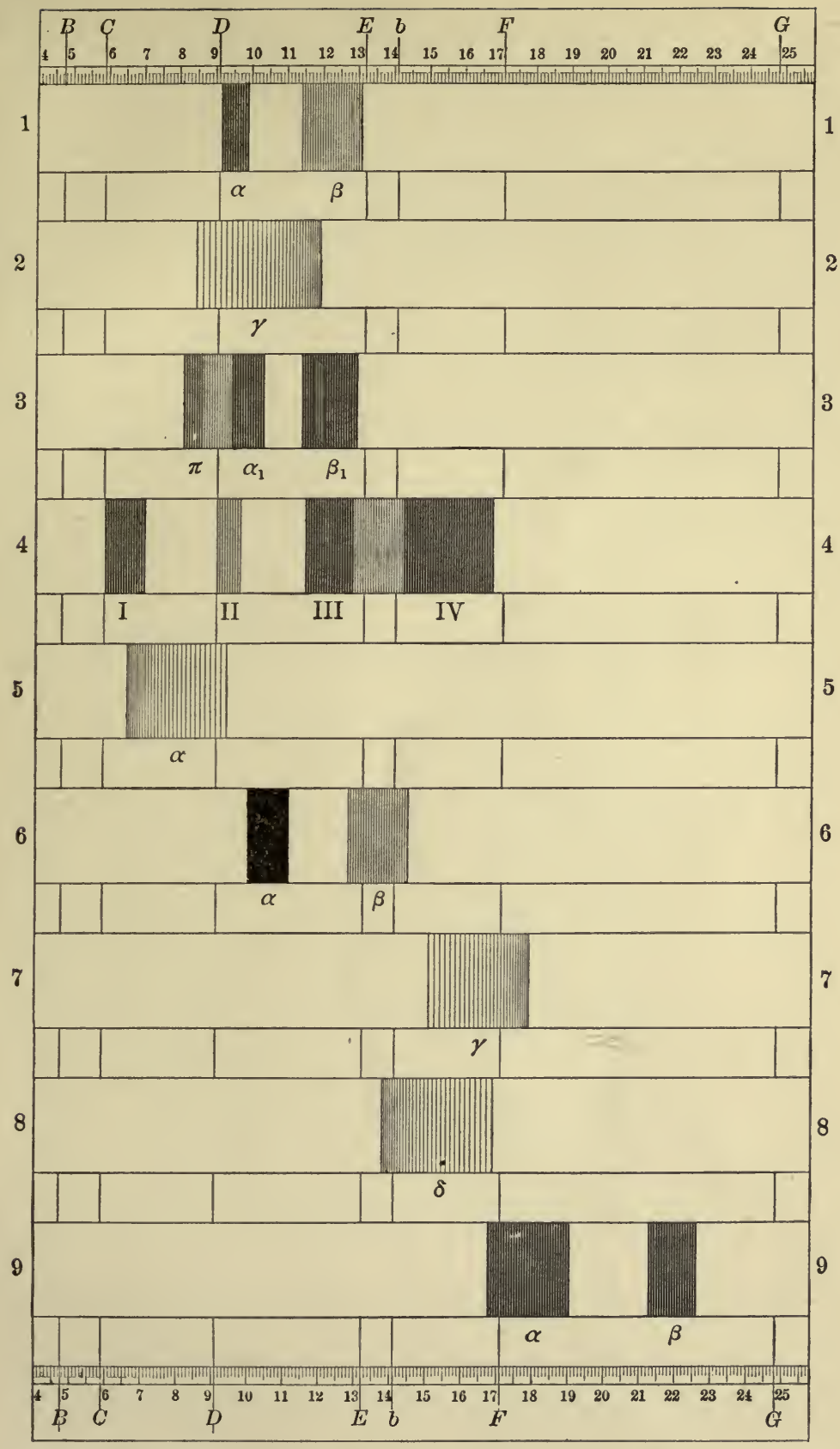

1, Absorption-spectrum of a solutinn of oxyhæmoglobin: 2, of $n$ solution of hæmoglobin: 3 , of a feebly alkaline solution of methrmoglobin; 4 , of a solution of hæmatin in acid ether (oxalic acid); 5 , of an alkaline solution of hrmatin; 6 , of an alkaline solution of hæm()chromogen: 7 , of an acid solution of urohilin: 8 , of an ammoniacal solution of urobilin, after the addition of zinc chloride solution; 9 , of a solution of lutein (ethereal extract of the yolk of egg). 

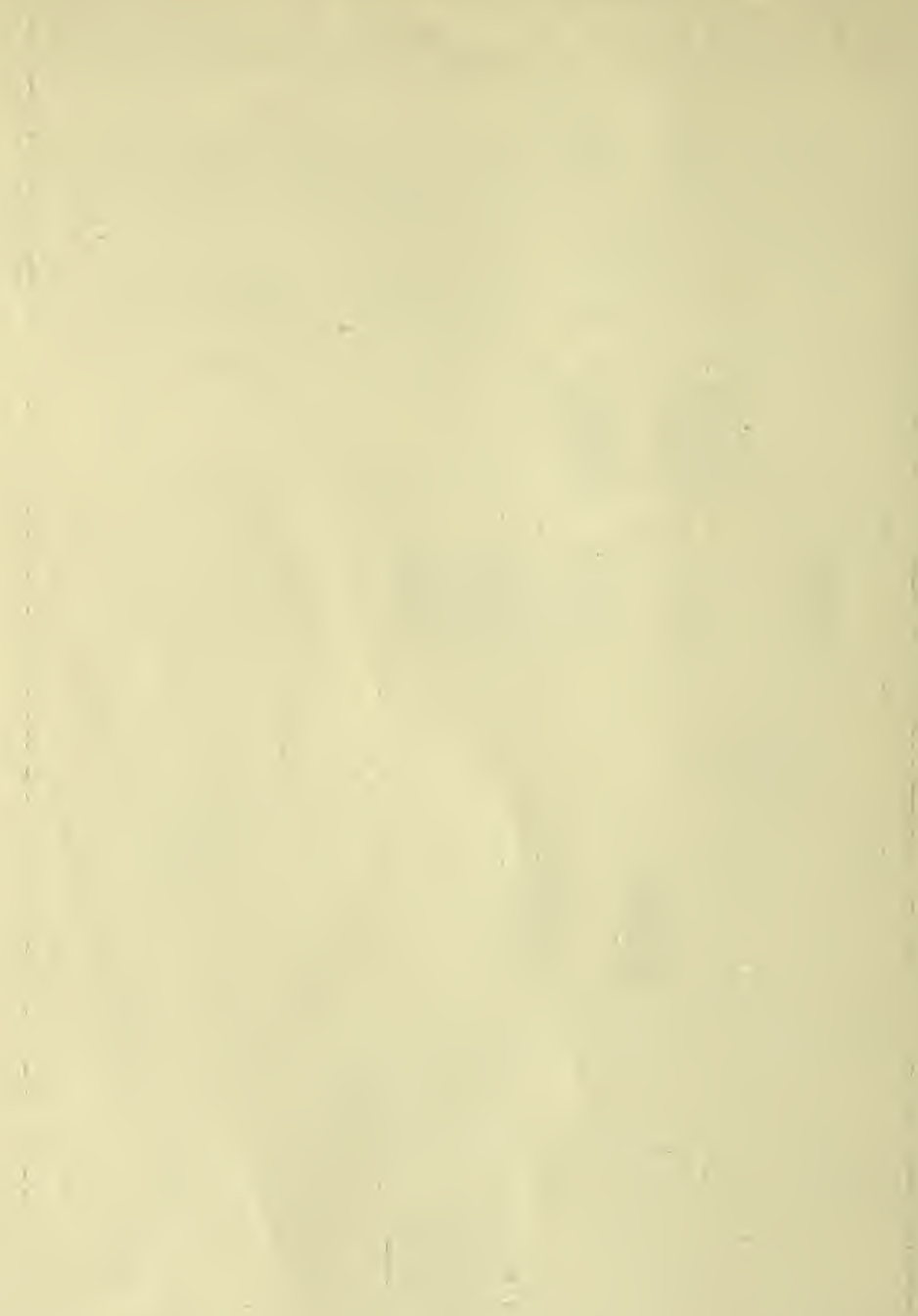


\section{INDEX.}

A.

ACCIPENSERIN, 53

Acetic acid, 90

in stomach contents, 134

tests for, 134

Acetone, 91, 280, 282

estimation of, 284

tests for, 282

Achroödextrin, 123

Acid, acetic, 90, 134

adenylic, 95

alloxyproteinic, 287

amino-valerianic, 80

antoxyproteinic, 287

arachinic, 437

aspartic, 81, 205

avivitellinic, 451

benzoic, 94

bilianic, 166

bilirubinic, 172

biliverdinic, 173

butyric, 90,134

capronic, 90

carbamic, 238

carnic, 369

chenocholalic, 167

chenotaurocholic, 164

chitonic, 87

cholalic, 165

cholanic, 166, 167

choleic, 167

choleo-camphoric, 166

cholesteric, 166

choloidinic, 166

cholonic, 162

chondroitin-sulphuric, 49, 403

cilianic, 166

citric, 439

crotonic, 91,280

cysteinic, 84,168

damalic, 277

damaluric, 277

dehydrocholalic, 166

dehydrocholeic, 167

desoxycholalic, 167

diacetic, 91,280

dialuric, 100,106

diamino-trioxydodecanis, 89

dioxy-phenyl-acetic, 274

fellic, 167
Acid, ferri-albuminic, 423

formic, 90

galactonic, 68

gluconic, 269

glucuronic, 268

glutaminic, 81, 206

glutaric, 92

glycerin-phosphoric, 76, 314

glycocholic, 83, 162

glycolic, 91

glycosuric, 274

guanidin-butyric, 81

guano-biliary, 163

guanylic, 95,426

hæmatinic, 348

hippuric, 83, 94

homogentisinic, 274

hydrochloric, 129

hydrocinnamic, 93

hydroparacumaric, 85, 216

hyocholalic, 167

hyoglycocholic, 163

hyotaurocholic, 164

ichthulinic, 452

inosinic, 95, 385

isobilianic, 166

kynurenic, 277

kynuric, 277

lactic, 91, 284

laurinic, 437

leucinic, 91

lithofellic, 167

lithuric, 276

lysuric, 89

mannonic, 67

manno-saccharinic, 67

mesoxalic, 106

methyl-hydantoinic, 84

mucinic, 68

myristinic, 178

nucleinic, 94

oleic, 75

ornithuric, 83,89

oxalic, $92,106,254$

oxaluric, 254

oxy-butyric, 91, 279

oxy-hydroparacumaric, 91

oxy-proteinic, 287

oxy-pyrrolidin carbonic, 81

oxy-tricarballylic, 439

palmitic, 75,90 
Acid, parabanic, 103, 254

paralactic, 284

para-oxy-benzoic, 93

para-oxy-phenyl-acetic, 93, 216, 274

para-oxy-phenyl-glycolic, 93, 274

para-oxy-phenyl-lactic, 92, 273

para-oxy-phenyl-propionic, 93, 216,273

phenaceturic, 83,94

phenyl-acetic, 93, 216

phenyl-propionic, 93, 216

phospho-carnic, 369

phyllocyanic, 21

plasminic, 97

propionic, 90

pyrocholesteric, 166

pyrrolidin carbonic, 81

rhodizonic, 378

saccharinic, 65, 269

saccharo-lactonic, 65

sarcylic, 95

skatol-carbonic, 86, 216, 267

sperma-nucleinic, 93

stearic, 75,90

succinic, 92

tartronic, 106

taurocarbaminic, 84, 287

taurocholic, 83, 163

tetraoxy-amido-capronic, 49

thyminic, 96

thymo-nucleinic, 95

trioxy-phenyl-propionic, 273

triticonucleinic, 95

tyrosin-hydantoinic, 84

uramino-benzoic, 84

uric, 103, 105

urocaninic, 277

urochloralic, 270

uroferric, 287

uroleucinic, 276

uroproteic, 287

uroxanthinic, 280

urrhodinic, 280

ursocholeic, 167

valerianic, 90

xanthylic, 95

yeast-nucleinic, 95

Acids, aromatic oxy-, 273

biliary, 159

Adamkiewicz' reaction, 37

Addison's disease, 459

Adenin, 101, 384

isolation of, 381

tests for, 384

Adenylic acid, 95

Adipocere, 411

Adipose tissue, 408

analysis of, 410

origin of, 410

significance of, 412

Adrenal glands, 459

Adrenalin, Takamine's, 460
Alanin, 80

in the urine, 285

Albamin, 44, 448

Albumen of birds' eggs, 446

albumins of, 447

analysis of, 446

ovalbumins of, 448

ovomucoid of, 449

tata, 446

Albumin of Bence Jones, 305

Albuminates, 59

alkaline, 59

of iron, 423

Albuminoids, 57

digestion of, 188

Albumins, 27, 47

behavior toward alcohol, 34

neutral salts, 31

polarized light, 29

classification of, 45

cleavage products of, 80

coagulated, 60

coagulation of, 30

color-reactions of, 35

crystallization of, 28

decomposition of, 38

denaturization of, 31

derived, 59

diffusion of, 29

digestion of, 181

elementary composition of, 27

estimation of, in the blood, 333

in milk, 436

in plasma, 333

in urine, 306

globulins, 47

glucoalbumins, 48

histons, 51

keratins, 50

molecular size of, 45

native, 47

nucleins, 56

nucleoalbumins, 53

of the albumen, 447

of the blood, 330

of the liver, 421

of the lymph, 361

of the milk, 433

of the muscle-tissue, 365

of the nerve-tissue, 388

of the urine, 301

of the yolk, 451

peptones, 61

precipitation of, 34

protamins, 52

proteids, 55

reaction of. 27

special, 34

solubility of, 28

structural composition of, 38

synthesis of, 26

tests for, in the urine, 302

Albuminuria, 301 
Albuminuria, physiological, 301

Albumoid, 405 isolation of, 405

Albumoses, 59

analysis of, 195, 196

in the blood, 339

in the urine, 305

primary, 60,195

secondary, 60, 195, 196

reactions of, 197

tests for, in the urine, 305

Aldehydase, 119

Aldoses, 64

Aleuron crystals, 28

Alkapton, 275

Allantoic acid, 455

Allantoin, 106, 256

in the urine, 256

isolation of, 256

Alloxan, 103, 106, 254

Alloxuric bases, 100

Alloxyproteinic acid, 287

Amino-acids, 80

in the urine, 285

Amino-valerianic acid, 80

Ammonia, estimation of, in the blood, 340

in the urine, 244

Ammonium purpurate, 106

Amniotic fluid analysis of, 362

Amphopeptone, 182

Amylases, 117

Amylodextrin, 71

Amyloid, 58

vegetable, 73

Amylolysis, 123, 179

Amylolytic ferment of pancreatic juice, 151

Amylopsin, 151

Amylum, 71

Animal cell, 318 fat, 408

Anti-bodies in the blood, 340

Antiferments, 112

Antipeptone, 61, 201

Aqueous humor, analysis of, 361, 396

Arabinose, 68

Arachinic acid, 437

Arbacin, 53

Arbutin, 24

Arginin, 88

in the spleen, 427

in the urine, 285

Arnold's test for diacetic acid, 281

Aromatic constituents of the urine, 273 oxy-acids, 91

Arterin, 343 in the urine, 273

Asparagin, 206

Aspartic acid, 81, 205

isolation of, 207

Autolysis, 114, 193

Avivitellinic acid, 451
B.

BACTERIAL action in the intestinal tract, 211

decomposition of biliary constituents, 217

of fats, 216

Bence Jones' albumin, 305

Benzoic acid, 94

isolation of, from hippuric acid, 272

Bezaar stones, 167

Bile, 155

acids of (see also Biliary acids), 159

amount of, 157

chemical composition of, 158

cholesterin in, 176

crystallized, of Platner, 161

general properties of, 157

iron in, 178

mucinous body of, 159

pigments of, 170

in the urine, 313

secretion of, 156

Bilianic acid, 166

Biliary acids, 160

formation of, 160

in the urine, 314

isolation of, 160

tests for, 161

constituents, bacterial decomposi-

tion of, 217

iron, 178

Bilicyanin, 175

Bilifuscin, 172, 175

Bilihumin, 176

Biliprasin, 172, 175

Bilipurpurin, 176

Bilirubin, 170

isolation of, 174

tests for, 173

Bilirubinic acid, 172

Biliverdin, 172, 174 isolation of, 175

Biliverdinic acid, 173

Bilixanthin, 176

Biuret, 35, 242

reaction, 35

Blood, 322

amount of, 325

chemical composition of, 327

examination of, 325

coagulation of, 335

color of, 323

extractives of, 340

fat in, 340

fibrin, 337

fibrin-ferment in, 335

fibrinogen in, 330

glucose in, 340

glycogen in, 340

leucocytes of, 341

odor of, 324 
Blood, physical characteristics of, 323 pigments of, 344

in urine, 310

plaques of, 322

plasma, 323,329

reaction of, 325

red corpuscles of, 322,343

serum, 323,334

serum-albumin of, 332

serum-globulin of, 330

specific gravity of, 324

taste of, 324

urea in, 340

Blood-pigments in the urine, 310

Boas' method of estimating lactic acid, 135

test for hydrochloric acid, 131 for lactic acid, 134

Boiling test for albumin, 303

Bone, 405 analysis of, 406

Bone-marrow, 407

Böttcher's crystals, 443

Brain-tissue, analysis of, 387

Brunner's glands, secretion of, 152

Bufidin, 419

Butter, 430

Buttermilk, 430

Butyric acid, 90, 134

in stomach contents, 134

tests for, 134

C.

Cachexia strumipriva, 458

Cadaverin, 89 in the urine, 316

Caffein, 101

Cane-sugar, 69

Carbamic acid, 238

Carbohæmoglobin, 352

Carbohydrates, 63 digestion of, 123, 179

fermentation of, 67

of the urine, 291 synthesis of, in plants, 23

Carbon dioxide hæmoglobin, 352 monoxide hæmoglobin, 352

Carnic acid, 369

Carniferrin, 369

Carnin, 102

in muscle-tissue, 384

isolation of, 381

properties of, 384

tests for, 385

Carnosin, 90, 385

Cartilage, 402

albumoid in, 405

analysis of, 403

chondroitin-sulphuric acid in, 403

chondromucoid in, 404

embryonic, 402

mineral salts in, 405
Casein. See Caseinogen.

Caseinogen, 433

digestion of, 191

estimation of, 436

isolation of, 436

origin of, 436

properties of, 433

Caseoses, 60

Castoreum, 419

Cell-globulins, 342

Celluloses, 73

Cement-substance of teeth, 407

Cerealose, 70

Cerebrin, 391

isolation of, 392

properties of, 391

Cerebron, 390

Cerebrosides in nerve-tissue, 390

Cerebrospinal fluid, analysis of, 362

Cerumen, 419

Cetin, 75

Cetylid, 392

Charcot-Leyden crystals, 220, 443

Cheese, 430

Chenocholalic acid, 167

Chenotaurocholic acid, 164

Chitin, 408

Chitonic acid, 87

Chitosamin, 87

Chitose, 87

Chlorides, estimation of, in urine, 233

Chlorophane, 400

Chlorophyl, 20

chemical nature of, 21

granules, 20

hydride, 24

Cholalic acid, 165 isolation of, 167

Cholanic acid, 166

Cholecyanin, 175

Choleglobins, 178

Choleic acid, 167

Choleocamphoric acid, 166

Cholesteric acid, 166

Cholesterilins, 176

Cholesterin, 78, 176

in nerve-tissue, 394

in the bile, 176

in the urine, 314

isolation of, 177

tests for, 177

Choletelin, 176

Cholin, 77

Choloidinic acid, 166

Cholonic acid, 162

Chondrin, 404

Chondroitin, 49

Chondroitin-sulphuric acid, 49

in cartilage, 403

isolation of, 404

properties of, 403

Chondromucoid, 404

isolation of, 404 
Chondrosin, 49

Choroid, 400

Chromogen of adrenal glands, 459

Chromophanes, 400

Chyle, analysis of, 361

Chymosin, 141 estimation of, 143

in the gastric juice, 141

in the pancreatic juice, 152 isolation of, 143

tests for, 143

Chymosinogen, 141

estimation of, 143

test for, 143

Cilianic acid, 166

Citric acid in milk, 439

Clupein, 52

Coagulating ferments, 119

Coagulation of blood, 335 rapidity of, 339

of milk, 434 theories of, 335

of proteins, 30

Collagen, 58

Colloidal platinum, 20

Colostrum, 440

Compound glycocolls in the urine, 270

Conalbumin, 448

Conjugate glucuronates in urine, 268 sulphates, estimation of, 235 in the urine, 232

Copper test for uric acid, 250

Cornea, 396

Corpora lutea, 445

Cream, 433

$a$-Crotonic acid, 91

Crystalline lens, 397 albumins of, 397 albumoid of, 397 crystallins of, 397

Crystallins, 397

Cyan-hæmoglobin, 353

Cyanurin, 265

Cyclopterin, 53

Cysteïn, 84, 287 in the urine, 287

Cysteïnic acid, 84

Cystin, 81, 288 estimation of, 290 in the kidneys, 428

in the liver, 426

in the urine, 288

isolation of, 290

preparation of, 290

properties of, 288

transformation to taurin, 289

Cystinuria associated with diaminuria, 288

Cytosin, 98

D.

Damalic acid, 277

Damaluric acid, 277
Dare's method of estimating the alkalinity of blood, 326

Dehydrocholalic acid, 166

Dehydrocholeic acid, 167

Denaturization, 30, 31

Dennigès' test for acetone, 283

Dentin, 407 for uric acid, 250

Derived albumins, 59

Descemet's membrane, 396

Desoxycholalic acid, 167

Deutero-albumoses, 196, 197, 200

Dextrins, 72

in the urine, 300

Dextrose. See Glucose.

Diacetic acid, 91, 280, 281

in the urine, 281

tests for, 281

Dialuric acid, 100, 106

Diamino-acids, 39, 87

Diamino-nitrogen, 87

Diamins in the urine, 316 isolation of, 316

Diaminuria, 316

Diastases, 117

Diazo reaction, 310

Diethylene diamin, 443

Differential density method of estimating sugar, 298

Digestion, 179

analysis of products of, 195

gastric, 182

products of, 195

of albuminoids, 188

of albumins, 181

of carbohydrates, 179

of fats, 192

of proteids, 187

tryptic, 185

products of, 195

Digestive glands, 426

Di-oxy-phenyl-acetic acid in urine, 274

Disaccharides, 68

Diureids, 104

Ductless glands, 456

Dulcite, 64

Dunlop's method of estimating oxalic acid, 261

Dyslysin, 166

E.

EAR, the, 400

Egg-albumins, 447

Eggs, 445

albumen of, 446

albumins of, 447

fats of, 452

incubation of, 453

lecithins of, 453

lipochromes of, 452

ovomucoid in, 449

ovovitellin in, 451

shell of, 445 
Eggs, yolk of, 449

Ehrlich's diazo reaction, 310

Elastin, 57

Elastoidin, 57

Elastoses, 60

Eleidin granules, 414

Emydin, 450

Enamel of teeth, 407

Encephalin, 393

Enteric juice, 152

amount of, 154

enterokinase in, 154

erepsin in, 155

ferments of, 154

prosecretin in, 155

secretin in, 155

Enterokinase, 154

Enzymes. See Ferments.

Eosinophilic crystalloids, 28

Epiguanin, 102

Epinephrin, Abel's, 460

Episarcin, 102

Erepsin, 117, 155, 189

Ereptic digestion, 189

Erythrodextrin, 123

Esbach's reagent, 307

Ethylenimin, 443

Ethyl sulphide in the urine, 287

Euglobulin, 331

Excretin in the feces, 223

Extractives of the blood, 340

of the liver, 426

of the lymph, 362

of the milk, 439

of the nerve-tissue, 394

of the saliva, 125

of the thyroid gland, 459

Eye, 396

\section{F.}

FAT, 74, 408

analysis of, 410

animal, 408

bacterial decomposition of, 216

chemistry of, 74

digestion of, 192

estimation of, in the milk, 438

of birds' egg, 452

of the blood, 340

of the liver, 425

of the lymph, 359

of the milk, 437

of the muscle-tissue, 386

of the urine, 314

origin of, 410

in milk, 437

significance of, 412

synthesis of, in plants, 25

Fatty acids, 90

estimation of, in urine, 278 isolation of, from urine, 278

degeneration, 425

infiltration, 425
Fatty tissue. See Adipose tissue.

Feces, 219

amount of, 219

bacteria in, 220

chemical composition of, 219

color of, 219

consistence of, 219

crystals in, 220

excretin in, 223

form of, 219

hydrobilirubin in, 222

macroscopical constituents of, 219

microscopical constituents of, 219

odor of, 219

reaction of, 219

serolin in, 223

stercobilin in, 223

stercorin in, 223

Fehling's method of estimating sugar, 296

solution, 294

test for sugar, 294

Fellic acid, 167

Fermentation, acetic, 213

alcoholic, 213

butyric, 213

lactic, 213

method of estimating sugar, 298

test for sugar, 294

Ferments, 110

amylolytic, 117

chemical composition of, 115

classification of, 116

coagulating, 119

general properties of, 112

reactions of, 115

glucolytic, 119

inverting, 117

lipolytic, 117

mode of action of, 116

of the adrenal glands, 461

of the blood, 339

of the enteric juice, 154

of the gastric juice, 135

of the liver, 424

of the lymph, 361

of the milk, 440

of the muscle-tissue, 370

of the pancreatic juice, 147,427

of the urine, 315

oxidizing, 118

proteolytic, 117

reducing, 119

reversible action of, 114

tissue, 114

which cause the cleavage of glucosides, 118

of nucleinic acids, 118 of urea, 117

split off carbon dioxide, 118

transform amion-acids to amides, 117

Ferratin, 423 
Ferri-albuminic acid, 423

Ferrocyanide test for albumin, 303

Fertilization of eggs, 453

Fibrin, 59, 337

estimation of, 338

-ferment, 335 chemical nature of, 337

isolation of, 336

properties of, 337

test for, 337

formation of, 337

isolation of, 338

of Henle, 442

properties of, 337

test for, in the urine, 306

Fibrinogen, 330

isolation of, from the blood, 330

properties of, 330

Fibrinoglobulin, 335

Fibroïn, 57

Fibrous tissue, elastic, 402

reticulated, 402

white, 401

yellow, 402

Fish-scales, 408

Floridins, 345, 356

Folin's method of estimating urea, 244 preformed ammonia in urine, 244

uric acid, 251

Food-stuffs of plants, 22

Fructose. See Lavulose.

Fuscin, 400

\section{G.}

Gadus histon, 52

Galactonic acid, 68

Galactose, 67

Gallois' test for inosit, 378

Gases of the blood, 328

of the stomach contents, 144

of the urine, 315

Gastric contents, acetic acid in, 134

butyric acid in, 134

lactic acid in, 133

total acidity of, 127

digestion, 182

juice, 126

acidity of, 127,129

amount of, 126

analysis of, 127

chemical composition of, 127

chymosin, 141

chymosinogen, 141

digestive power of, 126

ferments of, 135

gases of, 144

hydrochloric acid of, 129

lactic acid in, 133

pepsin, 137

pepsinogen, 141

pro-enzymes of, 135

sulphocyanides in, 144

$? 1$
Gelatin, 58

Gelatoses, 60

Gels, 29

Gerhardt's test for diacetic acid, 282

for urobilin, 309

Giacosa's pigment, 265

Glands, adrenal, 459

digestive, 420, 426

ductless, 456

lymph, 427

mammary, 428

reproductive, 441

salivary, 120

Globin, 345

isolation of, 346

Globulinoses, 60

Globulins, 47

Glucite, 64

Gluco-albumins, 48

Gluco-albumose, 200

Glucocyamidin, 108

Glucocyamin, 108

Glucolysis, 119

Glucolytic ferments, 119

Gluconic acid, 269

Glucoproteids, 48

Glucosamin, 87

Glucose, 67, 291

in the blood, 340

in the liver, 425

in the muscle-tissue, 375

in the urine, 291 estimation of, 296

tests for, 293

Glucosides in nerve-tissue, 390

synthesis of, in plants, 24

Glucosuria, 291

Glucuron, 269

Glucuronates, conjugate, 268

Glucuronic acid, 268

in the blood, 340

in the urine, 268

origin of, 269

properties of, 269 tests for, 270

Glutamin, 206

Glutaminic acid, 81, 206

Glutin, 58 isolation of, 207

Glutinoids, 57

Glutokyrin, 186

Glutolin, 339

Glycerin-phosphoric acid, 76

in the urine, 314

Glycin. See Glycocoll.

Glycocholic acid, 83, 162 isolation of, 163

Glycocoll, 80, 169, 207

in the urine, 285

isolation of, 170, 207

relation to hippuric acid, 207

test for, 207

Glycocolls, compound, $270 \curvearrowright$ 
Glycogen, 72, 371

estimation of, 374,425

formation of, 371

in the blood, 340

in the liver, 424

in the muscle-tissue, 371

isolation of, 374,425

properties of, 424

significance of, 371

Glycosuric acid, 274

Gmelin's test, 173

Guanidin-butyric acid, 81

Guanin, 101

in muscle-tissue, 383

isolation of, 381

tests for, 384

Guano-biliary acid, 163

Guanylic acid, 95, 426

Gums, 73

isolation of, 426

Gunning's test for acetone, 283

Günzburg's test for hydrochloric acid, 131

\section{H:}

\section{HAMATIN, 347}

in the urine, 311

isolation of, 347

Hæmatinic acids, 348

Hæmatochlorine, 455

Hrematogen, 421, 451

Hæmatoidin, 355

Hæmatoporphyrin, 311, 354

in the urine, 311

preparation of, 354

Hæmin, 347, 349 preparation of, 347

Hæmoalkalimeter, 326

Hæmochromogen, 346 isolation of, 346

Hæmocyanin, 356

Hæmoglobin, 344 amount of, 351 isolation of, 346 properties of, 346

Hæmoglobinometer of Sahli, 352

Hæmoglobins, 57

Hæmolysis, 79

Hæmometer of Dare, 351 of Fleischl, 351

Hæmopyrrol, 348

Hammerschlag's method of determining the specific gravity of blood, 324

Heller's test for albumin, 302 for blood, 311

Hemi-albumose, 42

Hemicelluloses, 73

Hemipeptone, 42

Henle's fibrin, 442

Hepatin, 421

Hetero-albumose, 197

Heterolysis, 114

\author{
Heteroxanthin, 101 \\ Hexobioses, 68 \\ Hexon-bases, 87 \\ in antipeptone, 186 \\ Hexoses, 64 \\ Hippomelanin, 313 \\ Hippuric acid, 83, 94, 270 \\ estimation of, 272 \\ isolation of, 272 \\ of the urine, 270 \\ origin of, 270 \\ properties of, 271 \\ synthesis of, 271 \\ Histidin, 89
}

Histons, 51

test for, in the urine, 306

Histozyme, 117

Hoffmann's test for tyrosin, 204

Homocerebrin, 392

Homogentisinic acid in the urine, 274 isolation of, 275

Hopkin's method of estimating uric acid, 251

Hoppe-Seyler's test for xanthin, 383

Hormone, 146, 155

Huppert's test, 173

Hyalins, 50

Hyalogens, 50

Hyalomucoid, 398

Hydrations in animal life, 19

Hydrazons, 65

Hydrobilirubin in the feces, 218, 222

Hydrocele fluid, analysis of, 362

Hydrochloric acid of gastric juice, 129

combined, 131

free, 129

origin, 129

quantitative estimation of, 131

secretion of, 129

significance of, 130

tests for, 130

Hydrocinnamic acid, 93

Hydrogen sulphide in the urine, 315

Hydroparacumaric acid, 85

in the intestinal contents, 216

in the urine, 273

isolation of, 222

Hydroquinon, 93

in the urine, 262

Hydrosals, 29

Hyocholalic acid, 167

Hyoglycocholic acid, 163

Hyotaurocholic acid, 164

Hypobromite method of estimating urea, 241

Hypoxanthin, 383

in muscle-tissue, 383

isolation of, 381

Hypoxanthylic acid, 95 
I.

ICHTHIDIN, 450

Ichthin, 450

Ichthulin, 450, 452

Ichthulinic acid, 452

Ichthylepidin, 408

Ignotin, 385

Ilasvay's reagent, 125

Incubation of eggs, 453

Indican, animal, 87, 264

estimation of, 266

in the urine, 264

origin of, 264

properties of, 265

tests for, 265

vegetable, 87

Indiglucin, 87

Indigo-blue, 87, 264

Indigo-purpurin, 265

Indigo-white, 87

Indirubin, 265

Indol, 86, 213 isolation of, 221

tests for, 214

Indoxyl, 86, 264

red, 264

sulphate. See Indicun.

Inosinic acid, 95 in muscle-tissue, 385

Inosit in muscle-tissue, 377

in the urine, 276

isolation of, 378

tests for, 378

Intestinal putrefaction, 216

Inulin, 72

Invertases, 117

Invertins, 117

Iron albuminate, 422

in the liver, 178,422

Isobilianic acid, 166

Isolactose, 70

Isoleucin, 80, 203

Isomaltose, 70

Ivory, 407

J.

JAFFE's test for indican, 265

for kreatinin, 258

Jecorin in nerve-tissue, 395

K.

Katalases, 118

Kathæmoglobin, 353

Kelling's test for lactic acid, 134

Kerasin See Homocerehrin.

Keratin in the skin, 414

Keratinoses, 60

Keratins, 50

Ketoses, 64

Kidneys, 428

Kjeldahl method of estimating nitrogen, 245
Kinases, 119

Knapp's method of estimating sugar, 296

Knop-Hüfner method of estimating urea, 243

Kreatin, 107, 257

in muscle-tissue, 379

in the urine, 257

isolation of, 380

origin of, 379

properties of, 257,379

relation to kreatinin, 257,380

Kreatinic leucomains, 107

Kreatinin, 107, 257

estimation of, 259

in muscle-tissue, 379

in the urine, 257

isolation of, 259

origin of, 257

properties of, 258,379

relation to kreatin, 257

synthesis of, 258

tests for, 258

Kreatins, 107

Kühne's amphopeptone, 182

antipeptone, 201

Kynurenic acid, 277

Kynuric acid, 277

Kynurin, 277

Kyrins, 61, 186

L.

LABORATORY exercises, 462

Lactalbumin, 435

estimation of, 436

isolation of, 436

Lactases, 117

Lactic acid, 133, 284

in the muscle-tissue, 375

estimation of, 377

isolation of, 377

origin of, 376

in the stomach contents, 133

in the urine, 284 estimation of, 135

isolation of, 284

properties of, 377

significance of, 376

tests for, 133

Lactobiose, 70

Lactoglobulin, 435

estimation of, 436

isolation of, 436

Lactose, 70, 298, 438

estimation of, 438

in the milk, 438

in the urine, 298

isolation of, 299

Lævulose, 67

in the urine, 299

Laiose, 299

Lanolin, 78 
Laurinic acid, 437

Lecithalbumins, 77

Lecithanes, 78

Lecithins, 76

digestion of, 193

in birds' eggs, 453

in nerve-tissue, 393

in the urine, 314

isolation of, 453

Legal's test for acetone, 282

Leichenwax. See Adipocire.

Leo's method of estimating hydrochloric acid, 132

Leucin, 80 sugar. See Laiose.

in the urine, 285

isolation of, 205

tests for, 202

Leucocytes, 341 chemical composition of, 342

Leucomains, 107

kreatinic, 107

xanthinic, 102

Leukonuclein, 427

Lichenin, 72

Lieben's test for acetone, 283

Liebermann's albumin reaction, 38

Liebermann-Burckhard's test for cholesterin, 177

Lignin, 73

v. Limbeck's method of estimating the alkalinity of the blood, 326

Lipase in the gastric juice 136 in the pancreatic juice, 151 in the urine, 315

Lipases, 117

Lipochrin, 400

Lipochromes, 76, 452

Lipochromic pigments, 452

Lipolysis, 192

Lipuria, 314

Lithofellic acid, 167

Lithuric acid, 276

Liver, 420

albuminates of, 422

albumins of, 421

chemical composition of, 421

extractives of, 426

fats of, 425

ferments of, 424

function of, 420

glucose of, 425

gly cogen of, 424

nucleins of, 422

Living matter, chemical changes in, 18

forces at work in, 17

general composition of, 17

Löwy's method of estimating the alkalinity of the blood, 326

Ludwig-Salkowski method of estimating xanthin-bases, 253

Luteins, the, of bird eggs, 452

\author{
Lymph, 357 \\ amount of, 359 \\ analyses of, 361,362 \\ chemical composition of, 359 \\ general properties of, 358 \\ Lymphagogues, 358 \\ Lymph-glands, 427 \\ Lysin, 88 \\ Lysuric acid, 89

\section{M.}

Maltase in the pancreatic juice, 152 in the saliva, 124

Maltases, 117

Maltobiose, 70

Maltodextrin, 72

Maltose, 70

in the urine, 300

Mammary glands, 428

Mannides, synthesis of, in plants, 25

Mannite, 25

Mannitides. See Mannides.

Mannose, 64

Meconium, 223

Melanins, 312

in the skin, 415

in the urine, 312

Melanogen, 313

Melanoidins, 415

Mesoporphyrin, 355

Mesoxalic acid, 106

Metalbumin, 360

Methæmoglobin, 353 sulphide, 354

Methyl-glycocoll, 108

Methyl-guanidin, 108

Methyl-hydantoin, 108

in muscle-tissue, 380

Methyl-hydantoinic acid, 85

Methyl-uracil, 100

Mett's method of estimating pepsin, 140

Milk, 429

albumins of, 433

amount of, 431

analysis of, 432

caseinogen, 433

chemical composition of, 432

citric acid in, 439

coagulation of, 434

extractives of, 439

fats in, 437

ferments in, 440

general characteristics of, 429

lactalbumin in, 435

lactoglobulin in, 435

lactose in, 438

reaction of, 431

skimmed, 431, 433

specific gravity of, 431

Millon's reaction, 37

Mineral ash, estimation of, in the urine, 233

Molisch's test, 38 
Mono-amino-acids, 39, 80

Monosaccharides, 64

Mono-ureids, 104

Mörner test for tyrosin, 205

Mucin, biliary, 159 salivary, 124

Mucinogen, 124

Mucinoids, 49

Mucins, 48 vegetable, 73

Mucoids, 49 corneal, 396

Mucous tissue, 401

Murexid, 106 test, 250

Musc, 419

Muscle-albumins, significance of, 368

Muscle-pigments, 371

Muscle-plasma, 365

Muscle-serum, 366

Muscle-stroma, 365, 370

Muscle-tissue, 364 adenin in, 384 albumins of, 365 analyses of, 364 carnin in, 384 carnosin in, 385 fat in, 386 ferments of, 370 gases in, 385 glucose in, 375 glycogen in, 371 guanin in, 383 hypoxanthin in, 383 inosinic acid in, 385 inosit in, 378 involuntary, 386 kreatin in, 379 kreatinin in, 379 lactic acid in, 375 myogen in, 366 myosin in, 366, 367 nitrogenous extractives of, 379 nucleins of, 369 phospho-carnic acid in, 369 pigments of, 371 plasma of, 365 serum, 366 stroma of, 365,370 xanthin in, 382 xanthin-bases in, 381

Myelin, 390

Myelin-bodies, 390

Myo-albumose, 369

Myogen, isolation of, 366 properties of, 366

Myogen-fibrin, insoluble, 367 soluble, 367

Myoglobulin, 369

Myoproteid, 369

Myosin, 367 isolation of, 367 properties of, 367
Myosin-fibrin, 368

Myosinogen, 369

Myosinoses, 60

Myricin, 75

Myristinic acid, 178 in the bile, 178

N.

Neossin, 51

Nerve-tissue, 387

albumins in, 388

cerebrin in, 391

cholesterin in, 394

encephalin in, 393

extractives of, 394

general chemical composition of, 388

homocerebrin in, 392

jecorin in, 395

lecithins in, 393

myelins in, 390

neuridin in, 394

neurokeratin in, 389

protagon in, 390

Neuridin in nerve-tissue, 394

Neurin, 77

Neurokeratin, 389

Neutral salts, behavior of albumins to, 31 sulphur, 286

Nitrates in the urine, 236

Nitric acid test for albumin, 302 oxide hæmoglobin, 353

Nitrites in the saliva, 125 test for, 125

Nitrogen, estimation of, 245

Nitrogenous equilibrium, 239

Nucleases, 118

Nucleinic acids, 94

bases. See Purin bases. cleavage products of, 94

Nuclein platelets of the blood, 351

Nucleins, 56 digestion of, 187 isolation of, 422 of the liver, 422

Nucleo-albumins, 53 test for, in the urine, 304

Nucleo-glucoproteid of the mammary gland, 428

of the pancreas, 428

Nucleo-histon, 341, 427 isolation of, 341

properties of, 341

Nucleon, 370

Nucleoproteids, 55 of the muscle-tissue, 369

Nylander's test for sugar, 293

0.

OBERMAYER's test for indican, 265

Oleic acid, 75 
Olein, 75

Oliguria, 226

Oocyanin, 446

Oorhodein, 445

Orcin test, 300

Ornithin, 88

Ornithuric acid, 83, 89

Osazons, 65

Osons, 65

Ossein, 405

Ovalbumin, 448 isolation of, 448

Ovaries, 445

Ovomucoid, 449 isolation of, 449

Ovovitellin, 451 isolation of, 451 properties of, 452

Ovum. See Eggs.

Oxalic acid, 92, 106, 254 estimation of, 255 in the urine, 254 origin of, 254

Oxaluric acid, 106, 254 in the urine, 254 origin of, 254

Oxidations in animal life, 19

Oxy-acids, aromatic, 91 in the urine, 273 isolation of, 273 tests for, 273

$\beta$-oxybutyric acid, 91, 279 estimation of, 281 relation to acetone, 280 to $a$-crotonic acid, 280 to diacetic acid, 280

Oxydases, 118 tests for, 279

in the liver, 424

in the saliva, 124

Oxygenases, 118

Oxyhæmocyanin, 345

Oxyhæmoglobin, 347,350

estimation of, 351

isolation of, 350

properties of, 350

Oxy-hydroparacumaric acid, 91

Oxyphenyl-ethylamin, 86

Oxyneurin, 77

Oxyprolin, 81

Oxypyrrolidin carbonic acid, 81

\section{P.}

Palmitic acid, 75

Palmitin, 75

Pancreas, 426

Pancreatic juice, 144

amount of, 146

chemical composition of, 147

ferments of, 147

general properties of, 146

significance of, 144
Pancreatic juice, specific gravity of, 147

Paralbumin, 360 zymogens of, 147

Paracaseïn, 434

Paracresol, 85, 93

in the urine, 262

Paraglobulin, 331

Paralactic acid in the urine, 284

Paralbumin, 360 isolation of, 284

Paranucleins, 56

Para-oxy-benzoic acid, 93

Para-phenyl-acetic acid, 85, 93 in the urine, 216

Para-oxy-phenyl-glycolic acid, 93 ir the urine, 216

Para-oxy-phenyl-lactic acid, 93 in the urine, 216

Para-oxy-phenyl-propionic acid, 85, 93 in the urine, 216

Paraxanthin, 101

Pekelharing's method of isolating pepsin, 140

Penta-methylene-diamin, 89, 316

Pentoses, 68

in the urine, 300 tests for, 300

Pepsin, 137 estimation of, 140 isolation of, 140 properties of, 138

Pepsinogen, 141 estimation of, 141 test for, 141

Peptic digestion, products of, 182

Peptids, 41, 61

Peptomelanin, 201, 415

Peptones, 61

Pericardial fluid, analysis of, 361

Peritoneal effusions, analysis of, 362

Peroxydases, 119

Pettenkofer's test, 161

Phenaceturic acid, 83, 94, 272 isolation of, 272 origin of, 272

properties of, 272

Phenol, 85, 93, 215, 262

estimation of, in the urine, 26.3

in the intestinal contents, 215

in the urine, 262

isolation of, 221

tests for, 215

Phenyl-acetic acid, 93 in the intestinal contents, 216 isolation of, 223

Phenyl-alanin, 81

in the urine, 285

Phenyl-ethylamin, 86

Phenyl-glucosazons, 66

Phenyl-hydrazin-test for sugar, 294

Phenyl-propionic acid, 93 in the intestinal contents, 216 isolation of, 223 
Phlebin, 343

Phloretin, 24

Phloridzin, 24

Phloroglucin test for pentoses, 301

Phosphates, estimation of, in urine, 233

Phospho-carnic acid, 369

Phosphoglobulins, 53

Photo-methæmoglobin, 354

Phyllocyanic acid, 21

Phylloporphyrin, 355

Phylloxanthin, 21

Phymatorhusin, 313, 415

Pigments of the bile, 170

of the blood, 344

of the muscle-tissue, 371

of the skin, 415

of the urine, 307

respiratory, 345

Pine-apple test for butyric acid, 134

Piperazin, 443

Piria's test for tyrosin, 204

Placenta, 455

Plaques, 343

Plasma, albumose-, 329

blood, 323,329

muscle-, 329

oxalate-, 329

peptone-, 329

salt-, 329

Plasminic acid, 97

Plasteins, 192

Platner's bile, 160

Pleural effusions, analyses of, 362

Polarimetric test for glucose, 295

Polypeptids, Fischer's, 41, 61

Polysaccharides, 70

Polyuria, 226

Prolin, 81

Prosecretin, 146, 155

Protagon, 390 isolation of, 390 properties of, 390

Protamins, 51, 52 in the spermatozoa, 444

Proteases, 117

Proteids, 55 digestion of, 187

Proteinochromes. See Tryptophane.

Proteins, 27

Proteases, 117

Proteolysis, 181 ereptic, 189

gastric, 182 tryptic, 185

Proteoses, 60

Prothrombin, 336

Proto-albumose, 197

Protones, 62

Protophyllin, 24

Pseudoglobulin, 331

Pseudohamoglobin, 351

Pseudomucin, 44
Pseudonucleins, 55

Pseudopepsin, 136

Ptomains, 108

acyclic, 108

cyclic, 108

in the intestinal contents, 216

in the urine, 315

Ptyalin, 122

action of, 123

chemical nature of, 124

isolation of, 123

Ptyalose, 70

Purin, 100

bases, 100

derivatives of the nucleinic acids, 100

Pus, analysis of, 363

Putrefaction, analysis of products of, 221

Putrescin, 88

in the urine, 316

Pyogenin, 390

Pyosin, 390

Pyrimidin derivatives of the nucleinic acids, 98

Pyrocatechin, 93

in the urine, 262

Pyrocatechin-reaction of adrenal glands, 459

Pyrocholesteric acid, 166

R.

Reductases, 119

Rennin. See Chymosin.

Reproductive glands, 441

Resorption of albumins, 189

of carbohydrates, 181

of fats, 192

Reticulin, 402, 427

Retina, 398

analysis of, 399

chromophanes in, 399

rhodopsin in, 399

Reversible action of ferments, 115

Rhamnose, 68

Rhodophane, 399

Rhodopsin, 399

Rigor mortis, 376

Rosenbach's reaction, 266

S.

SACCHARINIC acid, 65, 269

Saccharo-biose, 69

Saccharo-lactonic acid, 65

Saccharose, 69

Salamandrin, 419

Salicin, 24

Saligenin, 24

Saliva, 120

amount of, 120

analysis of, 122 
Saliva, chemical composition of, 121 chorda-, 121

digestive importance of, 124

extractives in, 125

ferments in, 124

gases in, 125

general characteristics of, 120

mineral constituents of, 125

mucin in, 124

nitrites in, 125

paralytic, 121

ptyalin in, 122

secretion of, 120

sulphocyanides in, 125

sympathetic, 121

Salkowski's test for cholesterin, 177

Salkowski-Volhard method of estimating chlorides, 233

Salmin, 53

Salmon, 52

Salmonucleinic acid, 95

Saponification, 76

Sarcin (see also Hypoxanthin).

Sarcylic acid, 95

Scherer's test for inosit, 378

for leucin, 204

for tyrosin, 205

Schiff's test for uric acid, 251

Schmiedeberg's histenzyme, 117

Sclerotic, 396

Scombrin, 53

Scombron, 56

Scyllite, 379

Sebum, 418

Secretin, 146, 155

Semen, 442

Serin, 80

general properties of, 442

Serolin in the feces, 223

Serum of the blood, 334 composition of, 334

Serum-albumin, 332

forms of, 333

in the urine, 301

isolation of, 333

of the blood, 332

test for, 304

Serum-globulin, 330

in the urine, 301

isolation of, 332

properties of, 331

test for, 304

Siderosis, 422

Skatol, 86, 214

isolation of, 221

tests for, 214

Skatol-amino-acetic acid. See 'I'ryptophan.

Skatol-carbonic acid, 86, 216, 267

isolation of, 221

test for, 221

Skatosin, 215

Skatoxyl, 86
Skatoxyl sulphate in the urine, 267 test for, 267

Skeletins, 408

Skin, 414 eleïdin granules in, 414

glands, 419

keratin in, 414

Smegma, 419

Smith's test, 173

Soaps, 76

Solanidin, 25

Solanin, 25

Sols, 29

Sorbite, 65

Spermanucleinic acid, 93

Spermatic fluid, 443

Spermatin, 443

Spermatozoa, 442, 444

analysis of, 444

chemical composition of, 444 protamins in, 444

Spermin, 442, 443

phosphate of, 443

Spirographin, 52

Spleen, 427

arginin in the, 427

Spongin, 58

Starch, digestion of, 123 granulose, 71

Starches, 71

Steapsin, 151

Stearic acid, 75

Stearin, 75

Stercobilin in the feces, 222

relation to urobilin, 222

Stercorin in the feces, 223

Stoke's reagent, 346

Stools (see also Feces). acholic, 219

Sturin, 53

Succinic acid, 91

Sulphates, estimation of, in urine, 234

conjugate, 261

Sulphocyanides in the gastric juice, 144

in the saliva, 125

test for, 125

Sulphohæmoglobin, 353

Sulphur, neutral, of the urine, 286

Sulphur-test, 38

Supporting tissues, 401

Suprarenin, v. Fürth's, 460

Sweat, 416

analysis of, 418

chemical composition of, 418

gases of, 418

general characteristics of, 417

significance of, 416

Synaptase, 118

Synovial fluid, 363

Synovin, 363

Syntonin, 59, 182 
T.

Tartronic acid, 106

Taurin, 84,168

isolation of, 169

relation to cystin, 168

Taurocarbaminic acid, 84

Taurocholic acid, 83, 163

Teeth, 407 isolation of, 165

Testicles, 441

Tetra-methylene-diamin, 88, 316

Theobromin, 102

Theophyllin, 102

Thioalbumose, 200

Thiosulphates in the urine, 286

Thrombin, 335

Thrombokinase, 336

Thymin, 100

Thyminic acid, 96

Thymo-nucleinic acid, 95

Thymus gland, 427

Thyreoglobulin, 457

Thyreo-nucleo-proteid, 458

Thyroid gland, 456

Thyroiodine, 456

Tollens' orcin test, 300 phloroglucin test, 301

Töpfer's method of estimating hydrochloric acid, 131

test for hydrochloric acid, 130

Transudates. See Lymph.

Triticonnucleinic acid, 95

Trypsin, 149 action of, 149

isolation of, 150

test for, 150

Trypsinogen, 149

Tryptic digestion, 185 products of, 196

Tryptophan, 81, 86, 208 tests for, 209

Tuberculosamin, 53

Tunicin, 408

Tyrosin, 81, 203

in the urine, 285

isolation of, 205

tests for, 204

Tyrosin-hydantoinic acid, 86

U.

UFFKLMANN's test for lactic acid, 133

Umilinff's reaction, 439

Uracil, 99

Urea, estimation of, 243

formation of, 82

in the blood, 340

in the urine, 236 isolation of, 243

origin of, 236

properties of, 241

synthesis of, 243

tests for, 242
Urea-nitrate, 241

Urea-oxalate, 241

Ureases, 117

Ureids, 104

Uric acid, 103, 105, 246

estimation of, 251

in the urine, 246

isolation of, 251

origin of, 246

properties of, 249

Urine, 224 tests for, 250

acetone in, 282

acidity of, 229

albumins in, 301

allantoin in, 256

amino-acids in, 285

ammoniacal decomposition of, 225

amount of, 226

aromatic constituents of, 261 oxy-acids of, 273

bile-pigments in, 313

blood-pigments in, 310

carbohydrates of, 291

chemical composition of, 230

chlorides in, 230

cholesterin in, 314

color of, 225

compound glycocolls in, 270

conjugate glucuronates in, 268 sulphates in, 232,261

cysteïn in, 287

cystin in, 288

dextrin in, 300

diacetic acid in, 281

fats in, 314

fatty acids in, 278

ferments in, 315

gases in, 315

general characteristics of, 224

glucose in, 291

hæmatin in. 311

hamatoporphyrin, 311

hippuric acid in, 270

homogentisinic acid in, 274

indican in, 264

inorganic constituents of, 230

inosit in, 276

kreatin in, 257

kreatinin in, 257

kynurenic acid in, 277

lactic acid, 284

lactose in, 298

lævulose in, 299

lecithin in, 314

leucin in, 285

lithuric acid in, 276

mineral ash, 233

maltose in, 300

melanins, 312

neutral sulphur in, 286

nitrates in, 236

nitrogenous constituents of, 236 
Urine, odor of, 225

organic constituents of, 236 ornithuric acid in, 273

oxalic acid in, 254

oxaluric acid in, 254

$\beta$-oxybutyric acid in, 279

paralactic acid in, 284

pentoses in, 300

phenaceturic acid in, 272

phenols in, 262

phosphates in, 231

pigments in, 307

ptomains in, 315

reaction of, 227

skatol-carbonic acid in, 267

skatoxyl sulphate in, 267

specific gravity of, 227

sulphates in, 231

tyrosin in, 285

urea in, 236

uric acid in, 246

urobilin in, 307, 309

urocaninic acid in, 276

urochrome in, 307

uroerythrin in, 308

urohæmatin in, 265.

uroleucinic acid in, 276

uroroseïn in, 267

volatile fatty acids in, 278

xanthin-bases in, 252

Urobilin, isolation of, 309

Jaffé's, 309

MacMunn's, 307

tests for, 309

Urocaninic acid, 276

Urochloralic acid, 270

Urochrome, 307 isolation of, 308

Urocyanin, 265

Uroerythrin, 308 isolation of, 309

Uroferric acid, 286

Urofuscohæmatin, 312

Uroglaucin, 265

Urohæmatin, 264 tests for, 265

Uroleucinic acid in the urine, 276

Uroproteic acid, 286

Uroroseïn, test for, 267

Uroroseïnogen, 267

Urorubin, 265

Urorubrohæmatin, 312

Urrhodin, 265

Ursocholeic acid, 167

Uterine milk, 441

\section{V.}

Vitellins, 54

Vitellolutein, 453
Vitellorubin, 453

Vitelloses, 60

Vitreous body, 398

analysis of, 398

W.

WANG's method of estimating indican, 266

Weidel's test for xanthin, 383

Welcker's method of estimating the total amount of blood, 325

Weyl's test for kreatinin, 258

Whey, acid, 438 sweet, 430

Witch's milk, 441

v. Wittich's method of isolating pepsin, 140

Wood, 73

X.

Xanthin, 101

in muscle-tissue, 382

in urine, 252

isolation of, 381

properties of, 382

tests for, 382

Xanthin-bases, 100, 252

estimation of, 253

in muscle-tissue, 381

isolation of, 253

of the urine, 252

origin of, 252

Xanthinic leucomains, 102

Xanthophane, 400

Xanthoproteic reaction, 37

Xanthopsin, 399

Xylose, 68

\section{Y.}

YeAST-NUCLEINIC acid, 95

Yolk of bird's eggs, 449

albumins of, 451

analysis of, 450

fats of, 452

hæmatogen in, 451

lipochromes of, 452

ovovitellin in, 451

platelets, 28,450

white, 449

Z.

ZyMase, 112 。

Zymogens, 111

Zymolysin, 154 



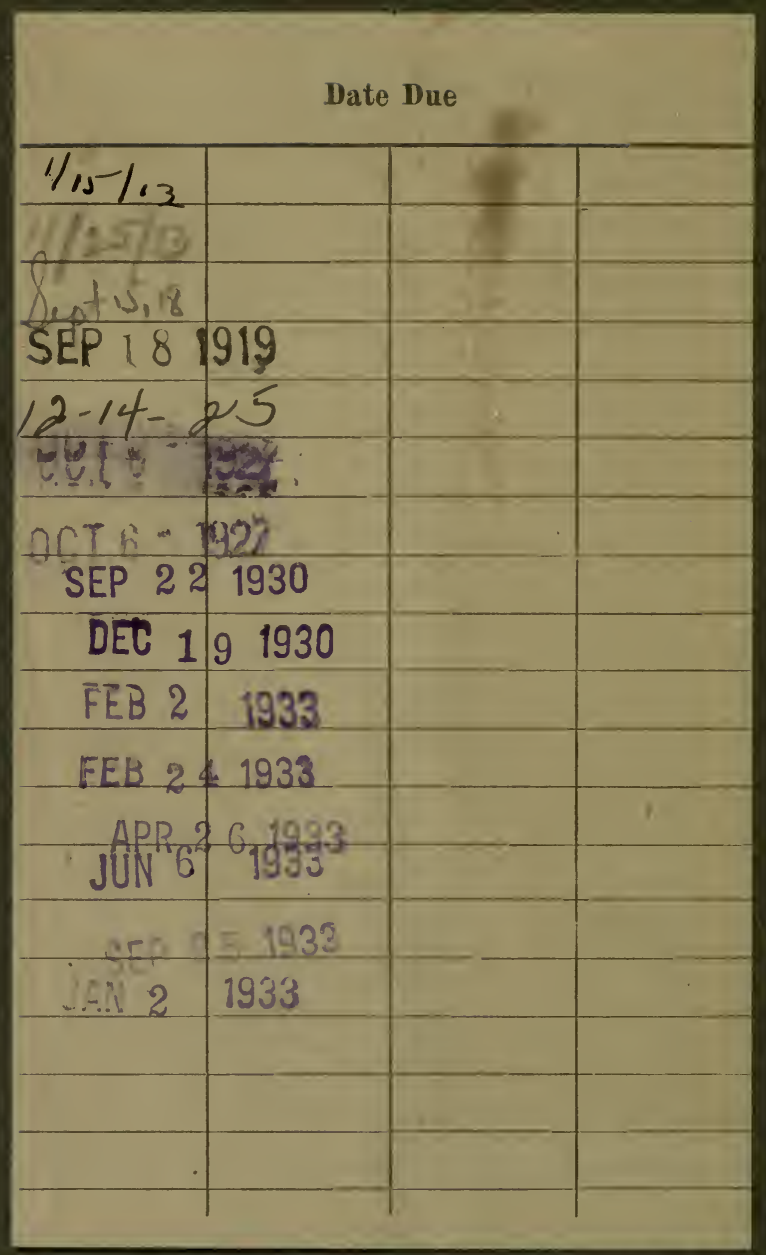




\section{3}

\section{LIBRARY RULES}

HOURS 11-12 A.M.

1. One book only will be issued on presentation of borrower's card.

2. Books may be retained for a period of four days. Renewal for four days more will be made upon request.

3. A fine of 5 cents per day will be charged on books not returned when due.

4. Borrowers will be charged for all injury to books.

LIBRARIAN'S HOURS

MONDAY AND FRIDAY, 9-12 A.M.

WEDNESDAY, 1 - 4 P.M. 
